(c) 2019

ARUN S. TADEPALLI

ALL RIGHTS RESERVED 


\title{
LIGHT ANTI-QUARK FLAVOR ASYMMETRY IN THE NUCLEON SEA AND THE NUCLEAR DEPENDENCE OF ANTI-QUARKS IN NUCLEI AT THE SEAQUEST EXPERIMENT
}

\author{
By \\ ARUN S. TADEPALLI \\ A dissertation submitted to the \\ School of Graduate Studies \\ Rutgers, The State University of New Jersey \\ In partial fulfillment of the requirements \\ For the degree of \\ Doctor of Philosophy \\ Graduate Program in Physics and Astronomy \\ Written under the direction of \\ Ronald A. Gilman \\ And approved by \\ New Brunswick, New Jersey
}

Oct, 2019 
ABSTRACT OF THE DISSERTATION

\title{
LIGHT ANTI-QUARK FLAVOR ASYMMETRY IN THE NUCLEON SEA AND THE NUCLEAR DEPENDENCE OF ANTI-QUARKS IN NUCLEI AT THE SEAQUEST EXPERIMENT
}

\author{
by ARUN S. TADEPALLI
}

\section{Dissertation Director: \\ Ronald A. Gilman}

Fermilab E906/SeaQuest is an experiment aimed at studying the anti-quark distributions of nucleons and nuclei. The experiment uses a $120 \mathrm{GeV} / \mathrm{c}$ proton beam extracted from the Main Injector at Fermilab to collide with various solid and cryogenic targets to study a variety of physics topics. The experiment takes advantage of the Drell-Yan process in order to probe specifically the high- $x$ anti-quark distributions of the target nucleus. The acceptance of the spectrometer is tuned to explore the unprecedentedly high Bjorken- $x$ region, thereby extending our knowledge of the anti-quark sea structure of nucleons and nuclei.

A new Intensity-Extrapolation method was developed to counter the rate dependence challenge at SeaQuest. Using this method, the ratio of cross sections of liquid deuterium and liquid hydrogen, $\sigma_{p d}(x) / 2 \sigma_{p p}(x)$, was measured in the range $0.1 \leq x_{T} \leq 0.45$. The results have been found to be consistently above 1 for all values of the measured range of $x_{T}$. Also, a first look at $\bar{d}(x) / \bar{u}(x)$ is presented. The trend of the data points indicate that the ratio is consistently above 1 for the measured range of $x_{T}$. These results (when combined with more data) will provide a key input in constraining many theoretical models that attempt to explain the origin of the nucleon sea.

Using the same method, the nuclear dependence of the per-nucleon cross section ratio, $R_{p A}$, of carbon, iron and tungsten versus deuterium studied as a function of $x_{T}, p_{T}$, and $x_{F}$ are reported. The results for $x_{T}$ are consistent with E772 indicating little nuclear de- 
pendence. A striking $A$ (atomic mass) dependence in which there is a depletion of low- $p_{T}$ dimuons and an excess of high- $p_{T}$ dimuons relative to deuterium is reported. Also, an $A$ dependence for the drop in $R_{p A}\left(x_{F}\right)$ for increasing $x_{F}$ is presented. An analysis with and without the cut $x_{T}>0.16$ (which removes the effects of the shadowing) gives consistent results indicating that the effects of shadowing are small. These results (when combined with more data) will address important questions on the modification of anti-quark distributions in nuclei and in modeling energy loss for partons traversing a cold nuclear medium. 
"Finally, may I add that the main purpose of my teaching has not been to prepare you for some examination-it was not even to prepare you to serve in the industry or the military. I wanted most to give you some appreciation of the wonderful world and the physicist's way of looking at it, which, I believe is a major part of the true culture of modern times."

- Richard P. Feynman, Feynman's Epilogue, The Feynman Lectures on Physics vol III 


\section{DEDICATION}

Dedicated to Nature... 


\section{ACKNOWLEDGEMENTS}

First and foremost, I would like to thank my parents, Sharada and Ramesh Tadepalli, for all that they have done for me as I made my way through graduate school. Together, they believed in me when the times were tough and through the ups and downs of this amazing journey. Their unconditional love and support have continuously fueled me and have helped me to accomplish this goal.

I would like to thank my lovely wife, Sumedha, for being there for anything and everything. Her unwavering faith in me, timely advice, and ever supportive nature helped make the end goal of this endeavor believable, exciting, and achievable. Without her cheerleading and push, I would not have finished writing this document.

I would like to express my sincere gratitude to my advisor Prof. Ron Gilman for his amazing mentoring, continued support, immense knowledge, and for patiently guiding me through this journey. He taught me to not fear making mistakes when exploring new ideas, but to learn from the challenges presented by these topics. Whether it was being a career panel member at the Frontiers and Careers conference or whether it was guiding me as his course teaching assistant, he remained my pillar of support and encouragement, and I will forever appreciate that.

I would like to express my sincere gratitude to Dr. Roy Holt for the amazing physics discussions, and invaluable life lessons that he shared. He has truly been an inspiring mentor and physicist. From his jovial attitude towards life (that magically leads to brilliant physics ideas), to his intuition into crisp physics results, I am grateful to have been guided in this journey by him.

I would like to express my sincere thanks to Dr. Paul Reimer. His love and enthusiasm for the subject are so contagious. I thank him for sparking my interest and for guiding me in my pursuit to obtain the $\bar{d}(x) / \bar{u}(x)$ result. I simply loved our one-on-one paper discussions and brainstorming sessions. His patience and willingness to help are qualities I admire and 
hope to acquire in my own practice.

I would like to express my sincere gratitude to Dr. Don Geesaman for being the best analysis partner that any student could ever have. I am so lucky and proud to have worked with him on all the physics topics. I simply loved our physics discussions and one-on-one live analysis sessions. We had so many physics discussions and I cherish and value each one. His fearlessness to dive deep into a topic to uncover a pearl of wisdom, is inspiring. I thank him for taking me along on this invaluable adventure countless times. Both the spokespersons have given their heart and soul to SeaQuest and I will forever appreciate that.

I would like to express my sincere gratitude to Prof. Jen-Chieh Peng for adopting me on as a UIUC member and for allowing me to attend UIUC weekly meetings and paper discussions. These meetings were a great source of new physics ideas and helped coordinate my analysis as well. I thank him for patiently answering my many questions regarding any physics topic.

My special thanks go to Dr. Kenichi Nakano for helping me with the many software issues encountered during analysis. Our many "short meetings" to discuss the progress of $\bar{d}(x) / \bar{u}(x)$ analysis were very helpful. His attention to detail, unwavering focus and useful tips helped accelerate my analysis.

I would like to express my sincere gratitude to Dr. Dave Christian for teaching me about drift chambers and debugging other hardware equipment. His magical insight into debugging deep technical problems is a super power that surprised me each time it was demonstrated.

I would like to thank Dr. Chuck Brown for his guidance and help. He was always ready for a stimulating physics discussion and never let go of a teachable moment. Working with him was competitive, fun and a great learning experience.

My sincere thanks goes to Dr. Kei Nagai for the analysis help he provided. He made my life much easier by helping write, understand, and organize an efficient analysis code. 
I would like to thank Dr. Po-Ju Lin for all the "fun" times at Lab 6 working on repairing and installing the new St 1 drift chamber. Working with 20 micron sense wire was itself difficult but his company made it bearable and fun. I would like to thank Dr. Joshua Rubin for all the inspiring and intellectually stimulating physics discussions. I truly enjoyed our Daddios' diner venting sessions and napkin discussions. I would like to thank Dr. Michelle Medeiros, Dr. Brian Tice, Dr. Kun Liu, Dr. Yen-chu Chen, Dr. Evan McClellan, Dr. Lamiaa El Fassi, Dr. Markus Diefenthaler and Dr. Grass Wang for the interesting discussions and potluck parties. I was lucky to have worked with a group of incredibly talented set of graduate students Florian Sanftl, Shou Miyasaka, Bryan Ramson, Shivangi Prasad, Jason Dove, Mae Hwee Teo, Bryan Kerns, Bryan Dannowitz, Bardia Nadim, Catherine Ayuso and Evan McClellan who helped me in one way or another. I would also like to thank my fellow graduate students: Aniket, Savvas, Navaneeth, Siva, Rijeesh and Prabhjot for all the good times.

My sincere thanks also go to my family members Ajay, Shailaja, Aria, Ashwin, Aditya, Om, Lakshmi and Gnaneshwar Chidella for making the journey memorable. Last but not least I would like to thank my in-laws Renuka and Bhanu Evani for their support and encouragement. 


\section{TABLE OF CONTENTS}

Abstract ........................... ii

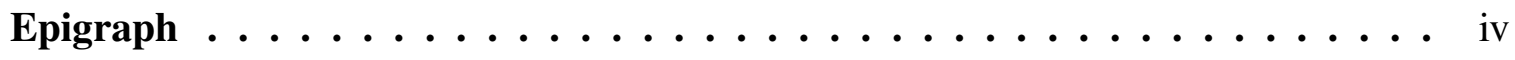

Dedication $\ldots \ldots \ldots \ldots \ldots \ldots \ldots \ldots \ldots \ldots \ldots$

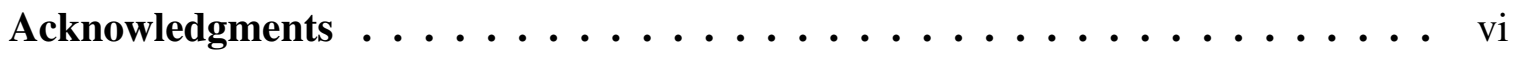

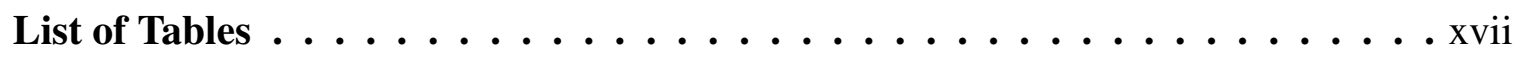

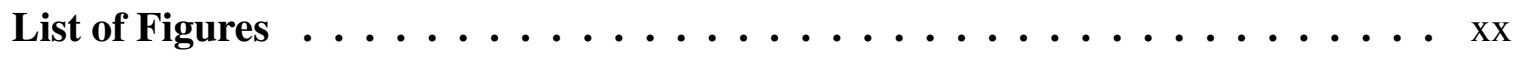

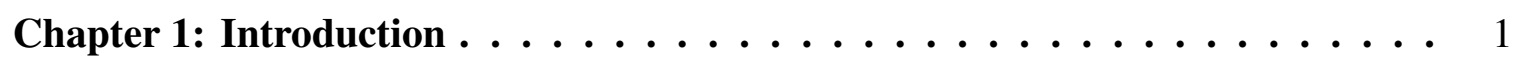

Chapter 2: What's the Matter? . . . . . . . . . . . . . . 3

$2.1 \mathrm{~B} u \mathrm{il} d \operatorname{ing} \mu \mathrm{p} t \mathrm{o} \tau \mathrm{h} e b \mathrm{lo} c \mathrm{k} s \ldots \ldots . \ldots \ldots$

2.1.1 The "Indivisible" Atom . . . . . . . . . . . . . 3

2.1.2 The Atomic Nucleus ................. 4

2.1.3 The Neutron .................. 5

2.1.4 The Elusive Neutrino . . . . . . . . . . . . . . 5

2.1.5 The Positron ................. 6

2.1.6 The Yukawa " $\mu$ meson" (Pion) ............. . 7 
2.1.7 The Birth of Quarks . . . . . . . . . . . . . . 7

2.1.8 Current Fundamental Building blocks _. . . . . . . . . . . 9

2.2 The Standard Model of Particle Physics _ . . . . . . . . . . . . 10

2.2.1 Quantum Chromodynamics . . . . . . . . . . . . . . 11

2.3 Probing Nucleon and Nuclear Structure $\ldots \ldots \ldots$

2.4 Elastic Scattering . . . . . . . . . . . . . . . . . . . . . . . . . . . 14

2.4.1 Nucleon Form Factors and Structure Functions _ . . . . . . . . 14

2.5 Deep Inelastic Scattering $\ldots \ldots \ldots \ldots \ldots$

2.6 Merging Quarks and Partons: The Quark-Parton Model . . . . . . . . . . 19

2.6.1 Structure Functions in the Quark-Parton Model . . . . . . . . 20

2.7 Accessing the Anti-Quark Distributions: The Drell-Yan Process . . . . . 24

2.7.1 Kinematics of the Drell-Yan Process . . . . . . . . . . . . 25

2.7.2 Leading Order Cross section of the Drell-Yan Process . . . . . . . 27

2.7.3 QCD Improved Drell-Yan Process … . . . . . . . . . . 28

2.8 Light Anti-Quark Flavor Asymmetry in the Nucleon Sea $\ldots \ldots \ldots$

2.8.1 The NMC Experiment $\ldots \ldots \ldots \ldots \ldots \ldots$

2.8.2 The NA51 Experiment $\ldots \ldots \ldots \ldots \ldots$

2.8.3 The E866/NuSea Experiment . . . . . . . . . . . . . . 35

2.8.4 Deducing $\bar{d}(x) / \bar{u}(x)$ from $\sigma_{p d}(x) / \sigma_{p p}(x) \ldots \ldots \ldots \ldots$

2.9 Origin of the Nucleon Sea $\ldots \ldots \ldots \ldots \ldots$

2.10 Role of SeaQuest - I . . . . . . . . . . . . . . . . . . . . . . . 44

2.11 Structure Functions of Nuclei $\ldots \ldots \ldots$. . . . . . . . . . 45

2.12 Nuclear $x$-landscape . . . . . . . . . . . . . . . . . . . . . . 48 
2.13 Dependence on Nuclear Properties . . . . . . . . . . . . . . . . . . . 49

2.13.1 Nuclear Dependence of $\mathrm{R}=\sigma_{L} / \sigma_{T} \ldots \ldots \ldots$. . . . . . 50

2.13.2 Dependence on Nuclear Mass $A \ldots \ldots$. . . . . . . . . . 50

2.13.3 Dependence on Average Nuclear Density $\rho \ldots . . . . . . .51$

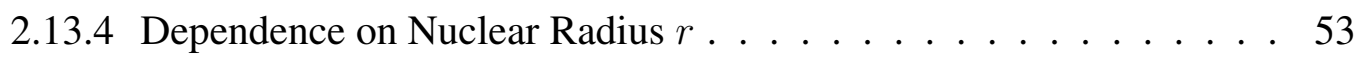

2.14 What happens if $x>1$. . . . . . . . . . . . 53

2.15 Nuclear Dependence of the Anti-Quark Sea . . . . . . . . . . . . . 55

2.15.1 Fermilab E772 experiment . . . . . . . . . . . . 55

2.15.2 The Fermilab NuSea/E866 Experiment . . . . . . . . . . . . . . . . 57

2.16 Role of SeaQuest - II . . . . . . . . . . . . . . . . . 58

Chapter 3: The SeaQuest Experiment . . . . . . . . . . . . . . . 59

3.1 Timeline of SeaQuest . . . . . . . . . . . . . . . . 61

3.1.1 Data Set Conditions . . . . . . . . . . . . . . . 62

3.1.2 Live Protons on each Target . . . . . . . . . . . . . . . . 64

3.2 The Proton Beam . . . . . . . . . . . . . . . . . . . 64

3.2.1 Micro-structure of the Beam . . . . . . . . . . . . . 67

3.3 Monitoring the Proton Beam Intensity . . . . . . . . . . . . . . 69

3.3.1 Beam Intensity Monitor . . . . . . . . . . . . . . . . . 69

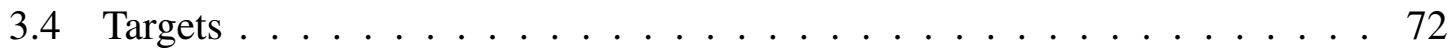

3.4 .1 Cryogenic Targets . . . . . . . . . . . . . . 72

3.4 .2 Solid targets ....................... 73

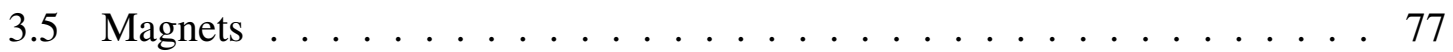


3.6 Hodoscopes . . . . . . . . . . . . . . . . . . . . . . . . 80

3.7 Drift Chambers . . . . . . . . . . . . . . . . 86

3.7.1 Selection of Gas for the Drift Chamber . . . . . . . . . . . . 88

3.7 .2 ASDQ cards . . . . . . . . . . . . . . . . . . 98

3.7.3 Level Shifter Boards . . . . . . . . . . . . . . . . . . . . . . . 99

3.7.4 TDC - Time to Digital Converter . . . . . . . . . . . 100

3.7 .5 RT curves . . . . . . . . . . . . . . . . . . . . 101

3.7 .6 Gas gain measurements . . . . . . . . . . . . . . . . 103

3.7.7 Monitoring Drift chamber efficiencies . . . . . . . . . . . 103

3.8 Proportional tubes $\ldots \ldots \ldots \ldots \ldots \ldots \ldots$

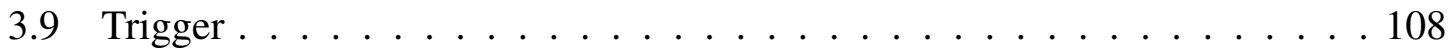

3.9 .1 Overall structure $\ldots \ldots \ldots \ldots \ldots$

3.9 .2 Trigger Firmware . . . . . . . . . . . . . . . . . . . . 109

3.9.3 Types of Trigger $\ldots \ldots \ldots \ldots \ldots \ldots \ldots$

3.10 Data Acquisition - DAQ . . . . . . . . . . . . . . . . . 114

3.10 .1 MainDAQ . . . . . . . . . . . . . . . . . . . . . 114

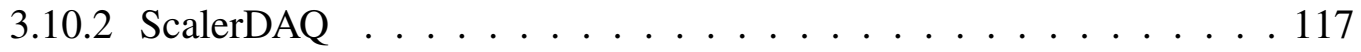

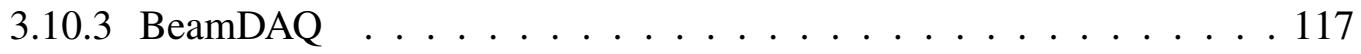

3.10.4 Slow Control Readout . . . . . . . . . . . . . . . . . . . . 118

3.11 Decoder . . . . . . . . . . . . . . . . . . 120

3.12 Online Monitoring . . . . . . . . . . . . . . . . . . 120

Chapter 4: Data Analysis . . . . . . . . . . . . . . . . . . 122 
4.1 Data sets . . . . . . . . . . . . . . . . . . . . . 122

4.2 Dimuon Reconstruction . . . . . . . . . . . . . . . . . . . . . . . 123

4.2.1 Pre-tracking analysis $\ldots \ldots \ldots \ldots . \ldots \ldots \ldots$

4.2.2 Single Track Reconstruction . . . . . . . . . . . . . . . . . . 129

4.2.3 Constructing Global Tracks . . . . . . . . . . . . . . . . 131

4.2.4 Single Track Vertex Reconstruction _ . . . . . . . . . . . . 134

4.2.5 Dimuon Vertex Reconstruction . . . . . . . . . . . . . . . . . 138

4.3 Monte Carlo Simulations . . . . . . . . . . . . . . . . . . . . . . . . . 140

4.3 .1 Generators . . . . . . . . . . . . . . . . . . . . . . . 140

4.3.2 "Clean" and "Messy" Monte Carlo simulations . . . . . . . . . . 142

4.4 Selections and Data Quality cuts . . . . . . . . . . . . . . . 148

4.4.1 Spill Level cuts . . . . . . . . . . . . . . . . . . . . . . . 148

4.4.2 Some R005 $\rightarrow$ R008 upgrades . . . . . . . . . . 150

4.4 .3 Event Level cuts _ . . . . . . . . . . . . . . . . . . . . 152

4.4.4 Track Level cuts . . . . . . . . . . . . . . . . . . . . . . . 153

4.4.5 Dimuon Level cuts . . . . . . . . . . . . . . . . . . . . . 155

4.4.6 Cuts designed to reduce random background . . . . . . . . . 157

4.4.7 Invariant Mass spectrum $\ldots \ldots \ldots \ldots \ldots$

4.4 .8 Combining data sets . . . . . . . . . . . . . . . . . . 159

4.5 Raw Dimuon Yields tables for different $x_{T}$ bins $\ldots \ldots \ldots$. . . . . . 164

4.6 Contamination and Density corrections: . . . . . . . . . . . . 167

4.7 Cross Section Ratios . . . . . . . . . . . . . . . . . . . . . 170

4.7.1 Statistical Errors for Individual CSR points _ . . . . . . . . 171 
4.8 The Intensity-Extrapolation method $\ldots \ldots \ldots \ldots \ldots$

4.8.1 kTracker Efficiency Corrections _ . . . . . . . . . . . . 174

4.8.2 Logic behind Fit function . . . . . . . . . . . . . . . . 175

Chapter 5: Results and Discussion . . . . . . . . . . . . . . . 178

5.1 Cross Section Ratios: $\sigma_{p d}(x) / 2 \sigma_{p p}(x) \ldots \ldots \ldots \ldots \ldots \ldots$

5.1.1 Systematic Uncertainties for $\sigma_{p d}(x) / 2 \sigma_{p p}(x) \ldots \ldots \ldots \ldots 180$

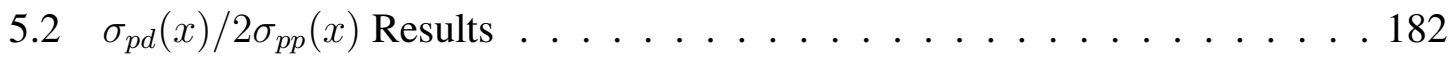

$5.2 .1 \sigma_{p d}(x) / 2 \sigma_{p p}(x)$ Comparison with E866 $\ldots \ldots \ldots \ldots \ldots$

5.2.2 Comparison with Statistical Parton model _ . . . . . . . . . 186

5.2.3 Tests for Stability of Intercepts _ . . . . . . . . . . . . 187

5.3 Extracting $\bar{d}(x) / \bar{u}(x)$ from $\sigma_{p d}(x) / 2 \sigma_{p p}(x) \ldots \ldots \ldots \ldots$

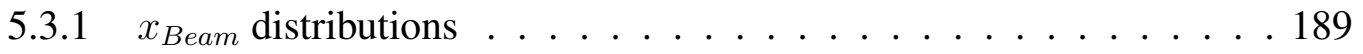

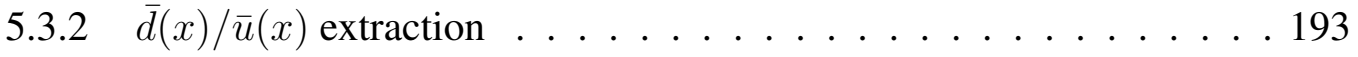

5.3.3 Statistical Uncertainties . . . . . . . . . . . . . . . . . . 196

5.3 .4 Systematic Uncertainties _ . . . . . . . . . . . . . . . 196

$5.4 \bar{d}(x) / \bar{u}(x)$ Results . . . . . . . . . . . . . . . . . . . . . . . . . 197

5.4.1 Comparison of $\bar{d}(x) / \bar{u}(x)$ with E866 . . . . . . . . . . . 198

5.4.2 Comparison of $\bar{d}(x) / \bar{u}(x)$ with Theoretical Models . . . . . . . 200

5.4 .3 Future Experiments . . . . . . . . . . . . . . . . . . . . 201

5.5 Nuclear Dependence: $R_{p A}=2 \sigma_{p A} / A \sigma_{p d} \ldots \ldots \ldots \ldots \ldots 2$

$5.5 .1 x_{T}$ dependence: . . . . . . . . . . . . . . 202

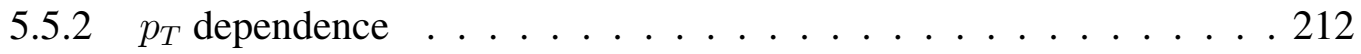


$5.5 .3 \quad x_{F}$ and $x_{B}$ dependence $\ldots \ldots \ldots \ldots \ldots \ldots \ldots$

5.5 .4 mass dependence . . . . . . . . . . . . . . . 216

Chapter 6: What's next? . . . . . . . . . . . . . . . . . 217

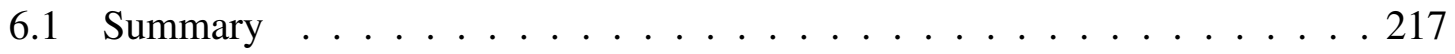

6.1.1 Light Anti-Quark Flavor Asymmetry in the Nucleon Sea $\ldots . .217$

6.1.2 Nuclear Dependence of Anti-Quarks . . . . . . . . . . . . . 218

6.2 Future Prospects . . . . . . . . . . . . . . . . . . . . . 219

6.2.1 Inclusion of Run IV, V and VI data . . . . . . . . . . . . . . 219

6.2.2 Improving Systematic Uncertainties . . . . . . . . . . . . 219

6.2.3 Calculating $\bar{d}(x)-\bar{u}(x) \ldots \ldots \ldots \ldots$

6.2.4 Nuclear dependence for $\mathrm{Fe} / \mathrm{C}$ and $\mathrm{W} / \mathrm{C} \ldots \ldots \ldots \ldots$

6.2.5 Intensity-Extrapolation method to study $J / \Psi$ physics . . . . . . . 220

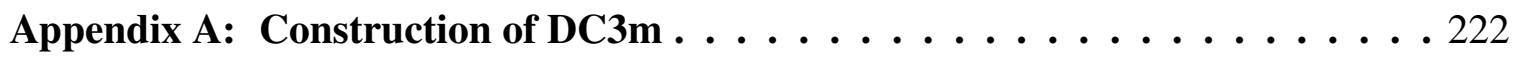

A.0.1 Stretching wires . . . . . . . . . . . . . . 223

A.0.2 Tension measurements . . . . . . . . . . . . . . . . 224

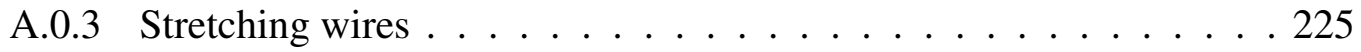

Appendix B: Dimuon Yields for Liquid and Solid targets . . . . . . . . . . 227

B.1 Table of live protons for total and individual data set . . . . . . . . 227

Appendix C: Intercepts for Nuclear dependence studies . . . . . . . . . . 230

C.1 $p_{T}$ dependence fits . . . . . . . . . . . . . 230

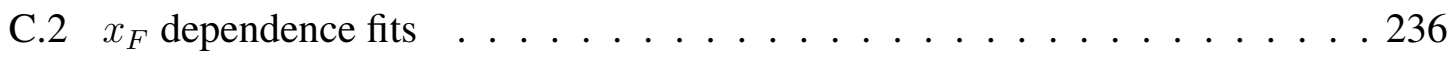


Appendix D: Search for Dark Photons at SeaQuest ․ . . . . . . . . . . 242

D.1 Why does the Dark Matter? . . . . . . . . . . . . . . . . . 242

D.2 Shedding Light on Dark Matter . . . . . . . . . . . . . . . . 243

D.2.1 Dynamics of Galaxies in Coma Clusters . . . . . . . . . . . 243

D.2.2 Rotational curves of galaxies . . . . . . . . . . . . . . . 244

D.2.3 Gravitational Lensing . . . . . . . . . . . . . . . . . . 244

D.2.4 Bullet Clusters . . . . . . . . . . . . . . . . . . . . . 245

D.2.5 Surveys of Cosmic Microwave Background (CMB) spectrum . . . 246

D.3 The Standard Model of Dark physics . . . . . . . . . . . . . . 249

D.3.1 What can Dark Matter be? . . . . . . . . . . . . . . . 249

D.3.2 Standard Model and Dark Sector coupling . . . . . . . . . . 250

D.4 SeaQuest Dark Photon Search Strategy . . . . . . . . . . . . . 251

D.5 Role of SeaQuest -III . . . . . . . . . . . . . . . . . . 253

D.5.1 Trigger Modification for Dark Photon Acceptance . . . . . . . . 254

D.5.2 Finishing up the Search for Dark Photons . . . . . . . . . 255

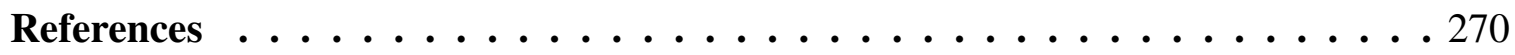




\section{LIST OF TABLES}

2.1 Definition of some kinematic quantities used in DIS . . . . . . . . . . . . 19

2.2 Summary of various experiments, beams used and their references. . . . . . 45

3.1 Table of data set conditions . . . . . . . . . . . . . . 64

3.2 Table of target position, target name, Raw POT and Live POT for full data set. . . . . . . . . . . . . . . . . 64

3.3 Characteristics of targets at SeaQuest. The "Spills/Cycle" for empty flask was changed from 1 to 2 in the latter part of the experiment [120]. . . . . . 74

3.4 Specifications of different hodoscope planes. The designation (L) and (R) refer to beam left or right and (T) and (B) refers to Top and Bottom. Z position is measured from the front face of FMAG. Values in the table are taken from $[125] . \ldots \ldots \ldots$. . . . . . . . . . . . . . 82

3.5 Configurations of Drift chambers used in different Runs [125]. . . . . . . . 89

3.6 Characteristics of SeaQuest drift chambers shown in Fig 3.27 . . . . . . . 92

3.7 Parameters of all chambers. Those of primed planes are almost the same as of unprimed planes. For the $x$ measuring planes, $z$ position is the distance measured from the front face of FMAG, while for $u$ and $v$ it denotes the offset relative to the $x$ plane.. Table taken from [120]. . . . . . . . . . . 93

3.8 Parameters of all proportional tubes. . . . . . . . . . . . 106

3.9 Roadsets used in SeaQuest and their descriptions. . . . . . . . . . . . . . 111

3.10 Trigger settings used in the SeaQuest experiment [120]. . . . . . . . . 113 
4.1 Table of "good" run and spill ranges. The definition of good is discussed here 4.5 . . . . . . . . . . . . . . . . . . . . . . . . . 123

4.2 Internal occupancy cuts for kTracker. $\ldots \ldots \ldots \ldots \ldots \ldots$

4.3 Residual windows for drift chambers . . . . . . . . . . . . . . . . 128

4.4 Energy loss parameterization constants for SeaQuest. . . . . . . . . . 136

4.5 Requirements for "good spill" eligibility. The definition of each quantity is given in the caption of the plots in the following pages. . . . . . . . . 149

4.6 Dimuon yields per intensity bin for $0.1<x_{T}<0.13 \ldots \ldots \ldots$. . . . . 164

4.7 Dimuon yields per intensity bin for $0.13<x_{T}<0.16 \ldots \ldots \ldots$

4.8 Dimuon yields per intensity bin for $0.16<x_{T}<0.195 \ldots \ldots \ldots \ldots$

4.9 Dimuon yields per intensity bin for $0.195<x_{T}<0.24 \ldots \ldots \ldots \ldots$

4.10 Dimuon yields per intensity bin for $0.24<x_{T}<0.29 . \ldots \ldots$. . . . 166

4.11 Dimuon yields per intensity bin for $0.29<x_{T}<0.35 \ldots \ldots \ldots$

4.12 Dimuon yields per intensity bin for $0.35<x_{T}<0.45 \ldots \ldots$. . . . 167

4.13 Weighted average quantities (weighted by live protons) for the entire data set.169

5.1 Table of cross section ratio values, statistical and systematic uncertainties. Total systematic uncertainty come from the choice of the fitting curve and the fluctuating QIE pedestal. . . . . . . . . . . . . . . 182

5.2 Other negligible contributions to systematic uncertainties. . . . . . . . 182

5.3 Table of $\bar{d}(x) / \bar{u}(x)$ values along with statistical and systematic uncertainties. CT10NLO PDF set was used in this extraction. The systematic uncertainties coming from the cross section ratio are propagated. Additional systematics from the value of PDF above 0.45 and below 0.1 not considered. . . . . . . . . . . . . . . . . . . 197

5.4 $R_{p A}$ values, statistical and systematic uncertainties for carbon. $F=p 0+$ $p 1 * I+p 2 * I^{2}$ (common pol2 fit) was used to estimate the systematic

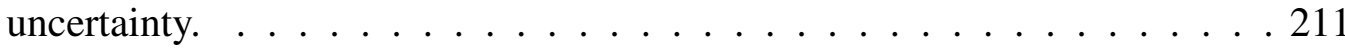


$5.5 R_{p A}$ values, statistical and systematic uncertainties for iron. . . . . . . . 211

5.6 $R_{p A}$ values, statistical and systematic uncertainties for tungsten. $\ldots \ldots 211$

B.1 Table of target position, target name, Raw POT and Live POT for full data set. . . . . . . . . . . . . . . . . . . . . 227

B.2 Table of target position, target name, Raw POT and Live POT for Roadset 57.228

B.3 Table of target position, target name, Raw POT and Live POT for Roadset 59.228

B.4 Table of target position, target name, Raw POT and Live POT for Roadset 62.228

B.5 Table of target position, target name, Raw POT and Live POT for Roadset 67.229

B.6 Table of target position, target name, Raw POT and Live POT for Roadset 70.229 


\section{LIST OF FIGURES}

2.1 Rutherford's gold foil alpha scattering experiment . . . . . . . . . . . 4

2.2 Cartoon of a photographic emulsion plate used in muon and pion detection cosmic ray experiments . . . . . . . . . . . . . 6

2.3 Examples of a Meson Octet (a), Baryon Octet (b) and Baryon decuplet (c)

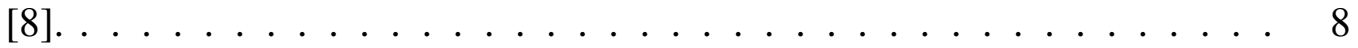

2.4 List of elementary particles $[22] \ldots \ldots \ldots$. . . . . . . . . . 10

2.5 Combination of red, blue and green making the overlap region "colorless" . 11

2.6 Behavior of QED and QCD coupling constants as a function of momentum transfer $\left(Q^{2}\right) \ldots \ldots \ldots \ldots \ldots \ldots$

2.7 Depiction of a typical fixed target scattering experiment . . . . . . . 13

2.8 Elastic scattering experiments by Hofstadter showing deviation between experimental cross section and Mott cross section [29]. . . . . . . . . . 15

2.9 Feynman diagram of deep inelastic scattering . . . . . . . . . . . . 17

2.10 Deviation between experimental cross section and elastic cross section observed in the case of deep inelastic scattering experiments conducted at SLAC [34]

2.11 Bands show Next-to-Next-to Leading Order Neural Network Parton Distribution Functions obtained in global analysis at scales $\mu^{2}=10 \mathrm{GeV}^{2}$ (left) and $\mu^{2}=10000 \mathrm{GeV}^{2}$ (right) [8]. One can see that as $Q^{2}$ (resolution) increases, the valence distributions decrease slightly and the gluon and sea quark distributions rise up rapidly revealing more structure at low- $x$. . . . 23

2.12 Cross section as a function of the invariant mass taken from [41] . . . . . . 24 
2.13 Collins-Soper frame is the center of mass frame of the dileptons produced in hadron-hadron collisions. The $\mathrm{Z}$ axis points along the beam direction. $h 1$ and $h 2$ are the colliding hadrons, $l$ and $l^{\prime}$ are the dileptons, $\theta$ is the polar angle and $\phi$ is the azimuthal angle. Figure taken from [43] . . . . . . . . . 26

2.14 Depiction of the Drell-Yan process [42] . . . . . . . . . . . . 27

2.15 Higher order corrections to the leading order Drell-Yan process that include additional gluon diagrams. . . . . . . . . . . . . . . . . . . . . 29

2.16 a) Feynman diagram of Deep Inelastic scattering process. b) Feynman diagram of the leading order Drell-Yan process c) Proton $F_{2}$ structure function data from various experiments from BCDMS, SLAC, NMC, HERMES at fixed $x$ with the CJ15 fits scaled by a factor $2^{i}$ [44] d) Proton induced DrellYan production from NA3 (triangles) at $400 \mathrm{GeV} / \mathrm{c}$ [45], E605 at $800 \mathrm{GeV} / \mathrm{c}$ [46], and E772 unpublished data [47]. The lines use NLO calculations for $\mathrm{p}+\mathrm{d}$ collisions at $800 \mathrm{GeV} / \mathrm{c}$ using CTEQ4m structure functions [48]. . . . 30

2.17 Feynman diagram of gluon splitting . . . . . . . . . . . 31

2.18 Plot to the right shows $F_{2}^{p}-F_{2}^{n}$ (scale to the right) and $\int_{x}^{1}\left(F_{2}^{p}-F_{2}^{n}\right) d x / x$ (scaled to the left). The circles (triangles) and open circles(open triangles) are the reevaluated (first published) values taken from [50] ([51]). The prediction of $1 / 3$ for a flavor symmetric sea is also shown as the dotted line on top left labeled QPM. . . . . . . . . . . . . . . . . . . . 33

2.19 Results of the NA51 experiment for $\bar{d} / \bar{u}$ at $\langle x=0.18\rangle$. Parton distribution functions that take the NA51 data into consideration are also shown. . . . . 34

2.20 Results from E866 experiment. Left plot shows the ratio of cross sections $\sigma_{p d}(x) / 2 \sigma_{p p}(x)$ and the right plot shows the extracted ratio $\bar{d}(x) / \bar{u}(x)$ [57].

2.21 Plot of $\bar{d}(x)-\bar{u}(x)$ for E866 [57] along with the calculations of several different theoretical models. the green triangle data points belong to HERMES [58] . . . . . . . . . . . . . . . . . . . . 38

2.22 Cartoon of the pion cloud model. The proton is expressed in terms of several different baryon virtual meson Fock states. CG are the Clebsch-Gordan coefficients. . . . . . . . . . . . . . . . . 39

2.23 Figure shows $\bar{d}(x)-\bar{u}(x)$ and $\bar{d}(x) / \bar{u}(x)$ that include a delicate balance between several competing mechanisms that include a $\pi \Delta+\pi N$ component (dashed) and a parameterized Pauli blocking component (dashed) and combined effect (solid) [62]. The E866/NuSea data points belong to [56] which is an analysis of a smaller data set compared to [57]. . . . . . . . . . 40 
$2.24 \bar{d}(x) / \bar{u}(x)$ prediction (grey band) for the meson cloud model [61]. Blue points are E866 data points. . . . . . . . . . . . . . . . . . . . . 41

$2.25 \bar{d}(x) / \bar{u}(x)$ for Chiral Quark model [67]. The red line and blue line include total CQM and LQM inputs in their calculations. . . . . . . . . . . . . . 42

$2.26 \bar{d}(x) / \bar{u}(x)$ (solid line) for Chiral Quark Soliton model [68]. The dashed curves labeled CTEQ4M, MRS(R2) and MRST are phenomenological PDF fits to the data before and after the E866 data points. . . . . . . . . . . . . 42

$2.27 \bar{d}(x) / \bar{u}(x)$ prediction (solid line) by the statistical parton model [66] for the E866 data at $Q^{2}=54 \mathrm{GeV}^{2}$.

2.28 The ratio of $F_{2}^{\mathrm{N}}(\mathrm{Fe}) / F_{2}^{\mathrm{N}}(\mathrm{D})$ as a function of Bjorken- $x$. From the EMC collaboration $[72,73]$. The shaded area represents the systematic uncertainty. The solid line is the expectation for the effect of Fermi motion in $F_{2}^{\mathrm{N}}(\mathrm{Fe})[74,75] \ldots \ldots \ldots \ldots \ldots \ldots \ldots \ldots$

2.29 Results from SLAC MIT Rochester group experiments conducted to confirm the results from the EMC experiment [76]. . . . . . . . . . . . 46

2.30 Plots of the $F_{2}^{\mathrm{A}}(x) / F_{2}^{\mathrm{A}}(x)$ for several different targets and experiments taken from [77]. The references for the various experiments in these plots

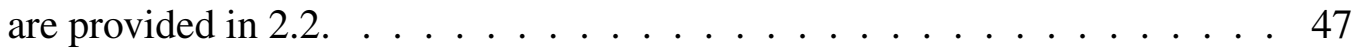

2.31 Separation of the regions in the ratio of structure functions taken from [93] . 48

2.32 Nuclear dependence as a function of mass number $A$. Plots are taken from [93]. Data in the left panel is from NMC (left panel) taken from [99] and E139 experiment (top right panel) performed at SLAC [82]. These plots show that the nuclear effects increase to a good approximation as a function of $\log (A)$. However, small deviations for $\mathrm{He}$ and $\mathrm{Li}$ for NMC data and $\mathrm{He}$ and $\mathrm{C}$ for SLAC-E139 data can be seen in the plots indicating that additional variables are required to explain the observed nuclear dependence. 51

2.33 Nuclear dependence as a function of nuclear density $\rho$. Data is from experiment E139 performed at SLAC [82] but plot taken from [93]. . . . . . . . 52

2.34 Slope of the EMC region $d \mathrm{R}_{\mathrm{EMC}} / d x$ plotted as a function of the scaled (by a factor of $(A-1) / A$ ) nuclear density. The scaling factor symbolizes the number of nucleons "witnessing" the scattering process. Plot taken from [87]. 53

2.35 Per nucleon cross section ratios of ${ }^{3} \mathrm{He},{ }^{4} \mathrm{He},{ }^{9} \mathrm{Be},{ }^{12} \mathrm{C},{ }^{63} \mathrm{Cu}$ and ${ }^{197} \mathrm{Au}$ as a function of $x$ for $\theta_{e}=18^{\circ}$. The horizontal lines indicate the plateau regions for NN SRC (Short Range Correlations) [88]. . . . . . . . . . . . . . . 54 
2.36 The EMC slopes versus the SRC scale factors taken from [103]. . . . . . . 55

2.37 Ratios of the Drell-Yan dimuon yields for C/LD2 (top left), Ca/LD2 (top right), Fe/LD2 (bottom left) and W/LD2 (bottom right) for the Fermilab E772 experiment [90]. The curves show predictions for the pion excess model, quark cluster model and rescaling model. . . . . . . . . . . . . . . 56

2.38 Ratios of the Drell-Yan dimuon yields as a function of mass, $x_{2}, x_{F}$ and $x_{1}$ for $\mathrm{Fe} / \mathrm{Be}$ (top row) and W/Be (bottom row) for the Fermilab NuSea/E866 experiment [91]. . . . . . . . . . . . . . . . . . 57

2.39 SeaQuest projections for nuclear dependence in sea quarks for Fe. The various models predictions were made for E772 kinematics $(800 \mathrm{GeV} / \mathrm{c}$ proton beam). . . . . . . . . . . . . . . . . 58

3.1 The SeaQuest Spectrometer . . . . . . . . . . . . . . . . . 60

3.2 Protons On Target (POT) plot for FY2014 - FY2017 time period. . . . . . . 62

3.3 Rough timeline of SeaQuest experiment. . . . . . . . . . . 63

3.4 Fermilab Accelerator Complex [118] . . . . . . . . . . . . . 65

3.5 Proton beam at the electrostatic septum [119] . . . . . . . . . . . 66

3.6 Micro structure of the beam along with examples of low intensity and high intensity buckets. ..................... 68

3.7 Depiction of the Čerenkov counter taken from $[120] \ldots \ldots 9$

3.8 Degraded backside of the Čerenkov counter mirror. . . . . . . . . . . . 70

3.9 Four snapshots 1800 RF buckets of the beam intensity delivered to SeaQuest. The red line shows the programmed inhibit threshold that is set to veto RF buckets above that intensity. . . . . . . . . . . . . . 71

3.10 The temperature of the condenser (red line, left scale) plotted as the $\mathrm{D}_{2}$ is cooled down. Blue dashed line gives the resistance of the level sensor inside the target flask. A rise in the value of the resistance indicates the formation of liquid [120]. . . . . . . . . . . . . . . 73

3.11 SeaQuest targets . . . . . . . . . . . . . . 75

3.12 Sideview of the target flask [122]. . . . . . . . . . . 76 
3.13 FMAG schematic view. The $5 \mathrm{~cm}$ (diameter) $\times 25 \mathrm{~cm}$ hole on the front face of the FMAG not shown in this picture [124]. . . . . . . . . . . . 78

3.14 Schematic view of KMAG [124] . . . . . . . . . . . . . . . 79

3.15 Depiction of hodoscopes and connected circuitry. . . . . . . . . . . . 80

3.16 H1X Hodoscope panels at St 1. . . . . . . . . . . . . . . . . 82

3.17 H1Y Hodoscope panels at St 1. . . . . . . . . . . . . . . 83

$3.18 \mathrm{H} 2 \mathrm{X}$ Hodoscope panels at St 2. . . . . . . . . . . . . 83

3.19 H2Y Hodoscope panels at St 2. . . . . . . . . . . . . . . . . . 84

3.20 H3X Hodoscope panels at St 3. . . . . . . . . . . . . . . . 84

$3.21 \mathrm{H} 4 \mathrm{X}$ Hodoscope panels at St 4 . . . . . . . . . . . . . . . 85

$3.22 \mathrm{H} 4 \mathrm{Y}$ Hodoscope panels at St $4 \ldots \ldots$. . . . . . . . . . . 85

3.23 Depiction of stages of creation of an avalanche taken from [126]. . . . . . 86

3.24 Drift time calculation schematic. . . . . . . . . . . . . . 87

3.25 Depiction of DC1.1 + DC1.2 configuration [124] . . . . . . . . . . . 89

3.26 positioning the wire using different techniques; printed circuits on G10 frame in the case of DC1.0, and feedthroughs in the case of DC1.1, DC3p

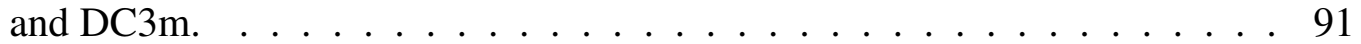

3.27 SeaQuest drift chambers . . . . . . . . . . . . . . . . . 94

3.28 Garfield simulations for SeaQuest drift chambers cells. . . . . . . . . . . . 95

3.29 Geometric depictions of D1X, D1U, D2X, D2U, D3pU, D3pX, D3mU and $\mathrm{D} 3 \mathrm{mX}$ planes ............................. 96

3.30 Cell structure of DC1.2 f . . . . . . . . . . . . . . 97

3.31 Cell structure of DC3p and DC3m . . . . . . . . . . . . . 97

3.32 Picture of an ASDQ card along with ribbon cables, ferrites (used for noise suppression) and cable supports. . . . . . . . . . . . . . . . 98 
3.33 Level Shifter Boards . . . . . . . . . . . . . . . . . . . . . . . . 99

3.34 Picture of a Time to Digital Converter $\ldots \ldots \ldots$. . . . . . 100

3.35 Common stop mode of a TDC $\ldots \ldots$. . . . . . . . . . 100

3.36 RT curve of DC3mU plane obtained from an iterative analysis. Since the SeaQuest TDCs adopted the "common stop" mode, a higher time (T) indicates a smaller $\mathrm{R}$ value. Top-left, top-right, bottom-left and bottom-right plots are results from first, second, third and fourth iterations respectively. Figure taken from $[132] . \ldots \ldots \ldots 1$

3.37 Plot of residuals of different chamber planes [120]. . . . . . . . . . 102

3.38 Gas gain measurements performed with $\mathrm{DC} 3 \mathrm{~m}\left(\mathrm{ArCO}_{2}\right.$ gas). The ${ }^{55} \mathrm{Fe}$ source is taped on different locations on the drift chamber in order to measure the pulse height. . . . . . . . . . . . . . . . 103

3.39 Gas gain measurements performed with $\mathrm{DC} 3 \mathrm{~m}\left(\mathrm{ArCO}_{2}\right.$ gas). Left (right) show the pulse height measurements at $2150 \mathrm{~V}(2230 \mathrm{~V})$ on the bottom middle part of the drift chamber. An average of 256 measurements of the pulse heights are made. Voltage is increased in steps until the peak size doesn't change and a voltage vs pulse height plateau is determined. . . . . . . . 104

3.40 Plot of chamber efficiencies. The $x$ axis shows different drift chamber planes and $y$ axis shows the corresponding efficiency [120]. . . . . . . . 104

3.41 Top view of a single proportional tube. . . . . . . . . . . . . 105

3.42 Residuals of proportional tubes. . . . . . . . . . . . . . . 106

3.43 Schematic of SeaQuest proportional tubes $[120] \ldots \ldots \ldots$. . . . . 107

3.44 Picture of Trigger Supervisor. . . . . . . . . . . . . . . . . . 108

3.45 Picture of a V1495 module [133] . . . . . . . . . . . . . . . 108

3.46 Schematic of SeaQuest trigger [133]. . . . . . . . . . . . . . . . 109

3.47 Schematic of NIM3 pseudo-random trigger. . . . . . . . . . . . 113

3.48 Picture of a Trigger Interface card. . . . . . . . . . . . . 114

3.49 Workflow of the MainDAQ [125] . . . . . . . . . . . . 115 
3.50 Daisy chain of the Trigger distribution. Readout is asynchronous. . . . . . . 116

3.51 Jump in the duty factor during Run 3 of data taking. . . . . . . . . . . . . 117

3.52 Online analysis of spill \# 1385729 [137]. The six individual plots show snippets of various aspects of the beam. For example, the top right plot shows the three Turns (red, blue and green) which indicate the stability of the beam in these turns. The right middle plot below shows the FFT (Fast Fourier Transform) of the beam. The prominent peak at $60 \mathrm{~Hz}$ arises due to the power supplies involved in the chain of electronics somewhere in the beam line. Information on a spill-by-spill basis is relayed back to the Control room and is used to tune beam for SeaQuest. . . . . . . . . . . . . 119

3.53 SeaScape hit distributions of DC2 from Run - 14406. The channels showing 0 hits on D2X and D2Up drift chamber planes are dead channels. . . . . 121

3.54 SeaScape hit distributions of $\mathrm{H} 1$ from Run - 14406. . . . . . . . . . . . . 121

4.1 kTracker flowchart . . . . . . . . . . . . . . 123

4.2 Depiction of hit removal from raw data. Blue arrow is the muon track. Red dots are the extra hits (top array) and tracked hit (bottom array). . . . . . . . 125

4.3 Cluster removal flowchart $[139] \ldots \ldots$. . . . . . . . . . . . . 126

4.4 Number of hits before and after hit removal on DC1.1 shown on a linear

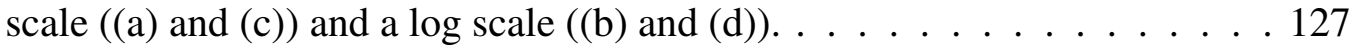

4.5 Building of tracklets in a drift chamber. . . . . . . . . . . . . 128

4.6 Sagitta method used for global track reconstruction. . . . . . . . . . . 131

4.7 Workflow of the Kalman filter process [125]. . . . . . . . . . . 133

4.8 Geometric depiction of the Kalman filter process [140]. . . . . . . . . . . . 134

4.9 Application of energy loss in kTracker. Blue curve is the momentum difference between the thrown momentum at the initial momentum - the thrown momentum at DC1. The most probable value in this curve is used in the kTracker. . . . . . . . . . . . . . . . . 135

4.10 Energy loss parameterization for SeaQuest. . . . . . . . . . . . . 136 
4.11 Application of energy loss in kTracker at the beginning and end of each slice with a $p_{T}$ kick at the middle of the slice. . . . . . . . . 138

4.12 Reconstructed vertex position blue position) for a fixed $z$ thrown position (dotted line) and mass [140]. . . . . . . . . . . . . . . . . . . 139

4.13 The acceptance reach of SeaQuest. As can be seen in the plot, the acceptance can be tuned to reach a Bjorken- $x$ region of $\sim 0.45 \ldots \ldots . \ldots 142$

4.14 Some reconstructed detector quantities from $m c \_d r e l l y a n \_L D 2 \_M 026 \_S 002$ Monte Carlo production generated from LD2 target. Top left is the dimuon vertex $y$ position, top right is the dimuon vertex $z$ position, bottom left is the dimuon vertex $x$ position and bottom right is the single muon $z$ vertex position. . . . . . . . . . . . . . . . . . . . . . 144

4.15 Some reconstructed detector quantities from $m c \_d r e l l y a n \_L D 2 \_M 026 \_S 002$ Monte Carlo production generated from LD2 target. Top left is the Bjorken $x$ of the target, top right is the dimuon $z$ component of the momentum, bottom left if the $\cos (\theta)$ of the dimuon and bottom right is the transverse momentum $p_{T}$ of the dimuon. . . . . . . . . . . . . . . . 145

4.16 Some reconstructed detector quantities from $m c \_d r e l l y a n \_L D 2 \_M 026 \_S 002$ Monte Carlo production generated from LD2 target. Top left is the Feynman$x$ of the dimuon, top right is the position of the $\mu^{+}$at DC1, bottom left and bottom right are the $\chi^{2}$ of the $\mu^{+}$at the target and the beam dump location respectively. . . . . . . . . . . . . . . . . . 146

4.17 Figure shows the number of messy hit tracks (on a log scale) given the number of clean hits $=15,16$ etc in different color schemes. For example, the 17 hit clean track (orange) has more 16 hit tracks compared to 18 hit messy tracks. That means, it is more likely to drop a hit while embedding with noise than gain a hit. The case is similar with 16 hit track. . . . . . . 147

4.18 Flowchart of defining the status of an RF bucket. Typically, the status is "Inhibited", "Busy" or "Live" depending on the whether the RF bucket was inhibited (by QIE VETO) or if the TS was busy and not able to read or is live and able to be recorded [132]. . . . . . . . . . . . . . . . 149

4.19 Flowchart of data taking in one spill $[141] . \ldots \ldots \ldots \ldots$

4.20 Target Beam dump separation plot for R008 data production with docid\#2111-

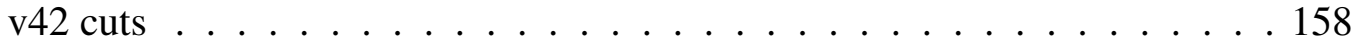

4.21 An invariant mass spectrum plot for LD2. The fit incorporates $J / \psi, \psi^{\prime}$ and Drell-Yan messy Monte Carlo along with a background spectrum. . . . . 159 
4.22 Roadset comparison of $p_{T}$ for (a) $\mathrm{LH}_{2}$, Empty flask and $\mathrm{LD}_{2}$ and b) No target, Fe, C and W targets. A slightly larger mean and RMS for the empty flask and no target (possibly due to random backgrounds) compared to $\mathrm{LH}_{2}$ and $\mathrm{LD}_{2}, \mathrm{C}, \mathrm{Fe}$, and $\mathrm{W} . \mathrm{LH}_{2}$ and $\mathrm{LD}_{2} p_{T}$ distributions also have a slight difference $(\approx 1.5 \%)$ in their distributions which is within the resolution. . . 160

4.23 Roadset comparison of $x_{T}$ for (a) $\mathrm{LH}_{2}$, Empty flask and $\mathrm{LD}_{2}$ and b) No target, $\mathrm{Fe}, \mathrm{C}$ and $\mathrm{W}$ targets. . . . . . . . . . . . . . . . . . 161

4.24 Roadset comparison of $x_{F}$ for (a) $\mathrm{LH}_{2}$, Empty flask and $\mathrm{LD}_{2}$ and b) No target, Fe, $\mathrm{C}$ and $\mathrm{W}$ targets. . . . . . . . . . . . . . . . 162

4.25 Roadset comparison of $d z$ (dimuon vertex) for (a) $\mathrm{LH}_{2}$, Empty flask and $\mathrm{LD}_{2}$ and b) No target, Fe, $\mathrm{C}$ and $\mathrm{W}$ targets. As can be seen in the violet and green curves, there are slightly more counts coming from near the front face of the beam dump (possibly randoms from the dump that satisfy the target cuts) and further upstream (flask end cap). . . . . . . . . . . . . 163

4.26 $\mathrm{LD}_{2} / \mathrm{LH}_{2}$ ratio of intercepts for linear and quadratic fits plot for mc_drellyan_LD2_M026_S002_m and mc_drellyan_LH2_M026_S002_messy GEANT4 Monte Carlo productions made using $\mathrm{LD}_{2}$ and $\mathrm{LH}_{2}$ targets. . . . . . . . . . . . . . . . 174

4.27 $\mathrm{LD}_{2} / \mathrm{LH}_{2}$ ratio as a function of the trigger intensity. As can be seen in the plot, the ratio of cross sections $\sigma_{p d} / 2 \sigma_{p p}$ (y-axis) exhibits an intensity dependence $\left(x\right.$-axis). The plot is made for $0.1<x_{T}<0.45 . \ldots 177$

5.1 CSR vs $x_{T}$ for different roadsets. . . . . . . . . . . . . . . 178

5.2 CSR as a function of $x_{T}$. Note that $p 10, p 11, p 20$ and $p 21$ are common for all fits. . . . . . . . . . . . . . . . . . 179

5.3 CSR intercepts for each $x_{T}$ bin along with the fit results. Note that $p 10$, $p 11, p 20$ and $p 21$ are common for all fits. . . . . . . . . . . . . 180

$5.4 \sigma_{p d}(x) / 2 \sigma_{p p}(x)$ vs $x_{T}$ plot along with PDF predictions. The red points are SeaQuest data points which are plotted along with the systematics band shown at the bottom. The data points are consistently above 1 for all $x_{T}$ and agree quite well with the PDF predictions in the low- $x$ region up to $x_{T} \approx$ 0.25. Since there are no data in the high- $x$ region to constrain the PDFs, it is natural to expect differences between the E906 and PDF predictions which are data driven. A small overall normalization correction is being studied by the collaboration. It is estimated to be from $0-3 \%$, and arises when taking into account the dead time experienced by low intensity events. 184 
$5.5 \sigma_{p d}(x) / 2 \sigma_{p p}(x)$ vs $x_{T}$ plot with E866 data only. The red data points are SeaQuest and black data points are for E866/NuSea. The systematic uncertainty for E866 is $<1 \%$ and not shown here [57]. A small overall normalization correction is being studied by the collaboration. It is estimated to be from $0-3 \%$, and arises when taking into account the dead time experienced by low intensity events. . . . . . . . . . . . . . . . . 185

5.6 $\sigma_{p d}(x) / 2 \sigma_{p p}(x)$ vs $x_{T}$ plot comparison with the statistical parton model [66, 164]. Red points are E906 data points. The green band is for the statistical parton model predictions for SeaQuest beam energy (not acceptance). A small overall normalization correction is being studied by the collaboration. It is estimated to be from $0-3 \%$, and arises when taking into account the dead time experienced by low intensity events. . . . . . . . . . . . . 186

5.7 CSR vs $x_{T}$ for different trigger intensities with 8 bins for each intensity

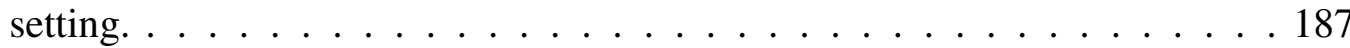

5.8 CSR vs $x_{T}$ for different number of trigger intensity bins. . . . . . . 188

$5.9 x_{\text {Beam }}$ distributions for $\mathrm{LH}_{2}$. The three colors correspond to the three trigger intensity regions; low (blue), intermediate (magenta) and high (black). All plots are normalized to their integral. . . . . . . . . . . . . 190

$5.10 x_{\text {Beam }}$ distributions for $\mathrm{LH}_{2}$. The three colors correspond to the three trigger intensity regions; low (blue), intermediate (magenta) and high (black). All plots are normalized to their integral. . . . . . . . . . . . . . . 191

$5.11 x_{\text {Beam }}$ distributions for $\mathrm{LD}_{2}$. The three colors correspond to the three trigger intensity regions; low (blue), intermediate (magenta) and high (black). All plots are normalized to their integral. . . . . . . . . . . . . . . 192

$5.12 x_{\text {Beam }}$ distributions for $\mathrm{LD}_{2}$. The three colors correspond to the three trigger intensity regions; low (blue), intermediate (magenta) and high (black). All plots are normalized to their integral. . . . . . . . . . . . . . . . 193

$5.13 \bar{d}(x) / \bar{u}(x)$ plot for SeaQuest. Next-to-Leading Order extraction was done using CT10NLO PDF set. . . . . . . . . . . . . . . . . . 198 
$5.14 \bar{d}(x) / \bar{u}(x)$ comparison between SeaQuest and NuSea experiments. The red data points are for SeaQuest and the black triangles are for NuSea. Systematic uncertainty band for SeaQuest is shown at the bottom of the plot. Systematic uncertainty band for E866 is not shown in the plot. SeaQuest data points indicate an increase with increasing $x_{T}$ unlike NuSea [57] which indicates an overturn and a statistically limited drop below 1. It should be noted that the small overall normalization correction was not considered in the extraction process. However this does not change the overall trend of the SeaQuest data points. . . . . . . . . . . . . . . . . . . . 199

$5.15 \bar{d}(x) / \bar{u}(x)$ for SeaQuest along with curves for the meson cloud model (blue band) and the statistical parton model (green band) [165, 164]. Black triangles are E866 data points. Systematic uncertainty band for E866 is not shown [57]. . . . . . . . . . . . . . . . . . . 200

$5.16 R_{p A}$ for carbon for different $x_{T}$ bins. $F=p 0+\left(p 10+p 11 *\left\langle x_{T}\right\rangle\right) *$ inte + $\left(p 20+p 21 *\left\langle x_{T}\right\rangle\right) * i n t e^{2}$ fit was used for the extrapolation. Note that $p 10$, $p 11, p 20$ and $p 21$ are common for all fits. . . . . . . . . . . . . 202

$5.17 R_{p A}$ for carbon for different $x_{T}$ bins. $F=p 0+\left(p 10+p 11 *\left\langle x_{T}\right\rangle\right) *$ inte + $\left(p 20+p 21 *\left\langle x_{T}\right\rangle\right) * i n t e^{2}$ fit was used for the extrapolation. Note that $p 10$, $p 11, p 20$ and $p 21$ are common for all fits. . . . . . . . . . . . . 203

5.18 $R_{p A}$ for iron for different $x_{T}$ bins. $F=p 0+\left(p 10+p 11 *\left\langle x_{T}\right\rangle\right) *$ inte + $\left(p 20+p 21 *\left\langle x_{T}\right\rangle\right) * i n t e^{2}$ fit was used for the extrapolation. Note that $p 10$, $p 11, p 20$ and $p 21$ are common for all fits. . . . . . . . . . . . . . . . 204

$5.19 R_{p A}$ for iron for different $x_{T}$ bins. $F=p 0+\left(p 10+p 11 *\left\langle x_{T}\right\rangle\right) *$ inte + $\left(p 20+p 21 *\left\langle x_{T}\right\rangle\right) * i n t e^{2}$ fit was used for the extrapolation. Note that $p 10$, $p 11, p 20$ and $p 21$ are common for all fits. . . . . . . . . . . . . 205

$5.20 R_{p A}$ for tungsten for different $x_{T}$ bins. $F=p 0+\left(p 10+p 11 *\left\langle x_{T}\right\rangle\right) *$ inte $+\left(p 20+p 21 *\left\langle x_{T}\right\rangle\right) *$ inte $^{2}$ fit was used for the extrapolation. Note that $p 10, p 11, p 20$ and $p 21$ are common for all fits. . . . . . . . . . . . 206

$5.21 R_{p A}$ for tungsten for different $x_{T}$ bins. $F=p 0+\left(p 10+p 11 *\left\langle x_{T}\right\rangle\right) *$ inte $+\left(p 20+p 21 *\left\langle x_{T}\right\rangle\right) * i n t e^{2}$ fit was used for the extrapolation. Note that $p 10, p 11, p 20$ and $p 21$ are common for all fits. . . . . . . . . . 207 
$5.22 R_{p A}$ vs $x_{T}$ for carbon (left), iron (center) and tungsten (right). No isoscalar corrections have been applied to the targets. Only statistical uncertainties are shown. A small overall normalization correction is being studied by the collaboration. It is estimated to be from $0-3 \%$, and arises when taking into account the dead time experienced by low intensity events. $F=p 0+$ $\left(p 10+p 11 *\left\langle x_{T}\right\rangle\right) * i n t e+\left(p 20+p 21 *\left\langle x_{T}\right\rangle\right) * i n t e^{2}$ fit was used for the extrapolation. . . . . . . . . . . . . . . 208

$5.23 R_{p A}$ vs $x_{T}$ for carbon (left), iron (center) and tungsten (right). No isoscalar corrections have been applied to the targets. Blue data points belong to SeaQuest and red data points belong to E772 [90]. Only statistical uncertainties shown. . . . . . . . . . . . . . . . . 208

5.24 $R_{p A}$ vs $p_{T}$ for carbon (left), iron (center) and tungsten (right). Statistical uncertainties shown only. $F=p 0+(p 10+p 11 *\langle p T\rangle) *$ inte $+(p 20+$ $p 21 *\langle p T\rangle) *$ inte $^{2}$ fit was used for the extrapolation. . . . . . . . . . 213

5.25 Depiction of the drop of the ratio $R_{p A}\left(x_{F}\right) \ldots \ldots \ldots . \ldots \ldots$

5.26 Depiction of a parton losing energy while traversing a cold nuclear medium. 214

$5.27 R_{p A}$ vs $x_{F}$ for carbon (left), iron (center) and tungsten (right). $F=p 0+$ $p 1 * I+p 2 * I^{2}$ (common pol2 fit) was used for extrapolation since it has a better reduced $\chi^{2}$ compared to the other fits. . . . . . . . . . . . 215

$5.28 R_{p A}$ vs $x_{F}$ for carbon (left), iron (center) and tungsten (right) with $x_{T}>$ 0.16 cut (red). $F=p 0+p 1 * I+p 2 * I^{2}$ (common pol 2 fit) was used for extrapolation in both the cases. The results are consistent within uncertainties indicating that the effects of shadowing are negligible. . . . . . . . . 215

$5.29 R_{p A}$ vs $x_{B}$ for carbon (left), iron (center) and tungsten (right). $F=p 0+$ $p 1 * I+p 2 * I^{2}$ (common pol2 fit) was used for extrapolation. . . . . . . . 216

$5.30 R_{p A}$ vs mass for carbon (left), iron (center) and tungsten (right). . . . . . . 216

A.1 Depiction of stretching a wire taken from [132]. . . . . . . . . . . . 224

A.2 Pulse generator and Oscilloscope used in the wire tension measurement . . 225

A.3 Magnet support system used in tension measurement . . . . . . . . 226

A.4 Cartoon of the setup used to measure the tension of the wires . . . . . . 226 


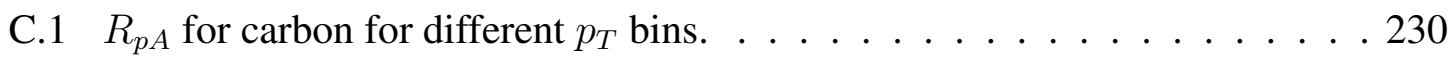

C.2 $R_{p A}$ for carbon for different $p_{T}$ bins along with the fit results. . . . . 231

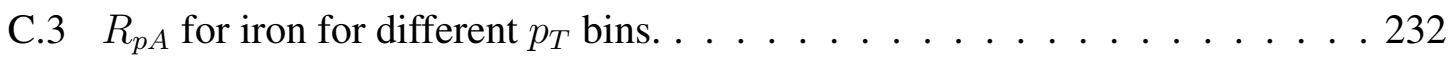

C.4 $R_{p A}$ for iron for different $p_{T}$ bins along with the fit results. $\ldots \ldots . .233$

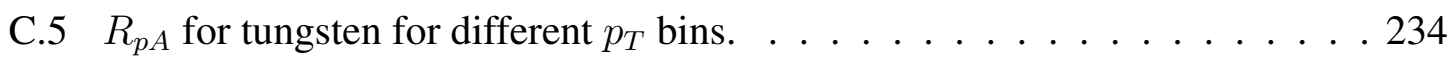

C.6 $R_{p A}$ for tungsten for different $p_{T}$ bins along with the fit results. . . . . 235

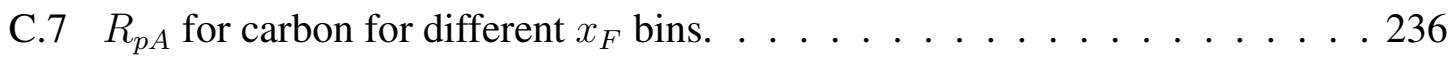

C.8 $R_{p A}$ for carbon for different $x_{F}$ bins along with the fit results. . . . . 237

C.9 $\quad R_{p A}$ for iron for different $x_{F}$ bins. $\ldots \ldots \ldots \ldots \ldots \ldots \ldots \ldots$

C.10 $R_{p A}$ for iron for different $x_{F}$ bins along with the fit results. . . . . . 239

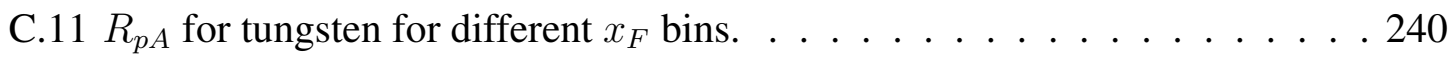

C.12 $R_{p A}$ for tungsten for different $x_{F}$ bins along with the fit results. . . . . 241

D.1 Energy budget of the Universe . . . . . . . . . . . . . . . 242

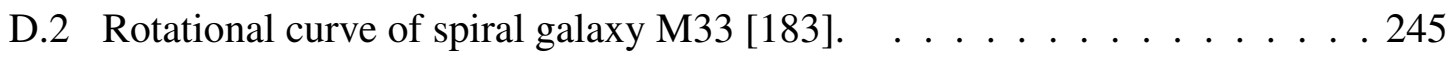

D.3 Gravitational lensing of Abell 2218 Galaxy cluster. Credits: NASA, Andrew Fruchter and the ERO team [184]. . . . . . . . . . . . . . 246

D.4 Red and yellow contours show X-ray maps of the collision of baryonic matter in a bullet cluster. Green contours show the gravitational lensing of X-rays [185]. . . . . . . . . . . . . . . . . . . . . 247

D.5 The detailed, all-sky picture of the infant universe created from nine years of Wilkinson Microwave Anisotropy Probe data (WMAP). Fig. shows a temperature range of $\pm 200 \mu \mathrm{K}$ [188] . . . . . . . . . . . . . 248

D.6 Seven year temperature power spectrum from Wilkinson Microwave Anisotropy Probe [189]. . . . . . . . . . . . . . . . . . . . . . . . . 248

D.7 Coupling between Standard Model sector and the Dark Sector . . . . . 250 
D.8 Cartoon of a dark photon production-propagation-decay event . . . . . . 252

D.9 Dark photon production mechanisms . . . . . . . . . . 253 


\section{Chapter 1}

\section{Introduction}

Understanding nucleon and nuclear structure is one of the central goals of nuclear physics. Experiments continue to peel the layers of the nucleon's rich inner-substructure each day. The nucleus is a dynamical QCD composite object which displays a multitude of phenomenon based on individual and collective behavior of its building blocks; quarks, gluons, protons and neutrons (collectively called nucleons).

Nucleon structure as it is understood today has been deduced in stages over a period of several decades of worldwide experimental and theoretical effort. There were several elastic scattering experiments with electron and muon beams conducted starting in the 50's which were crucial for the establishment of the nucleon as a composite object. With the wealth of information obtained from scattering experiments, a complicated picture of the proton emerged. Initially, the proton was thought to be made up of just three massive constituent quarks, before the form of the strong QCD force was known. This 'simple' modeling of the interplay has found several experimental observations "wanting" of more

dynamics in the nucleon. In the words of D. F. Geesaman and P. E. Reimer [1], "Despite what one may hear, the proton is never just three valence quarks and glue."

Every nucleon has a sea of quark anti-quark pairs and glue. The nucleon sea plays an important role in determining several global properties of the nucleon. It is the goal of SeaQuest to specifically access the anti-quark sea structure of the nucleon and nuclei. SeaQuest takes advantage of the Drell-Yan process in which a quark from one hadron annihilates with an anti-quark from another hadron producing a virtual photon which eventually decays into a dilepton. This process provides unique access to the sea structure thereby ad- 
dressing some key physics goals of interest to theoretical and experimental communities alike.

This thesis is organized as follows:

- Chapter 2 builds up from the current fundamental building blocks to aspects of nucleon and nuclear structure. It brings into context, some key physics goals of the SeaQuest experiment:

- Light anti-quark flavor asymmetry of the nucleon sea, and

- Nuclear dependence of anti-quarks in nuclei

- Chapter 3 discusses aspects of the SeaQuest spectrometer.

- Chapter 4 discusses dimuon track reconstruction and the careful design of the analysis cuts in identifying Drell-Yan dimuons coming from the target region. It also discusses the new Intensity-Extrapolation method developed to counter the rate dependence effects coming from a high intensity beam coupled with detector and track reconstruction algorithm limitations.

- Chapter 5 discusses the:

- $\sigma_{p d}(x) / 2 \sigma_{p p}(x)$ cross section ratio result obtained using the Intensity-Extrapolation method. It also gives a "first look" at the $\bar{d}(x) / \bar{u}(x)$ extracted using the the procedure described in the chapter.

- nuclear dependence of the per-nucleon cross section ratio, $R_{p A}$ as a function of Bjorken- $x$ of the target or $x_{T}$, transverse momentum of the dimuon or $p_{T}$, Feynman- $x$ or $x_{F}$, and Bjorken- $x$ of the beam parton or $x_{B}$.

- Chapter 6 summarizes the findings and gives a future outlook on how to use this thesis in gaining an insight into additional physics topics. 


\section{Chapter 2}

\section{What's the Matter?}

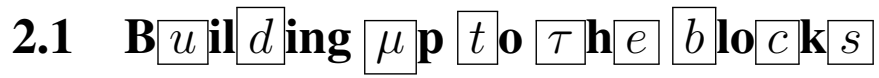

\subsubsection{The "Indivisible" Atom}

Understanding nature and the behavior of its fundamental constituents has been the central goal of human pursuit for centuries. Great intellectual insights, ingenious experiments, clever reasoning, and sometimes trial and error have paved the way to great discoveries in modern physics. The quest to understand the building blocks of nature dates back to before 1700 B.C.E. Ancient Indian civilizations believed that matter around us is made up of five gross elements, i.e. Pruthvi, (earth), Apas (water), Agni (Fire), Vayu (air) and Aakasha (space) and all observable phenomena could be explained by a conscious interplay of these five "fundamental" elements in time with Brahman as the ultimate underlying reality of it all. Ancient Greek civilization (around 500 B.C.E.) believed that matter around us is made up of Earth, Water, Air, Fire, and Aether and that these elements were built up by "indivisible atomos". Scientists all over the world have come a long way since then, in terms of discovering a deeper sub-structure to the observable universe ${ }^{1}$ and understanding new physical laws underlying complex emergent phenomena.

During the earlier part of the 19th century, John Dalton proposed that all matter was made up of atoms. These atoms were indivisible and indestructible fundamental building blocks and all substances were a mere combination of different kind of atoms. Although the essence of this theory is somewhat correct, it had its limitations in terms of understand-

\footnotetext{
${ }^{1}$ Observable here means matter that can be currently made in a lab.
} 


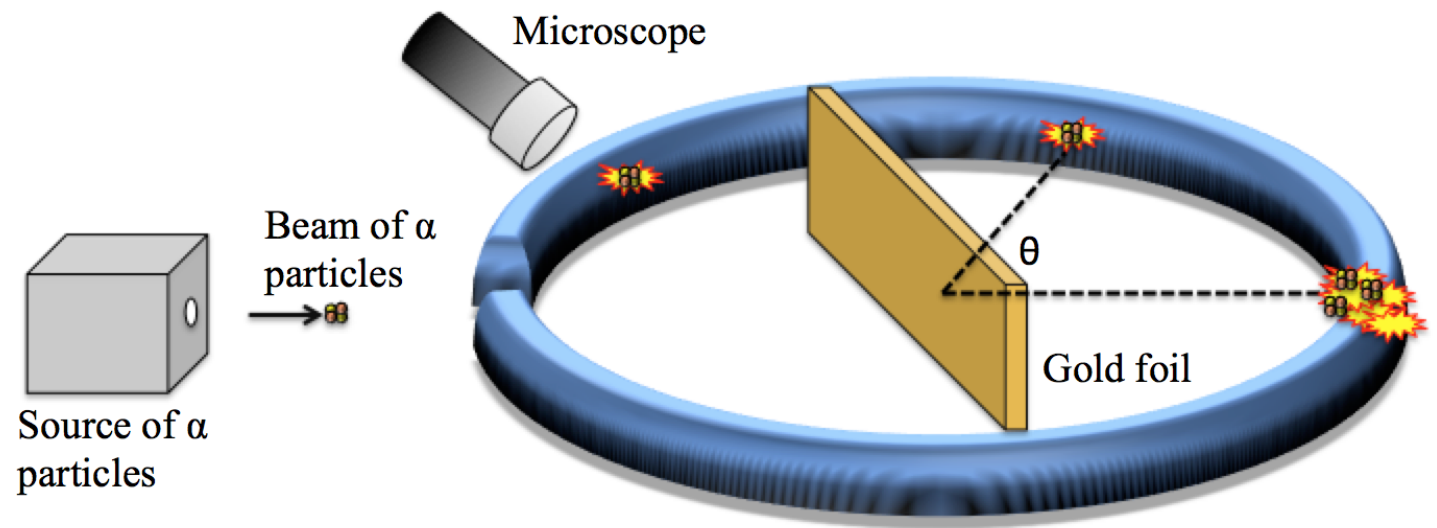

Fluorescent screen

Figure 2.1: Rutherford's gold foil alpha scattering experiment

ing the atomic weights and predicting new elements. Over the years, several new chemical elements were discovered in chemistry experiments conducted all over the world. Dmitri Mendeleev classified these elements by organizing the then known 56 chemical elements into a table of increasing atomic mass and predictable chemical properties called the periodic table. Although, the existence of several new elements was predicted that would fill the gaps in the periodic table, it was not obvious why the elements in a certain column had similar chemical properties. This was perhaps a hint of an underlying atomic sub-structure that explains the chemical and physical properties of various substances. With J.J. Thomson's cathode ray experiments and the discovery of the electron, John Dalton's view of the atomic world was proven to be inaccurate. J.J. Thomson postulated that electrically neutral atoms consist of two types of charges ( + and -$)$. He also proposed that the negative charges were evenly embedded in a positively charged clump of matter, similar to seeds in a watermelon or a plum pudding.

\subsubsection{The Atomic Nucleus}

In an experiment demonstrated by Hans Geiger and Ernst Marsden under the direction of Ernst Rutherford, a beam of $\alpha$ particles were fired onto a gold foil and scintillations on the 
screen, behind the foil, were recorded [2]. Most of the $\alpha$ particles went straight through with minimal deflection to their path. However, some of the alpha particles were deflected by almost $180^{\circ}$. Figure 2.1 shows a depiction of the experimental setup used in the famous gold foil experiment. The electric fields created by the prediction of Thomson's plum pudding model were not strong enough to cause such a large deflection. Rutherford concluded that the deflection of these $\alpha$ particles could not be due to multiple deflections from the gold foil, but rather due to the presence of a strong electric field in a tiny core located inside the atom. His team also continued to investigate the structure of the nucleus using several targets with various thicknesses. They found that Hydrogen nuclei were emitted in all the experiments. This led Rutherford to postulate that the the positive core in the nucleus was a compilation of several integer numbers of hydrogen nuclei. The term proton was given to this positively charged fundamental unit of matter. With this, the atomic structure of a tiny compact positive core with orbiting electrons emerged [3].

\subsubsection{The Neutron}

The question then came up as to how the nucleus was held together, in spite of having positive charges localized to such a small volume. Also, in order to explain the mass of the Helium atom which had two units of positive charge and four units of mass, Rutherford postulated the existence of another subatomic particle, called the neutron. With the discovery of the neutron by James Chadwick in 1932 [4] and the advent of the strong nuclear force, one could explain how the nucleus was tightly bound and held together in spite of the powerful electromagnetic forces that would otherwise tear the nucleus apart.

\subsubsection{The Elusive Neutrino}

Around the same time, Wolfgang Pauli postulated the existence of a new particle in order to explain the continuous energy spectra of the electron in experiments where an unstable nucleus decays by emitting $\beta^{-}$rays. According to Pauli, if there were only two particles 
emitted in the decay of the neutron, the energy spectrum of the emitted electron would have a sharp peak as allowed by the kinematics of the decay process.

$$
n \rightarrow p+e^{-}+\text {unknown }
$$

But instead, the spectrum was continuous with the challenge of having an undetected particle released in the process ${ }^{2}$. This led Pauli to postulate the existence of a 'neutrino', which escaped detection, but was still a particle emitted in the process.

Up until the early 1930's the question "what is the world made up of?" had a seemingly simple answer. It was made up of protons, neutrons (collectively called as nucleons) in a tiny positive core, with the electrons orbiting around it. To this list, two other particles were also added. The photon (postulated by Max Plank in 1900 to explain the black body radiation problem) and the elusive neutrino.

\subsubsection{The Positron}

Paul Dirac predicted the existence of the positron from his famous relativistic kinematic equations. Until then, the simple equation, “ $E^{2}=p^{2} c^{2}+m^{2} c^{4}$ " was thought to be solely linear with a unique solution. Dirac interpreted the negative multiplicative component of $E=$ $\pm \sqrt{p^{2} c^{2}+m^{2} c^{4}}$ as another particle with the

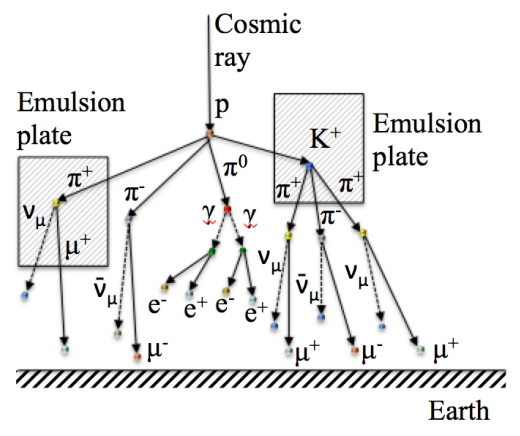
same mass but negative energy. Even though the interpretation at that time was not accurate, this led Dirac to hypothesize the existence of

Figure 2.2: Cartoon of a photographic emulsion plate used in muon and pion detection cosmic ray experiments another particle with the same mass as the electron, but with the opposite charge. Carl Anderson and collaborators discovered the positron

${ }^{2}$ Later identified as a $\bar{\nu}_{e}$. 
in their experiments and this was the discovery of an antiparticle [5].

\subsubsection{The Yukawa " $\mu$ meson" (Pion)}

Hideki Yukawa predicted that the nucleus was held together due to the existence of a "force carrier" which could communicate the force between two nucleons. He called them " $\mu$ mesons" (which had a mass around $100 \mathrm{MeV}$ that he obtained from back of the envelope

calculations). Based on Heisenberg's uncertainty principle arguments, $\Delta E \Delta t \sim \frac{\hbar}{2}$ where $\Delta E$ is the energy borrowed and $\Delta t$ is the time it is borrowed. If the carrier of the strong force were traveling the width of the nucleon $(1 \mathrm{fm})$ at speed $c$, taking $\hbar=197 \mathrm{MeVfm} / c$ and $\Delta t=1 \mathrm{fm} / c$, we get the mass of the exchanged particle to be roughly $100 \mathrm{MeV}$. In a series of cosmic ray experiments which were conducted in the upper part of the earth's atmosphere, physicists used photographic emulsions plates that recorded the trajectories from which one could deduce the mass and charge of these "traced out" particles. The muon (which is different from Yukawa's predicted " $\mu$ meson") was detected during these experiments. Strongly interacting particles such as the pion ( $\pi^{0}$ and $\left.\pi^{ \pm}\right)$and kaon $\left(K^{0}\right.$ and $K^{ \pm}$) were also discovered along with a plethora of new unstable particles from these cosmic ray experiments ( $\eta, \phi, \rho$ and so on). Figure 2.2 shows a cartoon of the emulsion plate experiments conducted in the upper atmosphere. The situation was similar to a hundred years ago where in there was a great need to classify the detected particles in order to bring out underlying symmetries.

\subsubsection{The Birth of Quarks}

In 1964, Murray Gell-Mann and George Zweig independently proposed that all hadrons are in fact composed of even more elementary constituents called "quarks" or "aces" [6, 7]. According to this method of classification, hadrons are bound states of three quarks (or three anti-quarks) and mesons are bound states of a quark and an anti-quark. At that time, 
only three quarks were introduced that became a representation of the SU(3) group ${ }^{3}$. The $\mathrm{u}$ (up), d (down) and s (strange) quarks were introduced as elementary constituents and the "Eightfold way" octets and decuplets of baryons and mesons emerged naturally as a permutation and combination of the quantum numbers of these quarks.

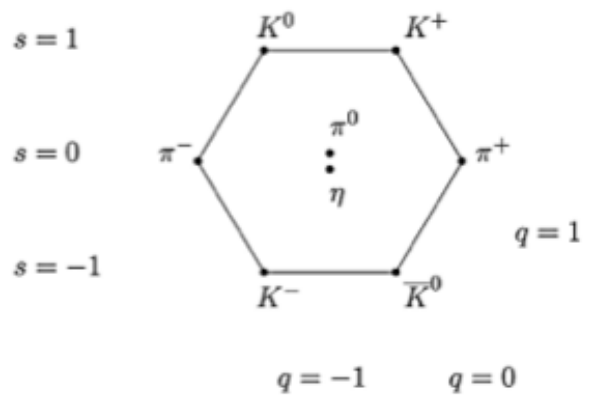

a)

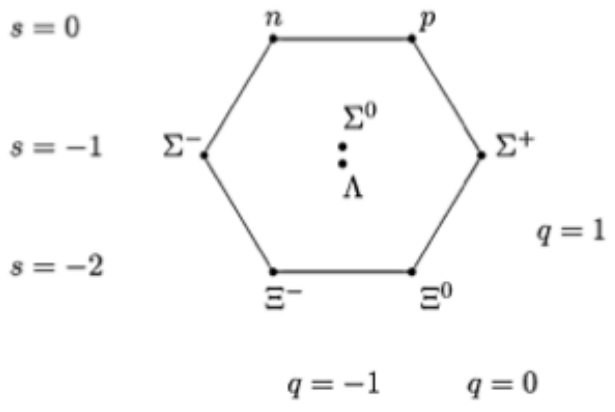

b)

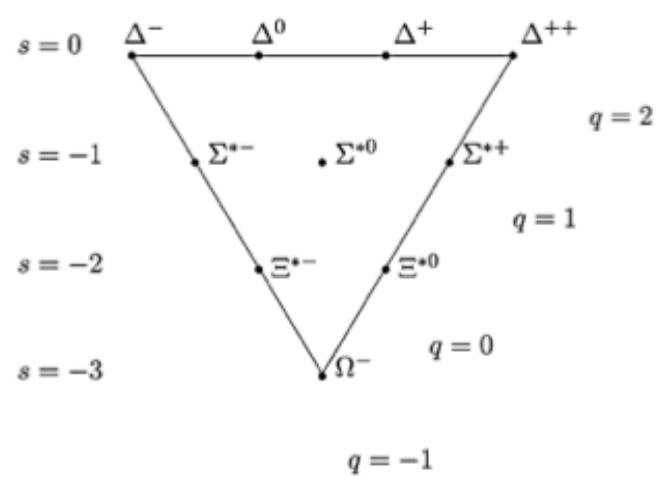

c)

Figure 2.3: Examples of a Meson Octet (a), Baryon Octet (b) and Baryon decuplet (c) [8].

$$
u=\left(\begin{array}{l}
1 \\
0 \\
0
\end{array}\right) ; d=\left(\begin{array}{l}
0 \\
1 \\
0
\end{array}\right) ; s=\left(\begin{array}{l}
0 \\
0 \\
1
\end{array}\right)
$$

These particles are placed on downward-sloping diagonal lines of charge $(\mathrm{Q}=-1,0,1$, etc) and horizontal lines of "strangeness" ( $s=-1,0,1$, etc). Elementary constituents of

\footnotetext{
${ }^{3}$ We will not describe group theory here.
} 
mesons and baryons were assigned these above stated quantum numbers and placed at the intersection points of geometric lines. This allowed the organization of a multitude of particles into Meson and Baryon octets and decuplets. Figure 2.3 shows examples of a meson octet, a baryon octet and a baryon decuplet chart. Based on the missing members on the decuplet chart, Gell-Mann predicted the existence (and also calculated the mass) of a new particle with strangeness $=-3$ ( $\Omega^{-}$baryon) [9]. With the discovery of this new particle, Gell-Mann's “mathematical entities” called quarks, became considered as possible real particles and this gave the first hint of a deeper "simpler" underlying substructure to the multiplicity of observed baryons and mesons.

Even though this method cleverly brought out the simplicity behind the observed complexity of short lived particles, several important questions remained unanswered. These include "why are the quarks not observed in isolation?" and "how can the $\Delta^{++}$(a spin $3 / 2$ particle which has three up quarks in the same spin state) exist in clear violation of the Pauli principle?". The first question was answered with the help of a phenomenon called quark confinement, and the second with the invention of an additional interaction called the "color".

\subsubsection{Current Fundamental Building blocks}

The 1970's and 80's were an important period for the growth of nuclear and particle physics alike. With improvements on the theoretical side as well as detector technologies, a large number of building blocks were detected and added to the list of new particles. Until then, the universe was made up of only the $\mathrm{u}, \mathrm{d}$ and s quarks and $\mathrm{e}, \nu_{e}, \mu$, and $\nu_{\mu}$ leptons. With the discovery of the charm quark (c) in $1975[10,11]$, tau $(\tau)$ lepton and its corresponding tau neutrino $\left(\nu_{\tau}\right)$ in 1975 [12], indirect existence of the gluon from the observation of three jet events in 1976 [13], bottom or beauty quark (b) in 1977 [14], neutral and charged electroweak gauge bosons $\left(Z^{0}\right.$ and $\left.W^{ \pm}\right)$in $1983[15,16,17]$, top quark (t) in $1996[18,19]$ and a Higgs boson in 2012 [20,21], a new picture of the fundamental building blocks that 
make up the universe began to emerge.

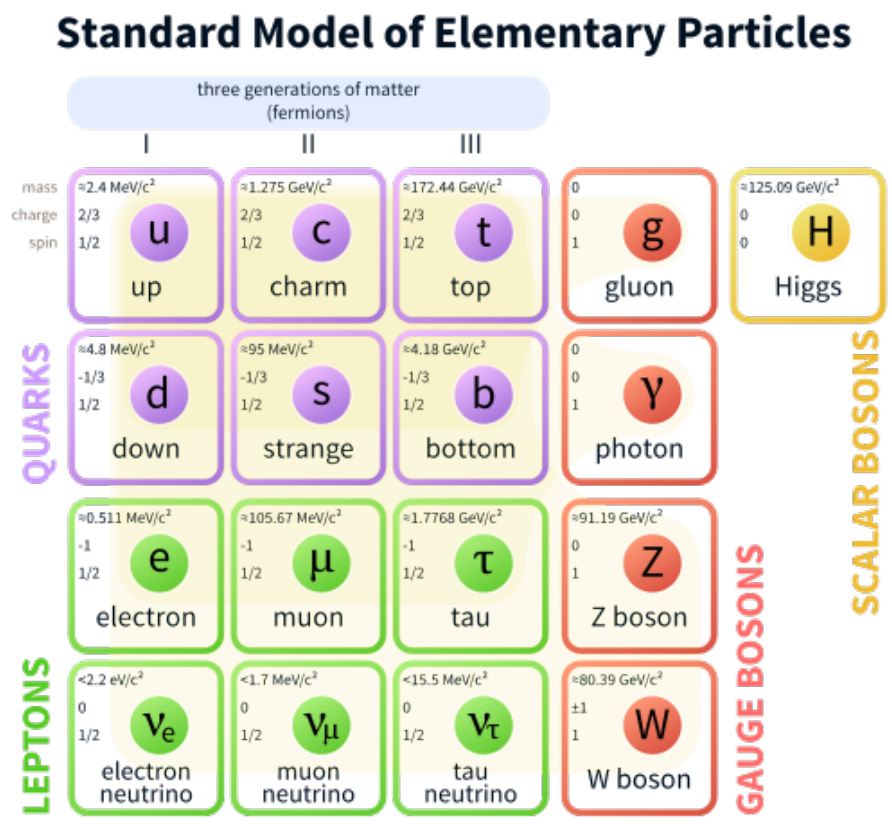

Figure 2.4: List of elementary particles [22]

\subsection{The Standard Model of Particle Physics}

According to the Standard model of Particle Physics, the observable universe around us is made up of three kinds of particles: quarks, leptons and gauge bosons and a Higgs boson. There are six flavors of quarks (along with their corresponding anti-particles) and six leptons (and their anti-leptons). The gauge bosons are the force communicators between elementary particles. Figure 2.4 shows a chart of all the current fundamental building blocks, their mass, charge and spin. The photon is the force carrier of the electromagnetic force, the $W^{ \pm}$and $Z^{0}$ are carriers of the weak force, the gluon is the carrier of the strong nuclear force and the graviton (which hasn't been found yet) is presumably the carrier of gravitational force. Quarks and leptons are fermions (which obey Fermi-Dirac statistics) and the force mediators are bosons (which obey Bose-Einstein statistics). A full discussion of all the forces is beyond the scope of this thesis and can be found in [23, 24, 25]. Some 
aspects of the strong nuclear force which are relevant to nuclear structure will be discussed.

\subsubsection{Quantum Chromodynamics}

Quantum Chromodynamics (QCD) is the gauge theory that describes the interactions of colored particles (quarks and gluons). It is the $\mathrm{SU}(3)_{\text {color }}$ component of $\mathrm{SU}(3)_{\text {color }} \times \mathrm{SU}(2)_{\text {weak }} \times \mathrm{U}(1)_{\gamma}$ Standard Model of particle physics [26]. It has many analogous features and key differences with Quantum Electrodynamics (QED), which describes the interactions between electrically

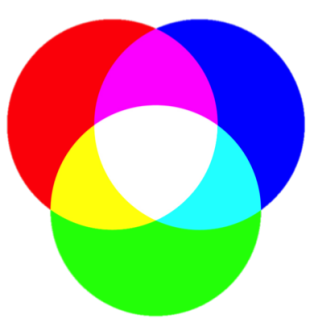
charged particles. According to QCD, besides electrical charge, Figure 2.5: Combistrongly interacting objects carry an additional type of charge called color ${ }^{4}$. There exists three types of color $(r-r e d, g-$ green, $\mathrm{b}$ - blue) along with their respective anti-colors. A combination of nation of red, blue and green making the overlap region "colorless"

three colors, three anti-colors or a color and the same anti-color makes a composite entity, such as a meson, baryon, tetra-quark or a penta-quark "colorless". Figure 2.5 shows an analogy of how the combination of three primary colors (red, blue and green) can make the canvas colorless, possibly supporting the choice of naming scheme of this new degree of freedom. Gluons carry a color and an anti-color. Unlike the photon (quanta of the electromagnetic field) which is electrically neutral, the gluon carries color and can interact with other gluons due to this feature.

The dynamics of the strong interaction are described by the QCD Lagrangian given by:

$$
\mathcal{L}_{Q C D}=\sum_{q} \bar{\psi}_{q, a}\left(i \gamma^{\mu} \partial_{\mu} \delta_{a b}-g_{s} \gamma^{\mu} t_{a b}^{C} \mathcal{A}_{\mu}^{C}-m_{q} \delta_{a b}\right) \psi_{q, b}-\frac{1}{4} F_{\mu \nu}^{A} F^{A \mu \nu}
$$

where $\gamma^{\mu}$ is the Dirac $\gamma$-matrix, the $\psi, \bar{\psi}_{q, a}$ are the quark-field spinors for a quark of flavor $q$ and mass $m_{q}$, with a color index that sums from $a=1$ to $N_{C}=3$ (since the quarks come in three colors), $\mathcal{A}_{\mu}^{C}$ correspond to the gluon fields with $\mathrm{C}$ running from 1 to $N_{C}^{2}-1=8$

\footnotetext{
4"Color" in QCD has nothing to do with color associated with vision.
} 
(since the gluons come in 8 color anti-color combinations), $t_{a b}^{C}$ are the eight $3 \times 3$ GellMann matrices of the SU(3) color group, $g_{s}$ is the QCD coupling constant and finally the field tensor $F_{\mu \nu}^{A}$ is given by:

$$
F_{\mu \nu}^{A}=\partial_{\mu} \mathcal{A}_{\nu}-\partial_{\nu} \mathcal{A}_{\mu}-g_{s} f_{A B C} \mathcal{A}_{\mu}^{B} \mathcal{A}_{\nu}^{C}
$$

where $f_{A B C}$ are the structure constants of the SU(3) group. The QCD coupling constant $g_{s}$ $=\sqrt{4 \pi \alpha_{s}}$ where $\alpha_{s}$ is given by

$$
\alpha_{s}=\frac{g^{2}(\mu)}{4 \pi}=\frac{12 \pi}{\left(33 N_{C}-2 n_{f}\right) \ln \left(\frac{\mu^{2}}{\Lambda_{Q C D}^{2}}\right)}
$$

where $N_{C}$ is the number of colors, $n_{f}$ is the number of flavors of quarks in the summation, $\mu$ is the renormalization scale, $\Lambda_{Q C D}$ is the QCD scale ( $\left.\sim 200 \mathrm{MeV}\right)$ [8]. The coupling constant of a force is an indicator of the strength of the interaction. In QED, the electromagnetic coupling constant ( $\alpha_{e} \approx 1 / 137$ ) has a weak dependence on $Q^{2}$, the resolution of the probe. As an electromagnetic probe approaches the test charge, the force it feels becomes greater due to the absence of screening from the surrounding polarized vacuum. As seen in Figure 2.6, the $\alpha_{Q E D}$ approaches a Landau pole (where the coupling constant becomes infinity) with increasing $Q^{2}$ values. Contrary to this, from equation 2.5, one can see that the strong force coupling constant $\left(\alpha_{s}\right)$ has a logarithmic decrease with increasing resolution. The logarithmic

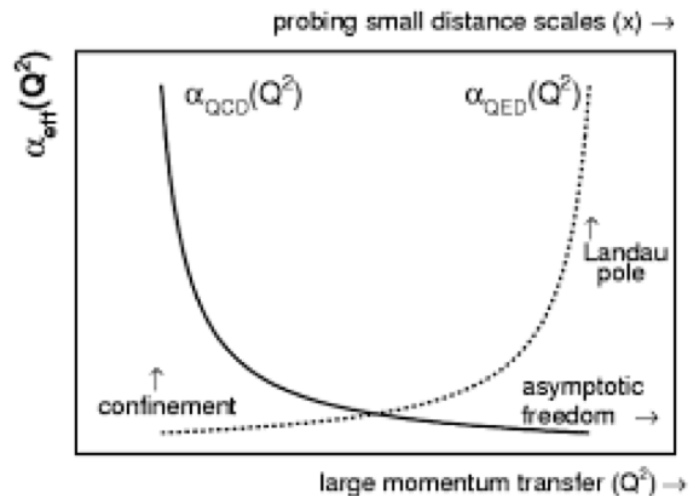

Figure 2.6: Behavior of QED and QCD coupling constants as a function of momentum transfer $\left(Q^{2}\right)$ decrease of the coupling constant implies that as a probe (which is colored) approaches another colored entity (surrounded by other colored objects induced in the QCD medium), 
the strong force decreases. Colored objects feel almost no force when close together, a phenomenon known as 'asymptotic freedom', discovered by Politzer, Gross and Wilczek $[27,28]$. However, as the distance between them grows, the coupling constant becomes very large. This is the principle that makes it difficult for quarks to isolate themselves from a nuclear medium ('quark confinement'). It is due to these important and crucial features that the nucleus can be used to study quark interactions but individual quarks cannot be isolated from the nucleus.

\subsection{Probing Nucleon and Nuclear Structure}

Understanding nucleon and nuclear structure is one of the central goals of nuclear physics. The nucleus is a dynamical QCD composite object which displays a multitude of phenomenon based on individual and collective behavior of its building

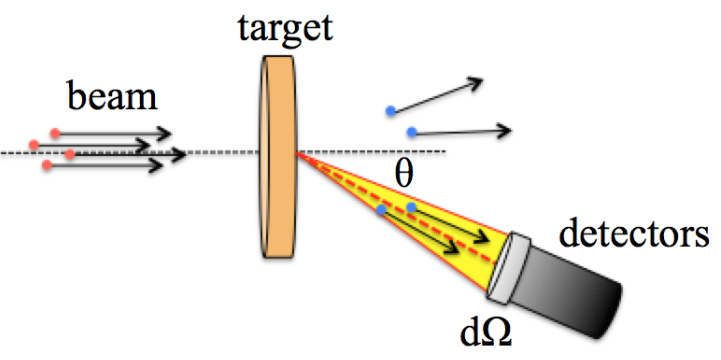
blocks, quarks, gluons, protons and neutrons (collectively called nucleons).

Much of the wealth of information on Figure 2.7: Depiction of a typical fixed target scattering experiment nucleon and nuclear structure comes from scattering experiments. Figure 2.7 shows the cartoon of a typical fixed target scattering experiment. The essence of these experiments is similar to Rutherford's $\alpha$ ray gold foil experiment where a:

- Beam and target of known parameters (particle type, energy, spin etc.) and whose structure we would like to study, are chosen.

- Beam particles are incident (or collide) on (with) the target and detector hits are recorded.

- Data are analyzed and cross sections are calculated. 
- Physics of the internal structure of the target (or beam) is extracted and interpreted.

Nucleon structure as it is understood today, has been deduced in stages over a period of several decades of worldwide theoretical and experimental effort. Several elastic scattering experiments with electron and muon beams were conducted in the 50's, which were crucial for the establishment of the nucleon as a composite object. Until then, the nucleon was first thought of as a point particle without spin. Then, spin and a spatial extent were added with the goal to determine the charge and magnetization distributions (that arise due to the internal motion of these charges). With higher momentum transfer $Q^{2}$ i.e. increased resolution $(\sim \hbar / Q)$, a deeper rich inner sub-structure of the nucleus emerged.

\subsection{Elastic Scattering}

\subsubsection{Nucleon Form Factors and Structure Functions}

To begin with, Rutherford's differential scattering formula is shown below:

$$
\left(\frac{d \sigma}{d \Omega}\right)_{\text {Ruth }}=\left(\frac{z Z e^{2}}{16 \pi \epsilon_{0} E_{k i n}}\right)^{2} \frac{1}{\sin ^{4}\left(\frac{\theta}{2}\right)}
$$

where $Z$ is the central charge of the nucleus, $z$ is the charge of the $\alpha$ particle, $E_{k i n}$ is the kinetic energy $y^{5}$ of the $\alpha$ particle, and $\theta$ is the angle of deflection in the lab frame. In this expression, the target and the beam particles are assumed to be non-relativistic structureless point particles with spin 0 that undergo an elastic collision. The recoil of the heavy target nucleus is neglected and first Born approximation is used for the incident and outgoing beam particle. However, when the spin of the particles are considered and helicity conserved, the formula for the differential cross section changes to:

$$
\left(\frac{d \sigma}{d \Omega}\right)_{\mathrm{Mott}}=\left(\frac{d \sigma}{d \Omega}\right)_{\mathrm{Ruth}}\left(1-\beta^{2} \sin ^{2}\left(\frac{\theta}{2}\right)\right)\left(\frac{E^{\prime}}{E}\right)
$$

\footnotetext{
${ }^{5}$ In order for the target particle to achieve a resolution of $>1 \mathrm{fm}$, electrons of several hundreds of $\mathrm{MeV}$ energy are required.
} 
where $E\left(E^{\prime}\right)$ is the energy of the incident (scattered) particle and $\beta=v / c$ where $v$ is the speed of the incident particle. When the electric and magnetic interaction between an electron and a point like proton is taken into consideration along with the elastic recoil, the cross section changes to:

$$
\left(\frac{d \sigma}{d \Omega}\right)_{\mathrm{expt}}=\left(\frac{d \sigma}{d \Omega}\right)_{\mathrm{Mott}}\left[1+\tau \tan ^{2}\left(\frac{\theta}{2}\right)\right]
$$

where $\tau=\frac{Q^{2}}{4 M^{2} c^{2}}$. The first elastic scattering experiments were conducted with electron beams on liquid hydrogen targets by Robert Hofstadter [29] in order to understand proton electric and magnetic form factors.

A deviation of experimental cross section from theory predictions were observed (as shown in Figure 2.8) which were crucial to establishing that the nucleon is a composite object and not a point particle. Traditionally, an additional term $F(\vec{q})$ is usually added to the Mott cross section to include the spatial extent of the nucleus. This term usually called the "form factor" accounts for the structure of the hadron's 'deviation' from point particle ${ }^{6}$.

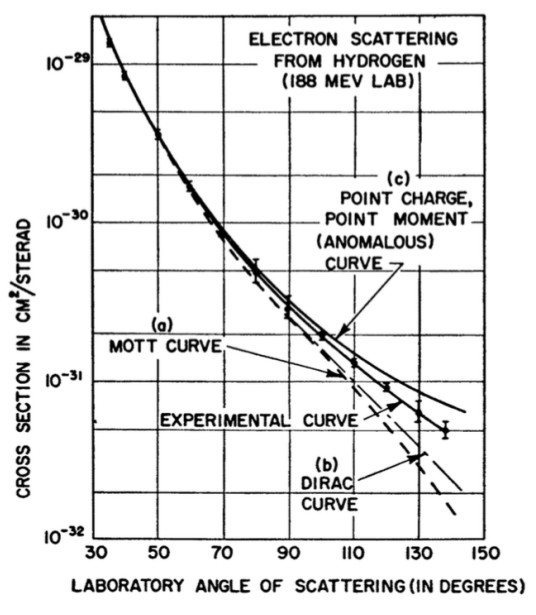

$$
\left(\frac{d \sigma}{d \Omega}\right)_{\text {expt }}=\left(\frac{d \sigma}{d \Omega}\right)_{\text {Mott }}\left|F\left(\overrightarrow{q^{2}}\right)\right|^{2}
$$

Figure 2.8: Elastic scattering experiments by Hofstadter showing deviation between experimental cross section and Mott cross section [29].

When the spatial extent of the nucleon is taken into consideration, two form factors are required to describe the structure of the nucleon and the cross section is given by the Rosen-

\footnotetext{
${ }^{6} F(\vec{q})$ becomes 1 for a point particle without any spatial extent.
} 
bluth formula [30]:

$$
\left(\frac{d \sigma}{d \Omega}\right)_{\mathrm{expt}}=\left(\frac{d \sigma}{d \Omega}\right)_{\mathrm{Mott}}\left[\frac{G_{E}^{2}\left(Q^{2}\right)+\tau G_{M}^{2}\left(Q^{2}\right)}{1+\tau}+2 \tau G_{M}^{2}\left(Q^{2}\right) \tan ^{2}\left(\frac{\theta}{2}\right)\right]
$$

where $\tau=\frac{Q^{2}}{4 M^{2} c^{2}}, G_{E}$ and $G_{M}$ are Sachs electric and magnetic form factors which depend on $Q^{2}$ [31]. At low $Q^{2}$ (where the scattering is still in the elastic regime), the proton (and neutron) electric and magnetic form factors can be approximately described by the dipole form factor:

$$
\begin{aligned}
G_{E}^{p}\left(Q^{2}\right) & =\frac{G_{M}^{p}\left(Q^{2}\right)}{\mu_{p}}=\frac{G_{M}^{n}\left(Q^{2}\right)}{\mu_{n}}=G_{D}\left(Q^{2}\right) \\
G_{D}\left(Q^{2}\right) & =\frac{1}{\left(1+\frac{Q^{2}}{0.71 \mathrm{GeV}^{2}}\right)^{2}}
\end{aligned}
$$

where $\mu_{p}$ and $\mu_{n}$ are the nuclear magnetic moments of the proton and the neutron. As can be seen in in the above equation, the surprising fact that experiments have observed a strong $Q^{2}$ dependence [32] in the cross section $\left(\propto Q^{-8}\right)$ for elastic scattering experiments suggested that proton has a rich inner substructure beyond that of a spin $1 / 2$ point particle.

\subsection{Deep Inelastic Scattering}

High-energy lepton nucleon scattering plays an important role in determining the structure of the nucleon. When the energy given to the target nucleon is slightly increased, new resonance states of the nucleon (quasi-elastic region) become possible hinting at a composite structure. The invariant mass squared of the hadronic state becomes:

$$
W^{2}=M^{2}+2 P \cdot q+q^{2}=M^{2}+2 M \nu+q^{2}
$$

where $M$ is the mass of the nucleon, $W$ is the invariant mass of the composite system, $P$ is the four momentum vector of the incoming nucleon, $q$ is the four momentum vector of 


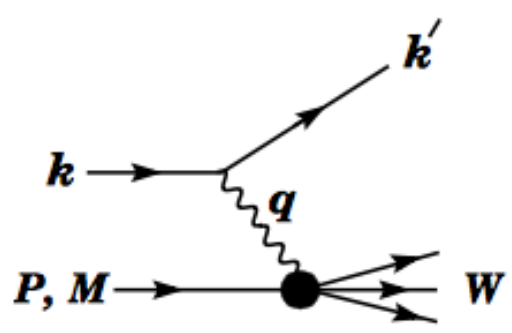

Figure 2.9: Feynman diagram of deep inelastic scattering

the virtual photon exchanged, and Lorentz-invariant quantity $\nu=\frac{2 P \cdot q}{M}=E-E^{\prime}$. For an elastic scattering process, $W=M$. Then $q^{2}+2 M \nu=0$. For an inelastic process, $q^{2}+2 M \nu>0$ as $W>M$. If one were to define $-q^{2}=Q^{2}$, and a variable $x=\frac{Q^{2}}{2 M \nu}$, then $x=1$ for elastic processes and $0<x<1$ for inelastic processes. The inelastic cross section is now written in terms of two structure functions $W_{1}$ and $W_{2}$ as [33]:

$$
\left(\frac{d \sigma}{d \Omega}\right)_{\mathrm{expt}}=\left(\frac{d \sigma}{d \Omega}\right)_{\mathrm{Mott}}\left[W_{2}\left(Q^{2}, \nu\right)+2 W_{1}\left(Q^{2}, \nu\right) \tau \tan ^{2}\left(\frac{\theta}{2}\right)\right]
$$

where $W_{1}$ and $W_{2}$ are the structure functions that summarize all the information about the internal structure of the target nuclei. It is interesting to note that the structure functions now depend on two variables $\left(Q^{2}\right.$ and $\nu$ ) instead of just one ( $Q^{2}$ as in Equation 2.10). Experiments conducted with a much more energetic beam of electrons also show a surprising disagreement with expectations for elastic scattering cross sections [34]. As discussed in the previous section, Equation 2.9 when divided by the point proton Mott cross section reveals a form factor of the probed object. The initial expectation at that time was that the nucleon was an extended object with a diffused charge distribution. As can be seen in Figure 2.10, the dipole form factor (in Equation 2.10) falls off rapidly (as a function of $Q^{-8}$ ) where as $\sigma_{\text {expt }} / \sigma_{\text {Mott }}$ for the inelastic case has a weak dependence on $Q^{2}$. This showed that the electron was consistently scattering off of structureless point particles (above a certain $W$ threshold) inside the nucleon, a viewpoint quite different from initial expectation. 


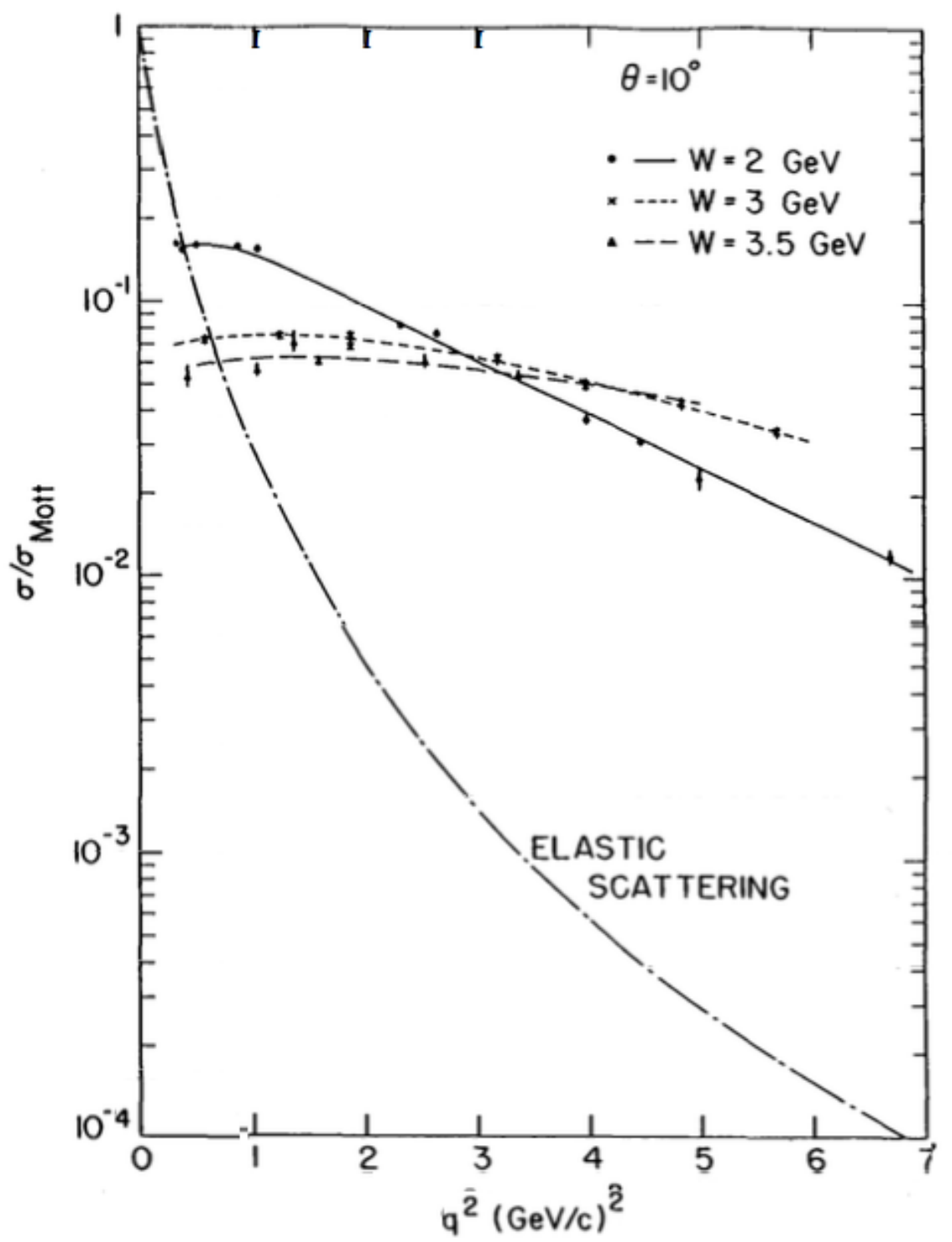

Figure 2.10: Deviation between experimental cross section and elastic cross section observed in the case of deep inelastic scattering experiments conducted at SLAC [34]. 


\begin{tabular}{|c|c|}
\hline Variable & Definition \\
\hline$P$ & Four momentum vector of the nucleon \\
\hline$q$ & $\begin{array}{l}\text { Four momentum vector of the exchanged } \\
\text { gauge boson }\end{array}$ \\
\hline$k=(E, \vec{k}), k^{\prime}=\left(E^{\prime}, \overrightarrow{k^{\prime}}\right)$ & $\begin{array}{l}\text { Four momentum vectors of the incoming and } \\
\text { outgoing lepton }\end{array}$ \\
\hline$\nu=\frac{q \cdot P}{M}=E-E^{\prime}$ & $\begin{array}{l}\text { Energy loss of the lepton in the nucleon rest } \\
\text { frame }\end{array}$ \\
\hline$Q^{2}=-q^{2}=2\left(E E^{\prime}-\vec{k} \cdot \overrightarrow{k^{\prime}}\right)$ & Momentum transfer to the nucleon \\
\hline$W^{2}=(P+q)^{2}=M^{2}+2 M \nu+q^{2}$ & Invariant mass squared of the hadronic state \\
\hline$s=(P+k)^{2}$ & $\begin{array}{l}\text { Center-of-mass energy squared of the lepton } \\
\text { nucleon system }\end{array}$ \\
\hline$\theta$ & $\begin{array}{l}\text { Scattered angle of the lepton in the nucleon } \\
\text { rest frame }\end{array}$ \\
\hline$x_{B j}$ or $x=\frac{Q^{2}}{2 M \nu}$ & Bjorken Scaling variable \\
\hline
\end{tabular}

Table 2.1: Definition of some kinematic quantities used in DIS

\subsection{Merging Quarks and Partons: The Quark-Parton Model}

James Bjorken conjectured that in the limit $Q^{2}, \nu \rightarrow \infty$ with the ratio $\omega=\frac{2 M \nu}{q^{2}}$ held fixed, the two quantities $\nu W_{1}$ and $W_{2}$ should both become functions of $\omega$ only. That is:

$$
\begin{aligned}
\lim _{Q^{2, \nu} \rightarrow \infty} 2 M W_{1}\left(\nu, q^{2}\right) & =F_{1}(\omega) \\
\lim _{Q^{2, \nu} \rightarrow \infty} \nu W_{2}\left(\nu, q^{2}\right) & =F_{2}(\omega)
\end{aligned}
$$

It is this property that is referred to as "Bjorken scaling" where the structure functions scale approximately as a function of one variable instead of two in the Bjorken limit [35]. 
Richard Feynman immediately recognized the connection between partons in high energy collisions, Bjorken scaling and the weak $q^{2}$ dependence of the cross sections (as seen in Figure 2.10) [33]. He proposed that the proton was made up of point like partons from which the electrons scattered incoherently. He considered the infinite momentum frame; a reference frame in which the proton is moving infinitely fast, the rest masses and the transverse momentum of these partons are small compared to the longitudinal momentum and can be ignored. These partons are assumed not to interact with one another while the virtual photon was being exchanged (called 'impulse approximation ${ }^{7}$ ') essentially to first order giving a momentary glimpse of the proton structure as a function of the sum of the longitudinal momenta of various partons. The unknown interacting electromagnetic spin 1/2 partons were later identified with quarks as confirmed by the Callan-Gross relation $[36]^{8}$. It is interesting to note that the proton which was initially modeled to be made up of three quarks has been remodeled to include a background of gluons and quark anti-quark pairs $[34,37]$.

\subsubsection{Structure Functions in the Quark-Parton Model}

In the infinite momentum frame, under the impulse approximation, the nucleon can be decomposed into 'free' partons which carry a certain fraction $\xi$ of the nucleon's total longitudinal momentum. This term $\xi$ is mathematically equivalent to the Bjorken scaling variable $x_{B j}$ mentioned in the previous section ${ }^{9}$. If the nucleon is built up of different kinds of quark

\footnotetext{
${ }^{7}$ According to the impulse approximation, the time taken for the partons to interact with each other is Lorentz dilated and large compared to the time it takes to exchange a boson with the incident beam particle. Hence these particles are essentially momentarily 'free' but are bound to the interactions of rest of the nucleon immediately afterwards.

${ }^{8} 2 F_{1}(\omega)=\omega F_{2}(\omega)$ for a spin $1 / 2$ electromagnetic current and 0 for a spin 0 current.

${ }^{9}$ Here on, the Bjorken scaling variable $x_{B j}$ will be referred to as just $x$.
} 
flavors, then the structure functions $F_{1}\left(x, Q^{2}\right)$ and $F_{2}\left(x, Q^{2}\right)$ are given by

$$
\begin{aligned}
& F_{2}\left(x, Q^{2}\right)=x \sum_{i} e_{i}^{2}\left[q_{i}\left(x, Q^{2}\right)+\bar{q}_{i}\left(x, Q^{2}\right)\right] \\
& F_{2}\left(x, Q^{2}\right)=2 x F_{1}\left(x, Q^{2}\right)
\end{aligned}
$$

where $e_{i}$ is the charge of the struck quark and $q_{i}\left(x, Q^{2}\right)$ is a parton number density of a certain kind of a parton $i$ to have a fraction of momentum $x$ between $x$ and $x+d x$ at a given $Q^{2}$. If we consider only light quarks $(u, d, s)$ for the case of proton and neutron, the structure functions for lepton-nucleon scattering are:

$$
\begin{aligned}
& F_{2}^{l p}\left(x, Q^{2}\right)=x\left[\frac{1}{9}\left(d^{p}+\bar{d}^{p}\right)+\frac{4}{9}\left(u^{p}+\bar{u}^{p}\right)+\frac{1}{9}\left(s^{p}+\bar{s}^{p}\right)\right] \\
& F_{2}^{l n}\left(x, Q^{2}\right)=x\left[\frac{1}{9}\left(d^{n}+\bar{d}^{n}\right)+\frac{4}{9}\left(u^{n}+\bar{u}^{n}\right)+\frac{1}{9}\left(s^{n}+\bar{s}^{n}\right)\right]
\end{aligned}
$$

where $u^{p, n}\left(\bar{u}^{p, n}\right)$ are the parton distribution functions ${ }^{10}$ of up (anti-up) quarks in the proton and neutron ${ }^{11}$. Combining data from various experiments and making global fits allows the extraction of individual parton distribution functions. Additional global constraints are put on the parton distribution functions to conserve the overall net quark numbers. For up quarks,

$$
\int_{0}^{1}(u(x)-\bar{u}(x)) d x=2
$$

For down quarks

$$
\int_{0}^{1}(d(x)-\bar{d}(x)) d x=1
$$

For strange quarks

$$
\int_{0}^{1}(s(x)-\bar{s}(x)) d x=0
$$

\footnotetext{
${ }^{10}$ The dependence of parton distribution functions on $\left(x, Q^{2}\right)$ is ignored for brevity.

${ }^{11}$ Discussion of additional structure functions obtained from neutrino and anti-neutrino deep inelastic scattering are beyond the scope of this thesis.
} 
Using charge symmetry (which is different from isospin symmetry ${ }^{12}$ ), one can access the neutron structure functions as well 13 by assuming that.

$$
\begin{gathered}
u^{p}\left(x, Q^{2}\right)=d^{n}\left(x, Q^{2}\right), \\
d^{p}\left(x, Q^{2}\right)=u^{n}\left(x, Q^{2}\right), \\
s^{p}\left(x, Q^{2}\right)=s^{n}\left(x, Q^{2}\right) .
\end{gathered}
$$

Since the anti-quarks contribute mostly to the sea of the nucleon, typically one identifies the "valence" region as

$$
q_{v}(x)=q(x)-q_{s}(x)=q(x)-\bar{q}_{s}(x)
$$

where $q_{v}(x)$ or $q_{v a l e n c e}(x)$ are the valence quark distributions and $q_{s}(x)$ or $q_{s e a}(x)=\bar{q}_{s}(x)$ are the sea quark distributions. It is impossible to point to any individual quark and identify whether it belongs to the sea or valence region given its quantum numbers. Some textbooks define "valence" quarks from a 'constituent quark' point of view as the three quarks that are responsible for the quantum numbers of proton. This is a purely model and resolution $\left(Q^{2}\right)$ dependent statement. In a model where the sea quarks and gluons are ignored perhaps this might be true. We now know that there are many nucleon properties (such as spin) that may have substantial contributions from transverse and orbital angular momentum of gluons and the sea. An example of NNPDF parton distribution functions decomposed into the valence and sea distributions is shown in Fig 2.11. One can see that the distribution for the valence quarks peak around $x f(x) \approx 0.25$ and those for the sea at a much smaller $x$ values. The evolution of these PDFs from one energy scale to another can be calculated using the Dokshitzer-Gribov-Lipatov-Altarelli-Parisi (DGLAP) equations [38, 39, 40].

\footnotetext{
${ }^{12}$ Charge symmetry states that if the mass of the up and down quark are the same then there is nothing in the QCD Lagrangian that distinguishes the two, hence physics has to be invariant under interchanging up and down quarks.

${ }^{13}$ Since there are no free neutron targets that are readily available for scattering.
} 

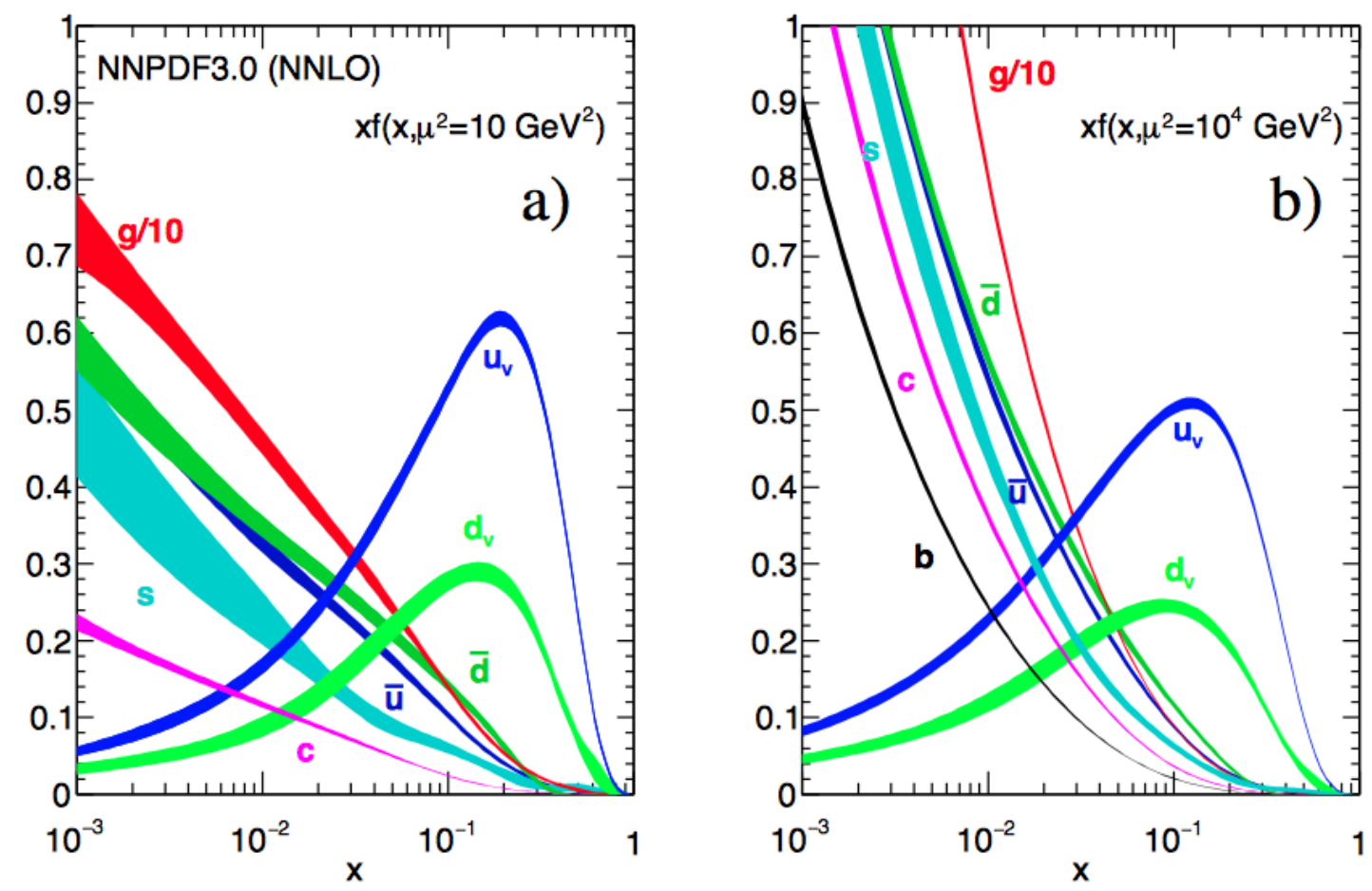

Figure 2.11: Bands show Next-to-Next-to Leading Order Neural Network Parton Distribution Functions obtained in global analysis at scales $\mu^{2}=10 \mathrm{GeV}^{2}$ (left) and $\mu^{2}=10000$ $\mathrm{GeV}^{2}$ (right) [8]. One can see that as $Q^{2}$ (resolution) increases, the valence distributions decrease slightly and the gluon and sea quark distributions rise up rapidly revealing more structure at low- $x$. 


\subsection{Accessing the Anti-Quark Distributions: The Drell-Yan Process}

The nucleon is a bound state QCD system and non-perturbative and perturbative effects play a key role in understanding nucleon structure. $98 \%$ of the nucleon's mass comes from something other than the rest mass of the valence quarks. This suggests that gluon and sea dynamics has to be important in explaining the global properties of the nucleon (such as mass, spin etc).

In 1970, Christenson et al. measured a production of massive lepton pairs in $29 \mathrm{GeV} / c$ proton Uranium collisions at high energies [41]. Two prominent features can be noted in their cross section versus invariant mass of dimuons as shown in Figure 2.12 .

- A shoulder near an invariant mass around 3.1 $\mathrm{GeV}$ (which we now know is because of the $J / \psi$ particle and its resonance states).

- An underlying continuum that fell rapidly as a function of the invariant mass.

Sidney Drell and Tung-Mow Yan interpreted this in the framework of the parton model as a parton from one hadron annihilating with an anti-parton from another hadron forming a virtual photon that decays into a lepton anti-lepton $\left(l^{+} l^{-}\right)$[42].

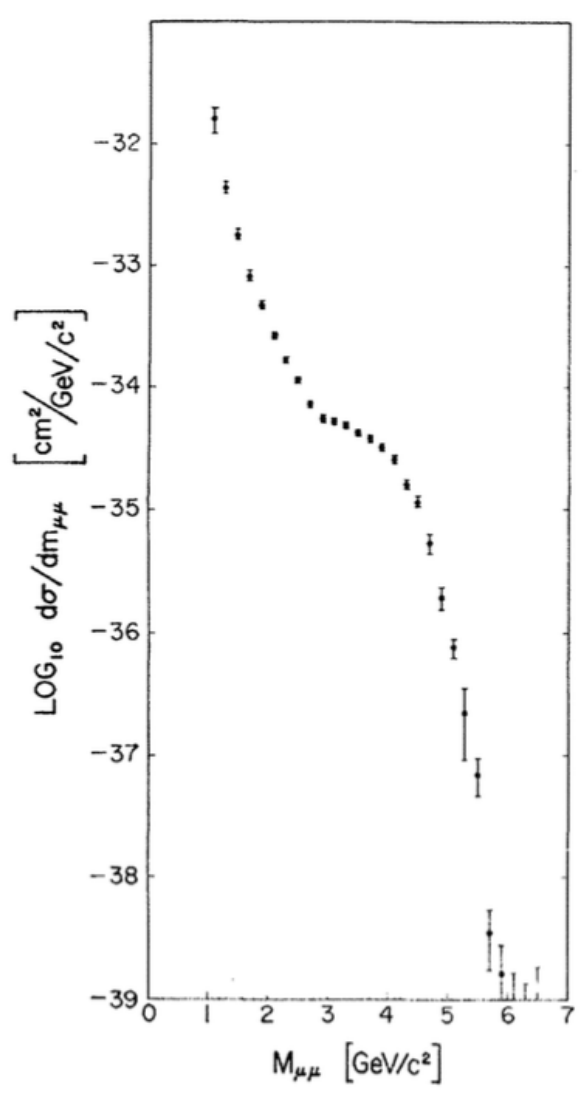

Figure 2.12: Cross section as a function of the invariant mass taken from [41]

$$
q \bar{q} \rightarrow \gamma^{*} \rightarrow l^{+} l^{-}
$$




\subsubsection{Kinematics of the Drell-Yan Process}

Generally, a lot of hadrons accompany the dileptons $\left(e^{+} e^{-}, \mu^{+} \mu^{-}\right.$or $\left.\tau^{+} \tau^{-}\right)$produced in the Drell-Yan process. Tracks of these dileptons are reconstructed and momenta extracted. SeaQuest basically:

- Found the measured 4 momentum of the muons in the lab frame

- Added them to find the 4 momentum of the virtual photon in the lab frame

- Squared the 4 momentum of the virtual photon to get the mass

- Boosted the 4 momentum to the Collins-Sopher frame

- Calculated $x_{F}$

- Calculated $x_{B}$ and $x_{T}$ from $x_{F}$ and mass

The combined properties of the dileptons reflect the properties of the virtual photon $\left(\gamma^{*}\right)$ that decayed from quark anti-quark annihilation. Using the Mandelstam variable $s$ and the dimuon momenta, one can calculate several variables of the virtual photon (such as longitudinal momentum $p_{l}$, invariant mass of the virtual photon, $M_{\gamma^{*}}$, energy of the virtual photon $E, \tau$, rapidity $y$ and $x_{F}$ or Feynman- $x$ ) by the following equations:

$$
\begin{gathered}
\tau=M_{\gamma^{*}}^{2} / s=x_{\text {beam }} x_{\text {target }} \\
y=\frac{1}{2} \ln \left(\frac{E+p_{l}}{E-p_{l}}\right) \\
x_{\text {Beam }}=\frac{P_{\text {target }} * P_{\text {sum }}}{P_{\text {target }} * P_{\text {cms }}} \\
x_{\text {Target }}=\frac{P_{\text {beam }} * P_{\text {sum }}}{P_{\text {beam }} * P_{\text {cms }}}
\end{gathered}
$$




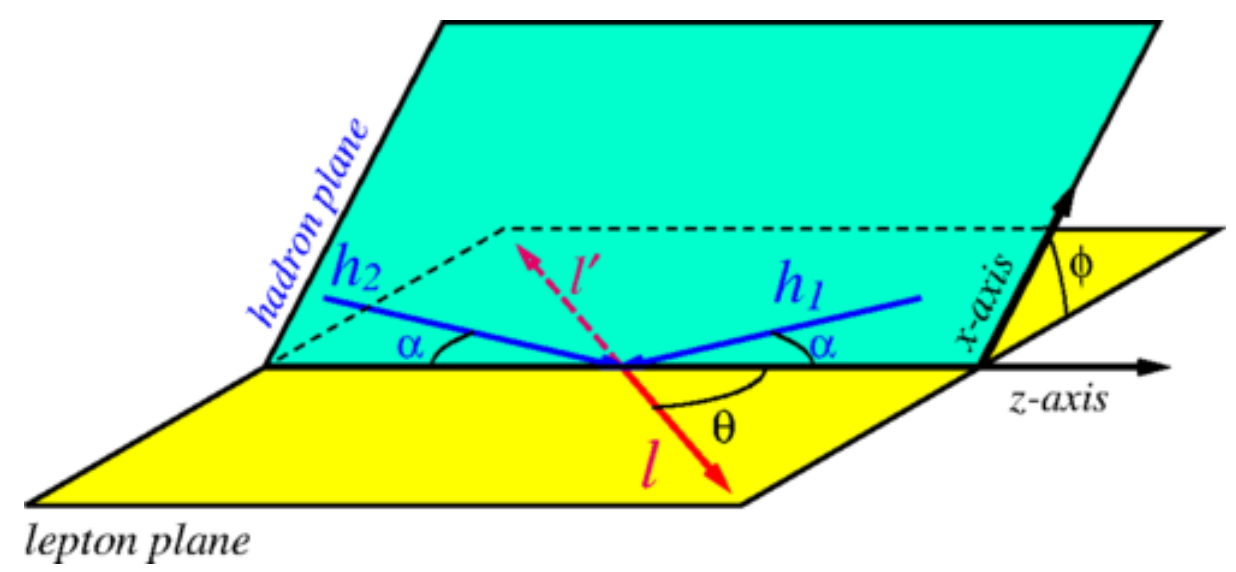

Figure 2.13: Collins-Soper frame is the center of mass frame of the dileptons produced in hadron-hadron collisions. The $\mathrm{Z}$ axis points along the beam direction. $h 1$ and $h 2$ are the colliding hadrons, $l$ and $l^{\prime}$ are the dileptons, $\theta$ is the polar angle and $\phi$ is the azimuthal angle. Figure taken from [43].

where $P_{\text {beam }}=\left(0,0, \sqrt{E_{\text {beam }}^{2}-m_{p}^{2}}, E_{\text {beam }}\right), P_{\text {target }}=\left(0,0,0, m_{p}\right), P_{\text {cms }}=P_{\text {beam }}+$ $P_{\text {target }}$ and $P_{\text {sum }}=P_{\text {pos }}+P_{\text {neg }}$ where $P_{\text {pos }}$ and $P_{\text {neg }}$ are the four vectors of the $\mu^{+}$and $\mu^{-}$ respectively. It should be noted that E866 experiment used $p_{l}^{\max }=\sqrt{s} / 2$.

$$
\begin{array}{r}
x_{F}=\frac{p_{l}}{p_{l}^{\text {max }}} \approx x_{\text {beam }}-x_{\text {target }} \\
p_{l}^{\text {max }}=\frac{\sqrt{s}}{2}\left(1-\frac{m_{\gamma^{*}}^{2}}{s}\right)
\end{array}
$$

These variables could also be used to extract information about the annihilating quark antiquark pair. Additional variables of the virtual photon (such as transverse momentum $p_{T}, \theta$ and $\phi$ ) can also be calculated from the momenta of the dileptons. The geometric depiction of some of these variables can be seen in Figure 2.13. 


\subsubsection{Leading Order Cross section of the Drell-Yan Process}

The scattering cross section of two charged, spin $1 / 2$, point particles is given by ${ }^{14}$

$$
d^{2} \sigma=\frac{4 \pi \alpha^{2} e^{2}}{3 M^{2}}
$$

In the case of $q \bar{q}$ annihilation, the $M^{2}$ in Equation 2.27 is simply the mass of the virtual photon. Additionally, a factor of $1 / 3$ in introduced to account for all three colors. Equation 2.27 becomes

$$
d^{2} \sigma=\frac{4 \pi \alpha^{2} e^{2}}{9 M^{2}}=\frac{4 \pi \alpha^{2} e^{2}}{9 x_{\text {beam }} x_{\text {target }} s}
$$

adding the terms for the convolution of the par-

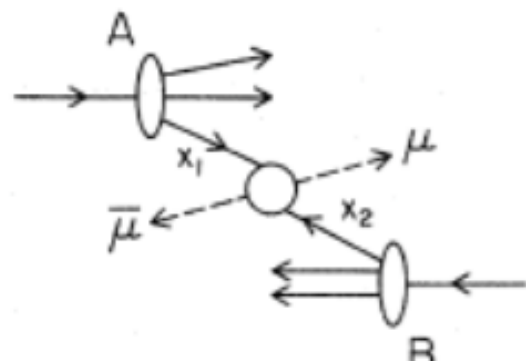

B ton distribution functions of the beam and the tar$\begin{array}{ll}\text { get hadron to the expression, the leading order }{ }^{15} & \text { Figure 2.14: Depiction of the Drell- } \\ \text { Yan process [42] }\end{array}$ Drell-Yan scattering cross section (depicted in Fig-

$$
\underline{Q^{2}=x_{1} x_{2} s}
$$

ure 2.14) is given by

$$
\frac{d^{2} \sigma}{d x_{\text {beam }} d x_{\text {targ }}}=\frac{4 \pi \alpha^{2}}{9 x_{\text {beam }} x_{\text {targ }} s} \sum_{i \in\{u, d, s, \ldots\}}^{n} \underbrace{e_{i}^{2}\left[f_{i}\left(x_{\text {beam }}\right) \bar{f}_{i}\left(x_{\text {targ }}\right)\right.}_{(1)}+\underbrace{\bar{f}_{i}\left(x_{\text {beam }}\right) f_{i}\left(x_{\text {targ }}\right)}_{(2)}]
$$

The leading order cross section is also written in terms of $M^{2}$ and $x_{F}$ as

$$
\frac{d^{2} \sigma}{d M^{2} d x_{F}}=\frac{4 \pi \alpha^{2}}{9 M^{4}} \frac{x_{\text {beam }} x_{\text {target }}}{x_{\text {beam }}+x_{\text {target }}} \sum_{i \in\{u, d, s, \ldots\}}^{n} e_{i}^{2}\left[f_{i}\left(x_{\text {beam }}\right) \bar{f}_{i}\left(x_{\text {targ }}\right)+\bar{f}_{i}\left(x_{\text {beam }}\right) f_{i}\left(x_{\text {targ }}\right)\right]
$$

\footnotetext{
${ }^{14}$ Equation 2.27 is an $s$-channel process where a lepton and an anti-lepton annihilate into another lepton + anti-lepton, whereas equation 2.8 is a $t$-channel process where a virtual photon is exchanged between the nucleus and the scattered lepton.

${ }^{15}$ Leading order cross section considers the simplest picture which ignores the contribution of the higher order processes in $\alpha_{s}$, the strong coupling constant.
} 
where $f_{i}\left(x_{\text {beam }}\right)$ and $\bar{f}_{i}\left(x_{\text {targ }}\right)$ are the quark distributions, $x_{\text {beam }}$ and $x_{\text {targ }}$ are the fractions of longitudinal momentum carried by the participating beam and target quarks, respectively, $s$ is the square of the center of mass energy, $\alpha$ is the fine structure constant revealing the electromagnetic nature of the process, $e_{i}$ is the quark flavor's charge and the sum is over all the quark flavors $(u, d, s, c, t, b)$. At large values of $x$, the quark distributions are dominated by the valence regions, and at small $x$ the quark distributions are dominated by the sea.

\subsubsection{QCD Improved Drell-Yan Process}

The leading order formula gives reasonable results for the mass and $x_{T}$ dependence but yields only $\approx 50 \%$ of the observed cross section. Higher order corrections to the leading order Drell-Yan process that include additional gluon emission and absorption lines (as shown in Figure 2.15) account for the remainder of the cross section. Deep inelastic scattering and Drell-Yan scattering provide complimentary information. As shown in the Feynman diagram in a) in Figure 2.16, the exchanged virtual photon does not differentiate between quarks and anti-quarks. Since the Drell-Yan process involves the annihilation of one anti-quark, one can choose the acceptance of the spectrometer to gain unique sensitivity to anti-quark distributions. The quark parton distributions from DIS are used as an input into Equation 2.29 to extract complimentary information on the anti-quark structure of the nucleon. When modern fits typically parametrize parton distributions and simultaneously fit all data, one observes that (Figure 2.16) points of a particular $x_{T}\left(x_{F}\right)$ value in left (right) plots exhibit a scaling behavior. Several competing mechanisms such as gluon splitting to quark anti-quark pairs, quarks radiating and absorbing gluons and gluons recombining all effectively contribute to an overall scaling violation and a slope of these data points. 

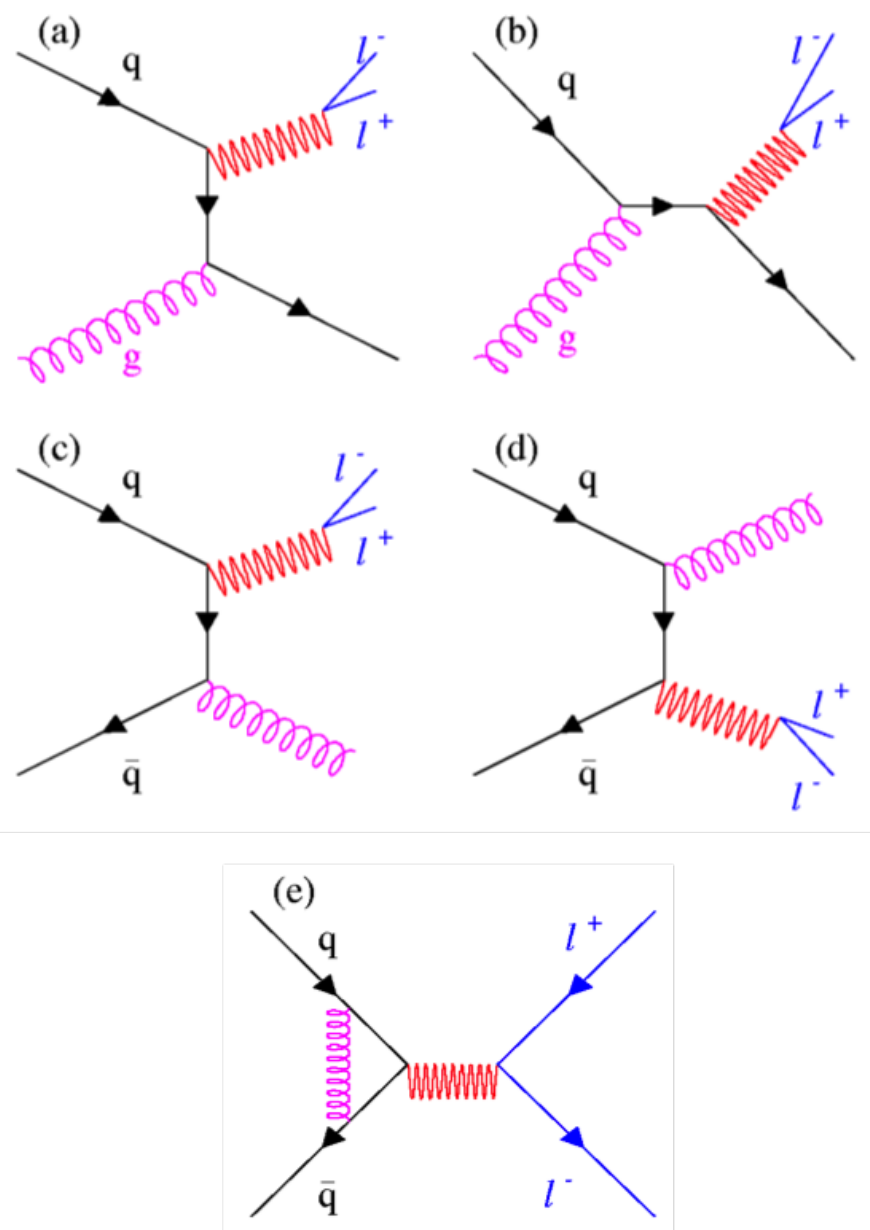

Figure 2.15: Higher order corrections to the leading order Drell-Yan process that include additional gluon diagrams. 

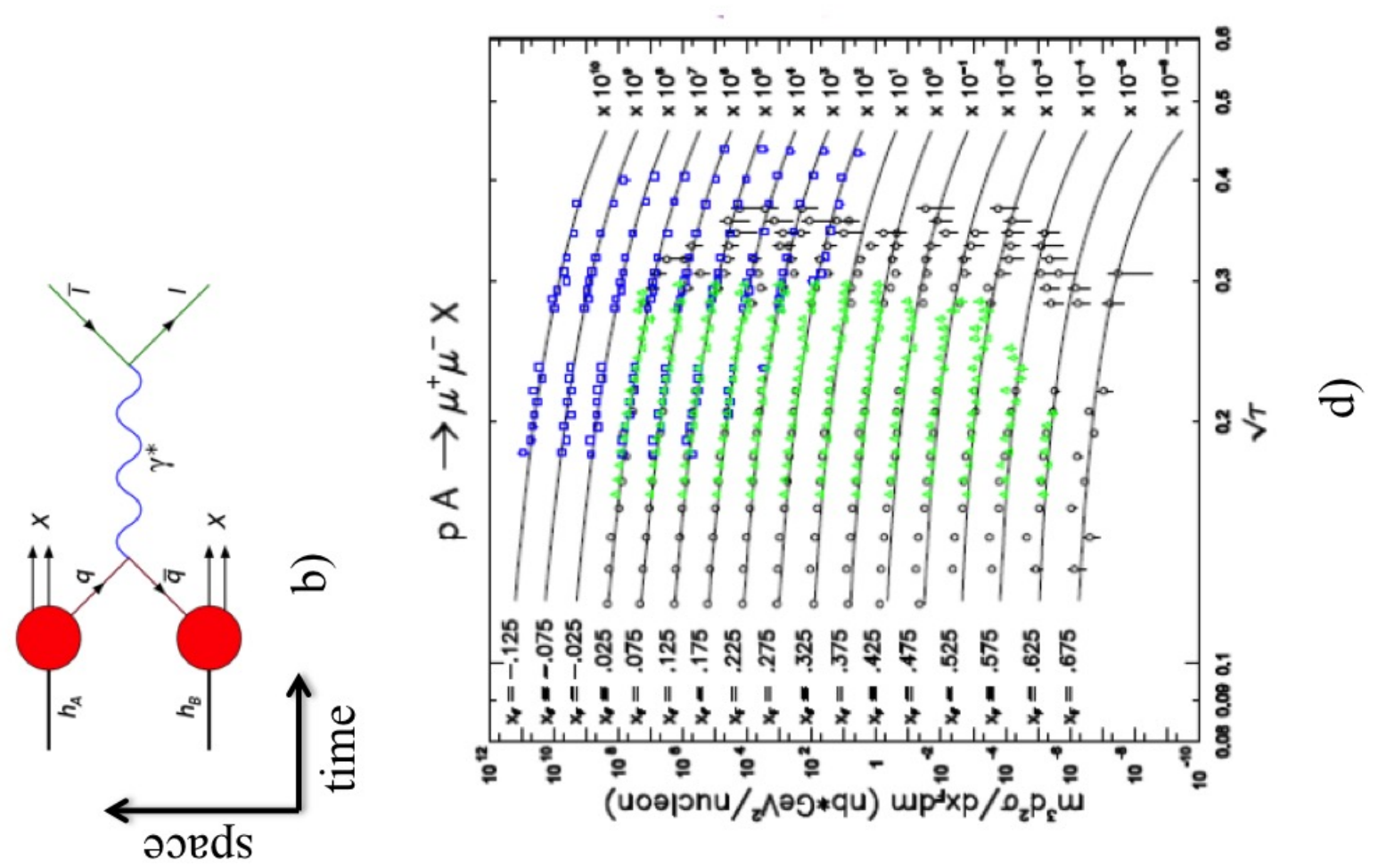

0ิ

总芯定

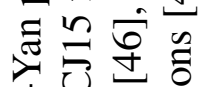

1

จ

0 品

屯

형

on $\overline{0} \approx$

$\exists \ddot{x}$

Ð 0 过

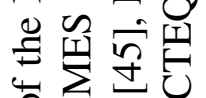

눈

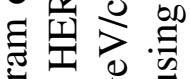

5 i 9

:

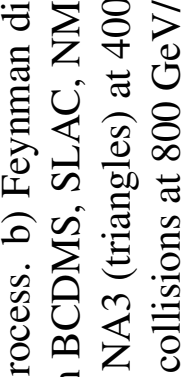

పี छ

我 +

힐
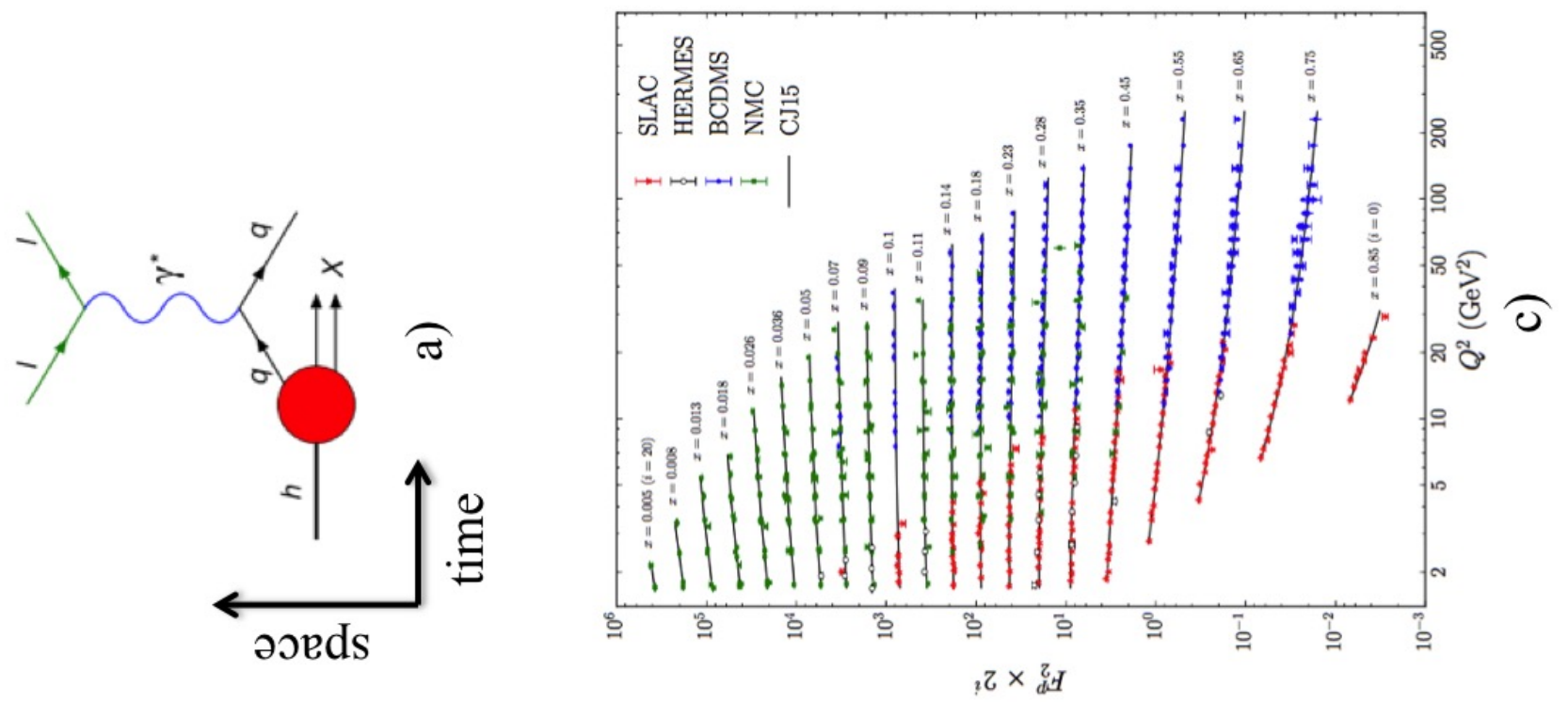

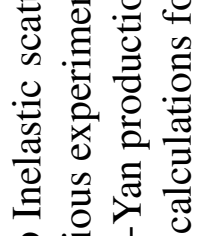

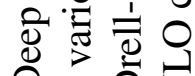

气宫之

फ

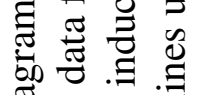

응 ㅎำ

ส

छ

氛。守

现考

ช

穴玨总

휴

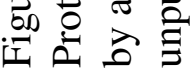




\subsection{Light Anti-Quark Flavor Asymmetry in the Nucleon Sea}

Although no known symmetry constraint requires them to be the same, until the early 1990 's it was assumed that the nucleon sea is light quark flavor symmetric i.e. $\bar{u}(x)=$ $\bar{d}(x)$. As gluons do not couple to flavor and the masses of $u$ and $d$ quarks are similar and small compared to the $\Lambda_{Q C D}$ scale, the flavor symmetric nucleon sea was thought to be generated from gluon splitting (shown in Figure 2.17). Subsequent experiments showed that the nucleon sea is not just flavor asymmetric but must have a non-perturbative origin.

\subsubsection{The NMC Experiment}

The Gottfried Sum Rule is a generalized sum rule in QCD which offers an insight into the structure of the

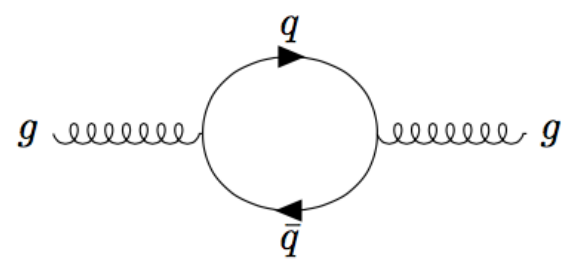
nucleon [49]. This sum rule assumes that the proton and the neutron's quark distributions are related by Figure 2.17: Feynman diagram of gluon splitting charge symmetry i.e. the up quark distributions in the proton are identical to the down quark distributions in the neutron and the down quark distributions in the proton are identical to the up quark distributions in the neutron. It also assumes that the nucleon is made up of only the light quarks $(u, d$ and $s)$ and the strange quark distributions are the same in the proton and the neutron. In such a case, if one were to take the difference in the proton and neutron leading order structure functions (assuming strange quark distributions are the same) and perform the Gottfried integral $S_{G}$

$$
\begin{aligned}
F_{2 p}(x)-F_{2 n}(x) & =x\left[\frac{4}{9}\left(u^{p}(x)+\bar{u}^{p}(x)\right)+\frac{1}{9}\left(d^{p}(x)+\right.\right. \\
\left.\bar{d}^{p}(x)\right) & \left.-\frac{4}{9}\left(u^{n}(x)+\bar{u}^{n}(x)\right)-\frac{1}{9}\left(d^{n}(x)+\bar{d}^{n}(x)\right)\right]
\end{aligned}
$$


where $u^{p}\left(\bar{u}^{p}\right), d^{p}\left(\bar{d}^{p}\right), u^{n}\left(\bar{u}^{n}\right)$ and $d^{n}\left(\bar{d}^{n}\right)$ are the up and down quark (anti-quark) distributions in the proton and the neutron. Since $u$ and $d$ are charge symmetric partners, $u^{p}=d^{n}$, $d^{p}=u^{n}, \bar{u}^{p}=\bar{d}^{n}$ and $\bar{d}^{p}=\bar{u}^{n}$

$$
\frac{1}{x}\left(F_{2 p}(x)-F_{2 n}(x)\right)=\left[\frac{1}{3}\left(u^{p}(x)-d^{p}(x)\right)+\frac{1}{3}\left(\bar{u}^{p}(x)-\bar{d}^{p}(x)\right)\right]
$$

The up quark distributions can be further broken down into valence and the sea distributions

$$
\frac{1}{x}\left(F_{2 p}(x)-F_{2 n}(x)\right)=\left[\frac{1}{3}\left(u_{v}^{p}(x)+u_{\text {sea }}^{p}(x)-d_{v}^{p}(x)-d_{\text {sea }}^{p}(x)\right)+\frac{1}{3}\left(\bar{u}^{p}(x)-\bar{d}^{p}(x)\right)\right]
$$

Assuming a perturbative mechanism, $u_{\text {sea }}^{p}(x)=\bar{u}^{p}(x)$ and $d_{\text {sea }}^{p}(x)=\bar{d}^{p}(x)$

$$
\frac{1}{x}\left(F_{2 p}(x)-F_{2 n}(x)\right)=\left[\frac{1}{3}\left(u_{v}^{p}(x)-d_{v}^{p}(x)\right)+\frac{2}{3}\left(\bar{u}^{p}(x)-\bar{d}^{p}(x)\right)\right]
$$

The Gottfried integral is

$$
S_{G}=\int_{0}^{1} \frac{1}{x}\left(F_{2 p}(x)-F_{2 n}(x)\right) d x=\int_{0}^{1} \frac{1}{3}\left(u_{v}^{p}(x)-d_{v}^{p}(x)\right) d x+\int_{0}^{1} \frac{2}{3}\left(\bar{u}^{p}(x)-\bar{d}^{p}(x)\right) d x
$$

Since the proton contains two up quarks and one down quark, $\int_{0}^{1} \frac{1}{3}\left(u_{v}^{p}(x)-d_{v}^{p}(x)\right) d x=\frac{1}{3}$. Hence,

$$
S_{G}=\frac{1}{3}+\int_{0}^{1} \frac{2}{3}\left(\bar{u}^{p}(x)-\bar{d}^{p}(x)\right) d x
$$

A purely perturbative origin of the nucleon sea would imply that $\int_{0}^{1} \bar{u}(x) d x$ and $\int_{0}^{1} \bar{d}(x) d x$ distributions in the proton are identical reducing the Gottfried integral $S_{G}$ to $1 / 3$. Any deviation from this value reflects a non-perturbative contribution.

In 1991, the NMC (New Muon Collaboration) experiment at CERN published an evaluation of the Gottfried sum rule $[50,51]$. The experiment used a $90 \mathrm{GeV}$ and $280 \mathrm{GeV}$ 
muon beam incident on liquid hydrogen and liquid deuterium targets ${ }^{16}$. They reported a value of $S_{G}=\int_{0.004}^{0.8}\left(F_{2}^{p}-F_{2}^{n}\right) d x / x=0.221 \pm 0.008$ (stat) \pm 0.019 (syst) at a value of $Q^{2}$ $=4 \mathrm{GeV}^{2}$. The values for $F_{2}^{p}-F_{2}^{n}$ were extrapolated on either ends of $x$ and a total integral of $S_{G}=\int_{0}^{1} \frac{1}{x}\left(F_{2}^{p}-F_{2}^{n}\right) d x=0.235 \pm 0.026$ was reported (shown in Figure 2.18). This was the first clear evidence of a violation of the Gottfried sum rule. It was also suggested that perhaps the assumed charge symmetry could be broken $[52,53]$ or that there is a non negligible contributions from the small- $x$ region.

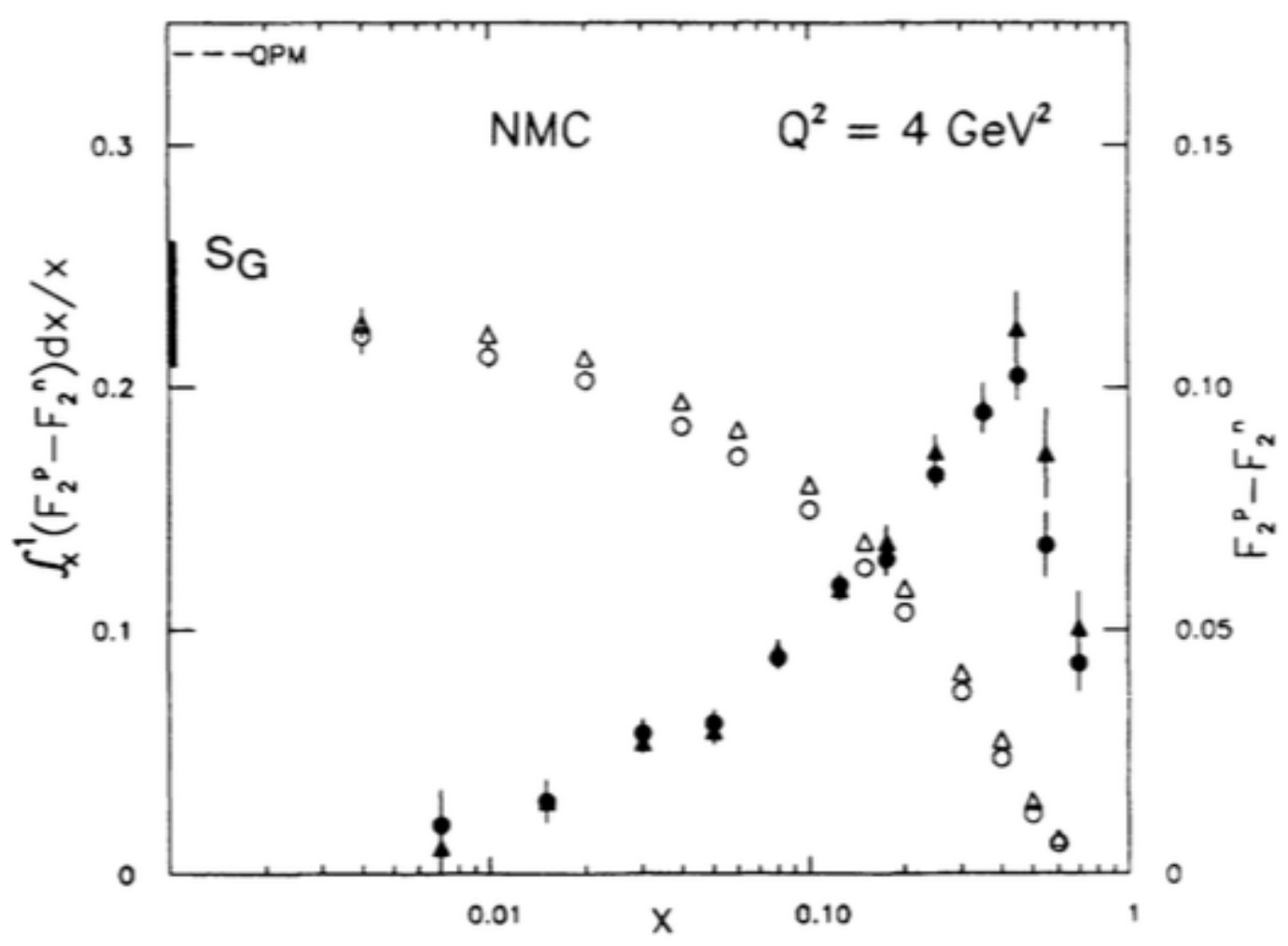

Figure 2.18: Plot to the right shows $F_{2}^{p}-F_{2}^{n}$ (scale to the right) and $\int_{x}^{1}\left(F_{2}^{p}-F_{2}^{n}\right) d x / x$ (scaled to the left). The circles (triangles) and open circles(open triangles) are the reevaluated (first published) values taken from [50] ([51]). The prediction of $1 / 3$ for a flavor symmetric sea is also shown as the dotted line on top left labeled QPM.

\footnotetext{
${ }^{16}$ Since there are no free neutron targets, experiments typically use a deuterium target and a hydrogen target and then subtract the proton part and nuclear effects in deuterium.
} 


\subsubsection{The NA51 Experiment}

It was suggested by Ellis and Stirling that one could take advantage of the Drell-Yan process to disentangle the two possible scenarios (non-symmetric sea parton distributions and isospin symmetry breaking) reported by the NMC experiment [54]. NA51 (North Area 51) experiment performed at CERN measured the Drell-

Yan reaction cross sections with a $450 \mathrm{GeV} / \mathrm{c}$ proton beam on liquid hydrogen and liquid deuterium targets [55]. The experiment considered a cross section asymmetry given by

$$
A_{D Y}=\frac{\sigma_{p p}-\sigma_{p n}}{\sigma_{p p}+\sigma_{p n}}=2 \frac{\sigma_{p p}}{\sigma_{p d}}-1
$$

under the assumption that the deuteron is a simple convolution of the proton and neutron i.e. $\sigma_{p d}=$ $\sigma_{p p}+\sigma_{p n}$. Assuming a parton model formalism,

$$
A_{D Y}=\frac{\left(4 u_{v}-d_{v}\right)(\bar{u}-\bar{d})+\left(u_{v}-d_{v}\right)(4 \bar{u}-\bar{d})}{\left(4 u_{v}+d_{v}\right)(\bar{u}+\bar{d})+\left(u_{v}+d_{v}\right)(4 \bar{u}+\bar{d})}
$$

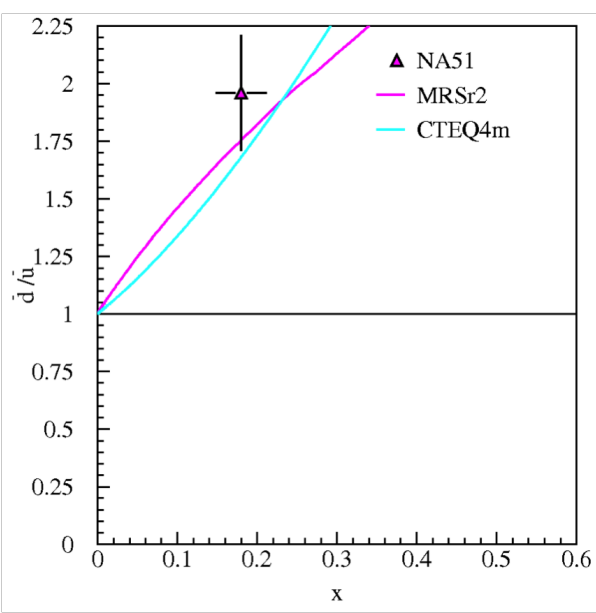

Figure 2.19: Results of the NA51 experiment for $\bar{d} / \bar{u}$ at $\langle x=0.18\rangle$. Parton distribution functions that take the NA51 data into consideration are also shown.

where $u_{v}$ and $d_{v}$ are the valence quark distributions and $\bar{u}$ and $\bar{d}$ are the anti-quark distributions. If we take $\lambda_{v}(x)=u_{v}(x) / d_{v}(x)$ and $\lambda_{s}=\bar{u}(x) / \bar{d}(x)$, then Equation 2.34 becomes

$$
A_{D Y}=\frac{\left(4 \lambda_{v}-1\right)\left(\lambda_{s}-1\right)+\left(\lambda_{v}-1\right)\left(4 \lambda_{s}-1\right)}{\left(4 \lambda_{v}+1\right)\left(\lambda_{s}+1\right)+\left(\lambda_{v}+1\right)\left(4 \lambda_{s}+1\right)} .
$$

The values reported by the experiment at a rapidity $y \approx 0$ and $\langle x\rangle=0.18$ are (shown in Fig. 2.19)

$$
A_{D Y}=\frac{\sigma_{p p}-\sigma_{p n}}{\sigma_{p p}+\sigma_{p n}}=-0.09 \pm 0.02 \text { (stat) } \pm 0.025 \text { (syst) }
$$




$$
\lambda_{s}=\left.\frac{\bar{u}}{\bar{d}}\right|_{\langle x=0.18\rangle}=0.51 \pm 0.04(\text { stat })+0.05 \text { (syst) }
$$

or

$$
\left.\frac{\bar{d}}{\bar{u}}\right|_{\langle x=0.18\rangle}=1.96 \pm 0.15(\text { stat })+0.19 \text { (syst) }
$$

\subsubsection{The E866/NuSea Experiment}

The E866/NuSea experiment was the first to measure an $x$-dependence of the ratio $\bar{d}(x) / \bar{u}(x)$ over a range $0.015<x<0.35[56,57]$. The experiment used the $800 \mathrm{GeV}$ proton beam extracted from the Tevatron at Fermilab and liquid hydrogen and liquid deuterium targets. Approximately 360,000 Drell-Yan dimuon pairs remained after analysis cuts on the data. The data from this experiment put several tight constraints on non-perturbative models that attempt to explain the origin of the nucleon sea.

\subsubsection{Deducing $\bar{d}(x) / \bar{u}(x)$ from $\sigma_{p d}(x) / \sigma_{p p}(x)$}

For a forward spectrometer, the $q\left(x_{\text {beam }}\right) \bar{q}\left(x_{\text {target }}\right)$ term dominates the $q\left(x_{\text {target }}\right) \bar{q}\left(x_{\text {beam }}\right)$ term in the expression for the cross section. Hence the latter term can be ignored and the cross sections can be written as:

$$
\sigma^{p p} \propto \frac{4}{9} u_{p}\left(x_{\text {beam }}\right) \bar{u}_{p}\left(x_{\text {targ }}\right)+\frac{1}{9} d_{p}\left(x_{\text {beam }}\right) \bar{d}_{p}\left(x_{\text {targ }}\right)
$$

where $u_{p}\left(x_{\text {beam }}\right)\left[d_{p}\left(x_{\text {beam }}\right)\right]$ and $u_{p}\left(x_{\text {targ }}\right)\left[d_{p}\left(x_{\text {targ }}\right)\right]$ are the up [down] quark distributions in the proton beam and target respectively. Similarly,

$$
\sigma^{n p} \propto \frac{4}{9} u_{p}\left(x_{\text {beam }}\right) \bar{u}_{n}\left(x_{\text {targ }}\right)+\frac{1}{9} d_{p}\left(x_{\text {beam }}\right) \bar{d}_{n}\left(x_{\text {targ }}\right)
$$


Ignoring the nuclear effects and assuming that a deuteron is a convolution of a free neutron and a free proton,

$$
\sigma^{p d} \approx \sigma^{p n}+\sigma^{p p}
$$

Hence, dividing by $\sigma^{p p}$, we get

$$
\frac{\sigma^{p d}}{\sigma^{p p}}=1+\frac{\sigma^{p n}}{\sigma^{p p}}
$$

Assuming charge symmetry, in which the proton and neutron are identical particles but with $u$ and $d$ quarks interchanged, $u_{p}(x)=d_{n}(x)$ and similarly for the anti-quarks, $u_{n}(x)=$ $d_{p}(x)$, the cross section formula can be rewritten as

$$
\frac{\sigma^{p d}}{\sigma^{p p}}=1+\frac{\frac{4}{9} u\left(x_{\text {beam }}\right) \bar{d}\left(x_{\text {targ }}\right)+\frac{1}{9} d\left(x_{\text {beam }}\right) \bar{u}\left(x_{\text {targ }}\right)}{\frac{4}{9} u\left(x_{\text {beam }}\right) \bar{u}\left(x_{\text {targ }}\right)+\frac{1}{9} d\left(x_{\text {beam }}\right) \bar{d}\left(x_{\text {targ }}\right)}
$$

Dividing the numerator and denominator with $\frac{4}{9} u\left(x_{\text {beam }}\right) \bar{u}\left(x_{\text {targ }}\right)$, we get:

$$
\frac{\sigma^{p d}}{2 \sigma^{p p}}=\frac{1}{2} \underbrace{\left[\frac{1+\frac{d\left(x_{\text {beam }}\right)}{4 u\left(x_{\text {beam }}\right)}}{1+\frac{d\left(x_{\text {beam }} \overline{\bar{d}}\left(x_{\text {targ }}\right)\right.}{4 u\left(x_{\text {beam }}\right) \bar{u}\left(x_{\text {targ }}\right)}}\right]}_{1}] \underbrace{\left[1+\frac{\bar{d}\left(x_{\text {targ }}\right)}{\bar{u}\left(x_{\text {targ }}\right)}\right]}_{2}
$$

Naively, we would expect $u\left(x_{\text {beam }}\right) \approx 2 d\left(x_{\text {beam }}\right)$. So $d\left(x_{\text {beam }}\right) / 4 u\left(x_{\text {beam }}\right) \approx 1 / 8$. Thus, term (1) in Equation $2.42 \approx 1$. Thus, the equation is simplified to

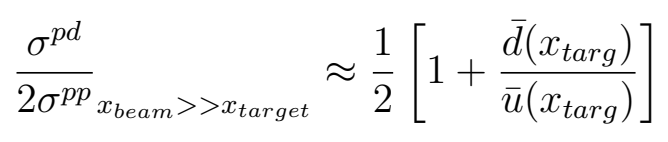

As seen in equation 2.43, the Drell-Yan process has unique sensitivity to anti-quark distributions in the target nucleus even in kinematic regions which are dominated by valence quarks. This sensitivity is not offered by deep inelastic scattering experiments as they cannot distinguish quarks from antiquarks. By comparing the Drell-Yan cross section ratios from proton-proton and proton-deuterium reactions one can gain insight into the anti-quark structure of the nucleon. 
The NuSea experiment measured the cross sections on $\mathrm{LD}_{2}$ and $\mathrm{LH}_{2}$ targets and the ratio $\bar{d}(x) / \bar{u}(x)$ was extracted using an iterative procedure [57]. Taking an initial value of $\bar{d}(x) / \bar{u}(x)$, the iterative process calculated $\sigma_{p d} / 2 \sigma_{p p}$ at leading order, adjusted it for the spectrometer acceptance and compared this quantity with the measurement. This procedure was repeated until the calculated $\sigma_{p d} / 2 \sigma_{p p}$ value agreed with the observed cross section. Plots of the ratio of cross sections $\sigma_{p d} / 2 \sigma_{p p}$ (left) and $\bar{d}(x) / \bar{u}(x)$ (right) show two
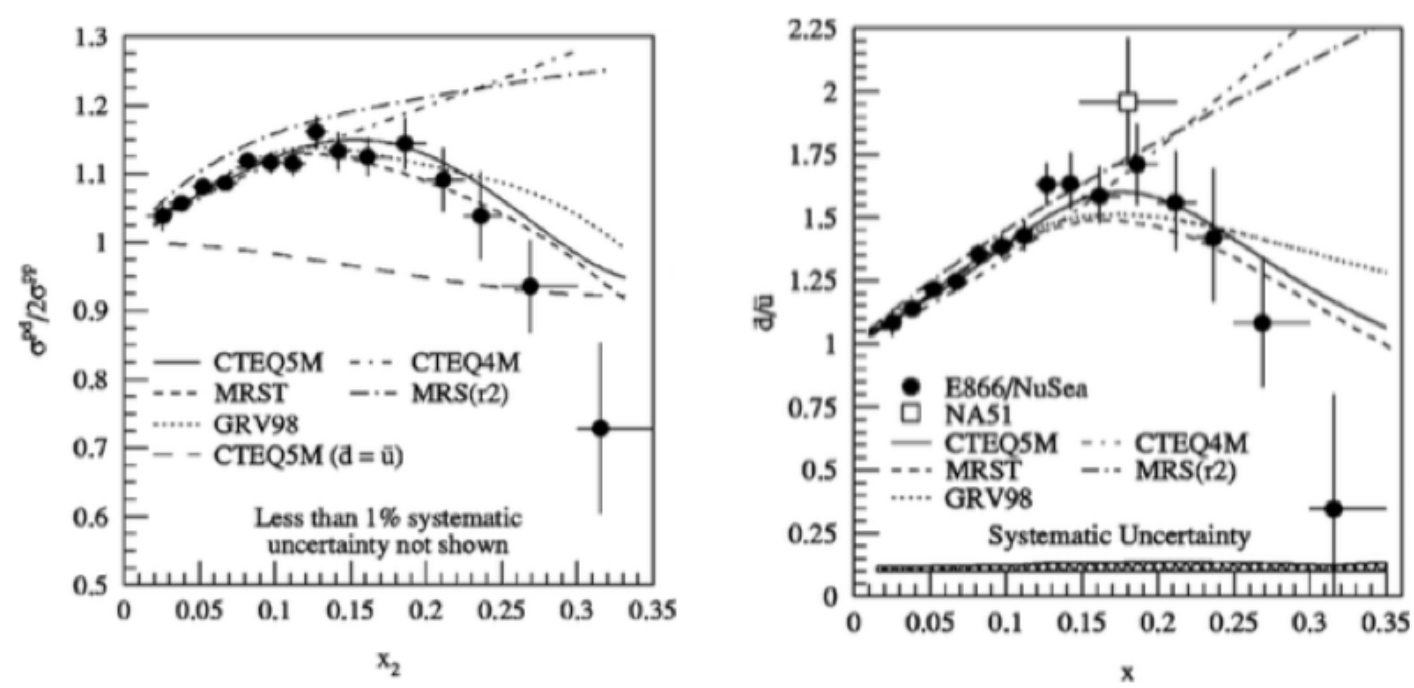

Figure 2.20: Results from E866 experiment. Left plot shows the ratio of cross sections $\sigma_{p d}(x) / 2 \sigma_{p p}(x)$ and the right plot shows the extracted ratio $\bar{d}(x) / \bar{u}(x)$ [57].

prominent features. The ratio seems to rise up until $x \approx 0.18$ and surprisingly starts falling down to a value below 1 (with limited statistical precision) near $x \approx 0.25$. After the quantity $\bar{d}(x) / \bar{u}(x)$ is extracted, the value of $\bar{d}(x)+\bar{u}(x)$ is taken from parameterizations and the quantity $\bar{d}(x)-\bar{u}(x)$ is calculated. $\bar{d}(x)-\bar{u}(x)$ is a unique quantity, which gives a direct insight into the non-perturbative asymmetric sea contribution ${ }^{17}$.

\footnotetext{
${ }^{17}$ There could also be a non-perturbative symmetric sea component as in case of the meson cloud model where a virtual $\pi^{0}$ cloud contains an equal amount of $u \bar{u}$ and $d \bar{d}$.
} 


\subsection{Origin of the Nucleon Sea}

The results from NuSea experiment for $\bar{d}(x) / \bar{u}(x)$ and $\bar{d}(x)-\bar{u}(x)$ (later confirmed by HERMES with limited statistical uncertainty [58]) put constraints on models that attempt to explain the nucleon sea and the observed flavor asymmetry. Fig. 2.21 shows a plot of $\bar{d}(x)-\bar{u}(x)$ for E866 along with the calculations of several different theoretical models. Initially, the sea was assumed to be generated perturbatively by gluon splitting. Field and Feynman suggested that the presence of an "additional" valence $u$ quark in a proton could lead to the suppression of the gluon splitting to $u \bar{u}$ relative to $d \bar{d}$ by a mechanism known as Pauli blocking [59].

Ross and Sachradja reported that the perturbative contribution to the $\bar{d}(x)-\bar{u}(x)$ is very small [60] and argued by taking the parameterizations suggested by Field and Feynman in [59] that Pauli blocking is not important. A purely perturbative mechanism is unable to account for the flavor asymmetry observed by NuSea. Therefore, this asymmetry must be of a non-perturbative origin. Several theoretical models were proposed to explain the origin of the nucleon sea as well as the flavor asymmetry. The pion cloud model rewrites the proton (under one meson approxima-

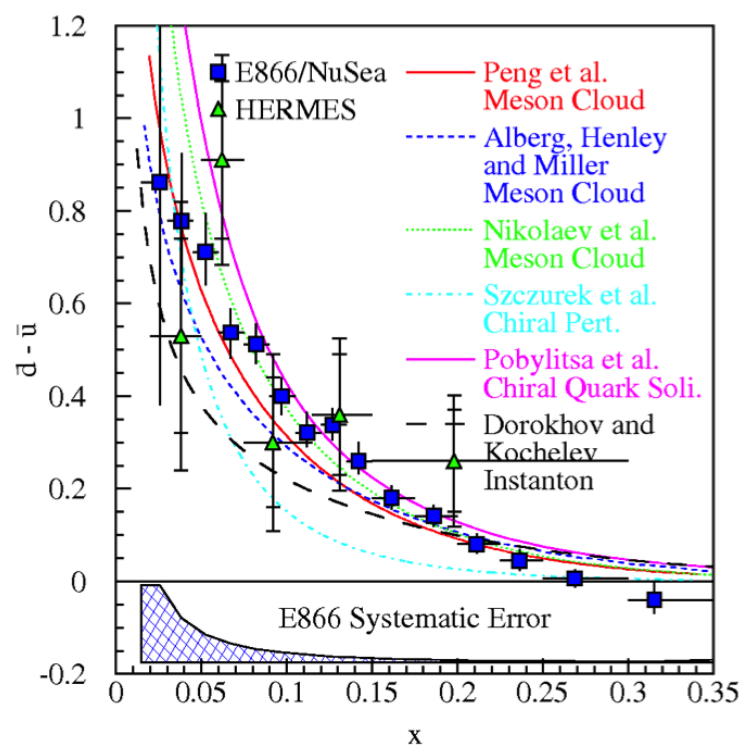

Figure 2.21: Plot of $\bar{d}(x)-\bar{u}(x)$ for E866 [57] along with the calculations of several different theoretical models. the green triangle data points belong to HERMES [58] tion) as a linear combination of several different fluctuations of baryon virtual meson Fock states. If $p_{0}$ is the bare proton with a 
symmetric sea, the proton can be written as:

$$
|p\rangle=\alpha\left|p_{0}\right\rangle+\beta\left|p_{0} \pi^{0}\right\rangle+\gamma\left|n \pi^{+}\right\rangle+\delta\left|\Delta^{++} \pi^{-}\right\rangle+\ldots
$$

If one were to consider the Clebsch-Gordan (CG) coefficients of different baryon virtual meson Fock states, as shown in Fig. 2.22 the lower energy state $\left|n \pi^{+}\right\rangle$has a larger ClebschGordan coefficient than the higher energy state $\left|\Delta^{++} \pi^{-}\right\rangle$. One would expect a $\bar{d}>\bar{u}$ for SeaQuest's $x$ range but cannot intuitively imagine an overturn of the ratio at $x \approx 0.25$. The meson cloud model predicts an overturn at a later value due to a shift in the mechanism where $\left|\Delta^{++} \pi^{-}\right\rangle$dominates $\left|n \pi^{+}\right\rangle$although not at $x \approx 0.25$ [61]. The meson cloud

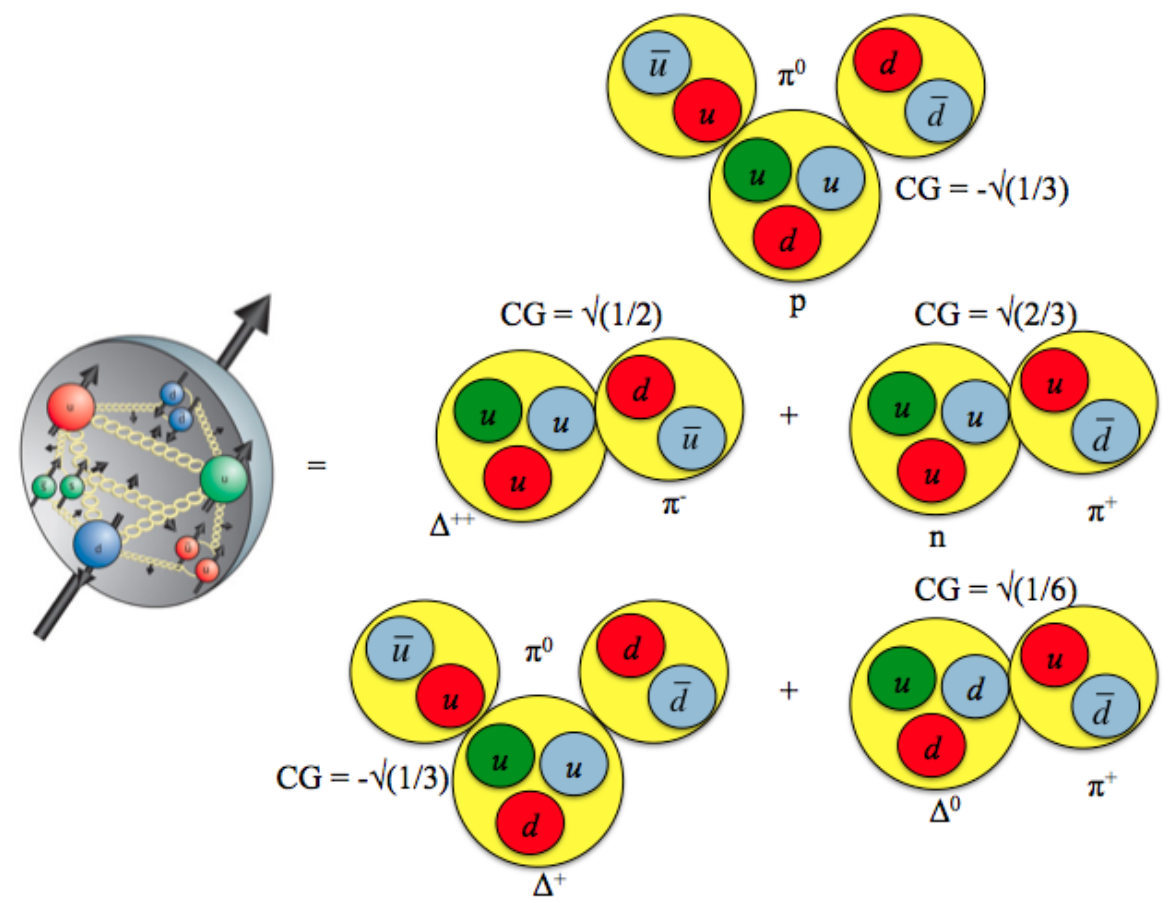

Figure 2.22: Cartoon of the pion cloud model. The proton is expressed in terms of several different baryon virtual meson Fock states. CG are the Clebsch-Gordan coefficients.

model ( $\pi, \omega, \rho$ etc.) which incorporates other baryon virtual meson Fock states is able to reproduce the $\bar{d}(x)-\bar{u}(x)$ difference but predicts that the ratio will cross 1 at a larger value of $x$ compared to NuSea [63,64]. Also, another challenge in this model is to find the exact place to truncate the hadronic expression in Equation 2.44. As shown in Fig. 2.23, 

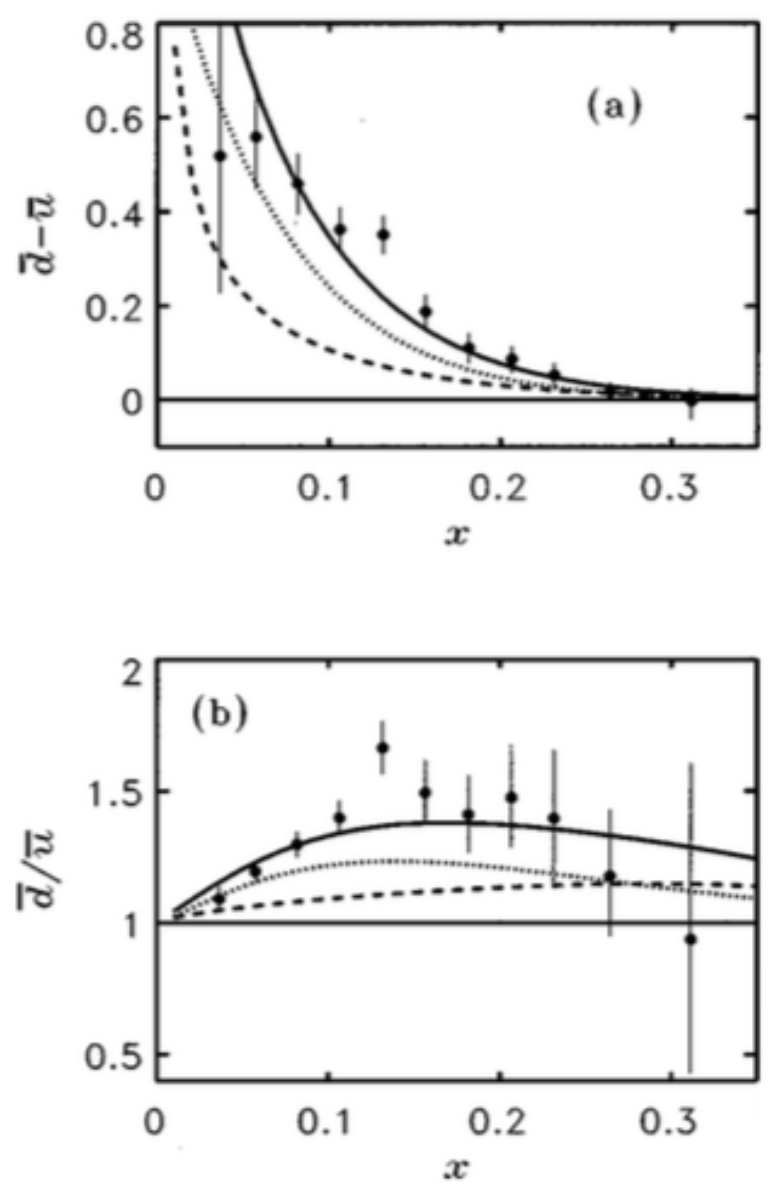

Figure 2.23: Figure shows $\bar{d}(x)-\bar{u}(x)$ and $\bar{d}(x) / \bar{u}(x)$ that include a delicate balance between several competing mechanisms that include a $\pi \Delta+\pi N$ component (dashed) and a parameterized Pauli blocking component (dashed) and combined effect (solid) [62]. The E866/NuSea data points belong to [56] which is an analysis of a smaller data set compared to [57].

some analyses report results that suggest a delicate balance between several competing mechanisms that include a $\pi \Delta, \pi N$ and a parameterized Pauli blocking component [62]. Chiral perturbation theory suggests that the constituent quarks couple to goldstone bosons $\left(u \rightarrow d \pi^{+}\right.$and $d \rightarrow u \pi^{-}$) and that the excess of $\bar{d}$ is simply due to the presence of an 'extra' $u$ constituent quark [65]. The prediction for this model falls short in explaining all the asymmetry seen in Fig. 2.21. Statistical parton distributions model which consider the nucleon as a gas of massless partons (quarks, anti-quarks and gluons) in equilibrium at a given temperature in a finite volume predicts a monotonic increase in the $\bar{d}(x) / \bar{u}(x)$ 


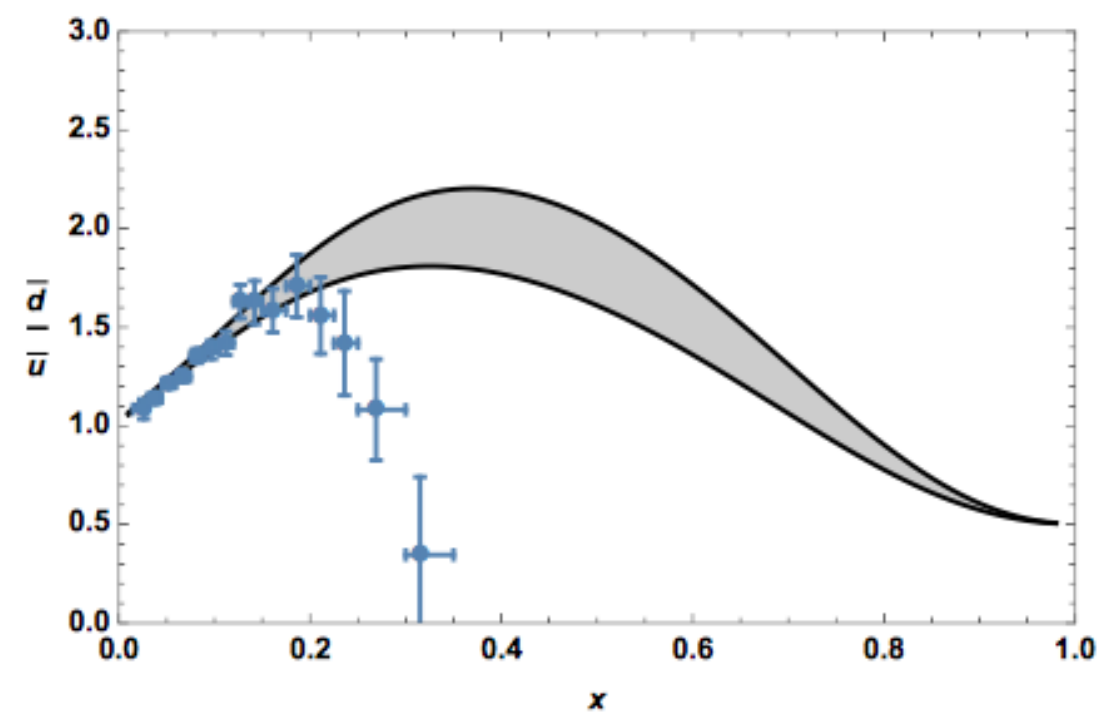

Figure 2.24: $\bar{d}(x) / \bar{u}(x)$ prediction (grey band) for the meson cloud model [61]. Blue points are E866 data points.

ratio [66]. The ratio $\bar{d}(x) / \bar{u}(x)$ is shown for several other non-perturbative models such as Chiral Quark model [67], Chiral Quark Soliton model [68], Instanton induced models [69] in Figures 2.25, 2.26, and 2.27. A full discussion of these models is beyond the scope of this thesis. While some models are able to reproduce $\bar{d}(x)-\bar{u}(x)$, they are unable to predict the surprising overturn and drop below 1 in the ratio (with limited statistical precision) observed at $x \approx 0.25$ by NuSea. It is evident that higher precision data is needed in the range $0.15<x<0.45$ region to map out the overturn at $x \approx 0.18$ and confirm the seeming drop below 1 at $x \approx 0.25$. 


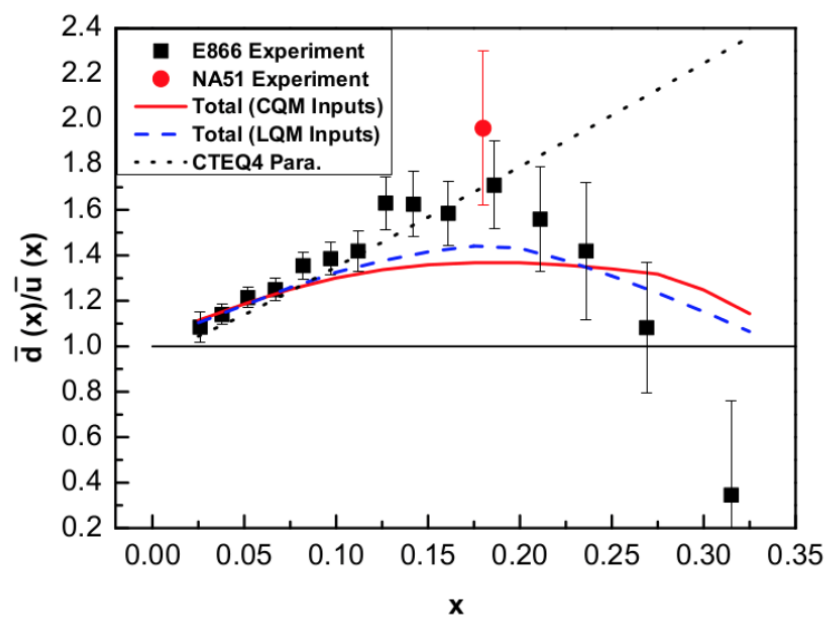

Figure 2.25: $\bar{d}(x) / \bar{u}(x)$ for Chiral Quark model [67]. The red line and blue line include total CQM and LQM inputs in their calculations.

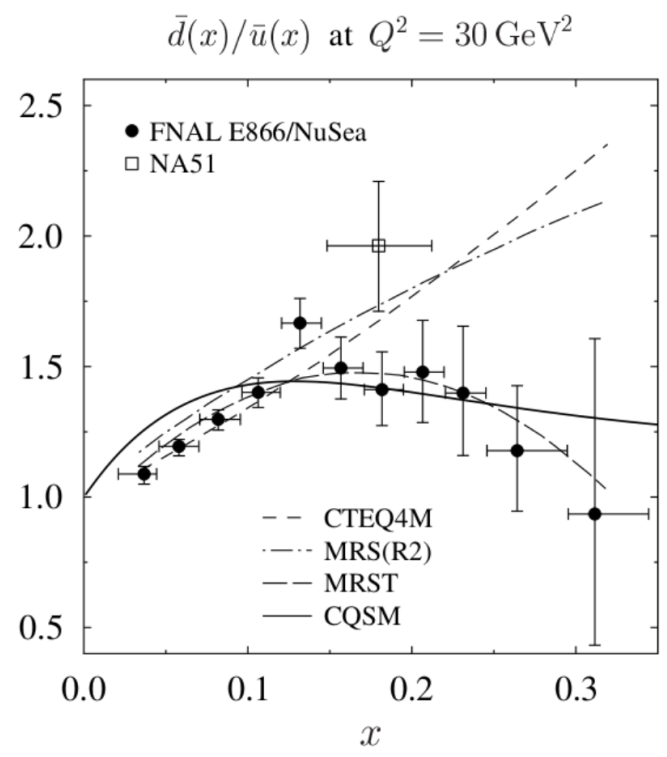

Figure 2.26: $\bar{d}(x) / \bar{u}(x)$ (solid line) for Chiral Quark Soliton model [68]. The dashed curves labeled CTEQ4M, MRS(R2) and MRST are phenomenological PDF fits to the data before and after the E866 data points. 


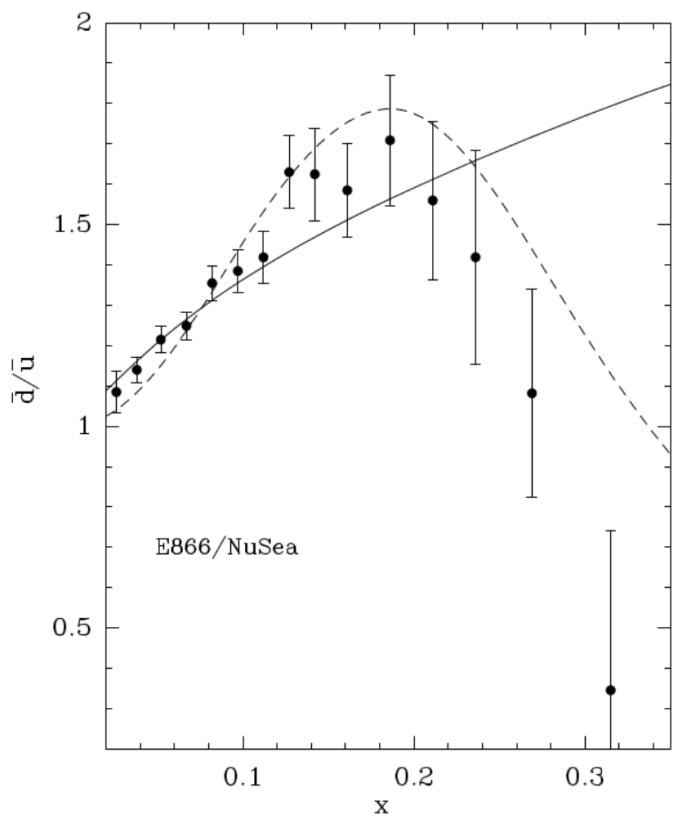

Figure 2.27: $\bar{d}(x) / \bar{u}(x)$ prediction (solid line) by the statistical parton model [66] for the E866 data at $Q^{2}=54 \mathrm{GeV}^{2}$. 


\subsection{Role of SeaQuest - I}

SeaQuest is a fixed target experiment that takes advantage of the Drell-Yan process to access the nucleon anti-quark structure [70]. The experiment uses a $120 \mathrm{GeV}$ proton beam extracted from the Main Injector at Fermilab to collide with liquid hydrogen and deuterium targets. SeaQuest is able to probe the region $0.1<x<0.45$ with higher statistical precision compared to the previous Drell-Yan experiment, NuSea.

- Since the Drell-Yan signal scales as $1 / s$, at the same $x_{\text {Beam }}$ and $x_{\text {Target }}$ with 7 times lower beam energy than NuSea $(800 \mathrm{GeV})$, SeaQuest has higher statistics compared to $\mathrm{NuSea}$ (given the same number of protons on target).

- An important background for the experiment consists of the muons coming from the decay of $J / \Psi$ particle. Since the production of $J / \Psi$ scales as $s[71]$, due to a lower beam energy, the background from $J / \Psi$ is relatively lowered by a factor of 7 compared to NuSea.

With higher statistical precision compared to NuSea, the experiment explored the high$x$ region constraining models that attempt to explain the generation of the nucleon sea, thereby shedding light on the anti-quark structure of the nucleon sea. 


\subsection{Structure Functions of Nuclei}

The typical binding energies of nuclei are on the order of several $\mathrm{MeV}$ per nucleon, and the Fermi momentum is $\sim 250 \mathrm{MeV} / \mathrm{c}$. Many expected that these small binding energies compared to the mass of the proton cannot cause large nuclear effects and nuclei can simply be considered as a convolution of protons and neutrons. Therefore, experiments simply used a heavy denser target in order increase the luminosity to study the structure functions of individual nucleons. Naively, one would expect that the nucleons in iron would just have to be corrected for Fermi motion of nucleons that arise due to their surrounding nuclear medium. When the ratio of nucleon structure functions for iron and deuterium $\left(F_{2}^{\mathrm{N}}(\mathrm{Fe}) / F_{2}^{\mathrm{N}}(\mathrm{D})\right)$ was compared, there was quite a deviation from this expectation (shown in Fig. 2.28). Surprisingly, the distributions of partons in nuclei differed significantly from those in free nucleons, an effect that was first discovered by the European Muon Collaboration in 1983 [72]. The ratio goes from $\sim 1.15$ at $x \approx 0.05$, reaches 1 at about $x \approx 0.3$ and goes down to 0.89 at $x \approx 0.65$. As can seen in Fig. 2.28, the ratio does not follow the predictions of the Fermi motion calculations. The result was quickly confirmed by the SLAC-MIT-Rochester group when they performed a reanalysis of data from aluminum and steel cell walls of liquid hydrogen and deuterium target flasks [76].

\begin{tabular}{|c|l|l|}
\hline Beam type & Experiments & References \\
\hline \hline$\mu$ experiments & BCDMS, EMC, NMC, E665 & {$[78],[72],[79],[80]$} \\
\hline$e^{-}$experiments & SLAC (E49, E87, E139, & {$[81],[76],[82],[83],[84]$,} \\
& E140), HERMES, JLAB & {$[85],[86],[87],[88]$} \\
\hline$\nu$ beam & CDHS & {$[89]$} \\
\hline Drell-Yan & E772, E866, E906 (SeaQuest) & {$[90],[91]$} \\
\hline
\end{tabular}

Table 2.2: Summary of various experiments, beams used and their references. 


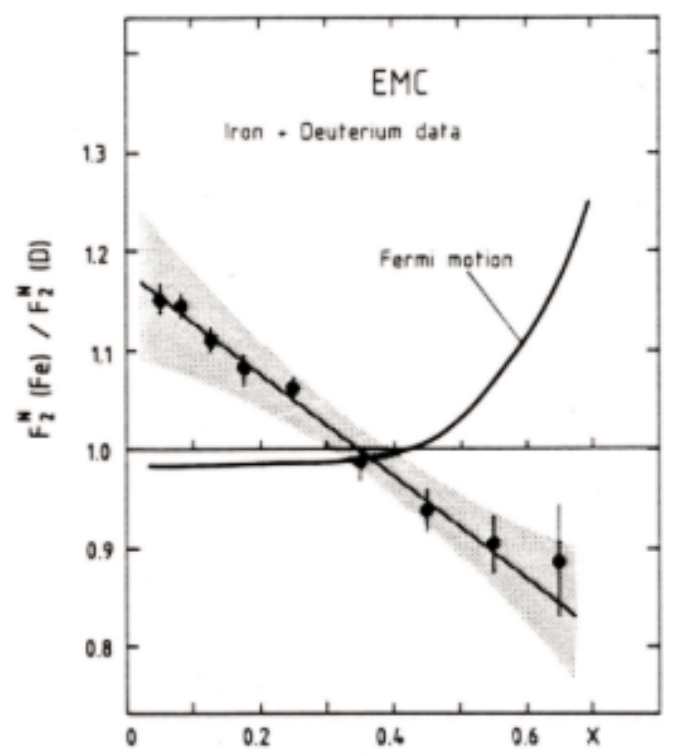

Figure 2.28: The ratio of $F_{2}^{\mathrm{N}}(\mathrm{Fe}) / F_{2}^{\mathrm{N}}(\mathrm{D})$ as a function of Bjorken- $x$. From the EMC collaboration $[72,73]$. The shaded area represents the systematic uncertainty. The solid line is the expectation for the effect of Fermi motion in $F_{2}^{\mathrm{N}}(\mathrm{Fe})[74,75]$.
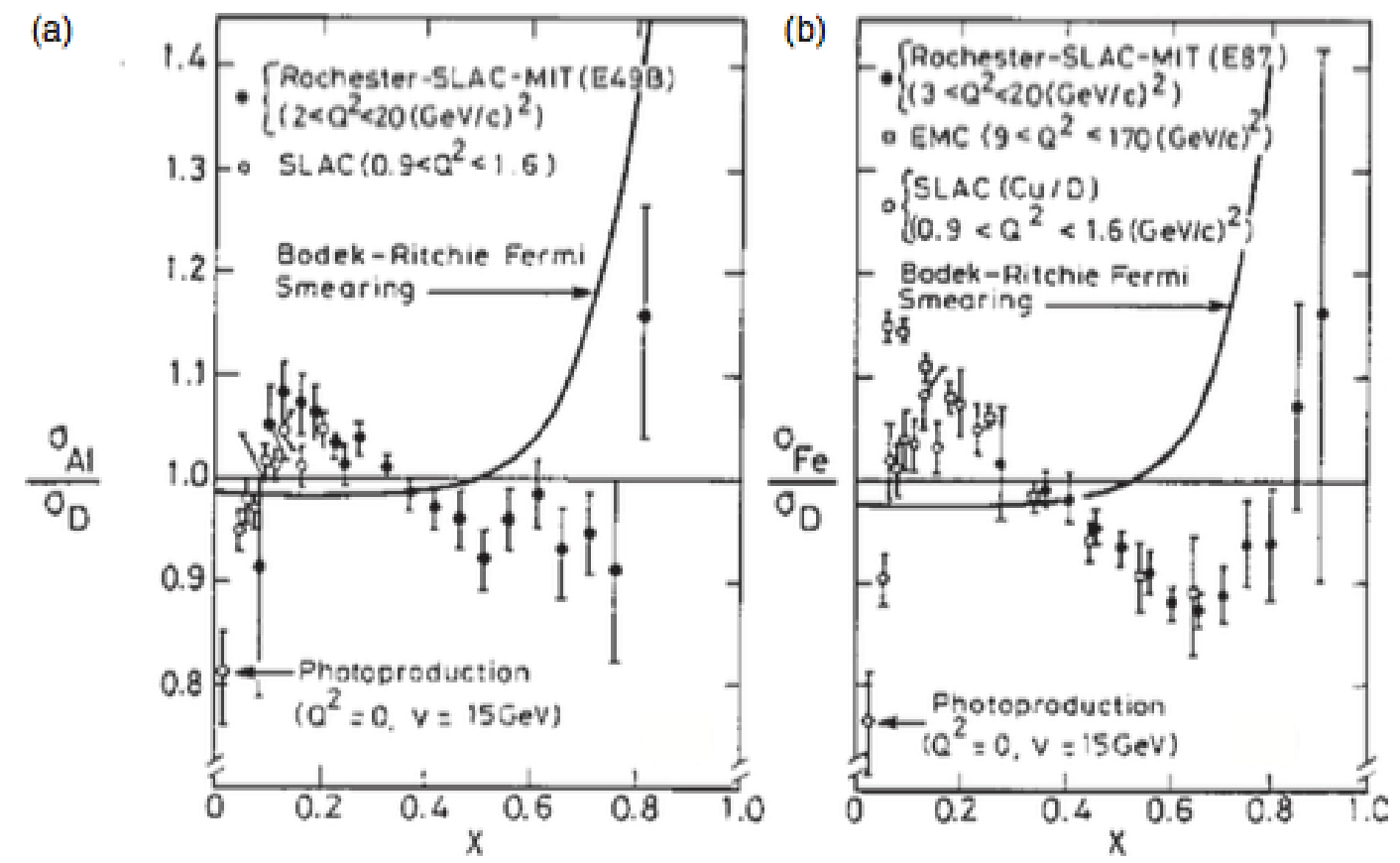

Figure 2.29: Results from SLAC MIT Rochester group experiments conducted to confirm the results from the EMC experiment [76]. 

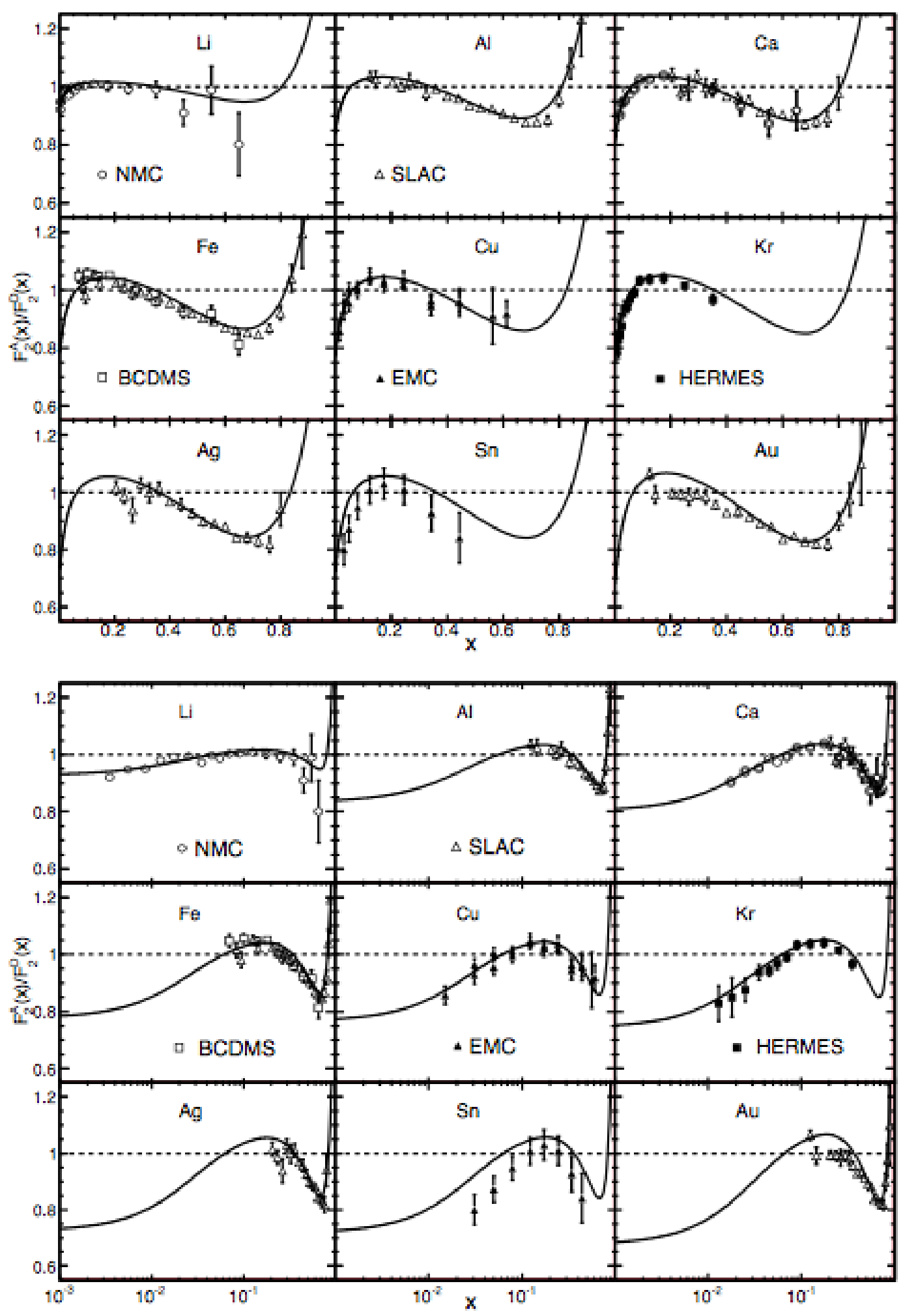

Figure 2.30: Plots of the $F_{2}^{\mathrm{A}}(x) / F_{2}^{\mathrm{A}}(x)$ for several different targets and experiments taken from [77]. The references for the various experiments in these plots are provided in 2.2. 


\subsection{Nuclear $x$-landscape}

The surprising results by EMC collaboration invoked a huge theoretical and experimental effort all over the world in understanding the observed features. Several experiments that utilized different kind of beams, targets and physics processes are summarized in table 2.2. Plots of the $F_{2}^{\mathrm{A}}(x) / F_{2}^{\mathrm{A}}(x)$ for several different targets (ranging from light to heavy nuclei) and experiments taken from [77] are shown in Fig. 2.30 [77]. All the experiments show an $x$-dependence on behavior of the $F_{2}^{\mathrm{A}}(x) / F_{2}^{\mathrm{A}}(x)$ with very little $Q^{2}$ dependence in the measured range [92]. The nuclear $x$-landscape is generally divided into four broad $x$-regions for $0<x<1$ as shown in Fig. 2.31.

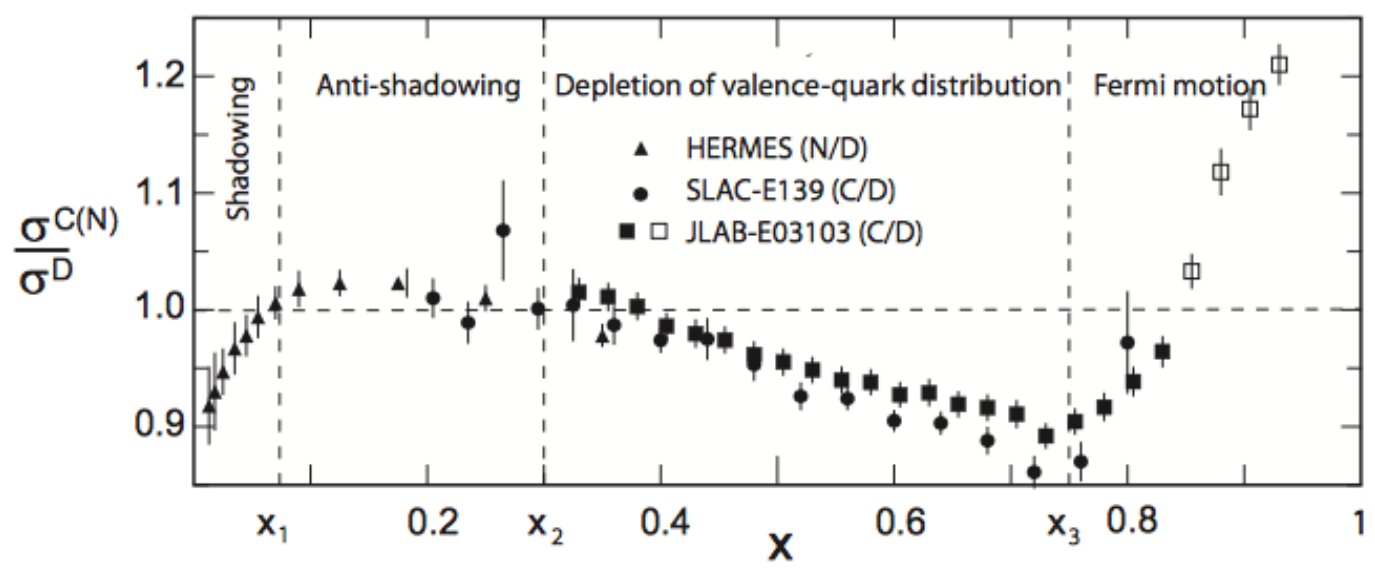

Figure 2.31: Separation of the regions in the ratio of structure functions taken from [93]

- Shadowing region $(0<x<0.06)$ : The structure function ratio is smaller than unity and decreases with decreasing $x$ (and perhaps reaches a saturation point [80]). The cause of a flux reduction is generally attributed to multiple interactions of the virtual photon that fluctuates to $q \bar{q}$ with the nucleus. From each interaction, the scattering amplitude acquires a negative phase that adds to the overall scattering amplitude of single interaction in a deconstructive way. Hence there is a reduction in the flux [94].

- Anti-shadowing region $(0.06<x<0.3)$ : The structure function ratio rises slightly 
above 1 . It could be due to a constructive interference of the scattering amplitudes discussed previously. In a quark-parton model picture, shadowing is due to parton recombination due to high gluon densities. Therefore, a depletion in low $x$-region appears as an excess in a slightly higher $x$-region. This phenomenon of parton recombination qualitatively explains both shadowing and anti-shadowing.

- EMC region $(0.3<x<0.8)$ : The structure function ratio linearly decreases with increasing $x$ up to 0.8 with the magnitude of the slope increasing with A. This region of depletion is usually called the "EMC region". The cause of this depletion is still being understood. A detailed discussion of the current status of the puzzle and the theoretical and experimental efforts can be found in Ref. [93, 95, 96, 97].

- Fermi motion region $(0.8<x<1)$ : The ratio of the structure functions increases rapidly in this region. The higher Fermi momentum allows the quarks to carry a larger fraction of the momentum. This increase is also attributed to a rapid decrease in the nucleon structure function as $x \rightarrow 1$ relative to deuterium.

\subsection{Dependence on Nuclear Properties}

Several experiments conducted after the initial discovery by the EMC collaboration revealed a universal $x$ dependence for a plethora of nuclei. Therefore, it is natural to ask the following questions: "Where does this nuclear dependence in nuclei come from? Is there a unique variable or quantity that can describe this dependence? Is there a universal model that explains this dependence for all values of $x$ ?". 


\subsubsection{Nuclear Dependence of $\mathbf{R}=\sigma_{L} / \sigma_{T}$}

The nuclear dependence of parton distributions have been studies by mainly charged lepton beams. The cross section formula is given by

$$
\frac{d^{2} \sigma}{d Q^{2} d x}=\frac{4 \pi \alpha^{2}}{Q^{4}} \frac{F_{2}\left(x, Q^{2}\right)}{x}\left[1-y-\frac{Q^{2}}{4 E^{2}}+\frac{y^{2}+Q^{2} / E^{2}}{2\left[1+R\left(x, Q^{2}\right)\right]}\right]
$$

where $Q^{2}$ and $x$ have the usual meaning, and $y=\frac{E-E^{\prime}}{E}$ in the target rest frame and $R\left(x, Q^{2}\right)$ is the ratio of the longitudinal $\left(\sigma_{L}\right)$ to transverse $\left(\sigma_{T}\right)$ photon absorption cross section. For a point like spin $1 / 2$ particle, the value of $R$ is 0 . Quark transverse momentum, quark masses and gluon radiation cause this value of $R$ to deviate from 0 [93]. $R$ is typically of the order 0.1 . The results of experiments initially indicated that this value could have an $A$ dependence but it was shown that $R^{F e}-R^{D}=0.001 \pm 0.018$ (stat) \pm 0.016 (syst) consistent with 0 [98]. This indicated that the higher twist effects and possible spin- 0 constituents in the nuclei do not appear to be different from those in nucleons.

\subsubsection{Dependence on Nuclear Mass $A$}

Nuclear dependence on $A$ was studied in detail by EMC [72], BCDMS [76], E665 [80], NMC [99] and E139 [82] experiments. Fig. 2.30 shows that the nuclear effects increase continuously with increasing nuclear mass $A$. The left panel of Fig 2.32 taken from [93] shows the nuclear dependence for two different $x$ values $(x=0.0125$ and $x=0.175)$ for the NMC experiment and the plot on the top right panel shows the ratio of cross sections $\sigma_{A} / \sigma_{D}$ for several targets. The bottom right panel shows the value $\alpha(x)$ extracted from a fit of the form $\sigma_{A} / \sigma_{D}=c(x) A^{\alpha(x)}$. These plots show that the nuclear effects increase to a good approximation as a function of $\log (A)$. However, small deviations for $\mathrm{He}$ and Li for NMC data and He and C for SLAC-E139 data can be seen in the plots. If nuclear dependence were to just depend on A, then all the points in the bottom right panel of Fig 2.32 would lie on a flat line. It can also be seen that the value $\alpha$ has an $x$ dependence and 
therefore, additional variables are required to explain the nuclear dependence.
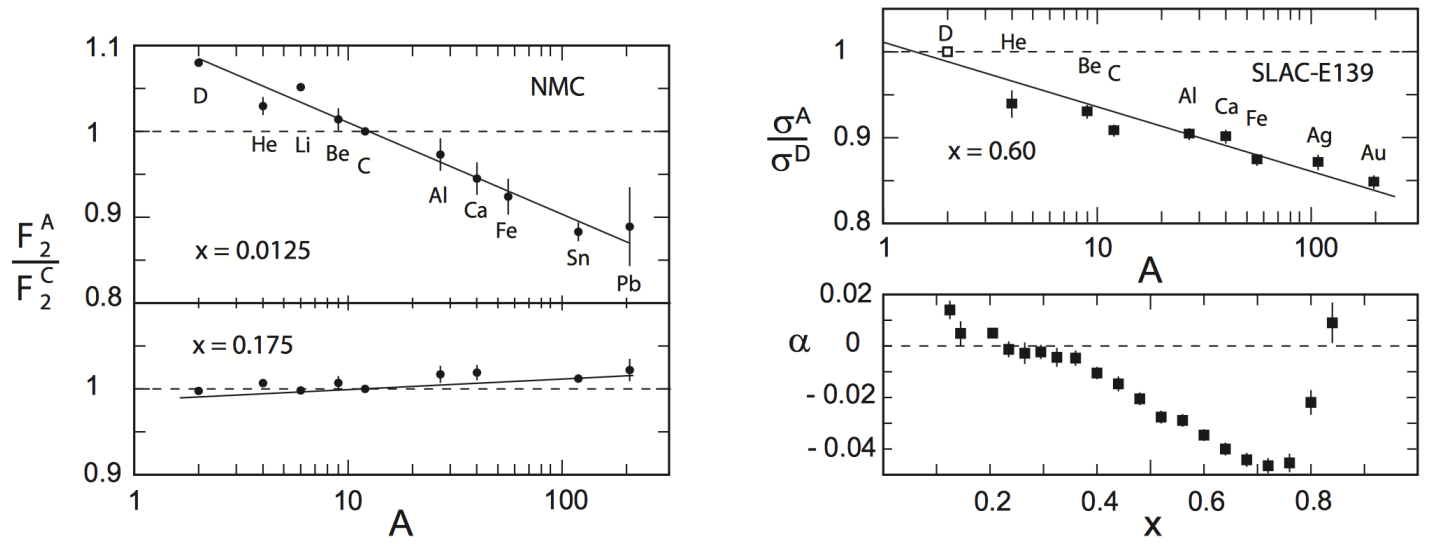

Figure 2.32: Nuclear dependence as a function of mass number $A$. Plots are taken from [93]. Data in the left panel is from NMC (left panel) taken from [99] and E139 experiment (top right panel) performed at SLAC [82]. These plots show that the nuclear effects increase to a good approximation as a function of $\log (A)$. However, small deviations for $\mathrm{He}$ and $\mathrm{Li}$ for NMC data and He and C for SLAC-E139 data can be seen in the plots indicating that additional variables are required to explain the observed nuclear dependence.

\subsubsection{Dependence on Average Nuclear Density $\rho$}

Similar plots for NMC and E139 data were made for nuclear dependence as a function of the average nuclear density $\rho$ (assuming a hard sphere distribution), where $\rho=3 A / 4 \pi R^{3}$ and $R$ is given by $R^{2}=5\left\langle r^{2}\right\rangle / 3$ where $\left\langle r^{2}\right\rangle$ is the root-mean-square radius of the charge distribution value taken from [100]. Fig 2.33 shows that the nuclear effects increase continuously with increasing nuclear density $\rho$. Left panel of Fig 2.33 taken from [93] shows the nuclear dependence for two different $x$ values $(x=0.0125$ and $x=0.175)$ for the NMC experiment and the plots on the right panel show the ratio of cross sections $\sigma_{A} / \sigma_{D}$ for several targets for two different $x$ values $(x=0.22$ and $x=0.6)$. The plot on the left shows that nuclear dependence in the low $x$ region is not well described by a linear function. However, plots in the high $x$ region increase to a good approximation as a function of $\rho$ with Be and He showing small deviations. It can also be seen that the value $\alpha$ 
has an $x$ dependence and therefore, additional variables are required to explain the nuclear dependence. A global analysis of SLAC and NMC data showed that the EMC effect can
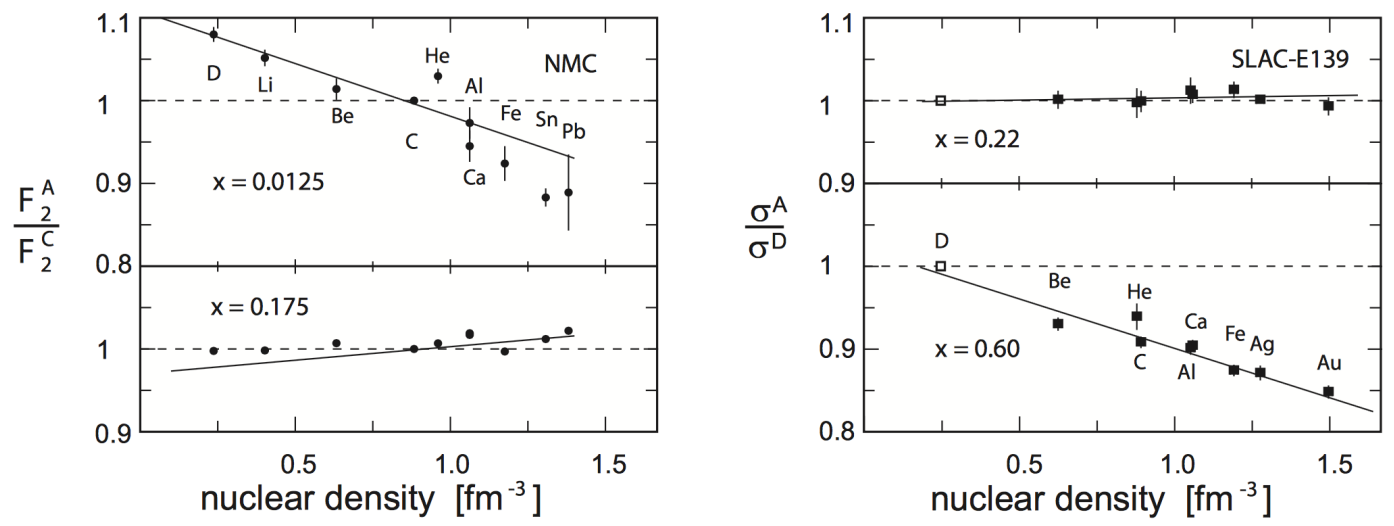

Figure 2.33: Nuclear dependence as a function of nuclear density $\rho$. Data is from experiment E139 performed at SLAC [82] but plot taken from [93].

be described by assuming that the slope of the EMC effect region (also called "strength of EMC effect") is roughly proportional to the average density of nuclei. While these fits well described heavier nuclei, they showed deviations for light nuclei. However, limited data available at that time for light nuclei made disentangling different models difficult. Surprising results from experiments conducted at Jefferson lab (E03103) in Hall C [87] on a series of light nuclei suggested that it is the local nuclear density that drives the modification and not the average nuclear density as initially expected. When the slope of the EMC region $d \mathrm{R}_{\mathrm{EMC}} / d x$ is plotted as a function of the scaled nuclear density ${ }^{18}$, the strength of the EMC effect of ${ }^{9} \mathrm{Be}$ and ${ }^{12} \mathrm{C}$ were similar even though the average nuclear density of ${ }^{9} \mathrm{Be}$ is much lower than ${ }^{12} \mathrm{C}$. This suggested that the simple mass or density scaling models (averaged global properties) break down for light nuclei.

\footnotetext{
${ }^{18}$ scaled by a factor of $(A-1) / A$ to symbolize the number of nucleons "witnessing" the scattering process.
} 


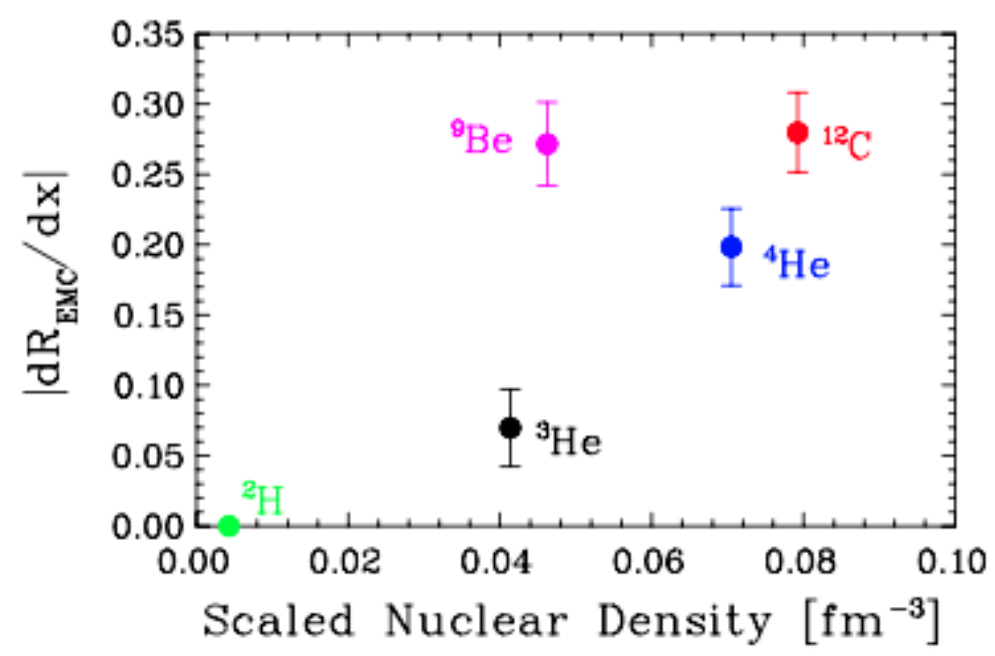

Figure 2.34: Slope of the EMC region $d \mathrm{R}_{\mathrm{EMC}} / d x$ plotted as a function of the scaled (by a factor of $(A-1) / A$ ) nuclear density. The scaling factor symbolizes the number of nucleons "witnessing" the scattering process. Plot taken from [87].

\subsubsection{Dependence on Nuclear Radius $r$}

Nuclear radius $r$ was also tried as a possible parameter to explain nuclear dependence. Analyses were performed on nuclei with same radius and different average nuclear density $\left({ }^{6} \mathrm{Li}\left(r=2.6 \mathrm{fm}, \rho=0.04 \mathrm{fm}^{-3}\right)\right.$ and $\left.{ }^{12} \mathrm{C}\left(r=2.5 \mathrm{fm}, \rho=0.09 \mathrm{fm}^{-3}\right)\right)$ and nuclei with different radius but same average nuclear density $\left({ }^{4} \mathrm{He}\left(r=1.7 \mathrm{fm}, \rho=0.09 \mathrm{fm}^{-3}\right)\right.$ and $\left.{ }^{12} \mathrm{C}\left(r=2.5 \mathrm{fm}, \rho=0.09 \mathrm{fm}^{-3}\right)\right)$ in order to differentiate the effects between $r$ and $\rho$. The analyses [101] performed revealed a rather complicated interplay between $r$ and $\rho$ not confirming a single variable that could describe the nuclear dependence for all $x$.

\subsection{What happens if $x>1$ ?}

The momentum distribution of nucleons can extend well beyond the Fermi-momentum region $\left(k_{F} \sim 250 \mathrm{MeV} / \mathrm{c}\right)$. In principle, since the nucleon moves in the nucleus, due to strong interactions, it can have a larger momentum than is possible for a free nucleon ${ }^{19}$.

\footnotetext{
${ }^{19}$ It is general practice to use the proton mass in defining $x$. In principle a quark could have up to the entire momentum of the nucleus and then have $x=M_{\text {nucleus }} / M_{\text {proton }}$ with that definition. A subtlety is that $M_{\text {nucleus }} / M_{\text {proton }}$ is not exactly the number of nucleons due to $1 \%$ binding effects.
} 
Hence, $x$ can be greater than 1 . High momentum nucleons are generated in the nucleus due to $N N$ interactions (tensor forces and short-range repulsive forces) at distances shorter than the average inter-nucleon distances. Under such circumstances, the momentum of the correlated nucleons is very high but the momentum of the center of mass of the $N N$ system is low. These are called Short Range Correlations (SRC).

For the first time, inclusive electron scattering experiments were done at Jefferson Lab in which cross sections ratios of ${ }^{4} \mathrm{He},{ }^{12} \mathrm{C}$ and ${ }^{56} \mathrm{Fe}$ over ${ }^{3} \mathrm{He}$ have been done in the range $Q^{2}>1.4 \mathrm{GeV}^{2}$ for two $x$ regions; $1.5<x<2$ and $x>2.25$ $[85,86]$. Another experiment (E02-019) was also conducted in Hall $\mathrm{C}$ of Jefferson Lab that investigated the higher $x$ region

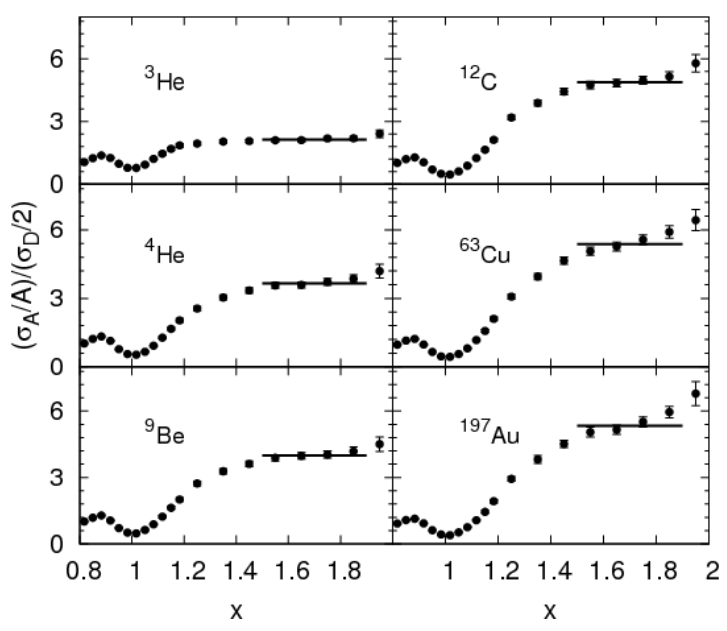
[88]. Some prominent features in the region $x>1$ include a) a plateau in the region $1.5<x<2$ b) a rise of the ratio of cross sections in the region $2<x<2.25$

Figure 2.35: Per nucleon cross section ratios of ${ }^{3} \mathrm{He},{ }^{4} \mathrm{He},{ }^{9} \mathrm{Be},{ }^{12} \mathrm{C},{ }^{63} \mathrm{Cu}$ and ${ }^{197} \mathrm{Au}$ as a function of $x$ for $\theta_{e}=18^{\circ}$. The horizontal lines indicate the plateau regions for NN SRC (Short Range Correlations) [88].

c) a rise for $x>2.25$. These regions are attributed to NN SRC, scattering off nucleons involved in moving NN SRC pairs and and $3 \mathrm{~N}$ SRC respectively. Fig 2.35 shows the cross section ratios of a) ${ }^{4} \mathrm{He}$, b) ${ }^{12} \mathrm{C}$ and c) ${ }^{56} \mathrm{Fe}$ as a function of $x$ for $Q^{2}>1.4 \mathrm{GeV}^{2}$. The horizontal lines in the plot indicate the plateau regions for $2 \mathrm{~N}$ and 3N SRC (Short Range Correlations). Several experiments were conducted since then and the height of the SRC plateau (also called "strength of SRC") is plotted as a function of $A$. It was suggested by [102] that there could be a connection between the strength of the EMC effect and the strength of the SRC. Fig 2.36 shows a plot of the EMC slopes versus the SRC scale factors [103]. It can be seen from the plot that 
there is a strong correlation between these two values.

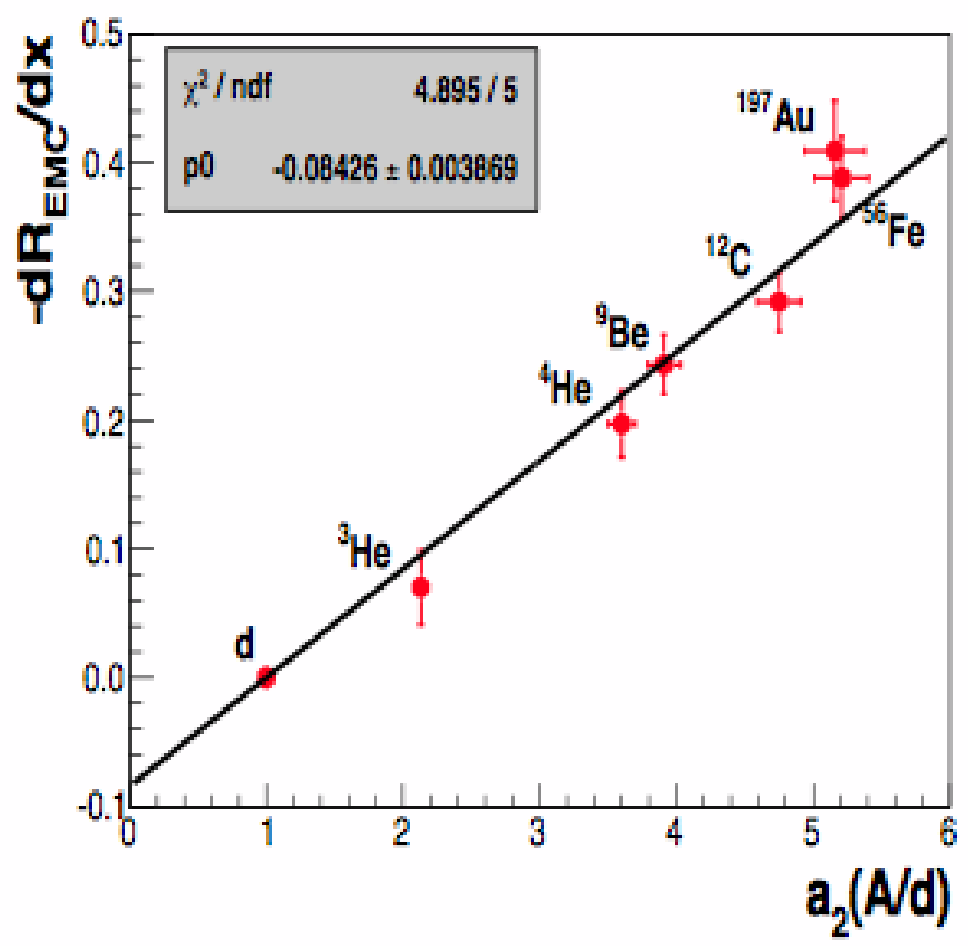

Figure 2.36: The EMC slopes versus the SRC scale factors taken from [103].

Several experiments at Jefferson Lab are aimed at determining the causation versus correlation connection between SRC and EMC effect. A detailed account is beyond the scope of this thesis. However, a summary of the current status and efforts can be found in $[93,104,105,103,106,107,108,109]$. The important question that pertains to this thesis is, "do the sea quarks also exhibit similar nuclear dependence?".

\subsection{Nuclear Dependence of the Anti-Quark Sea}

\subsubsection{Fermilab E772 experiment}

Deep Inelastic Scattering experiments conducted by several groups previously discussed showed that the quark structure of nucleons is modified for nucleons bound in a nucleus. However at high- $x$, DIS explores regions which are usually dominated by valence quarks. 
Therefore, one could ask the question whether the same nuclear effects are observed in the case of sea quarks. Since the Drell-Yan process gives unique access to the sea, one can answer this question for both valence ands sea quarks. Experiment E772 at Fermilab used an $800 \mathrm{GeV} / \mathrm{c}$ proton beam incident on $\mathrm{C}, \mathrm{Ca}, \mathrm{Fe}$ and $\mathrm{W}$ to study Drell-Yan reaction dimuons. The experiment measured the ratio of the Drell-Yan yield of $\mathrm{C} / \mathrm{LD}_{2}, \mathrm{Ca} / \mathrm{LD}_{2}$, $\mathrm{Fe} / \mathrm{LD}_{2}$ and $\mathrm{W} / \mathrm{LD}_{2}$. The plots show evidence of shadowing in the $x<0.08$. In the range above the shadowing region $(x>0.1)$, the experiment reported almost no nuclear dependence for the anti-quark ratio, although it was limited by statistical uncertainty for $x>0.2$. This thus implies no clear modification of the nucleon anti-quark sea in nuclei relative to deuterium [90]. The plots of the yields along with theory calculations predictions for pion excess model, quark cluster model and rescaling models are shown in Fig. 2.37. The disagreement between their data and pion excess predictions questions the possibility that anti-shadowing is not a sea-quark effect caused by the exchange of the so called "nuclear pions".

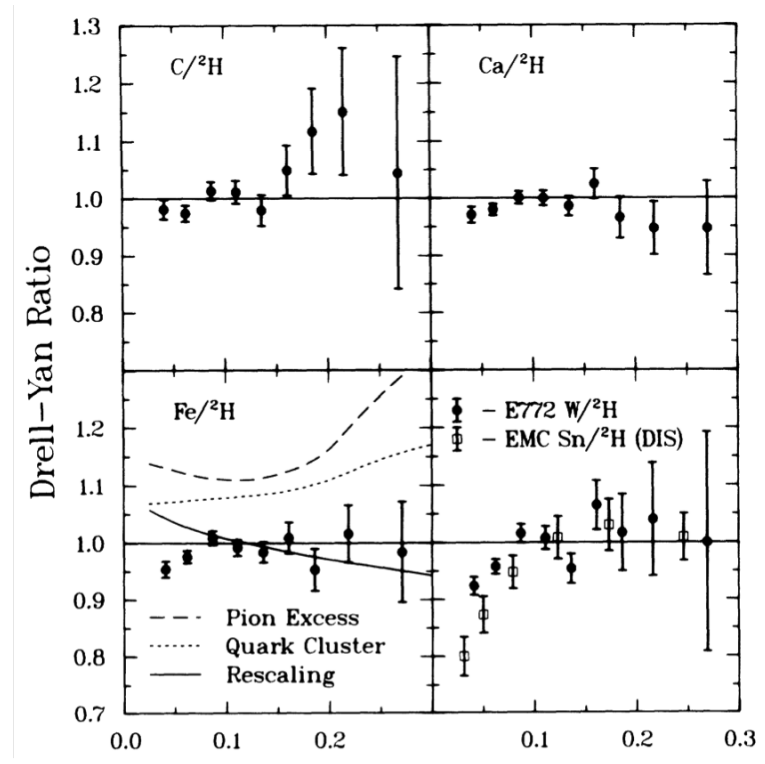

Figure 2.37: Ratios of the Drell-Yan dimuon yields for C/LD2 (top left), Ca/LD2 (top right), Fe/LD2 (bottom left) and W/LD2 (bottom right) for the Fermilab E772 experiment [90]. The curves show predictions for the pion excess model, quark cluster model and rescaling model. 


\subsubsection{The Fermilab NuSea/E866 Experiment}

Details of the NuSea experiment have already been discussed in section 2.8.3. The ratios of the Drell-Yan dimuon yields as a function of mass, $x_{2}, x_{F}$ and $x_{1}$ for Fe/Be (top row) and W/Be (bottom row) for the Fermilab NuSea/E866 experiment are shown in Fig. 2.38 [91]. The results are shown for a limited range of $x$ but similar to E772, they show no enhancement in the ratios as expected by the pion excess model and are consistent with 1 above the shadowing region. Much of the data for the anti-shadowing and the valence regions is

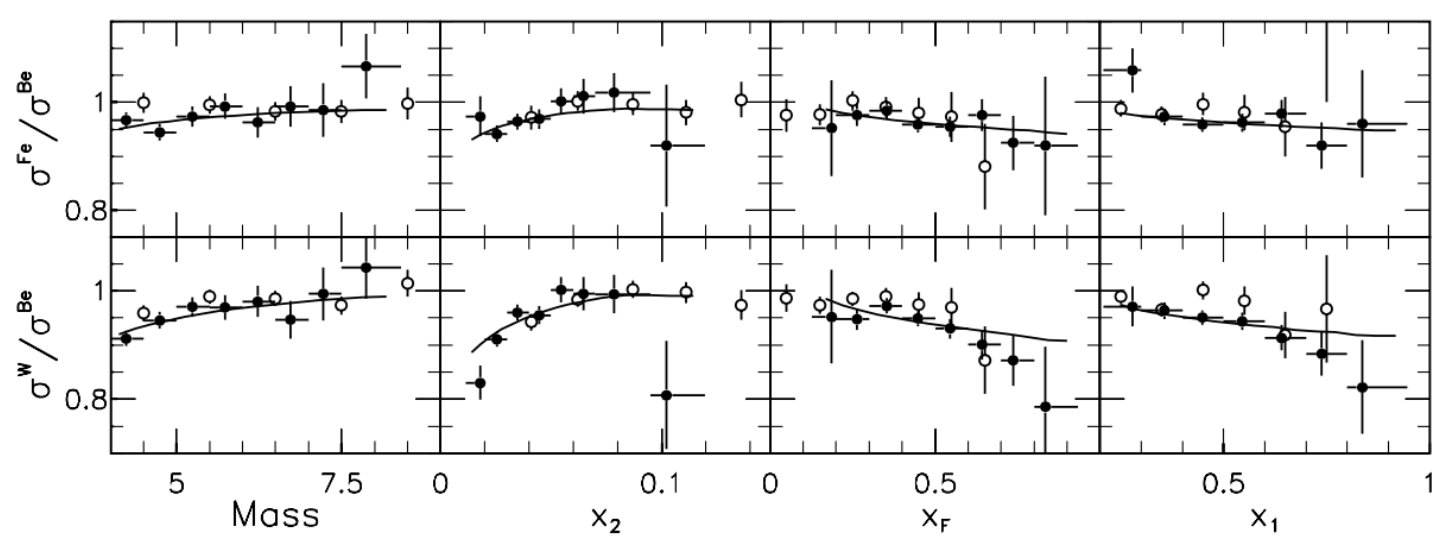

Figure 2.38: Ratios of the Drell-Yan dimuon yields as a function of mass, $x_{2}, x_{F}$ and $x_{1}$ for $\mathrm{Fe} / \mathrm{Be}$ (top row) and W/Be (bottom row) for the Fermilab NuSea/E866 experiment [91].

provided by DIS data which does not differentiate between quarks and anti-quarks and in regions which are dominated by valence quarks. One can ask the question "Do we see the same nuclear dependence for the sea quark distributions or is this just a valence effect?" It is possible that nuclear effects on sea quarks are completely different from those in the valence sector [110]. Higher precision data in the anti-shadowing region as well as larger $x$ than E772 could access would provide crucial information in differentiating the predictions for sea quarks, especially in the high- $x$ region. As shown in Fig 2.39, calculations by Berger and Coester [111, 112] and Jung and Miller [113], expect an enhancement in the ratio of Drell-Yan reaction cross sections where as those by Dieperink and Korpa [114] and Brown et. al. [115] expect a decrease. Smith and Miller [116] (not shown) predict 
no enhancement in the ratios where as Kulagin and Petti $[110,117]$ (not shown) a slight enhancement $(\sim 5 \%)$ that drops with increasing $x$. Drell-Yan data in the high- $x$ region is crucial in differentiating these models.

\subsection{Role of SeaQuest - II}

The experimental capability of SeaQuest has already been discussed in 2.10. By taking advantage of the Drell-Yan process, SeaQuest is able to investigate the modification of sea distributions in the range $0.1<x<0.45$ with higher statistical precision compared to E772 and E866, SeaQuest sheds light on the nuclear dependence of $\mathrm{C}, \mathrm{Fe}$ and $\mathrm{W}$ targets relative to deuterium. Data taken on these solid targets will give an insight into the nuclear modification of the quark distributions thereby confronting theoretical predictions for the high- $x$ region. The ratio of

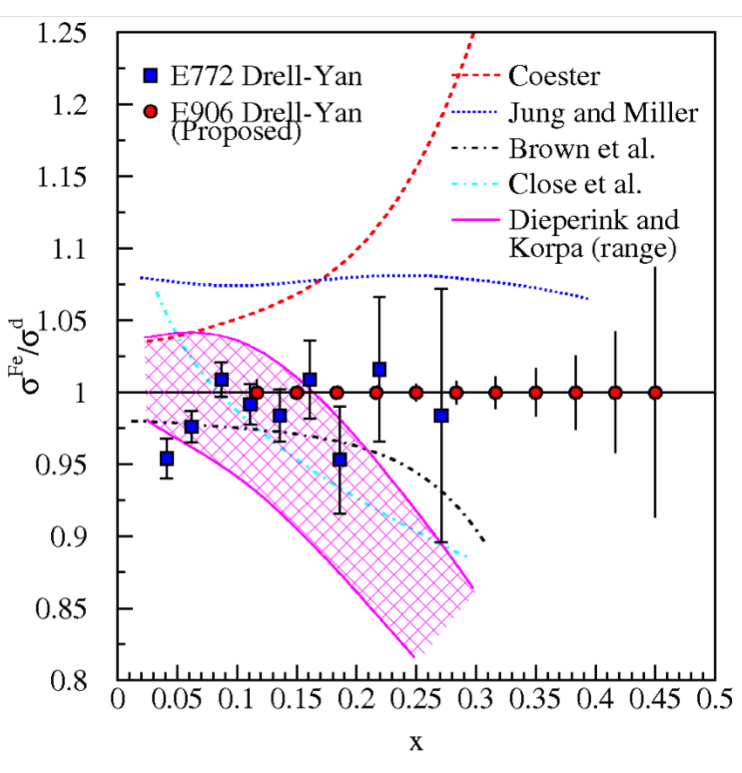

Figure 2.39: SeaQuest projections for nuclear dependence in sea quarks for Fe. The various models predictions were made for E772 kinematics $(800 \mathrm{GeV} / \mathrm{c}$ proton beam).

Drell-Yan dimuons yields of these three targets to liquid deuterium up to a region of 0.45 in Bjorken- $x$ is sensitive to the modifications of the anti-quark distributions in nuclei in the "anti-shadowing" and "EMC" region. Analysis of these dimuons (when combined with more data) will put constraints on various models that attempt to explain the EMC effect observed in Deep Inelastic scattering (DIS). 


\section{Chapter 3}

\section{The SeaQuest Experiment}

The Fermilab Experiment 906, also called "SeaQuest", is a quest into the anti-quark structure of nucleon and nuclei. The experiment takes advantage of the Drell-Yan process specifically in order to probe the high- $x$ anti-quark distributions. The Drell-Yan process is a rare process (as compared to pion production for example) when a proton strikes the target material. Typically Drell-Yan dimuons are swamped with muonic background coming from other physics processes that happen both in the target as well as the beam dump. Optimization of the target beam dump separation as well as sensitivity to the micro-structure of the proton beam play a crucial role in isolating the desired Drell-Yan signal from enormous backgrounds in this experiment.

The SeaQuest experiment is located at the KTeV Hall or enclosure 4 on the Neutrino Muon beamline (NM4) along the fixed target experiments in the accelerator complex. It uses the $120 \mathrm{GeV}$ proton beam (center of mass energy $\sqrt{s}=15.06 \mathrm{GeV}$ ) that is extracted from the Main Injector (MI) at Fermilab. These protons interact with several liquid and nuclear targets chosen to study a plethora of interesting physics topics. Some of the targets are chosen to make a direct comparison with previous Drell-Yan measurements (E605, E772 and E866). These targets are 8 - 13\% in interaction lengths. The "unused" proton beam interactions with the beam dump can also be used to study massive dark photons that are generated in the first few interaction lengths of the beam dump. Figure 3.1 shows a depiction of the SeaQuest spectrometer. Due to the wider acceptance of the spectrometer, SeaQuest explored an unprecedentedly large $x \sim 0.45$, thereby extending our knowledge of anti-quark distributions in nucleons and nuclei. The total spectrometer spans about 25 


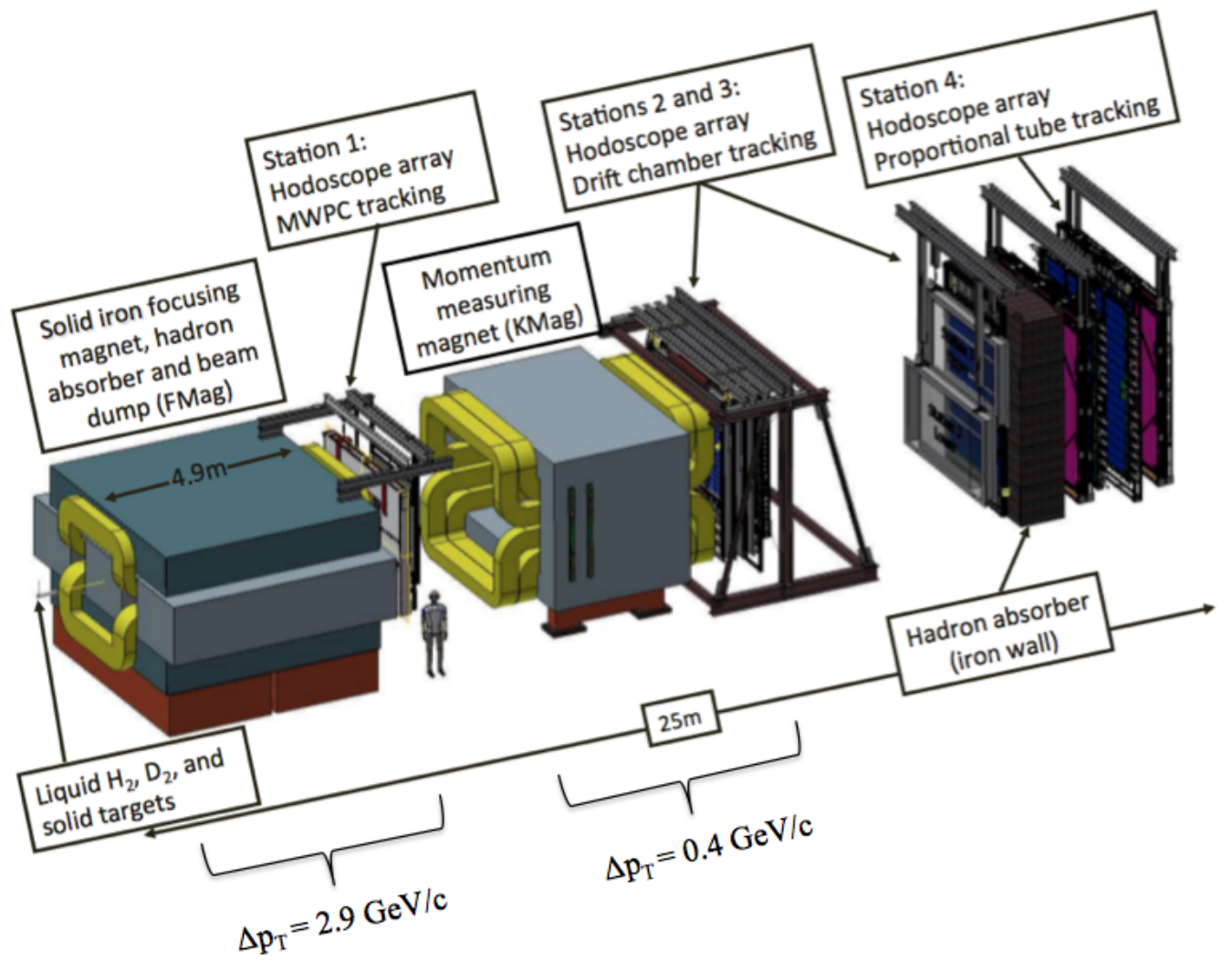

Figure 3.1: The SeaQuest Spectrometer 
meters from the target table to the final triggering station. The proton beam enters the spectrometer from the left (as shown in Fig. 3.1), interacts with the target material $\left(\mathrm{LH}_{2}\right.$ or liquid hydrogen, empty flask, $\mathrm{LD}_{2}$ or liquid deuterium, none, iron, carbon and tungsten) or beam dump. The empty flask and "no" target position are simply used to subtract away the background for liquid and solid targets respectively. The experiment is designed to isolate muons that are generated in the Drell-Yan interactions of the proton beam with the target. The muons thus generated go through the first closed aperture magnet (called FMAG). FMAG also serves as a beam dump for the protons in the beam that do not interact with the target material. It also acts as a "muon filter" by blocking off the hadronic debris thus created. The first magnet gives a $p_{T}$ kick of $\sim 2.9 \mathrm{GeV} / c$. The detector system contains a set of 4 "stations" in which the first three contain layers of drift chambers and scintillator hodoscopes used for tracking and triggering purposes and the fourth contains an array of proportional tubes and scintillator hodoscopes used for muon identification. After passing the first series of drift chambers and scintillator hodoscopes at Station 1, these muons pass through the second open aperture magnet (called KTeV magnet or simply KMAG). This magnet gives an additional $p_{T}$ kick of $\sim 0.4 \mathrm{GeV} / c$. The muons pass though the next two stations of drift chambers and hodoscopes. A $1 \mathrm{~m}$ long iron absorber wall in between Station 3 and Station 4 blocks off any additional strongly interacting particles which make it through the spectrometer. Finally the muons pass through the Station 4 (muon identification stage) of proportional tubes and hodoscopes. Details of all sub-systems will be discussed in subsequent sections.

\subsection{Timeline of SeaQuest}

A short commissioning of data (Run I) took place in Spring 2012. This run confirmed the functionality of all detector subsystems and brought up issues which were subsequently addressed during the next shutdown of the Main Injector. During analysis of the commissioning data, it was realized that track reconstruction from beam intensity beyond a certain 
level ( $\approx 6.0 \times 10^{4}$ protons/bunch) was nearly impossible.

Therefore, a Čerenkov detector was installed in the beamline to rectify the situation. This monitored the beam intensity on a bucket by bucket basis and has the ability to "silently" veto buckets (inhibit) with beam intensity above a chosen threshold. A new drift chamber was constructed at Fermilab and installed to make the top and bottom acceptance symmetric at St 3. The experiment resumed datataking in November, 2013. SeaQuest took data for nearly four years before successfully finishing in June, 2017. Highlights of the experiment timeline along with some upgrades are shown in Fig 3.3. Fig 3.2 shows the protons on target collected for

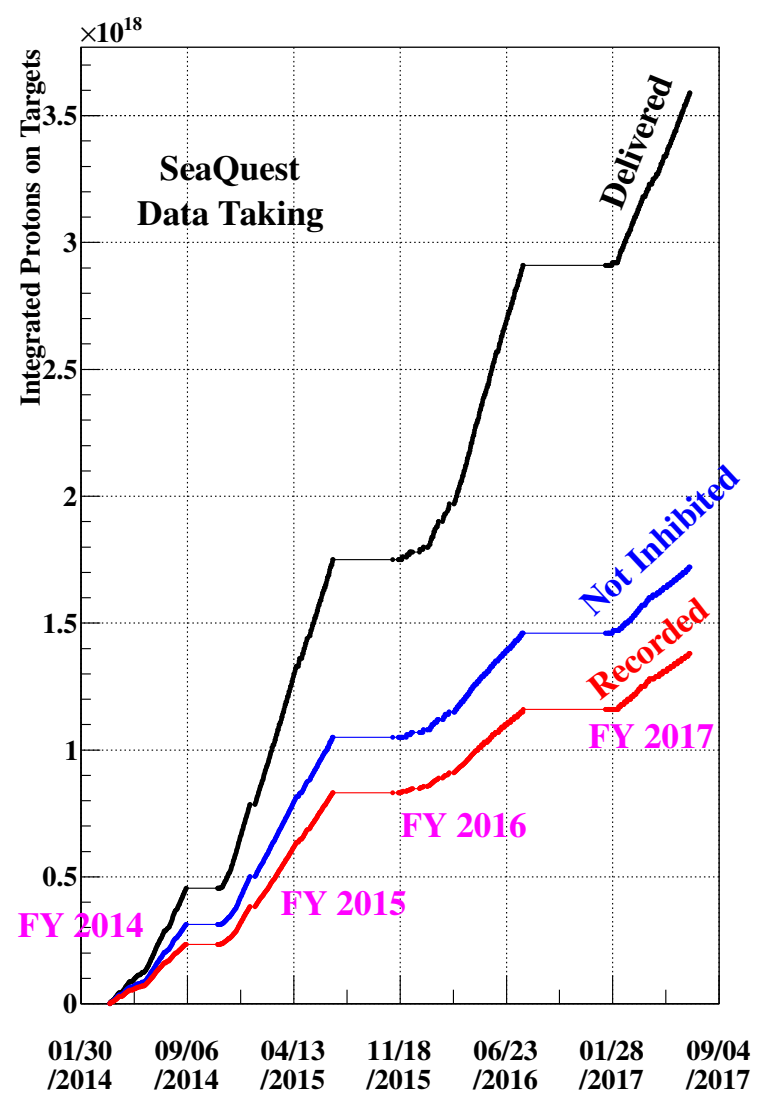

Figure 3.2: Protons On Target (POT) plot for FY2014 - FY2017 time period. several different fiscal years. The black, blue and red lines show the integrated delivered, not inhibited and recorded protons on target plotted as a function of time.

\subsubsection{Data Set Conditions}

Data was collected with a variety of beam, magnetic field and trigger settings. The data sets were given a unique name based on the trigger firmware (or "roadset") setting used at that time. Table 3.1 shows different roadsets and their corresponding run ranges, spill ranges, beam offset and magnetic field orientation. 


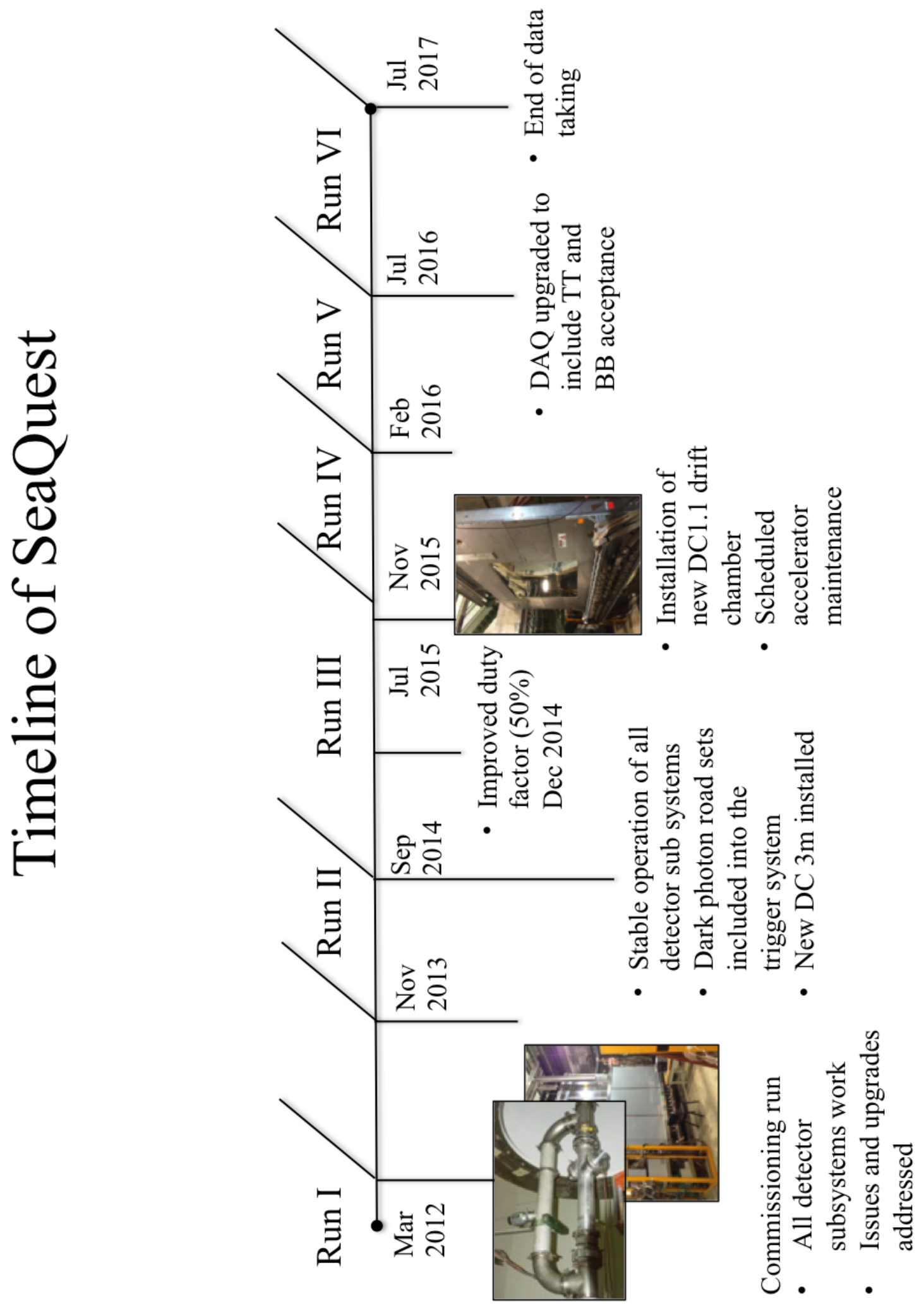




\begin{tabular}{|c|c|c|c|c|}
\hline Roadset & Run range & Spill range & beam offset & B orientation \\
\hline 57 & $8912-10420$ & $310955-370099$ & $0.4 \mathrm{~cm}$ & B \\
\hline 59 & $10421-10912$ & $370110-388469$ & $0.4 \mathrm{~cm}$ & B \\
\hline 62 & $11075-12435$ & $409547-482571$ & $1.6 \mathrm{~cm}$ & B flipped \\
\hline 67 & $12525-15789$ & $484746-676223$ & $1.6 \mathrm{~cm}$ & B flipped \\
\hline 70 & $15793-16076$ & $676498-696454$ & $1.6 \mathrm{~cm}$ & B flipped \\
\hline
\end{tabular}

Table 3.1: Table of data set conditions

\subsubsection{Live Protons on each Target}

The values for the total raw and live protons on target (POT) for each target are summarized in Table 3.2.

\begin{tabular}{|c|c|c|c|}
\hline \multicolumn{4}{|c|}{ Full data set } \\
\hline TargetPos & Target & Total Raw POT & Total Live POT \\
\hline 0 & All & $1.43 \mathrm{E}+18$ & $6.73 \mathrm{E}+17$ \\
\hline 1 & LH2 & $6.08 \mathrm{E}+17$ & $2.87 \mathrm{E}+17$ \\
\hline 2 & Empty & $1.17 \mathrm{E}+17$ & $5.78 \mathrm{E}+16$ \\
\hline 3 & LD2 & $3.05 \mathrm{E}+17$ & $1.37 \mathrm{E}+17$ \\
\hline 4 & None & $1.25 \mathrm{E}+17$ & $6.14 \mathrm{E}+16$ \\
\hline 5 & Fe & $6.69 \mathrm{E}+16$ & $3.07 \mathrm{E}+16$ \\
\hline 6 & C & $1.41 \mathrm{E}+17$ & $6.67 \mathrm{E}+16$ \\
\hline 7 & W & $6.74 \mathrm{E}+16$ & $3.13 \mathrm{E}+16$ \\
\hline
\end{tabular}

Table 3.2: Table of target position, target name, Raw POT and Live POT for full data set.

\subsection{The Proton Beam}

SeaQuest utilizes the $120 \mathrm{GeV}$ beam for all the Drell-Yan measurements. The origin of the beam to the gaining of $120 \mathrm{GeV}$ energy (shown in Fig 3.4) is described in this section [118].

- Radio Frequency Quadrupole (RFQ): A Radio Frequency Quadrupole accelerates the $\mathrm{H}^{+}$ions which are generated at the ion source to about $35 \mathrm{KeV}-750 \mathrm{KeV}$. The low energy beam is then transferred to the LINAC. It is in this section that the proton beam acquires a 53.1 MHz RF (Radio Frequency) structure. 


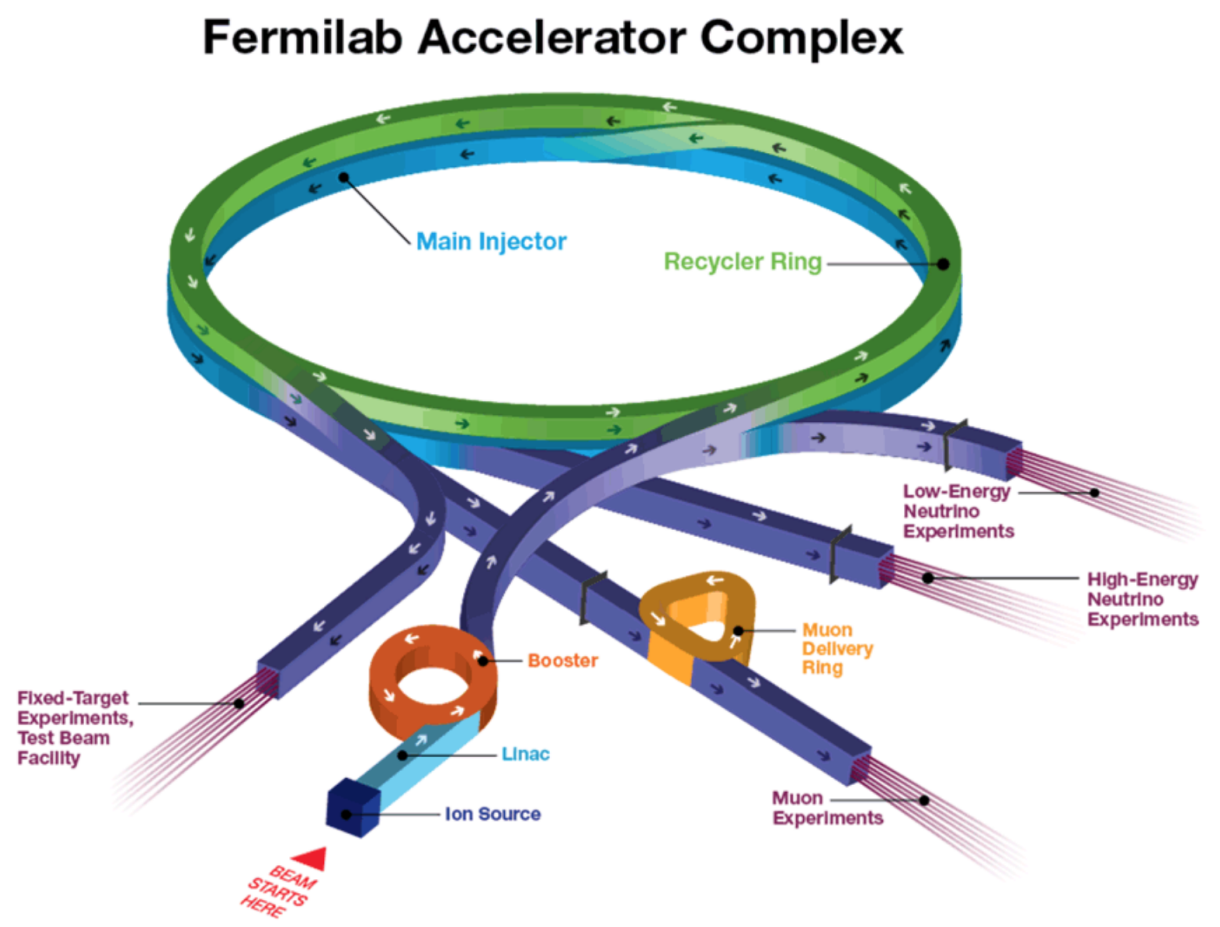

Figure 3.4: Fermilab Accelerator Complex [118]

- Linear Accelerator (LINAC): The LINAC further accelerates the particles to energies of about $400 \mathrm{MeV}$ after collision with the carbon foil (that is used to strip the $\left.e^{-}\right)$. The accelerated hydrogen ions are stripped off of the electrons and sent into the Booster ring.

- Booster: The Booster further accelerates the beam to $8 \mathrm{GeV}$ and passes it on to the recycler.

- Recycler Ring (RR): The recycler ring is sort of a storage place for the beam. Additional batches are combined in the recycler in order to create a more intense beam in a process called "slip stacking". During this combination, the beam intensity varies greatly from empty RF buckets to a high intensity RF buckets with a highly variable and not well controlled transverse structure.

- Main Injector (MI): The Main Injector (2 miles in circumference) is where protons are accelerated to $120 \mathrm{GeV}$. An electrostatic septum which uses strong electric fields 
is used to split the incoming beam into multiple beams. Figure 3.5 shows a depiction of such a septum where transversely oscillating proton beam is "sliced" and sent to experiments. The beam is delivered to NuMI (Neutrinos at the Main Injector) beam line, Switchyard beam line or the Muon campus from there on. SeaQuest is provided the $120 \mathrm{GeV}$ beam from the Switchyard beam line.

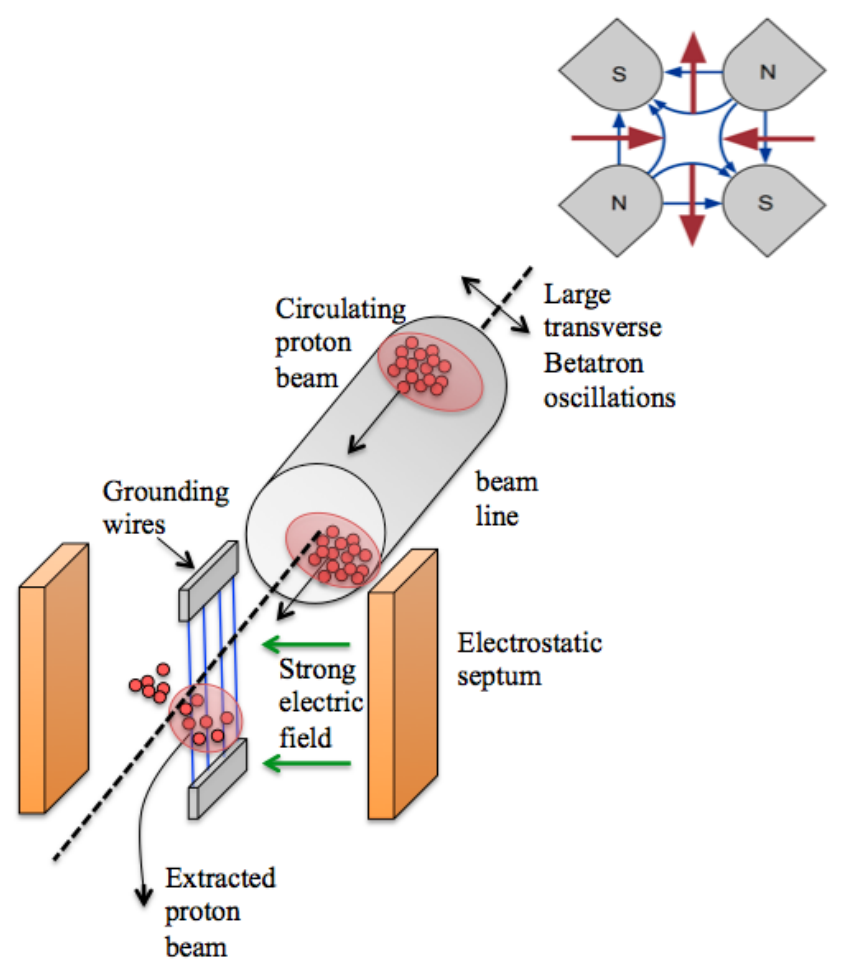

Figure 3.5: Proton beam at the electrostatic septum [119]

\section{What causes a "splat"?}

Fermilab Quadrupole magnets act as lenses focusing in one plane and defocusing in another. Net transport of the beam is achieved with a periodic placement of quadrupoles along the circumference of the accelerator [119]. Betatron oscillation (transverse oscillation of particles) of the proton beam is caused due to the alternate focusing/defocusing pattern in a circular accelerator. As mentioned in the previous section, additional batches 
of protons are combined in order to create a more intense beam in a process called "slipstacking". A combination of these perturbations and slip stacking creates beam with a large and not well controlled transverse structure. An electrostatic septum splits the beam into multiple beam lines as shown in Fig 3.5. The beam intensity varies from empty RF buckets to very high intensity ones. Sometimes the beam intensity is so high that the track reconstruction pattern recognition algorithm simply fails to identify any tracks due to too many detector hits. Under such circumstances, the detector undergoes a "splat". A beam monitor was installed for the very purpose of monitoring the beam intensity on a bucket-by-bucket basis. This serves the purpose of monitoring the intensity to count the number of protons on target as well as "silently" vetoing the buckets with high intensity. More details will be discussed in the section on the beam monitor.

\subsubsection{Micro-structure of the Beam}

As mentioned in the previous section, the proton beam retains the $53.1 \mathrm{MHz}$ structure that it initially acquires at the RFQ. The duration of each spill delivered to SeaQuest is under 5 seconds with a spill delivered about once a minute (60.1 seconds). Each spill contains six Booster injections (called trains) which typically spans the 2 mile circumference of the Main Injector. Each Booster injection (or train) contains 84 RF buckets ${ }^{1}$. Even though the Main Injector has a capacity to contain seven trains, one of the trains (along with a gap of 2 buckets between successive trains) is left empty to be used as a window to inject or extract the proton beam as well as an abort gap for the beam in emergency situations. The time between each successive bucket/bunch is $1 / 53.1 \mathrm{MHz}=18.8 \mathrm{~ns}$. There are a total of 369,000 turns in a spill but only 360,000 typically have beam in them. The spills are typically $20 \mathrm{~cm}$ long (in the lab frame) and $\sim 1 \mathrm{~cm}$ in diameter. Fig 3.6 shows a depiction of the micro-structure of the beam.

\footnotetext{
${ }^{1}$ The Harmonic number i.e. number of RF wavelengths of the machine is 84 for the Booster and $7 \times 84=$ 588 for the Main Injector.
} 


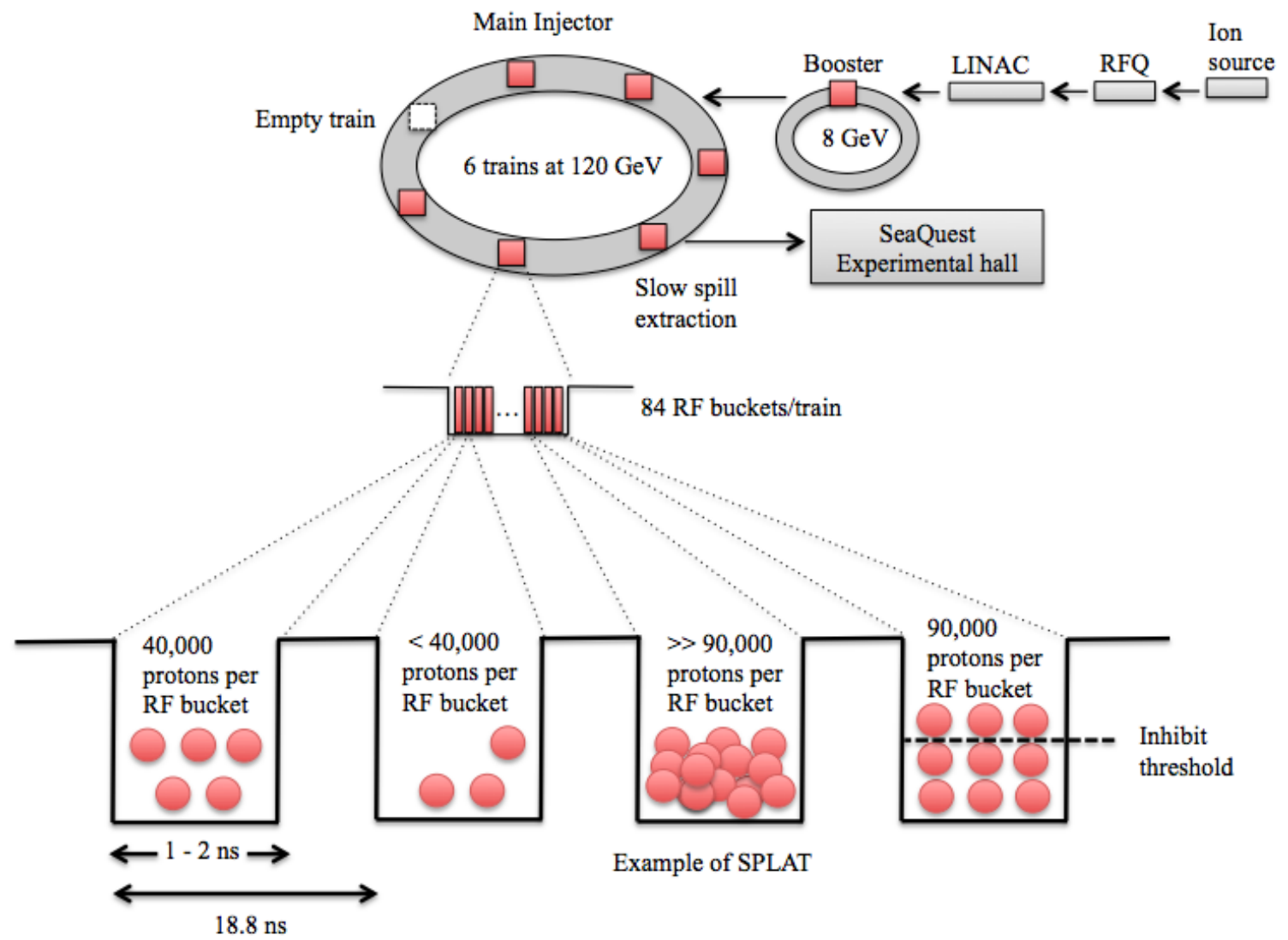

Figure 3.6: Micro structure of the beam along with examples of low intensity and high intensity buckets. 


\subsection{Monitoring the Proton Beam Intensity}

The intensity of the proton beam is measured at several places before reaching SeaQuest. Several Ion Chambers and SEMs (Secondary Emission Monitors) are installed and calibrated (using activation samples from thin copper foils with known cross sections placed in the path of the beam) for this very purpose. Secondary emission monitors are used in high energy physics for counting the number of charged particles that pass a particular region. They typically contain foils from which electrons are knocked out and counted. Their response is typically linear over a large dynamic range. However, they do not have individual bucket resolution. In particular, SeaQuest uses the SEM in the G2 enclosure to integrate the number of protons over the 5 s duration of the spill.

\subsubsection{Beam Intensity Monitor}

The Beam Intensity Monitor (BIM) is part of the upstream instrumentation package along with a SEM. As discussed in section 3.2, the BIM was installed to monitor the beam intensity on a bucket-by-bucket basis. It is able to sense when the beam intensity is above a certain programmable threshold and inhibits triggers in an adjustable window ( \pm 9 RF buckets) until the intensity falls below an acceptable level. Typically, the intensity level is programmed to be between 65,000 and 95,000 protons per bucket.

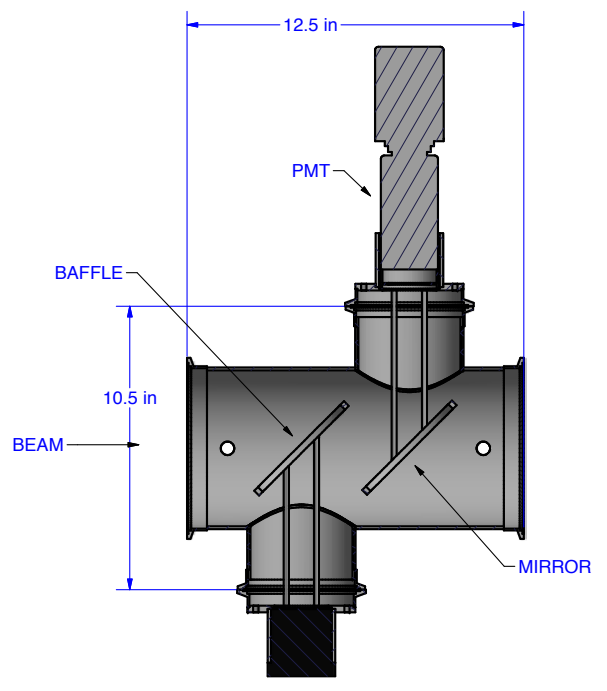

Figure 3.7: Depiction of the Čerenkov counter taken from [120] The beam intensity is measured using the beam Čerenkov counter that uses Argon (80\%) and $\mathrm{CO}_{2}$ (20\%) maintained at atmospheric pressure. A diagram of the Čerenkov counter is shown in Fig 3.7. 
The Čerenkov counter and the electronics were designed to have adequate time resolution and a linear response over a large dynamic range. A $45^{\circ}$ aluminized Kapton mirror that is held on an elliptical G10 frame directs the light to a photomultiplier tube positioned close to the mirror to collect all the light that falls on the face of the phototube. The mirror reflectivity is reduced due to radiation damage and had to be replaced several times during the experiment. Fig 3.8 shows

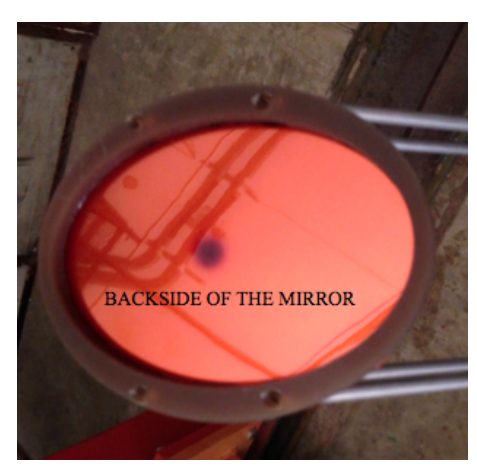

Figure 3.8: Degraded backside of the Čerenkov counter mirror. an example of a degraded mirror (back side). A baffle of black construction paper held parallel to the mirror ensures that the beam covers the same path length regardless of the beam position. Also the light generated in the part before the baffle is blocked by it. The QIE chip is in sync with the Main Injector RF clock and provides an ADC conversion every $18.8 \mathrm{~ns}$ (end of each bucket). The signal from the photomultiplier tube is collected and sent to a custom integrated circuit designed at Fermilab for the CMS (Compact Muon Solenoid) experiment at CERN Large Hadron Collider. This QIE (Charge Integrator and Encoder) is one in the family of circuits used first by the $\mathrm{KTeV}$ experiments. We measure the charge of the signal coming from the phototube which is proportional to the number of protons per RF bucket, assuming:

- The overall light collection efficiency is stable for the duration of the entire spill. The veto could have been re-calibrated on a spill-by-spill basis if we needed it to.

Along with monitoring the beam intensity on a bucket-by-bucket basis, the BIM interface module provides the following important information relevant to the experiment:

- Integrated beam for the entire spill: $\mathrm{QIE}_{\text {sum }}$.

- Integrated beam while inhibit is applied at the trigger logic level: inhibit_block_sum.

- Integrated beam during trigger dead time: trigger_sum_no_inhibit. 
- A snapshot of the beam intensity close to the trigger ( \pm 16 buckets): Fig 3.9.

- A complete record of the bucket-by-bucket intensity for the spill.

SeaQuest receives a beam intensity of $\sim 5 \times 10^{12}$ protons per spill. The bucket-by-bucket intensity varies greatly and a large number of background tracks can be produced from the high intensity buckets. These events can overwhelm the spectrometer, generate events which are simply unanalyzable and significantly increase the DAQ deadtime. The unique features of the BIM help in identifying and vetoing these buckets, thereby making it a crucial part of the spectrometer.
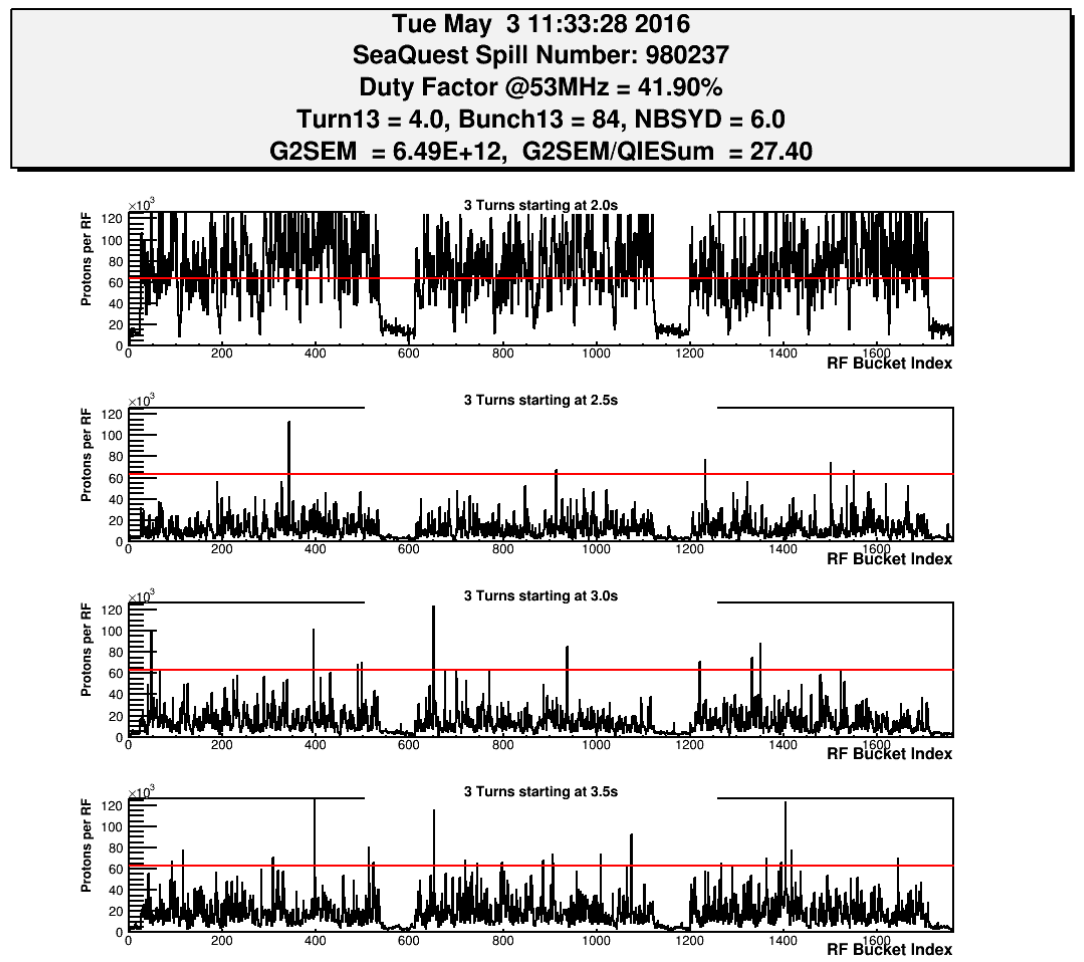

Figure 3.9: Four snapshots 1800 RF buckets of the beam intensity delivered to SeaQuest. The red line shows the programmed inhibit threshold that is set to veto RF buckets above that intensity. 


\subsection{Targets}

SeaQuest target system consists of two liquid targets (hydrogen and deuterium) and three solid targets (carbon, iron and tungsten). An empty flask and an empty solid target holder are used for background measurement ${ }^{2}$. These targets are mounted on a table that can translate in the $x$-direction over a range of $91.4 \mathrm{~cm}$. Motion of the target table is achieved by a lead screw which moves the table on the rails. A single step of the motor translates the table in the $x$-direction by $2.54 \mu \mathrm{m}$ and the positions of the targets are checked by magnetic proximity sensors attached to the target table. Data is taken on different targets in a programmable cyclic order as shown in Table 3.3. It typically takes 30 seconds to switch from one target to another and this happens in the 55 seconds after a spill when SeaQuest doesn't get beam. The purpose of this is to minimize the systematics associated with longterm change in experimental conditions. All targets are typically $7-15 \%$ in interaction length.

\subsubsection{Cryogenic Targets}

The liquid targets are $50.8 \mathrm{~cm}$ long and $7.62 \mathrm{~cm}$ in diameter and can contain 2.2 liters of liquid in them. The flask walls are made of $76 \mu$ m-thick stainless steel with $51 \mu \mathrm{m}$-thick stainless steel end caps. The liquid hydrogen (or $\mathrm{LH}_{2}$ or $\mathrm{H}_{2}$ ) target used "Ultra High Purity" gas which is $99.999 \%$ commercially pure. The gas used for deuterium (or $\mathrm{LD}_{2}$ or $\mathrm{D}_{2}$ ) target came from two sources:

- $95.8 \pm 0.2 \%$ gas that was used for bubble chamber experiments at Fermilab. This gas contained contamination from ${ }^{2} \mathrm{H}$ and ${ }^{1} \mathrm{H}$ primarily in $\mathrm{HD}$ molecules.

- $99.99 \%$ pure commercially available deuterium that was used during the latter part of the experiment.

\footnotetext{
${ }^{2}$ As mentioned before, the iron beam dump could also be used as a "target" to search for massive dark photons.
} 
In order to make sure that there is liquid in the flask (at a calculable density), the targets are operated at the vapor-liquid saturation curve. The pressure of vapor in the lines at the top of the flask is measured and that is used to regulate power to three $500 \Omega$ heater resistors. The resistance of these three resistors (located at the bottom, middle and top of the flask) also indicates the level of the liquid (Fig 3.10). A desired pressure is chosen and the heater power is regulated to maintain this pressure in the sensors. The liquid density (mentioned in Table 3.3) is calculated from the intercept of the saturation curve using the measured pressure [121].

\subsubsection{Solid targets}

Each of the solid targets is divided into three discs of $1 / 3$ the total thickness. The properties of these targets are mentioned in Table 3.3. These targets are placed $25.4 \mathrm{~cm}$ apart along the beam axis (with the exception of $\mathrm{Fe}(17.1 \mathrm{~cm})$ during data set 2$)$ on the beam axis as shown in Fig 3.28. This was done to somewhat mimic the spatial distribution of liquid targets and to minimize target depen-

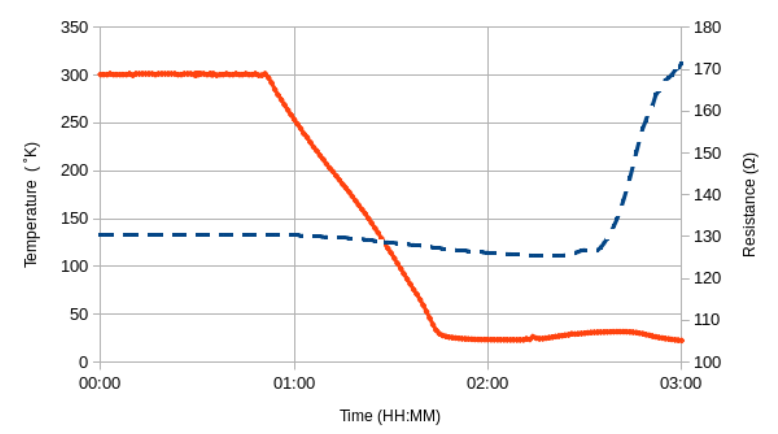

Figure 3.10: The temperature of the condenser (red line, left scale) plotted as the $\mathrm{D}_{2}$ is cooled down. Blue dashed line gives the resistance of the level sensor inside the target flask. A rise in the value of the resistance indicates the formation of liquid [120]. dent variation in spectrometer acceptance [120]. 


\begin{tabular}{|c|c|c|c|c|c|}
\hline Position & $\begin{array}{c}\text { Target } \\
\text { Material }\end{array}$ & $\begin{array}{c}\text { Target } \\
\text { Density } \\
\left(\mathrm{g} / \mathrm{cm}^{3}\right)\end{array}$ & $\begin{array}{c}\text { Thickness } \\
(\mathrm{cm})\end{array}$ & $\begin{array}{c}\text { Number of } \\
\text { Interaction } \\
\text { Lengths }\end{array}$ & $\begin{array}{c}\text { Typical } \\
\text { Spills/ } \\
\text { Cycle }\end{array}$ \\
\hline \hline 1 & $\mathrm{LH}_{2}$ & 0.071 & 50.8 & 0.069 & 10 \\
\hline 2 & Empty Flask & - & - & 0.0016 & 2 \\
\hline 3 & $\mathrm{LD}_{2}$ & 0.163 & 50.8 & 0.120 & 5 \\
\hline 4 & No Target & - & - & 0 & 2 \\
\hline 5 & Iron & 7.87 & 1.905 & 0.114 & 1 \\
\hline 6 & Carbon & 1.80 & 3.322 & 0.209 & 2 \\
\hline 7 & Tungsten & 19.30 & 0.953 & 0.096 & 1 \\
\hline
\end{tabular}

Table 3.3: Characteristics of targets at SeaQuest. The "Spills/Cycle" for empty flask was changed from 1 to 2 in the latter part of the experiment [120]. 


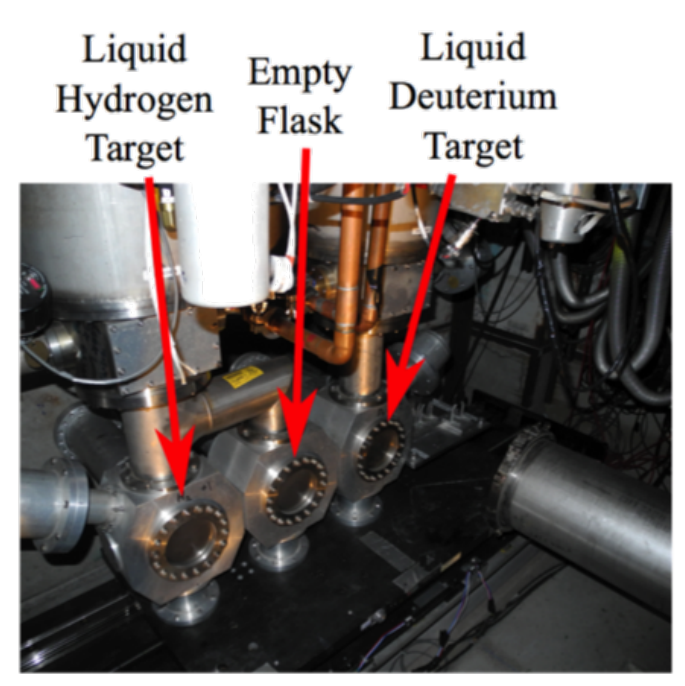

(a) Liquid targets

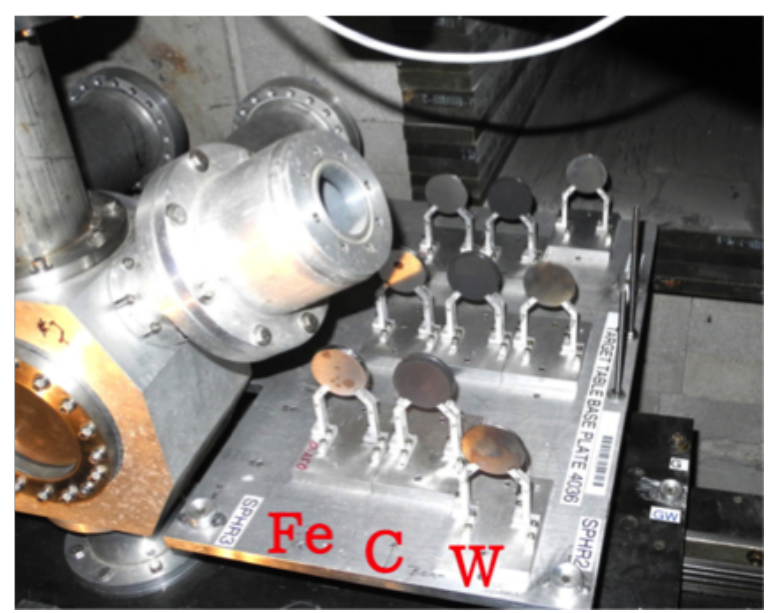

(b) Solid targets

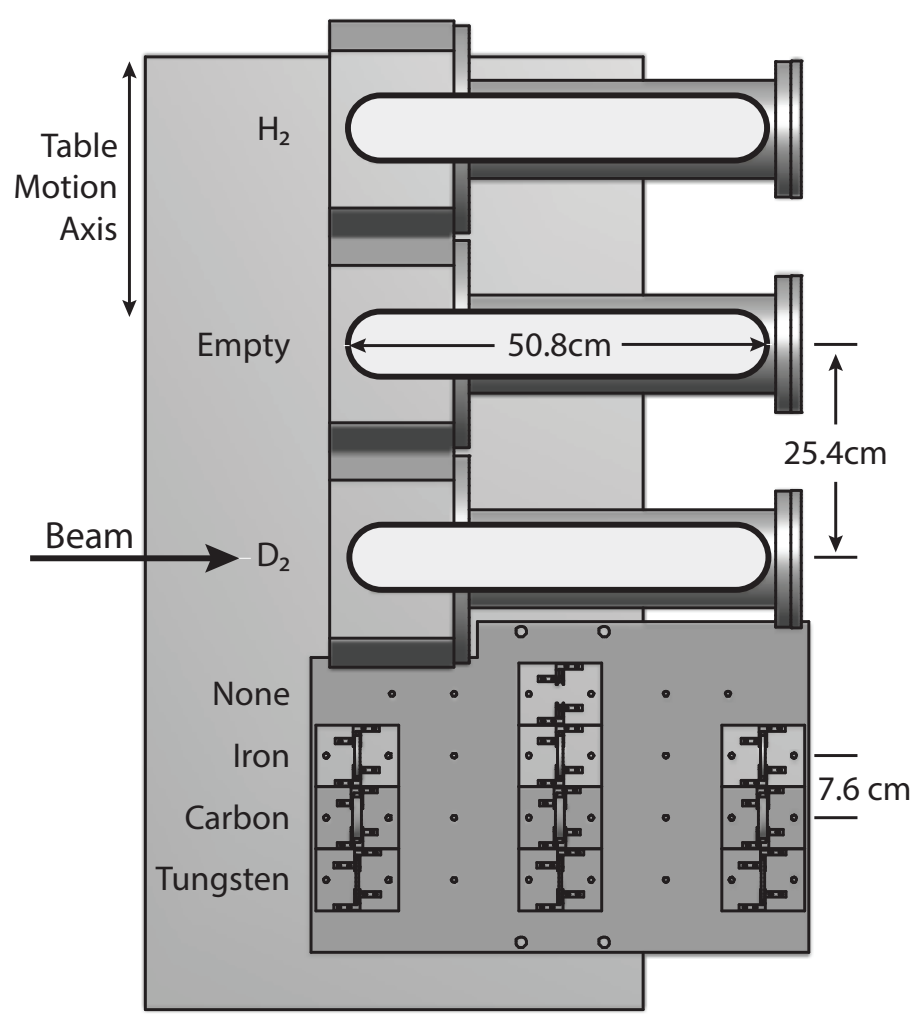

(c) Schematics of the target table

Figure 3.11: SeaQuest targets 


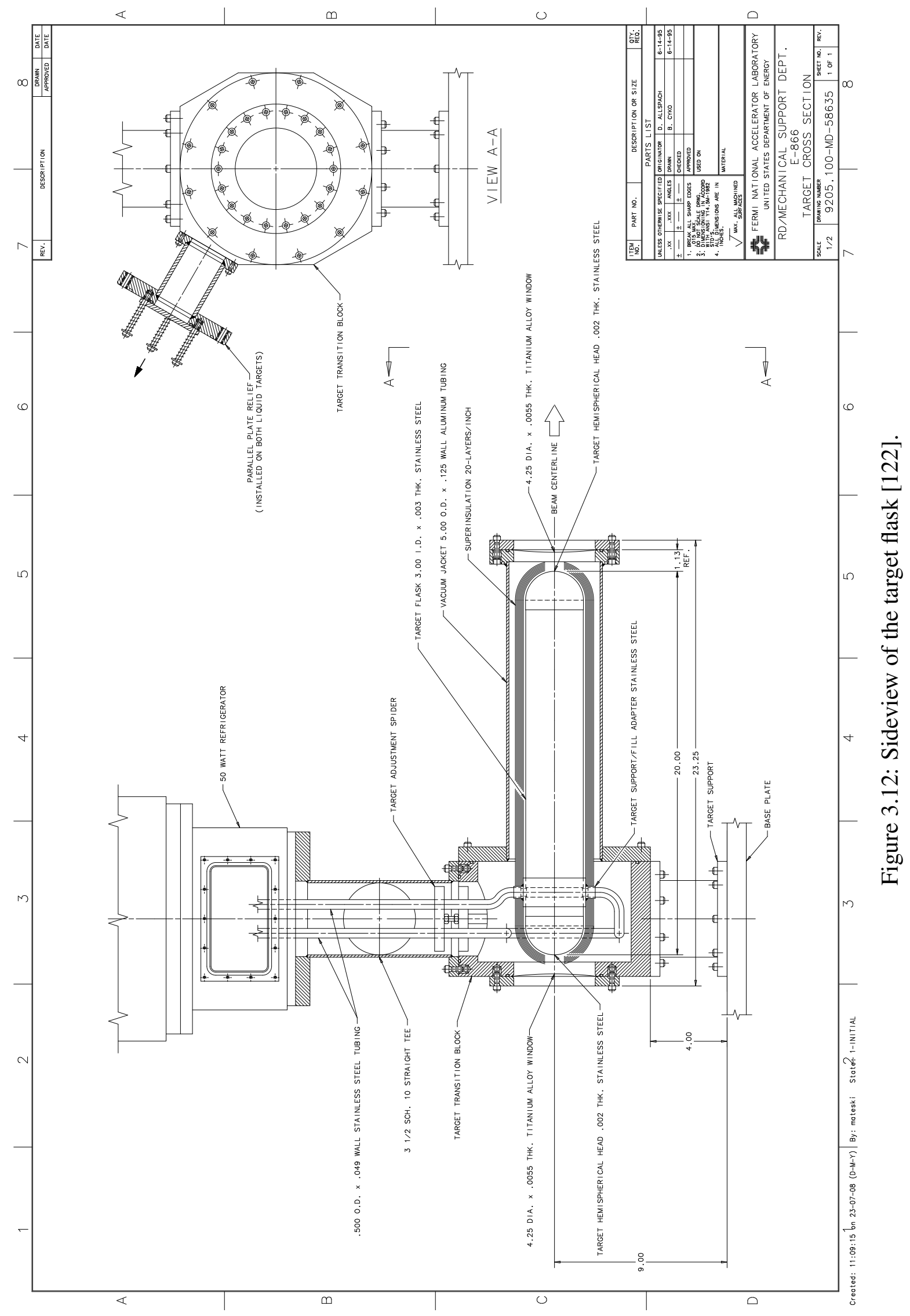




\subsection{Magnets}

SeaQuest uses two dipole magnets. The first magnet, known as Fe magnet or FMAG is made out of $43.2 \mathrm{~cm} \times 160 \mathrm{~cm} \times 503 \mathrm{~cm}_{\text {Iron slabs }}^{3}$. The iron was recycled from the Columbia University Nevis Laboratory Cyclotron in 1980. It uses the bedstead coils recovered from SM3 magnet used in the E866 experiment. As shown in Fig 3.13, bedstead coils (yellow) curl up and leave room for other detectors to be placed close to the magnet. FMAG was consistently maintained at $2000 \mathrm{~A}(25 \mathrm{~V}$ using $50 \mathrm{~kW}$ of power) to generate a magnetic field of $1.8 \mathrm{~T}$ (giving a total magnetic deflection of $3.07 \mathrm{GeV} / \mathrm{c}$ ) [120]. The control system relays back the signal from FMAG to the control room so that beam is not sent when its off preventing damage to the spectrometer. The FMAG also consists of blocks of iron that absorbs the beam that remains after interactions with the target. It has a $5 \mathrm{~cm}$ (diameter) $\times 25 \mathrm{~cm}$ hole along the beam axis in the front to prevent the deposited beam from back "splashing" afterwards. It also sweeps out the low momentum muons and pushes the high momentum (desired signal like) muons into acceptance. The field calibration of this magnet is done by aligning the reconstructed mass peak with the known mass of the $J / \psi$ (3097) particle.

The second magnet, $\mathrm{KTeV}$ magnet or KMAG is $300 \mathrm{~cm}$ long iron rectangular air-core magnet with $289 \mathrm{~cm} \times 203 \mathrm{~cm}$ high central gap. It was originally constructed by the E799/KTeV collaboration at Fermilab, using donated steel from the University of Maryland Cyclotron [120]. During data taking periods, KMAG was run at 1600 A (270 V using $430 \mathrm{~kW}$ of power) to generate a magnetic field of $0.4 \mathrm{~T}$ (giving a total magnetic deflection of $0.39 \mathrm{GeV} / \mathrm{c})$. Hall probe measurements were done by SeaQuest for central field calibration [123]. Similar to FMAG, the final value for the magnetic field is deduced from the measurement of the exact mass of the $J / \psi(3097)$ particle. The field of both magnets is oriented vertically $+y$ for the former and $-y$ for the latter part of the experiment. The change

\footnotetext{
${ }^{3}$ one interaction length for protons on Iron $=17 \mathrm{~cm}$. Hence all of FMAG is equivalent to 35 interaction lengths.
} 
was done to identify any left-right asymmetries present in the spectrometer. The vertical orientation of the magnetic field made the $x$-direction the bend plane and the $y$ direction the non-bend plane.
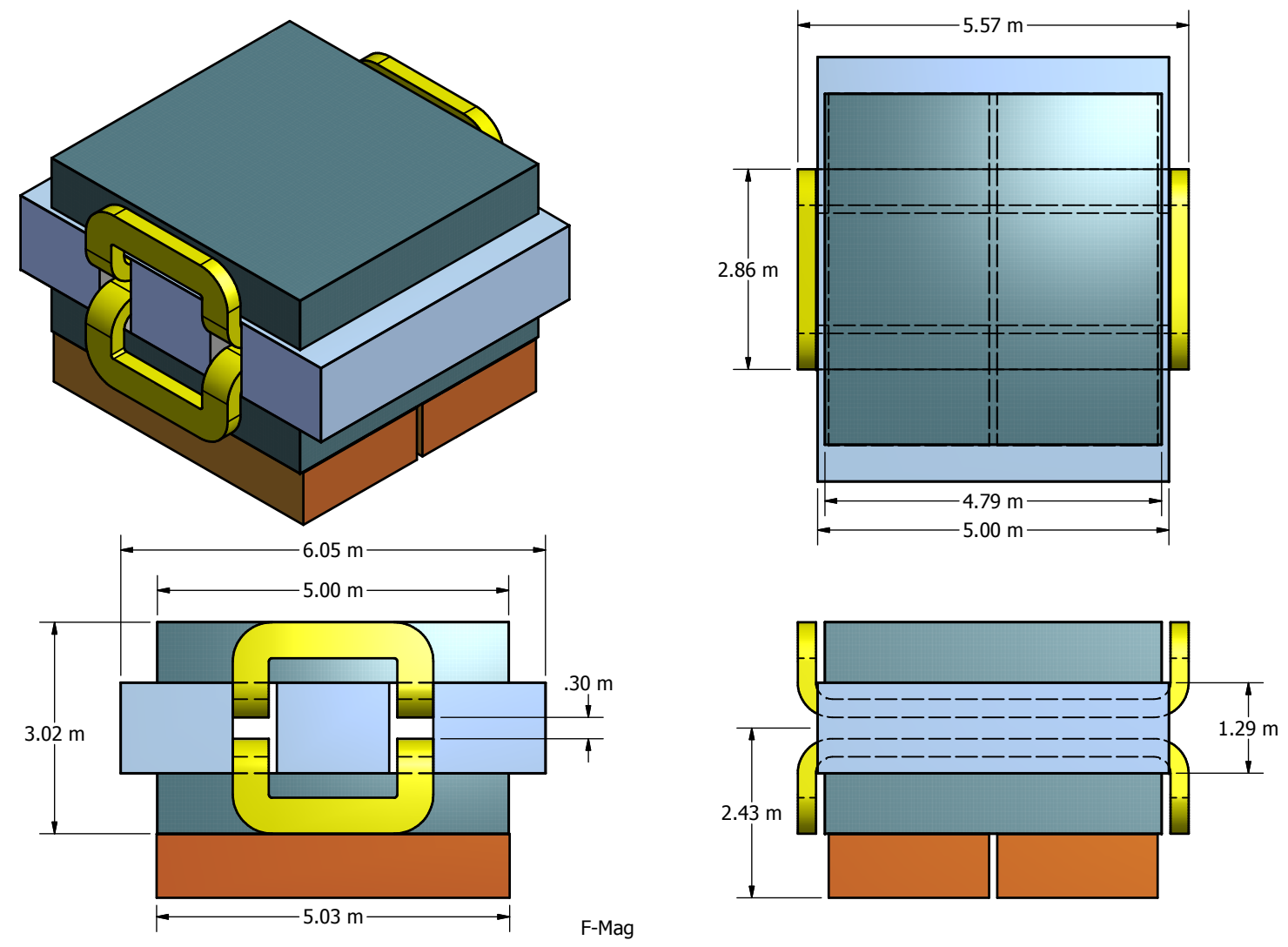

Figure 3.13: FMAG schematic view. The $5 \mathrm{~cm}$ (diameter) $\times 25 \mathrm{~cm}$ hole on the front face of the FMAG not shown in this picture [124]. 

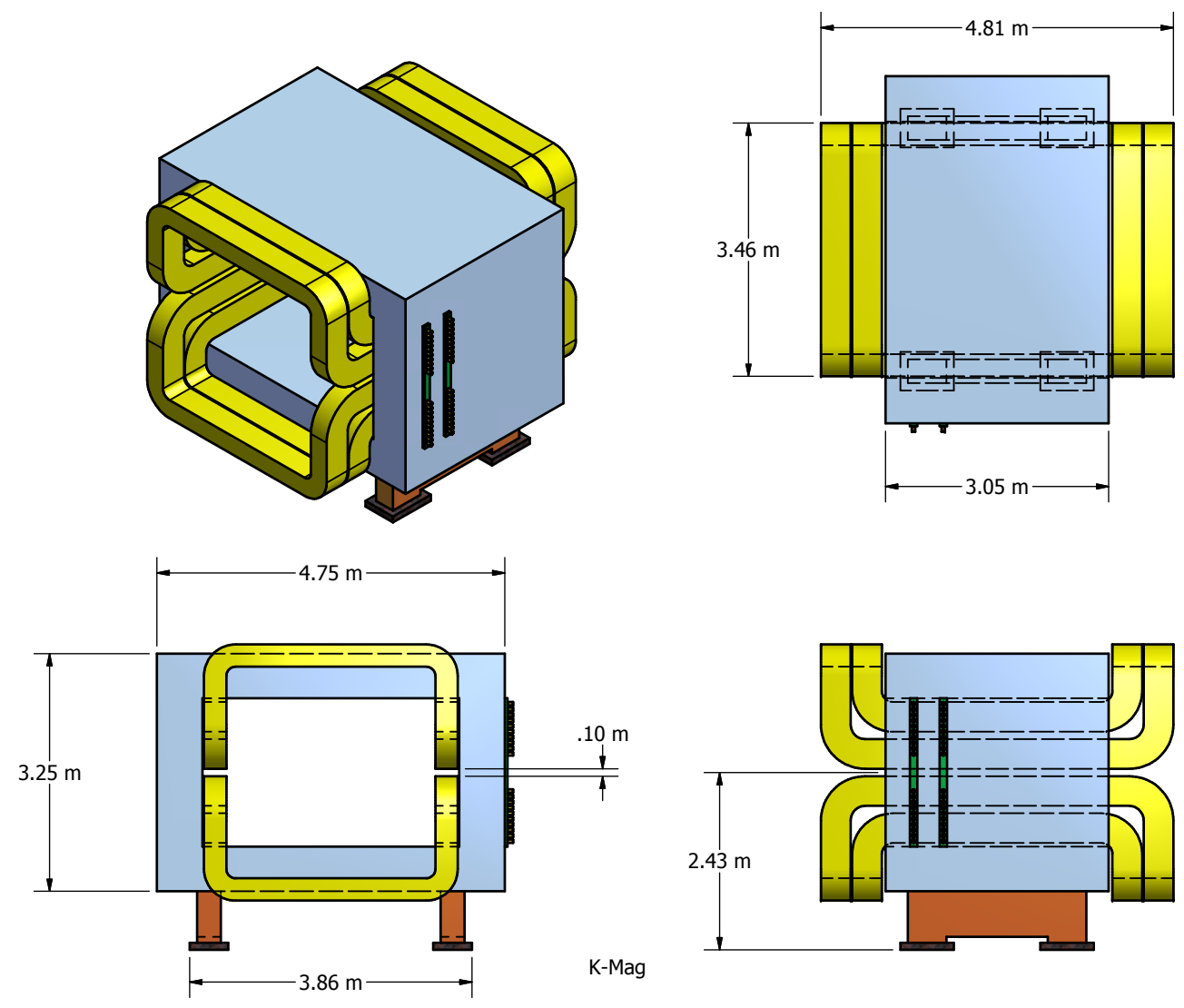

Figure 3.14: Schematic view of KMAG [124]. 


\subsection{Hodoscopes}

SeaQuest uses four tracking "Stations" for muon track identification, reconstruction and momentum measurement. They are labeled Station 1, Station 2, Station 3 and Station 4 respectively. Stations 1, 2 and 3 consists of drift chambers and hodoscope planes whereas Station 4 consists of proportional tubes and hodoscopes planes. Four hodoscope planes are

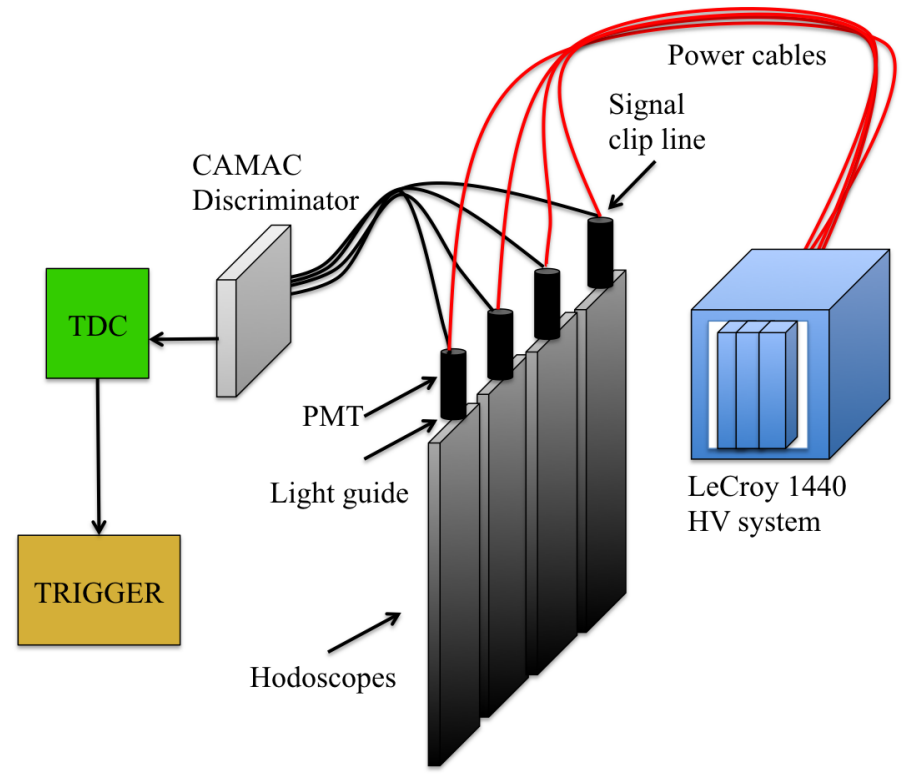

Figure 3.15: Depiction of hodoscopes and connected circuitry.

used as the primary trigger in SeaQuest spectrometer. Stations 1 and 2 used recycled hodoscopes from HERMES and St 3 and 4 use new Eljen EJ-200 scintillator material. Each hodoscope of scintillating material is covered by black paper to prevent light leaks. They are then attached to a plexi-glass light guide which is further connected to a photomultiplier tube. The PMTs are powered by LeCroy 1440 High Voltage supplies. The planes of hodoscopes are arranged vertically ( $x$-plane) and horizontally ( $y$-plane) to measure the $x$ and $y$ position respectively. All planes have a slight overlap to make sure there were no "holes" in the acceptance. Station 1 and 2 have a single $x$ plane. Station 3 has only one $x$ plane and Station 4 has two $y$ planes and one $x$ plane. The geometric specifications of each 
set are shown in Figures 3.16, 3.17, 3.18, 3.19, 3.20, 3.21, 3.22 and mentioned in Table 3.4. Each plane is divided into T/ B or Top/Bottom to indicate $+\mathrm{Y} /-\mathrm{Y}$ half of the spectrometer and L/R or (Left/Right) indicating $+\mathrm{X} / \mathrm{X}$ half of the $\mathrm{Y}$ planes. Due to the physical size of the panels, PMTs are placed on either end of the Top/Bottom and Left/Right panels of St 3 and St 4 .

\section{Hodoscope maintenance}

Hodoscopes were regularly monitored by taking efficiency runs. The count rates as a function of high voltage were observed for each individual channel and the voltage was set to be on the count-rate vs high voltage plateau. Occasionally, one or more of the hodoscope paddles would stop showing hits on SeaScape ${ }^{4}$. This could happen due to multiple reasons. These reasons were investigated and the problem solved. Some scenarios encountered are listed below:

- A channel in the LeCroy power supply could suddenly stop working for no obvious reason. A simple power cycling usually recovers the dead channels.

- The PMT base, which was designed to handle high rates, could get damaged due to radiation or over heating. In this case, the PMT was replaced with a spare (with clip line attached).

- Sometimes, the PMT itself could stop working. Under such circumstances, the PMT was replaced ${ }^{5}$.

- The CAMAC discriminator module which was attached to the PMTs would sometimes stop working. The module was simply replaced under such circumstances.

\footnotetext{
${ }^{4}$ SeaQuest software that is used to look at hit distributions of various detector elements

${ }^{5}$ Care was taken to make sure that the Silicone material between the plexi-glass light guide and the front face of the photomultiplier tube were touching in order to reduce light leaks.
} 


\begin{tabular}{c|c|c|c|c|c}
\hline \hline Detector & $\begin{array}{c}\text { Paddle width } \\
(\mathrm{cm})\end{array}$ & $\begin{array}{c}\text { Paddle length } \\
(\mathrm{cm})\end{array}$ & \# of paddles & $\begin{array}{c}\text { Width } \times \text { Height } \\
(\mathrm{cm})\end{array}$ & Z - position \\
\hline \hline H1T & 7.32 & 69.9 & 23 & $162 \times 69.85$ & 667.12 \\
H1B & 7.32 & 69.9 & 23 & $162 \times 69.85$ & 667.12 \\
H1L & 7.32 & 78.7 & 20 & $78.74 \times 140.12$ & 654.03 \\
H1R & 7.32 & 78.7 & 20 & $78.74 \times 140.12$ & 654.03 \\
H2T & 13.04 & 132 & 16 & $203.24 \times 150.00$ & 1421.06 \\
H2B & 13.04 & 132 & 16 & $203.24 \times 150.00$ & 1421.06 \\
H2L & 13.07 & 152 & 19 & $132.00 \times 241.29$ & 1402.86 \\
H2R & 13.07 & 152 & 19 & $132.00 \times 241.29$ & 1402.86 \\
H3T & 14.59 & 132 & 16 & $227.52 \times 167.64$ & 1958.51 \\
H3B & 14.59 & 132 & 16 & $227.52 \times 167.64$ & 1958.51 \\
H4T & 19.65 & 182.9 & 16 & $304.52 \times 182.88$ & 2234.50 \\
H4B & 19.65 & 182.9 & 16 & $304.52 \times 182.88$ & 2250.68 \\
H4Y1L & 23.48 & 152.4 & 16 & $152.40 \times 365.80$ & 2130.27 \\
H4Y1R & 23.48 & 152.4 & 16 & $152.40 \times 365.80$ & 2146.45 \\
H4Y2L & 23.48 & 152.4 & 16 & $152.40 \times 365.80$ & 2200.44 \\
H4Y2R & 23.48 & 152.4 & 16 & $152.40 \times 365.80$ & 2216.62 \\
\hline \hline
\end{tabular}

Table 3.4: Specifications of different hodoscope planes. The designation (L) and (R) refer to beam left or right and (T) and (B) refers to Top and Bottom. Z - position is measured from the front face of FMAG. Values in the table are taken from [125].

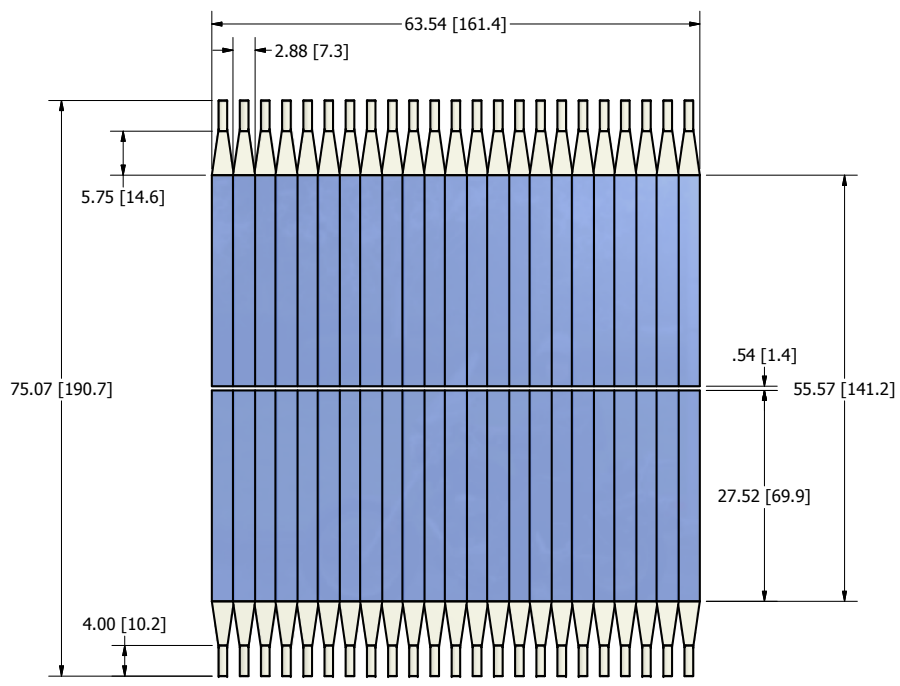

Hodoscope Array $1 \mathrm{X}$

Figure 3.16: H1X Hodoscope panels at St 1. 


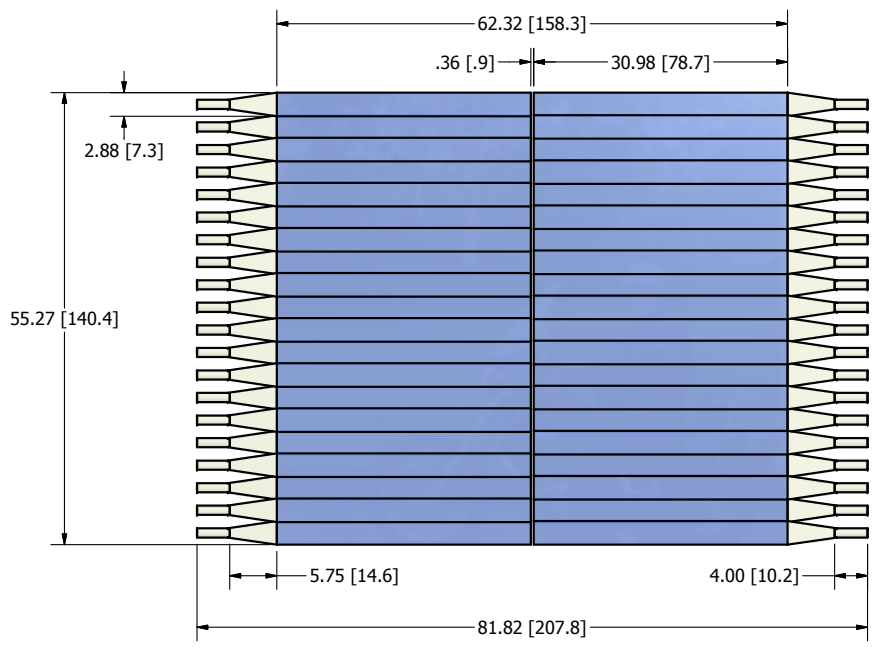

Hodoscope Array $1 Y$

Figure 3.17: H1Y Hodoscope panels at St 1.

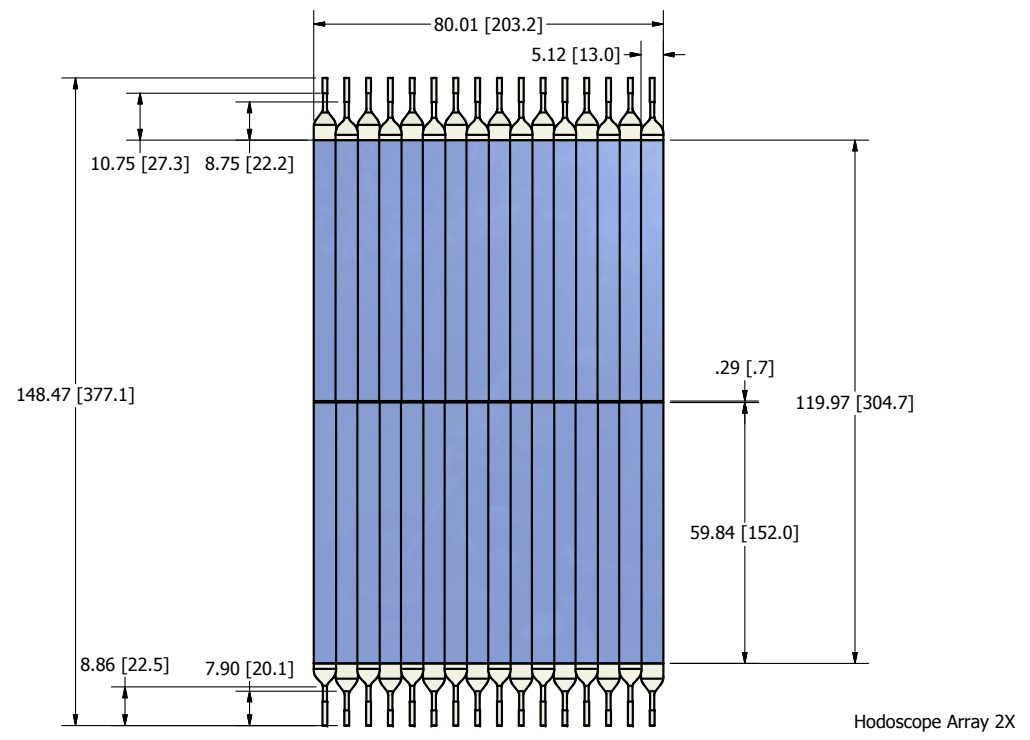

Figure 3.18: H2X Hodoscope panels at St 2. 


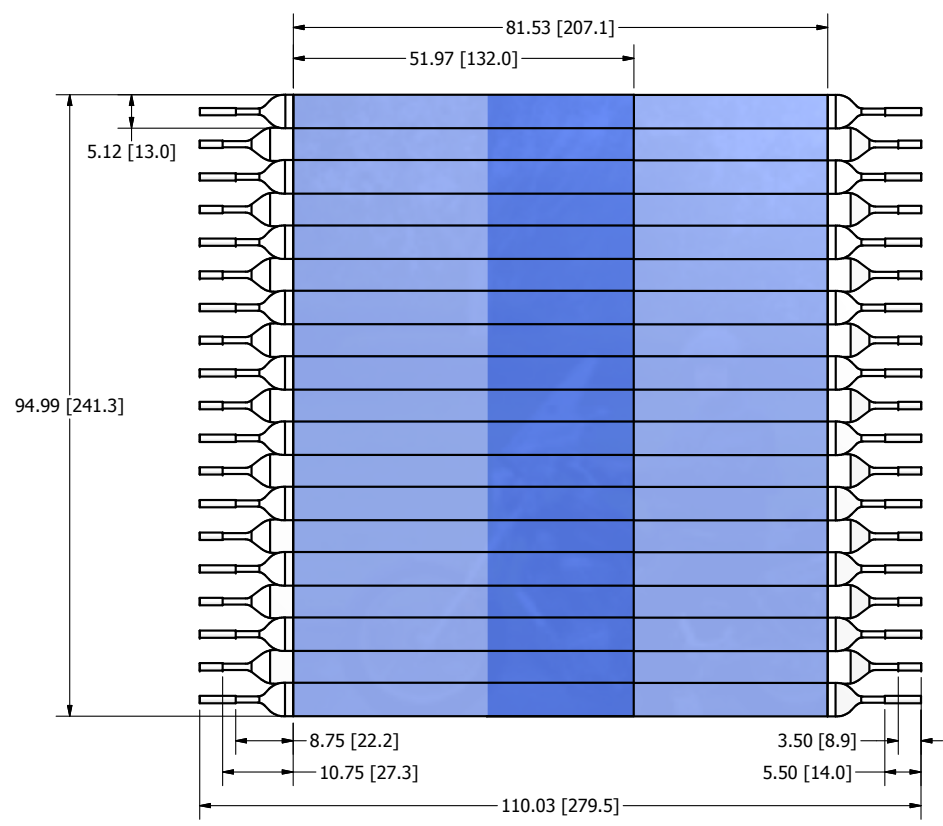

Hodoscope Array $2 Y$

Figure 3.19: H2Y Hodoscope panels at St 2.

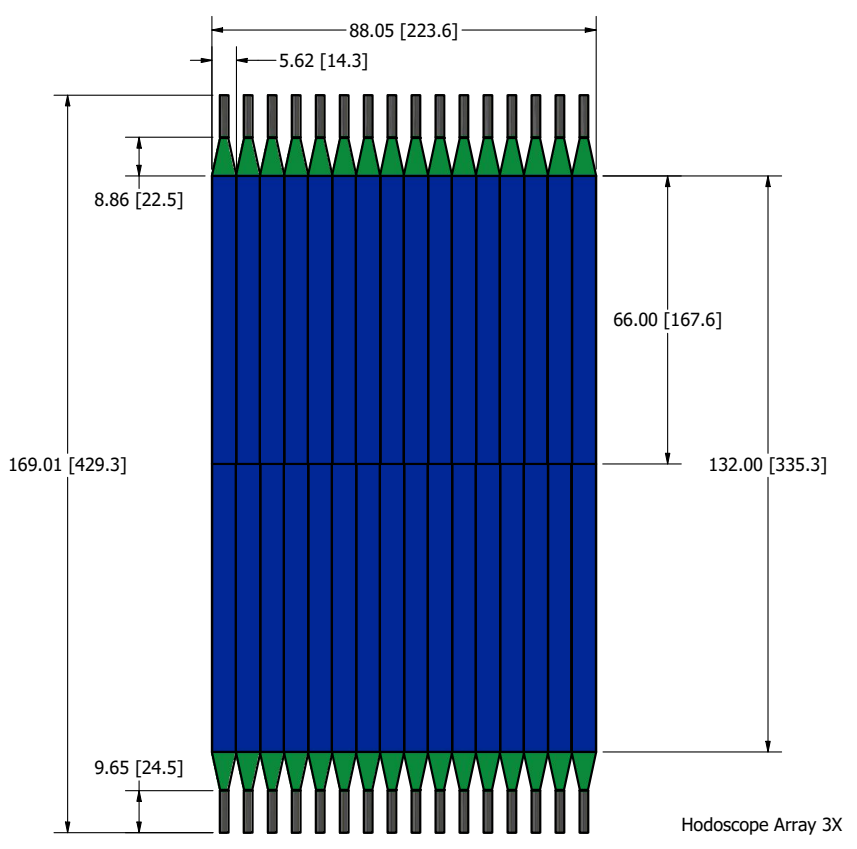

Figure 3.20: H3X Hodoscope panels at St 3. 


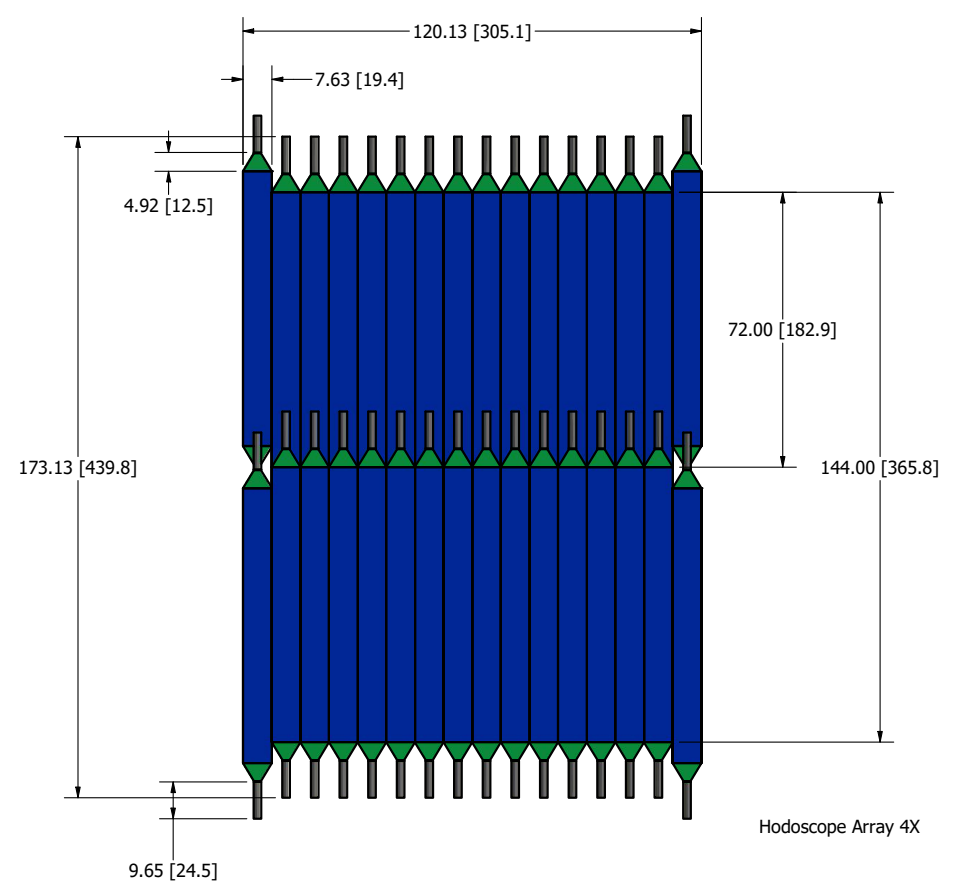

Figure 3.21: H4X Hodoscope panels at St 4.

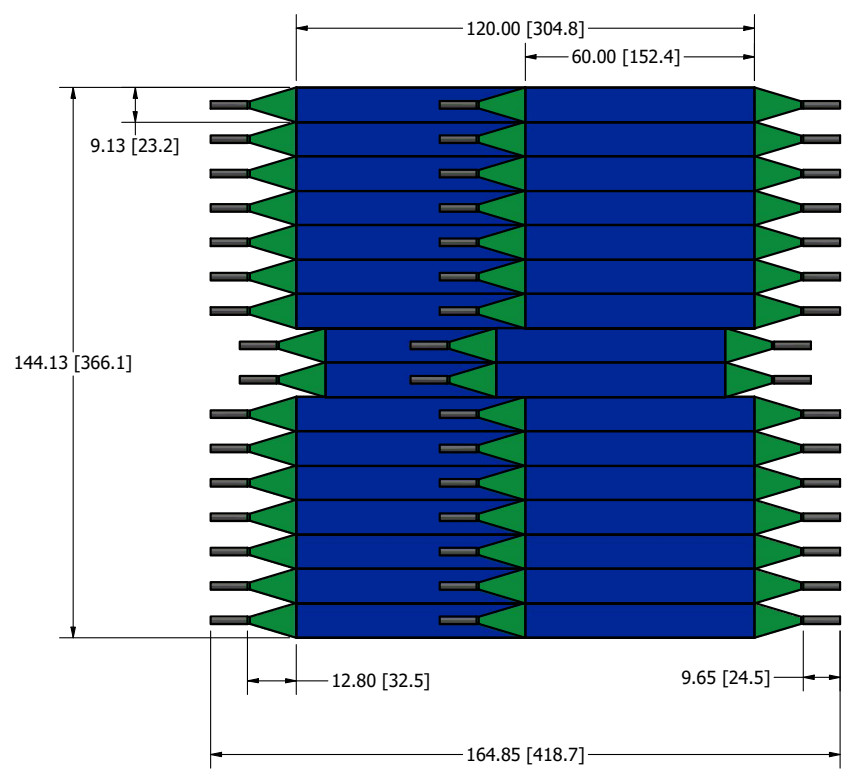

Hodoscope Array $4 \mathrm{Y}$

Figure 3.22: H4Y Hodoscope panels at St 4. 


\subsection{Drift Chambers}

Drift chambers at SeaQuest play a crucial role in detecting the passage of charged particles. The principle of operation is the same as that of a simple ionization detector. Whenever a charge particle passes through the gaseous medium of a drift chamber, certain number of electron-ion pairs are created depending on the $E / p$ ratio where $E$ is the electric field and $p$ is the pressure of the gas in the chamber. These 'primary electrons' are accelerated towards the positively charged anode wire. In the process, the electrons gain sufficient energy and knock out more 'secondary electrons' from gaseous atoms in their path. This multiplication happens within in just a few radii of the anode wire (depending on the strength of the field). Assuming we are in the voltage range that is close to the operational voltage of a drift chamber, a localized avalanche (proportional to the number of primary electrons) is created and the electrons are pulled towards the anode wire. The electrons can be col-
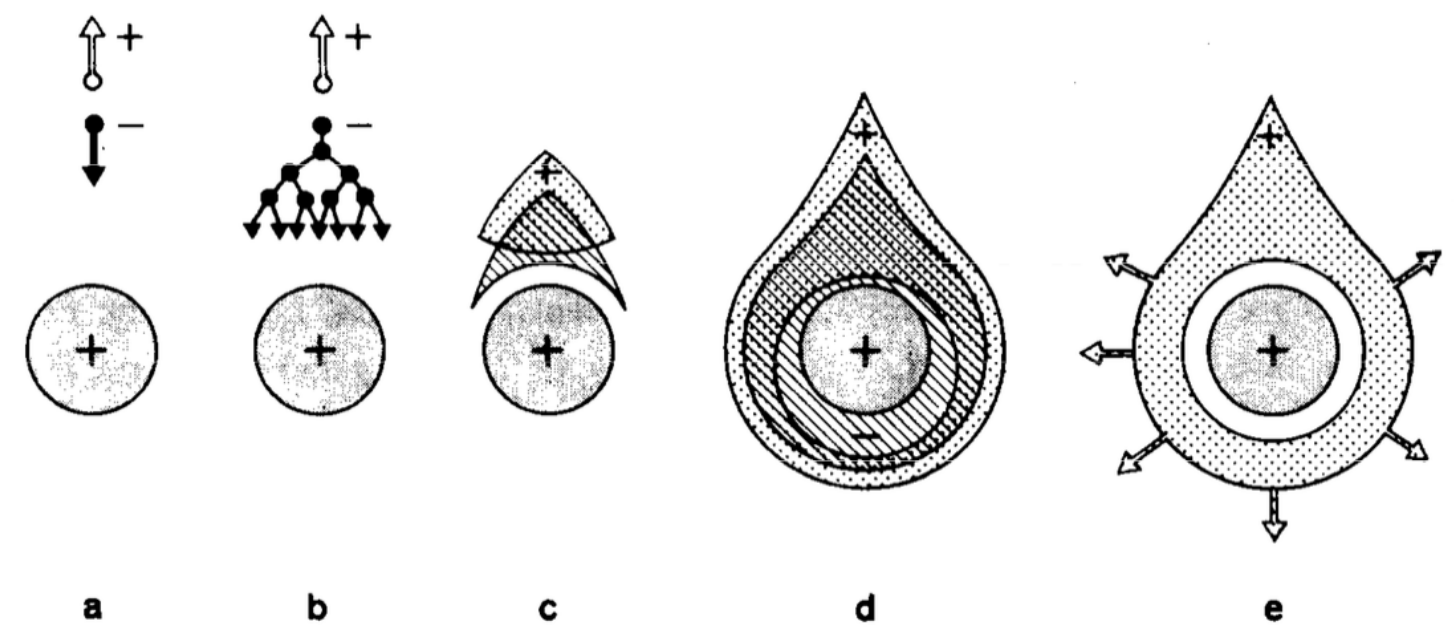

Figure 3.23: Depiction of stages of creation of an avalanche taken from [126].

lected very quickly $(\sim 1 \mathrm{~ns})$ while the positive charge that was pulled towards the anode wire along with the drop of negative charge begins drifting towards the cathode wire. The drift of the positive charge towards the cathode pulls stored energy from the anode causing a voltage drop, inducing a signal. It is this induced signal on the anode wire that is 
detected in the electronics. Figure 3.23 shows the different stages of the formation of the signal. Spatial information can be obtained from measuring the drift time of the electrons which are coming from an ionizing event. Fig 3.24 depicts such a measurement. A scintillation counter starts the timer of the passage of the charged particle, and the time difference between the hodoscope signal and signal created at the anode yields the drift time. From this time difference, the position of the passage of the charged particle is calculated. Several planes are arranged as a grid and the position information can be deduced from the timing information by using a distance to time (RT) curve for each plane. More information on the principles of operation of drift chambers can be found in $[126,127,128,129$, 130]. A drift chamber typically has a grid (or a cell) of different kinds of wires, each with a unique purpose:

- Anode wires: Small radius, often positive high voltage wires used to collect electrons and generate the avalanche.

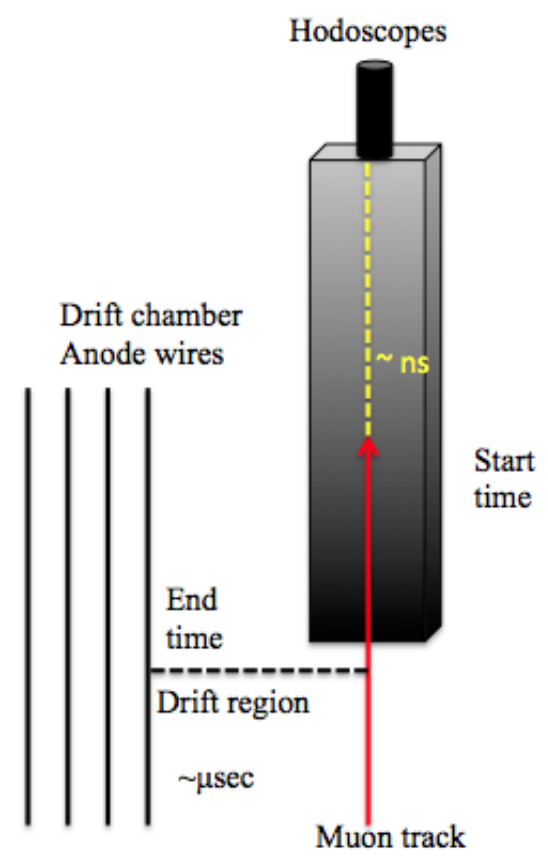

Figure 3.24: Drift time calculation schematic.

- Cathode wires: Large radius, usually ground wires (sometimes a thin foil in a plane) used to pull the positive ions away from the anode.

Some chambers are designed to operate without field or guard wires.

- Field wires: Used in shaping the field and removes any electromagnetic non uniformities in the inter-anode fields. It could also be placed in the middle of two neighboring anode wires to strengthen the electromagnetic field around that position. 
- Guard wires: Used in "electromagnetic blocking" of one section of the drift chamber from another.

\subsubsection{Selection of Gas for the Drift Chamber}

Photons produced in de-excitation of ionized gas atoms can in principle ionize other atoms, with electrons drifting and causing secondary avalanches. Hence, a "quencher" is added to absorb the photons and dissipate the energy through other channels such as thermal collisions and other modes of excitations. Small amounts of an electronegative gas can increase the gain by trapping the electrons before they reach the anode, preventing the unwanted avalanches. Usually noble gases are chosen due to their low requirement for electric field intensity. Due to its low cost and specific ionization, Argon is used in SeaQuest. But, continuous discharge occurs in pure Argon because of the high excitation energy. This causes the excited atoms in Argon to de-excite releasing $11.6 \mathrm{eV}$ thereby creating further avalanches. In order to prevent this, a small amount of polyatomic gas $\left(\mathrm{CH}_{4}\right.$ in the case of SeaQuest) is chosen as a quencher. Organic quenchers cause problems by dissociating into free radicals and depositing on the cathode wire. This causes a deposition of positive charge in the chamber due to slow dissipation and neutralization, which generates arcs in the chamber. A small amount of Methylal, or another non-polymeric compound, is added in order to increase the gain. Sometimes, small amounts of electronegative gases $\left(\mathrm{CF}_{4}\right.$ in case of SeaQuest) are also added to increase the gain or drift speed.

\section{Drift Chambers at SeaQuest}

Drift chambers at Station 1, 2 and 3 are used to measure precisely the $x$ and $y$ positions of muons from drift chamber planes located at different $z$ locations across the spectrometer. SeaQuest used a total of 10 drift chambers through out the duration of the experiment. These drift chambers present at the four stations are labeled DC1.1, DC1.2, DC2, DC3m.1, 
DC $3 \mathrm{~m} .2$ and DC $3 \mathrm{p}^{6}$. The experiment started out with the configuration DC1.1 + DC2 + DC3m.1 + DC3p during the commissioning run in 2012. However, the upper and lower halves at St 3 i.e. DC3m. 1 and DC3p were not symmetric. In order to make the acceptance symmetric, new drift chamber DC3m.2 was constructed at Fermilab and installed. Similarly, at Station 1, DC1.1 (Runs 1 - 3) was replaced with by a new larger chamber DC1.2 (Runs 4-6) with better expected rate handling capabilities. However, due to the many problems with DC1.2, DC1.1 was reinstalled along with DC1.2 in order to continue data taking in case DC1.2 runs into issues. Table 3.5 summarizes all the Run configurations.

\begin{tabular}{c|c|c|c|c}
\hline \hline Run period & Dates & St 1 & St 2 & St 3 \\
\hline Run 1 & 2012 Mar - 2012 Apr & DC1.1 & DC2 & DC3p + DC3m.1 \\
Run 2 & 2013 Nov - 2014 Aug & DC1.1 & DC2 & DC3p + DC3m.2 \\
Run 3 & 2014 Nov - 2015 Jul & DC1.1 & DC2 & DC3p + DC3m.2 \\
Run 4 & 2015 Nov - 2016 Mar & DC1.2 & DC2 & DC3p + DC3m.2 \\
Run 5 & 2016 Mar - 2016 Jul & DC1.1 + DC1.2 & DC2 & DC3p + DC3m.2 \\
Run 6 & 2016 Nov - 2017 Jul & DC1.1 + DC1.2 & DC2 & DC3p + DC3m.2 \\
\hline \hline
\end{tabular}

Table 3.5: Configurations of Drift chambers used in different Runs [125].

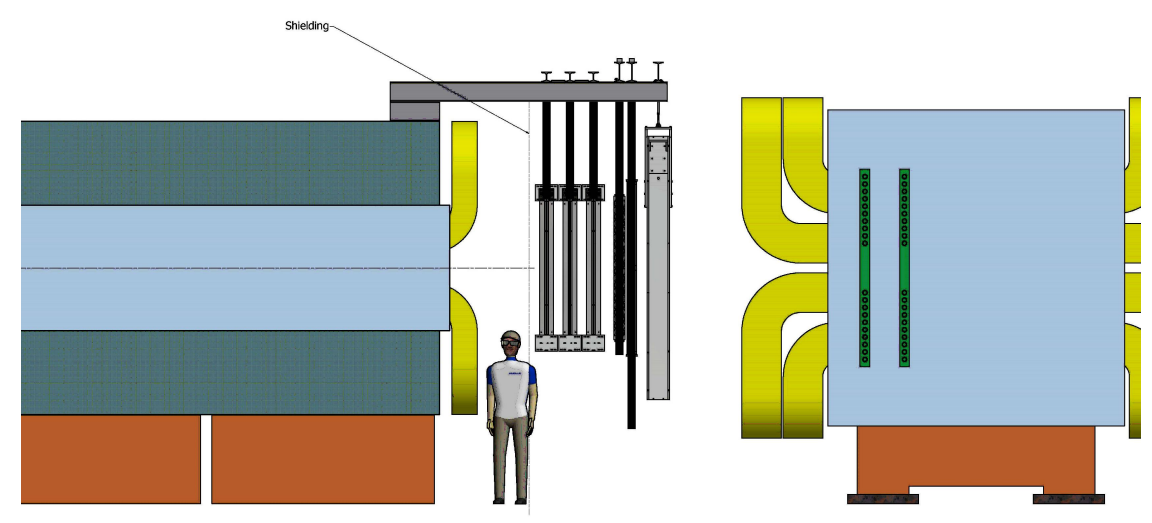

Figure 3.25: Depiction of DC1.1 + DC1.2 configuration [124].

\footnotetext{
${ }^{6}$ The $\mathrm{p}$ (plus) and $\mathrm{m}$ (minus) in DC3p and DC3m are to indicate the vertical position of these chambers relative to the beam $y$ axis.
} 


\section{Similarities:}

- The overall structure is common to all drift chambers. Each Station has drift chambers which have an $\mathrm{X}, \mathrm{X}^{\prime}$ plane for horizontal measurement of the position. They also have $\mathrm{U}, \mathrm{U}^{\prime}\left(\right.$ at $+14^{\circ}$ stereo angle) and V, V' ( $-14^{\circ}$ stereo angles $\left.^{7}\right)$.

- The primed and unprimed planes are shifted by half a cell width in order to resolve the left right ambiguity in detector hits.

- Every drift chamber plane is adjusted (by eye) with spectrometer surveys to be perpendicular to the $z$ axis.

- Drift chamber efficiency for all chambers was monitored on almost a daily basis. The nominal high voltage was chosen such that the drift chambers were at least $95 \%$ efficient. Chamber efficiency studies were done by studying the clean single-track event (FPGA4 with tight QIE threshold) at different high voltage settings. After establishing the tracks vs voltage efficiency plateau curve, the high voltage was lowered slightly in order to keep them on the "knee" instead of the plateau of the curve. Fig 3.40 shows an example of such an efficiency plot. DC1.1, DC2 (E605) and DC3m.1 (E866) chambers were used in previous Fermilab experiments almost three decades ago.

- Gas flowing through all drift chambers and proportional tubes (except DC 1.2) was P08:CF4 (Ar: $\mathrm{CH}_{4}: \mathrm{CF}_{4}$ in the ratio $88 \%: 8 \%: 4 \%$ ).

\section{Differences:}

- Due to its design, the sense plane of DC1.1 was held at a positive voltage compared to the other chambers where the cathode plane was held at a negative voltage instead.

\footnotetext{
${ }^{7} \tan \left( \pm 14^{\circ}\right)= \pm 0.25$. Makes it easy for calculations during design.
} 

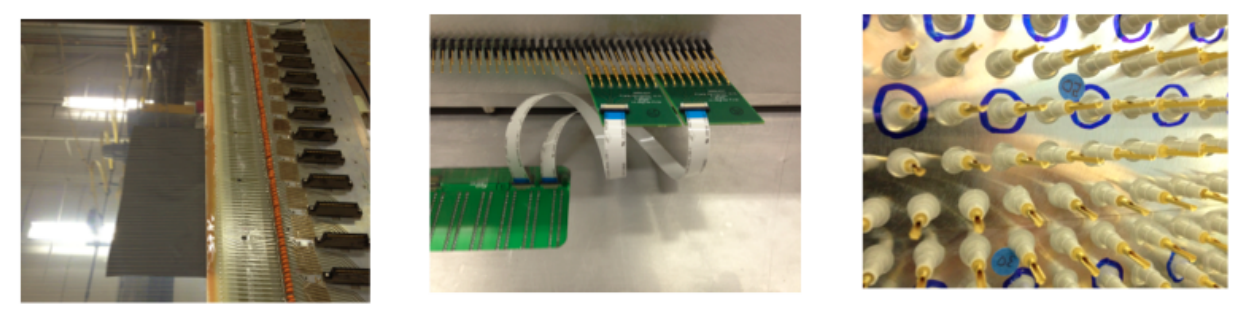

Figure 3.26: positioning the wire using different techniques; printed circuits on G10 frame in the case of DC1.0, and feedthroughs in the case of DC1.1, DC3p and DC 3m.

The cathode plane at DC1.1 is connected to the chamber frame making it hazardous to apply voltage to it unlike the other chambers.

- DC1.1 and DC2 use printed circuits on G10 frames to hold wires in place with epoxy where as DC1.2, DC3p and DC3m use feedthroughs with soldering to hold the wires in place.

- DC1.2 used Ar: $\mathrm{CF}_{4}: \mathrm{C}_{4} \mathrm{H}_{10}: \mathrm{C}_{3} \mathrm{H}_{8} \mathrm{O}_{2}$ in the ratio 81\%:5\%:12\%:2\% (flammable) gas. Additional precaution had to be taken while checking the overall drift chamber leak rate and purging the gas out in case of repairs.

- The type of wire used in stringing the sense planes are different for different chambers.

Table 3.6 summarizes the characteristics of all the SeaQuest drift chambers. During preparation, installation, commissioning and data taking, significant work was done on each chamber in terms of:

- Restringing loose and broken wires, removing broken fragments of wires or thin conducting materials that could cause a dead short. Some of the chambers are $\approx 30$ years old or more.

- Restoring dead channels by repairing or replacing faulty readout electronics.

- Repairing the Level Shifter Boards and High Voltage supplies in order to successfully take data from the drift chambers. 

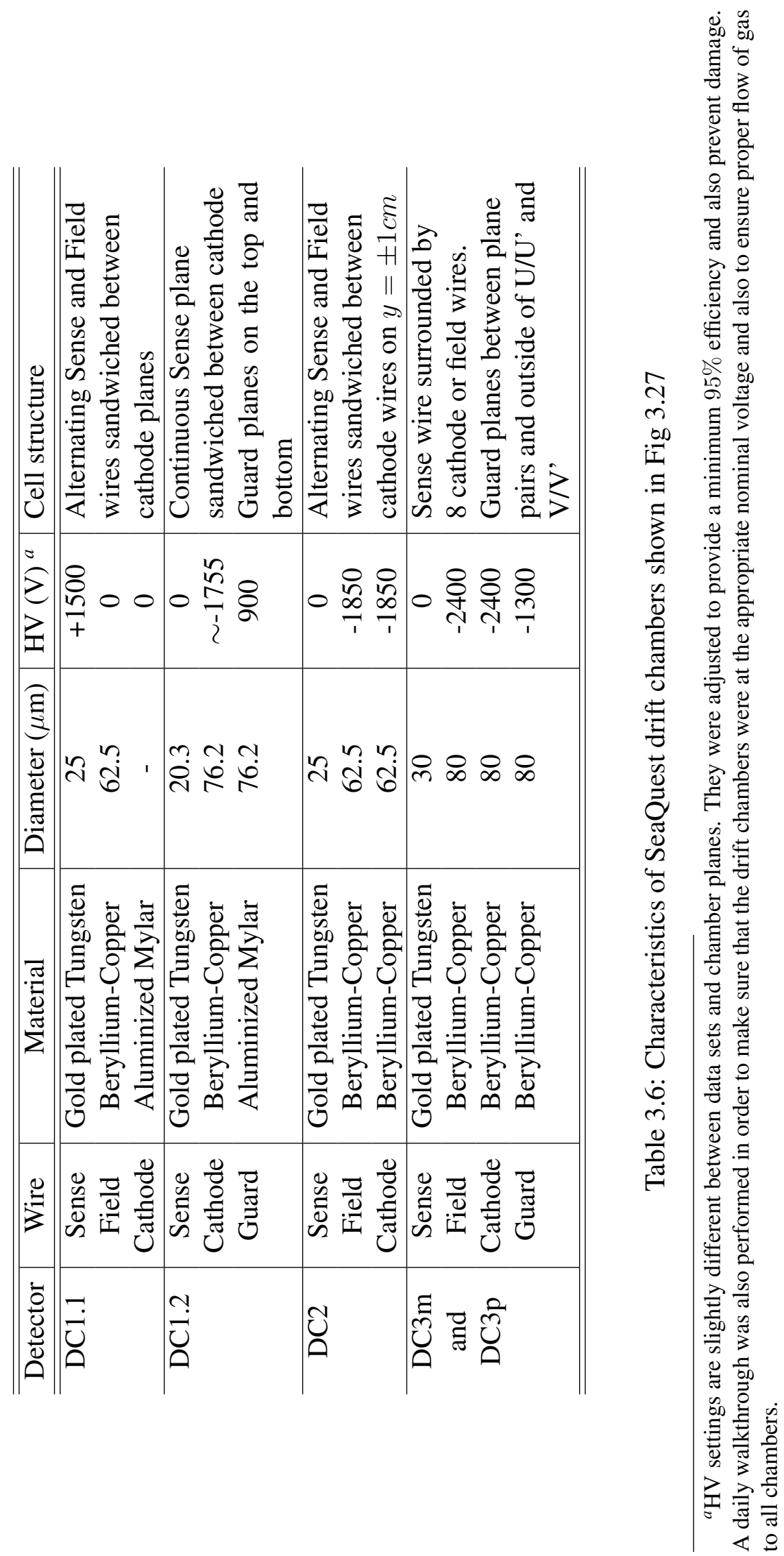


\begin{tabular}{|c|c|c|c|c|c|}
\hline Chamber & View & $\begin{array}{c}\text { No. } \\
\text { of } \\
\text { wires }\end{array}$ & $\begin{array}{c}\text { Cell } \\
\text { width } \\
(\mathrm{cm})\end{array}$ & $\begin{array}{c}\text { Width } \\
\times \text { height } \\
(\mathrm{cm})\end{array}$ & $\begin{array}{c}z \\
\text { Position } \\
(\mathrm{cm})\end{array}$ \\
\hline \hline DC1.1 & $x$ & 160 & 0.64 & $102 \times 122$ & 616 \\
& $u, v$ & 201 & 0.64 & $101 \times 122$ & \pm 20 \\
\hline DC1.2 & $x$ & 320 & 0.50 & $153 \times 137$ & 691 \\
& $u, v$ & 384 & 0.50 & $153 \times 137$ & \pm 1.2 \\
\hline DC2 & $x$ & 112 & 2.1 & $233 \times 264$ & 1347 \\
& $u, v$ & 128 & 2.0 & $233 \times 264$ & \pm 25 \\
\hline DC3p & $x$ & 116 & 2.0 & $232 \times 166$ & 1931 \\
& $u, v$ & 134 & 2.0 & $268 \times 166$ & \pm 6 \\
\hline DC3m.1 & $x$ & 176 & 1.0 & $179 \times 168$ & 1879 \\
& $u, v$ & 208 & 1.0 & $171 \times 163$ & \pm 19 \\
\hline DC3m.2 & $x$ & 116 & 2.0 & $232 \times 166$ & 1895 \\
& $u, v$ & 134 & 2.0 & $268 \times 166$ & \pm 6 \\
\hline
\end{tabular}

Table 3.7: Parameters of all chambers. Those of primed planes are almost the same as of unprimed planes. For the $x$ measuring planes, $z$ position is the distance measured from the front face of FMAG, while for $u$ and $v$ it denotes the offset relative to the $x$ plane.. Table taken from [120]. 


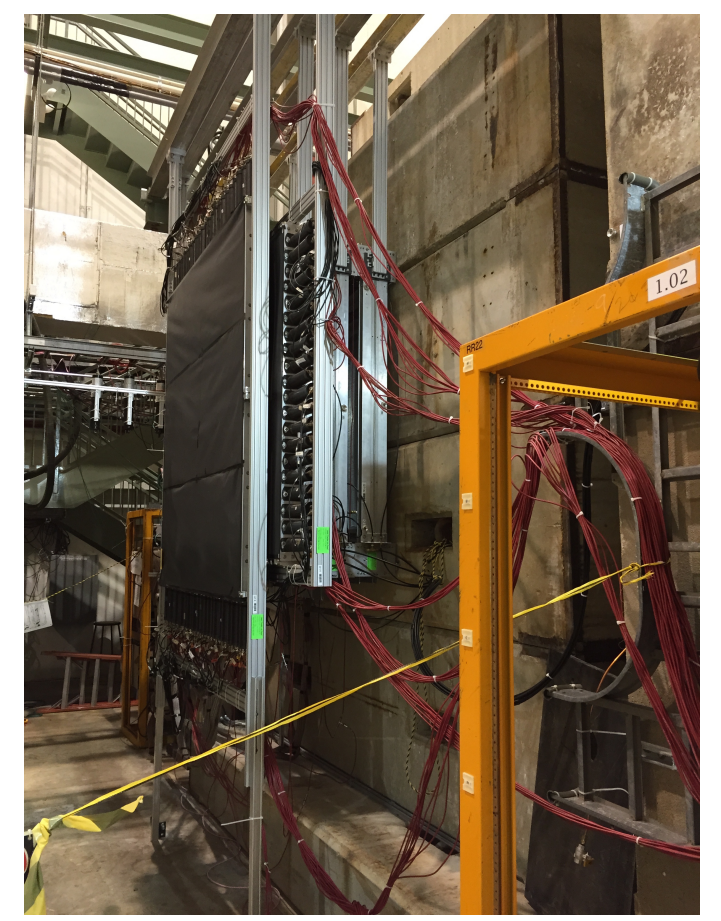

(a) DC1.1

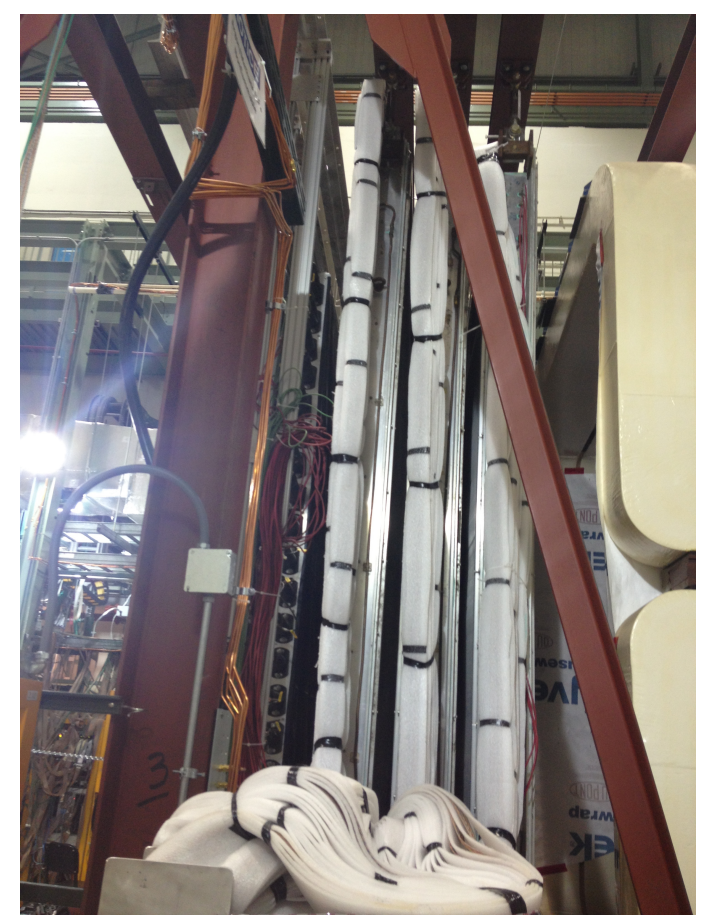

(a) $\mathrm{DC} 2$

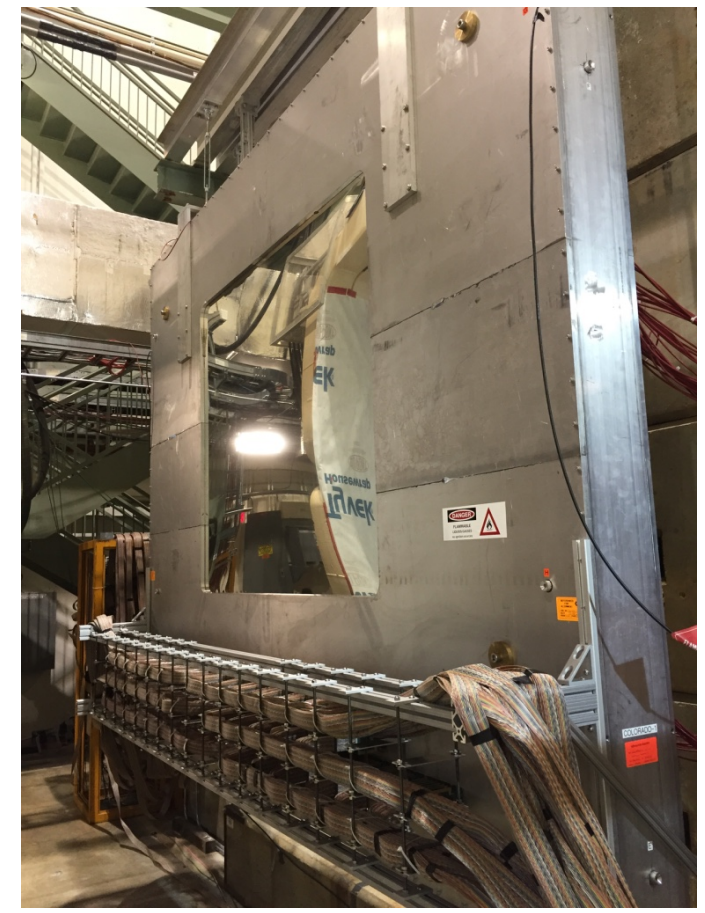

(b) DC1.2

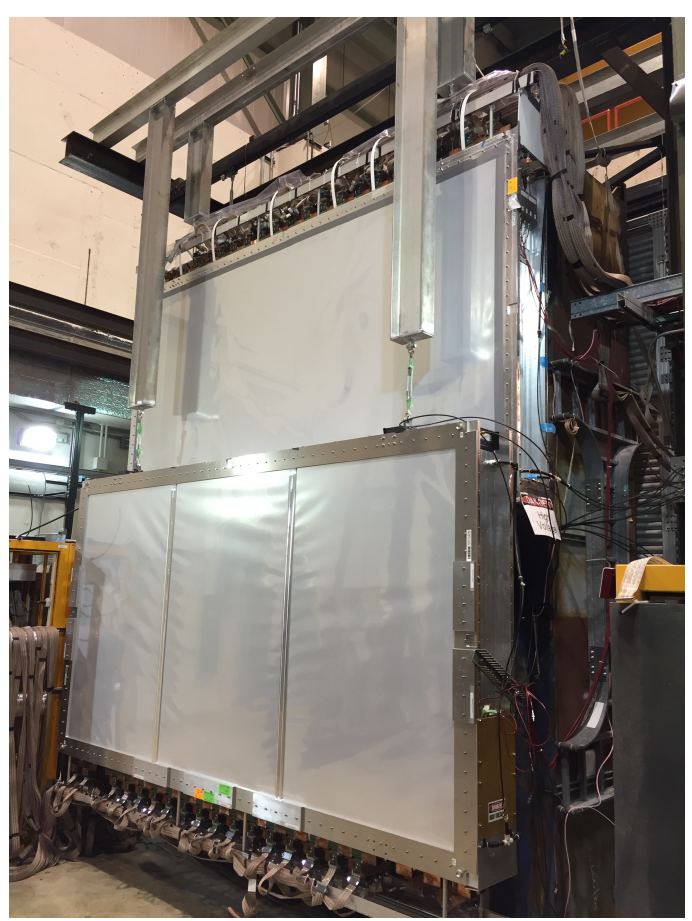

(b) DC3p (top) and DC3m (bottom)

Figure 3.27: SeaQuest drift chambers 


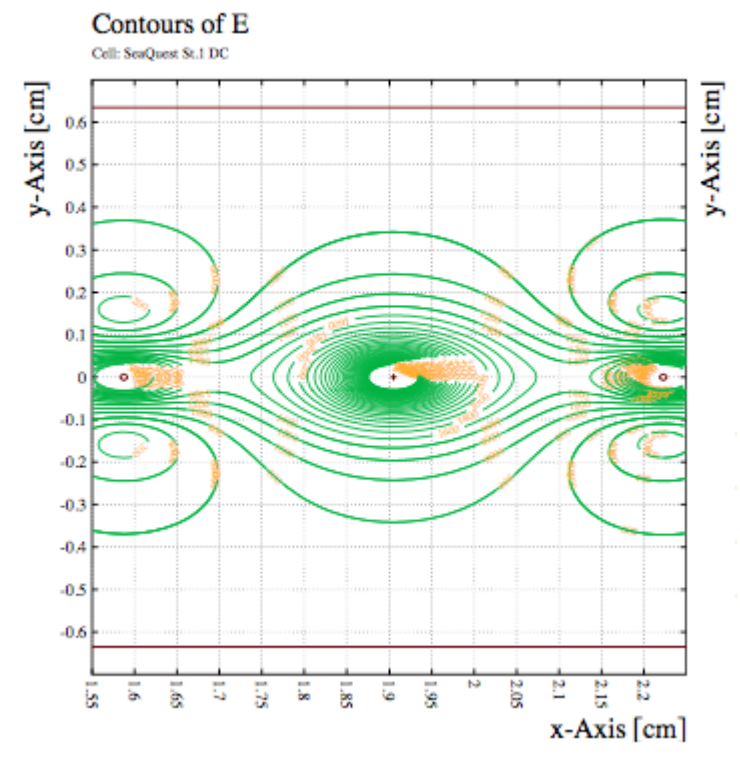

(a) DC1.1 Single cell electric field simulation.

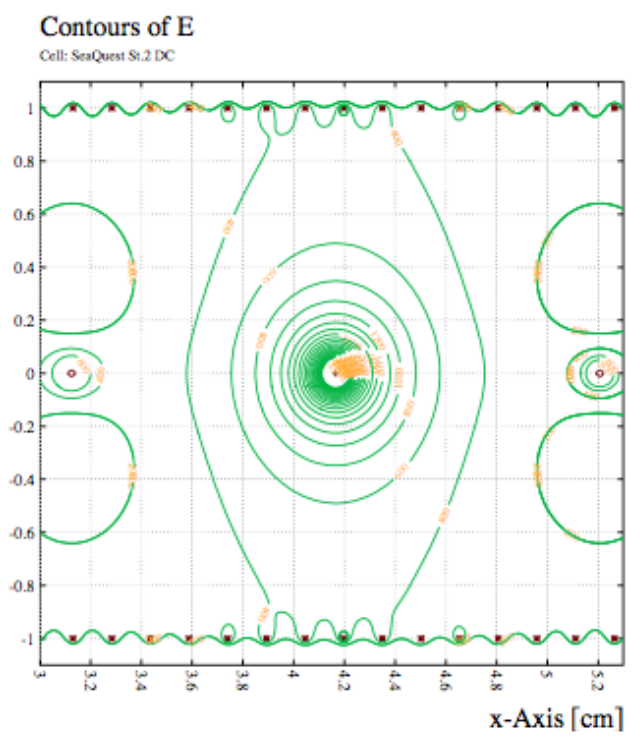

(a) DC2.1 Single cell electric field simulations.

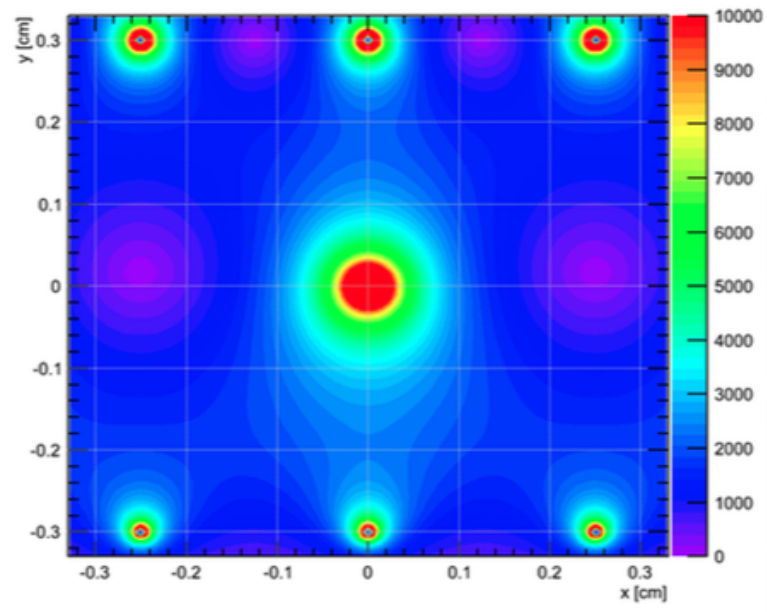

(b) DC1.2 Single cell electric field simulation [125].

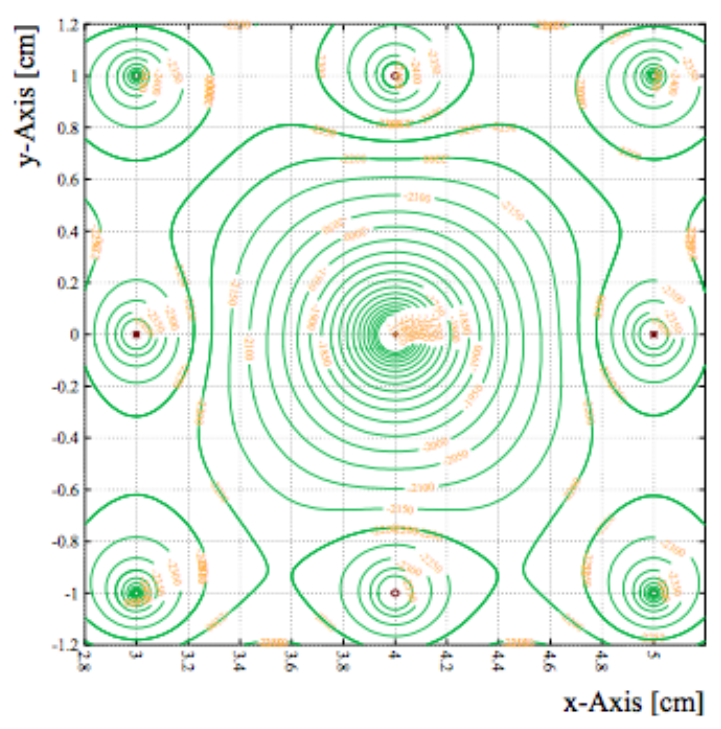

(b) DC3p (top) and DC3m (bottom) single cell electric field simulation.

Figure 3.28: Garfield simulations for SeaQuest drift chambers cells. 

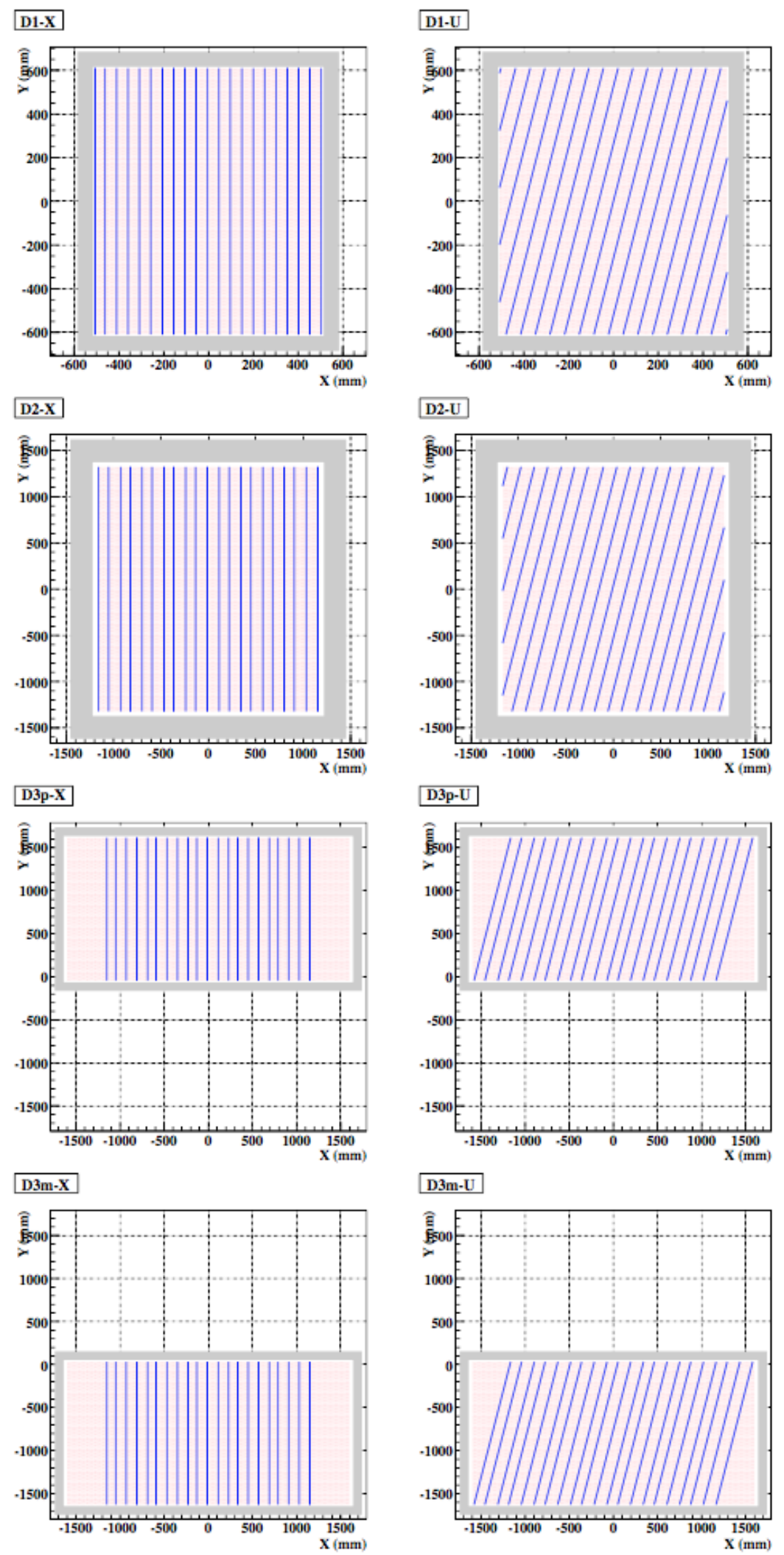

Figure 3.29: Geometric depictions of D1X, D1U, D2X, D2U, D3pU, D3pX, D3mU and D3mX planes 


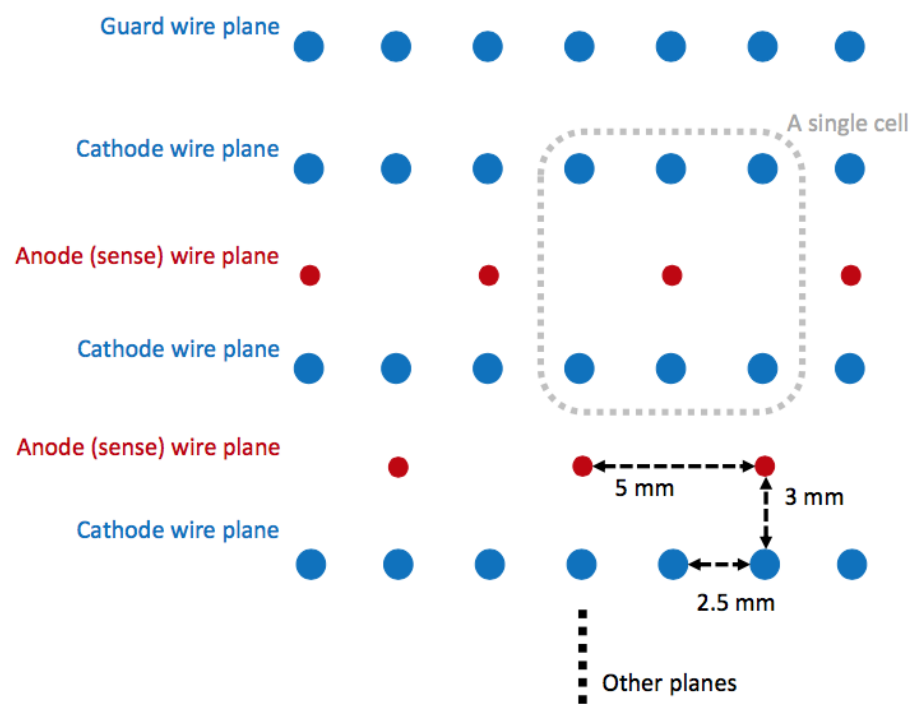

Figure 3.30: Cell structure of DC1.2

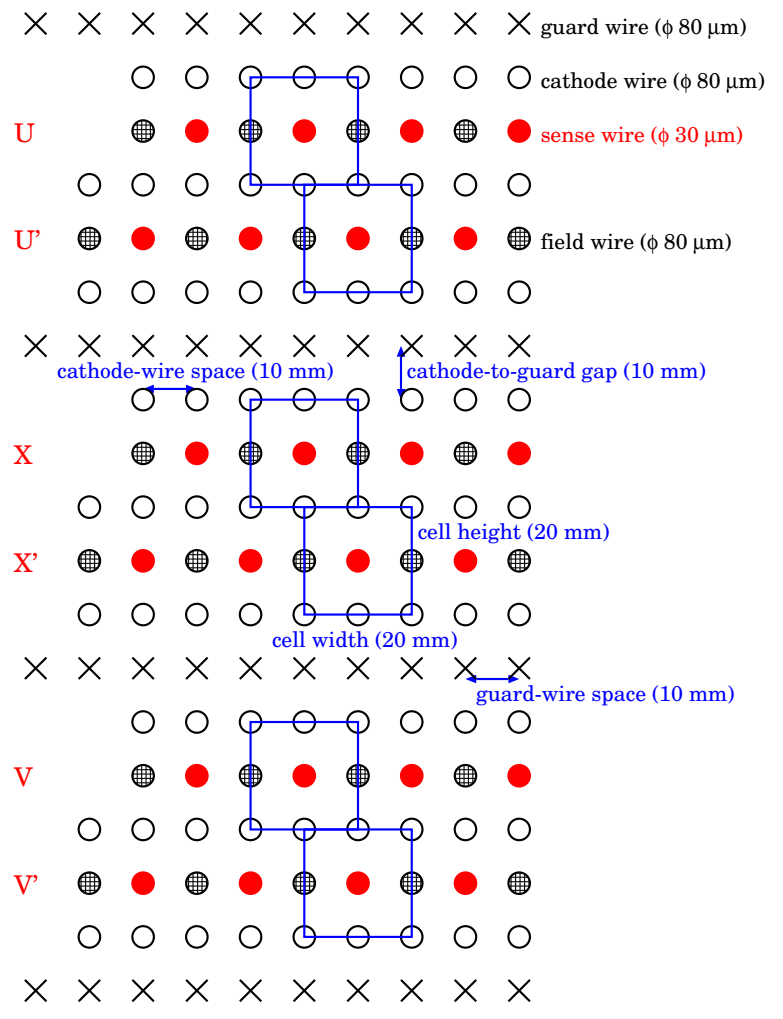

Figure 3.31: Cell structure of DC3p and DC3m 


\section{Drift Chamber Readout Electronics}

\subsubsection{ASDQ cards}

The signal from individual channels are collected using ASDQ (Amplifier Shaper Discriminator and Charge encoding (Q)) cards which are mounted directly on each drift chamber. These 8-channel ASDQ cards were developed at Fermilab for SeaQuest [120]. These cards (shown in Fig 3.32) have the following features:

- Preamplifier: At this stage, the raw signals received from the drift chamber are amplified, converting the charge input into a voltage output while minimizing the noise added to the signal.

- Ion tail cancellation: The tail of the signal that was amplified is removed. The signal is amplified even more.

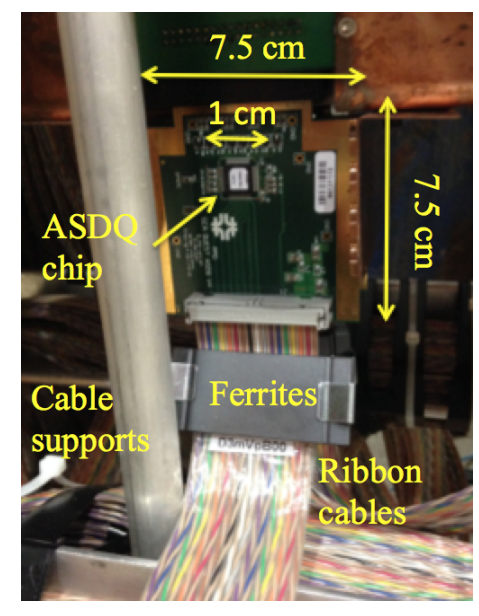

Figure 3.32: Picture of an ASDQ card along with rib-

- Baseline restoration: This step brings the baseline of bon cables, ferrites (used the amplified signal to zero. for noise suppression) and cable supports.

- Discriminator: If the signal coming from the BLR stage is above a programmable threshold, a differential signal is output. The threshold can be manually adjusted using special commands sent to the Level Shifter Boards.

- Charge encoding option: This option is not utilized for SeaQuest. This feature makes the width of the digital output signal proportional to the total charge of the raw signal.

The cards are also designed with protection from large spikes which have various causes. 


\subsubsection{Level Shifter Boards}

Level Shifter Board (LSB) is another specialized board developed at Fermilab for use at SeaQuest [120]. It has 64 channels and can accommodate signals from 8 ASDQ cards. It can also supply low voltages $(-3 \mathrm{~V}$ and $+3 \mathrm{~V})$ to ASDQ cards. The digital differential signals go into the Level Shifter Boards which are further converted to standard LVDS signals. A master board is selected per drift chamber and controlled using telnet commands. Other boards are connected in a daisy chain using RJ11 cables. The board also has the following features:

- Threshold level adjustment: The ASDQ threshold previously mentioned can be set using commands provided to the LSB via telnet using ethernet. These threshold values are 12 bit (0 - 4096) values that correspond to $0-10 \mathrm{mV}$ for the amplified signal that is generated at the BLR stage of the ASDQ card. When noise

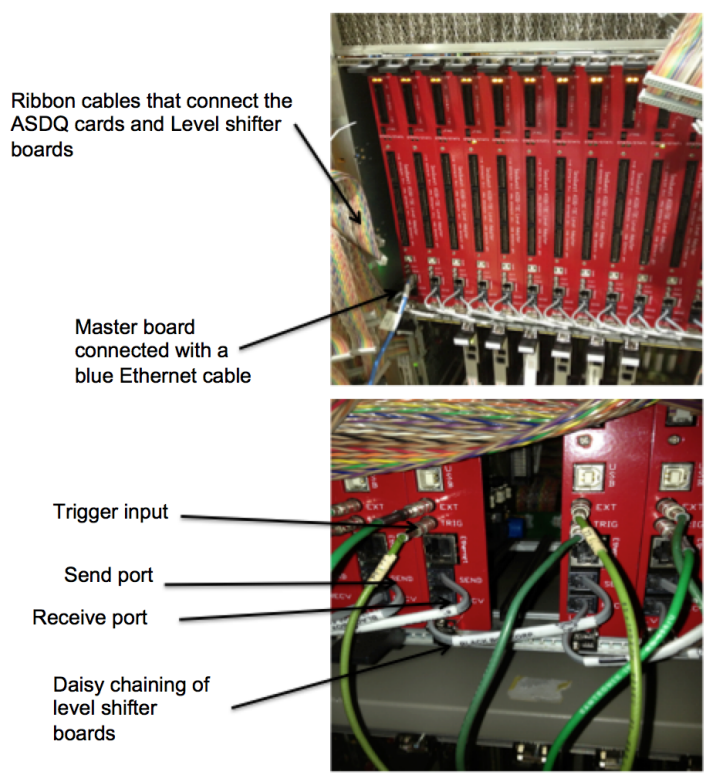
is induced in the system due to un-

Figure 3.33: Level Shifter Boards known reasons, one can adjust these thresholds to suppress it to continue data taking.

- Internal Test Pulser: The Level Shifter board also has the option to send test pulse similar to signals from an ASDQ card. This option was used extensively during commissioning period to try and find any mapping issues (only up till the ASDQ card) in the hardware chain of electronics as well as mapping files.

- Voltage Monitor: The voltage at different checkpoints can also be monitored to 
check if there are any issues.

\subsubsection{TDC - Time to Digital Converter}

The LVDS (low voltage differential signal) analog outputs from the drift chambers, hodoscopes and proportional tubes are sent to Time to Digital Converter (TDC) modules to be digitized and sent to the DAQ. This 64 channel TDC module has $6 \mathrm{U}$ VMEbus form factor and is equipped with a low-power radiation hardened Microsemi ProASIC3 Flash based FPGA (Field Programmable Gate Array) [131]. During Run 1, due to a lack of zero suppression, it was found that the data size was quite large resulting in significant DAQ deadtime. The TDC microcode was then upgraded to include zero-suppression resulting in reduced data volume,

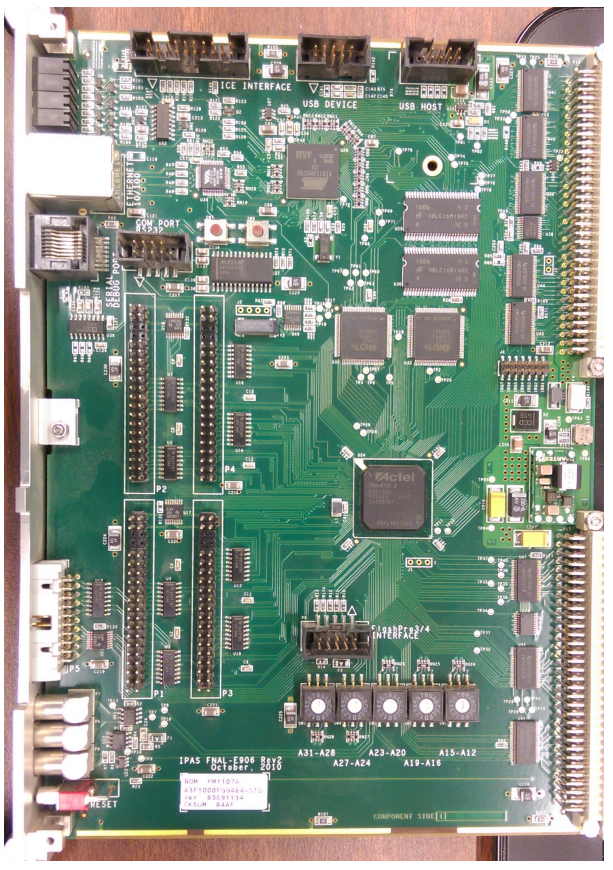

Figure 3.34: Picture of a Time to Digital Converter thereby improving DAQ efficiency.

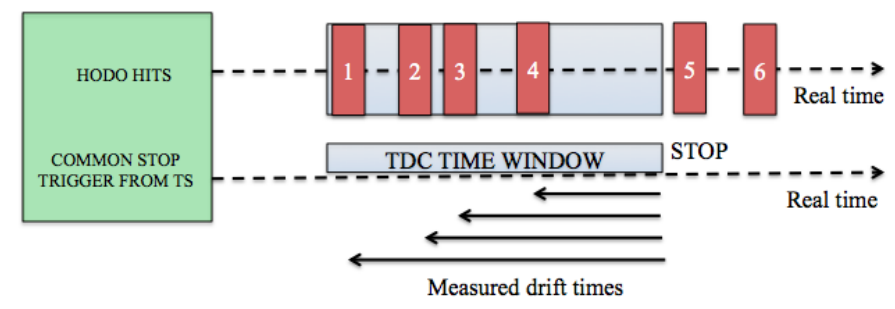

Figure 3.35: Common stop mode of a TDC

The TDCs typically operate in a 'Common Stop mode'. In this mode, the signals from the drift chambers (or scintillator hodoscopes) come in on individual channels and start timing a clock. The "COMMON STOP", a logical combination of hodoscope signals, stops the clock. The TDC has a time window and only reports time within the window. 
Multihit TDCs can report multiple hits on individual channel within the time window. Fig 3.35 shows a cartoon of the common stop mode. More information will be discussed in the section on DAQ.

\subsubsection{RT curves}

The drift time information obtained from the TDCs is converted to a drift distance using the RT curves, where $\mathrm{R}$ is the drift distance from the wire and $\mathrm{T}$ is the measured time. The hit position information is calculated from the curve. The value is calculated using an iterative procedure. An example of an RT curve for DC3mU plane is shown in Fig 3.36. Since the TDCs operate in a common stop mode, a larger time indicates a smaller R value. Top-left, top-right, bottom-left and bottom-right plots are results from first, second, third and fourth iterations respectively. Details about the analysis can be found in [132]. The drift chamber
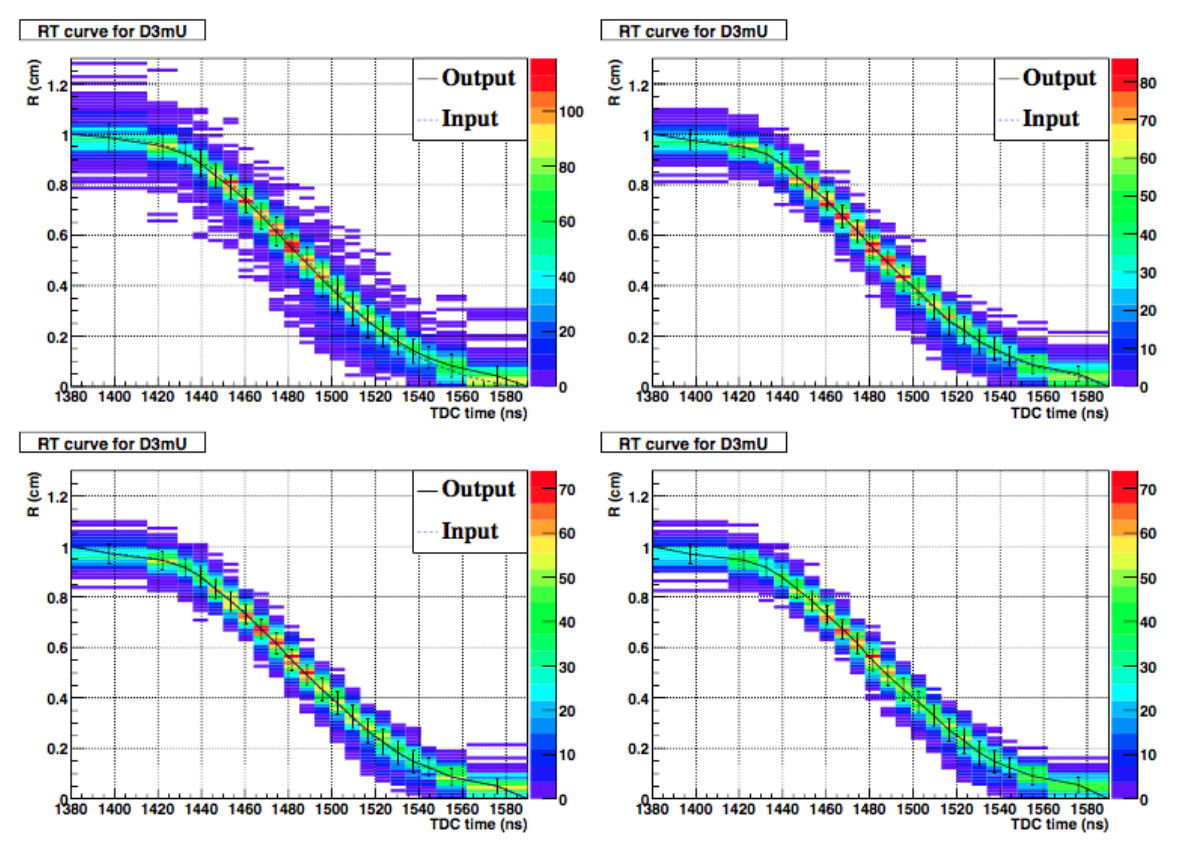

Figure 3.36: RT curve of DC3mU plane obtained from an iterative analysis. Since the SeaQuest TDCs adopted the "common stop" mode, a higher time (T) indicates a smaller R value. Top-left, top-right, bottom-left and bottom-right plots are results from first, second, third and fourth iterations respectively. Figure taken from [132]. 
residuals are calculated for each drift chamber plane given by the formula

$$
\text { residual }=r_{\text {drift }}-f\left(t_{\text {drift }}\right)
$$

where $r_{\text {drift }}$ is the drift distance obtained from track reconstruction, $f\left(t_{\text {drift }}\right)$ is the R-T curve and the $t_{\text {drift }}$ is the measured TDC time. Fig 3.37 shows the residuals calculated for different chamber planes. They peak around 0 and are $<400 \mu \mathrm{m}$ indicating that they are closer to the true chamber intrinsic RT value and are within requirement conditions.
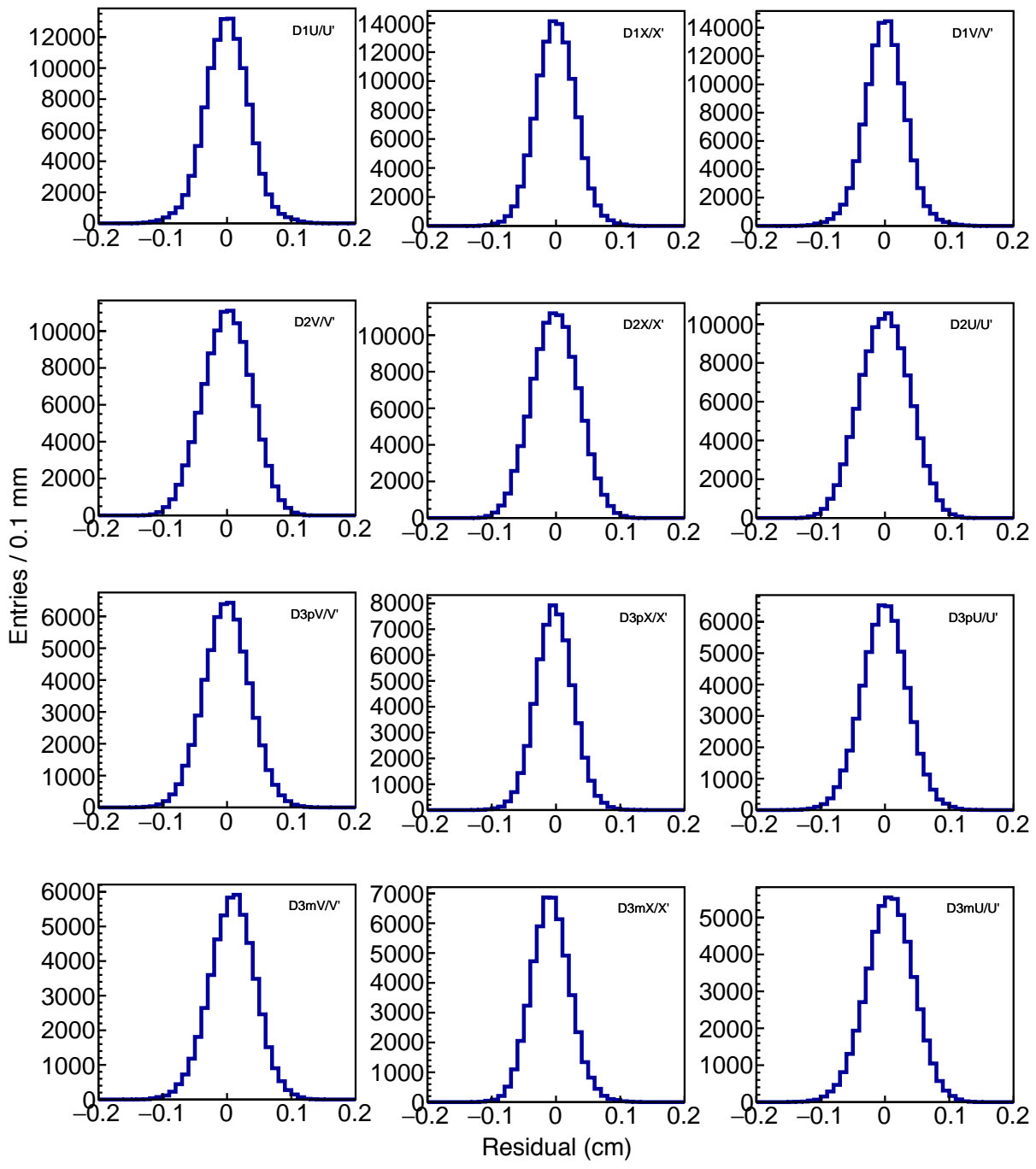

Figure 3.37: Plot of residuals of different chamber planes [120]. 


\subsubsection{Gas gain measurements}

Gas gain measurements were done on each chamber to roughly determine the operational voltage. An ${ }^{55} \mathrm{Fe}$ radioactive source is placed/taped on different locations of the drift chamber as shown in Fig 3.38. The signal thus generated is measured from the ASDQ card end directly by connecting to an oscilloscope. ${ }^{55} \mathrm{Fe}$ decays to ${ }^{55} \mathrm{Mn}$ by emitting $5.89 \mathrm{keV} K_{\alpha}$ X-rays.

A $\sim 3 \mathrm{keV}$ Argon escape peak as well as $5.89 \mathrm{keV}$ signals (signal of interest) are seen on the scope. An average of 256 measurements are taken of the $5.89 \mathrm{keV}$ pulse height for the corresponding voltage. The voltage of the drift chamber is increased in steps and the process is repeated until a voltage vs pulse height plateau is determined ${ }^{8}$.This roughly gives the operational voltage for the drift chamber. It is convenient to use the same procedure to deter-

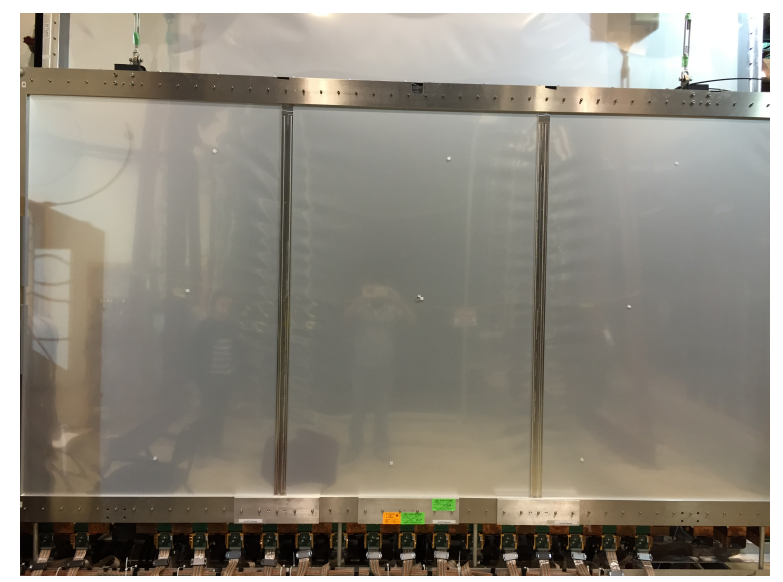

Figure 3.38: Gas gain measurements performed with $\mathrm{DC} 3 \mathrm{~m}\left(\mathrm{ArCO}_{2}\right.$ gas). The ${ }^{55} \mathrm{Fe}$ source is taped on different locations on the drift chamber in order to measure the pulse height.

mine the drift chamber efficiencies both in monitoring chamber performance with the experimental data and in setting the chamber operating voltages. Ultimately what is important is the efficiency of the chamber systems together to reconstruct tracks, so the track reconstruction efficiency is used to fine tune the operating voltages from those set in source studies to the final operating voltages. As a result, it is not necessary to interrupt data taking for efficiency measurements.

\subsubsection{Monitoring Drift chamber efficiencies}

\footnotetext{
${ }^{8}$ The pulse height no longer changes upon further increasing the drift chamber voltage but the peak broadens due to the saturation of the amplification on ASDQ card.
} 

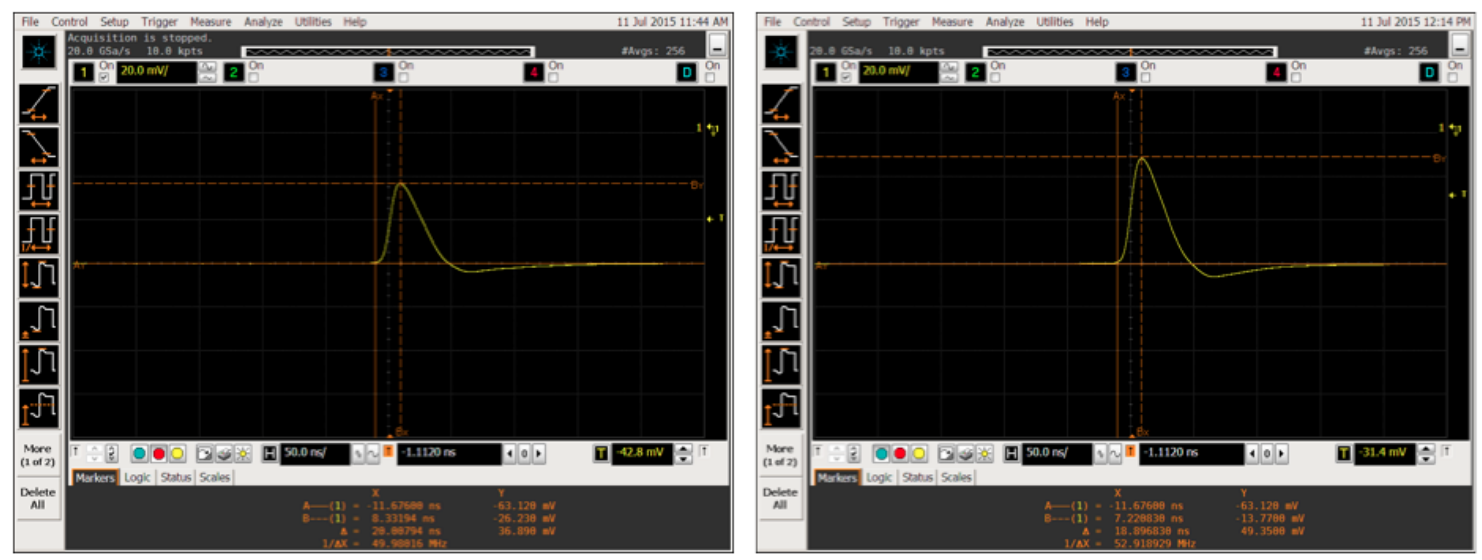

Figure 3.39: Gas gain measurements performed with $\mathrm{DC} 3 \mathrm{~m}\left(\mathrm{ArCO}_{2}\right.$ gas). Left (right) show the pulse height measurements at $2150 \mathrm{~V}(2230 \mathrm{~V})$ on the bottom middle part of the drift chamber. An average of 256 measurements of the pulse heights are made. Voltage is increased in steps until the peak size doesn't change and a voltage vs pulse height plateau is determined.

Monitoring drift chamber efficiencies requires an efficient technique that does not interrupt data taking. Single plane efficiencies were monitored on a daily basis using track reconstruction algorithm (kTracker). Muon tracks were reconstructed with drift chamber planes from Stations 1, 2 and 3 using the formula:

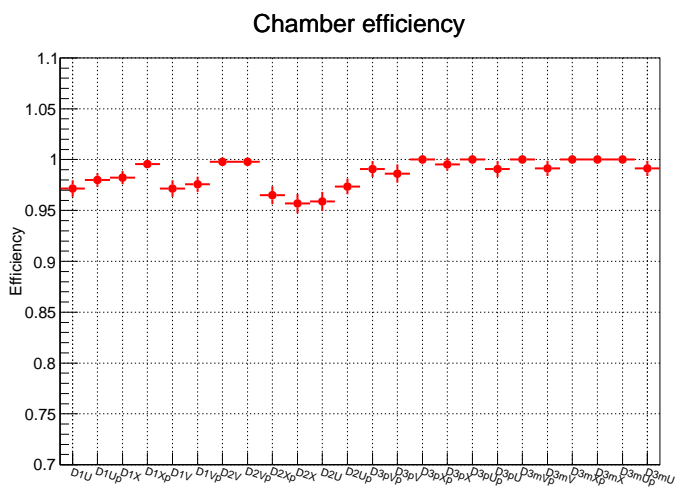

Figure 3.40: Plot of chamber efficiencies. The $x$ axis shows different drift chamber planes and $y$ axis shows the corresponding ef$\epsilon=\frac{N_{18}}{N_{17}+N_{18}}$ ficiency [120].

where $\epsilon$ is the single plane efficiency of a chosen drift chamber plane, $N_{17}$ and $N_{18}$ are the number of 17 and 18 hit reconstructed tracks respectively excluding and including the plane. Measurement results from the data taking period April 2014 - June 2015 are shown in Fig 3.40. The single plane efficiencies of all the planes are $>95 \%$ as desired. The measurements made used low intensity beam with fewer than $10^{4}$ protons per RF bucket. 
At nominal intensity, the efficiency might be much lower due to several rate dependent effects.

\subsection{Proportional tubes}

Muon identification is achieved using proportional tubes at Station 4, which is downstream of a $1 \mathrm{~m}$ long iron wall. This wall blocks off any strongly interacting hadronic debris. The electrons would shower and are thus scattered more significantly than muons. Therefore, having a small momentum dependent deflection of the track before and after passing through the iron wall is the signature that is used in muon identi-

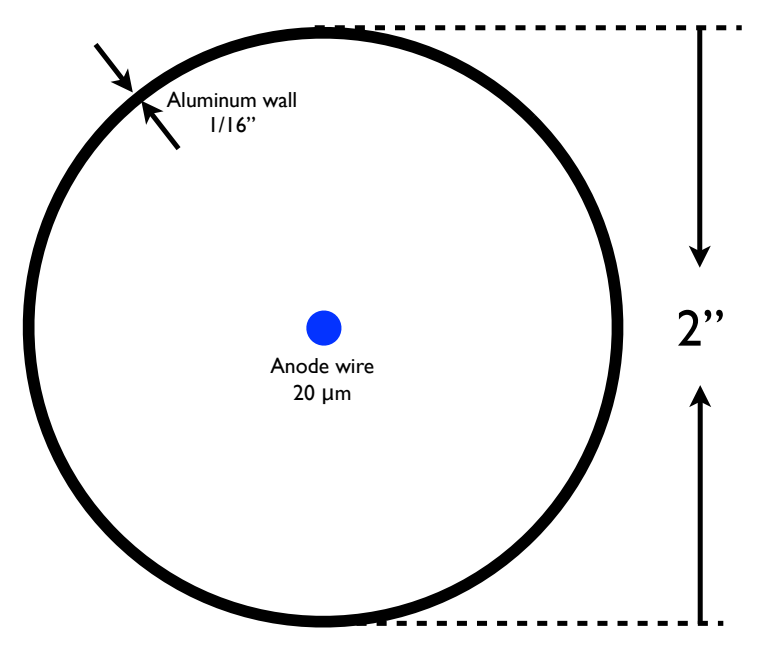

Figure 3.41: Top view of a single proportional tube. fication.

The Station 4 tracking detectors consist of 4 layers of proportional tube planes. Each plane is made of nine proportional tube modules and each module is assembled from 16 proportional tubes (12 ft long with 2 in diameter) as shown in Fig 3.41 and 3.43. The wall thickness of each tube is $1 / 16$ in. The central anode is made of gold-plated $20 \mu \mathrm{m}$ diameter Tungsten wire. All the proportional tube modules use the same gas as the drift chambers (P08:CF4 (Ar: $\mathrm{CH}_{4}: \mathrm{CF}_{4}$ in the ratio 88\%:8\%:4\%). The proportional tubes are oriented in the horizontal and vertical direction to measure in both the $y$ and $x$ direction. A typical muon track produces hits on two anode wires in each proportional tube plane. The principle of operation of a proportional tubes is similar to those of drift chambers and has been discussed already in Section 3.7. The groups of 16 proportional tube anode channels are read out using N-277 16 channel Amplifier/Discriminator cards with a common programmable threshold. The typical drift time of proportional tubes are $\sim 650 \mathrm{~ns}$. The 8 
hits from 4 proportional tube modules are used for muon identification in track reconstruction algorithms. Residual distributions of proportional tubes are shown in 3.42. They peak around 0 and widths are $<0.5 \mathrm{~mm}$ indicating that they are close to the true proportional tube RT value and are within requirement conditions. Other proportional tube parameters are summarized in Table 3.8 .

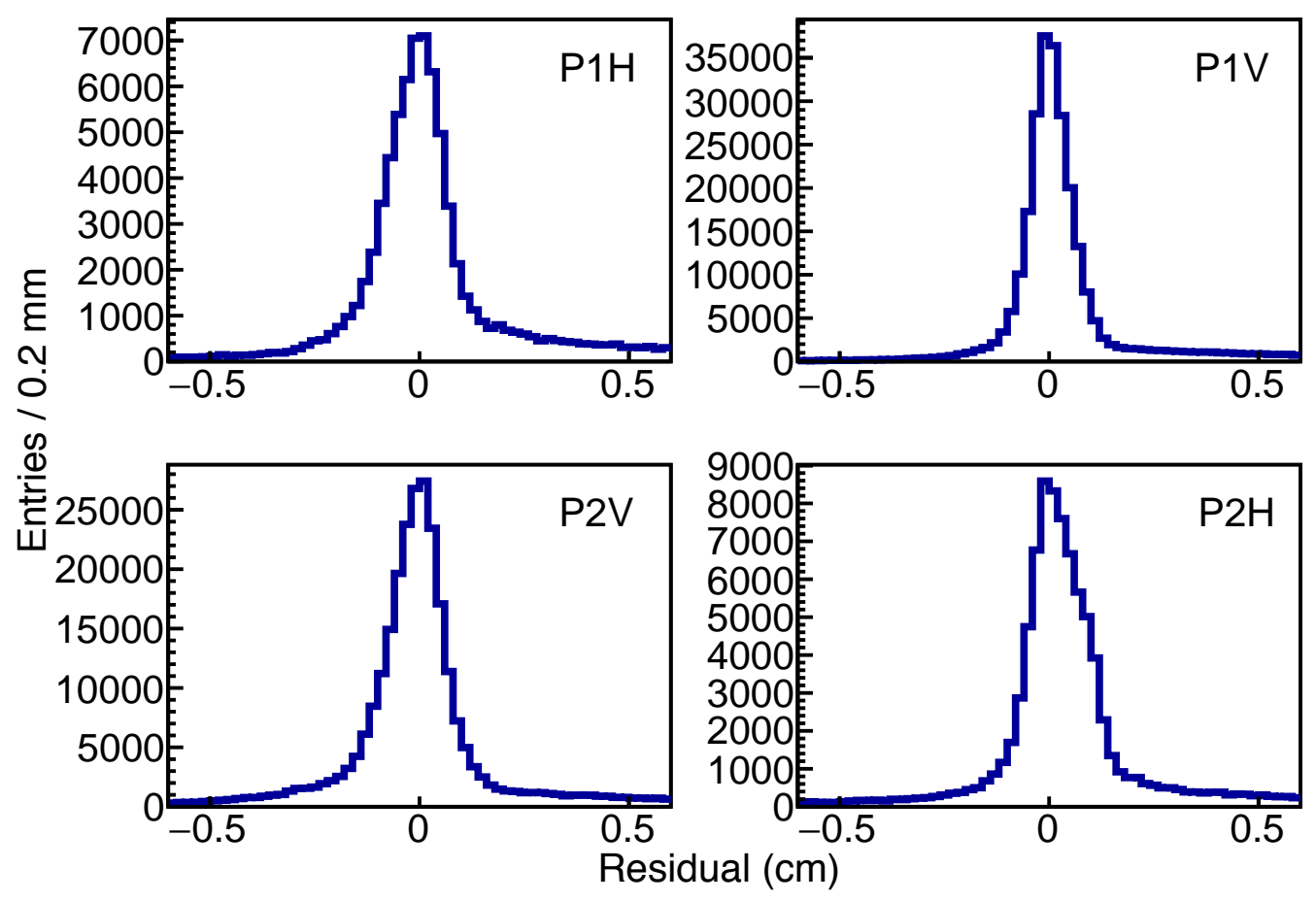

Figure 3.42: Residuals of proportional tubes.

\begin{tabular}{|c|c|c|}
\hline Plane & $\begin{array}{c}\text { Width } \times \text { Height } \\
\mathrm{cm} \times \mathrm{cm}\end{array}$ & $\begin{array}{c}z \text { position of forward } \\
(\text { backward) sub-plane }(\mathrm{cm})\end{array}$ \\
\hline \hline P1H & $368.3 \times 368.3$ & $2099(+4)$ \\
P1V & $368.3 \times 368.3$ & $2175(+4)$ \\
P2H & $368.3 \times 368.3$ & $2389(+4)$ \\
P2V & $368.3 \times 368.3$ & $2367(+4)$ \\
\hline
\end{tabular}

Table 3.8: Parameters of all proportional tubes. 


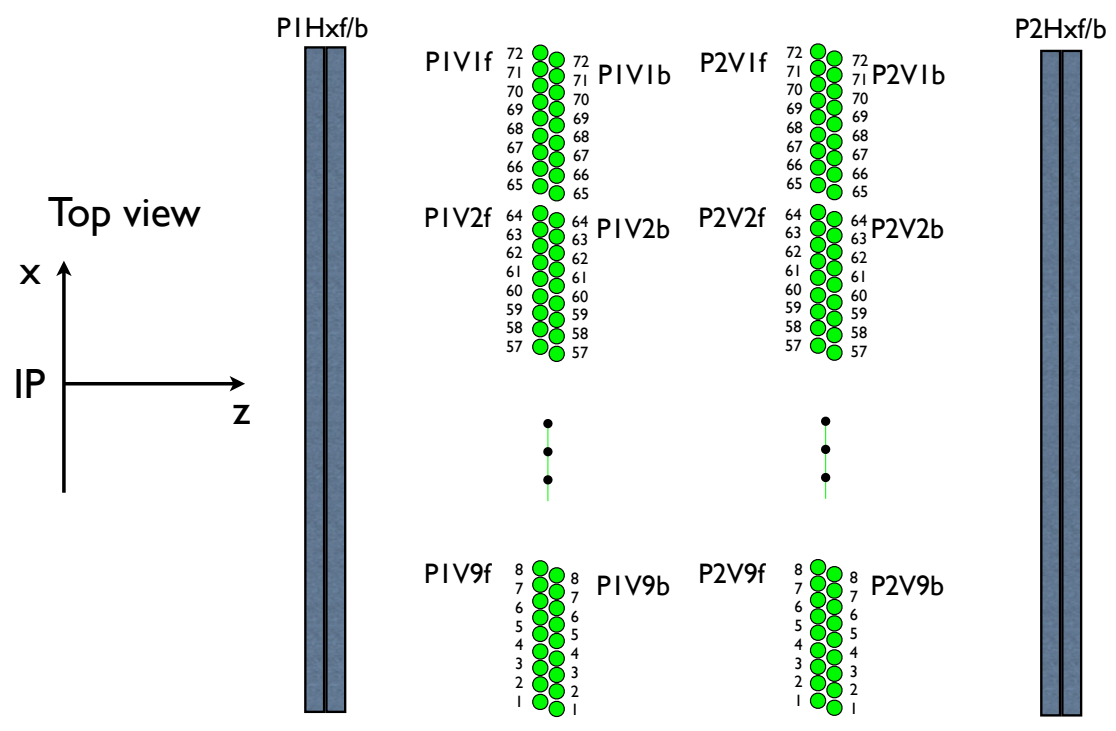

(a) $x z$ view of the proportional tube layout

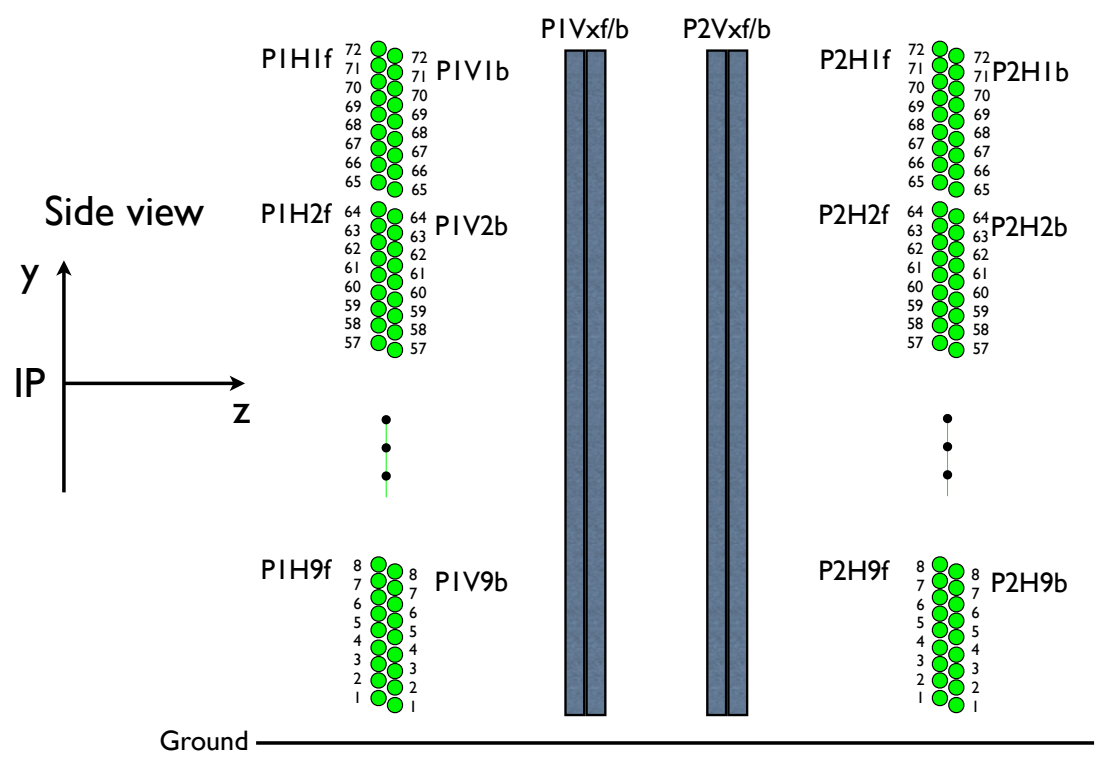

(b) $y z$ view of the proportional tube layout

Figure 3.43: Schematic of SeaQuest proportional tubes [120] 


\subsection{Trigger}

The Trigger is usually considered the 'heart' of the experiment and it is no different in the case of SeaQuest. Even though the trigger is optimized to accept high mass Drell-Yan dimuon events $\left(4-10 \mathrm{GeV} / \mathrm{c}^{2}\right)$ coming from the target $(\sim 10 \%$ interac-

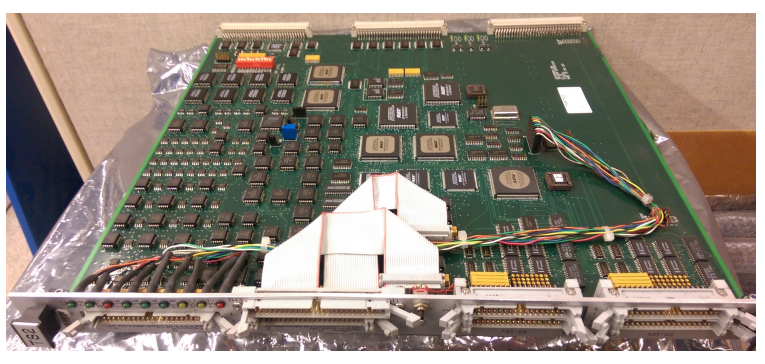

Figure 3.44: Picture of Trigger Supervisor. tion length), it is swamped by single muon backgrounds coming from cosmic muons, pion decays and quarkonia decays ${ }^{9}$ coming from upstream, the target and the beam dump $(\approx 30$ interaction lengths). Any muons having similar kinematics will satisfy the trigger as it doesn't distinguish between their source. Therefore, a careful design of the trigger plays a crucial role in selecting true Drell-Yan events of interest.

\subsubsection{Overall structure}

The SeaQuest trigger system consists of three "Levels" of nine V1495 modules (Level $0=4$, Level $1=4$, Level $2=1$ as shown in 3.45) and a Trigger Supervisor (TS) VME module (shown in Fig 3.44) designed at Jefferson Lab [134]. Each V1495 module includes an Altera EP1C 20F400C6 FPGA (shown in Fig 3.45). Discriminated signal from four hodoscope planes is used as an input for the trigger.

- Level 0: The four independent groups of "quadrants" (lower $x$ - lower bend plane, lower $y$ - lower non-bend plane, upper $x$ - upper bend plane, upper $y$ - upper non-

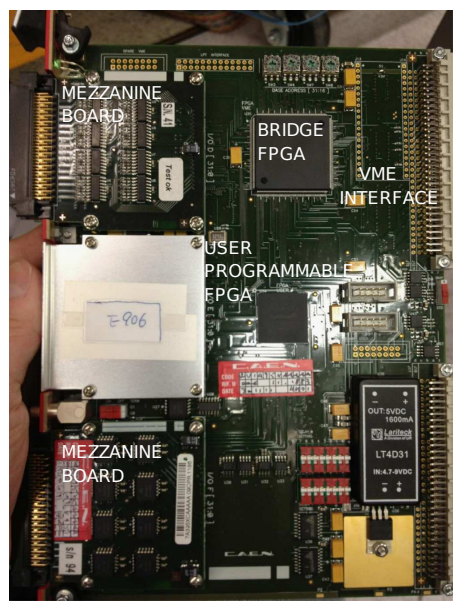

Figure 3.45: Picture of a V1495 module [133].

\footnotetext{
${ }^{9}$ In order to maintain a reasonable DAQ dead time, muons coming from dominant sources of background such as $\mathrm{J} / \psi$ decays are highly suppressed.
} 
bend plane) are used as inputs in Level 0 trigger signal

as shown in Fig 3.46. Level 0 can operate in two modes; "Production" mode and "Pulser" mode. During Production mode, the Level 0 simply passes on the input signals taken from the hodoscopes to the Level 1. In Pulser mode, Level 0 utilizes predesigned text files with arbitrary hit patterns as a diagnostic tool to check the functionality (detect loss of signal) of Level 1 and Level 2.

- Level 1: Level 1 also consists of four V1495 modules which take output signals coming from the 4 Level 0 boards. It is primarily responsible for finding four hit combination track candidates.

- Level 2: This is the "track correlator". Its forms all possible pairs of track candidates given by Level 1, makes firmware specified criteria and sends five output triggers to the Trigger Supervisor.
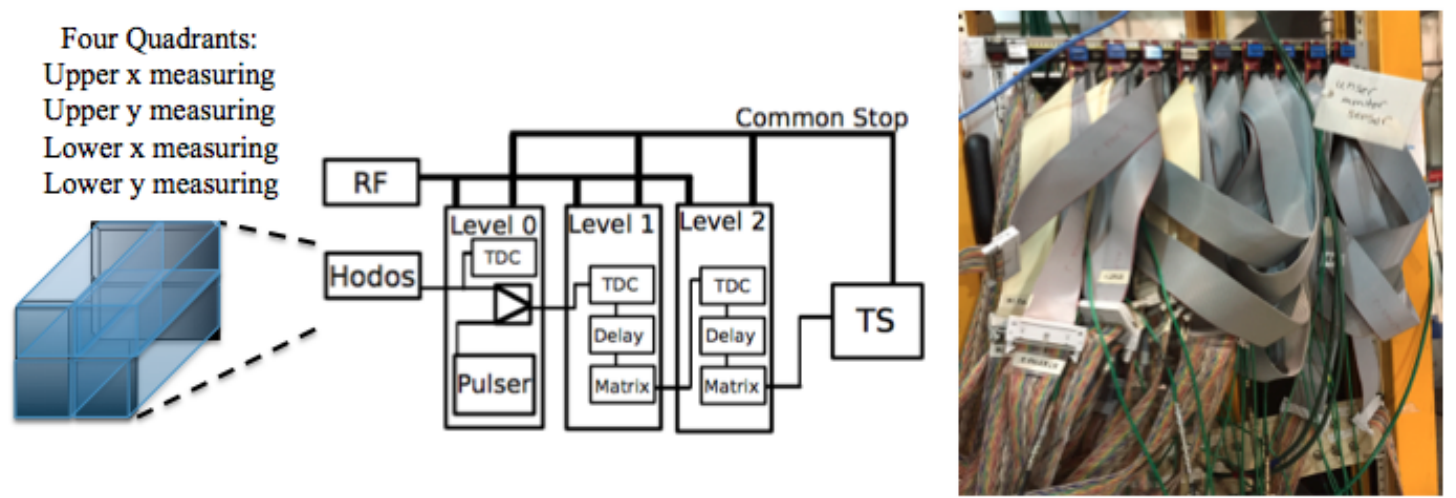

Figure 3.46: Schematic of SeaQuest trigger [133].

\subsubsection{Trigger Firmware}

The firmware for Level 1 and Level 2 are almost identical, differing in the logic pipeline. There are three main parts of the custom firmware written for SeaQuest; TDC block, delay adjustment pipeline and trigger matrix. 
- TDC Block: The TDC digitizes the signals and sends them to the V1495s. A four phase sampling scheme with a 212.4 MHz clock achieves $1 /(4 \times 212.4 \mathrm{MHz})=1.17$ ns resolution. A PLL (Phase Locked Loop) utilizes the Main Injector 53.1 MHz clock to generate the $212.4 \mathrm{MHz}$ clock. The Main Injector RF is synchronized with the RF structure of the proton beam. The TDC block generates four clocks from the fast $\operatorname{clock}\left(0^{\circ}, 90^{\circ}, 180^{\circ}, 270^{\circ}\right)$ and achieves a $1 /(4 \times 212.4 \mathrm{MHz})=1.17 \mathrm{~ns}$ resolution for the TDCs. This block is identical to Level 1 and Level 2 firmware designs.

- Delayed Pipeline: After the TDC hits are digitized, they are pipelined in $18.8 \mathrm{~ns}$ bins (16 clock-ticks of $1.17 \mathrm{~ns}$ ). The capacity of each bin is only one bit. If there are multiple hits on a single channel within one $18.8 \mathrm{~ns}$ bin, only the latest hit is kept. The delay adjustment pipeline also aligns the timing of the input signals and provides event storage. When the TS accepts a trigger, it sends a STOP signal to TDCs and V1495s. The pipeline is halted and the information is readout by the VME interface and recorded by the DAQ. The readout is zero-suppressed and is stored in a buffer $[135,133]$.

- Trigger Matrix: Trigger Matrix is essentially a look-up-table-based trigger logic implementation that is used for identifying possible track candidates. This feature is different between Level 1 and Level 2. The digitized hits from the TDC are sent to the Trigger Matrix. Level 1 trigger logic is assigned the task of identifying track candidates from combinations of the four hodoscope hit quadrants initially mentioned in this section. For each RF bin, the Level 1 look up tables has "trigger roads" which are combinations of roads of four hodoscope hits, one from each detector station, which are likely to come from the the paddles that are hit by Drell-Yan dimuons traversing the four stations of the spectrometer. The output bits are binned according to the charge and average $p_{x}$ of the track. These are sent as 24-bit words (twelve $0.5 \mathrm{GeV} / \mathrm{c}$ average $p_{x}$ bins $\times 2$ types of charge) as outputs by the Level 1 board. The Level 2 Trigger Matrix then checks all possible combinations against another look-up-table 
of possible "di-roads" events. The trigger fires even though there are multiple patterns coming from the same RF bucket. A quantity called "RoadID" is later assigned to each possible muon hit path. It is calculated using the formula:

$$
\operatorname{RoadID}=(\mathrm{H} 1-1) * 16^{3}+(\mathrm{H} 2-1) * 16^{2}+(\mathrm{H} 3-1) * 16+\mathrm{H} 4
$$

Where $\mathrm{H} 1, \mathrm{H} 2, \mathrm{H} 3$ and $\mathrm{H} 4$ are the hodoscope paddle numbers at Station 1, 2, 3 and 4. One muon is required to come from the upper half and the other from the lower half. Detailed GEANT-4 based Monte Carlo simulations have been performed in order to make these look up tables that are loaded into the Level 1 firmware. These roads have been fine tuned over the coarse of the experiment in terms of symmetrizing the top and bottom "road halves", removing noisy roads and adding additional roads which have some acceptance for dark photons. Table 3.9 gives a description of all the Roadsets. More information about the generation procedure of these roads and their implementation can be found in [135].

\begin{tabular}{|c|c|}
\hline Roadset & Description \\
\hline \hline 49 & Initial GMC roads, hot roads removed \\
\hline 57 & Added a few dark photon roads \\
\hline 59 & fastMC half FMAG-field roads \\
\hline 61 & Recompiled Roadset \# 57 with RF-Clocking \\
\hline 62 & Charge symmetry enforced, hot roads removed (mag field flip) \\
\hline 67 & Dark photon roads added and some hot roads removed from 67 \\
\hline 70 & $\begin{array}{r}\text { GMC roads to include new DC1.2 acceptance, proton straight-through roads } \\
\text { and dark photon roads added, some hot roads removed from } 67\end{array}$ \\
\hline 78
\end{tabular}

Table 3.9: Roadsets used in SeaQuest and their descriptions.

\subsubsection{Types of Trigger}

The types of various triggers used in SeaQuest are summarized in Table 3.10. Each trigger has a unique purpose and a rather brief description is provided of the purpose of these 
triggers. The trigger roads were changed several times throughout the duration of the experiment. An arbitrary number is given to a set of roads used for data taking (Roadset 57, 59, 62, 67, 70 and 78). All triggers except the main FPGA1 trigger were prescaled in order to simultaneously collect $\sim 1 \%$ data of that type of trigger. The prescale factor indicates how many of that type of triggers have to pass before the DAQ records one event that satisfied that particular type of trigger.

\section{FPGA Trigger}

- FPGA1 or MATRIX1: This is the main trigger of the experiment. This triggers requires oppositely charged muons that traverse the opposite vertical halves (Top + Bottom or Bottom + Top) of the spectrometer.

- FPGA2 or MATRIX2: This trigger requires opposite sign muons to travel the same vertical halves of the spectrometer (Top + Top or Bottom + Bottom).

- FPGA3 or MATRIX3: This trigger requires same sign muons to travel the opposite halves of the spectrometer. This trigger is used to estimate combinatoric background contributions

- FPGA4 or MATRIX4: This trigger requires single muons top or bottom half of the spectrometer.

- FPGA5 or MATRIX5: This trigger requires single muons top or bottom half of the spectrometer but with a $p_{x}>0.3 \mathrm{GeV} / \mathrm{c}$ cut.

\section{NIM Trigger}

During commissioning of the experiment, when the FPGA triggers were still under development, NIM-based trigger was used to debug spectrometer related issues.

- NIM1: NIM1 trigger is a coincidence of $\mathrm{H} 1 \mathrm{~T}+\mathrm{H} 2 \mathrm{~T}+\mathrm{H} 3 \mathrm{~T}+\mathrm{H} 4 \mathrm{~T}$ or $\mathrm{H} 1 \mathrm{~B}+\mathrm{H} 2 \mathrm{~B}+\mathrm{H} 3 \mathrm{~B}+\mathrm{H} 4 \mathrm{~B}$. It doesn't differentiate between the charge of the muons, hence it could be used to 
study gross spectrometer related issues (cosmic muons, debugging timing related issues, inefficient hodoscope paddles etc.)

- NIM2: N/A

- NIM3 or Pseudo Random Trigger: This trigger is a special pseudo-random rigger. It is a coincidence of RF clock with a $7.5 \mathrm{kHz}$ pulse signal produced by a gate generator which is prescaled. This trigger collects events when there is an overlap between the two clocks. It is used to randomly select a RF bucket to study the background. Events acquired from this trigger are embedded in the "clean" Monte Carlo productions in order to simulate and study the effects of background on various parameters and track reconstruction efficiencies.

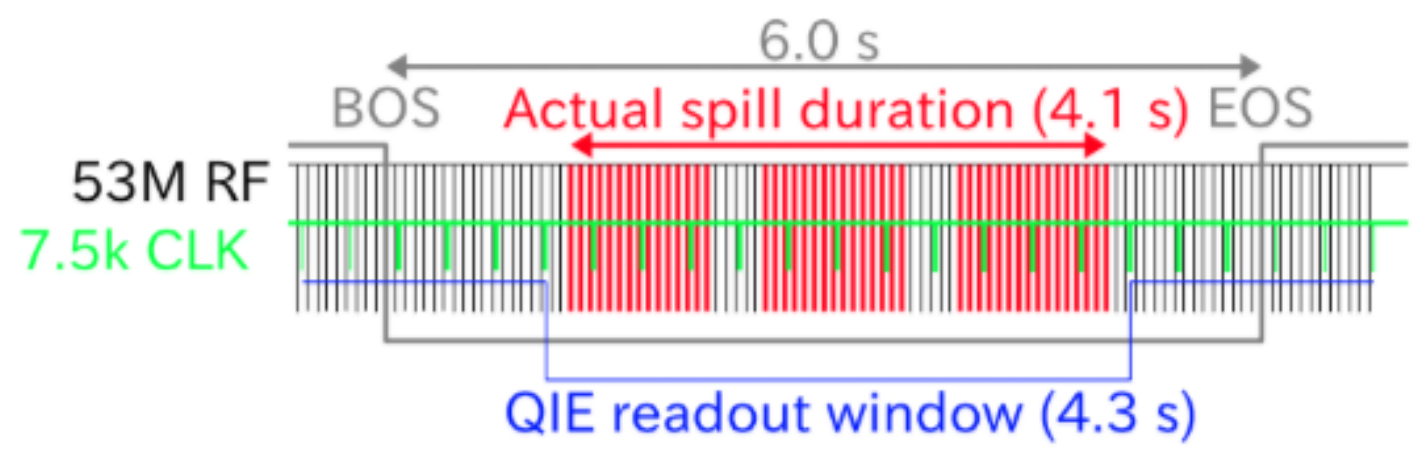

Figure 3.47: Schematic of NIM3 pseudo-random trigger.

\begin{tabular}{lclcl}
\hline \hline Name & Side & Charge & $p_{x}$ Req. & Notes \\
\hline Matrix 1 & TB/BT & $+-/-+$ & None & Main physics trigger \\
Matrix 2 & TT/BB & $+-/-+$ & None & Same-Side trigger \\
Matrix 3 & TB/BT & $++/--$ & None & Like-Charge trigger \\
Matrix 4 & T/B & $+/-$ & None & All singles trigger \\
Matrix 5 & T/B & $+/-$ & $p_{x}>3 \mathrm{GeV} / \mathrm{c}$ & High- $p_{T}$ singles trigger \\
\hline NIM 1 & Y coincidence & $+/-$ & - & - \\
NIM 2 & X coincidence & $+/-$ & - & - \\
NIM 3 & Random RF & $+/-$ & - & RF clock $+7.5 \mathrm{kHz}$ clock \\
\hline \hline
\end{tabular}

Table 3.10: Trigger settings used in the SeaQuest experiment [120]. 


\subsection{Data Acquisition - DAQ}

The SeaQuest data acquisition system is divided into three separate systems based on the timing and bandwidth requirements that cannot be met with a single central DAQ system. These three subsystems are called "MainDAQ or EventDAQ", "ScalerDAQ" and "BeamDAQ". Each of these systems have a unique purpose. The MainDAQ records the event-by-event

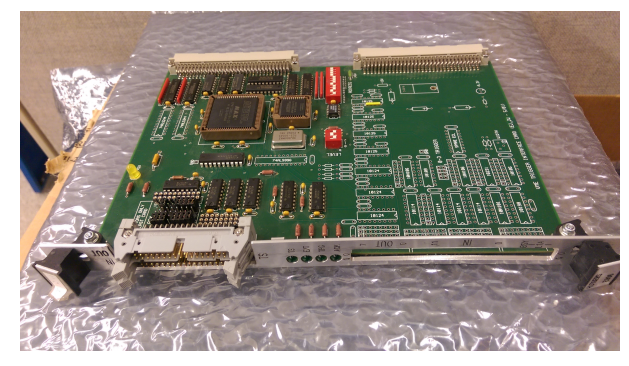

Figure 3.48: Picture of a Trigger Interface card. main detector information and trigger timing. The ScalerDAQ records the scaler information on a $7.5 \mathrm{kHz}$ clock and at the end of the spill. The BeamDAQ records information from the beam line Čerenkov detector. The MainDAQ and the ScalerDAQ use the VME-based CODA (CEBAF Online Data Aquisition) ${ }^{10}$ [136].

\subsubsection{MainDAQ}

The MainDAQ is triggered by either FPGA trigger matrix or NIM triggers. The MainDAQ consists of 14 VME crates with one of them being the Trigger Supervisor crate. The Trigger Supervisor (shown in Fig 3.44) receives trigger signals and fans them out to the rest of the 13 VME crates. These 13 crates have a VME processor or a Read Out Controller (ROC), Trigger Interface (TIR), and TDCs (typically 6 or 7 per crate although the capacity is 12) to accept signals from the drift chambers, hodoscopes and proportional tubes (shown in Fig 3.50). The Trigger Supervisor has 12 trigger slots of which the first five are NIM-based, the next 5 are FPGA-based and the remainder EOS (End Of Spill) and BOS (Beginning Of Spill). The flow of data is the following:

1. Signals are sent from the Hodoscope TDCs (section 3.7.4) to the V1495 Level-2 or NIM modules. The logic modules send signals to the TS inputs. TS prescales as

\footnotetext{
${ }^{10}$ CEBAF - Continuous Electron Beam Accelerator Facility
} 


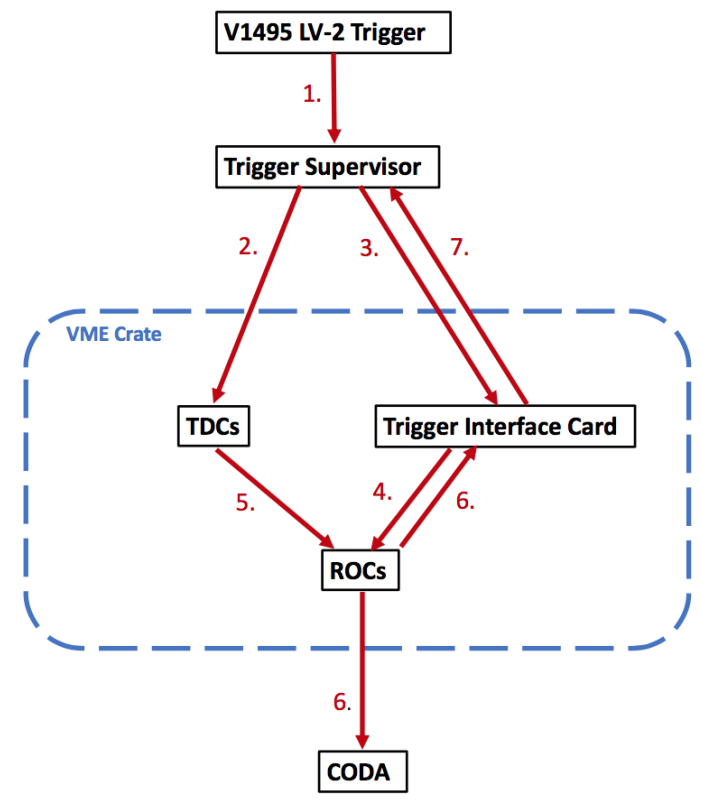

Figure 3.49: Workflow of the MainDAQ [125]

appropriate. If "BUSY", the trigger is dropped. If "NOT BUSY", trigger is accepted and processed, BUSY is set, outputs are generated, causing data to be read as follows.

2. LVL-1 accept is sent out to all TDCs. The TDCs stop data taking, and save the hit information in the ring buffer.

3. After being delayed by $32 \mu \mathrm{s}$, the TS outputs trigger to the 13 Trigger Interface Cards (TIR) once it accepted a trigger. This is the copy-in-progress (CIP) time.

4. After another $10 \mu \mathrm{s}$, each TI instructs the ROC to read out the TDCs.

5. The TDCs deliver the hit information to the ROC through the VME backplane. This process takes about $100 \mu \mathrm{s}$.

6. The TS is also responsible for sending the common stop signal. The ROCs start reading the TDCs and then tells the TIR that it has finished reading and the collected data is sent to MainDAQ via a private network. 
7. The TIR signals the TS that the ROC has finished reading all of the TDCs. The TS also sends a trigger to the QIE board that and the QIE retains data about the beam intensity from \pm 12 to $16 \mathrm{RF}$ buckets around the triggered bucket.

8. Once the TS receives an acknowledge from all the 13 ROCs, it clears the BUSY and is ready for the next trigger.

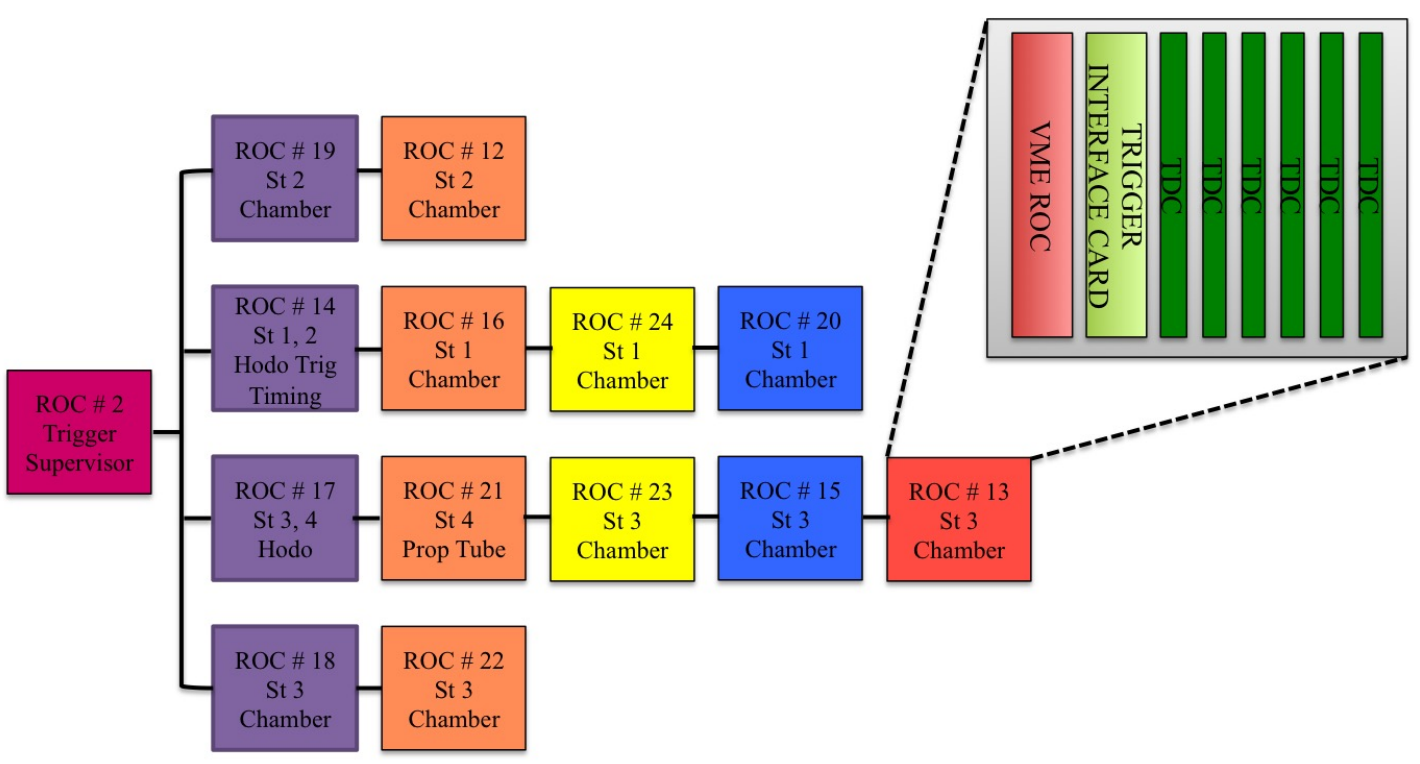

Figure 3.50: Daisy chain of the Trigger distribution. Readout is asynchronous.

This continues until a "run" is finished ${ }^{11}$. A new improved readout scheme has been implemented since Fall of 2016 in which the data is stored locally in the TDC modules during a spill and then transferred through the VME backplanes between spills reducing the readout time from $\approx 150 \mu$ s to $\approx 30 \mu \mathrm{s}$.

\footnotetext{
${ }^{11}$ It is important to note that run and Run are two different SeaQuest concepts. Run is the same as a data set taken with a particular experimental setting and run is an individual $1 \mathrm{hr}$ long short period of data taking.
} 


\subsubsection{ScalerDAQ}

The ScalerDAQ is a standalone DAQ that is designed to monitor the spectrometer, trigger and quality of the beam coming into SeaQuest. It is also driven by the JLab CODA system. The system is comprised of one VME crate that reads out four 32-channel VME scalers. The ScalerDAQ is designed to do the following:

- One of the channels counts the coincidence of $7.5 \mathrm{kHz}$ gate generator and the beam spill signal using the response of two unrelated hodoscopes (used to calculate the duty factor).

- The other three scalers (triggered by BOS and EOS) record trigger/inhibit/hodoscope/etc counts per spill. This includes the number of times the MainDAQ trigger is satisfied, and the rates of the hodoscope arrays.

\subsubsection{BeamDAQ}

The BeamDAQ is responsible for recording the $53 \mathrm{MHz}$ structure of the beam on a bucket-by-bucket basis (RF intensity information). It reads the data from the Čerenkov detector in the proton beam. Its calculation of the "Duty Factor" DF $=\frac{\langle I\rangle^{2}}{\left\langle I^{2}\right\rangle}$ is the primary measure of the quality of the beam that is relayed back to the accelerator operators for beam tuning. If this number is 1 , it means that the beam has a stable beam intensity throughout all the buckets in the spill. Due to improvements in the quality of the beam, a jump in the duty factor was noted during the middle of Run 3 of data taking. Four types of data are

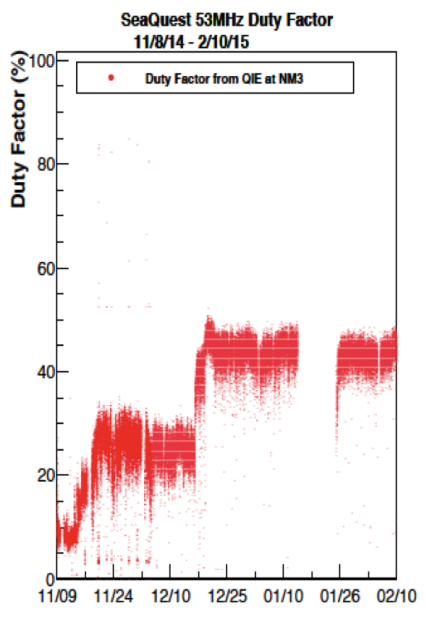

recorded by the QIE board during the spill: 
- Intensity of each individual RF bucket (which was measured to obtain QIEsum but not recorded since it was too big)

- Number of protons inhibited due to the high instantaneous intensity due to each inhibit generated.

- Number of protons missed as the BeamDAQ was busy during readout. This number excludes inhibited protons to avoid double counting.

- The sum of beam intensity, I, and the intensity squared, $\mathrm{I}^{2}$ for the spill. The duty factor is calculated using the formula discussed above previously.

The BeamDAQ begins the readout at the arrival of EOS signal and all the data is output to ASCII files.

\subsubsection{Slow Control Readout}

Slow control data are gathered when the EOS (End Of Spill) is delivered. The slow control data consist of accelerator, target, and environmental conditions during the spill that is acquired by utilizing EPICS (Experimental Physics and Industrial Control System) software package to communicate across various servers.

- The accelerator information describes the intensity and quality of the proton beam delivered, the configuration of the accelerator, and the status of FMAG and KMAG. The current on the magnets is constantly monitored by the accelerator operators as sending a high intensity beam directly to NM4 without sweeping away the debris could be detrimental to the experimental equipment. This data are collected by ACNET (Accelerator Control Network) and retrieved by SeaQuest.

- Target data such as target in the beam, target rotation pattern and pressures (used to calculate density) and temperatures of the cryogenics are read from an EPICS instance that has an interface with the target system. 


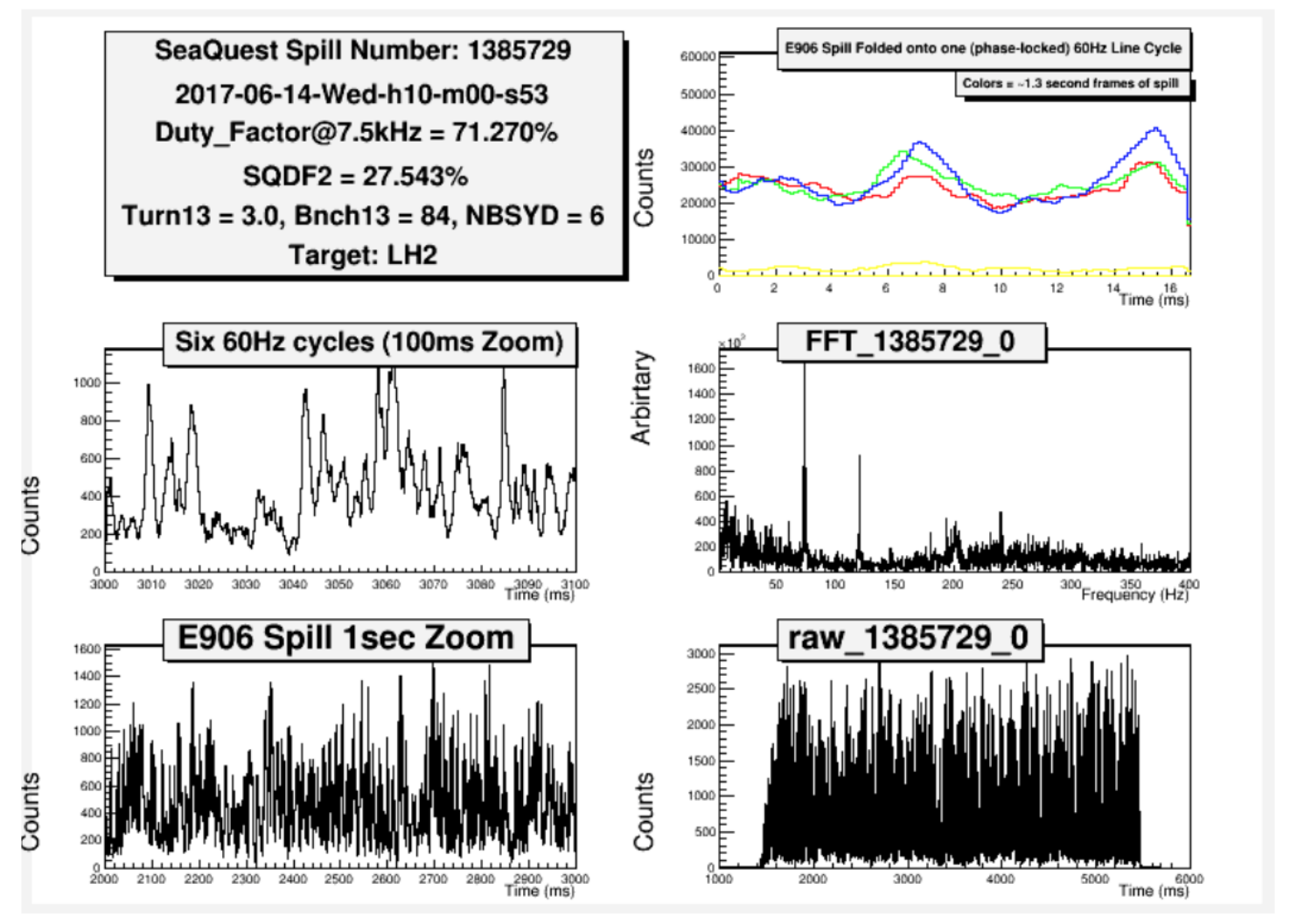

Figure 3.52: Online analysis of spill \# 1385729 [137]. The six individual plots show snippets of various aspects of the beam. For example, the top right plot shows the three Turns (red, blue and green) which indicate the stability of the beam in these turns. The right middle plot below shows the FFT (Fast Fourier Transform) of the beam. The prominent peak at $60 \mathrm{~Hz}$ arises due to the power supplies involved in the chain of electronics somewhere in the beam line. Information on a spill-by-spill basis is relayed back to the Control room and is used to tune beam for SeaQuest.

- Environmental conditions include temperatures (DAQ crates, temperatures of upper and lower parts of the experimental hall), pressure, and humidity by placing sensors through out the hall. Monitoring humidity is particularly important so that the flow of Nitrogen to the ASDQ cards of DC3p and DC3m could be increased in order to counter the increasing drift chamber leak current under high humidity situations. 


\subsection{Decoder}

The MainDAQ and ScalerDAQ CODA files and BeamDAQ ASCII files are stored on the SeaQuest servers and backed up by the tape storage service managed by Fermilab Computing Division. Each type of file requires a specific kind of parsing, processing and storage. This process is called as "decoding" and the software tool used to perform the mentioned functions is called the decoder. The decoded runs are stored on a MySQL server to be queried and used for analysis. Each run is is decoded into its own schema neatly organized into tables convenient to be retrieved for analysis. Data is also decoded in a "sampling mode" where it is used to look at quick results with the help of SeaScape. More information on the decoder can be found in [138].

\subsection{Online Monitoring}

SeaScape software is used to continuously monitor the status of detectors via hit distributions while data taking. Hodoscope, drift chamber, and proportional tube hit distributions, TDC spectra, multiplicity spectra, Detector, ScalarDAQ, ACNET variables, Slow control variables are some of the variables which can be viewed on this convenient software tool. As an example, SeaScape hit distributions of DC2 from Run - 14406 are shown in Figure 3.53. Two peaks which correspond to $\mu^{+}$(right) and $\mu^{-}$(left) can be seen in these hit distributions. These peaks are caused due to the presence of the FMAG magnetic field that pushes the $\mu^{+}$one way and $\mu^{-}$the other way. Similarly, two broad peaks on H1T (top) and H1B (bottom) which correspond to $\mu^{+}$(right) and $\mu^{-}$(left) can be seen in Figure 3.54. The bottom left and right plots show the hits on the Left and Right counters of H1Y hodoscopes which are peaked in the middle due to $\mu^{+}$(left) and $\mu^{-}$(right). It is important to note that the detector populations reversed when the magnetic fields were flipped. 

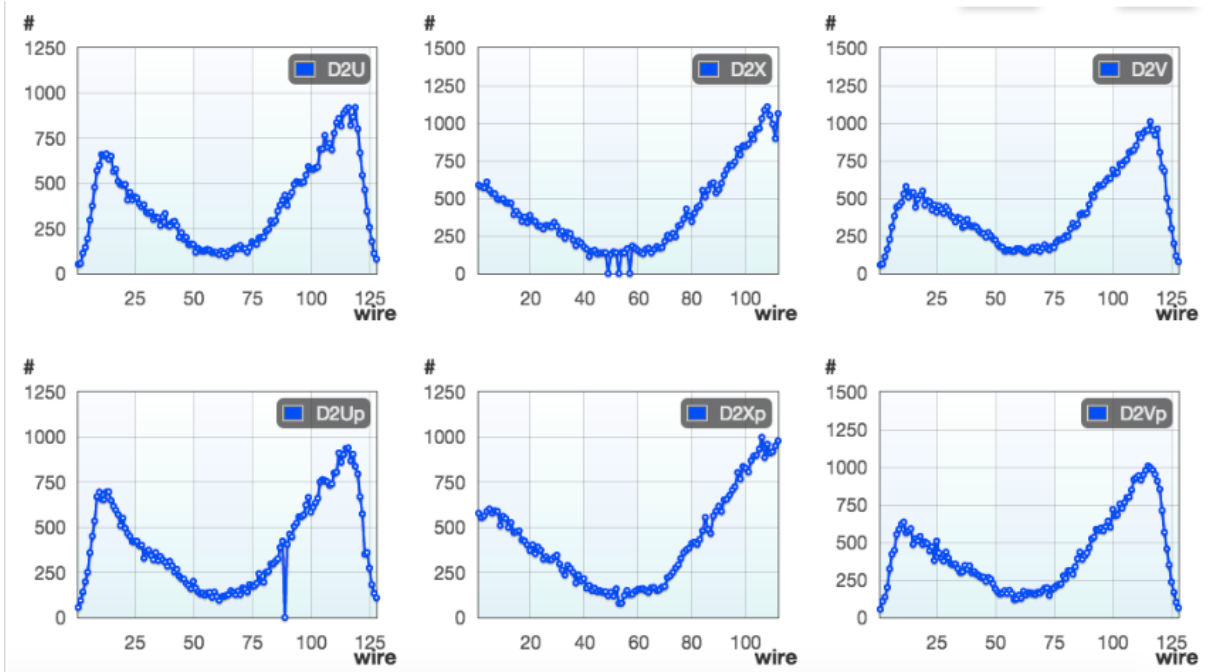

Figure 3.53: SeaScape hit distributions of DC2 from Run - 14406. The channels showing 0 hits on D2X and D2Up drift chamber planes are dead channels.
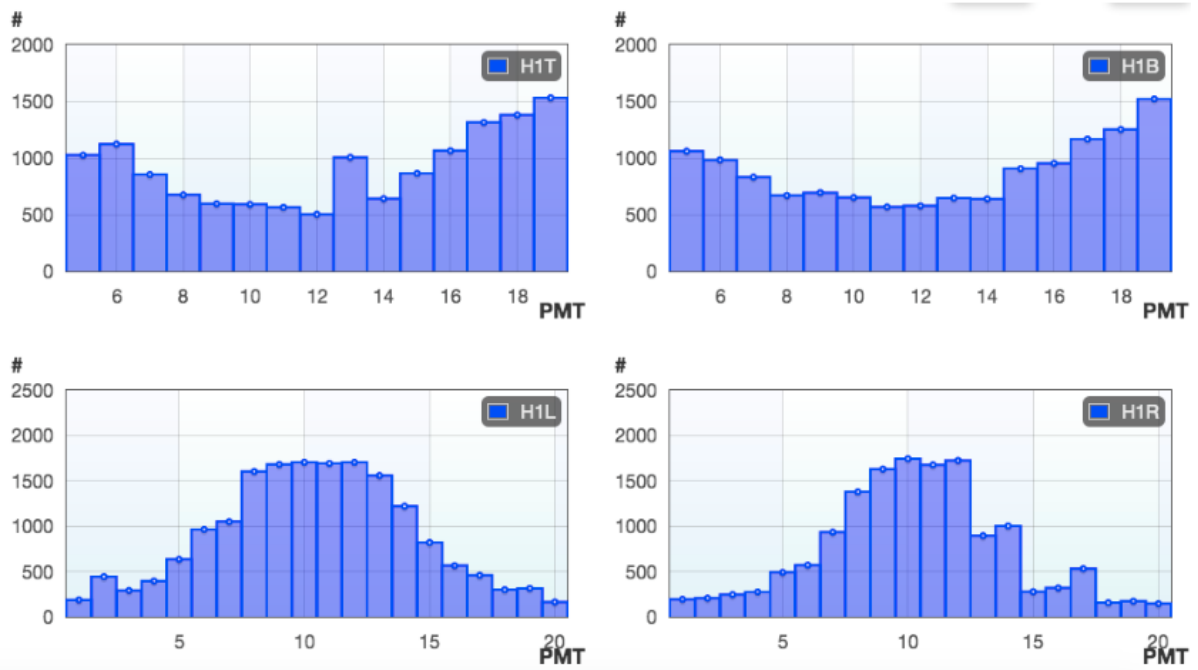

Figure 3.54: SeaScape hit distributions of H1 from Run - 14406. 


\section{Chapter 4}

\section{Data Analysis}

The physics of the experiment is hidden in the detector hits. The challenge of data analysis is to carefully isolate the signal from the background and extract the underlying physics. True Drell-Yan events from the target ( $\sim 10 \%$ interaction length) are swamped by backgrounds coming from various physics processes from upstream and the iron beam dump ( $\sim 90 \%$ interaction length). Therefore tracks have to reconstructed and clever analysis cuts have to be designed in order to carefully study the signal and accomplish the goals of the experiment.

\subsection{Data sets}

As discussed in the section on Trigger, the experiment successfully ran for almost four years taking six experimental runs with a variety of trigger settings. Table 4.1 summarizes the different settings used for data taking. The "Roadset" is just a name for a set of trigger roads used for that period of data taking. As can be seen in the table, the magnetic field was switched between Roadsets 62 and 67. Also, the vertical offset of the beam changed between Roadsets 59 and 62. These changes have been considered in Monte Carlo simulations, track reconstruction and analysis cuts. Results will be shown based on Run 2 and 3 data sets. 


\begin{tabular}{|c|c|c|c|c|}
\hline Roadset & Run range & Spill range & beam offset & B orientation \\
\hline 57 & $8912-10420$ & $310955-370099$ & $0.4 \mathrm{~cm}$ & $\mathrm{~B}+$ \\
\hline 59 & $10421-10912$ & $370110-388469$ & $0.4 \mathrm{~cm}$ & $\mathrm{~B}+$ \\
\hline 62 & $11075-12435$ & $409547-482571$ & $1.6 \mathrm{~cm}$ & $\mathrm{~B}+$ \\
\hline 67 & $12525-15789$ & $484746-676223$ & $1.6 \mathrm{~cm}$ & $\mathrm{~B}-$ (flipped) \\
\hline 70 & $15793-16076$ & $676498-696454$ & $1.6 \mathrm{~cm}$ & $\mathrm{~B}-$ (flipped) \\
\hline
\end{tabular}

Table 4.1: Table of "good" run and spill ranges. The definition of good is discussed here 4.5 .

\subsection{Dimuon Reconstruction}

The main track reconstruction program used by SeaQuest is named "kTracker" where the "k" stands for Kalman-Filter method used in reconstructing the dimuon vertex. This program was mainly developed by Kun Liu, a collaborator from Los Alamos National Laboratory. As shown in the workflow diagram in Fig 4.1, the entire tracking procedure can be divided into a few stages; pre-tracking analysis, track reconstruction and reaction vertex reconstruction.

\subsubsection{Pre-tracking analysis}

In order to optimize the time consuming tracking process, a few steps are taken that remove potential noise hits. These steps are a preparatory phase where noisy hits which have certain

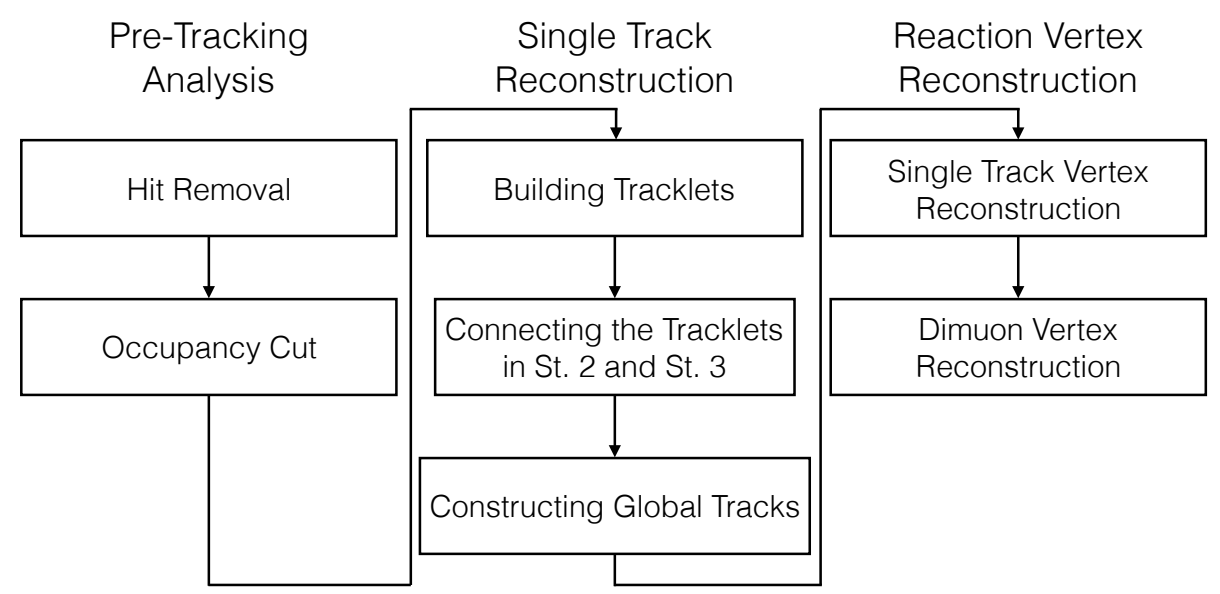

Figure 4.1: kTracker flowchart 
characteristics are identified and discarded before passing on to the tracker. Removing these extra hits significantly helps with track reconstruction process.

\section{"Extra" hit removal}

- Out-of-time hits: This stage removes the hits with TDC time falling out of a predefined TDC time window.

- After pulse removal: After pulse signals arise in the signal wire after the passage of the true signal. In such a case, the first signal is considered as the most likely to be the true signal.

- Random hits removal: Since the trigger is caused by hits on the hodoscopes, corresponding hits on the drift chamber are "matched" to a slightly larger hodoscope "geometric window". Drift chamber hits that do not have a match are dropped. This is done in the HodoscopeMasking (chamber-to-hodoscope) function in kTracker ${ }^{1}$.

- Hit cluster removal: A group of contiguous hits are called "hit clusters". An example of a size 4 cluster is depicted in Fig 4.3 [139]. These clusters are categorized into three types; electronic noise, cell edge hits and $\delta$ rays.

- Edge hits: When a muon passes in between two cells, it produces a signal on both the neighboring wires. These are called edge hits. In such a case, the hit with the larger drift distance is discarded.

- Electronic noise: When a charge particle passes through or maybe for some unknown reason (noisy ASDQ cards, noise from the VME back plane etc.) hits appear on a series of two or three wires. The time difference of the drift times is less than $10 \mathrm{~ns}$. These hits are discarded as coming from electronic noise.

- $\delta$ rays: These clusters (series of 3 or 4 contiguous hits) are created by $\delta$ rays (high energy electrons which are created when particles collide with primary

\footnotetext{
${ }^{1}$ Masking here means "masking out" the unmatched data.
} 
ions. These $\delta$ rays are capable of creating secondary ionizations. If a cluster consists of two or more contiguous hits and the average of their time difference is typically large (10 ns or larger), this is considered to be generated from a $\delta$ ray moving in the $\mathrm{X}-\mathrm{Y}$ plane. In such a case the two hit edges (which are the likely source of the $\delta$ ray) are stored and the others discarded. A flowchart of the process of identifying and removal of these hits is shown in Figure 4.3 [139]. Figure 4.4 shows histograms on a linear and log scale of the hits as a function of the St 1 occupancy before and after hit removal. Tests were performed to see how many of the true hits were removed by the cluster removal procedure. The following procedure was used in estimating that.

* Track reconstruction is performed on "raw data".

* Find a plane without any track associated hits (call it "empty plane”).

* Compare the raw data and check whether the empty plane has any hits near the track position on the empty plane $(3 \times$ hit resolution $)$. If such a hit exists, the "hit removal" procedure has removed a true hit. The estimated fraction of true hits removed has been found to be $<1 \%$

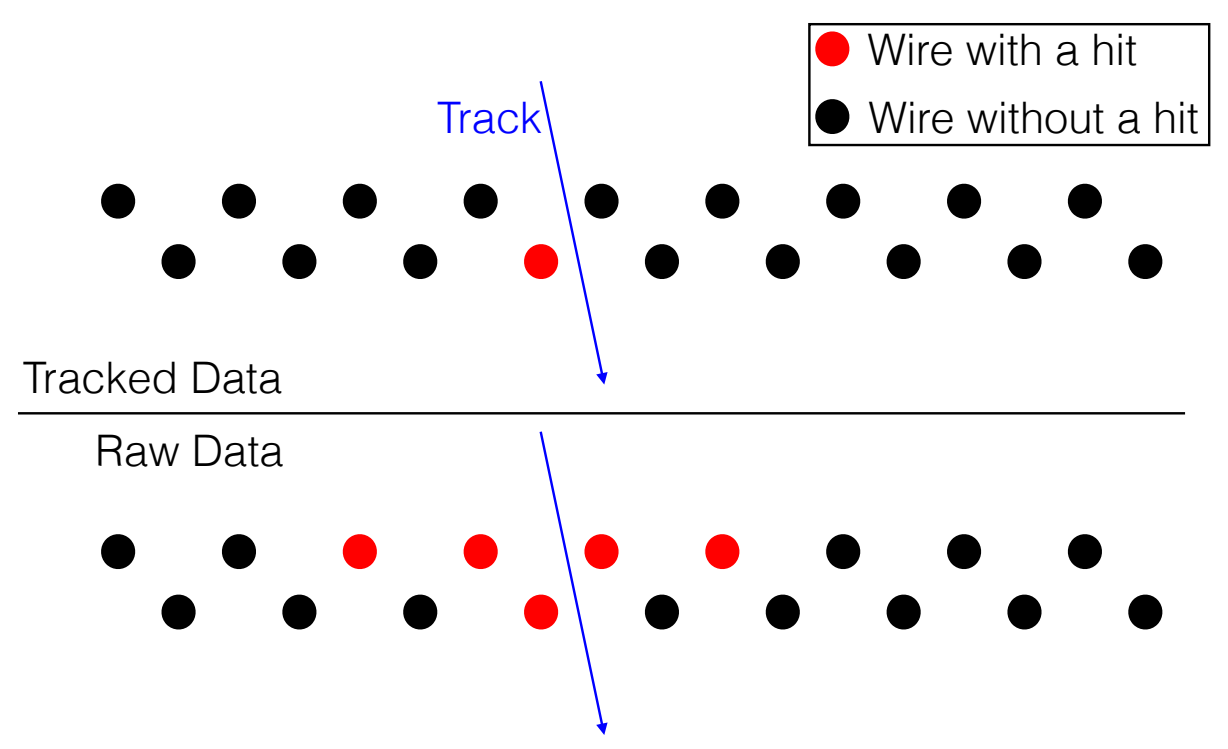

Figure 4.2: Depiction of hit removal from raw data. Blue arrow is the muon track. Red dots are the extra hits (top array) and tracked hit (bottom array). 


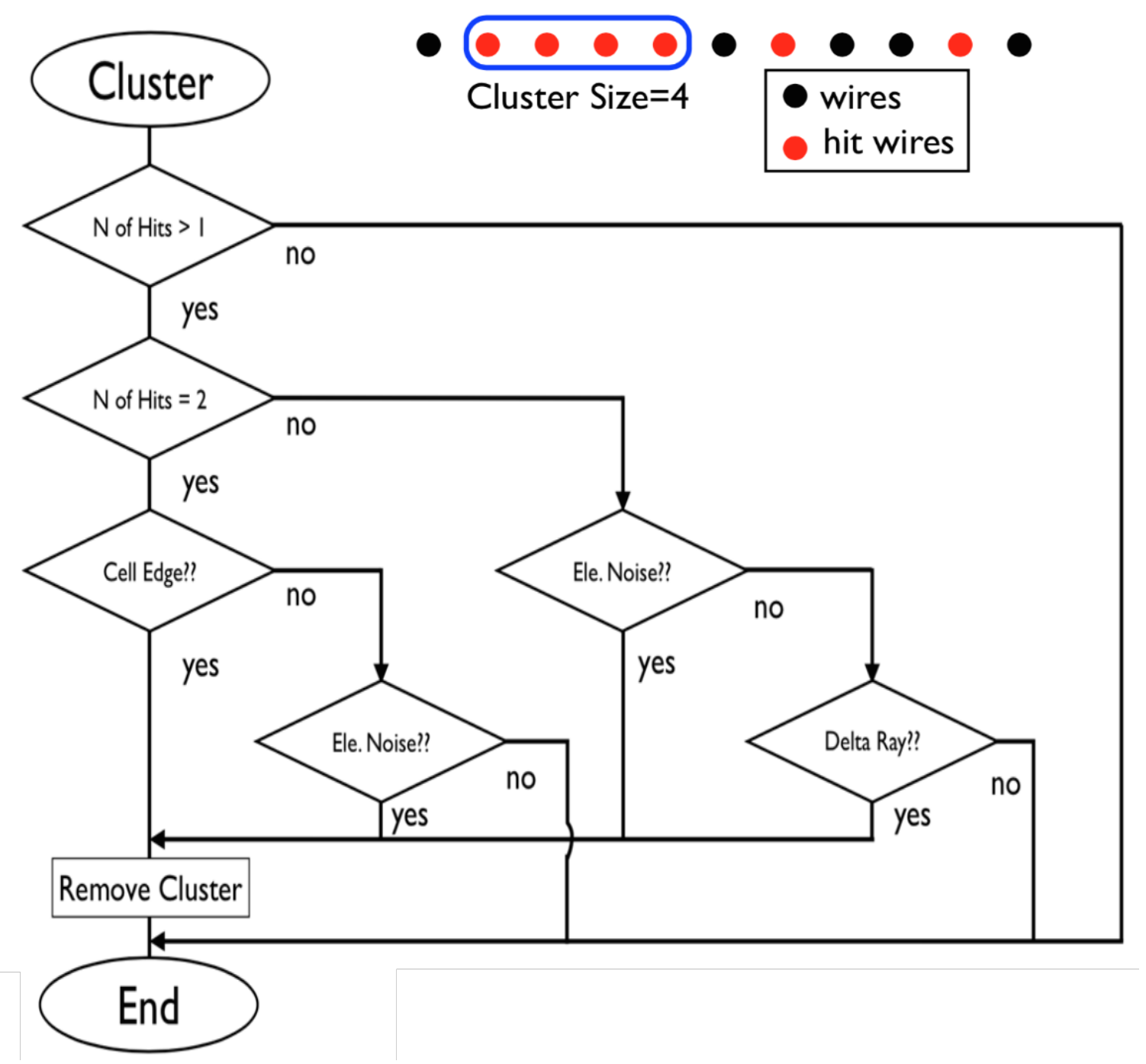

Figure 4.3: Cluster removal flowchart [139]

\section{Occupancy cuts}

Even after extra hit removal, some events include too many hits making the track reconstruction possibly difficult and time consuming. In order to remove those events, loose occupancy (multiplicity of hits on detectors) cuts are applied. The definition of occupancy corresponds to the number of hits after hit removal. Table 4.2 lists the upper limit for R008 data production. Additional trigger related functions are also used in trimming the raw data. If a set of $\mathrm{H} 1 \mathrm{X}, \mathrm{H} 2 \mathrm{X}, \mathrm{H} 3 \mathrm{X}$ and $\mathrm{H} 4 \mathrm{X}$ hodoscope in-time hits matches an enabled 


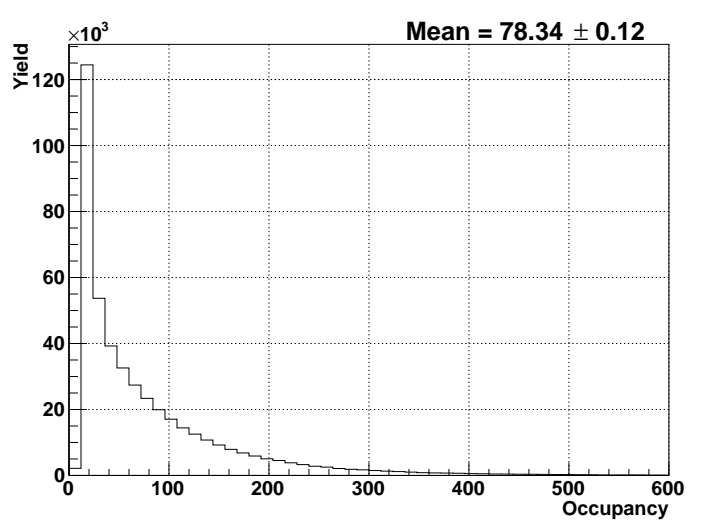

(a) Number of hits on DC1.1 before hit removal (linear scale).

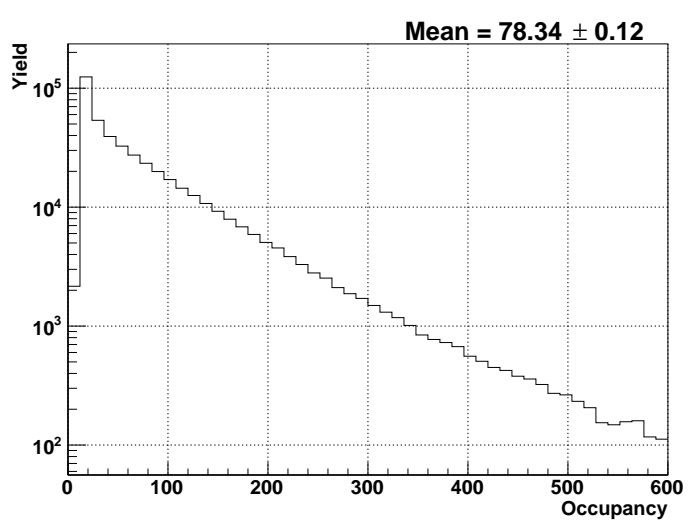

(c) Number of hits on DC1.1 before hit removal (log scale).

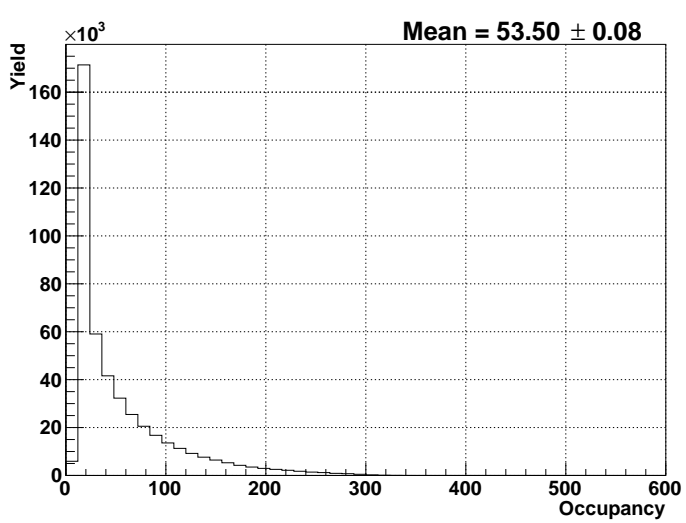

(b) Number of hits on DC1.1 after hit removal (linear scale).

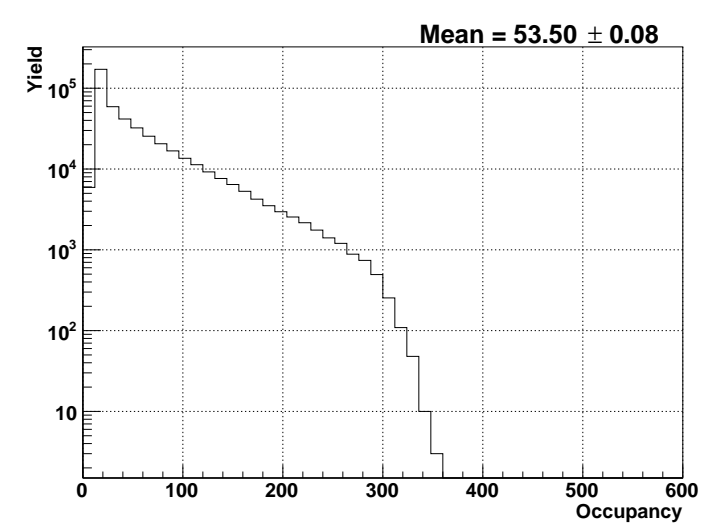

(d) Number of hits on DC1.1 after hit removal (log scale).

Figure 4.4: Number of hits before and after hit removal on DC1.1 shown on a linear scale ((a) and (c)) and a log scale ((b) and (d)).

road, the four hits in the set are extracted. The extracted hits of all such sets are used for the hodoscope masking. As a result every chamber hit used in the tracking is matched to an element of a fired road. The hodoscope hits not associated with any road candidates are removed. Also, if an event has five or more possible $\mu^{+}$or $\mu^{-}$roads, it is discarded since it takes up a lot of time to analyze such events. Proportional tube tracklets are also built as track seeds to be used for muon identification later [140]. 


\begin{tabular}{|c|c|}
\hline Detector & Occupancy cut \\
\hline DC1.1 & 350 \\
\hline DC1.2 & 350 \\
\hline DC2 & 170 \\
\hline DC3m & 140 \\
\hline DC $3 p$ & 140 \\
\hline
\end{tabular}

Table 4.2: Internal occupancy cuts for kTracker.

\section{Residual cuts}

Additional cuts on the drift chamber residuals are applied to reject random events in track reconstruction process. The values for the residuals and the corresponding drift chamber are mentioned in Table 4.3.

\begin{tabular}{|c|c|}
\hline Parameter & Residual value \\
\hline \hline DC1.1 & 0.1200 \\
\hline DC1.2 & 0.1200 \\
\hline DC2 & 0.1500 \\
\hline DC3m & 0.1400 \\
\hline DC3p & 0.1600 \\
\hline
\end{tabular}

Table 4.3: Residual windows for drift chambers
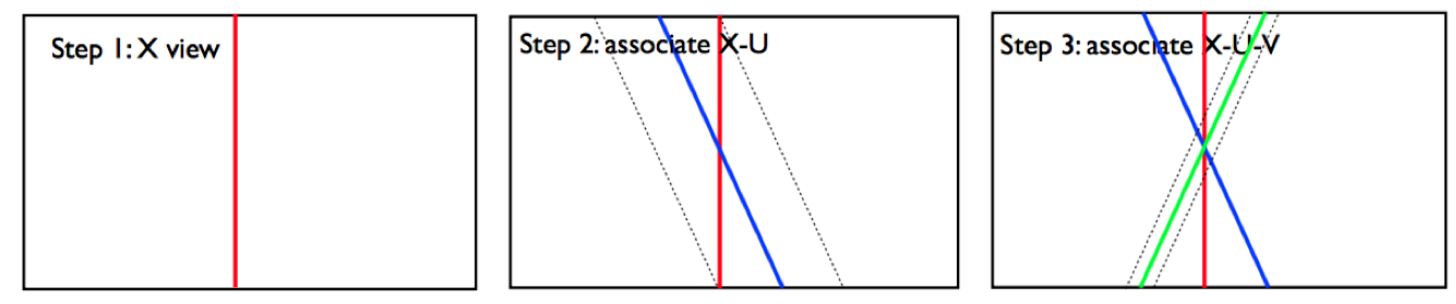

Figure 4.5: Building of tracklets in a drift chamber. 


\subsubsection{Single Track Reconstruction}

\section{Building tracklets in DC2 and DC3}

The next stage of track reconstruction is identifying tracklets inside individual drift chambers. The building up of tracklets starts from DC2 and DC3 due to the track being a linear segment without any magnetic field and relatively less noisy hits compared to further upstream drift chambers. Adjacent hits on the $\mathrm{XX}$ ' which have the difference between the elementIDs of a hits are selected as hit pairs. The next step is to find the UU' planes on hit pairs. As can be seen in Figure 4.5, the hit pairs on the UU' planes are searched in the window given by:

$$
u_{\text {center }}=x \cos \theta
$$

where $\theta$ is the inclination angle of the UU' plane and $x$ is the wire hit position on the $x$ plane. The width of the window is defined as:

$$
u_{w i n}=\frac{1}{2} L_{x} \sin \theta+t_{x}^{\max }\left|z_{u}-z_{x}\right| \cos \theta+t_{y}^{\max }\left|z_{u}-z_{x}\right| \sin \theta+2 \Delta u+\delta
$$

where $L_{x}$ is the length of the $\mathrm{X}$ wire, $\Delta u$ is the wire spacing of the $\mathrm{U}$ plane, $t_{x}^{\max }(=0.15)$ and $t^{\max _{y}}(=0.1)$ are the maximum possible tracklet slopes in the $\mathrm{X}-\mathrm{Z}$ and $\mathrm{Y}-\mathrm{Z}$ views, $z_{u}$ and $z_{x}$ are the $\mathrm{z}$ positions of the $\mathrm{U}$ and the $\mathrm{X}$ planes and $\delta(=5 \mathrm{~cm}$ for St. 1 and St. $2,=10$ cm for St. 3 drift chambers) is the extra contingency window [141]. All the hits within the $u_{\text {center }} \pm u_{\text {win }}$ are kept as hit pairs. The next step is to calculate the window for the VV' is calculated based on the mini-tracklets constructed using the $\mathrm{XX}^{\prime}$ and $\mathrm{UU}$ ' hit pairs. From the track slopes $t_{x}$ and $t_{y}$;

$$
\begin{aligned}
& u=x \cos \theta+t_{x}\left(z_{u}-z_{x}\right) \cos \theta+t_{y}\left(z_{u}-z_{x}\right) \sin \theta \\
& v=x \cos \theta+t_{x}\left(z_{v}-z_{x}\right) \cos \theta-t_{y}\left(z_{v}-z_{x}\right) \sin \theta
\end{aligned}
$$


The following relation should be satisfied for the correct combination of the hit pairs:

$$
u+v=2 x \cos \theta+t_{x}\left(z_{u}-z_{x}+z_{v}-z_{x}\right) \cos \theta+t_{y}\left(z_{u}-z_{v}\right) \sin \theta
$$

The search window for the $\mathrm{V}$ plane is calculated based on

$$
\begin{aligned}
v_{\text {center }} & =2 x \cos \theta-u \\
v & =t_{x}^{\max }\left|z_{u}+z_{v}-2 z_{x}\right| \cos \theta+t_{y}^{\max }\left|z_{u}-z_{v}\right|\left(z_{v}-z_{x}\right) \mid \sin \theta+2 \Delta v
\end{aligned}
$$

where $x$ and $u$ stand for the hit positions on $\mathrm{X}$ and $\mathrm{U}$ planes and the $\Delta v$ is the wire spacing in the V view [141]. Multiple triplets are allowed to use the same hits. The triplets are discarded if:

- the tracklet doesn't point towards to a fired hodoscope paddle in the neighboring $\mathrm{x}$-hodo station (with a slightly increased size of the paddle).

- the tracklet doesn't point to the target (loose cut)

- the tracklet has less than 4 hits.

- the $\chi^{2}$ is beyond a certain range (loose cut) or if the hits from a specific view are missing.

\section{Connecting tracklets in DC2 and DC3}

The next stage is to connect the tracklets in DC2 and DC3. All possible tracklets are taken and "back-partial" tracks are reconstructed between DC2 and DC3. If there are two partial tracks that have more than $1 / 3$ of the hits in common, they are regarded as the same track and the one with the smaller $\chi^{2}$ is kept. Additional loose cuts on the rough partial-track slopes and $\chi^{2}$ are also applied to ensure the quality of the track [141]. A tracklet combination is thrown away if the associated track doesn't seem to come from the 
target, or does not point towards the fired paddles at St 2, 3 and 4 hodoscopes. A muon identification cut is also used to require that the projected partial track has at least one matched hit at the Station 4 proportional tubes along with a small deflection is observed between the difference of the slopes caused due to the multiple scattering from the iron wall.

\subsubsection{Constructing Global Tracks}
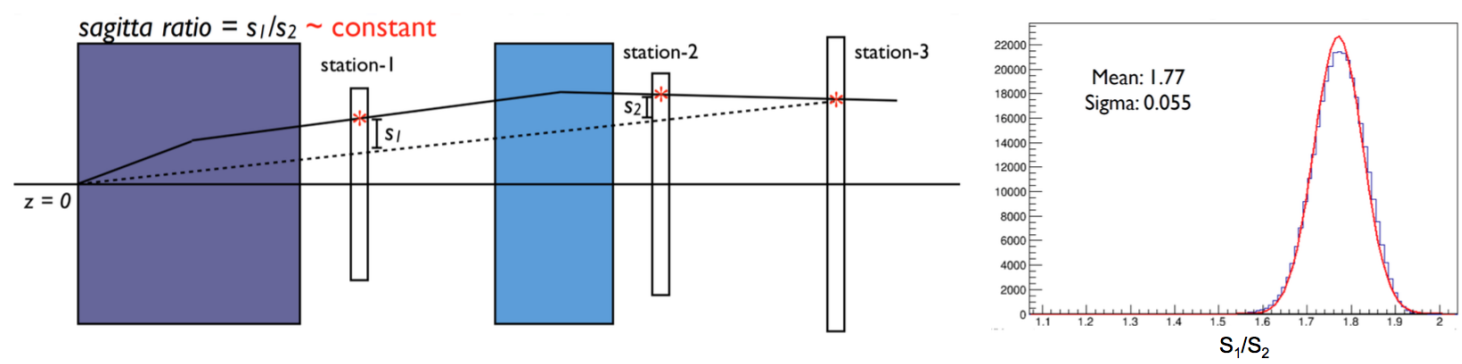

Figure 4.6: Sagitta method used for global track reconstruction.

The next step involves connecting the back partial tracks from DC2 - DC3 tracklets by projecting it back to DC1 drift chamber and pairing it with the appropriate DC1 tracklet to form a "global track". The search window for DC1 is determined using the sagitta ratio method. The sagitta is defined as the distance between the track and the line connecting $\mathrm{X}$ $=\mathrm{Y}=\mathrm{Z}=0$ and the DC3 triplet. Sagitta ratio which is independent of the momentum is calculated from Monte Carlo simulations. It is the ratio of the distance s1 and s2 as shown in Figure 4.6. From simulations, this value was found to be $1.77 \pm 0.055$. A track is defined by five parameters. $C / p_{x z}, t_{x z}, t_{y}, x_{0}$ and $y_{0}$ where $\mathrm{C}$ is the sign of the charge of the track and $p_{x z}=\sqrt{p_{x}^{2}+p_{y}^{2}}, t_{x}, t_{y}, x_{0}$ and $y_{0}$ are the momenta, slopes and intersections in the $\mathrm{X}-\mathrm{Z}$ and $\mathrm{Y}-\mathrm{Z}$ plane of the back partial track in the track. Since the B is oriented in the $y$ direction, the slopes and intersection in the Y-Z plane before and after KMAG are the same. The slope and intersection in the $\mathrm{X}-\mathrm{Z}$ plane are calculated using the formula: 


$$
\begin{aligned}
& t_{x}^{D C 1}=t_{x}+p_{T}(\text { kick }) \frac{C}{p_{x z}} \\
& x_{0}^{D C 1}=t_{x} z_{\text {bend }}+x_{0}-t_{x}^{D C 1} z_{\text {bend }}
\end{aligned}
$$

where $p_{T}($ kick $)$ is the kick provided by the $\mathrm{KMAG}=0.4016 \mathrm{GeV} / \mathrm{c}$ and $z_{\text {bend }}=1064.26 \mathrm{~cm}$, the $z$ position of the center of KMAG. To reconstruct global tracks, the bad hits are cleaned out in an iterative procedure. After fitting to a track candidate, the hit having the greatest residual is discarded if the residual is greater than three times the chamber resolution. The remaining hits are used again for a re-fit. This process is repeated again until all the hits of the possible global track have residuals smaller than three times the resolution $(7 \mathrm{~mm})$ [125]. Several other quality cuts are applied to ensure good track quality of the global track. A global track is discarded if:

- It doesn't point to fired $x$-hodoscope paddles in any station.

- $N_{\text {hits }}<13$

- $P<5 \mathrm{GeV} / \mathrm{c}$ or $P>100 \mathrm{GeV} / \mathrm{c}$

- $\chi^{2}>20$

- The track has less than 4 hits in one of the stations, or has one missing view

- Deflection caused by the $1 \mathrm{~m}$ long absorber wall is larger than expected

The tracks passing these cuts are passed on to the Kalman Filter algorithm [142]. The Kalman Filter is a recursive program that is intended to finding the optimum estimation of an unknown state vector according to some given initial conditions. The Kalman filter starts with a certain initial approximation for a given vector and refines the vector adding one measurement after another. The final optimum value is attained after including all the measurement in this recursive process [143]. It starts with an initial state vector with a 
specified uncertainty, propagates it based on the input physical model and projects an estimate of the variables. The workflow of the Kalman filter process is shown in 4.7 [125]. It combines the estimation with the measurements using a weighted average with more

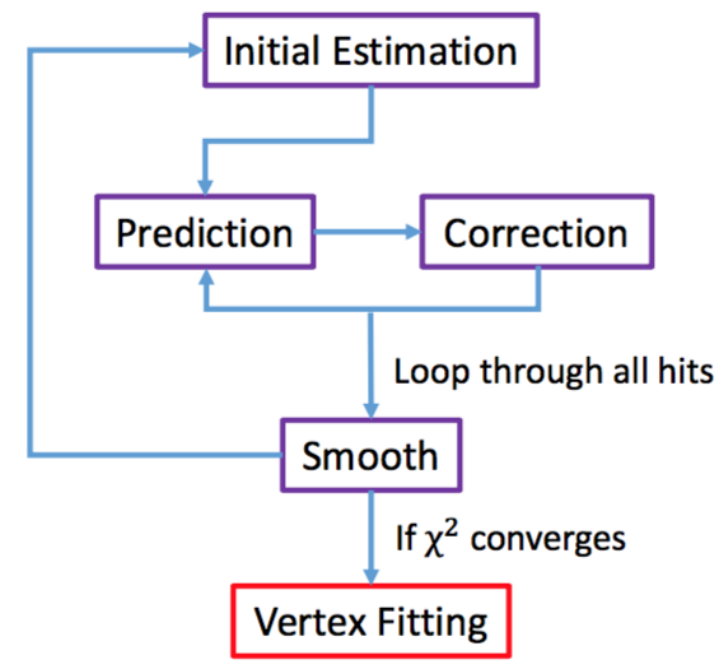

Figure 4.7: Workflow of the Kalman filter process [125].

weight given to the one with higher certainty. The combined and "newly" updated state vectors are taken and again propagated to generate a "newer" estimation of the state vector and this recursive process is repeated until the final measurement input is used. Figure 4.8 shows a geometric depiction of the implementation of the recursive process in which the $(k-1)^{t h}$ plane projects the state vector for the $k^{t h}$ plane, uses the measurements $m$ along with the uncertainties, adjusts the state vector accordingly and propagates it to the $(k+1)^{t h}$ plane [140]. In the case of SeaQuest, the state vector consists of the spatial coordinates of the muon and its three momenta. The evolution of the state vector corresponds to the parameters of the muon as it is propagated through the spectrometer. The hit positions at each detector (along with the detector resolutions as uncertainties) are treated as the measurement (and uncertainties) used as inputs for the Kalman filter. GEANT4 is used to simulate the propagation of the muons through the spectrometer. This package is also used in Monte Carlo simulations. As mentioned at the beginning of this section, the muon trajectory is 
reconstructed from $\mathrm{DC} 3 \rightarrow \mathrm{DC} 2 \rightarrow \mathrm{DC} 1 \rightarrow$ target region. This is done to get a straight track segment between DC3 and DC2 which free from magnetic fields and relatively less noise which leads to a better convergence of the Kalman filter estimation.

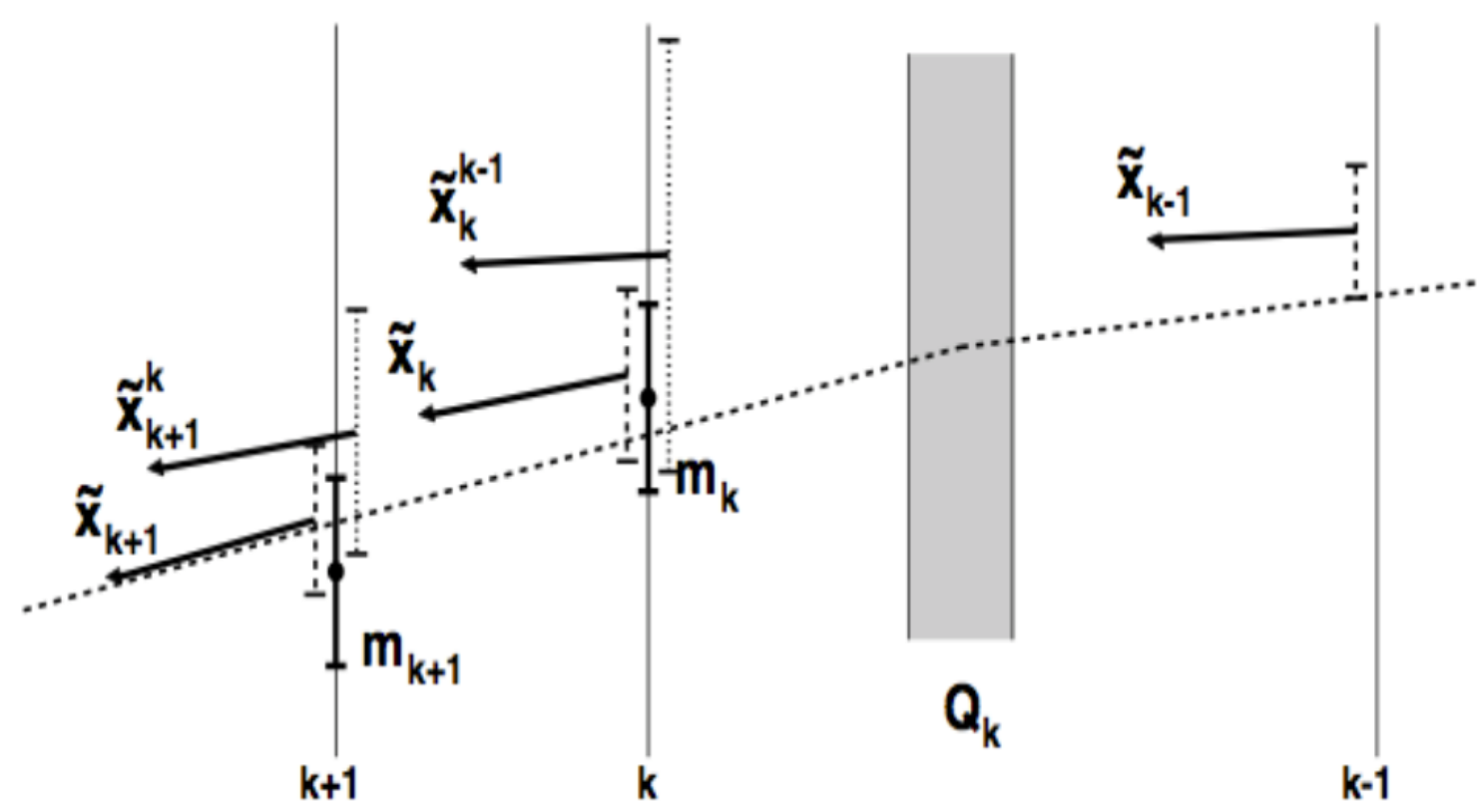

Figure 4.8: Geometric depiction of the Kalman filter process [140].

\subsubsection{Single Track Vertex Reconstruction}

Before going into the details of vertex finding, it is important to understand the application of energy loss while muons traverse the $5 \mathrm{~m}$ long iron beam dump. Since there is no way to determine the true energy lost by the traversing muon, energy loss modeling poses a challenge.

\section{Energy loss in GMC and kTracker:}

The energy loss is tackled differently in the GEANT4 based Monte Carlo simulations and kTracker. GEANT4 handles the energy loss where a random value from the Landau energy loss distribution for ionizing particles is applied to the thrown muon [144]. Figure 4.9 


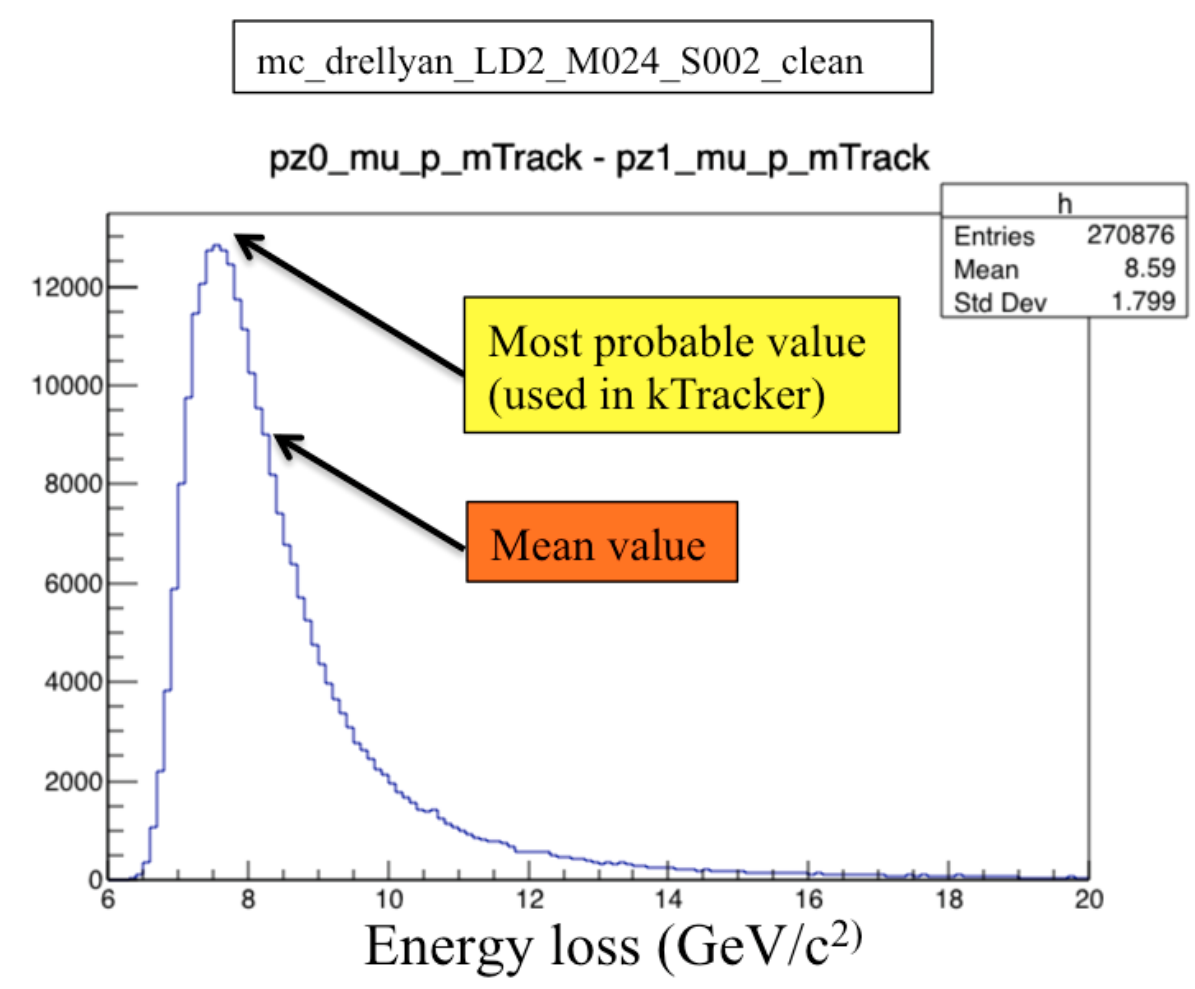

Figure 4.9: Application of energy loss in kTracker. Blue curve is the momentum difference between the thrown momentum at the initial momentum - the thrown momentum at DC1. The most probable value in this curve is used in the kTracker.

shows the momentum difference between the thrown value at the target and the first drift chamber DC1. The curve also shows the mean and the most probable value (used in the kTracker).

The parameterization was tuned for E906 energy range using E866/NuSea legacy code. Parameterization for the momentum range takes the most probable value for the energy loss into consideration for different energy ranges. As seen in Figure 4.10, for $20-112 \mathrm{GeV} / \mathrm{c}$, the energy loss is be monotonically increasing as designed to be so. An arbitrary choice exists between choosing the mean versus the most probable value for the energy loss. For an asymmetric $J / \psi$ peak, the choice of the value of energy loss decides if the mean or the peak value aligns with the actual $J / \psi$ peak at $3.097 \mathrm{GeV} / \mathrm{c}^{2}$. In the case of SeaQuest, the most probable value was chosen since it is easier to identify the peak rather than apply 
cuts and identify the mean of $J / \psi$. Table 4.4 and Figure 4.10 shows the parameterization constants and their plot as function of the muon energy range for SeaQuest. The magnitude of the energy loss inside the FMAG is given by

$$
E_{\text {loss }}=E_{0}+E_{1} p+E_{2} p^{2}+E_{3} p^{3}+E_{4} p^{4}
$$

where $E_{0}, E_{1}, E_{2}, E_{3}$ and $E_{4}$ are the parameterized energy loss constants and $p$ is the momentum of the penetrating muon. The $502 \mathrm{~cm}$ long iron beam dump in the FMAG is

\section{Energy loss vs muon momentum}

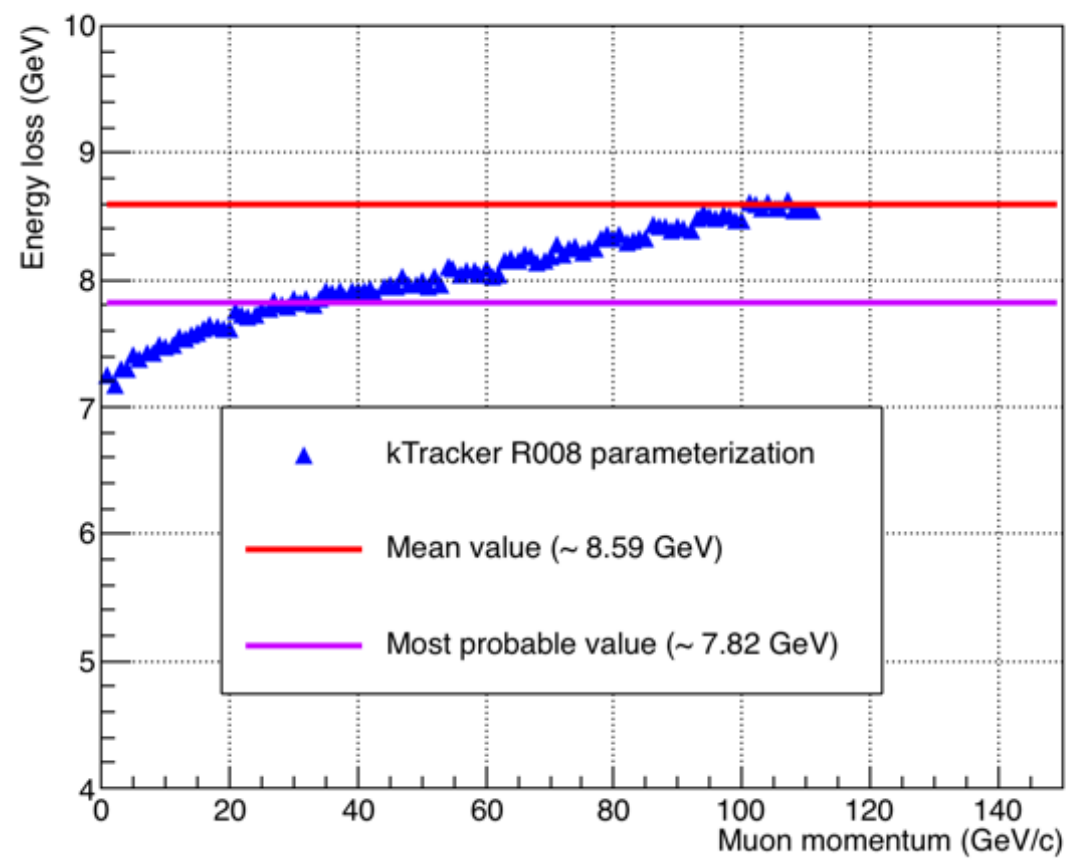

Figure 4.10: Energy loss parameterization for SeaQuest.

\begin{tabular}{|c|c|}
\hline Parameter & Value \\
\hline \hline$E_{0}$ & 7.18274 \\
\hline$E_{1}$ & 0.0361447 \\
\hline$E_{2}$ & -0.000718127 \\
\hline$E_{3}$ & $7.97312 \times 10^{-6}$ \\
\hline$E_{4}$ & $-3.05481 \times 10^{-8}$ \\
\hline
\end{tabular}

Table 4.4: Energy loss parameterization constants for SeaQuest. 
divided into 100 slices ( $5 \mathrm{~cm}$ steps) where each slice is divided into two sections. After giving the muon a $p_{T}$ kick at the center, the track slope changes changes from $t_{x 1}$ to $t_{x 2}$ given by the equation:

$$
t_{x 1}=t_{x 2}+s \cdot \frac{k i c k}{L_{F M A G}} \cdot \frac{L_{F M A G}}{N_{s}} \cdot \frac{1}{\sqrt{P_{1 x}^{2}+P_{1 z}^{2}}}
$$

where $k i c k=2.909 \mathrm{GeV} / \mathrm{c}, s$ is the charge sign of the track, $t_{x 1}$ and $t_{x 2}$ are the track slopes of the track in the X-Z plane at the downstream and upstream surfaces of the FMAG slices, $N_{s}$ is the number of slices in FMAG (= $100-5 \mathrm{~cm}$ slices) and $P_{1 x}$ and $P_{1 z}$ are the $x$ and $z$ momenta of the track at the downstream surface of the slice. Energy loss (according to the parameterization shown) is applied at the beginning of one slice, then a $p_{T}$ kick $=2.909$ $\mathrm{GeV} / \mathrm{c}$ is applied at the center and another energy loss (gain) is applied at the end of the slice. The swimming is divided to 2 parts. The first part is from $502.92 \mathrm{~cm}$ to $0 \mathrm{~cm}$. In this range we have iron and magnetic field. The whole range is divided to 100 steps, with all $z 0$ in this range a multiple of $5.0292 \mathrm{~cm}$. The second part is from $0 \mathrm{~cm}$ to $-500 \mathrm{~cm}$. It is divided into 200 steps, with all $z 0$ in this range multiples of $2.5 \mathrm{~cm}$. The three momentum at the center of the FMAG slice $P_{2}$ is given by:

$$
\begin{aligned}
P_{2} & =P_{1}+7.18274+\left(0.0361447 P_{1}-0.000718127 P_{1}^{2}\right. \\
& \left.+7.97312 \times 10^{-6} P_{1}^{3}-3.05481 \times 10^{-8} P_{1}^{4}\right) \cdot \frac{1}{L_{F M A G}} \cdot \frac{1}{T_{1}}
\end{aligned}
$$

where $L_{F M A G}$ is the length of FMAG and $T_{1}$ is the trajectory of a track at the former half of the FMAG slice given by:

$$
T_{1}=\left(\frac{t_{x 1} \cdot L_{F M A G}}{2 \cdot N_{s}}, \frac{t_{y} \cdot L_{F M A G}}{2 \cdot N_{s}}, \frac{L_{F M A G}}{2 \cdot N_{s}}\right)
$$


Similarly, the 3-momenta at the upstream surface the FMAG slice would be

$$
\begin{gathered}
P_{3}=P_{2}+7.18274+\left(0.0361447 P_{2}-0.000718127 P_{2}^{2}\right. \\
\left.+7.97312 \times 10^{-6} P_{2}^{3}-3.05481 \times 10^{-8} P_{2}^{4}\right) \cdot \frac{1}{L_{F M A G}} \cdot \frac{1}{T_{1}} \\
T_{2}=\left(\frac{t_{x 2} \cdot L_{F M A G}}{2 \cdot N_{s}}, \frac{t_{y} \cdot L_{F M A G}}{2 . N_{s}}, \frac{L_{F M A G}}{2 \cdot N_{s}}\right)
\end{gathered}
$$

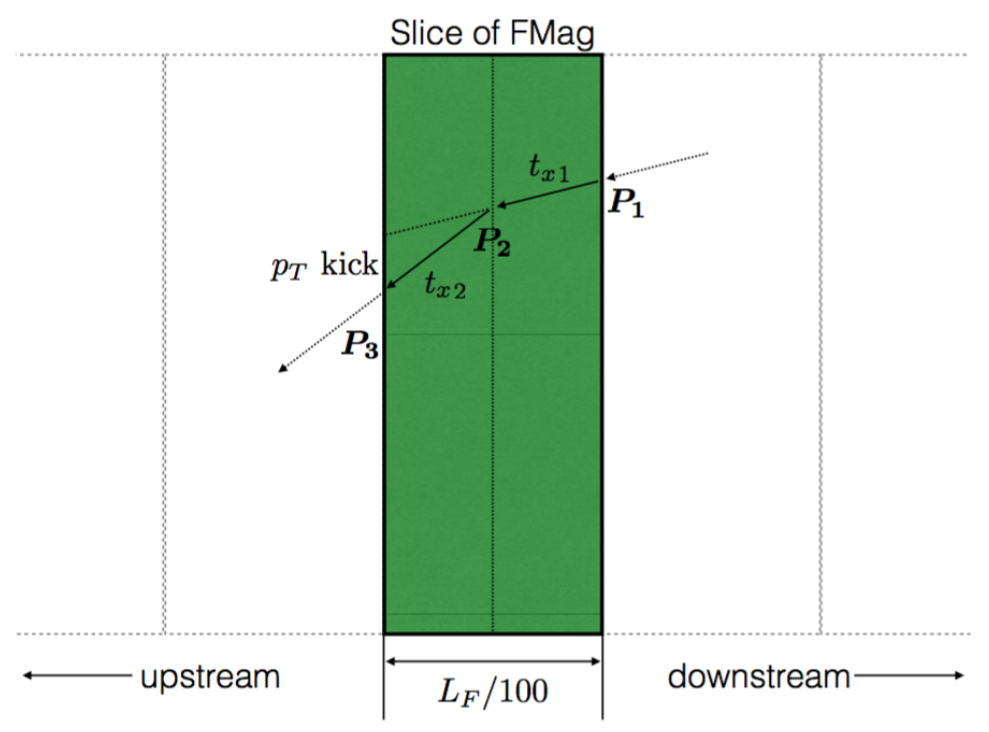

Figure 4.11: Application of energy loss in kTracker at the beginning and end of each slice with a $p_{T}$ kick at the middle of the slice.

This process is continued until the muon traverses the entirety of FMAG. The point with closest approach to the beam line is considered to be the position of the single muon vertex.

\subsubsection{Dimuon Vertex Reconstruction}

The dimuon vertex finding employed in SeaQuest also uses the extended Kalman filter process. After obtaining the $\mu^{+}$and $\mu^{-}$tracks using the procedure described above, all the combinations are tried out for a suitable dimuon vertex. The dimuon vertex position is reconstructed following a Kalman filter procedure as well [143]. The first guess of the 
$\mathrm{h}$

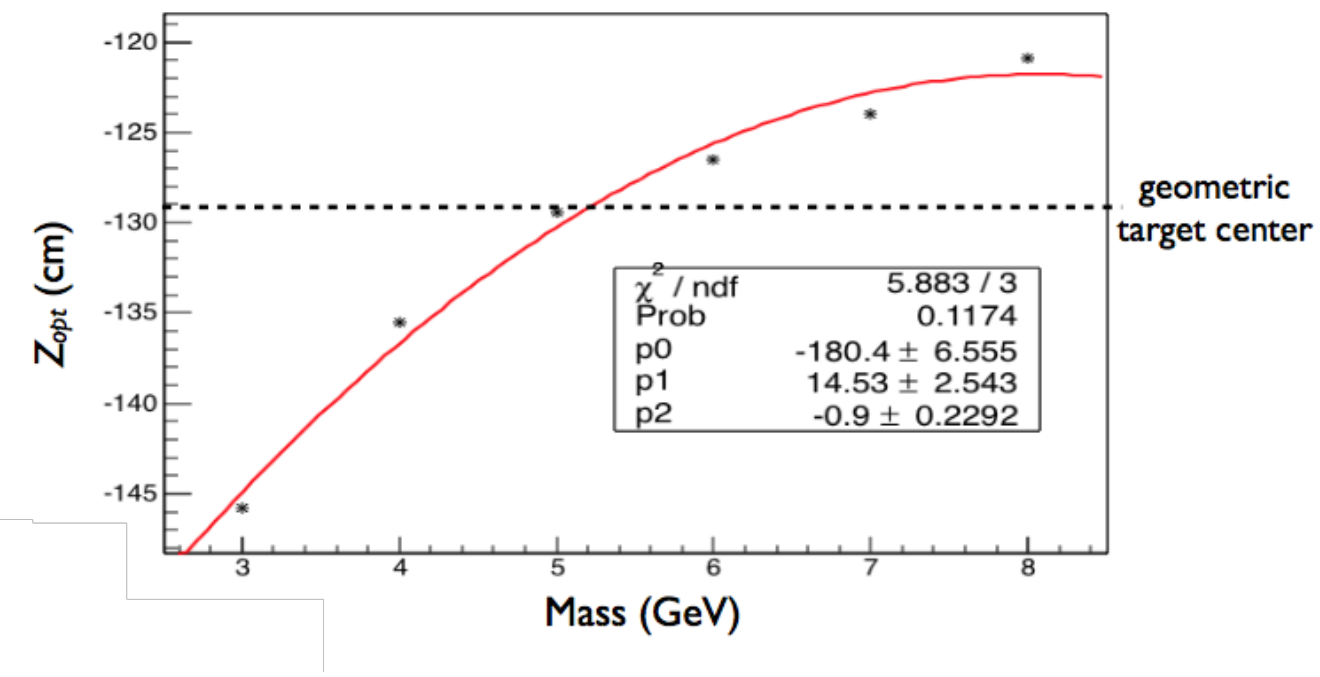

Figure 4.12: Reconstructed vertex position blue position) for a fixed $z$ thrown position (dotted line) and mass [140].

vertex position is just simply the average $z$ position of the single muon vertices (distance of closest approach - DOCA). This value is used as an input and the Kalman filter method updates the dimuon vertex position until the $\chi^{2}$ converges and the result passes some quality checks.

\section{Vertex optimization condition}

So far only the energy loss has been taken into consideration in the trace back procedure used to get tracks. However, multiple scattering (and other effects which are not accounted for by energy loss) of muons from iron also plays a role in the reconstructed position of the dimuon vertex. An additional factor is used to correct the mass and vertex position based on Monte Carlo simulations. If the dimuon is "believed" to be coming from the target with the condition on both muons as:

$$
\chi_{\text {target }}^{2}<1.5 \chi_{\text {dump }}^{2}
$$


then the vertex optimization condition is applied to the dimuon. The optimization formula is given by:

$$
z \_v e r t \_o p t=-305.465+104.731 m-24.3589 m^{2}+2.5564 m^{3}-0.0978876 m^{4}
$$

After we use the optimization, the effect is that for low mass it puts the dimuon vertex downstream and for high mass it is puts a dimuon vertex value upstream. It is important to note that this optimization is applied only for target likely dimuons. A different optimization formula has to be applied for events which might be coming from the dump [140].

\subsection{Monte Carlo Simulations}

The Monte Carlo simulation used for SeaQuest is called GMC (GEANT4 based Monte Carlo). GMC is a convenient tool that can be used to study various parameters. It can be used to study tracker reconstruction parameters and efficiencies, detector hit populations for Drell-Yan signals and background events, in tweaking analysis cuts and many other tasks. Some details of the GMC will be discussed in this section.

\subsubsection{Generators}

GMC has four types of generators:

- Dimuon generator: The primary purpose of the dimuon generator is to generate dimuons with a particular specified distribution in chosen kinematic variables. Three options could generate dimuons. They could come from Drell-Yan, $J / \psi$ or $\psi^{\prime}$ decays. For Drell-Yan dimuons the procedure followed is as follows:

1. $2 \mathrm{GeV} / c^{2}<$ mass $<10 \mathrm{GeV} / c^{2}$ (flat distribution) is used.

2. $-1<x_{F}<1$ (flat distribution) is used. 
3. $p_{T}^{2}>0$ and $p_{T}$ distributions (which are parameterized for $800 \mathrm{GeV}$ ) as mentioned in [46] with the intention that they could re-weighted for $120 \mathrm{GeV}$.

4. $0<x_{B}<1$ is used.

5. $0<x_{T}<1$ is used.

6. A sigWeight (weighting of dimuons) calculated according to the Drell-Yan cross section multiplied by the K-Factor.

7. PDFs could be chosen with flexibility.

8. Acceptance is chosen to be either $4 \pi$ or spectrometer acceptance.

For $J / \psi$ or $\psi^{\prime}$ dimuons, the following options are chosen instead:

- mass $=3.097 \mathrm{GeV}(J / \psi)$ or $3.686 \mathrm{GeV}\left(\psi^{\prime}\right)$ is used

- $p_{T}^{2}>0$ and $p_{T}$ distributions as mentioned in [71] are used.

- Gun generator: This generator just creates a $120 \mathrm{GeV}$ proton in front of the target. All kinds of primary and secondary particles $(\pi, K$ etc.) are generated while a "gun generator simulated proton" interacts with the target material. Typically simulating and reconstruction of tracks coming from $10^{7}$ protons which interact with the target material takes several days. Hence, it is very difficult and time consuming to simulate a real time spill $\left(5 \times 10^{12}\right.$ protons/spill $)$. Hence, this kind of generator is practically used to study relative population of charged particles in detector regions, pion decays in flight, and other aspects.

- $\eta$ generator: The GMC has recently also been modified to include the $\eta$ generator. This throws the $\eta$ particles which take into consideration the PYTHIA generated distributions. The eta decays into a photon and a dark photon (A') which could decay into a dimuon. The $\eta-2 \mu$ mass is used as a phase space to allow for the dark photon to be generated which eventually decays into a dimuon. 


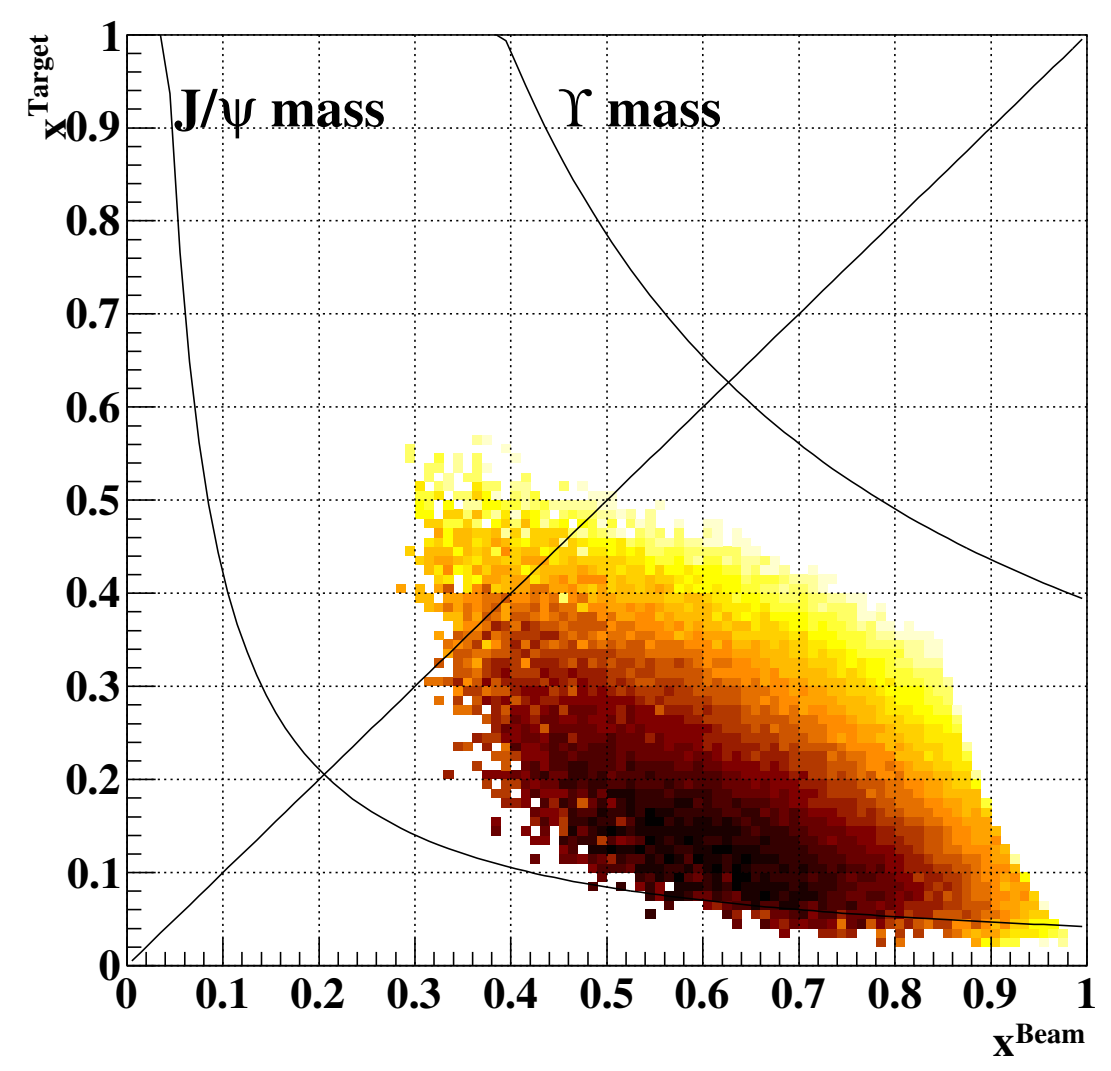

Figure 4.13: The acceptance reach of SeaQuest. As can be seen in the plot, the acceptance can be tuned to reach a Bjorken- $x$ region of $\sim 0.45$

- Single muon generator: Although this kind of generator is very useful, it is not often used in SeaQuest.

The output of all these results are stored as mTrack and mDimuon tables (which have the thrown values) and kTrack and kDimuon tables (which have reconstructed values). The acceptance plot for the SeaQuest spectrometer is shown in Figure 4.13. This shows that the spectrometer has access to the high- $x$ region.

\subsection{2 "Clean" and "Messy" Monte Carlo simulations}

As mentioned in the Trigger section, the Random RF trigger (NIM3) collects data when there is an overlap of the Main Injector RF clock and the $7.5 \mathrm{kHz}$ clock. This data is used 
as a "snippet" for studying the effects of background. The generated Drell-Yan or $J / \psi$ or $\psi^{\prime}$ Monte Carlo production is free from any noise or background coming from other muons. The NIM3 data is embedded in clean Monte Carlo production in order to simulate background and its effects such as reconstruction efficiencies and resolution of quantities are studied. In order to simulate "realistic" conditions, hits are smeared in a Gaussian way in the Monte Carlo and 6\% are dropped to mimic chamber efficiencies. This process in called "Realization".

- For Clean MC productions, Realization is turned on but no NIM3 embedding is done.

- For Messy MC productions, Realization is turned on before embedding the events and then the newly created ROOT file is re-tracked.

Various quantities were compared for these kinematic variables and effects of noise in the detectors is studied. Some basic detector quantities are shown in Figures 4.14, 4.15 and 4.16. Many such plots are compared and a comprehensive list of analysis cuts were developed for identifying high mass Drell-Yan events coming from the target region.

When a clean track is embedded with noise hits, it is possible that a 17-hit track could have its "gap" filled with a noise hit and become an 18-hit perfect track. But, it is also possible that the 17-hit track could lose one of the hits due to there being very high occupancy events which confuse the tracker. Figure 4.17 shows the number of messy hit tracks (on a log scale) given the number of clean hits $=15,16$ etc in different color schemes. For example, the 17 hit clean track (orange) has more 16 hit tracks compared to 18 hit messy tracks. That means, it is more likely to drop a hit while embedding with noise than gain a hit. The case is similar with 16 hit track. 

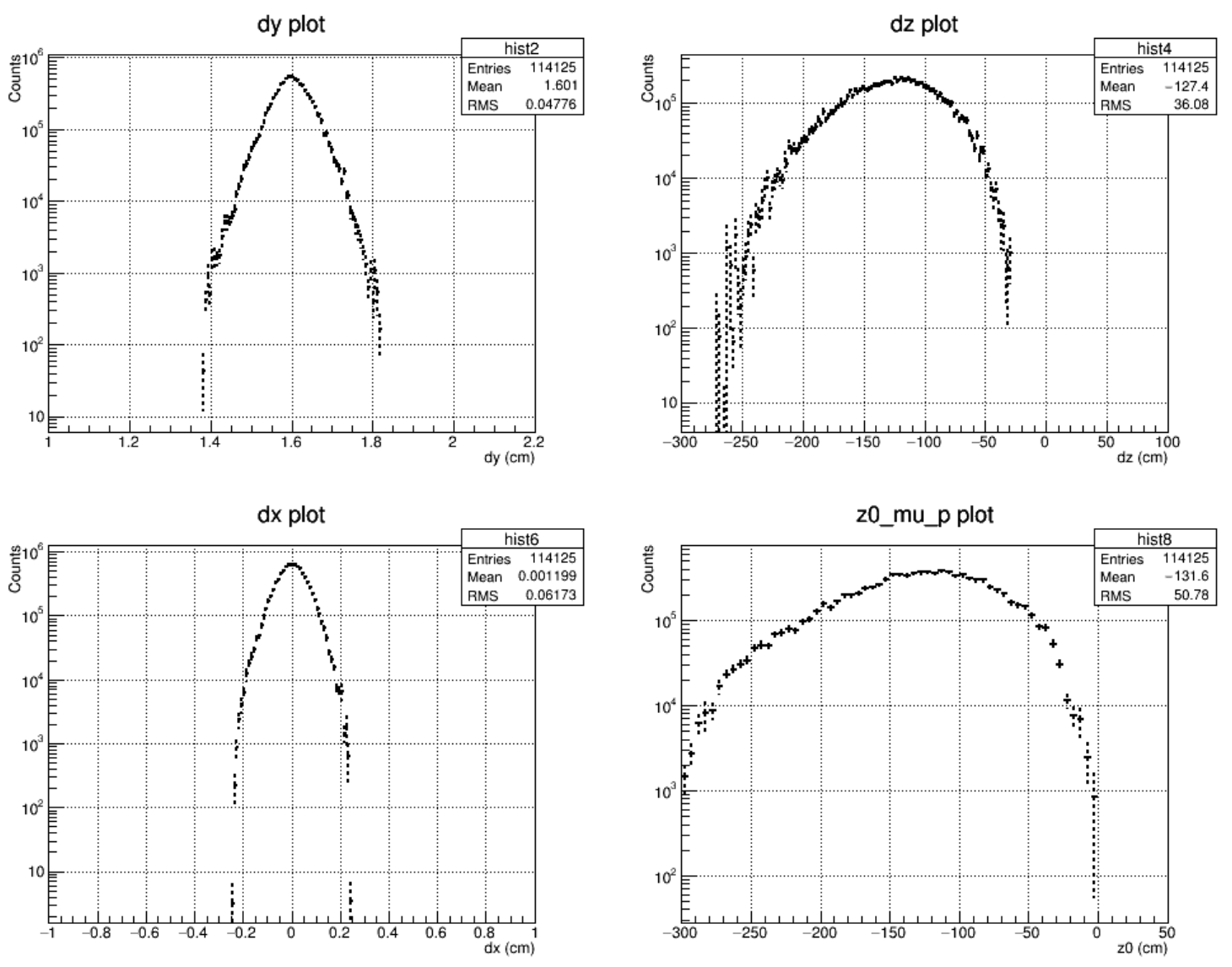

Figure 4.14: Some reconstructed detector quantities from $m c \_d r e l l y a n \_L D 2 \_M 026 \_S 002$ Monte Carlo production generated from LD2 target. Top left is the dimuon vertex $y$ position, top right is the dimuon vertex $z$ position, bottom left is the dimuon vertex $x$ position and bottom right is the single muon $z$ vertex position. 

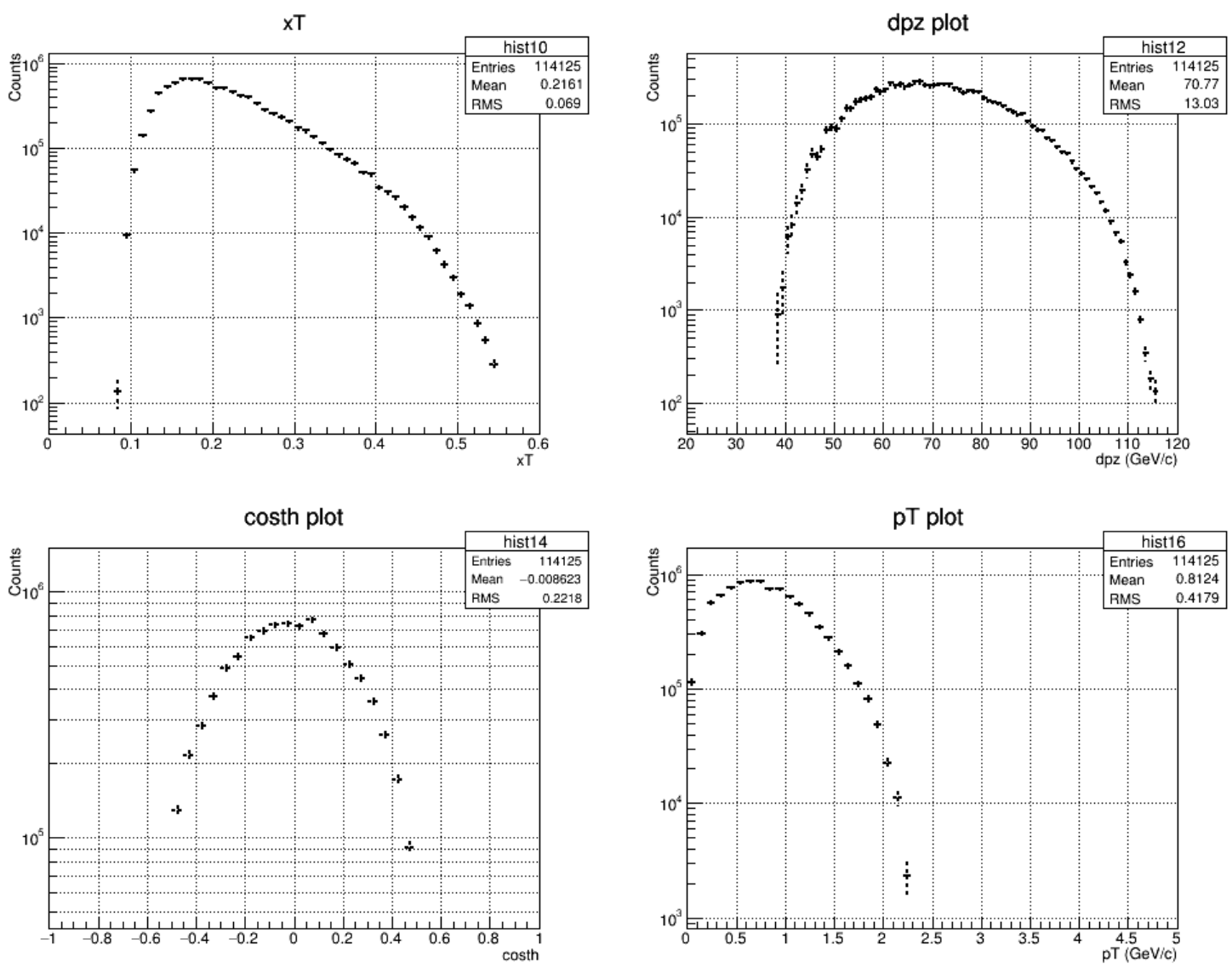

Figure 4.15: Some reconstructed detector quantities from $m c \_d r e l l y a n \_L D 2 \_M 026 \_S 002$ Monte Carlo production generated from LD2 target. Top left is the Bjorken $x$ of the target, top right is the dimuon $z$ component of the momentum, bottom left if the $\cos (\theta)$ of the dimuon and bottom right is the transverse momentum $p_{T}$ of the dimuon. 

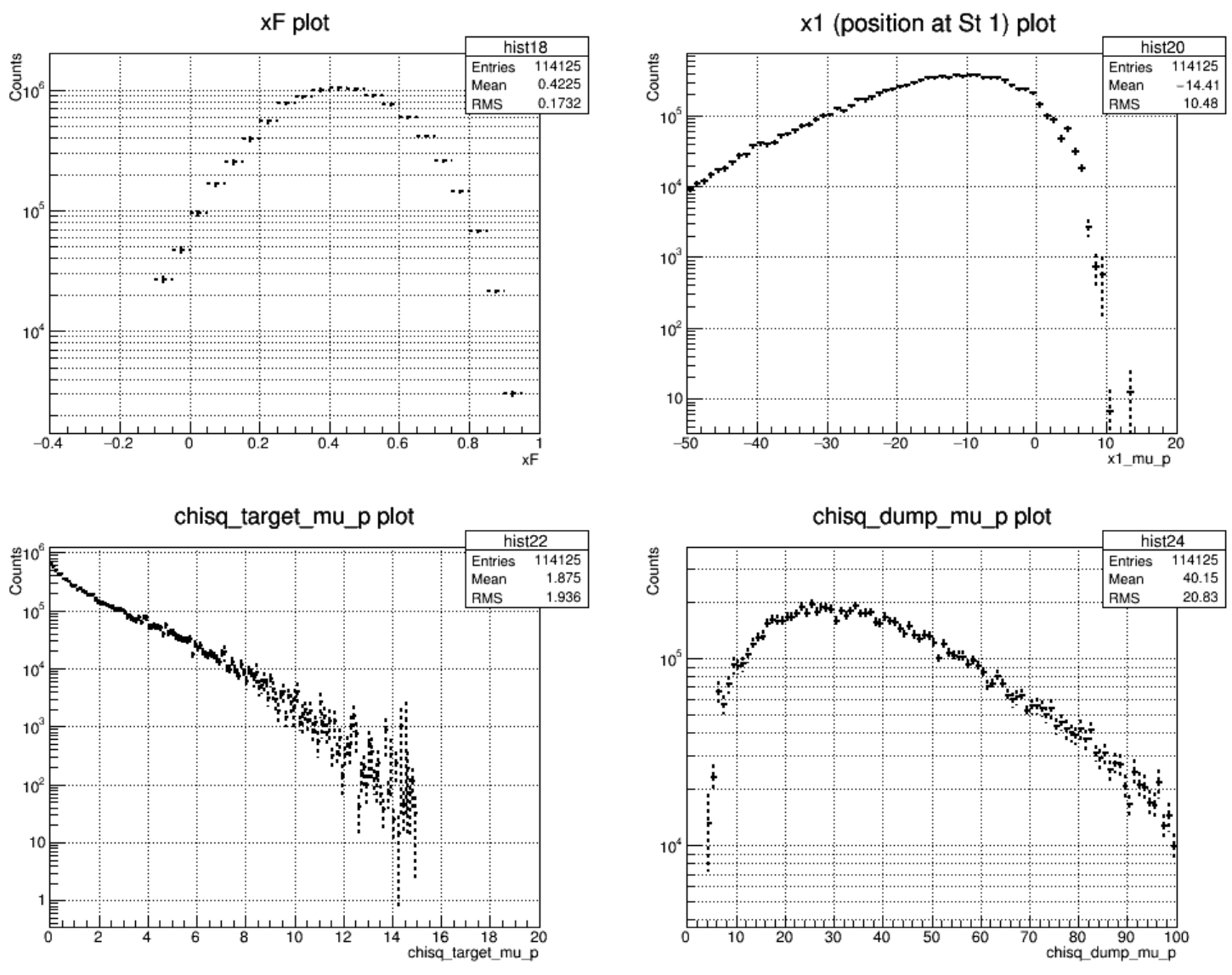

Figure 4.16: Some reconstructed detector quantities from $m c \_d r e l l y a n_{-} L D 2 \_M 026 \_S 002$ Monte Carlo production generated from LD2 target. Top left is the Feynman- $x$ of the dimuon, top right is the position of the $\mu^{+}$at DC1, bottom left and bottom right are the $\chi^{2}$ of the $\mu^{+}$at the target and the beam dump location respectively. 


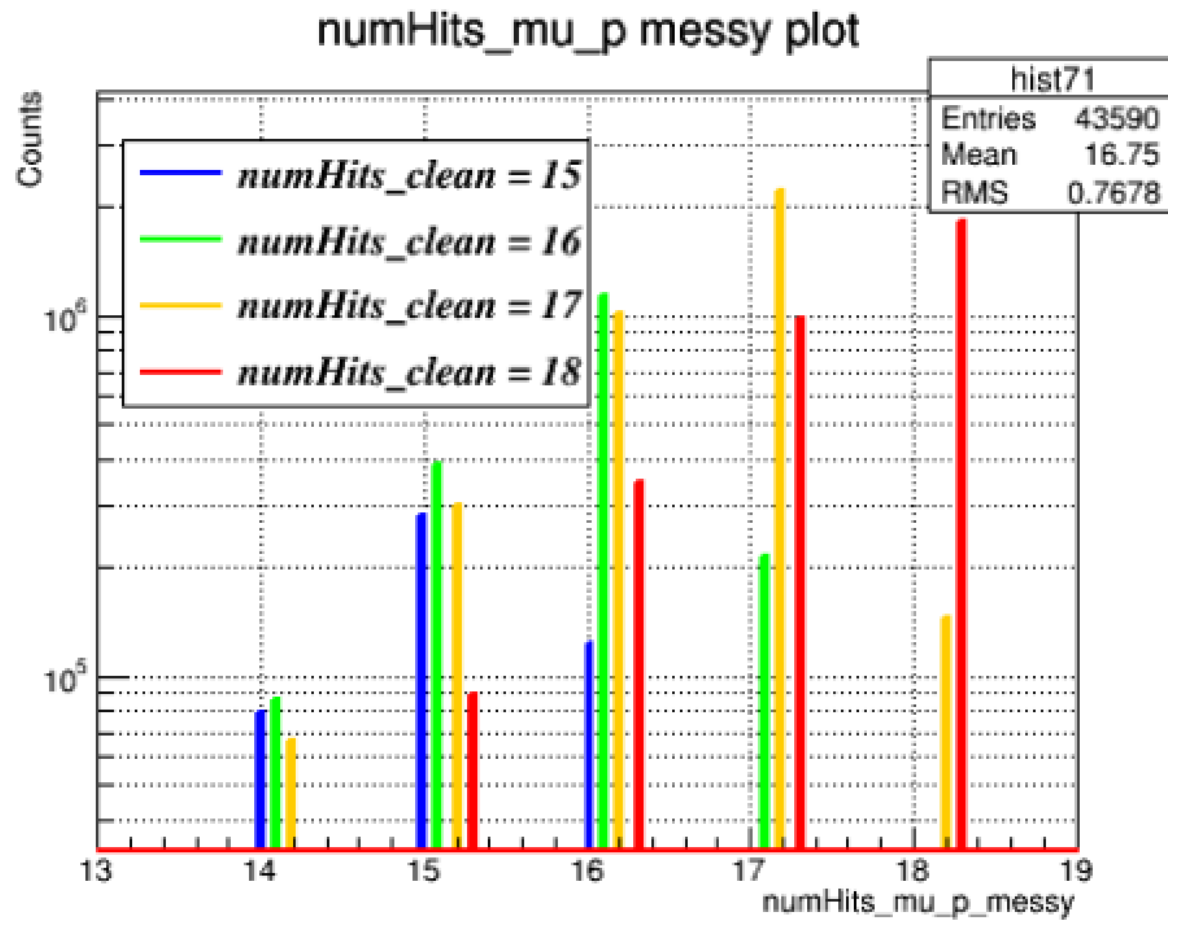

Figure 4.17: Figure shows the number of messy hit tracks (on a log scale) given the number of clean hits $=15,16$ etc in different color schemes. For example, the 17 hit clean track (orange) has more 16 hit tracks compared to 18 hit messy tracks. That means, it is more likely to drop a hit while embedding with noise than gain a hit. The case is similar with 16 hit track. 


\subsection{Selections and Data Quality cuts}

\subsubsection{Spill Level cuts}

Quality cuts are applied on several levels in order to gather analyzable data. The cuts can be categorized into spill level and event level (track level and dimuon level) cuts. A convenient data quality bit is used for spills which satisfy certain minimum requirements. These spills need not necessarily have a dimuon in them but are eligible for data analysis. Table 4.5 shows a list of all the cuts which are applied for the Spill.dataQuality $=0$ bit. Plots are categorized into different roadsets with the scheme as: a) = Roadset 57, b= Roadset 59, c) $=$ Roadset $62, \mathrm{~d})=$ Roadset 67 and $\mathrm{e})=$ Roadset 70 . For the sake of avoiding repetition, the definition of each quantity is given in the caption of plots. Any observation in particular is also noted. Even though the plots are made using R006 data production [145], and the analysis is done on R008 production, the differences in the overall global trend of various quantities between these two data productions as a function of time is small. Spill and data taking flowcharts are shown in Figure 4.18 and Figure 4.19. 


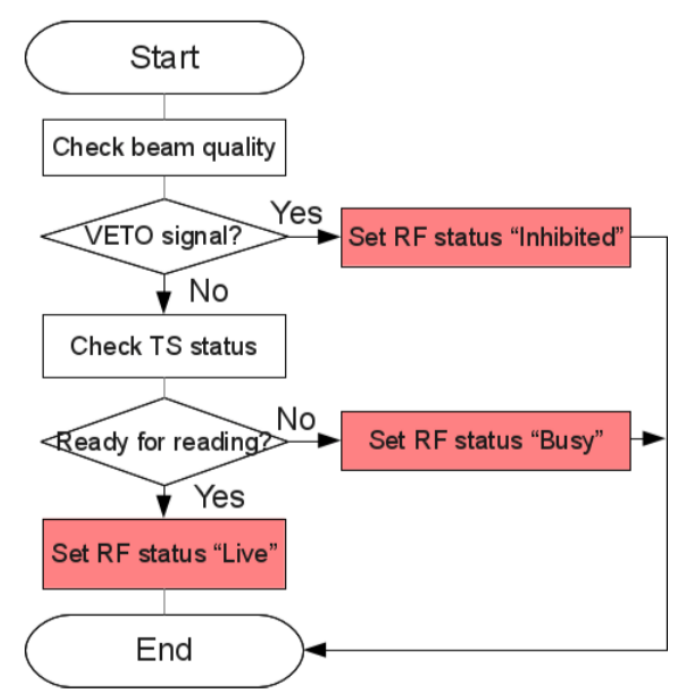

Figure 4.18: Flowchart of defining the status of an RF bucket. Typically, the status is "Inhibited", "Busy" or "Live" depending on the whether the RF bucket was inhibited (by QIE VETO) or if the TS was busy and not able to read or is live and able to be recorded [132].

\begin{tabular}{|c|c|c|c|c|}
\hline Quantity & Roadset & $57 \& 59$ & 61 & $62,67 \& 70$ \\
\hline \hline TargetPos & & {$[1,7]$} & {$[1,7]$} & {$[1,7]$} \\
\hline TargetPos.2 & & $=$ TargetPos & $=$ TargetPos & $=$ TargetPos \\
\hline AcceptedFPGA1 & & N/A & N/A & N/A \\
\hline AfterInhFPGA1 & & N/A & N/A & N/A \\
\hline TSGo & & {$[1 \mathrm{e} 3,8 \mathrm{e} 3]$} & {$[1 \mathrm{e} 3,12 \mathrm{e} 3]$} & {$[100,6000]$} \\
\hline AcceptedFPGA1 & & {$[1 \mathrm{e} 3,8 \mathrm{e} 3]$} & {$[1 \mathrm{e} 3,12 \mathrm{e} 3]$} & {$[100,6000]$} \\
\hline AfterInhFPGA1 & & {$[1 \mathrm{e} 3,30 \mathrm{e} 3]$} & {$[1 \mathrm{e} 3,1000 \mathrm{e} 3]$} & {$[100,10000]$} \\
\hline AcceptedFPA1/AfterInhFPGA1 & & {$[0.2,0.9]$} & {$[0.0,0.9]$} & {$[0.2,1.05]$} \\
\hline FMAG & & {$[1950,2050]$} & {$[200,500]$} & {$[1950,2050]$} \\
\hline KMAG & & {$[1550,1650]$} & {$[1550,1650]$} & {$[1550,1650]$} \\
\hline G2SEM & & {$[2 \mathrm{e} 12,1 \mathrm{e} 13]$} & {$[2 \mathrm{e} 12,1 \mathrm{e} 13]$} & {$[2 \mathrm{e} 12,1 \mathrm{e} 13]$} \\
\hline QIEsum & & {$[4 \mathrm{e} 10,1 \mathrm{e} 12]$} & {$[4 \mathrm{e} 10,1 \mathrm{e} 12]$} & {$[4 \mathrm{e} 10,1 \mathrm{e} 12]$} \\
\hline Inhibit & & {$[4 \mathrm{e} 9,1 \mathrm{e} 11]$} & {$[4 \mathrm{e} 9,1 \mathrm{e} 11]$} & {$[4 \mathrm{e} 9,2 \mathrm{e} 11]$} \\
\hline Busy & & {$[15,1 \mathrm{e} 11]$} & {$[4 \mathrm{e} 9,1 \mathrm{e} 11]$} & {$[4 \mathrm{e} 9,1 \mathrm{e} 11]$} \\
\hline Duty Factor & & $>0$ & {$[15,60]$} & {$[10,60]$} \\
\hline N of tracks/spill & & $>0$ & $>0$ \\
\hline
\end{tabular}

Table 4.5: Requirements for "good spill" eligibility. The definition of each quantity is given in the caption of the plots in the following pages. 


\subsubsection{Some $\mathrm{R005} \rightarrow \mathrm{R} 008$ upgrades}

In R005 data production (used for release of the preliminary result), there were many unknown and unsolved issues with data quality, fundamental experimental parameters, kTracker optimization conditions, and rate dependence corrections that were later realized and implemented in subsequent data productions. These issues caused the re-tracking of data from R005 to R008 software version. Therefore, a re-analysis is necessary to extract the improved yields and physics results. Some of the important implementations are discussed below:

- Beam offset for different roadsets: It was found that the proton beam has a Y-offset of $\approx 0.4 \mathrm{~cm}$ for $\operatorname{RS} 57,59$ and $\approx 1.6 \mathrm{~cm}$ for $\mathrm{RS} 62,67,70$ and 78 . More information about the discovery and implementation of the offset in data and Monte Carlo productions can be found in $[146,147,148]$. This offset wasn't accounted for in R005 productions. It has been incorporated in R007 and subsequent data productions.

- Updated KMAG position and magnetic field calibration constants: Information can be found in [149].

- Recovery of 1/3 of RS 67: Reprocessing the dataset recovered some data which failed reconstruction during the R005 production. Information can be found in [150].

- Purity of Deuterium: Updated values since R005 data production [151]

- Analysis cuts: To be discussed in detail in Section 4.4.4.

- Tracker upgrades: Changing the vertex optimization condition, including cuts on drift chamber residuals, inclusion of time window for the removal of after-pulses, improved alignment, modification of $\phi$ range and many more. More detailed information can be found on the Fermilab redmine seaquest-ktracker/activity page. 


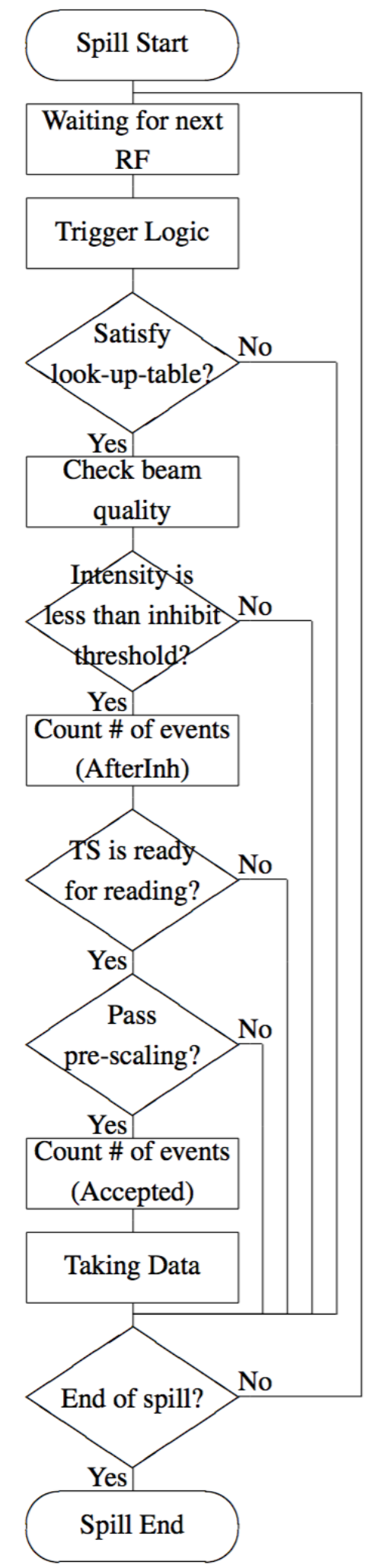

Figure 4.19: Flowchart of data taking in one spill [141]. 


\subsubsection{Event Level cuts}

The analysis strategy is to include as many target Drell-Yan dimuons as possible. Significant work has been done in optimizing the cuts to achieve proper target beam dump separation and removing randoms by comparison with low and high DC1 occupancy distributions. Six different sets of plots $\left(\mathrm{LD}_{2}\right.$ data, $\mathrm{LH}_{2}$ data, Empty flask data, $\mathrm{LD}_{2}$ and $\mathrm{LH}_{2}$ (target and beam dump) messy Monte Carlo productions) were compared in order to optimize the cuts to get target Drell-Yan dimuons. In this section, a brief description of the variables used in the analysis and the analysis cuts imposed on those variables will be discussed. It should be noted that the beam offset changed between Roadsets 57, 59 and 62, 67, 70 and 78 and the cuts have to be modified accordingly. Also, the magnetic field was flipped at the beginning of RS 67. This can be noted in the various spectrometer variable and momentum distributions.

- targetPos: The position of the target should be either $1\left(\mathrm{LH}_{2}\right), 2$ (Empty flask) or 3 $\left(\mathrm{LD}_{2}\right)$

- Occupancy: The occupancy of the event at DC1, DC2, DC3 and the combination should be less than a certain value to study reconstructible meaningful events. The associated cuts are

$$
\begin{aligned}
& -\mathrm{D} 1<400 \\
& -\mathrm{D} 2<400 \\
& -\mathrm{D} 3<400 \\
& -\mathrm{D} 1+\mathrm{D} 2+\mathrm{D} 3<1000
\end{aligned}
$$

- Trigger requirement: This cut is applied to ensure that the $\mu^{+}$and $\mu^{-}$come completely either from the top or bottom halves of the spectrometer. Eventually, the product of the vertical positions of $\mu^{+}$and $\mu^{-}$at DC3 should be less than zero so that 
they satisfy the $\mathrm{B} / \mathrm{T}$ or $\mathrm{T} / \mathrm{B}$ requirement of the FPGA1 dimuon trigger. The associated cuts are:

$$
\begin{aligned}
& -y 1_{-} \mu^{+} * y 3_{-} \mu^{+}>0 \\
& -y 1_{-} \mu^{-} * y 33_{-} \mu^{-}>0 \\
& -y 3{ }_{-} \mu^{+} * y 33_{-} \mu^{-}<0
\end{aligned}
$$

\subsubsection{Track Level cuts}

- $z 0$ : These are the variables that define the distance of closest approach to the beam line. The selection criteria is used to minimize the contributions coming from the upstream instrumentation package and the downstream tail of events coming from the iron beam dump. The associated cuts are:

$$
\begin{aligned}
& --320 \mathrm{~cm}<z 0_{-} \mu^{+}<-5 \mathrm{~cm} \\
& --320 \mathrm{~cm}<z 0_{-} \mu^{-}<-5 \mathrm{~cm}
\end{aligned}
$$

- $x T, y T, x D, y D$ : The variables are the projected $x$ and $y$ positions at $z=-129 \mathrm{~cm}$ $(x T$ and $y T)$ and $z=42 \mathrm{~cm}(x D$ and $y D) . z=+42 \mathrm{~cm}$ is chosen as it is one interaction length $(17 \mathrm{~cm})$ from the hole in the front face of the beam dump $(25 \mathrm{~cm})$. The associated cuts for both $\mu^{+}$and $\mu^{-}$are:

$$
\begin{aligned}
& -x T * x T+(y T-\text { beamoffset }) *(y T-\text { beamoffset })<320 \mathrm{~cm}^{2} \\
& -x D * x D+(y D \text { - beamoffset }) *(y D-\text { beamoffset })<1100 \mathrm{~cm}^{2} \\
& \text { - } x D * x D+(y D-\text { beamoffset }) *(y D-\text { beamoffset })>16 \mathrm{~cm}^{2}
\end{aligned}
$$

- KMAG $p_{T}$ kick: $p x 1$ and $p x 3$ are the $x$ component of the three momenta at DC1 and DC3 respectively. This quantity is influenced by the magnetic field between DC1 and DC3. To ensure that the correct $p_{T}$ is applied at KMAG cuts are applied for both, $\mu^{+}$and $\mu^{-}$. The associated cuts are: 
- $\operatorname{abs}(\operatorname{abs}(\mathrm{px} 1-\mathrm{px} 3)-0.416)<0.008 \mathrm{GeV} / \mathrm{c}$

- abs(py1 - py3) $<0.008 \mathrm{GeV} / \mathrm{c}$

- $\operatorname{abs}(\mathrm{pz} 1-\mathrm{pz} 3)<0.08 \mathrm{GeV} / \mathrm{c}$

- chisq_upstream, chisq_target,chisq_dump: This is a key parameter used to separate target tracks from dump tracks. Three $\chi^{2}$ 's are given out when the track is forced to go through $x=y=0$ and $z=-490 \mathrm{~cm},-129 \mathrm{~cm}$ and $42 \mathrm{~cm}$ [152]. For Drell-Yan dimuons, coming from the target region $\chi^{2}$ for the target should be less than the $\chi^{2}$ for the dump and upstream. Also, an upper limit on the track $\chi^{2}$ is placed to restrict the possibility of "poorly" reconstructed tracks which still made it through the loose upper limits of the internal tracker cuts. The associated cuts for both, $\mu^{+}$and $\mu^{-}$are:

- chisq_target $<1.5 *$ chisq_upstream

- chisq_target $<1.5 *$ chisq_dump

- chisq_target $<15$

- abs(chisq_target_ $\mu^{+}+$chisq_target_ $\mu^{-}-$chisq_dimuon $)<2$

- numHits: A track typically has 16,17 or 18 hits as some hits can be dropped due to hardware efficiency, track reconstruction inefficiency and other rate dependence effects. It is unlikely that a "good" track has 13 hits. Also, based on Monte Carlo studies, an upper limit is placed on the overall number of hits on each track for the dimuon (sum of hits on both the tracks) and the number of hits of both the tracks at DC1.1. The associated cuts are:

$$
\begin{aligned}
& \text { - } \text { numHits_ }_{-} \mu^{+}>13 \\
& \text { - } \text { numHits_}_{-} \mu^{-}>13 \\
& \text { - numHits } \mu^{+}+\text {numHits_ } \mu^{-}>29 \\
& \text { - numHits_ } \mu^{+}{ }_{2} a t_{-} D C 1+\text { numHits_ } \mu^{-}{ }_{-} a t_{-} D C 1>8
\end{aligned}
$$


- chisq/(numHits -5$)$ : The track is specified by 5 variables $\left(x_{B}, x_{T}\right), p_{T}, \phi, \theta$ which are also the degrees of freedom of the track. Therefore, an upper limit is placed on the $\chi^{2} / \mathrm{NDF}$ in order to ensure good quality reconstructed tracks. The associated cuts for both, $\mu^{+}$and $\mu^{-}$are:

$$
\text { - chisq/(numHits }-5)<12
$$

- $p z 1$ : This variable is the $z$ component of the three-momentum of a muon at DC1. The associated cuts are:

$$
\begin{aligned}
& -9<p z 1 \_\mu^{+}<75 \mathrm{GeV} / \mathrm{c} \\
& -9<p z 1 \_\mu^{-}<75 \mathrm{GeV} / \mathrm{c}
\end{aligned}
$$

- Rejecting cross-over muons: The cuts ensure that the vertical position of the muons at DC3 is higher than at St1 thereby ensuring that they do not cross over from the top half to the bottom half of the spectrometer.

$$
\begin{aligned}
& \text { - } y 1_{\mu^{+}} / y 3_{\mu^{+}}<1 \\
& \text { - } y 1_{\mu^{-}} / y 3_{\mu^{-}}<1
\end{aligned}
$$

\subsubsection{Dimuon Level cuts}

- $d x, d y, d z$ : These are the variables that define the dimuon vertex position from vertex finding. The selection criteria try to minimize the contributions coming from the upstream instrumentation package and the downstream tail of events coming from the iron beam dump. The associated cuts are:

$--280 \mathrm{~cm}<d z<-5 \mathrm{~cm}$

- $a b s(d y$ - beamoffset $)<0.22 \mathrm{~cm}$ (beam offset $=0.4 \mathrm{~cm}$ or $1.6 \mathrm{~cm}$ depending on the roadset)

- $a b s(d x)<0.25 \mathrm{~cm}$ 
- $d x * d x+(d y$ - beamoffset $) *(d y$ - beamoffset $)<0.06 \mathrm{~cm}^{2}$

- $d p x, d p y, d p z:$ These are the variables that define the dimuon three momenta at vertex position after re-tracking. The associated cuts are:

$$
\begin{aligned}
& \text { - } a b s(d p x)<1.8 \mathrm{GeV} / \mathrm{c} \\
& \text { - } a b s(d p y)<2 \\
& \text { - } 38 \mathrm{GeV} / \mathrm{c}<a b s(d p x)<116 \mathrm{GeV} / \mathrm{c} \\
& \text { - } d p x * d p x+d p y * d p y<5(\mathrm{GeV} / \mathrm{c})^{2}\left(\text { loose cut on } p_{T}^{2}\right)
\end{aligned}
$$

- mass: This variable is the calculated dimuon mass. For Drell-Yan dimuons, the associated cut is:

$$
-4.2 \mathrm{GeV} / \mathrm{c}^{2} \text {; mass ; } 8.8 \mathrm{GeV} / \mathrm{c}^{2}
$$

- $x_{F}$ : This variable is the calculated Feynman- $x$. For Drell-Yan dimuons, the associated cut is:

$$
-0.1<x_{F}<0.95
$$

- $x_{T}$ : This variable is the calculated Bjorken- $x$. For Drell-Yan dimuons coming from the target, the associated cut is:

$$
-0.1<x_{T}<0.58
$$

- $\cos (\theta)$ : This variable is the polar angle in the Collins-Soper frame. For Drell-Yan dimuons, the associated cut is:

$$
\text { - } \operatorname{abs}(\cos (\theta))<0.5
$$

- trackSeparation: This variable is the distance between the points of closest approach between $\mu^{+}$and $\mu^{-}\left(z_{-} \mu^{+}-z_{-} \mu^{-}\right)$. For Drell-Yan dimuons coming from the target region, the associated cut is: 
- abs(trackSeparation) $<270 \mathrm{~cm}$

- chisq_dimuon: This variable is the $\chi^{2}$ obtained when forcing both muons to go through the dimuon vertex. An upper limit is placed to ensure that "good" reconstructed dimuons are filtered for analysis. For Drell-Yan dimuons coming from the target region, the associated cut is:

- chisq_dimuon $<18$

- Trigger Intensity: Trigger Intensity is the simply the number of protons in the triggered RF bucket. The formula used for calculating the trigger intensity (\# of protons in the triggered RF bucket - RF00) range is the following:

TriggerIntensity $=($ RF00 - pedestal $) * \frac{\text { G2SEM }}{(\text { QIEsum }- \text { pedestal } * \text { buckets } * \text { turns })}$

$$
\text { PotPerQie }=\frac{\text { G2SEM }}{(\text { QIEsum }- \text { pedestal } * \text { buckets } * \text { turns })}
$$

The QIE module reads out the pedestal value for all the buckets (including empty buckets). In a spill, the number of buckets are 588 and the number of turns (Main Injector turn number) are 369,000. A QIE pedestal value of 36.2 was taken for spill range $<450,000$ and 32.6 there after. The associated cuts are:

- $($ RF00 - pedestal $) *$ PotPerQie $>0$

- $($ RF00 - pedestal $) *$ PotPerQie $<64000$

\subsubsection{Cuts designed to reduce random background}

Most of the "fire of muons" coming from the beam dump are concentrated near the beam line. Low and high DC1 occupancy distributions and Monte Carlo and data 
distributions are compared and a limit is placed on the vertical component of the momentum and the $x$ position of muons at $\mathrm{DC} 1$.

- $\operatorname{abs}\left(x 1_{-} \mu^{+}+x 1_{-} \mu^{-}\right)<42 \mathrm{~cm}$

- $\operatorname{abs}\left(p y 1_{-} \mu^{+}\right)>0.02 \mathrm{GeV} / \mathrm{c}$

- $\operatorname{abs}\left(p y 1_{-} \mu^{-}\right)>0.02 \mathrm{GeV} / \mathrm{c}$

\section{Target Beam Dump Separation}

Figure 4.20 shows a plot of events coming from the target and the beam dump for Roadset 67 data separated by target and beam dump analysis cuts.

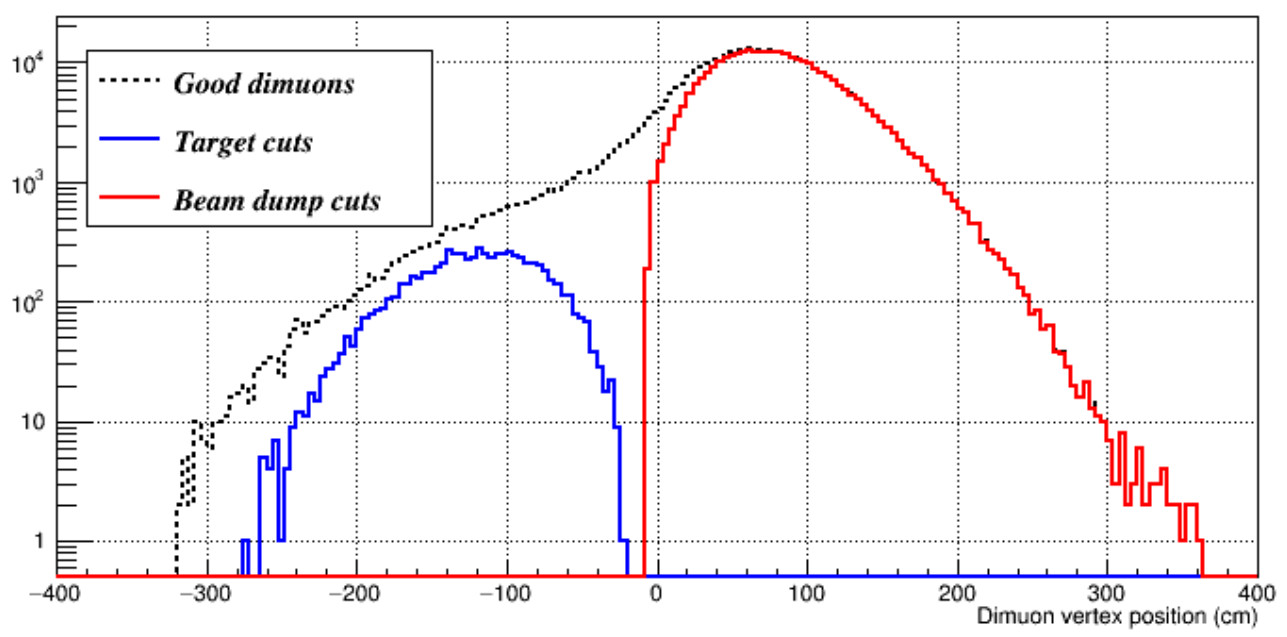

Figure 4.20: Target Beam dump separation plot for R008 data production with docid\#2111-v42 cuts

\subsubsection{Invariant Mass spectrum}

Figure 4.21 shows the invariant mass spectrum of LD2. The fit incorporates $J / p s i, \psi^{\prime}$ and Drell-Yan Monte Carlo along with a background spectrum which is simulated only using the FPGA4 events. As can be seen in the figure, the region above $4.2 \mathrm{GeV}$ has mostly DrellYan events. The fit for other targets also looks similar but with a different background. 


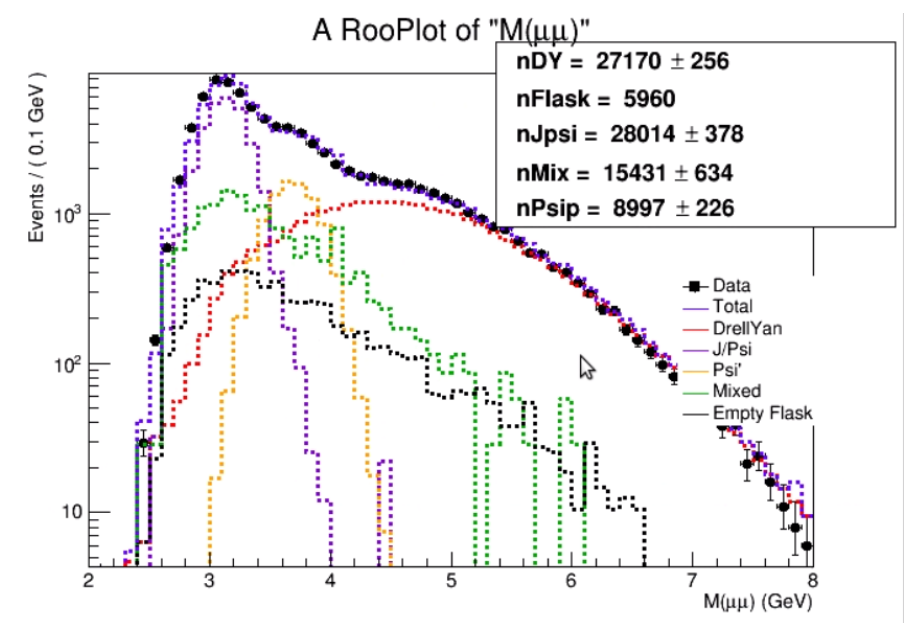

Figure 4.21: An invariant mass spectrum plot for LD2. The fit incorporates $J / \psi, \psi^{\prime}$ and Drell-Yan messy Monte Carlo along with a background spectrum.

\subsubsection{Combining data sets}

Plots of various kinematic variables were made and some minor differences between different roadsets in the high mass region (mass $>4.2 \mathrm{GeV} / \mathrm{c}$ ) for the same target were noted. This could be due to low statistics in some of the data sets. However, these differences are within resolution of that respective quantity. Therefore, all of Run II and Run III data (57, 59, 62, 67 and 70) for the high mass region (mass $>4.2 \mathrm{GeV} / \mathrm{c}$ ) can safely be combined for further studies. Other differences between liquid targets and empty flask data for $p_{T}$ distributions, trackSeparation, $d z$ and $z 0$ distributions have been pointed out. These differences could possibly be due to randoms, single muons from the beam dump that satisfy the target cuts, and upstream beam instrumentation package which become more prominent for empty flask data compared to $\mathrm{LH}_{2}$ or $\mathrm{LD}_{2}$ data. Only a few are shown in Figures 4.22, $4.23,4.24$ and 4.25 . The prominent features of the plots are noted in the captions to avoid repetition. 


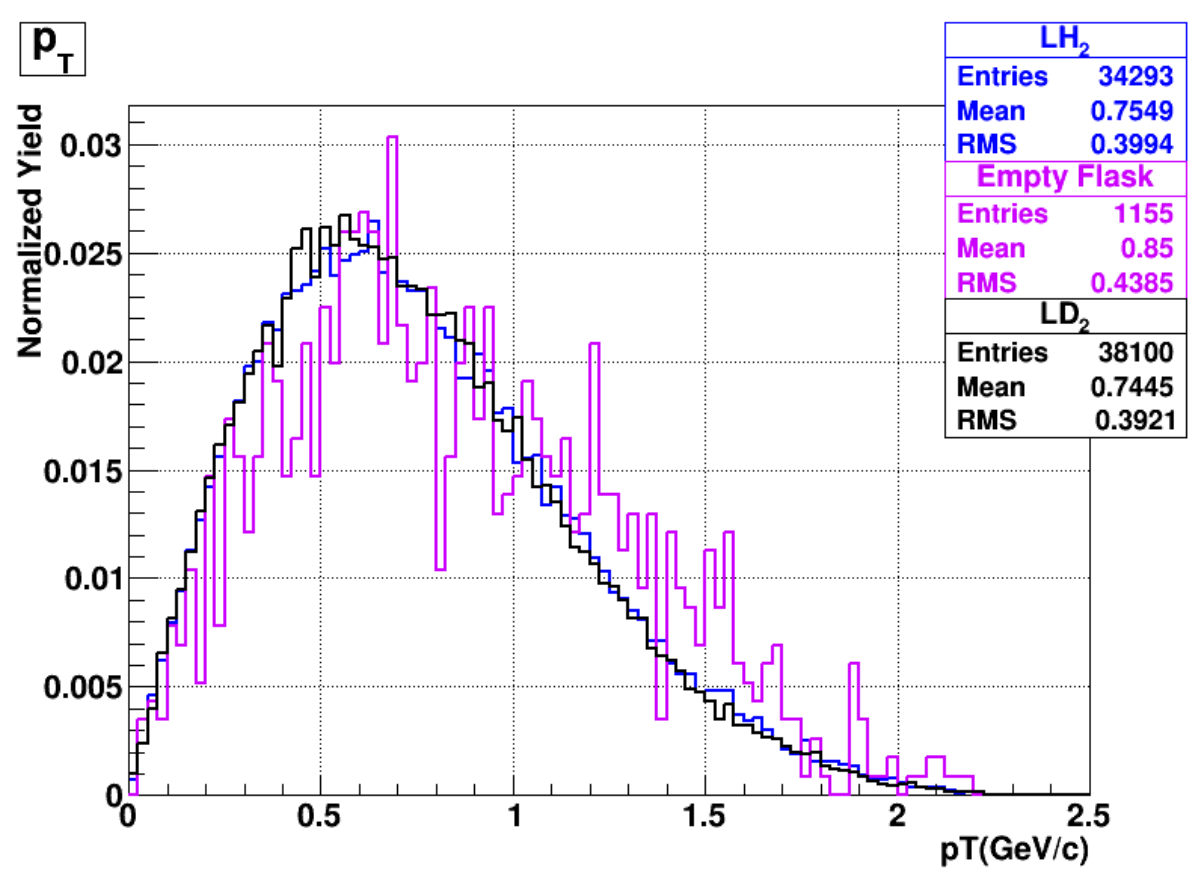

(a) Liquid targets

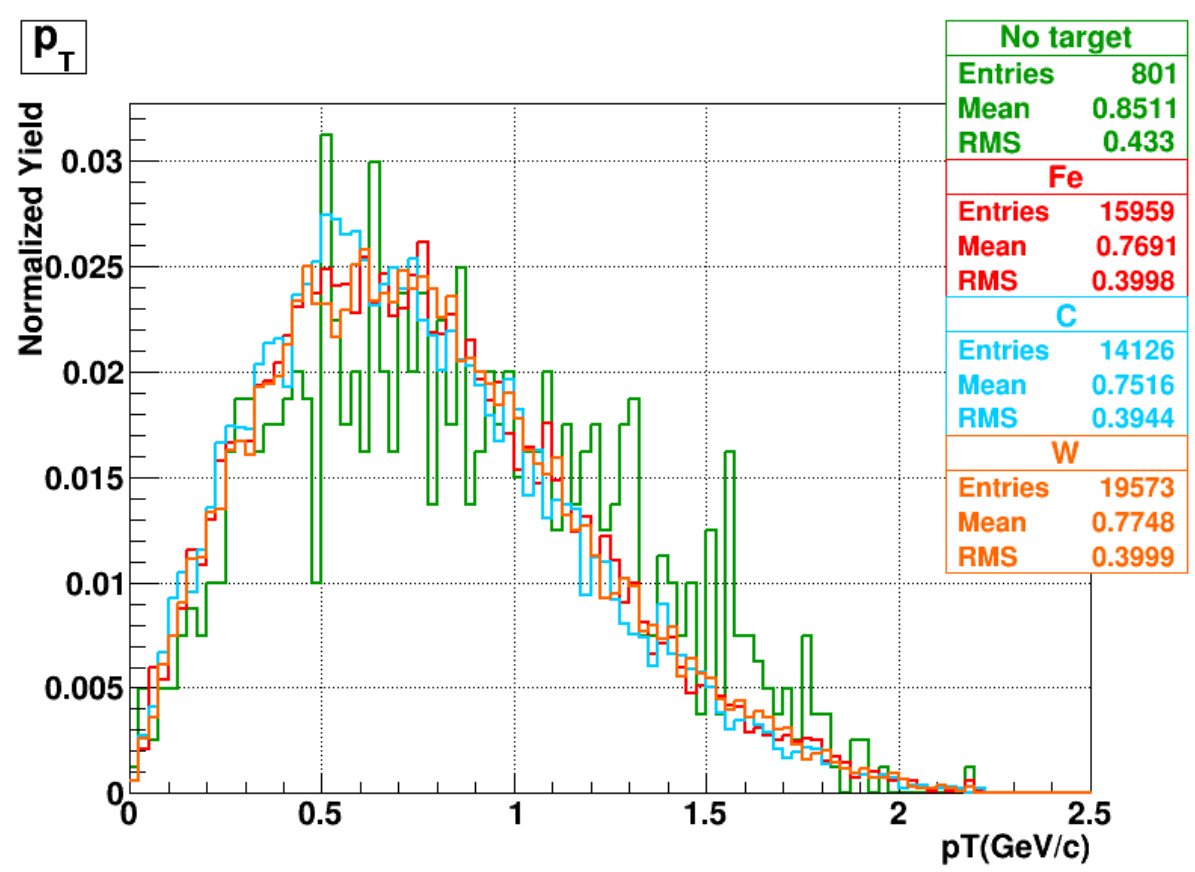

(b) Solid targets

Figure 4.22: Roadset comparison of $p_{T}$ for (a) $\mathrm{LH}_{2}$, Empty flask and $\mathrm{LD}_{2}$ and b) No target, $\mathrm{Fe}, \mathrm{C}$ and $\mathrm{W}$ targets. A slightly larger mean and RMS for the empty flask and no target (possibly due to random backgrounds) compared to $\mathrm{LH}_{2}$ and $\mathrm{LD}_{2}, \mathrm{C}$, Fe, and $\mathrm{W} . \mathrm{LH}_{2}$ and $\mathrm{LD}_{2} p_{T}$ distributions also have a slight difference $(\approx 1.5 \%)$ in their distributions which is within the resolution. 


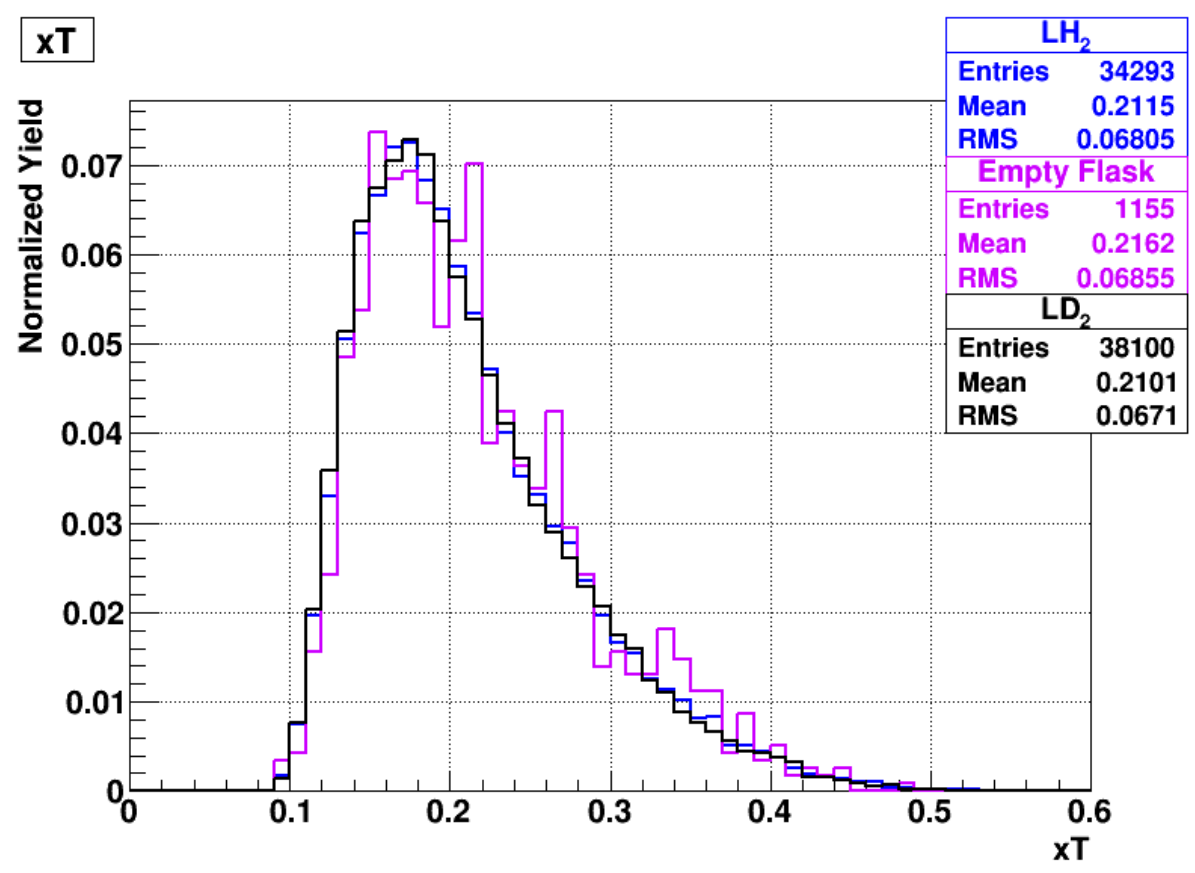

(a) Liquid targets

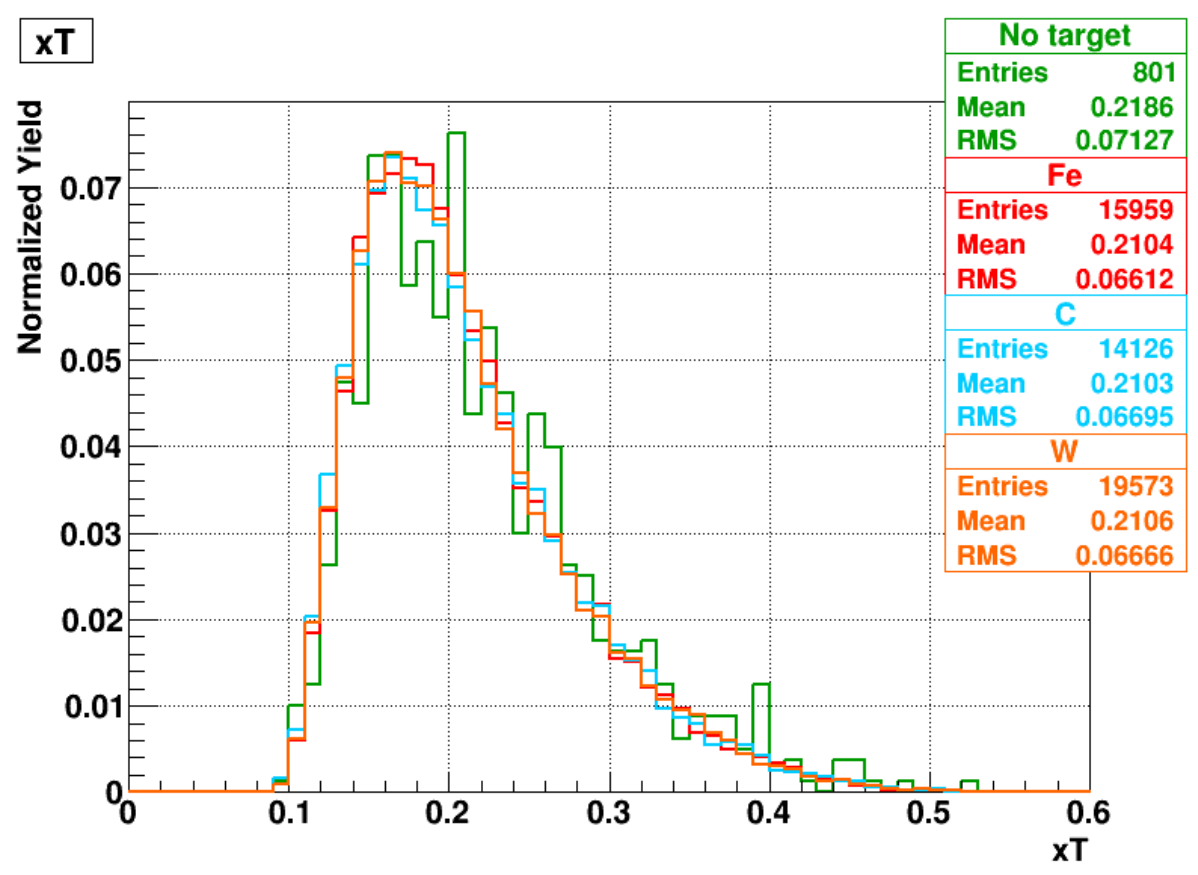

(b) Solid targets

Figure 4.23: Roadset comparison of $x_{T}$ for (a) $\mathrm{LH}_{2}$, Empty flask and $\mathrm{LD}_{2}$ and b) No target, $\mathrm{Fe}, \mathrm{C}$ and $\mathrm{W}$ targets. 


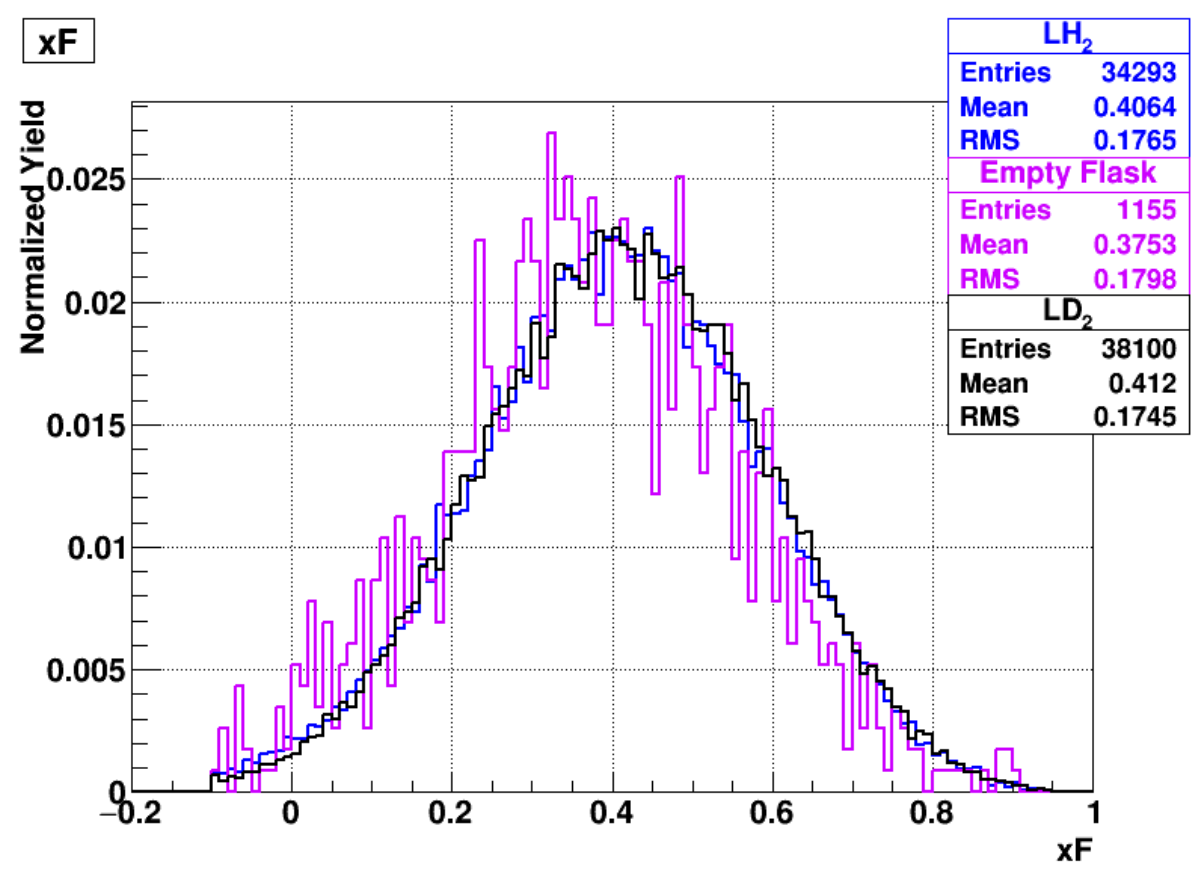

(a) Liquid targets

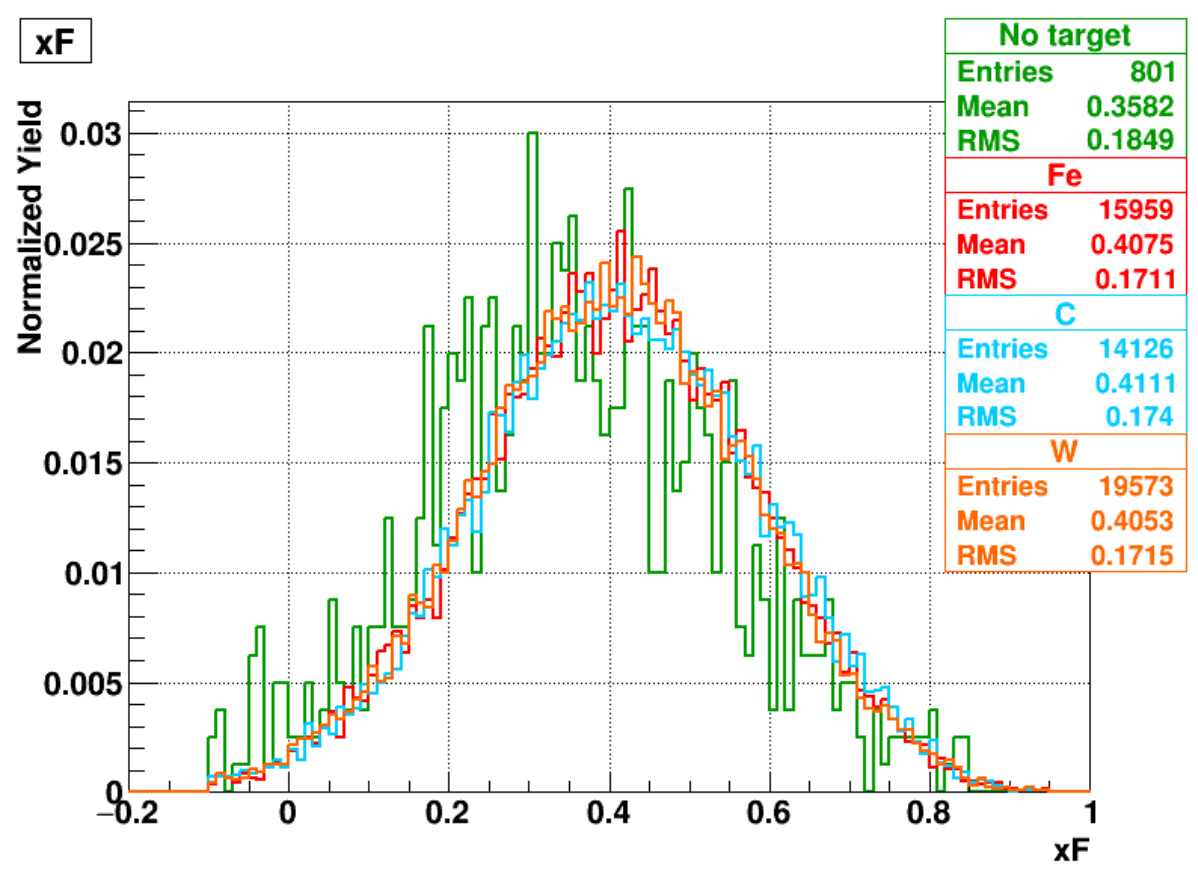

(b) Solid targets

Figure 4.24: Roadset comparison of $x_{F}$ for (a) $\mathrm{LH}_{2}$, Empty flask and $\mathrm{LD}_{2}$ and b) No target, $\mathrm{Fe}, \mathrm{C}$ and $\mathrm{W}$ targets. 


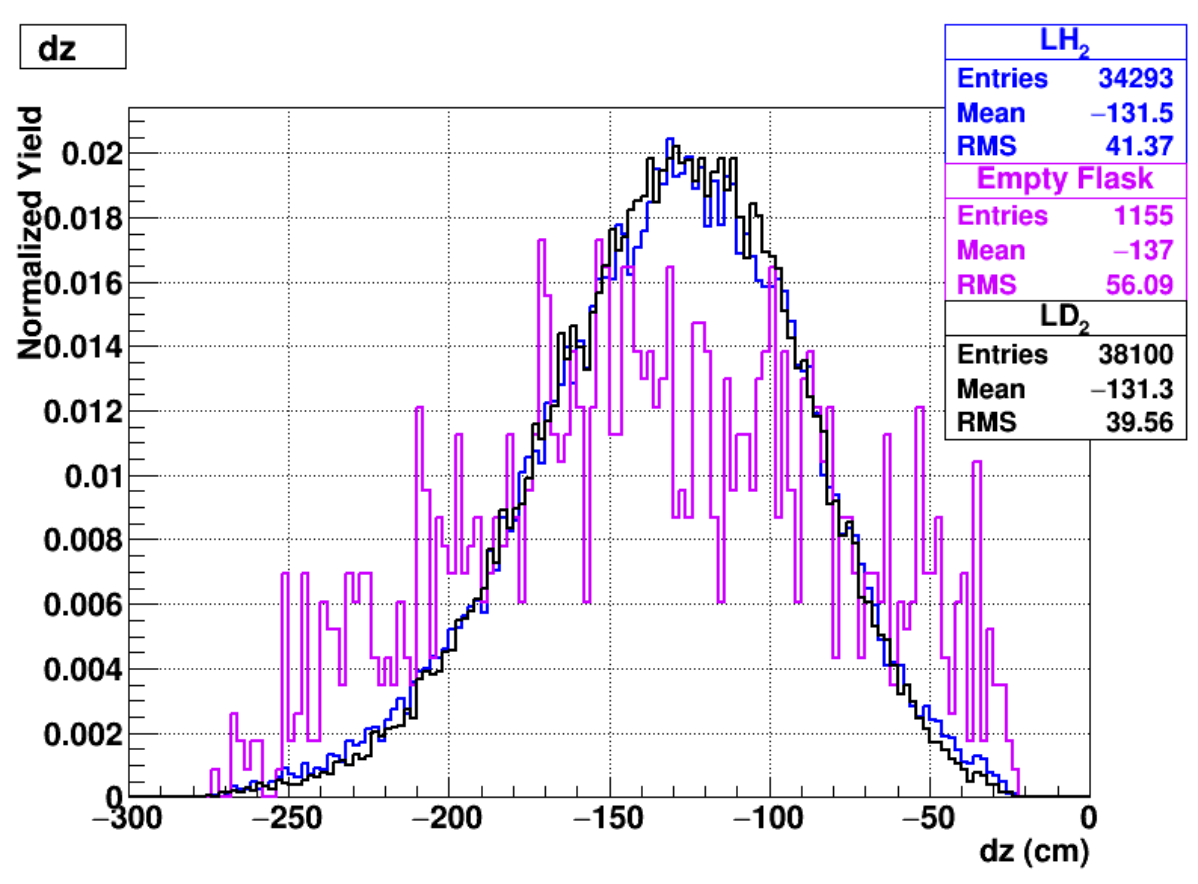

(a) Liquid targets

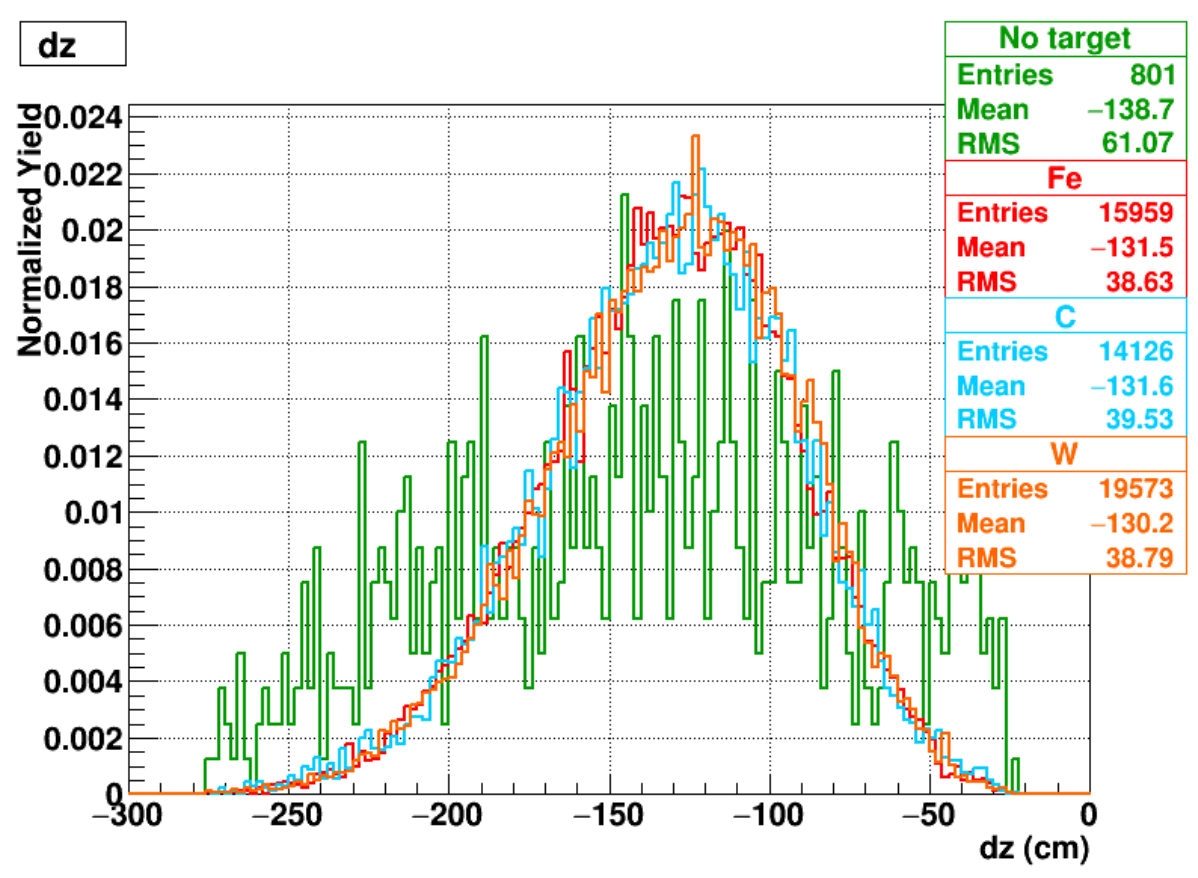

(b) Solid targets

Figure 4.25: Roadset comparison of $d z$ (dimuon vertex) for (a) $\mathrm{LH}_{2}$, Empty flask and $\mathrm{LD}_{2}$ and b) No target, $\mathrm{Fe}, \mathrm{C}$ and $\mathrm{W}$ targets. As can be seen in the violet and green curves, there are slightly more counts coming from near the front face of the beam dump (possibly randoms from the dump that satisfy the target cuts) and further upstream (flask end cap). 


\subsection{Raw Dimuon Yields tables for different $x_{T}$ bins}

\begin{tabular}{|c|c|c|c|c|c|c|c|c|}
\hline \multicolumn{8}{|c|}{ Run II \& Run III $\left(0.1<x_{T}<0.13\right)$} \\
\hline Intensity min & Intensity max & LH $_{2}$ & EMPTY & LD $_{2}$ & None & Fe & C & W \\
\hline 0 & 64000 & 1959 & 50 & 2323 & 36 & 886 & 885 & 1117 \\
\hline 0 & 8000 & 272 & 9 & 344 & 7 & 127 & 137 & 162 \\
\hline 8000 & 16000 & 412 & 9 & 475 & 8 & 197 & 180 & 236 \\
\hline 16000 & 24000 & 396 & 8 & 460 & 7 & 156 & 169 & 213 \\
\hline 24000 & 32000 & 309 & 7 & 372 & 4 & 144 & 142 & 161 \\
\hline 32000 & 40000 & 211 & 10 & 301 & 5 & 99 & 101 & 144 \\
\hline 40000 & 48000 & 181 & 4 & 178 & 1 & 71 & 76 & 95 \\
\hline 48000 & 56000 & 90 & 2 & 113 & 1 & 52 & 43 & 64 \\
\hline 56000 & 64000 & 88 & 1 & 80 & 3 & 40 & 37 & 42 \\
\hline
\end{tabular}

Table 4.6: Dimuon yields per intensity bin for $0.1<x_{T}<0.13$.

\begin{tabular}{|c|c|c|c|c|c|c|c|c|}
\hline \multicolumn{8}{|c|}{ Run II \& Run III $\left(0.13<x_{T}<0.16\right)$} \\
\hline Intensity min & Intensity max & LH $_{2}$ & EMPTY & LD $_{2}$ & None & Fe & C & W \\
\hline 0 & 64000 & 5847 & 198 & 6663 & 123 & 2818 & 2472 & 3436 \\
\hline 0 & 8000 & 903 & 23 & 1040 & 14 & 431 & 377 & 520 \\
\hline 8000 & 16000 & 1203 & 47 & 1393 & 22 & 615 & 492 & 691 \\
\hline 16000 & 24000 & 1071 & 33 & 1312 & 20 & 520 & 475 & 644 \\
\hline 24000 & 32000 & 923 & 32 & 1058 & 22 & 415 & 407 & 567 \\
\hline 32000 & 40000 & 643 & 22 & 705 & 22 & 337 & 288 & 397 \\
\hline 40000 & 48000 & 515 & 18 & 522 & 11 & 240 & 202 & 275 \\
\hline 48000 & 56000 & 320 & 15 & 386 & 6 & 158 & 129 & 225 \\
\hline 56000 & 64000 & 264 & 8 & 247 & 6 & 102 & 102 & 117 \\
\hline
\end{tabular}

Table 4.7: Dimuon yields per intensity bin for $0.13<x_{T}<0.16$. 


\begin{tabular}{|c|c|c|c|c|c|c|c|c|}
\hline \multicolumn{8}{|c|}{ Run II \& Run III $\left(0.16<x_{T}<0.195\right)$} \\
\hline Intensity min & Intensity max & LH $_{2}$ & EMPTY & LD $_{2}$ & None & Fe & C & W \\
\hline 0 & 64000 & 8055 & 248 & 9015 & 175 & 3920 & 3355 & 4706 \\
\hline 0 & 8000 & 1220 & 24 & 1484 & 19 & 571 & 522 & 714 \\
\hline 8000 & 16000 & 1738 & 56 & 1902 & 29 & 847 & 720 & 918 \\
\hline 16000 & 24000 & 1470 & 48 & 1714 & 26 & 757 & 654 & 889 \\
\hline 24000 & 32000 & 1249 & 30 & 1396 & 25 & 584 & 513 & 756 \\
\hline 32000 & 40000 & 893 & 38 & 976 & 34 & 467 & 357 & 546 \\
\hline 40000 & 48000 & 665 & 22 & 700 & 22 & 308 & 242 & 408 \\
\hline 48000 & 56000 & 492 & 15 & 491 & 8 & 235 & 219 & 284 \\
\hline 56000 & 64000 & 328 & 15 & 352 & 12 & 151 & 128 & 191 \\
\hline
\end{tabular}

Table 4.8: Dimuon yields per intensity bin for $0.16<x_{T}<0.195$.

\begin{tabular}{|c|c|c|c|c|c|c|c|c|}
\hline \multicolumn{8}{|c|}{ Run II \& Run III $\left(0.195<x_{T}<0.24\right)$} \\
\hline Intensity min & Intensity max & LH $_{2}$ & EMPTY & LD $_{2}$ & None & Fe & C & W \\
\hline 0 & 64000 & 7457 & 250 & 8342 & 172 & 3687 & 3244 & 4551 \\
\hline 0 & 8000 & 1102 & 25 & 1325 & 21 & 562 & 505 & 665 \\
\hline 8000 & 16000 & 1428 & 46 & 1807 & 25 & 746 & 617 & 964 \\
\hline 16000 & 24000 & 1387 & 43 & 1639 & 27 & 710 & 606 & 870 \\
\hline 24000 & 32000 & 1183 & 52 & 1259 & 32 & 571 & 506 & 689 \\
\hline 32000 & 40000 & 896 & 30 & 885 & 30 & 441 & 397 & 545 \\
\hline 40000 & 48000 & 655 & 27 & 680 & 18 & 328 & 282 & 396 \\
\hline 48000 & 56000 & 458 & 17 & 450 & 9 & 205 & 186 & 255 \\
\hline 56000 & 64000 & 348 & 10 & 297 & 10 & 124 & 145 & 167 \\
\hline
\end{tabular}

Table 4.9: Dimuon yields per intensity bin for $0.195<x_{T}<0.24$. 


\begin{tabular}{|c|c|c|c|c|c|c|c|c|}
\hline \multicolumn{8}{|c|}{ Run II \& Run III $\left(0.24<x_{T}<0.29\right)$} \\
\hline Intensity min & Intensity max & LH $_{2}$ & EMPTY & LD $_{2}$ & None & Fe & C & W \\
\hline 0 & 64000 & 4829 & 174 & 5315 & 112 & 2268 & 2019 & 2787 \\
\hline 0 & 8000 & 709 & 19 & 883 & 19 & 397 & 297 & 433 \\
\hline 8000 & 16000 & 949 & 30 & 1105 & 10 & 436 & 390 & 546 \\
\hline 16000 & 24000 & 888 & 20 & 985 & 16 & 423 & 407 & 535 \\
\hline 24000 & 32000 & 752 & 34 & 808 & 20 & 366 & 325 & 446 \\
\hline 32000 & 40000 & 567 & 19 & 575 & 15 & 256 & 234 & 329 \\
\hline 40000 & 48000 & 442 & 19 & 419 & 14 & 172 & 170 & 209 \\
\hline 48000 & 56000 & 304 & 19 & 311 & 5 & 123 & 107 & 172 \\
\hline 56000 & 64000 & 218 & 14 & 229 & 13 & 95 & 89 & 117 \\
\hline
\end{tabular}

Table 4.10: Dimuon yields per intensity bin for $0.24<x_{T}<0.29$.

\begin{tabular}{|c|c|c|c|c|c|c|c|c|}
\hline \multicolumn{8}{|c|}{ Run II \& Run III $\left(0.29<x_{T}<0.35\right)$} \\
\hline Intensity min & Intensity max & LH $_{2}$ & EMPTY & LD $_{2}$ & None & Fe & C & W \\
\hline 0 & 64000 & 2750 & 87 & 3143 & 60 & 1304 & 1169 & 1612 \\
\hline 0 & 8000 & 428 & 7 & 499 & 4 & 195 & 178 & 244 \\
\hline 8000 & 16000 & 550 & 10 & 672 & 8 & 288 & 240 & 334 \\
\hline 16000 & 24000 & 494 & 11 & 596 & 13 & 245 & 225 & 305 \\
\hline 24000 & 32000 & 415 & 17 & 508 & 8 & 204 & 168 & 244 \\
\hline 32000 & 40000 & 295 & 12 & 341 & 9 & 151 & 140 & 179 \\
\hline 40000 & 48000 & 235 & 8 & 240 & 5 & 97 & 98 & 148 \\
\hline 48000 & 56000 & 195 & 12 & 165 & 5 & 78 & 74 & 91 \\
\hline 56000 & 64000 & 138 & 10 & 122 & 8 & 46 & 46 & 67 \\
\hline
\end{tabular}

Table 4.11: Dimuon yields per intensity bin for $0.29<x_{T}<0.35$. 


\begin{tabular}{|c|c|c|c|c|c|c|c|c|}
\hline \multicolumn{1}{|c|}{ Run II \& Run III $\left(0.35<x_{T}<0.45\right)$} \\
\hline Intensity min & Intensity max & $\mathrm{LH}_{2}$ & EMPTY & $\mathrm{LD}_{2}$ & None & $\mathrm{Fe}$ & $\mathrm{C}$ & $\mathrm{W}$ \\
\hline 0 & 64000 & 1348 & 53 & 1435 & 40 & 578 & 537 & 760 \\
\hline 0 & 8000 & 205 & 8 & 247 & 2 & 98 & 93 & 127 \\
\hline 8000 & 16000 & 298 & 8 & 304 & 3 & 101 & 129 & 167 \\
\hline 16000 & 24000 & 256 & 5 & 276 & 4 & 125 & 99 & 134 \\
\hline 24000 & 32000 & 183 & 6 & 228 & 6 & 93 & 76 & 128 \\
\hline 32000 & 40000 & 148 & 7 & 135 & 2 & 66 & 64 & 76 \\
\hline 40000 & 48000 & 114 & 10 & 111 & 9 & 43 & 33 & 56 \\
\hline 48000 & 56000 & 83 & 5 & 81 & 9 & 29 & 27 & 36 \\
\hline 56000 & 64000 & 61 & 4 & 53 & 5 & 23 & 16 & 36 \\
\hline
\end{tabular}

Table 4.12: Dimuon yields per intensity bin for $0.35<x_{T}<0.45$.

\subsection{Contamination and Density corrections:}

After getting the raw dimuon yields, some corrections have to be applied before comparing the per proton yield for the targets. The corrections applied are as follows:

- Beam attenuation correction: Since the interaction length is different for the liquid targets, the beam that falls on the entire target (and the remainder that goes into the beam dump) is not the same for both the targets.

- Target contamination corrections: The liquid targets are $50.8 \mathrm{~cm}$ long and $7.62 \mathrm{~cm}$ in diameter and can contain 2.2 liters of liquid in them. The flask walls are made of $76 \mu \mathrm{m}$-thick stainless steel with $51 \mu \mathrm{m}$-thick stainless steel end caps. The liquid Hydrogen (or $\mathrm{LH}_{2}$ or $\mathrm{H}_{2}$ ) target used "Ultra High Purity" gas which is $99.999 \%$ commercially pure. The gas used for Deuterium (or $\mathrm{LD}_{2}$ or $\left.\mathrm{D}_{2}\right)$ target came from two sources:

- $95.8 \pm 0.2 \%$ gas that was used for bubble chamber experiments at Fermilab. This gas contained contamination from ${ }^{2} \mathrm{H}$ and ${ }^{1} \mathrm{H}$ primarily in $\mathrm{HD}$ molecules.

- $99.99 \%$ pure commercially available deuterium that was used during the latter part of the experiment. 
The liquid target contamination and densities corrections method has been adopted from [153]. The volume of HD molecule is roughly $9.4 \%$ higher in volume than the $\mathrm{D} 2$ or $\mathrm{H} 2$ molecule and the density of $\mathrm{H}$ used in HD has to be adjusted accordingly. The density of deuterium and hydrogen were taken to be $0.164 \mathrm{gm} / \mathrm{cc}$ and $0.0708 \mathrm{gm} / \mathrm{cc}$ [154]. Relative to pure deuterium, the volume of the mixed deuterium is ${ }^{2}$ :

$$
\begin{aligned}
& V_{c}=\text { FractionD }_{2} * 1.0+\text { FractionHD } * 1.094+\text { Fraction }_{2} * 1.221 \\
& =0.918+0.082 * 1.094+0 * 1.221=1.007708 \\
& \left\langle V_{c}\right\rangle=\frac{\text { Live }_{\text {cont }} * 1.007708+\text { Live }_{\text {pure }} * 1}{\text { Live }_{\text {Total }}}
\end{aligned}
$$

If we call the contamination as $c$ (which is $8.2 \%$ ), the weighted (by LIVE:G2SEM) average of several quantities is given by:

$$
\langle D\rangle=\frac{\text { Live }_{\text {cont }} *(1-c)+\text { Live }_{\text {pure }} * 1}{\text { Live }_{\text {Total }}}
$$

where $\langle D\rangle$ is the molecular fraction of $\mathrm{D}$ of pure deuterium in the contaminated $\mathrm{LD}_{2}$

$$
\langle H D\rangle=\frac{\text { Live }_{\text {cont }} *(c)+\text { Live }_{\text {pure }} * 0}{\text { Live }_{\text {Total }}}
$$

where $\langle H D\rangle$ is the molecular fraction of HD in contaminated deuterium.

$$
1 /\langle\lambda\rangle=\frac{L * \rho_{D} * \sigma_{D} * N_{a v o} * c / 2}{\left\langle V_{c}\right\rangle * M_{D}}+\frac{L * \rho_{D} * \sigma_{H} * N_{a v o} *(1-c / 2)}{\left\langle V_{c}\right\rangle * M_{D}}
$$

where $N_{a v o}$ is the Avogadro's number

$$
T_{D}^{D}=L * \rho_{D} *\left\langle V_{c}\right\rangle *\left[\langle D\rangle+\frac{\langle H D\rangle}{2}\right]
$$

\footnotetext{
${ }^{2}$ The small contamination of $\mathrm{H}$ is ignored $\left(\right.$ Fraction $\left.\mathrm{H}_{2}=0\right)$
} 


$$
\begin{gathered}
T_{H}^{D}=L * \rho_{D} *\left\langle V_{c}\right\rangle *\left[\frac{\langle H D\rangle}{2}\right] \\
T_{H}^{H}=L * \rho_{H} \\
A_{H}=\rho_{H} *\left(1-e^{-L / \lambda_{H}}\right) * 1 / L \\
A_{D}=\rho_{D} *\left(1-e^{-L /\left\langle\lambda_{D}\right\rangle}\right) * 1 / L
\end{gathered}
$$

$T_{A}^{A}$ and $A_{A}$ for solid targets are calculated in a similar way as 4.25 and 4.26. The table below shows these constants averaged for the whole data set. The dimuon yields are

\begin{tabular}{|c|c|}
\hline Quantity & Weighted average value \\
\hline$T_{D}^{D}$ & 8.0112 \\
\hline$T_{H}^{D}$ & 0.2437 \\
\hline$T_{H}^{H}$ & 3.5966 \\
\hline$A_{D}$ & 0.9451 \\
\hline$A_{H}$ & 0.9662 \\
\hline$V_{c}$ & 1.0056 \\
\hline C (avg contamination) & $5.91 \%$ \\
\hline
\end{tabular}

Table 4.13: Weighted average quantities (weighted by live protons) for the entire data set.

combined for all the roadsets for all the three targets. 


\subsection{Cross Section Ratios}

The yields for individual targets can be written as:

$$
\begin{aligned}
\text { Yield }_{L H_{2}}= & T_{H}^{H} * N_{A} * P_{H} * A_{H} * \sigma_{p p} * \varepsilon_{H} / M_{H} \\
\text { Yield }_{L D_{2}}= & \left\langle T_{D}^{D}\right\rangle * N_{A} * P_{D} * A_{D} * \sigma_{p d} * \varepsilon_{D} / M_{D} \\
& +\left\langle T_{H}^{D}\right\rangle * N_{A} * P_{D} * A_{D} * \sigma_{p p} * \varepsilon_{D} / M_{H} \\
\text { Yield }_{A}= & T_{A}^{A} * N_{A} * P_{A} * A_{A} * \sigma_{p A} * \varepsilon_{A} / M_{A}
\end{aligned}
$$

and the formula used in the calculation of the ratio of cross sections $\sigma_{p d} / 2 \sigma_{p p}$ is given by:

$$
\frac{\sigma_{p d}}{2 \sigma_{p p}}=\frac{T_{H}^{H} M_{D}}{2\left\langle T_{D}^{D}\right\rangle M_{H}} \cdot\left\{\left[\frac{\frac{Y_{L D 2}}{P_{D} *\left\langle A_{D}\right\rangle}-\frac{Y_{M T}}{P_{M T}}}{\frac{Y_{L H 2}}{P_{H} * A_{H}}-\frac{Y_{M T}}{P_{M T}}}\right]-\frac{\left\langle T_{H}^{D}\right\rangle}{T_{H}^{H}}\right\}
$$

Similarly, the formula used in the calculation of the ratio $R_{p A}$ which is the per nucleon cross section ratio is given by:

$$
R=\frac{2}{A} \cdot \frac{\sigma_{p A}}{\sigma_{p d}}=\frac{2}{A} \frac{\left\langle T_{D}^{D}\right\rangle}{T_{A}^{A}} \frac{M_{A}}{M_{D}}\left\{\left[\frac{\frac{Y_{A}}{P_{A} * A_{A}}-\frac{Y_{N O}}{P_{N O}}}{\frac{Y_{L D 2}}{P_{D} *\left\langle A_{D}\right\rangle}-\frac{Y_{M T}}{P_{M T}}-\frac{\left\langle T_{H}^{D}\right\rangle}{T_{H}^{H}}\left[\frac{Y_{L H 2}}{P_{H} * A_{H}}-\frac{Y_{M T}}{P_{M T}}\right]}\right]\right\}
$$

where the variables used in the equation are:

$Y_{L H 2}$ - Yield on LH2 target in events

$Y_{L D 2}$ - Yield on LD2 target in events

$Y_{A}$ - Yield on solid target A target in events

$Y_{N O}$ - Yield on none target in events

$T_{H}^{H}$ - Thickness of hydrogen in LH2 target $(\mathrm{gm} / \mathrm{cm} 2)$

$T_{H}^{D}$ - Thickness of hydrogen in LD2 target $(\mathrm{gm} / \mathrm{cm} 2)$

$T_{D}^{D}$ - Thickness of deuterium in LD2 target $(\mathrm{gm} / \mathrm{cm} 2)$

$T_{A}^{A}$ - Thickness of solid target $(\mathrm{gm} / \mathrm{cm} 2)$ 
$M_{H}$ - Atomic mass of hydrogen $=1.008$

$M_{D}$ - Atomic mass of deuterium $=2.014$

$M_{A}$ - Atomic mass of solid target $\mathrm{C}=12.000, \mathrm{Fe}=55.845, \mathrm{~W}=183.84$

$N_{A}$ - Avogadros number $=6.0221409 \mathrm{e} 23$

$P_{D}$ - Number of protons on LD2 target

$P_{H}$ - Number of protons on LH2 target

$P_{N O}$ - Number of protons on None target

$P_{M T}$ - Number of protons on empty target

$P_{A}$ - Number of protons on solid target A

$A_{D}$ - Attenuation of protons on LD2 target

$A_{H}$ - Attenuation of protons on LH2 target

$A_{A}$ - Attenuation of protons on solid target

$A$ - Number of nucleons on the solid target

$\varepsilon_{H}$ - Spectrometer efficiency on LH2 target

$\varepsilon_{D}$ - Spectrometer efficiency on LD2 target

$\varepsilon_{M T}$ - Spectrometer efficiency on empty target

$\sigma_{H}-$ Cross section for Hydrogen $=32.2 \mathrm{e}-27$

$\sigma_{D}-$ Cross section for Deuterium $=46.6$ e -27

\subsubsection{Statistical Errors for Individual CSR points}

\section{Liquid targets:}

The cross section for the ratio is calculated using the formula:

$$
\frac{\sigma_{p d}}{2 \sigma_{p p}}=\frac{T_{H}^{H} M_{D}}{2\left\langle T_{D}^{D}\right\rangle M_{H}} \cdot\left\{\left[\frac{\left(\frac{a}{A_{D}}-c\right)}{\left(\frac{b}{A_{H}}-c\right)}\right]-\frac{\left\langle T_{H}^{D}\right\rangle}{T_{H}^{H}}\right\}
$$

where $a=Y_{\text {ield }}{ }_{L D 2} / L I V E: G 2 S E M_{L D 2}, b=Y_{\text {ield }}{ }_{L H 2} / L I V E: G 2 S E M_{L H 2}$ and $c=$ Yield $_{\text {empty }} / L I V E: G 2 S E M_{\text {empty }}$. We compute the errors manually in order to take 
into account the additional correlated factor that arises due to the common factor $c$ that is subtracted out in the numerator and denominator. This common factor $c$ isn't taken into account by ROOT for example while calculating errors.

$$
\begin{aligned}
\frac{\partial F}{\partial a} & =\frac{T_{H}^{H} M_{D}}{2\left\langle T_{D}^{D}\right\rangle M_{H}} \cdot \frac{1}{\left(\frac{b}{A_{H}}-c\right)} \cdot \frac{1}{\left\langle A_{D}\right\rangle} \\
\frac{\partial F}{\partial b} & =\frac{T_{H}^{H} M_{D}}{2\left\langle T_{D}^{D}\right\rangle M_{H}} \cdot \frac{\left(\frac{a}{\left\langle A_{D}\right\rangle}-c\right)}{\left(\frac{b}{A_{H}}-c\right)^{2}} \cdot \frac{1}{A_{H}} \\
\frac{\partial F}{\partial c} & =\frac{T_{H}^{H} M_{D}}{2\left\langle T_{D}^{D}\right\rangle M_{H}} \cdot \frac{\left(\frac{a}{\left\langle A_{D}\right\rangle}-\frac{b}{A_{H}}\right)}{\left(\frac{b}{A_{H}}-c\right)^{2}}
\end{aligned}
$$

Eventually, errors are added in quadrature using the formula:

$$
\sigma_{F}=\sqrt{\underbrace{\left(\frac{\partial F}{\partial a} \sigma_{a}\right)^{2}}_{1}+\underbrace{\left(\frac{\partial F}{\partial b} \sigma_{b}\right)^{2}}_{2}+\underbrace{\left(\frac{\partial F}{\partial c} \sigma_{c}\right)^{2}}_{3}}
$$

where the $\sigma_{a}, \sigma_{b}$ and $\sigma_{c}$ are the errors in the histograms for $a, b$ and $c\left(\mathrm{LD}_{2}, \mathrm{LH}_{2}\right.$ and empty flask which are scaled by their total Live protons).

\section{Solid targets:}

The cross section for the ratio is calculated using the formula:

$$
R=\frac{2}{A} \cdot \frac{\sigma_{p A}}{\sigma_{p d}}=\frac{2}{A} \cdot \frac{\left\langle T_{D}^{D}\right\rangle}{T_{A}^{A}} \cdot \frac{M_{A}}{M_{D}}\left\{\left[\frac{\frac{a}{A_{A}}-d}{\frac{b}{\left\langle A_{D}\right\rangle}-c-\frac{\left\langle T_{H}^{D}\right\rangle}{T_{H}^{H}}\left[\frac{e}{A_{H}}-c\right]}\right]\right\}
$$

where $a=$ Yield $_{A} / L I V E: G 2 S E M_{A}, b=Y_{\text {ield }}$ LD2 $/ L I V E: G 2 S E M_{L D 2}$ and $c=$ Yield $_{M T} / L I V E: G 2 S E M_{M T}, d=$ Yield $_{N O} / L I V E: G 2 S E M_{N O}$ and $e=$ 
Yield $_{L H 2} / L I V E: G 2 S E M_{L H 2}$. The errors are computed manually.

$$
\begin{aligned}
& \frac{\partial R}{\partial a}=\frac{2}{A} \cdot \frac{\left\langle T_{D}^{D}\right\rangle}{T_{A}^{A}} \cdot \frac{M_{A}}{M_{D}} \cdot \frac{1}{A_{A}} \cdot \frac{1}{\sigma_{p d}} \\
& \frac{\partial R}{\partial b}=\frac{2}{A} \cdot \frac{\left\langle T_{D}^{D}\right\rangle}{T_{A}^{A}} \cdot \frac{M_{A}}{M_{D}} \cdot \frac{1}{\left\langle A_{D}\right\rangle} \frac{\sigma_{p A}}{\sigma_{p d}^{2}} \\
& \frac{\partial R}{\partial c}=\frac{2}{A} \cdot \frac{\left\langle T_{D}^{D}\right\rangle}{T_{A}^{A}} \cdot \frac{M_{A}}{M_{D}} \cdot \frac{\sigma_{p A}}{\sigma_{p d}^{2}} \cdot\left(\frac{\left\langle T_{H}^{D}\right\rangle}{T_{H}^{H}}-1\right) \\
& \frac{\partial R}{\partial d}=\frac{2}{A} \cdot \frac{\left\langle T_{D}^{D}\right\rangle}{T_{A}^{A}} \cdot \frac{M_{A}}{M_{D}} \cdot \frac{1}{\sigma_{p d}} \\
& \frac{\partial R}{\partial e}=\frac{2}{A} \cdot \frac{\left\langle T_{D}^{D}\right\rangle}{T_{A}^{A}} \cdot \frac{M_{A}}{M_{D}} \cdot \frac{\sigma_{p A}}{\sigma_{p d}^{2}} \frac{\left\langle T_{H}^{D}\right\rangle}{T_{H}^{H}} \cdot \frac{1}{A_{H}}
\end{aligned}
$$

Eventually, errors are added in quadrature using the formula:

$$
\sigma_{R}=\sqrt{\underbrace{\left(\frac{\partial R}{\partial a} \sigma_{a}\right)^{2}}_{1}+\underbrace{\left(\frac{\partial R}{\partial b} \sigma_{b}\right)^{2}}_{2}+\underbrace{\left(\frac{\partial R}{\partial c} \sigma_{c}\right)^{2}}_{3}+\underbrace{\left(\frac{\partial R}{\partial d} \sigma_{d}\right)^{2}}_{4}+\underbrace{\left(\frac{\partial R}{\partial e} \sigma_{e}\right)^{2}}_{5}}
$$

where the $\sigma_{a}, \sigma_{b}, \sigma_{c}, \sigma_{d}$ and $\sigma_{e}$ are the errors in the histograms for $a, b, c, d$ and $e\left(\mathrm{~A}, \mathrm{LD}_{2}\right.$, empty flask, none and $\mathrm{LH}_{2}$ which are scaled by their total LIVE protons).

\subsection{The Intensity-Extrapolation method}

The idea of the "Intensity-Extrapolation method" is pretty simple. We plot the ratio of cross sections (normalized by LIVE:G2SEM) from two targets as a function of the number of protons in the triggered bucket (also known as trigger intensity). There is a slope for the Drell-Yan dimuon yield ratio that could be caused by aspects of rate dependence such as:

- Different accidental backgrounds

- Relative tracking efficiency difference

- DAQ dead time differences 
Rate dependence vanishes at beam intensity $=0$. We extract the intercept at 0 which is free from accidental background and rate dependence. The advantage of the IntensityExtrapolation method is that it is purely data driven. We directly confront data (dimuon yield ratio after contamination corrections) and ask it to take us to a place where the ratio of cross sections is free from rate dependence effects. We have tested and are continuing to test it by changing the number of bins, adjusting cuts, changing intensity ranges, checking stability as a function of cuts and many other checks which are presented in the next chapter.

\subsection{1 kTracker Efficiency Corrections}

We do not apply any kTracker efficiency corrections as the ratio of tracker efficiencies ratio goes to 1 at 0 intensity. Studies showed [155] that the tracker efficiency ratio indeed goes to 1 for linear and quadratic fits as shown in Figure 4.26.

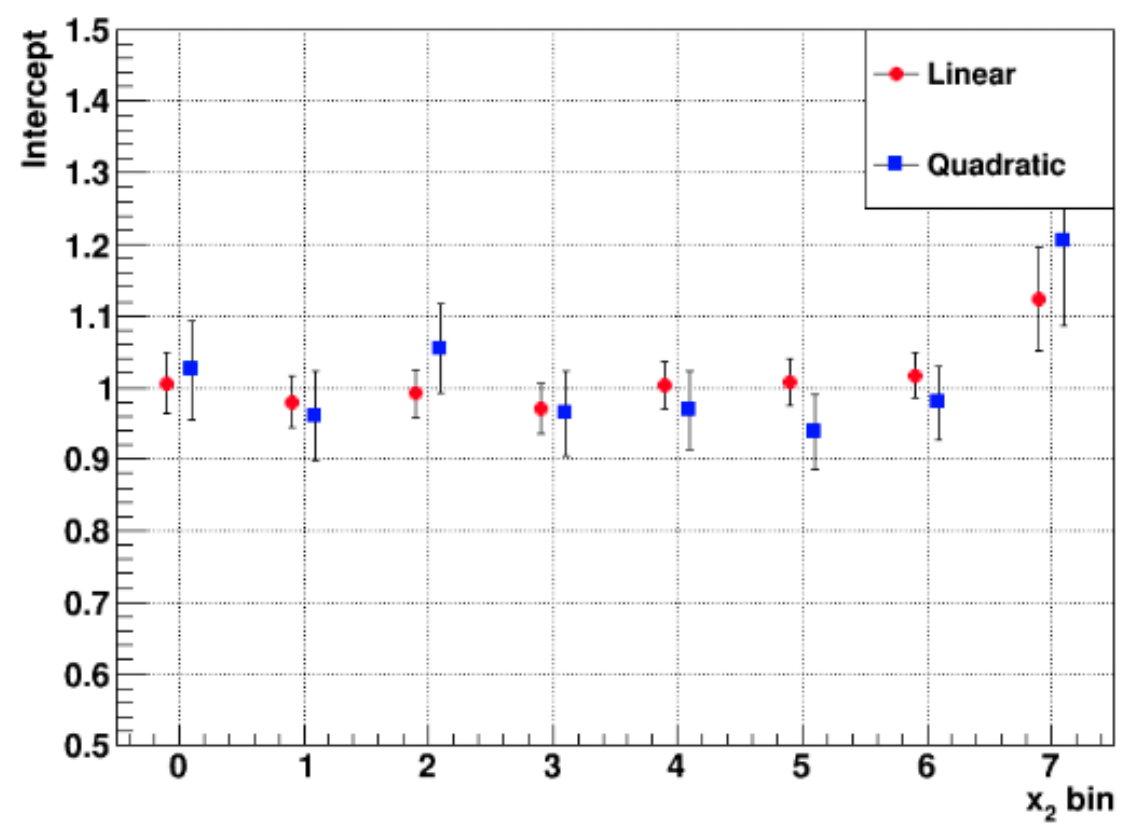

Figure 4.26: $\mathrm{LD}_{2} / \mathrm{LH}_{2}$ ratio of intercepts for linear and quadratic fits plot for mc_drellyan_LD2_M026_S002_messy and mc_drellyan_LH2_M026_S002_messy GEANT4 Monte Carlo productions made using $\mathrm{LD}_{2}$ and $\mathrm{LH}_{2}$ targets. 


\subsubsection{Logic behind Fit function}

This section is mostly an excerpt from [156].

\section{Empirical vs Theoretical Shapes}

It is surely better, as long as possible, that we construct a "theoretical shape" of the intensity dependence and adopt it in the fitting. For example, the individual target yields (not ratio) can be expressed with a signal term and a background term as follows:

$$
N_{\text {dimuons }}=\alpha_{\text {signal }} \cdot I+\alpha_{\text {background }} \cdot I^{2}
$$

But, to validate this expression, various effects must be formulated or corrected, such as

- Beam-intensity profile,

- Reconstruction ( tracking) efficiency and

- Categorization of random backgrounds.

Indeed the investigation by Jason Dove, a SeaQuest collaborator from UIUC, showed that the intensity dependence in our data doesn't match with the theoretical shape at present. It indicates that we haven't yet understood every effect individually. Therefore we are trying to use an "empirical" (or "phenomenological") shape in the fitting. Actually it should be the key advantage of the intensity-extrapolation method. It is because all effects as a whole will vanish at $I=0$ and thus they need not be formulated nor corrected one-by-one. A difficulty in using the empirical shape is "the choice of fit shape". In our present analysis condition, lots of candidates are not distinguishable as they result in the similarly good $\chi^{2} / \mathrm{NDF}$ values. Given the condition, we need to limit them with "reasonable" constraints and assign the systematic error "properly". 


\section{More vs Fewer Parameters}

A choice of the number of parameters can be straightforward when our data themselves can distinguish the correct one. For example, we can reject any shape when its probability is small like $<1 \%$ by $\chi^{2} / \mathrm{NDF}$. But our data cannot do that at present. A typical problem about this choice is "second order polynomial vs first order polynomial". The effect ( syst. error) of mis-choice (i.e. fitting shape $\neq$ true shape) was estimated by numerical simulation in pages 4-8 of [157]. The conclusion was that we should adopt the less-strict one, where its stat. error becomes larger but covers the true value in case of mis-choice. We parameterized the $x_{T}$ dependence (as well as the intensity dependence) and carried out a two-dimensional fit. It was because the fits to the $x_{T}$ bins resulted in too-large statistical error, simply due to the statistical error of our current data. The parameterization enforces a smooth change of the rate dependence over $x_{T}$ and then the resultant statistical error be-

comes small. We selected "pol1" for the $x_{T}$ dependence, where the average of $x_{T}$ per bin was taken as the fitting variable. Figure 4.27 shows the fit for all data and all $x_{T}$ bins

\section{Fit Function Adopted}

The final fit function is:

$$
F=p 0+\left(p 10+p 11 *\left\langle x_{T}\right\rangle\right) * \text { inte }+\left(p 20+p 21 *\left\langle x_{T}\right\rangle\right) * i n t e^{2}
$$

where $\mathrm{p} 10, \mathrm{p} 11, \mathrm{p} 20$ and $\mathrm{p} 21$ are common to all $x_{T}$ bins and $\left\langle x_{T}\right\rangle$ is the average value of that particular $x_{T}$ bin and inte is the trigger intensity. The results for the cross section ratio intercepts using this Intensity-Extrapolation method are presented in the next chapter. 


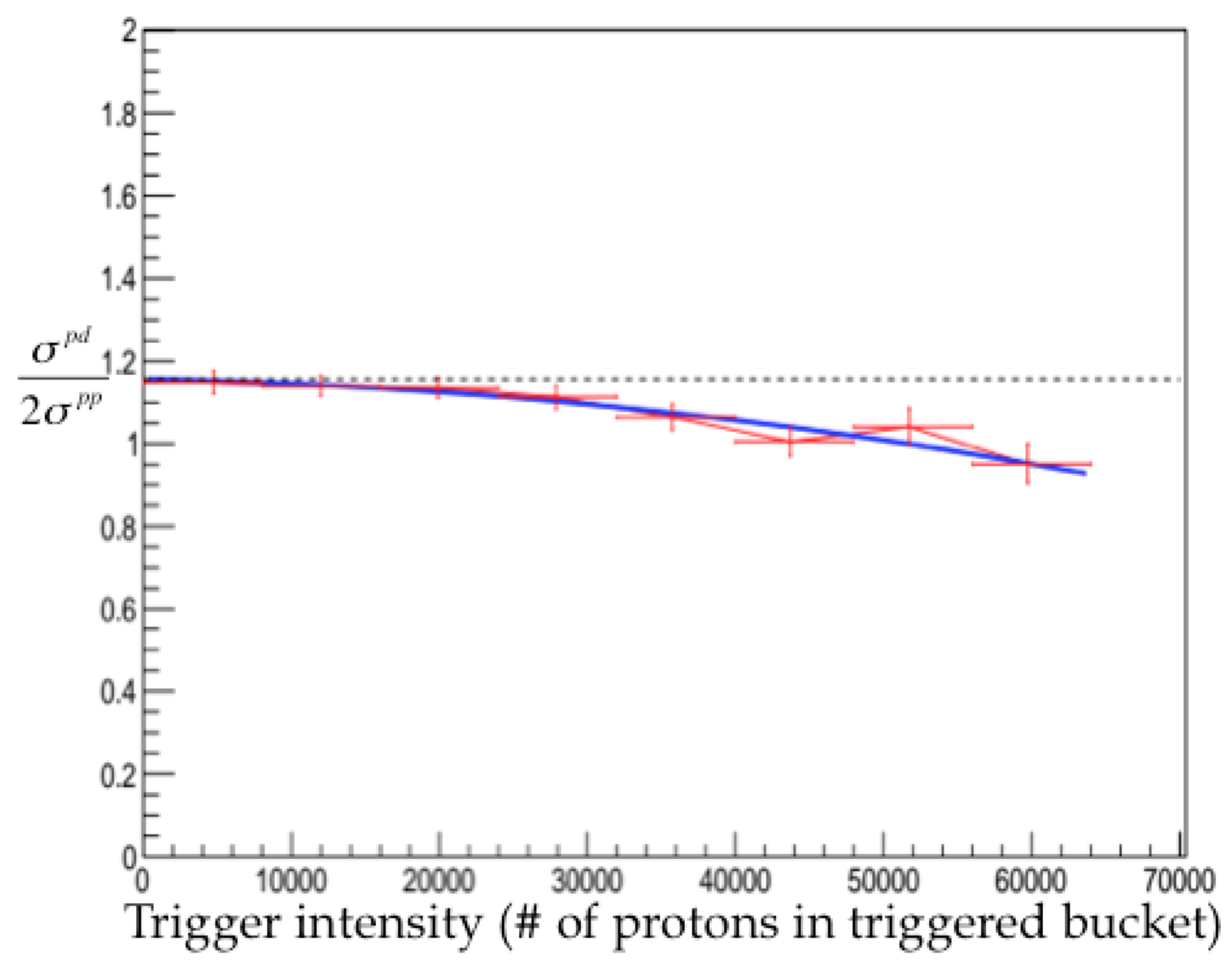

Figure 4.27: $\mathrm{LD}_{2} / \mathrm{LH}_{2}$ ratio as a function of the trigger intensity. As can be seen in the plot, the ratio of cross sections $\sigma_{p d} / 2 \sigma_{p p}$ ( $y$-axis) exhibits an intensity dependence ( $x$-axis). The plot is made for $0.1<x_{T}<0.45$. 


\section{Chapter 5}

\section{Results and Discussion}

\subsection{Cross Section Ratios: $\sigma_{p d}(x) / 2 \sigma_{p p}(x)$}

As a proof of principle, the intensity extrapolation technique was studied with individual data sets and the concept was demonstrated to work as shown in Fig. 5.1. One can see that the data points for roadset 70 are systematically lower than the other roadsets for higher $x_{T}$ values. Roadset 70 is a small
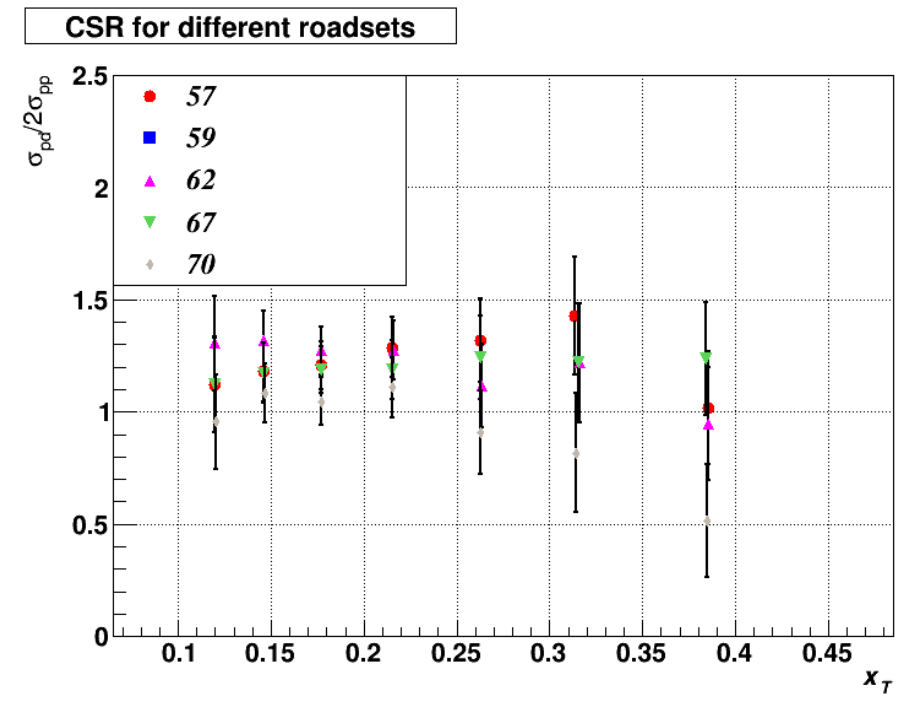

Figure 5.1: CSR vs $x_{T}$ for different roadsets. data set $(\approx 275$ "good" data runs compared to roadset 67 which is $\approx 3000$ "good" data runs). After all analysis cuts, roadset 70 has $<20 \mathrm{LH}_{2}$ and $\mathrm{LD}_{2}$ events and handful of empty flask events per each of the 8 trigger intensity bins. It has been shown through numerical simulations by Kenichi Nakano, a SeaQuest collaborator from Tokyo Institute of Technology, that cross section ratio values are systematically lower $(\sim 15 \%)$ for low statistics data sets with a Gaussian treatment compared to a Poissonian treatment [158] ${ }^{1}$. Also the size of the uncertainties would be smaller by $\sim 10 \%$ due to this treatment. How-

\footnotetext{
${ }^{1}$ The result arises from the asymmetry of a Poisson distribution, with the most probable result being less than the mean result.
} 
ever, we need not worry about extrapolation to small individual data sets as after the data sets are merged, the Gaussian criteria is met and one final cross section ratio is calculated. SeaQuest has collected enough data to separate out the entire dataset into different $x_{\text {Target }}$ bins. The intercepts from extrapolation for each bin are shown in Figures 5.2 and 5.3.

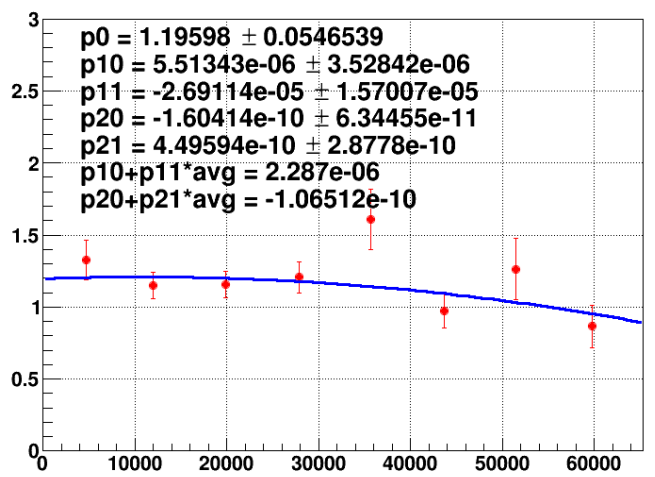

(a) $0.1<x_{T}<0.13$

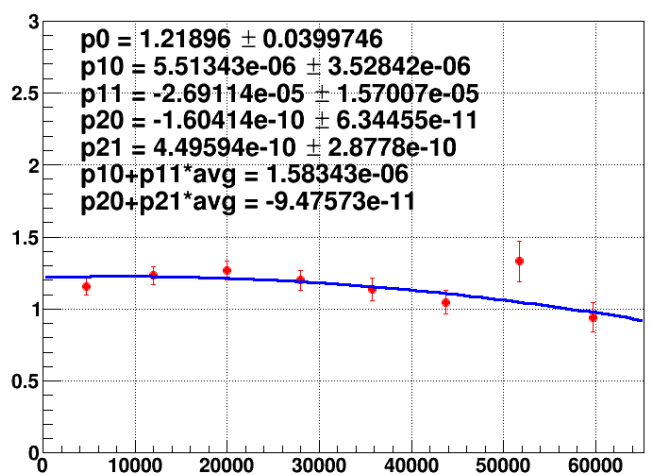

(b) $0.13<x_{T}<0.16$

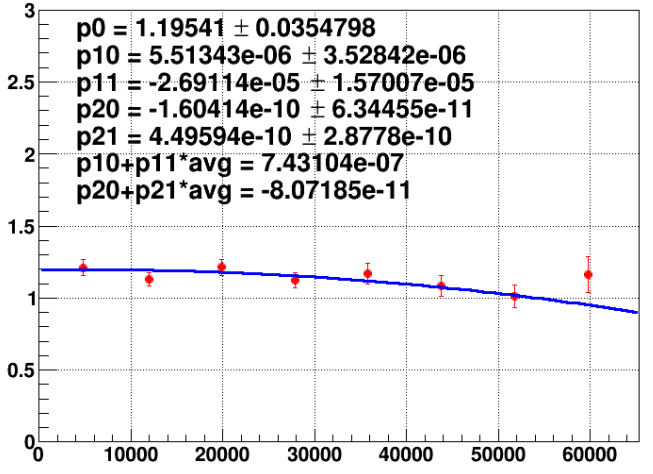

(c) $0.16<x_{T}<0.195$

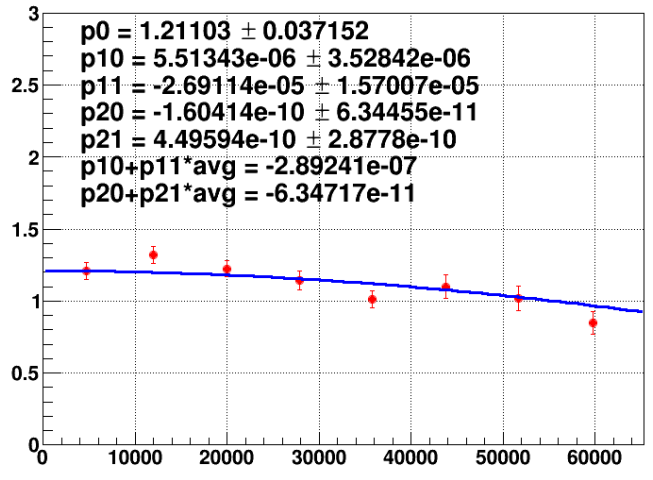

(d) $0.195<x_{T}<0.24$

Figure 5.2: CSR as a function of $x_{T}$. Note that $p 10, p 11, p 20$ and $p 21$ are common for all fits. 


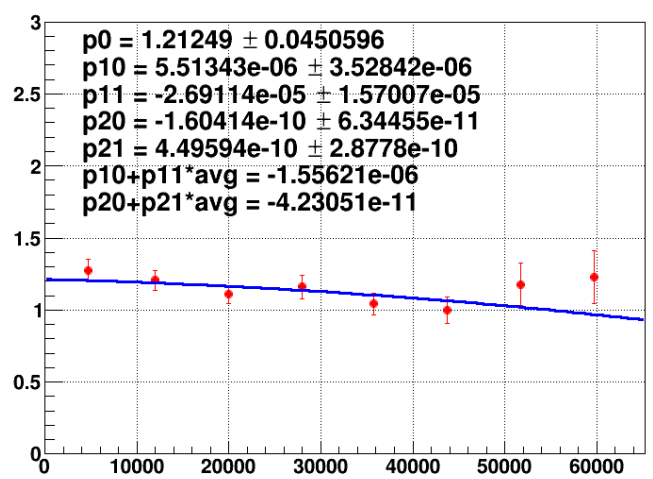

(a) $0.24<x_{T}<0.29$

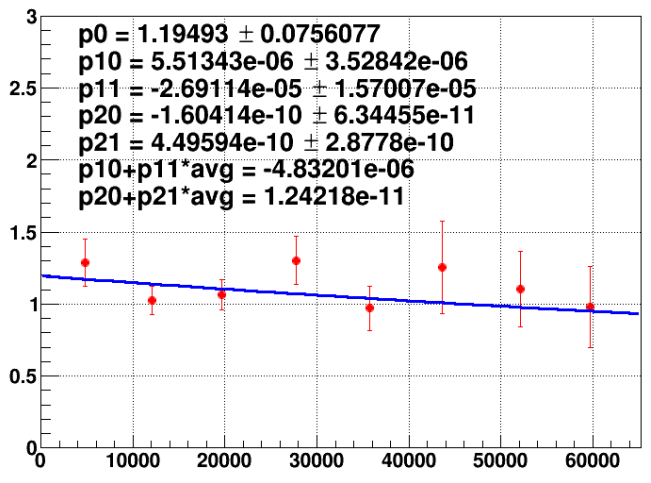

(c) $0.35<x_{T}<0.45$

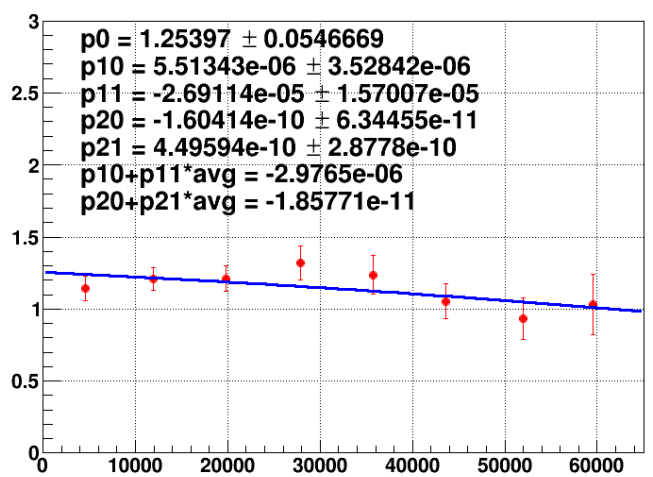

(b) $0.29<x_{T}<0.35$
Fit result:

chi2 / ndf $=48.0914 / 45=1.0687$

par $0:-1.60414 \mathrm{e}-10+-6.34455 \mathrm{e}-11$

par 1: $4.49594 \mathrm{e}-10+-2.8778 \mathrm{e}-10$

par 2 : $5.51343 \mathrm{e}-06+-3.52842 \mathrm{e}-06$

par $3:-2.69114 \mathrm{e}-05+-1.57007 \mathrm{e}-05$

par $4: 1.19598+-0.0546539$

par $5: 1.21896+-0.0399746$

par $6: 1.19541+-0.0354798$

par $7: 1.21103+-0.037152$

par $8: 1.21249+-0.0450596$

par $9: 1.25397+-0.0546669$

par 10: $1.19493+-0.0756077$

(d) Fit results

Figure 5.3: CSR intercepts for each $x_{T}$ bin along with the fit results. Note that $p 10, p 11$, $p 20$ and $p 21$ are common for all fits.

\subsubsection{Systematic Uncertainties for $\sigma_{p d}(x) / 2 \sigma_{p p}(x)$}

Some sources of systematic uncertainties considered are shown below:

1. Contamination of the $J / \psi$ and $\psi$ ' tail into the Drell-Yan mass region: We estimated the $J / \psi$ and $\psi^{\prime}$ tail contamination into the Drell-Yan mass region using mass fits in [159]. The bin by bin contamination of $J / \psi$ in the DY mass region for $\mathrm{LH}_{2}$ and $\mathrm{LD}_{2}$ is almost $0 \%$. The bin by bin contamination of $\psi^{\prime}$ in the DY mass region is a maximum of $1 \%$ (for lowest xT bin) for $\mathrm{LH}_{2}$ and $1.29 \%$ for $\mathrm{LD}_{2}$ (for lowest $x_{T}$ bin). It was shown that the bin by bin contamination is very small for the lowest bin and therefore this source can be safely ignored. 
2. Deuterium contamination: The purity of deuterium is $95.8 \pm 0.2 \%$ by numbers of atoms (or $91.8 \% \mathrm{D}_{2}$ and $8.2 \%$ of $\mathrm{HD}$ in mole fractions). Two sets of intercepts were calculated. One with $95.8+0.2$ and another with $95.8-0.2$ and the change in the intercepts compared to the nominal values is negligible.

3. Target Length difference between $\mathbf{L D}_{2}$ and $\mathbf{L H}_{2}$ flasks: It was mentioned in [57] that the uncertainty in the difference between flask lengths is $0.2 \%$. Two sets of intercepts were calculated with $50.8+0.002$ and $50.8-0.002$ and the change is negligible (5th significant digit in the intercept). Hence this systematic uncertainty can be safely ignored.

4. Accuracy of live protons (= G2SEM): The fluctuation in the G2SEM/QIEsum ratio was observed to be $0.16 \%$ [160]. So we adjusted the trigger intensity values to be + $0.16 \%$ and $-0.16 \%$ and calculated the fit parameters. The observed changes in the intercepts are negligible.

5. Fluctuating QIE pedestal: The QIE pedestal is calculated by looking at the QIE readout when the G2SEM value $=0$. However, this value could change when there is beam. So we assumed that 34 is the nominal value and used \pm 4 as the uncertainty. The difference between nominal value intercepts and higher/lower value pedestal intercepts are added in quadrature divided by $\sqrt{2}$.

6. Systematics from Choice of Fitting curve: This is the major source of systematic uncertainty. Different curves are chosen and the intercepts are calculated from these functional forms. A particular logic behind this choice is also briefly mentioned.

(a) $\mathrm{F}=\mathrm{p} 0 * \cos (\mathrm{I} /(\mathrm{p} 10+\mathrm{p} 11 * \mathrm{x} 2 \mathrm{avg}))$ : Tests a completely different shape particularly at intensity $\sim 0$.

(b) $\mathrm{F}=\mathrm{p} 0+\mathrm{p} 1 * \mathrm{I}+\mathrm{p} 2 * \mathrm{I}^{2}$ (Common Pol2 without the $x_{T}$ dependence). This checks the initial assumption of a dependence on $x_{T}$. 
All the fits are added in quadrature (without dividing by $\sqrt{2}$ as they probe independent aspects i.e. $x_{2}$ dependence and a new type of intensity dependent fitting function). Remember, there is no one unique choice given our statistics!

Finally, all the systematic uncertainties for each individual $x_{T}$ bin are added in quadrature using the formula:

$$
\sigma_{\text {sys }}\left(x_{T}\right)=\sqrt{\sum_{i}\left(\sigma_{i}\left(x_{T}\right)\right)^{2}} .
$$

\begin{tabular}{|c|c|c|c|}
\hline$x_{T}$ bin & CSR - Fit 5c & $\sigma_{\text {stat }}$ & $\sigma_{\text {syst }}$ \\
\hline $0.1-0.13$ & 1.1959 & 0.0546 & 0.0403 \\
\hline $0.13-0.16$ & 1.2189 & 0.0399 & 0.0256 \\
\hline $0.16-0.195$ & 1.1954 & 0.0354 & 0.0112 \\
\hline $0.195-0.24$ & 1.2110 & 0.0371 & 0.0053 \\
\hline $0.24-0.29$ & 1.2124 & 0.0450 & 0.0239 \\
\hline $0.29-0.35$ & 1.2539 & 0.0546 & 0.0406 \\
\hline $0.35-0.45$ & 1.1949 & 0.0756 & 0.0757 \\
\hline
\end{tabular}

Table 5.1: Table of cross section ratio values, statistical and systematic uncertainties. Total systematic uncertainty come from the choice of the fitting curve and the fluctuating QIE pedestal.

\begin{tabular}{|c|c|c|}
\hline Source of systematic uncertainty & Value & Reference \\
\hline Target length $(0.2 \%$ flask length diff $)$ & negligible & {$[57]$} \\
\hline Accuracy of live protons $(=$ G2SEM) & negligible & {$[160]$} \\
\hline Deuterium contamination systematics $(0.2 \%)$ & negligible & {$[151]$} \\
\hline$J / \psi$ tail and $\psi$ ' tail contamination & negligible & {$[159]$} \\
\hline
\end{tabular}

Table 5.2: Other negligible contributions to systematic uncertainties.

\section{$5.2 \sigma_{p d}(x) / 2 \sigma_{p p}(x)$ Results}

Figure 5.4 shows the $\sigma_{p d}(x) / 2 \sigma_{p p}(x)$ cross section ratio as a function of the $x_{\text {Target }}$. The red points are SeaQuest data points which are plotted along with the systematics band shown at the bottom. Some observations regarding the data points are noted below:

- SeaQuest data points are consistently above 1 for all $x_{T}$. 
- Also plotted together with SeaQuest data points are three other PDF predictions; CT10 NLO (CTEQ-TEA collaboration²) [161], CT14 NLO [162] and MMHT NLO (Martin, Motylinski, Harland-Lang, Thorne [163]). The data points seem to agree quite well with the PDF predictions in the low- $x$ region up to $x_{T} \approx 0.2$. Since there are no data in the high- $x$ region to constrain the PDFs, it is natural to expect differences between SeaQuest and the PDF predictions which are data driven.

- The systematic uncertainty for the fourth $x_{T}$ bin is small compared to the other bins. It is "natural" to some extent because of the nature of simultaneous fitting used in the Intensity-Extrapolation method. This bin has the most statistics and also, it is constrained by the other outer bins when a simultaneous fit is applied.

- A small overall normalization correction is being studied by the collaboration. It is estimated to be from $0-3 \%$, and arises when taking into account the dead time experienced by low intensity events.

\footnotetext{
${ }^{2}$ CTEQ-TEA is Coordinated Theoretical-Experimental Project on QCD - Tung Et Al collaboration
} 


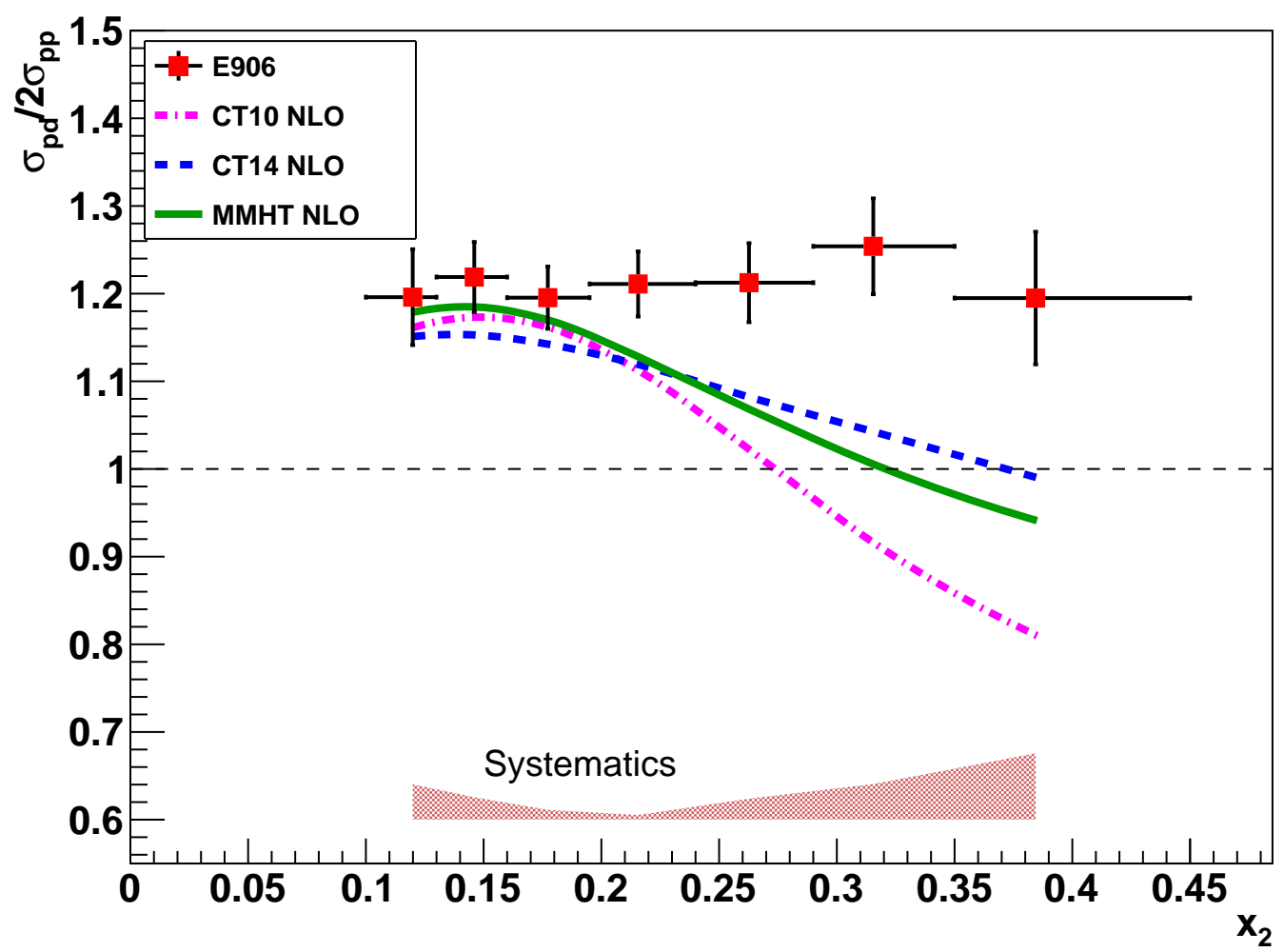

Figure 5.4: $\sigma_{p d}(x) / 2 \sigma_{p p}(x)$ vs $x_{T}$ plot along with PDF predictions. The red points are SeaQuest data points which are plotted along with the systematics band shown at the bottom. The data points are consistently above 1 for all $x_{T}$ and agree quite well with the PDF predictions in the low- $x$ region up to $x_{T} \approx 0.25$. Since there are no data in the high- $x$ region to constrain the PDFs, it is natural to expect differences between the E906 and PDF predictions which are data driven. A small overall normalization correction is being studied by the collaboration. It is estimated to be from $0-3 \%$, and arises when taking into account the dead time experienced by low intensity events. 


\subsection{1 $\sigma_{p d}(x) / 2 \sigma_{p p}(x)$ Comparison with E866}

Typically, SeaQuest data are compared with E866/NuSea data. One would expect some differences between the two experimental cross section ratios due to:

- differences in beam energies ( $800 \mathrm{GeV}$ for E866 compared to $120 \mathrm{GeV}$ for SeaQuest),

- differences in acceptance, and

- differences in $x_{\text {Beam }}$ distributions that are accessed for the same given $x_{T}$.

The SeaQuest collaboration is considering the size of different effects listed above in understanding the differences between the two sets of data points.

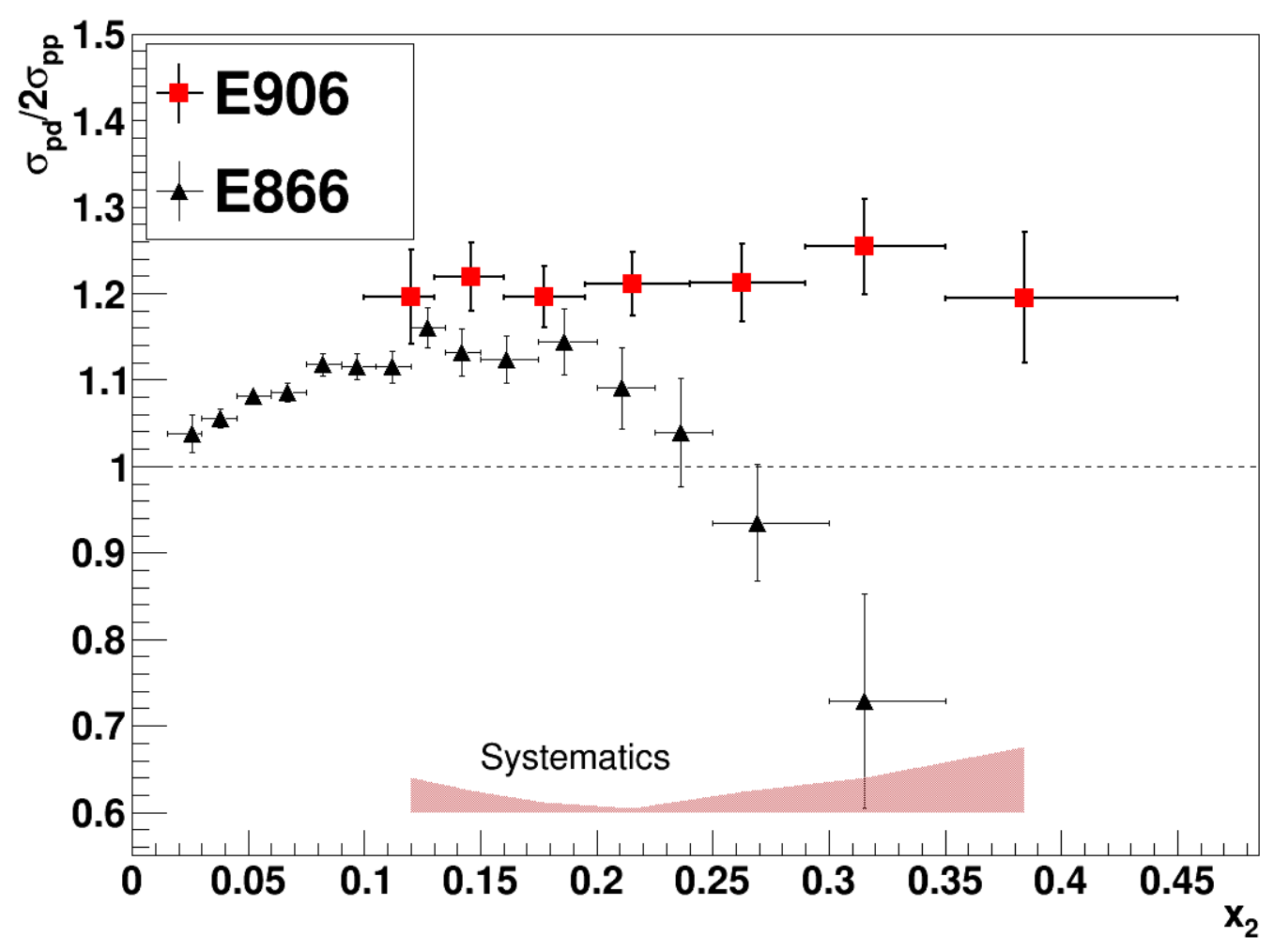

Figure 5.5: $\sigma_{p d}(x) / 2 \sigma_{p p}(x)$ vs $x_{T}$ plot with E866 data only. The red data points are SeaQuest and black data points are for E866/NuSea. The systematic uncertainty for E866 is $<1 \%$ and not shown here [57]. A small overall normalization correction is being studied by the collaboration. It is estimated to be from $0-3 \%$, and arises when taking into account the dead time experienced by low intensity events. 


\subsubsection{Comparison with Statistical Parton model}

Fig. 5.6 shows the comparison of SeaQuest cross section ratio values with those of the statistical parton model. The statistical parton model considers the nucleon as a gas of massless partons (quarks, anti-quarks and gluons) in equilibrium at a given temperature in a finite volume [66]. As one can see in Fig. 5.6, there is good agreement between the SeaQuest data and statistical parton model predictions. It should be noted that the data points for the statistical parton model are calculated for the SeaQuest beam energy but do not take into account the SeaQuest acceptance.

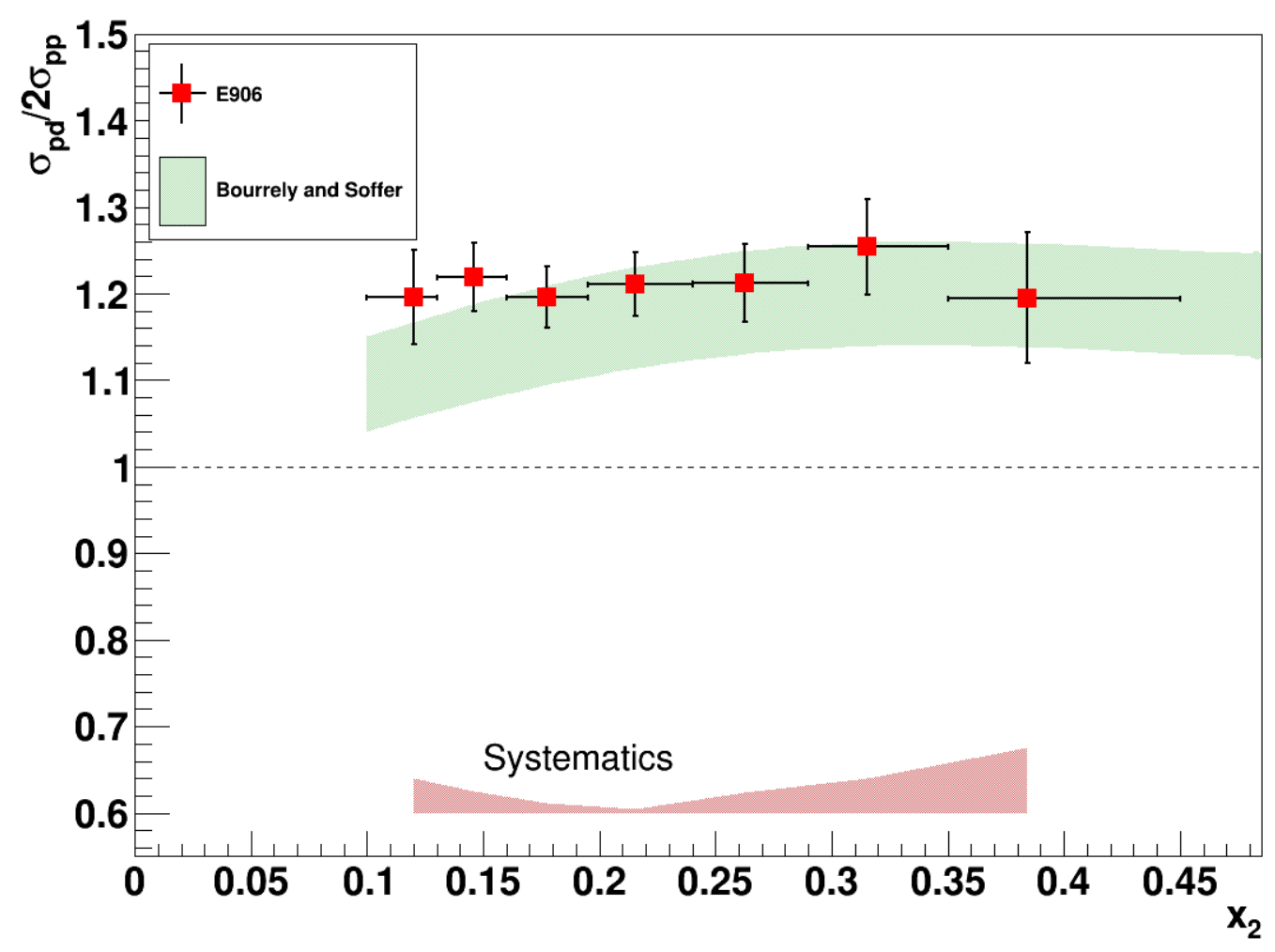

Figure 5.6: $\sigma_{p d}(x) / 2 \sigma_{p p}(x)$ vs $x_{T}$ plot comparison with the statistical parton model [66, 164]. Red points are E906 data points. The green band is for the statistical parton model predictions for SeaQuest beam energy (not acceptance). A small overall normalization correction is being studied by the collaboration. It is estimated to be from $0-3 \%$, and arises when taking into account the dead time experienced by low intensity events. 


\subsubsection{Tests for Stability of Intercepts}

Several tests were also done in order to check the stability of the cross section ratio intercepts. Fig. 5.7 shows the cross section ratio plotted as a function of different intensity ranges ranging from 0 - 64000. As can be seen from the plot, the data points are distributed around the nominal value leading to consistent results within uncertainties. The cross section ratio intercepts were also studied by varying several analysis cuts (such as $d z$, mass etc) and the intercepts were consistent within uncertainties.

\section{CSR for different Intensities}

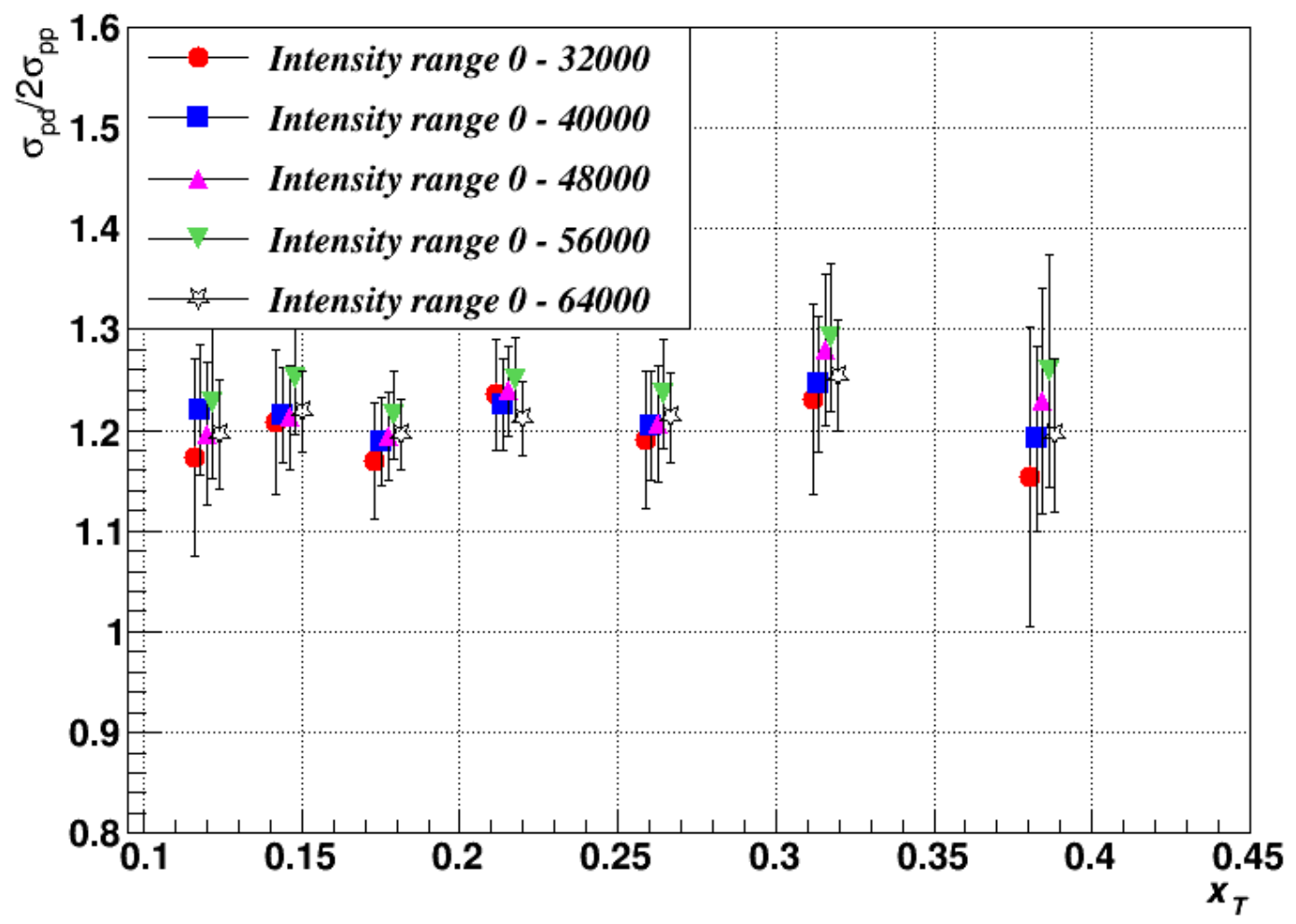

Figure 5.7: CSR vs $x_{T}$ for different trigger intensities with 8 bins for each intensity setting. 


\section{CSR for different \# of Intensity bins}

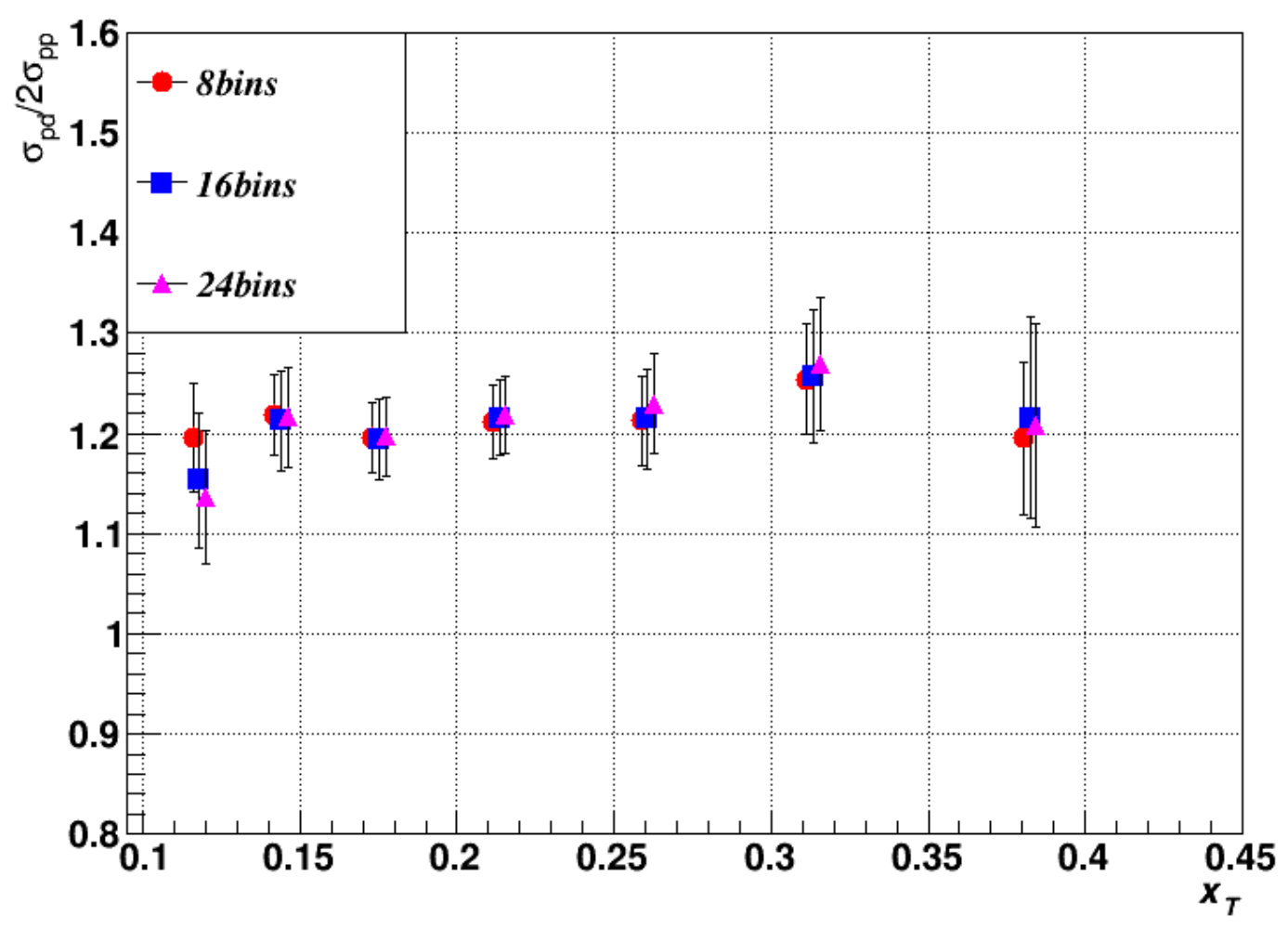

Figure 5.8: CSR vs $x_{T}$ for different number of trigger intensity bins. 


\subsection{Extracting $\bar{d}(x) / \bar{u}(x)$ from $\sigma_{p d}(x) / 2 \sigma_{p p}(x)$}

\subsection{1 $x_{\text {Beam }}$ distributions}

The Intensity-Extrapolation method provides us with the cross section ratio intercepts which are essentially "free" from rate dependence. Therefore one has to also consider the proper $x_{\text {Beam }}$ distributions to use while extracting the $\bar{d}(x) / \bar{u}(x)$ ratio. $\mathrm{LH}_{2}$ and $\mathrm{LD}_{2}$ data are separated into three intensity ranges; Low trigger intensity, Intermediate trigger intensity and high trigger intensity. Small differences have been observed between these intensities as can be seen in Figures 5.9, 5.10, 5.11 and 5.12.

In principle, these distributions could be corrected for rate dependence and perhaps the ratio $\bar{d}(x) / \bar{u}(x)$ can be extracted. Or the difference between the high and low intensity distribution extracted $\bar{d}(x) / \bar{u}(x)$ could be taken into systematic uncertainty. These differences are small compared to the systematic uncertainty arising from the extraction process and for the sake of simplicity, this thesis will consider the entire uncorrected-distribution for the $\bar{d}(x) / \bar{u}(x)$ extraction process.

Typically theoretical models use the cross section ratio, $\left\langle x_{\text {Beam }}\right\rangle$ and $\left\langle x_{F}\right\rangle$ provided by SeaQuest and employ their model based assumptions or use them in global fits for the $\bar{d}(x) / \bar{u}(x)$ extraction. However, SeaQuest has the advantage that we have the knowledge of the exact non-gaussian $x_{\text {Beam }}$ distributions. The extraction process for a Leading Order extraction is described in the next section. 


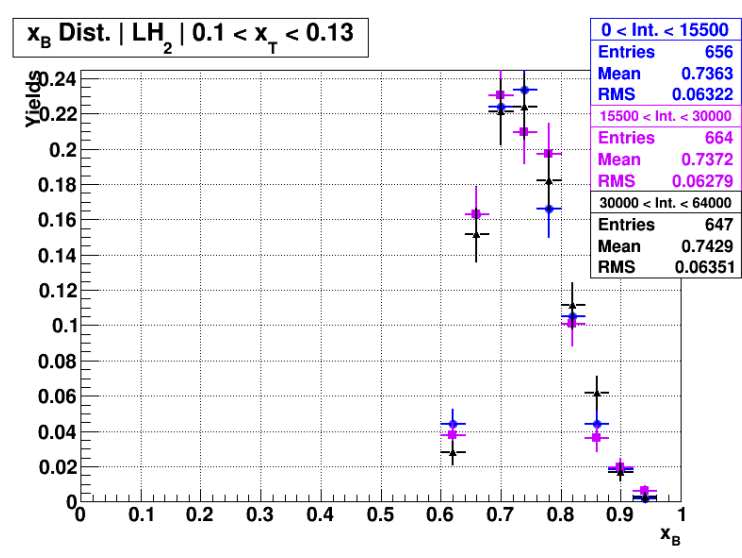

(a) $0.1<x_{T}<0.13$

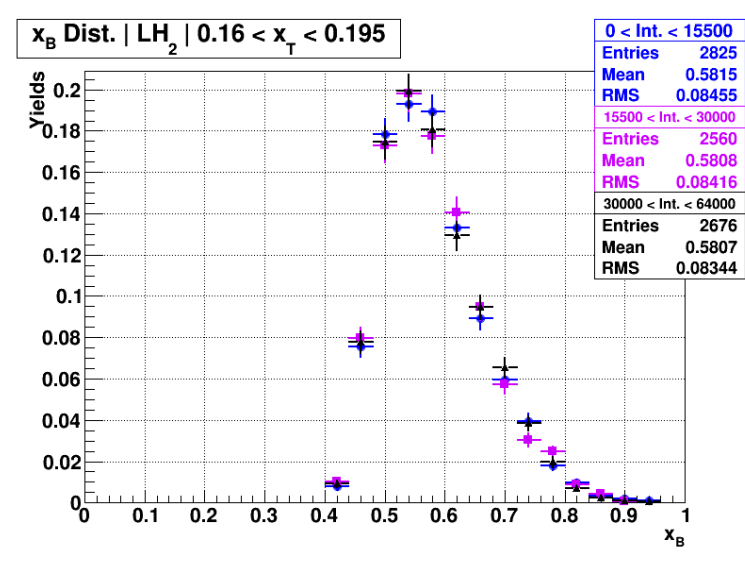

(c) $0.16<x_{T}<0.195$

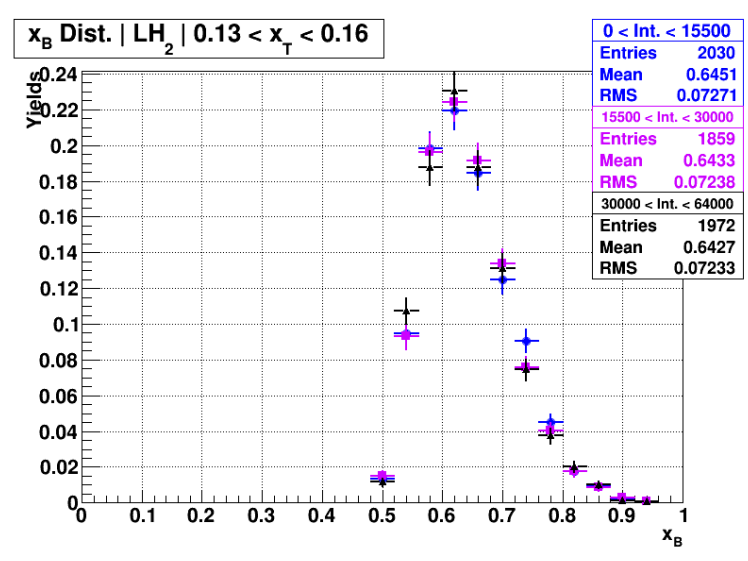

(b) $0.13<x_{T}<0.16$

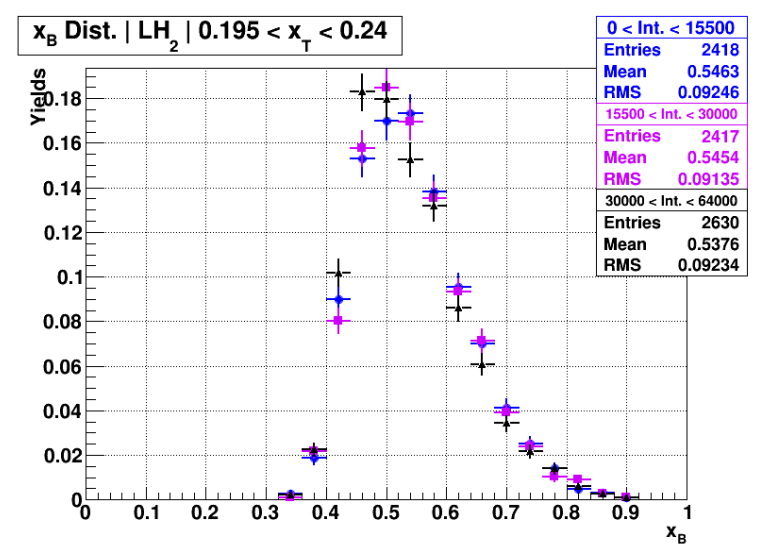

(d) $0.195<x_{T}<0.24$

Figure 5.9: $x_{\text {Beam }}$ distributions for $\mathrm{LH}_{2}$. The three colors correspond to the three trigger intensity regions; low (blue), intermediate (magenta) and high (black). All plots are normalized to their integral. 


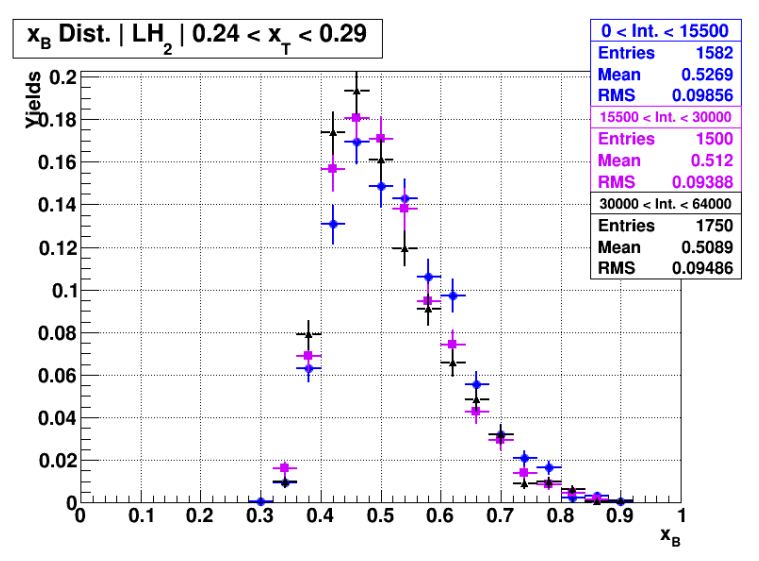

(a) $0.24<x_{T}<0.29$

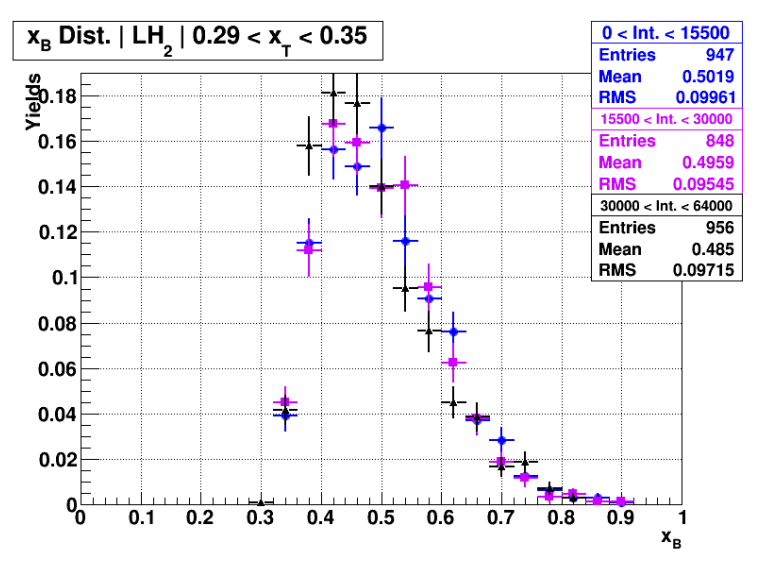

(b) $0.29<x_{T}<0.35$

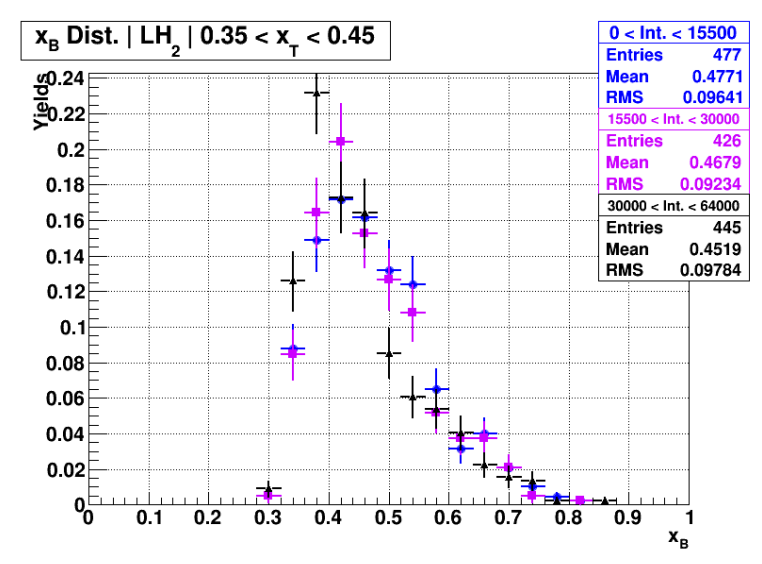

(c) $0.35<x_{T}<0.45$

Figure 5.10: $x_{\text {Beam }}$ distributions for $\mathrm{LH}_{2}$. The three colors correspond to the three trigger intensity regions; low (blue), intermediate (magenta) and high (black). All plots are normalized to their integral. 


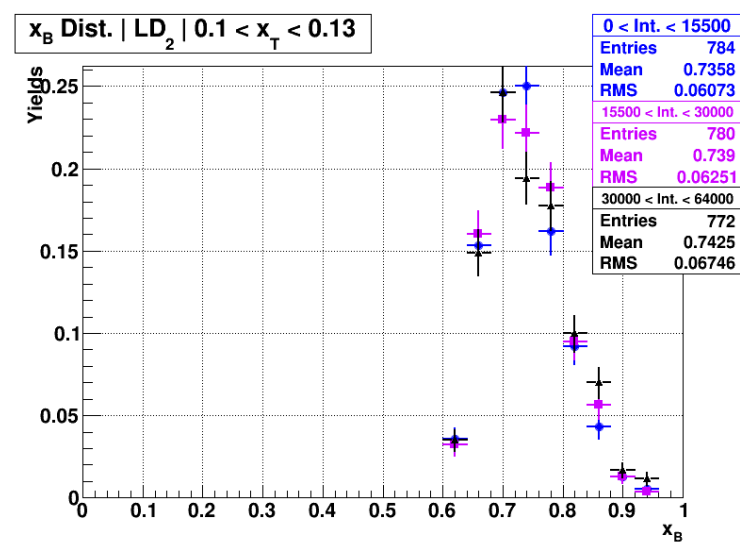

(a) $0.1<x_{T}<0.13$

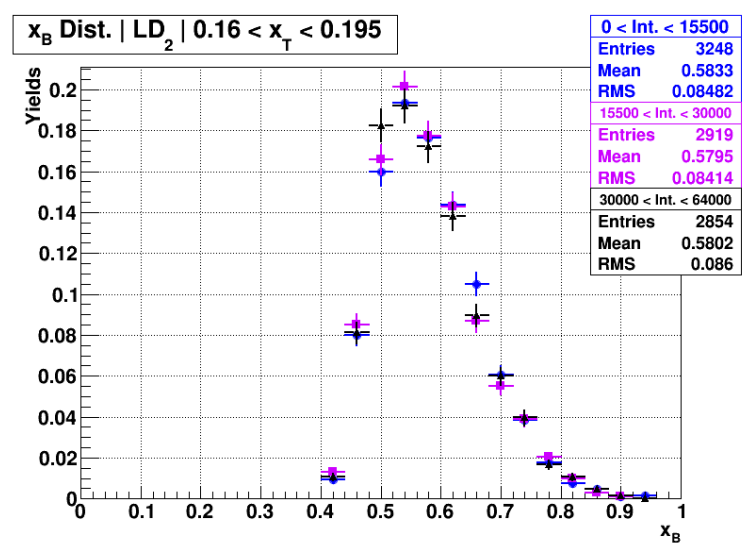

(c) $0.16<x_{T}<0.195$

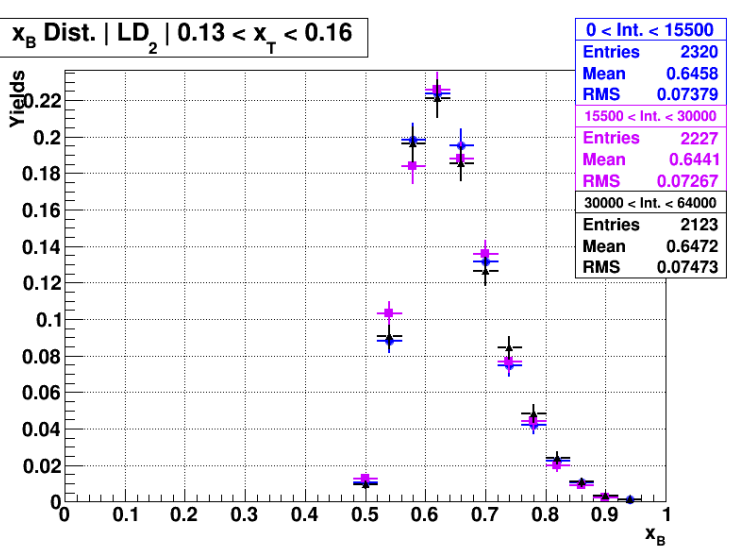

(b) $0.13<x_{T}<0.16$

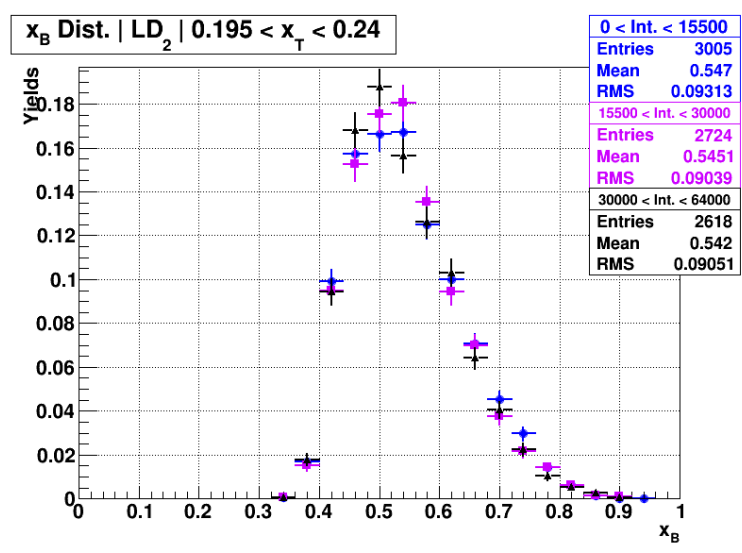

(d) $0.195<x_{T}<0.24$

Figure 5.11: $x_{\text {Beam }}$ distributions for $\mathrm{LD}_{2}$. The three colors correspond to the three trigger intensity regions; low (blue), intermediate (magenta) and high (black). All plots are normalized to their integral. 


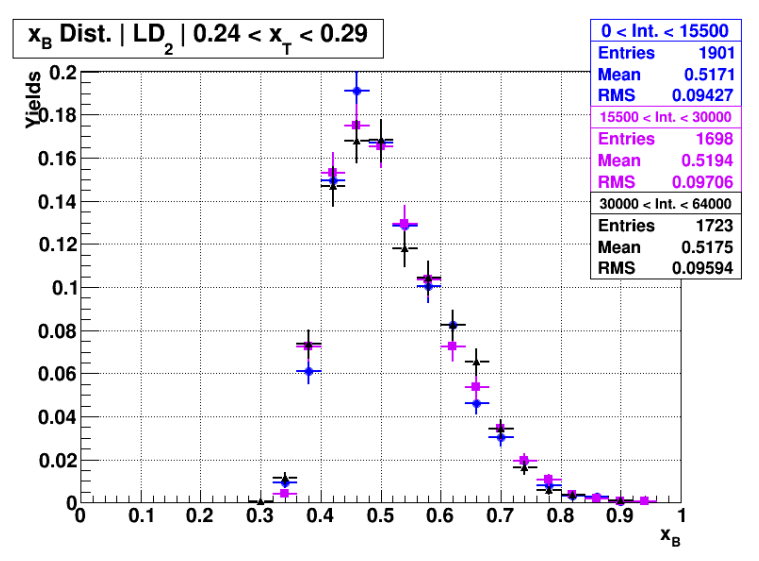

(a) $0.24<x_{T}<0.29$

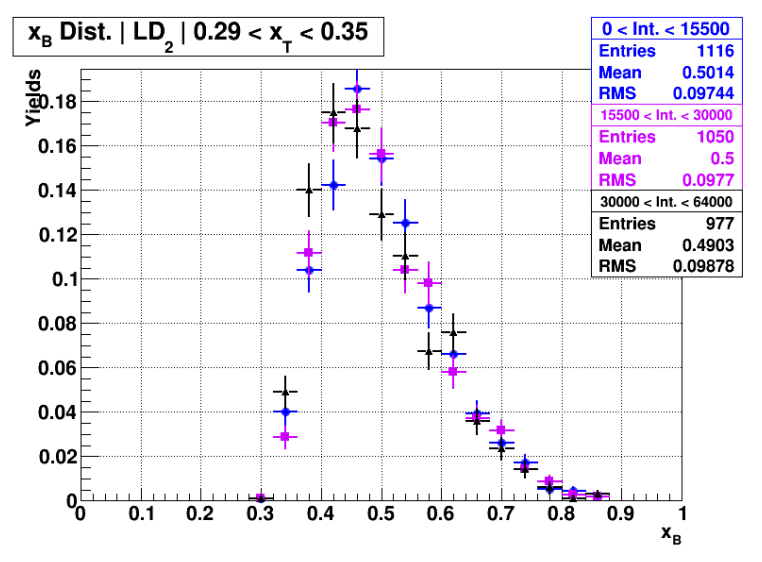

(b) $0.29<x_{T}<0.35$

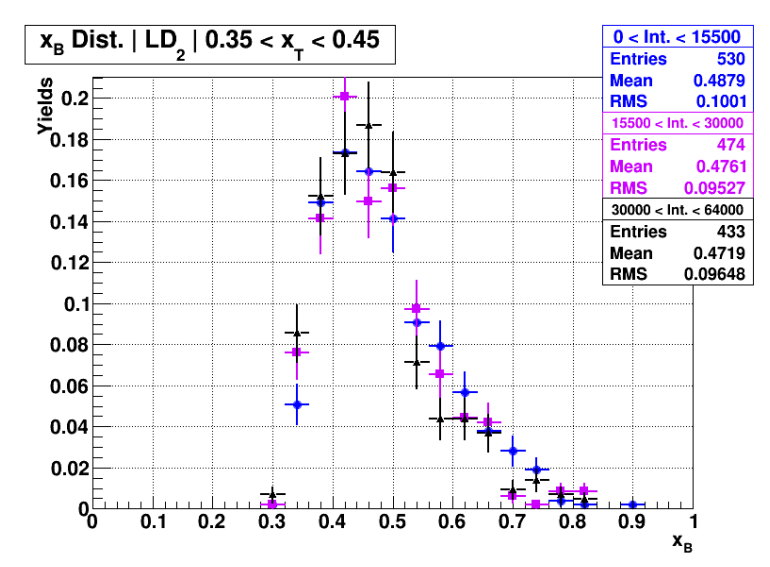

(c) $0.35<x_{T}<0.45$

Figure 5.12: $x_{\text {Beam }}$ distributions for $\mathrm{LD}_{2}$. The three colors correspond to the three trigger intensity regions; low (blue), intermediate (magenta) and high (black). All plots are normalized to their integral.

\subsection{2 $\bar{d}(x) / \bar{u}(x)$ extraction}

The original version of the code for the $\bar{d}(x) / \bar{u}(x)$ extraction used in the E866 experiment was written in FORTRAN by Dr. Paul Reimer, the co-spokesperson of SeaQuest. The code for the $\bar{d}(x) / \bar{u}(x)$ extraction used in this analysis was written by Dr. Shou Miyasaka, Prof. Kenichi Nakano (and tweaked by Dr. Kei Nagai) by SeaQuest collaborators from Tokyo Institute of Technology. It was developed for the previous preliminary release of the SeaQuest results. Instead of rewriting the entire code, it was simply integrated with other code so as to grab the cross section ratio intercepts and extract the desired ratio. The 
extraction code has the capability to do a LO (Leading Order) as well as an NLO (Next-toLeading Order) extraction. The code can be adjusted to accommodate different PDF sets as well.

The extraction of $\bar{d}(x) / \bar{u}(x)$ is done using an iterative procedure with many underlying assumptions:

- Charge symmetry is assumed i.e. $u_{p}(x)=d_{n}(x)$ and vice versa.

- $\bar{d}(x)+\bar{u}(x)$ is fixed by the chosen PDF set.

- $\sigma_{p d} \approx \sigma_{p p}+\sigma_{p n}$. The deuteron is simply a convolution of a proton and neutron without the inclusion of any nuclear effects.

- $c(x)=\bar{c}(x)$ and $s(x)=\bar{s}(x)$.

- The $c$ and $s$ in proton and neutron are the same.

- Contributions from the heavier quarks such as top and bottom are ignored.

The essential steps of the extraction code are given as follows:

1. The cross section formula is written in terms of the parton distribution functions.

2. Inputs for $u(x), d(x), s(x), c(x)$ and $\bar{d}(x)+\bar{u}(x)$ are taken from the PDF set used.

3. $x_{B}$ value for each dimuon is used and an initial guess at $\bar{d}(x) / \bar{u}(x)$ is made.

4. From the mass, $x_{B}$ and $x_{T}$, the cross section ratio is calculated and compared to the data cross section ratio (intercepts from Intensity-Extrapolation method).

5. These two values are compared and the iterative process is repeated until the difference between them is $<10^{-4}$.

6. This is done for all the dimuons of interest.

7. The value of $\bar{d}(x) / \bar{u}(x)$ for a particular $x_{T}$ bin is a distribution of values. 
8. The average value of the ratio $\bar{d}(x) / \bar{u}(x)$ in each bin is the value of $\bar{d}(x) / \bar{u}(x)$ for the bin.

For the LO extraction, the cross sections of $\sigma_{p p}, \sigma_{p n}$ and $\sigma_{p d}$ can be written as:

$$
\begin{aligned}
\sigma_{p p} & \propto 4 u_{p}\left(x_{B}\right) \bar{u}_{p}\left(x_{T}\right)+4 \bar{u}_{p}\left(x_{B}\right) u_{p}\left(x_{T}\right)+d_{p}\left(x_{B}\right) \bar{d}_{p}\left(x_{T}\right) \\
& +\bar{d}_{p}\left(x_{B}\right) d_{p}\left(x_{T}\right)+8 c_{p}\left(x_{B}\right) \bar{c}_{p}\left(x_{T}\right)+2 s_{p}\left(x_{B}\right) s_{p}\left(x_{T}\right) \\
\sigma_{p n} & \propto 4 u_{p}\left(x_{B}\right) \bar{u}_{n}\left(x_{T}\right)+4 \bar{u}_{p}\left(x_{B}\right) u_{n}\left(x_{T}\right)+d_{p}\left(x_{B}\right) \bar{d}_{n}\left(x_{T}\right) \\
& +\bar{d}_{p}\left(x_{T}\right) d_{n}\left(x_{B}\right)+8 c_{p}\left(x_{B}\right) \bar{c}_{n}\left(x_{T}\right)+2 s_{p}\left(x_{B}\right) s_{n}\left(x_{T}\right) \\
\sigma_{p d} & =\sigma_{p p}+\sigma_{p n}
\end{aligned}
$$

After using charge symmetry arguments, $\sigma_{p n}$ can be rewritten as:

$$
\begin{aligned}
\sigma_{p n} & \propto 4 u_{p}\left(x_{B}\right) \bar{d}_{p}\left(x_{T}\right)+4 \bar{u}_{p}\left(x_{B}\right) d_{p}\left(x_{T}\right)+d_{p}\left(x_{B}\right) \bar{u}_{p}\left(x_{T}\right) \\
& +\bar{d}_{p}\left(x_{T}\right) u_{p}\left(x_{B}\right)+8 c_{p}\left(x_{B}\right) \bar{c}_{p}\left(x_{T}\right)+2 s_{p}\left(x_{B}\right) s_{p}\left(x_{T}\right)
\end{aligned}
$$

For simplicity, the subscript $p$ will be dropped. The $\bar{u}(x)$ and $\bar{u}(x)$ are calculated using the expressions below:

$$
\begin{aligned}
& \bar{u}(x)=\frac{[\bar{u}(x)+\bar{d}(x)]_{\mathrm{PDF}}}{\left[\frac{\bar{d}(x)}{\bar{u}(x)}\right]_{\mathrm{EST}}+1} \\
& \bar{d}(x)=\left[\frac{\bar{d}(x)}{\bar{u}(x)}\right]_{\mathrm{EST}} \cdot \bar{u}(x)
\end{aligned}
$$

where $\left[\frac{\bar{d}(x)}{\bar{u}(x)}\right]_{\mathrm{EST}}$ is the estimate value of the ratio and $[\bar{d}(x)+\bar{u}(x)]_{\mathrm{PDF}}$ is the sum which is restricted by the PDF. An initial value for the ratio is used to start off the iteration process. Each subsequent iteration takes the value from the previous iteration in calculating the cross section ratio. The values of the $\bar{d}(x) / \bar{u}(x)$ for the ranges outside of $0.1<x_{T}<0.45$ are set to be 1 in this case. These values are plugged into the above equations and the cross section 
ratio is calculated. This is compared to the original intercept value and the iterative process is repeated. The NLO extraction involves a more complicated equation for the calculation of the cross section ratios.

\subsubsection{Statistical Uncertainties}

As can be seen equation 5.3.2, the terms in one $x_{T}$ bin involves terms coming from another $x_{T}$ bin (for example, the $u\left(x_{T}\right) \bar{u}\left(x_{B}\right)$ and $\bar{u}\left(x_{T}\right) u\left(x_{B}\right)$ terms for a given $x_{T}$ ). Therefore the statistical uncertainties are calculated by changing the statistical uncertainty in another bin and calculating the change in the considered bin. This process is repeated for all the bins and the differences in the change of the considered bin are added in quadrature. The procedure is described below ${ }^{3}$ :

1. Shift the cross section ratio at bin $j$ by its statistical uncertainty upwards.

2. Calculate the $\bar{d} / \bar{u}_{\mathrm{NEW}+}$ at the $i$ th bin.

3. Take the difference between $\bar{d} / \bar{u}_{\mathrm{NEW}+}$ and $\bar{d} / \bar{u}_{\mathrm{OLD}}$

4. Shift the cross section ratio at bin $j$ by its statistical uncertainty downwards.

5. Calculate the $\bar{d} / \bar{u}_{\mathrm{NEW}}$ - at the $i$ th bin.

6. Take the difference between $\bar{d} / \bar{u}_{\mathrm{NEW}-}$ and $\bar{d} / \bar{u}_{\mathrm{OLD}}$

7. Take the average of the two values, $\bar{d} / \bar{u}_{\mathrm{NEW}+}$ and $\bar{d} / \bar{u}_{\mathrm{NEW}-}$. This is the statistical uncertainty for the $j$ th bin.

\subsubsection{Systematic Uncertainties}

Only one source of systematic uncertainty is considered here:

- Systematics coming from the cross section ratio. The systematic uncertainties for this source is calculated in the same way as the statistical uncertainties.

\footnotetext{
${ }^{3}$ The procedure in calculating the statistical and systematic uncertainties is adapted from [141]
} 
- Systematic uncertainty coming from the $x_{T}<0.1$ and above $x_{T}>0.45$ region is not considered in this analysis.

\section{$5.4 \bar{d}(x) / \bar{u}(x)$ Results}

Fig. 5.13 gives a "first look" at the $\bar{d}(x) / \bar{u}(x)$ ratio extracted using the technique mentioned in the previous section. The SeaQuest collaboration is still considering refining the technique and the underlying assumptions in the extraction, so the numbers shown not the final values. Table 5.3 show the values of $\bar{d}(x) / \bar{u}(x)$ along with the systematic and statistical uncertainties for the Next-to-Leading Order extraction of $\bar{d}(x) / \bar{u}(x)$ from the ratio of cross sections. The results indicate that the data points are above 1 for all $x_{T}$ regions and are monotonically rising within the measured $x_{T}$ range. It should be noted that the small overall normalization correction that was mentioned earlier was not considered in the extraction process. However this doesn't change the trend of the data points.

\begin{tabular}{|c|c|c|c|c|c|}
\hline$\left\langle x_{T}\right\rangle$ & $\bar{d}(x) / \bar{u}(x)$ & $\sigma_{\text {stat }}^{+}$ & $\sigma_{\text {stat }}^{-}$ & $\sigma_{\text {syst }}^{+}$ & $\sigma_{\text {syst }}^{-}$ \\
\hline 0.1199 & 1.4178 & 0.0948 & 0.0822 & 0.0780 & 0.0759 \\
\hline 0.1460 & 1.4734 & 0.0762 & 0.0751 & 0.0527 & 0.0517 \\
\hline 0.1773 & 1.4668 & 0.0792 & 0.0905 & 0.0254 & 0.0267 \\
\hline 0.2156 & 1.5323 & 0.0900 & 0.0972 & 0.0162 & 0.0162 \\
\hline 0.2627 & 1.5993 & 0.1179 & 0.1234 & 0.0610 & 0.0627 \\
\hline 0.3155 & 1.8115 & 0.1535 & 0.1457 & 0.1196 & 0.1164 \\
\hline 0.3844 & 1.8425 & 0.2810 & 0.2398 & 0.2601 & 0.2480 \\
\hline
\end{tabular}

Table 5.3: Table of $\bar{d}(x) / \bar{u}(x)$ values along with statistical and systematic uncertainties. CT10NLO PDF set was used in this extraction. The systematic uncertainties coming from the cross section ratio are propagated. Additional systematics from the value of PDF above 0.45 and below 0.1 not considered. 


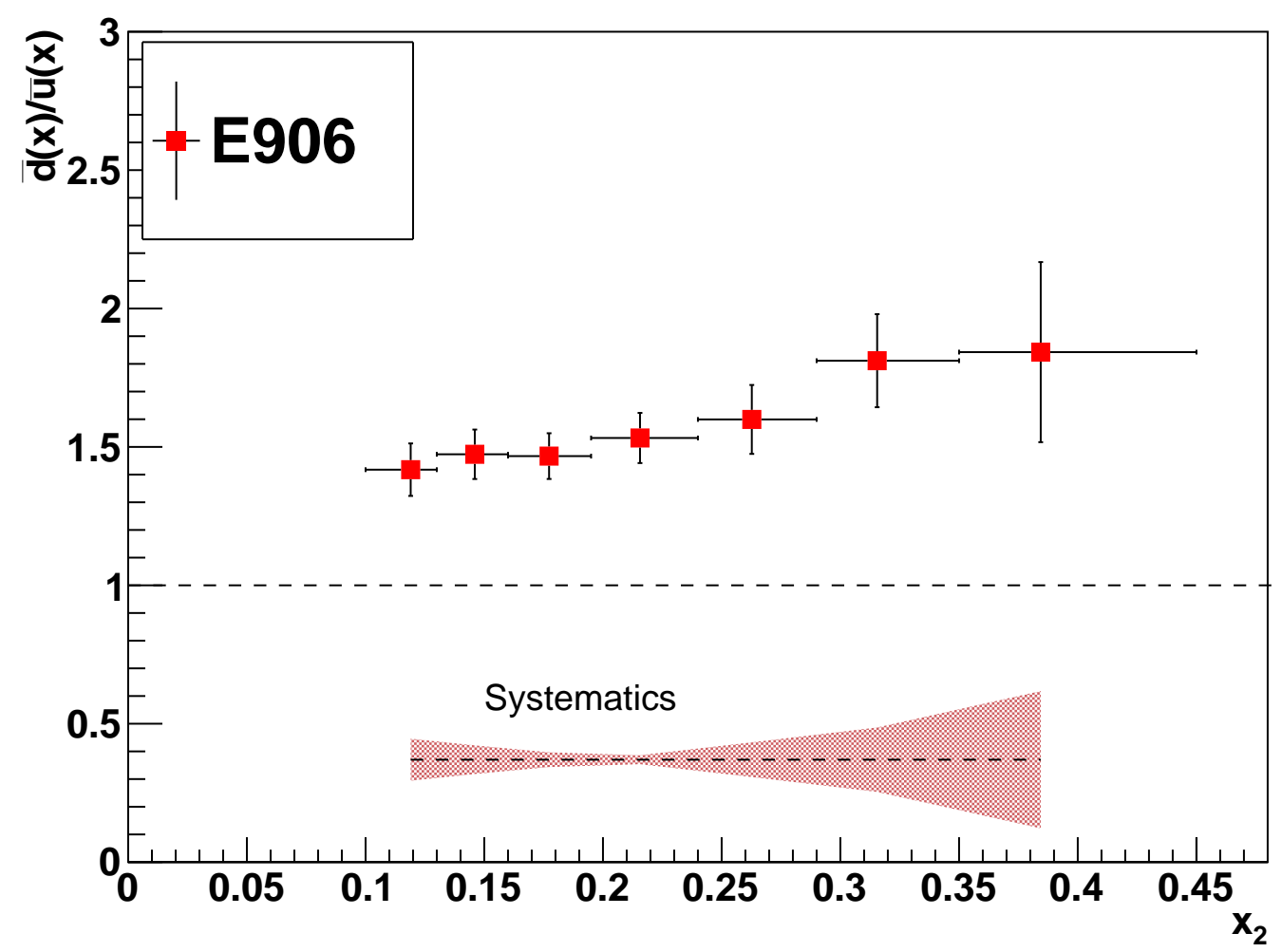

Figure 5.13: $\bar{d}(x) / \bar{u}(x)$ plot for SeaQuest. Next-to-Leading Order extraction was done using CT10NLO PDF set.

\subsubsection{Comparison of $\bar{d}(x) / \bar{u}(x)$ with E866}

Two main questions come up while comparing with the $\bar{d}(x) / \bar{u}(x)$ ratio with E866 [57]. Is the overturn at $\approx 0.2$ as observed in E866 real? And does the ratio go below 1 at $x_{T} \approx 0.3$ like E866? Some key differences between the two experiments are:

- SeaQuest is statistically more robust in the region $x_{T}>0.2$ compared to E866.

- The two experiments ran at different beam energies giving rise to a different value of $\left\langle Q^{2}\right\rangle$ per $x_{T}$ bin. The $\left\langle Q^{2}\right\rangle$ for $\mathrm{E} 866$ is $\approx 56 \mathrm{GeV}^{2}$ and the $\left\langle Q^{2}\right\rangle$ for SeaQuest is $\approx 19 \mathrm{GeV}^{2}$. Therefore, one has to evolve in $\left\langle Q^{2}\right\rangle$ to compare the two $\bar{d}(x) / \bar{u}(x)$ ratios. But studies have shown that the $\left\langle Q^{2}\right\rangle$ evolution does not contribute much to such a big difference as seen by the two experiments. 
- The two experiments have different acceptances.

- The two experiments access different $x_{\text {Beam }}$ distributions for the same given $x_{T}$.

From the trend of the results shown in Fig. 5.14, the $\bar{d}(x) / \bar{u}(x)$ ratio has a monotonic rise unlike the E866 experiment which shows an overturn and a (statistically limited) drop below 1.

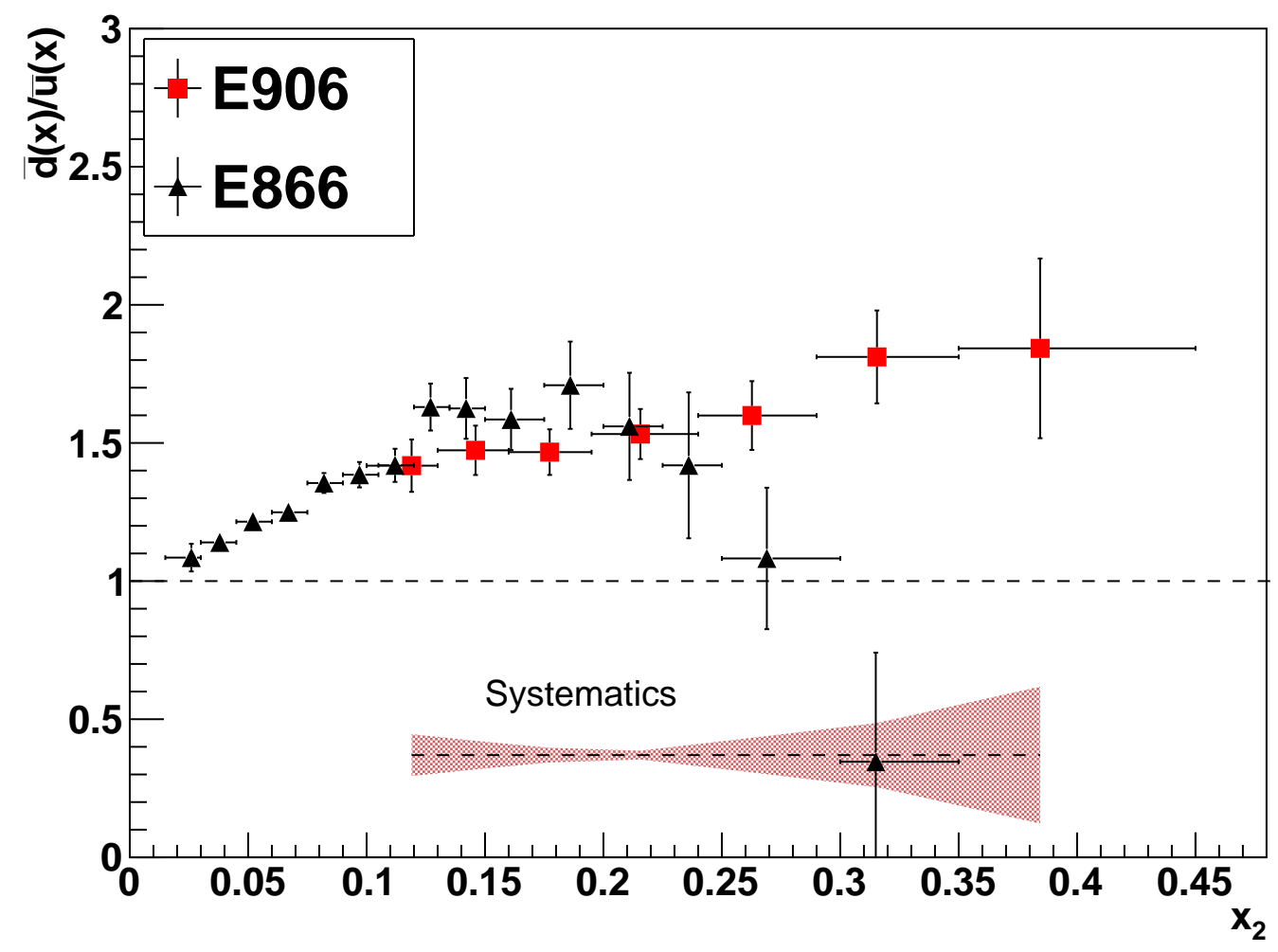

Figure 5.14: $\bar{d}(x) / \bar{u}(x)$ comparison between SeaQuest and NuSea experiments. The red data points are for SeaQuest and the black triangles are for NuSea. Systematic uncertainty band for SeaQuest is shown at the bottom of the plot. Systematic uncertainty band for E866 is not shown in the plot. SeaQuest data points indicate an increase with increasing $x_{T}$ unlike NuSea [57] which indicates an overturn and a statistically limited drop below 1 . It should be noted that the small overall normalization correction was not considered in the extraction process. However this does not change the overall trend of the SeaQuest data points. 


\subsubsection{Comparison of $\bar{d}(x) / \bar{u}(x)$ with Theoretical Models}

At present, these two models (statistical parton model and the pion cloud model) follow the same trend as SeaQuest data points. However, data from SeaQuest could be used to tweak the parameters for other models as well.

1. The statistical parton model [66] considers the nucleon as a gas of massless partons (quarks, anti-quarks and gluons) in equilibrium at a given temperature in a finite volume. It predicts a monotonic increase in the $\bar{d}(x) / \bar{u}(x)$ ratio. In the measured region, their data band agrees with SeaQuest.

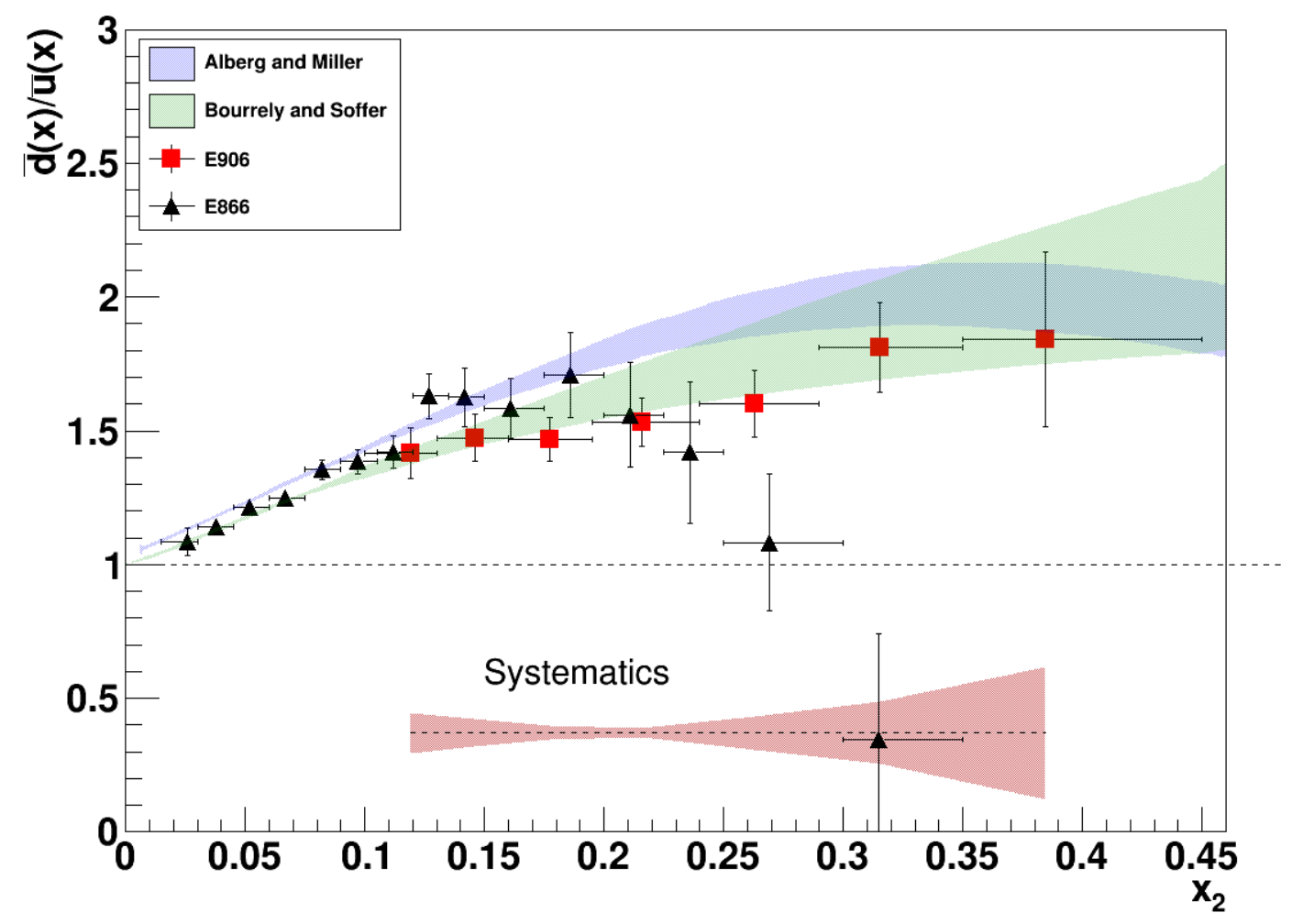

Figure 5.15: $\bar{d}(x) / \bar{u}(x)$ for SeaQuest along with curves for the meson cloud model (blue band) and the statistical parton model (green band) [165, 164]. Black triangles are E866 data points. Systematic uncertainty band for E866 is not shown [57].

2. The meson cloud model [61] rewrites the proton (under one meson approximation) as a linear combination of several different fluctuations of baryon virtual meson Fock 
states. If $p_{0}$ is the bare proton with a symmetric sea, the proton can be written as:

$$
|p\rangle=\alpha\left|p_{0}\right\rangle+\beta\left|p_{0} \pi^{0}\right\rangle+\gamma\left|n \pi^{+}\right\rangle+\delta\left|\Delta^{++} \pi^{-}\right\rangle+\ldots
$$

Although the meson cloud model predicts a drop in the ratio at a much higher value of $x$, in the measured region, their data band agrees with SeaQuest data trend.

\subsubsection{Future Experiments}

While statistical parton model and the meson cloud model agree with the SeaQuest $\bar{d}(x) / \bar{u}(x)$ trend, our data could be used to tweak the parameters for other theoretical models as well. However, each of these models also have a prediction for the spin and angular momentum contribution of the anti-quarks and experiments at Jefferson Lab, Fermilab, RHIC, CERN and perhaps JPARC are planned to pursue this measurement $[1,166]$. 


\subsection{Nuclear Dependence: $R_{p A}=2 \sigma_{p A} / A \sigma_{p d}$}

\subsection{1 $x_{T}$ dependence:}

A similar approach is taken for the nuclear targets in calculating the $R_{p A}$ for carbon, iron, and tungsten for different $x_{T}$ bins. The fit results can be found in Figures 5.16 and 5.17 for carbon, 5.18 and 5.19 for iron, and 5.20, and 5.21 for tungsten. A small overall normalization correction is being studied by the collaboration. It is estimated to be from $0-3 \%$, and arises when taking into account the dead time experienced by low intensity events.

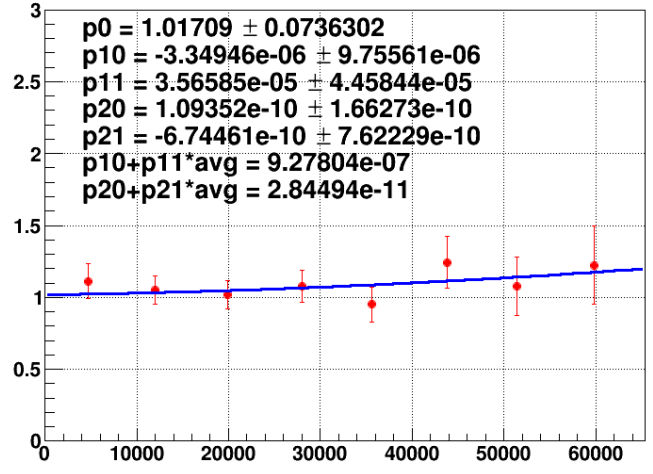

(a) $0.1<x_{T}<0.13$

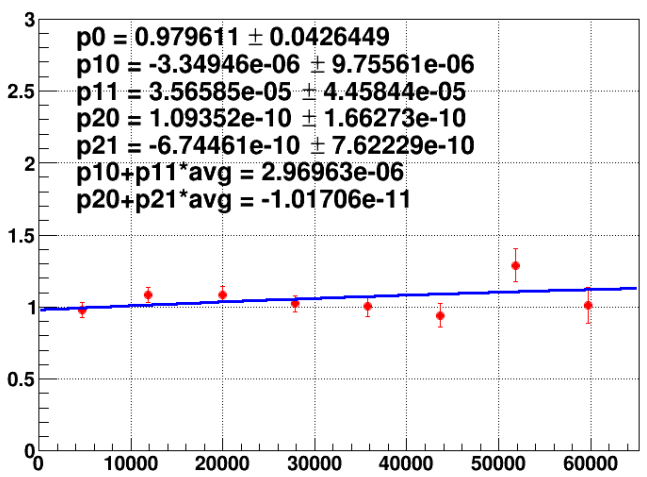

(c) $0.16<x_{T}<0.195$

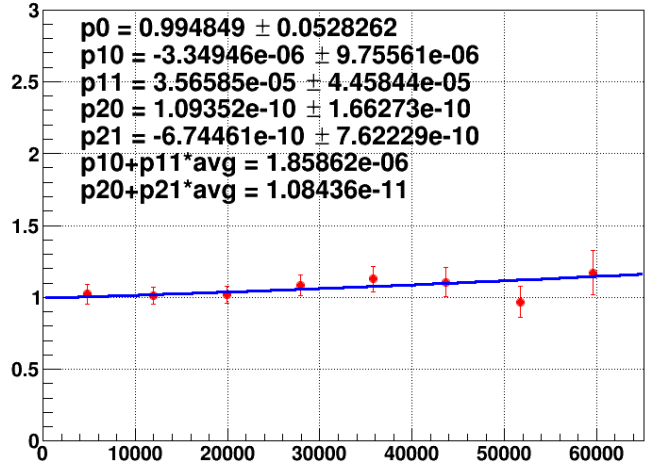

(b) $0.13<x_{T}<0.16$

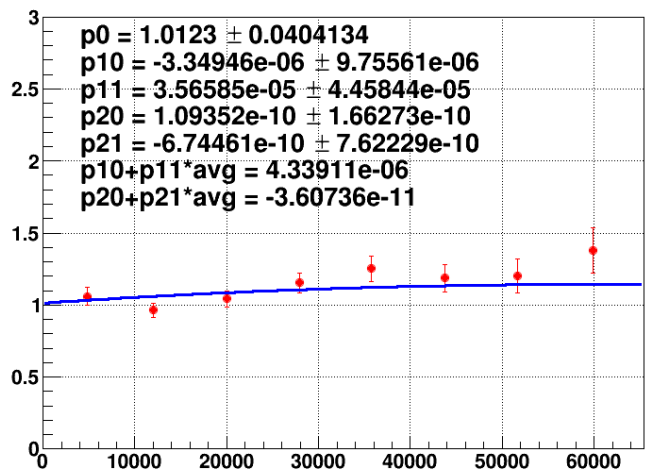

(d) $0.195<x_{T}<0.24$

Figure 5.16: $R_{p A}$ for carbon for different $x_{T}$ bins. $F=p 0+\left(p 10+p 11 *\left\langle x_{T}\right\rangle\right) *$ inte + $\left(p 20+p 21 *\left\langle x_{T}\right\rangle\right) * i n t e^{2}$ fit was used for the extrapolation. Note that $p 10, p 11, p 20$ and $p 21$ are common for all fits. 


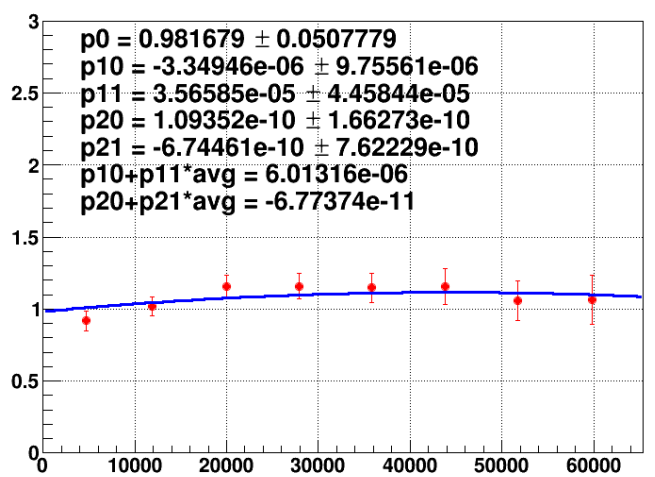

(a) $0.24<x_{T}<0.29$

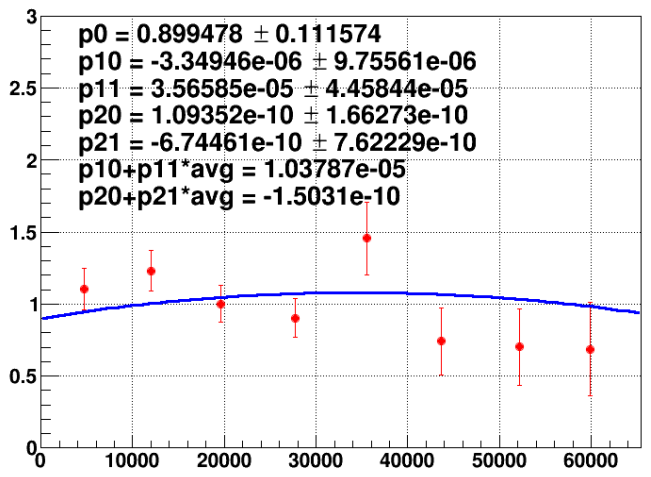

(c) $0.35<x_{T}<0.45$

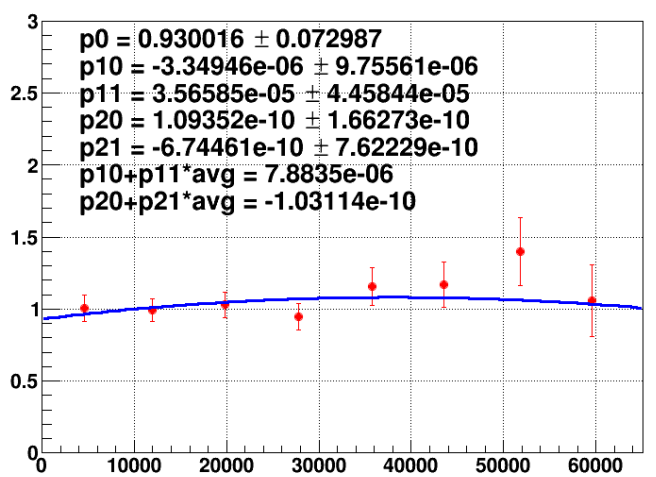

(b) $0.29<x_{T}<0.35$

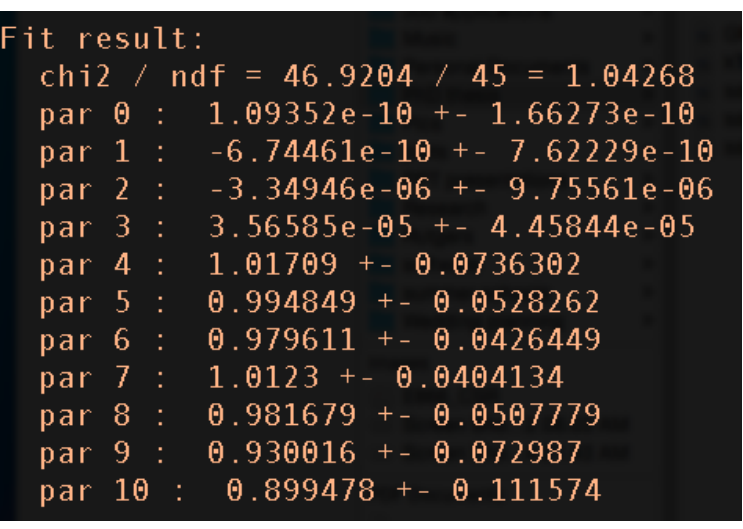

(d) Fit results

Figure 5.17: $R_{p A}$ for carbon for different $x_{T}$ bins. $F=p 0+\left(p 10+p 11 *\left\langle x_{T}\right\rangle\right) *$ inte + $\left(p 20+p 21 *\left\langle x_{T}\right\rangle\right) * i n t e^{2}$ fit was used for the extrapolation. Note that $p 10, p 11, p 20$ and $p 21$ are common for all fits. 


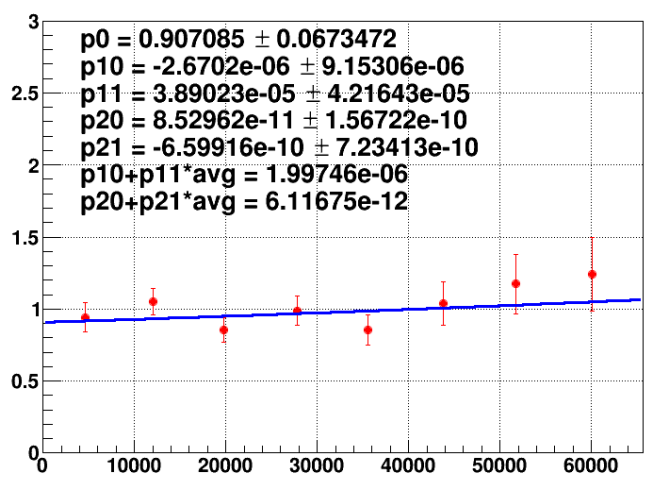

(a) $0.1<x_{T}<0.13$

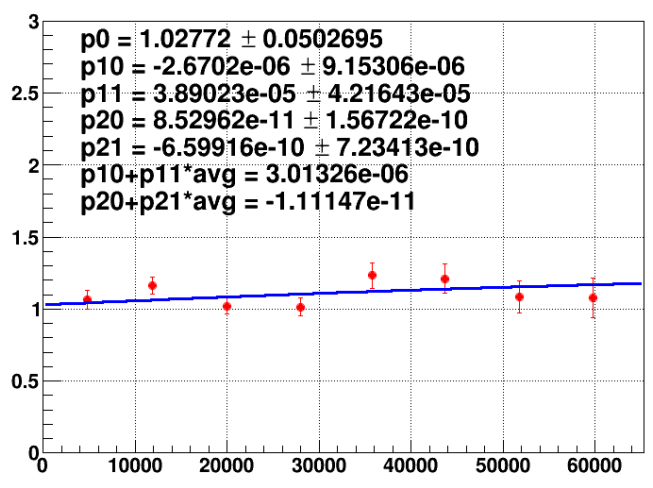

(b) $0.13<x_{T}<0.16$

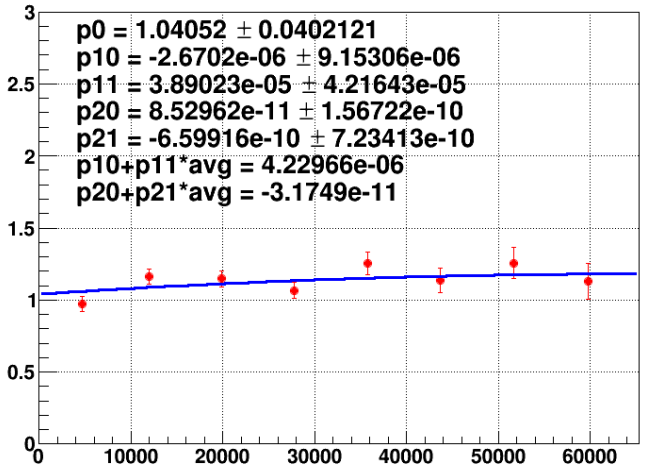

(c) $0.16<x_{T}<0.195$

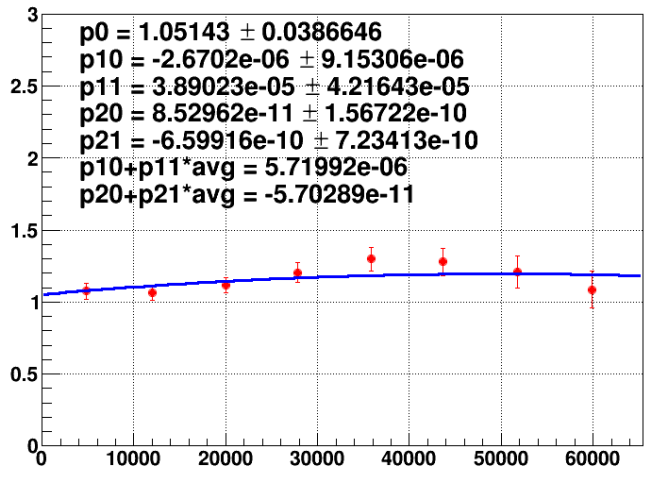

(d) $0.195<x_{T}<0.24$

Figure 5.18: $R_{p A}$ for iron for different $x_{T}$ bins. $F=p 0+\left(p 10+p 11 *\left\langle x_{T}\right\rangle\right) *$ inte + $\left(p 20+p 21 *\left\langle x_{T}\right\rangle\right) * i n t e^{2}$ fit was used for the extrapolation. Note that $p 10, p 11, p 20$ and p21 are common for all fits. 


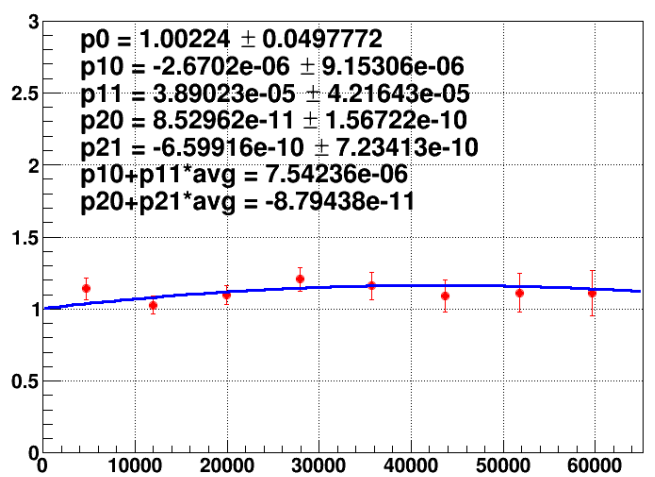

(a) $0.24<x_{T}<0.29$

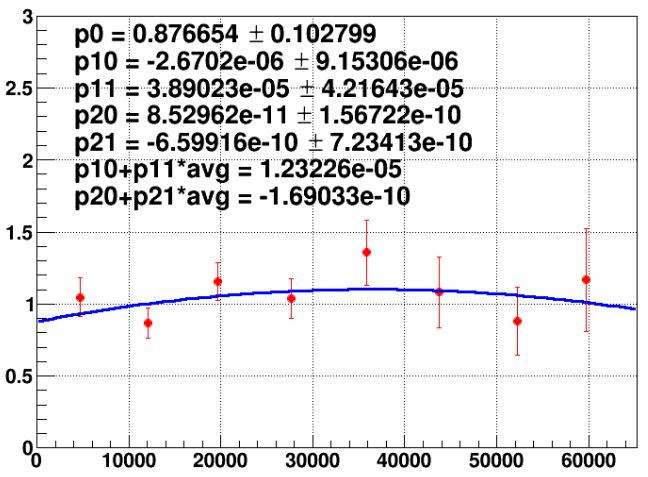

(c) $0.35<x_{T}<0.45$

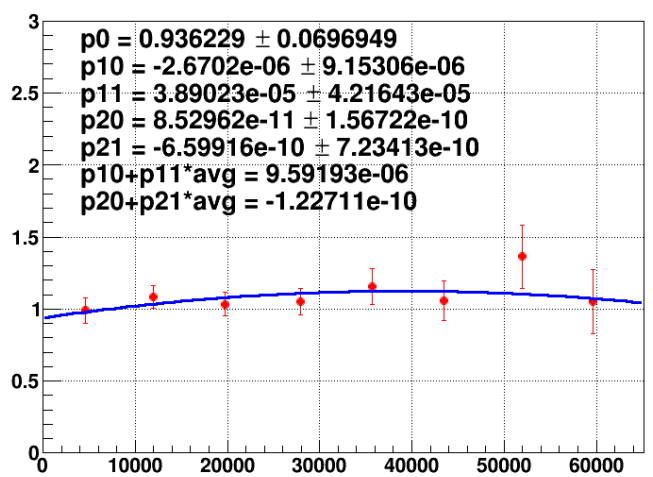

(b) $0.29<x_{T}<0.35$

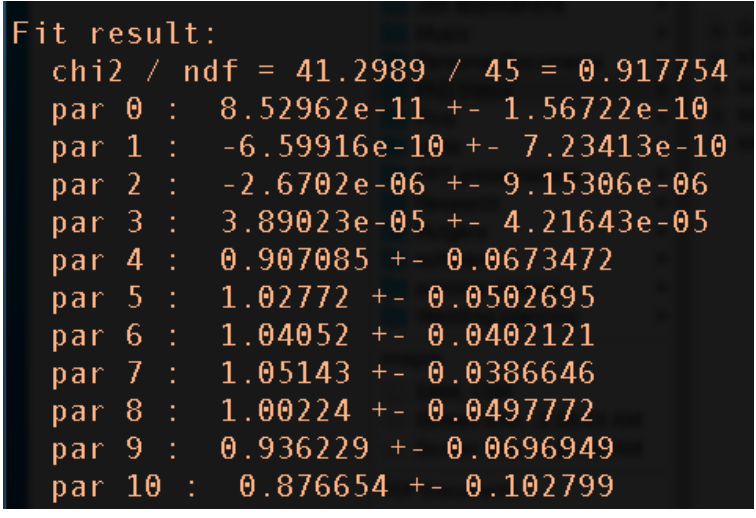

(d) Fit results

Figure 5.19: $R_{p A}$ for iron for different $x_{T}$ bins. $F=p 0+\left(p 10+p 11 *\left\langle x_{T}\right\rangle\right) *$ inte + $\left(p 20+p 21 *\left\langle x_{T}\right\rangle\right) * i n t e^{2}$ fit was used for the extrapolation. Note that $p 10, p 11, p 20$ and p21 are common for all fits. 


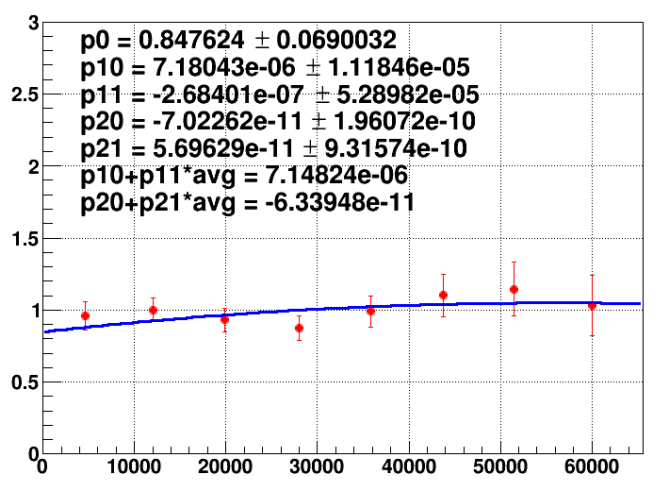

(a) $0.1<x_{T}<0.13$

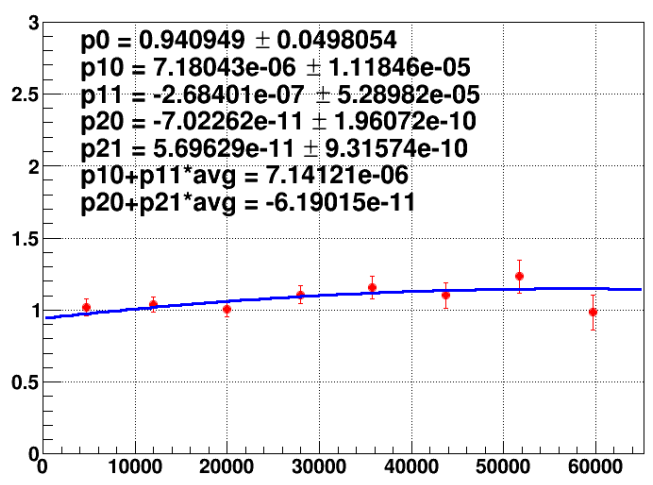

(b) $0.13<x_{T}<0.16$

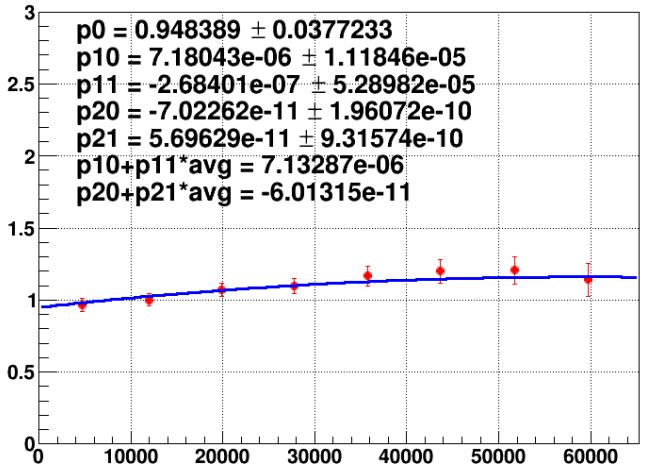

(c) $0.16<x_{T}<0.195$

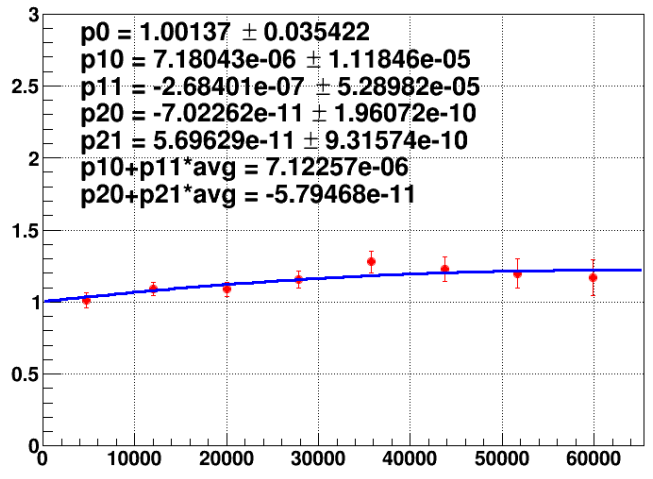

(d) $0.195<x_{T}<0.24$

Figure 5.20: $R_{p A}$ for tungsten for different $x_{T}$ bins. $F=p 0+\left(p 10+p 11 *\left\langle x_{T}\right\rangle\right) *$ inte + $\left(p 20+p 21 *\left\langle x_{T}\right\rangle\right) * i n t e^{2}$ fit was used for the extrapolation. Note that $p 10, p 11, p 20$ and $p 21$ are common for all fits. 


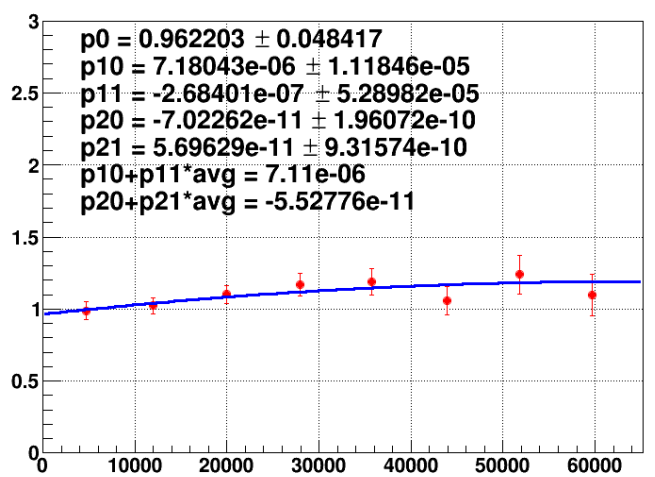

(a) $0.24<x_{T}<0.29$

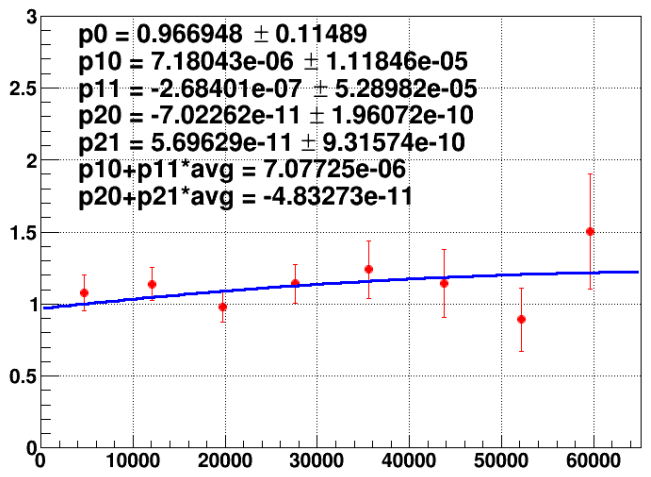

(c) $0.35<x_{T}<0.45$

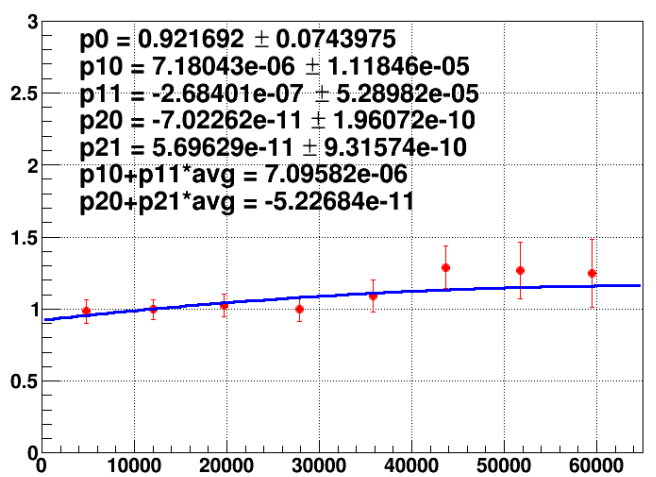

(b) $0.29<x_{T}<0.35$

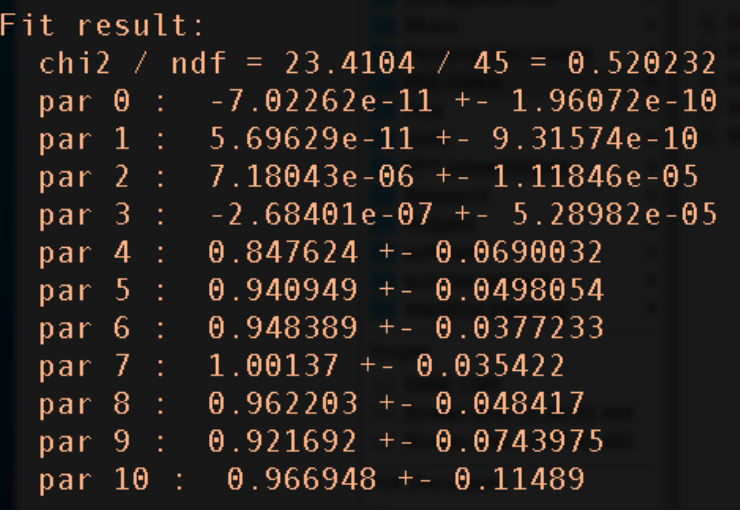

(d) Fit results

Figure 5.21: $R_{p A}$ for tungsten for different $x_{T}$ bins. $F=p 0+\left(p 10+p 11 *\left\langle x_{T}\right\rangle\right) *$ inte + $\left(p 20+p 21 *\left\langle x_{T}\right\rangle\right) * i n t e^{2}$ fit was used for the extrapolation. Note that $p 10, p 11, p 20$ and $p 21$ are common for all fits. 

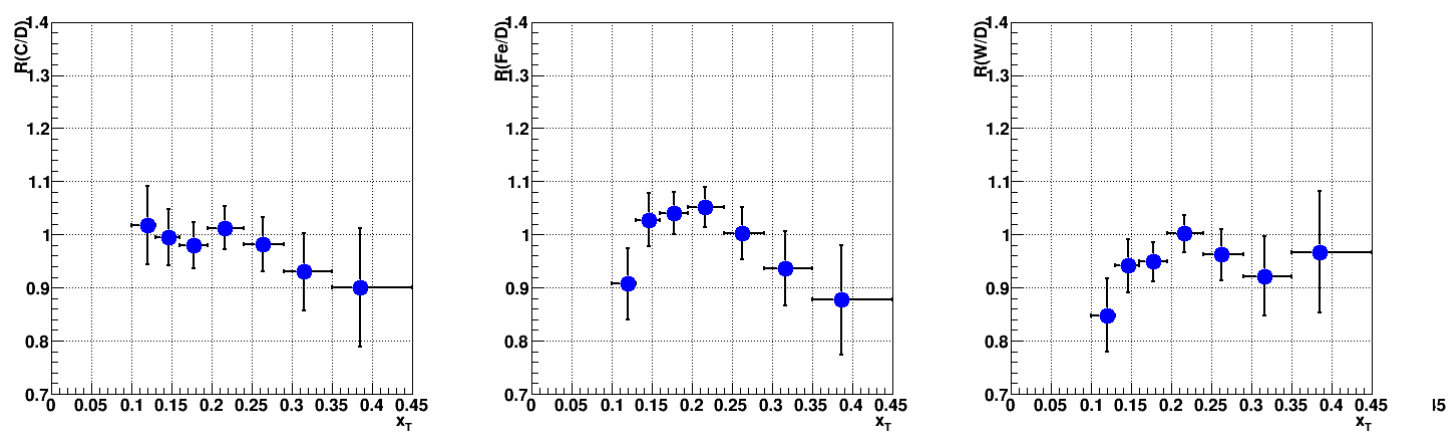

Figure 5.22: $R_{p A}$ vs $x_{T}$ for carbon (left), iron (center) and tungsten (right). No isoscalar corrections have been applied to the targets. Only statistical uncertainties are shown. A small overall normalization correction is being studied by the collaboration. It is estimated to be from $0-3 \%$, and arises when taking into account the dead time experienced by low intensity events. $F=p 0+\left(p 10+p 11 *\left\langle x_{T}\right\rangle\right) *$ inte $+\left(p 20+p 21 *\left\langle x_{T}\right\rangle\right) * i n t e^{2}$ fit was used for the extrapolation.

Fig. 5.22 shows a panel of the per nucleon cross section ratio comparison for $\mathrm{C} / \mathrm{LD}_{2}$ (left), $\mathrm{Fe} / \mathrm{LD}_{2}$ (center) and $\mathrm{W} / \mathrm{LD}_{2}$ (right) for Drell-Yan dimuons. It is important to note that no isoscalar corrections have been applied for the targets. Some target-to-target and overall observations for the entire nuclear landscape are listed below:

\section{Comparison with E772}
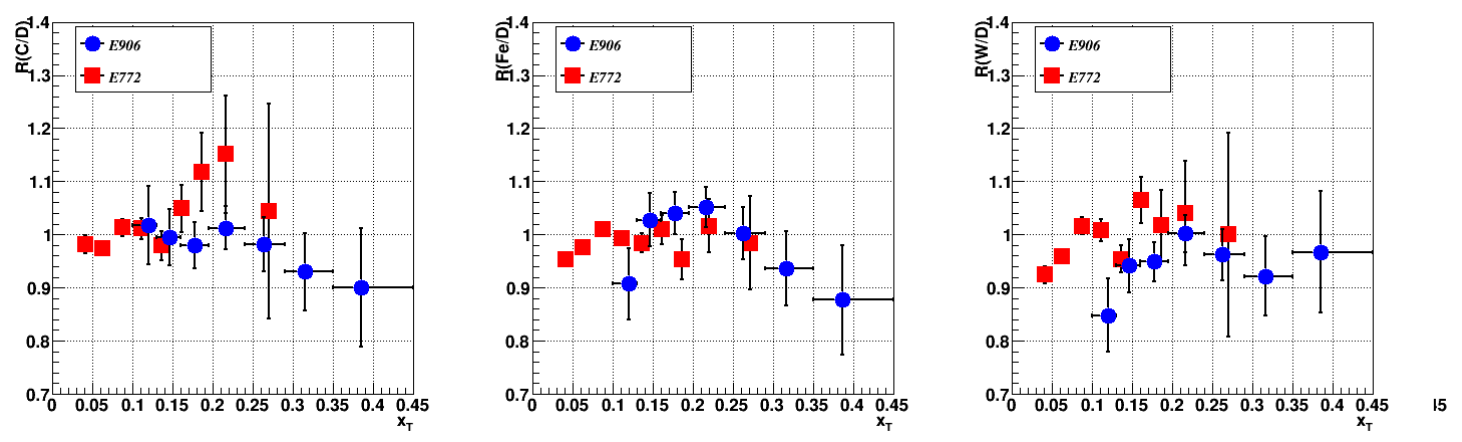

Figure 5.23: $R_{p A}$ vs $x_{T}$ for carbon (left), iron (center) and tungsten (right). No isoscalar corrections have been applied to the targets. Blue data points belong to SeaQuest and red data points belong to E772 [90]. Only statistical uncertainties shown.

- These results seem to be mostly consistent with E772 data for the common measured 
regions below $x_{T} \approx 0.2$. Above $x_{T}>0.2$ the uncertainties overlap for both the experiments.

- A slight drop off is seen for iron and tungsten in the region $0.1<x_{T}<0.13$ which is later than the usual DIS shadowing region. This is also a kinematic bin which is on the edge of spectrometer acceptance and a slight excess of $\mathrm{LD}_{2}$ events (signal and background) compared to the solid targets could induce such a feature.

- Although limited by statistical uncertainty, the ratio $R_{p A}$ may begin to gradually drop off at $x_{T} \sim 0.25$ but is statistically consistent with 1 . More statistics in the high- $x$ region will confirm this drop off.

- The data from all three targets is limited by statistics to make any predictions about the EMC region $\left(0.3<x_{T}<0.45\right.$ is the available reach of SeaQuest). More statistics in the high- $x$ region could help understand the "seeming" drop.

- A pure pion excess model, or a pure quark cluster model, or calculations by Jung and Miller [113] predict an enhancement (for E772 kinematics [90]) in the ratio due to the exchange of the so called "nuclear pions". These predict almost $10-25 \%$ enhancement above unity at $x_{T} \sim 0.25$. Although the predictions for $120 \mathrm{GeV} / \mathrm{c}$ SeaQuest beam could be different, all the three targets do not exhibit such an enhancement.

- When looking at a particular $x_{T}$ region on the 'nuclear landscape', one has to consider the implications of physics mechanisms in other regions as well. For example, the theories that explain the EMC region have implications for the anti-shadowing region as well. Similarly, the theories that explain the shadowing region have implications for the anti-shadowing region. Therefore, one has to consider a composite model which involves a combination of several different mechanisms. It is also possible that the effects of one mechanism could be masked by another mechanism. Therefore, one cannot simply rule out or accept a particular mechanism by looking 
at its predictions for only one region. Data from all three targets seem to show agreement with the predictions made by Kulagin and Petti [117] which is a composite model that takes into account the Fermi motion and nuclear binding (FMB), off-shell correction (OS), nuclear coherent correction (NS) and the nuclear meson correction (PI) into consideration.

\section{Systematic Uncertainties}

Same sources of systematic uncertainties have been considered as in 5.1.1. Most of the sources were found to be negligible. The QIE pedestal fluctuation has not been considered as a source of systematic uncertainty since it is very small compared to the systematics from the fitting curve. Also, $\mathrm{F}=\mathrm{p} 0 * \cos (\mathrm{I} /(\mathrm{p} 10+\mathrm{p} 11 * \mathrm{x} 2 \mathrm{avg}))$ has not been used for the estimation of systematic uncertainty since it has a poor reduced $\chi^{2}$.

1. Systematics from Choice of Fitting curve: This is the major source of systematic uncertainty. Only this curve has been chosen for the calculation of systematic uncertainty

(a) $\mathrm{F}=\mathrm{p} 0+\mathrm{p} 1 * \mathrm{I}+\mathrm{p} 2 * \mathrm{I}^{2}$ (Common Pol2 without the $x_{T}$ dependence). This checks the initial assumption of a dependence on $x_{T}$. 


\begin{tabular}{|c|c|c|c|}
\hline$x_{T}$ bin & CSR - Fit 5c & $\sigma_{\text {stat }}$ & $\sigma_{\text {syst }}($ Fittingcurve $)$ \\
\hline $0.1-0.13$ & 1.0170 & 0.0736 & 0.0292 \\
\hline $0.13-0.16$ & 0.9948 & 0.0528 & 0.0196 \\
\hline $0.16-0.195$ & 0.9796 & 0.0426 & 0.0098 \\
\hline $0.195-0.24$ & 1.0123 & 0.0404 & 0.0022 \\
\hline $0.24-0.29$ & 0.9816 & 0.0507 & 0.016 \\
\hline $0.29-0.35$ & 0.9300 & 0.0729 & 0.0344 \\
\hline $0.35-0.45$ & 0.8995 & 0.1115 & 0.0555 \\
\hline
\end{tabular}

Table 5.4: $R_{p A}$ values, statistical and systematic uncertainties for carbon. $F=p 0+p 1 *$ $I+p 2 * I^{2}$ (common pol2 fit) was used to estimate the systematic uncertainty.

\begin{tabular}{|c|c|c|c|}
\hline$x_{T}$ bin & CSR - Fit 5c & $\sigma_{\text {stat }}$ & $\sigma_{\text {syst }}$ (Fittingcurve $)$ \\
\hline $0.1-0.13$ & 0.907 & 0.0673 & 0.0366 \\
\hline $0.13-0.16$ & 1.0277 & 0.0502 & 0.0246 \\
\hline $0.16-0.195$ & 1.0405 & 0.0402 & 0.0116 \\
\hline $0.195-0.24$ & 1.0514 & 0.0386 & 0.0032 \\
\hline $0.24-0.29$ & 1.00022 & 0.0497 & 0.0218 \\
\hline $0.29-0.35$ & 0.936 & 0.0696 & 0.0432 \\
\hline $0.35-0.45$ & 0.8766 & 0.1028 & 0.0696 \\
\hline
\end{tabular}

Table 5.5: $R_{p A}$ values, statistical and systematic uncertainties for iron.

\begin{tabular}{|c|c|c|c|}
\hline$x_{T}$ bin & CSR - Fit 5c & $\sigma_{\text {stat }}$ & $\sigma_{\text {syst }}($ Fittingcurve $)$ \\
\hline $0.1-0.13$ & 0.8476 & 0.0690 & 0.0033 \\
\hline $0.13-0.16$ & 0.9409 & 0.0498 & 0.0024 \\
\hline $0.16-0.195$ & 0.9483 & 0.0377 & 0.0012 \\
\hline $0.195-0.24$ & 1.0013 & 0.0354 & 0.0001 \\
\hline $0.24-0.29$ & 0.9622 & 0.0484 & 0.0017 \\
\hline $0.29-0.35$ & 0.9216 & 0.0743 & 0.0032 \\
\hline $0.35-0.45$ & 0.9669 & 0.1148 & 0.0055 \\
\hline
\end{tabular}

Table 5.6: $R_{p A}$ values, statistical and systematic uncertainties for tungsten. 


\subsection{2 $p_{T}$ dependence}

Transverse momentum of the dimuon, $p_{T}$, is nearly an independent variable that makes measurements in the transverse direction while other variables such as $x_{T}, x_{B}$ and $x_{F}$ tell us about the longitudinal direction. Multiple interactions of the parton traversing a nuclear medium leads to the broadening of the transverse momentum since each of the interaction is accompanied with an energy loss. With minimal final state interactions, the Drell-Yan process is an ideal probe to study broadening of the transverse momentum which could give an insight into induced energy loss [167] and could used as a sensitive probe for properties of matter created in heavy ion collisions. E772, E789 and E866 experiments studied $p_{T}$ broadening with $800 \mathrm{GeV}$ proton beam [48, 168, 91, 169]. SeaQuest is able to provide data at a relatively lower energy compared to the above experiments in order to study the energy dependence of transverse momentum broadening. Fig. 5.24 shows the ratio $R_{p A}$ as a function of $p_{T}$ for $\mathrm{C} / \mathrm{LD}_{2}, \mathrm{Fe} / \mathrm{LD}_{2}$, and $\mathrm{W} / \mathrm{LD}_{2}$. The data show a clear $p_{T}$ dependence and some observations are noted below:

- The ratio $R_{p A}$ is consistent with 1 for $\mathrm{C} / \mathrm{LD}_{2}$.

- For iron, there seems to be a slight reduction in the per nucleon cross section ratio in the low- $p_{T}$ region coupled with an enhancement in the high- $p_{T}$ region. This effect is more prominent in tungsten (which is a heavier nuclei).

- When we integrate over $p_{T}$, the ratio is 1 which is consistent with the interpretation that events at low $p_{T}$ are "pushed" to a higher $p_{T}$ value.

\section{Comparison with NuSea/E866}

In the E866 experiment, the cross section ratios as a function of $p_{T}$ were measured for $\mathrm{Fe} / \mathrm{C}, \mathrm{Fe} / \mathrm{Be}, \mathrm{W} / \mathrm{C}$ and W/Be. Similar behavior was seen in those ratios as well where there is a drop in the low- $p_{T}$ region and an enhancement in the high- $p_{T}$ region. This behavior 

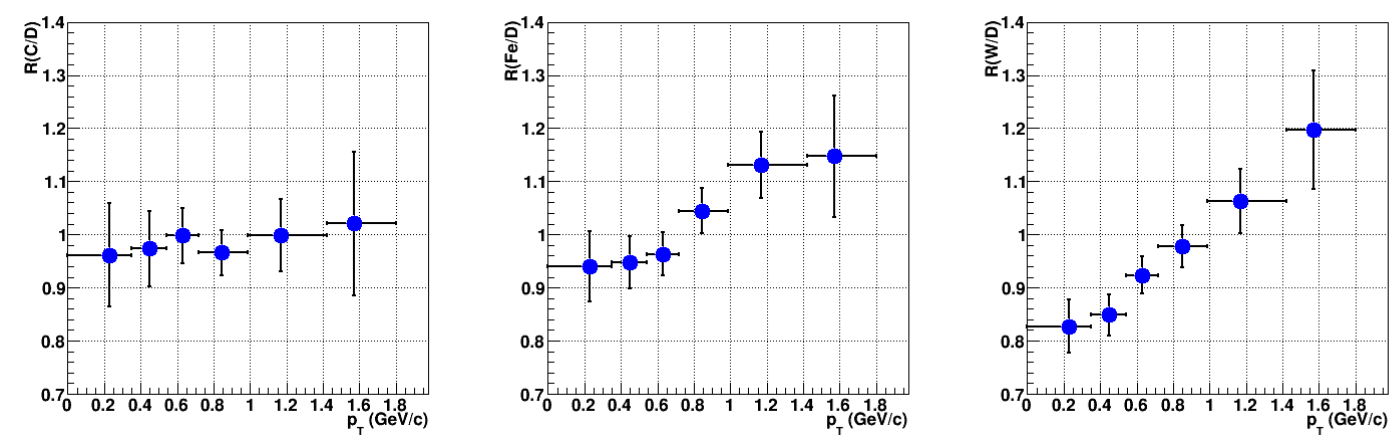

Figure 5.24: $R_{p A}$ vs $p_{T}$ for carbon (left), iron (center) and tungsten (right). Statistical uncertainties shown only. $F=p 0+(p 10+p 11 *\langle p T\rangle) *$ inte $+(p 20+p 21 *\langle p T\rangle) *$ inte $^{2}$ fit was used for the extrapolation.

of a slight reduction in the per nucleon cross section ratio coupled with a slight increase in the high $p_{T}$ region was noted as being a characteristic of multiple scattering of the incident parton in the nucleus [91].

\subsection{3 $x_{F}$ and $x_{B}$ dependence}

Parton energy loss in p-A collisions serves as an important tool in exploring the properties of the Quark Gluon Plasma (QGP). With minimal final state interactions, the dimuon pair arising from the Drell-Yan process in $\mathrm{p}-\mathrm{A}$ collisions will help set a baseline relative to energy loss in QGP.

Feynman- $x$ or $x_{F}$ is another variable of interest that sheds light on the longitudinal structure of the initial state of the interacting quark. If there is no energy loss of the incoming parton (which could be very small in deuterium), then the $x_{\text {Beam }}$ distributions are centered around a particular $x_{\text {Beam }}$ value. However, if the beam parton
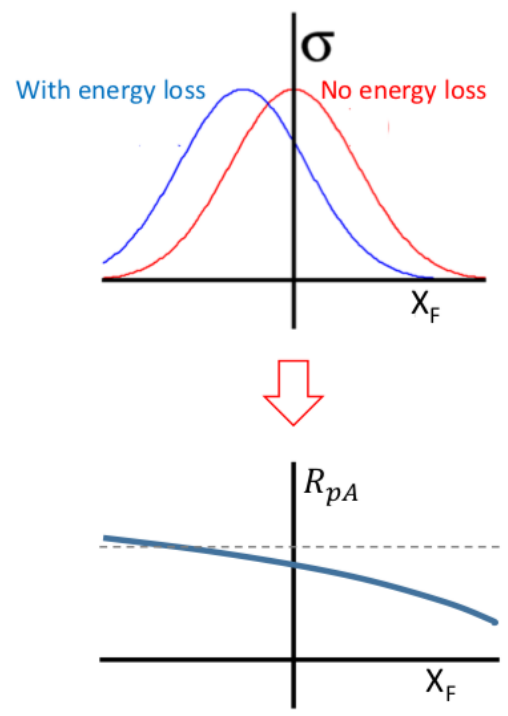

Figure 5.25: Depiction of the drop of the ratio $R_{p A}\left(x_{F}\right)$ is subject to initial-state energy loss in a heavier nucleus, 
the $x_{\text {Beam }}$ distributions in this nucleus are shifted making the ratio of the per-nucleon cross section $R_{p A}$ have an overall slope as a function of $x_{\text {Beam }}$ as seen in Fig. 5.25. A decrease in the $R_{p A}$ value with increasing $x_{F}$ is typically attributed to the energy loss of the incoming parton due to its interaction with the cold nuclear medium (depiction in Fig. 5.26) according to some theoretical models [170, 171, 172, 173, 174].

Previous experiments $[91,175]$ found a slight depletion in the high $x_{F}$ region which could also be attributed to shadowing effects coming from the low- $x$ region. Therefore, data outside of the shadowing region is essential to decouple the effects of shadowing and parton energy loss in cold nuclear matter [176]. SeaQuest is sensitive to the region which is well outside of the effects of shadowing and data taken on C, Fe and W targets can be used to place upper limits on the energy loss of a fast parton traversing a cold nucleus. Fig. 5.27 shows the ratio $R_{p A}$ vs $x_{F}$ for $\mathrm{C} / \mathrm{LD}_{2}, \mathrm{Fe} / \mathrm{LD}_{2}$ and $\mathrm{W} / \mathrm{LD}_{2}$ for SeaQuest data. Some observations regarding the $x_{F}$ dependence for all three targets relative to deuterium are listed below:

- The ratio $R_{p A}$ is consistent with 1 for carbon.

- The ratio $R_{p A}$ has an $A$-dependence where the value of $R_{p A}$ decreases with increasing $x_{F}$ and $A$ for iron and tungsten.

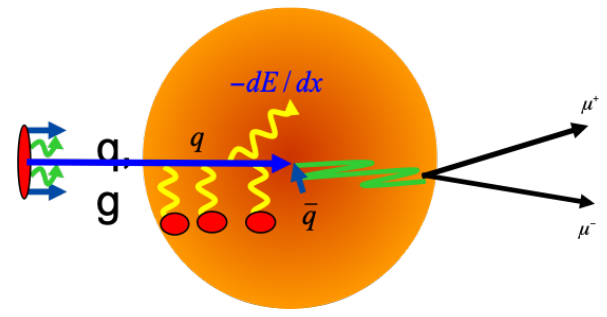

Figure 5.26: Depiction of a parton losing energy while traversing a cold

- Since high $x_{F}$ corresponds to a low $x_{T}$, a denuclear medium. pletion at high- $x_{F}$ could also be due to a depletion coming from the $A$ dependence of depletion in the shadowing region of the target nucleus. Hence a cut $x_{T}>0.16$ was applied to move out of the shadowing region. Fig. 5.28 shows the ratio $R_{p A}$ vs $x_{F}$ for $\mathrm{C} / \mathrm{LD}_{2}, \mathrm{Fe} / \mathrm{LD}_{2}$ and $\mathrm{W} / \mathrm{LD}_{2}$ with and without the $x_{T}$ cut. The results are consistent within uncertainties with and without the $x_{F}$ cut indicating that the effects of 
shadowing are negligible.

- $x_{\text {Beam }}$ also exhibits similar behavior as $x_{F}$.
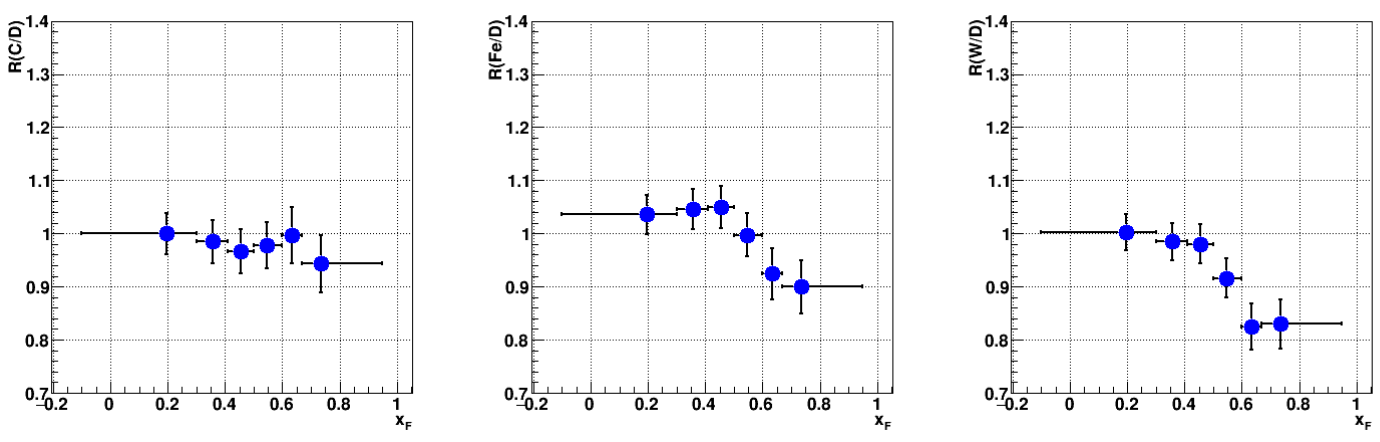

Figure 5.27: $R_{p A}$ vs $x_{F}$ for carbon (left), iron (center) and tungsten (right). $F=p 0+p 1 *$ $I+p 2 * I^{2}$ (common pol2 fit) was used for extrapolation since it has a better reduced $\chi^{2}$ compared to the other fits.
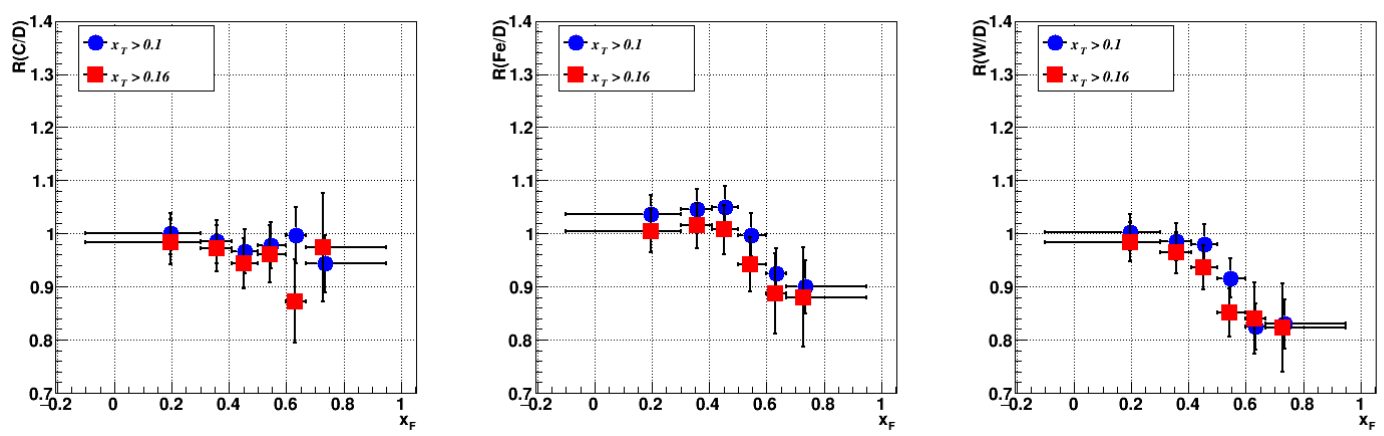

Figure 5.28: $R_{p A}$ vs $x_{F}$ for carbon (left), iron (center) and tungsten (right) with $x_{T}>0.16$ cut (red). $F=p 0+p 1 * I+p 2 * I^{2}$ (common pol2 fit) was used for extrapolation in both the cases. The results are consistent within uncertainties indicating that the effects of shadowing are negligible. 

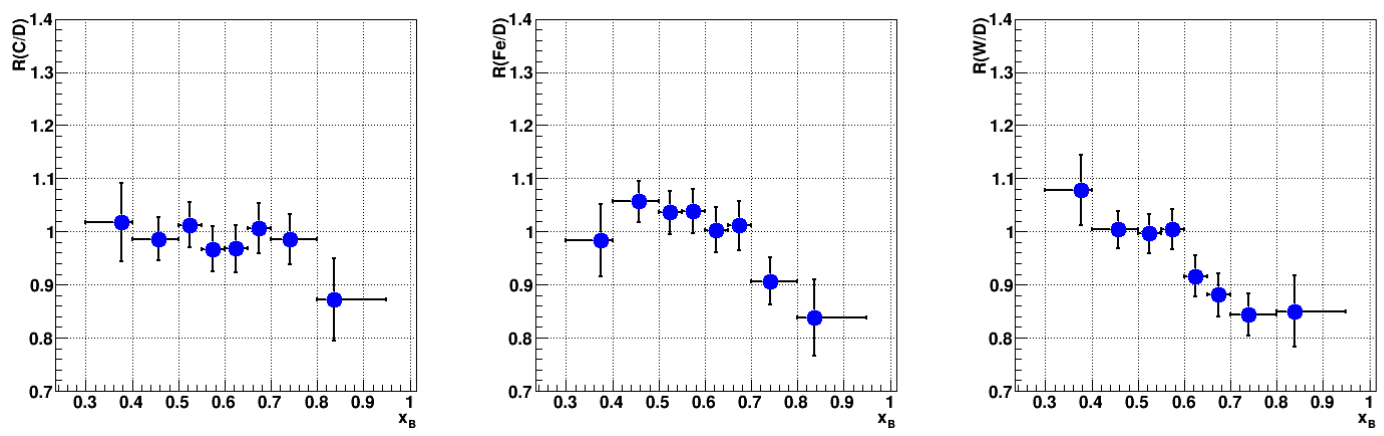

Figure 5.29: $R_{p A}$ vs $x_{B}$ for carbon (left), iron (center) and tungsten (right). $F=p 0+p 1 *$ $I+p 2 * I^{2}$ (common pol2 fit) was used for extrapolation.

\subsection{4 mass dependence}

The $R_{p A}$ for mass is consistent with 1 for all three targets except tungsten which has a slightly more prominent dip at around $4.5 \mathrm{GeV}$.
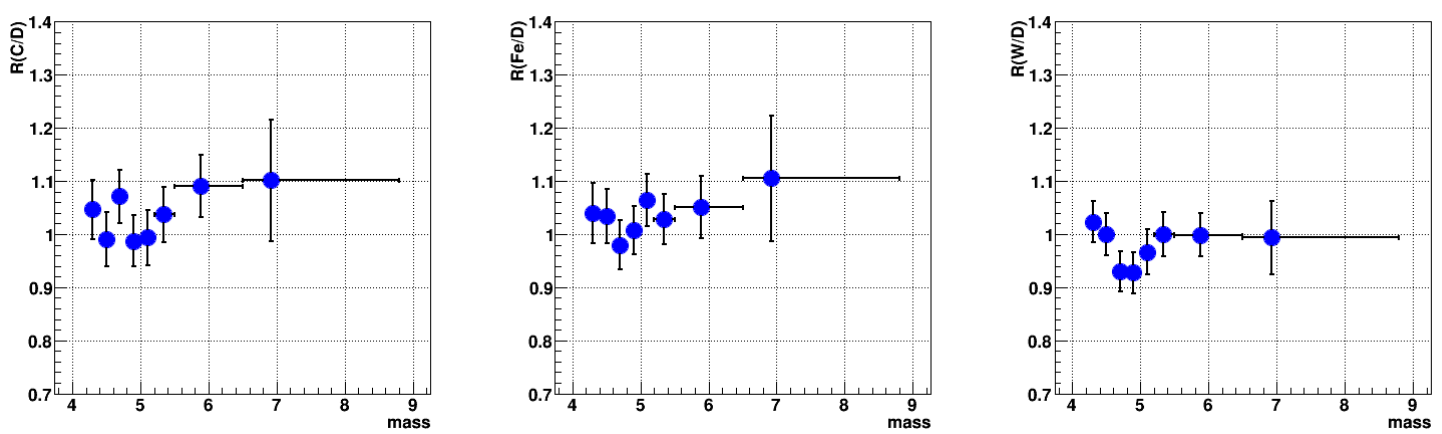

Figure 5.30: $R_{p A}$ vs mass for carbon (left), iron (center) and tungsten (right). 


\section{Chapter 6}

\section{What's next?}

\subsection{Summary}

Understanding the structure and behavior of the constituents of matter is one of the central goals of modern physics. Many experiments all over the world continue to peel the layers of nature's rich sub-structure each day. SeaQuest is one such experiment that has potential for great discovery. SeaQuest is a quest into the anti-quark structure of the nucleon and nuclei. The experiment takes advantage of the Drell-Yan process specifically in order to probe the high- $x$ anti-quark distributions. Ratio of cross sections of different targets is the key to probing the sea structure of the nucleon and various other nuclei.

\subsubsection{Light Anti-Quark Flavor Asymmetry in the Nucleon Sea}

Dealing with rate dependence effects are a challenge to any experiment. A new IntensityExtrapolation method was developed in which the ratio of cross sections are plotted as a function of the number of protons in the triggered bucket. Extrapolation to 0 is performed since all the rate dependence effects simply vanish there. Using this method:

- The ratio of cross sections $\sigma_{p d}(x) / 2 \sigma_{p p}(x)$ has been measured for $0.1<x_{T}<0.45$. The values are consistently above 1 for the measured range.

- A first look at the iteratively extracted $\bar{d}(x) / \bar{u}(x)$ is also presented. The trend of the data points indicate that the ratio is consistently above 1 for $0.1<x<0.45$. This extraction has many underlying assumptions and the SeaQuest collaboration is 
considering the best method to extract $\bar{d}(x) / \bar{u}(x)$ from the ratio of cross sections at the present time.

These results (when combined with more data) will provide a key input in constraining many theoretical models that attempt to explain the origin of the nucleon sea.

\subsubsection{Nuclear Dependence of Anti-Quarks}

Using the same Intensity-Extrapolation method, the nuclear dependence of the per-nucleon cross section ratio $R_{p A}$ of $\mathrm{C} / \mathrm{LD}_{2}, \mathrm{Fe} / \mathrm{LD}_{2}$ and $\mathrm{W} / \mathrm{LD}_{2}$ as a function of $x_{T}, p_{T}, x_{F}, x_{B}$ and mass was reported.

- The nuclear dependence of the per nucleon ratio of cross sections all three targets for $x_{T}$ are basically consistent with results reported by E772. No enhancement is seen in the high- $x$ region as expected by some theoretical models which take into consideration the exchange of the so called "nuclear pions". However, the results seem to be consistent with a composite model which take several nuclear effects into consideration. More statistics in the high- $x$ region will help answer an important question about the observation of the "EMC effect" for anti-quarks.

- A striking $A$ dependence in which there is a depletion of low- $p_{T}$ dimuons and an excess of high- $p_{T}$ dimuons relative to deuterium is reported.

- Similarly, an $A$ dependence for the drop in $R_{p A}$ for increasing $x_{F}$ is also noted. An analysis cut $x_{T}>0.16$ (which removes the effects of shadowing) reports consistent results without the cut indicating that the effects of shadowing are small.

These results (when combined with more data) will address important questions on the modification of anti-quark distributions in nuclei and in modeling energy loss for partons traversing a cold nuclear medium. 


\subsection{Future Prospects}

\subsubsection{Inclusion of Run IV, V and VI data}

The results shown in the thesis are based on just $\approx 30 \%$ of the data. Run 4,5 and 6 have the new DC1.2 installed in them. The new DCs yield a wider acceptance and more data specifically aimed at the high- $x$ region. One can use this data to do a crosscheck of Run II and III results presented in this thesis. With the inclusion of this data, one can address many interesting questions that are aimed at the high- $x$ region.

\subsubsection{Improving Systematic Uncertainties}

The sources of systematic uncertainties that were considered were discussed. The major source of systematic uncertainty for all the studies comes from the choice of the fitting function. The uncertainties cannot identify a unique fitting function at present. However, with the inclusion of more data, the fits will be more constrained and the systematic uncertainties coming from the choice of the fitting function will be further reduced.

\subsubsection{Calculating $\bar{d}(x)-\bar{u}(x)$}

After the extraction of $\bar{d}(x) / \bar{u}(x)$, one could use the CT10NLO $[\bar{d}(x)+\bar{u}(x)]_{P D F}$ values to calculate the rough value of $\bar{d}(x)-\bar{u}(x)$ for different $x_{T}$ values. This could tell us if the value crosses 0 (as seen by E866) and provide an additional comparison with various non-perturbative theoretical models that are driven to explain $\bar{d}(x)-\bar{u}(x)$.

\subsubsection{Nuclear dependence for $\mathrm{Fe} / \mathrm{C}$ and $\mathrm{W} / \mathrm{C}$}

One could use the Intensity extrapolation method in the same way to study nuclear dependence for several quantities in the per-nucleon ratio of cross sections for Fe/C and W/C. These measurements could extend the E866 results to higher- $x$ and a lower beam energy. 


\subsubsection{Intensity-Extrapolation method to study $J / \Psi$ physics}

An extension of this work involves adjusting the analysis cuts to gain an insight into interesting $J / \Psi$ physics as well. For example:

- $2.7<$ mass $<3.2$

- $0.4<x_{F}<0.95$

- $0.05<x_{T}<0.1$

By doing this, one can study the ratio of cross sections of deuterium and hydrogen, nuclear dependence (of $x_{\text {Target }}, x_{\text {Feynman }}, p_{T}$, mass and $x_{\text {Beam }}$ ) of $\mathrm{C} / \mathrm{LD}_{2}, \mathrm{Fe} / \mathrm{LD}_{2}, \mathrm{~W} / \mathrm{LD}_{2}, \mathrm{Fe} / \mathrm{C}$ and W/C. All these topics have generated considerable theoretical interest in the physics community. Carrying out this program will involve an estimate of the contamination of the $\Psi^{\prime}$ and the Drell-Yan dimuon tails in the above specified kinematic regions. 


\section{Appendices}




\section{Appendix A}

\section{Construction of DC3m}

DC3m drift chamber was constructed at Fermilab in order to symmetrize the top and bottom acceptance at Station 3. The chamber was constructed with the help of many collaborators under the initiative of Japanese group. Section 3.7 discusses many aspects that go into the design of a drift chamber. I got a chance to get involved in the assembly, wire stretching (sense, cathode, field and guard wires) and tension measurements and commissioning stage of this drift chamber. The stages involved in the fabrication of the drift chamber has been discussed in detail in [132]. Only some details of the construction procedure are discussed.

DC3m drift chamber has four planes of guard wires, six planes of alternating sense and field wires and nine planes of cathode wires. In total, it has 768 sense wires, 774 field wires, 2315 cathode wires and 1296 guard wires. Sense wires are gold plated tungsten with Rhenium with a diameter of $30 \mu \mathrm{m}$. These wires provide three dimensional information about the trajectory of the particle. Guard/Field/Cathode wires are gold plated beryllium copper wires with a diameter of $80 \mu \mathrm{m}$. Cathode wires are thicker compared to sense wires and reinforce the electric field. Guard wires separate different layers of electric fields inside a drift chamber. After creating a clean workspace, the chamber frame was assembled. The internal corners of the frame was sealed with RTV (Room Temperature Vulcanizer). The holes which hold the feedthroughs were thoroughly cleaned with a steam cleaner followed by cotton swabs. The feedthroughs were then inserted into these holes and sealed with RTV to prevent gas leaks. Four different types of feedthroughs were installed depending on the type of wire and its tilt. 


\section{A.0.1 Stretching wires}

After assembling the chamber frames, wires are stretched between the chamber walls. A total of 5154 ( 768 sense wires and 4386 cathode wires) were assembled. The tension of the wires are calculated beforehand taking into consideration the mechanical sag of the drift chambers and the electrostatic forces between wires. As discussed in [127], the expression for minimum Tension $T_{C}$ is given by

$$
T_{C}=\frac{1}{4 \pi \epsilon_{0}}\left(\frac{C V_{0} L}{s}\right)
$$

where $\epsilon_{0}$ is the permittivity of free space, $C$ is the single-wire capacitance per unit length, $L$ is the wire length, and $s$ the wire spacing. The capacitance $C$ is given by

$$
T_{C}=\frac{2 \pi \epsilon_{0}}{(\pi l / s)-\ln (2 \pi a / s)}
$$

After installing the feedthroughs, the chamber was ready for wiring. The steps involved in stretching the wires are discussed below:

1. After choosing the type of wire that needs to be stretched, the wire is inserted on one end of the chamber and held in place using a wire grabber. It is then run across the chamber volume with the help of a pulley system to prevent kinking or damaging the wire.

2. The wire is then soldered on one side to hold it in place

3. An appropriate weight is attached to apply tension to the wire on the other end. For sense wires, a tension of $85 \mathrm{gf}$ (gram-force) was applied. A tension of 130 gram force was applied to the other types of wires. Too little solder might not be able to hold the wire in place while too much solder prevents the attachment of the readout read out electronics which have a small diameter. 
4. After attaching the weight, the wire is soldered in place and this process is repeated.

5. Any extra wire is cut off and additional amounts of solder are shaved off.

6. After stretching the wires on an entire plane, the tension of these wires were measured and the wires that had a tension outside the $10 \%$ tolerance limit were reported bad and eventually removed. The bad wires were replaced the following day after changing their feedthroughs, and their tension was checked again.

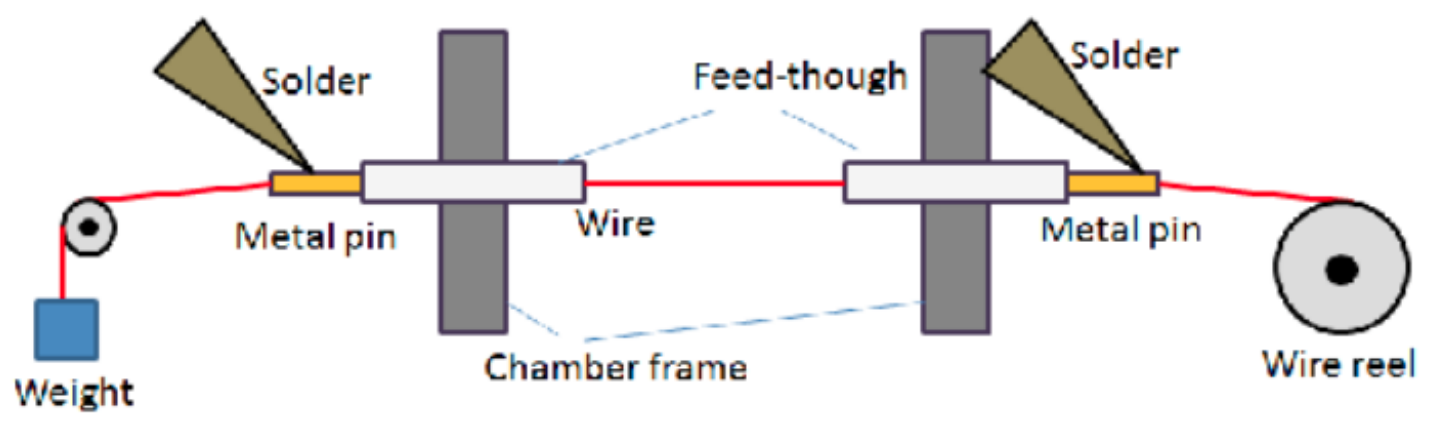

Figure A.1: Depiction of stretching a wire taken from [132].

The entire process takes about 2 mins per cathode wire and 4 mins for sense wire (due to sense wire having a smaller diameter and difficult to handle).

\section{A.0.2 Tension measurements}

Building a drift chamber which has over 5000 wires requires a quick and reliable method to measure the wire tension. One way is to induce mechanical oscillations in the wire by passing A.C. current at close to the resonant frequency or a short pulse through it, in the presence of a magnetic field, and study the induced current in the wire. The wire undergoes harmonic oscillations and the induced current in the wire is maximum for the resonance frequency. The tension of the wires at the resonance frequency can be extracted using the formula:

$$
T=4 f^{2} L^{2} \mu
$$


where $T$ is the tension of the wire, $L$ is the length, $f$ is the resonance frequency of the fundamental mode (lowest frequency of periodic oscillation) and $\mu$ is the linear mass density of the wire. When an external magnetic field is applied, the charged particles in the wire experience Lorentz force given by

$$
\vec{F}_{\text {magnetic }}=q \vec{v}_{d} \times \vec{B}
$$

where $v_{d}$ is the drift velocity of the charged particles in the wire and $B$ is the external magnetic field. This force acting on the wire induces transverse mechanical oscillations. As a result, the area of the circuit containing the wire changes. The voltage of the induced current signal can be calculated using Faraday's law of electromagnetic induction.

$$
\varepsilon=-\frac{d \Phi(t)}{d t}
$$

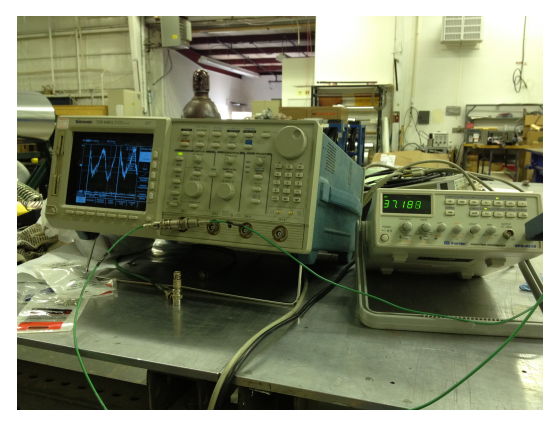

Figure A.2: Pulse generator and Oscilloscope used in the wire tension measurement

Where $\Phi$ is the magnetic flux through the circuit containing the wire. Figure 4 shows a pulse generator and oscilloscope used in the setup involved in measuring the tension of the wires. Figure 5 shows a cartoon of the setup used in the process of tension measurement. The magnet is placed underneath or on top of the wire, and a pulse with a known frequency is applied to the wire.

\section{A.0.3 Stretching wires}

When the input frequency corresponds to the frequency of the mechanical oscillations, resonance occurs and the amplitude of the transverse vibration of the wire becomes a maximum. The goal of the tension measurement is to determine this input frequency which corresponds to the frequency of the fundamental mode. The difficulty in the tension measurements is to isolate the induced current signal from any kind of noise in the setup. 
There were mainly two sources of noise in the output signal that we encountered during preliminary measurements. The first was an $80 \mathrm{~Hz}$ noise (sinusoidal) coming from the signal generator and the second was $10 \mathrm{kHz}$ noise whose source was unknown. The former was removed by taking the average of several samples of the output signal. The latter was removed by attaching a low pass filter which

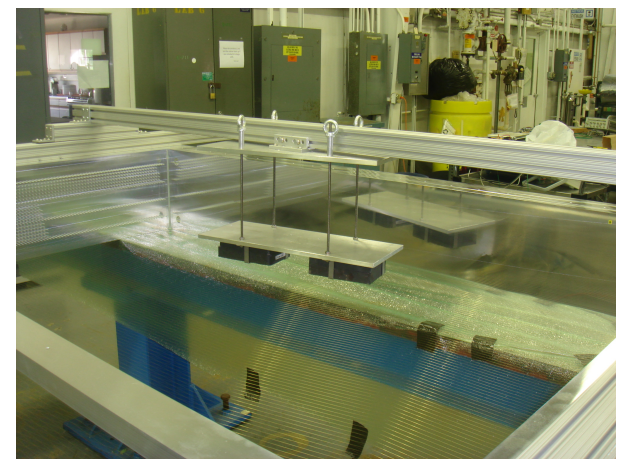

Figure A.3: Magnet support system used in tension measurement blocked out $10 \mathrm{kHz}$ noise. The setup was able to locate the resonance of the induced current to within $0.15 \mathrm{~Hz}(0.15 \%$ of the tolerance) for the field/guard/cathode wires and $0.15 \mathrm{~Hz}$ ( $0.15 \%$ of the tolerance) for the sense wires. Several other measurements such as numbers of magnets used and their position with regards to the wire's planes were made before designing the magnet support system that was later designed and installed on the chamber frame for the main tension measurements. After finishing construction at Lab 6, the chamber was hooked up with electronics, underwent high voltage training, gas training and was moved to the experimental hall and eventually installed in place [177].

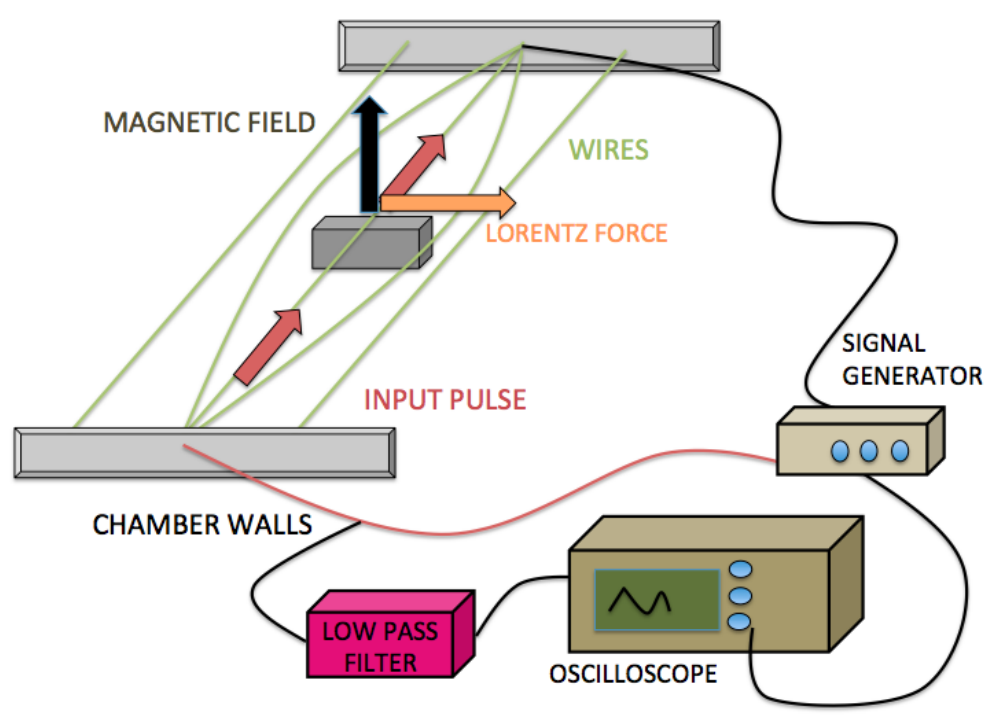

Figure A.4: Cartoon of the setup used to measure the tension of the wires 


\section{Appendix B}

\section{Dimuon Yields for Liquid and Solid targets}

\section{B.1 Table of live protons for total and individual data set}

The values for the total (and individual) live protons used in the analysis for each roadset have been taken from data2/production/list/R008 are shown in Tables 2 - 7. Live protons are obtained after applying the Spill.dataQuality $=0$ bit for individual roadsets. The conditions for "good spills" are given on the website mentioned at the end of Section 4.4.4.

\begin{tabular}{|c|c|c|c|}
\hline \multicolumn{5}{|c|}{ Full data set } \\
\hline TargetPos & Target & Total Raw POT & Total Live POT \\
\hline 0 & All & $1.43 \mathrm{E}+18$ & $6.73 \mathrm{E}+17$ \\
\hline 1 & LH2 & $6.08 \mathrm{E}+17$ & $2.87 \mathrm{E}+17$ \\
\hline 2 & Empty & $1.17 \mathrm{E}+17$ & $5.78 \mathrm{E}+16$ \\
\hline 3 & LD2 & $3.05 \mathrm{E}+17$ & $1.37 \mathrm{E}+17$ \\
\hline 4 & None & $1.25 \mathrm{E}+17$ & $6.14 \mathrm{E}+16$ \\
\hline 5 & Fe & $6.69 \mathrm{E}+16$ & $3.07 \mathrm{E}+16$ \\
\hline 6 & C & $1.41 \mathrm{E}+17$ & $6.67 \mathrm{E}+16$ \\
\hline 7 & W & $6.74 \mathrm{E}+16$ & $3.13 \mathrm{E}+16$ \\
\hline
\end{tabular}

Table B.1: Table of target position, target name, Raw POT and Live POT for full data set. 


\begin{tabular}{|c|c|c|c|}
\hline \multicolumn{4}{|c|}{ Roadset 57 } \\
\hline TargetPos & Target & Raw POT & Live POT \\
\hline 0 & All & $1.81844 \mathrm{e}+17$ & $9.26687 \mathrm{e}+16$ \\
\hline 1 & LH2 & $7.82876 \mathrm{e}+16$ & $4.01315 \mathrm{e}+16$ \\
\hline 2 & Empty & $8.19295 \mathrm{e}+15$ & $4.46133 \mathrm{e}+15$ \\
\hline 3 & LD2 & $4.13282 \mathrm{e}+16$ & $2.01604 \mathrm{e}+16$ \\
\hline 4 & None & $1.24442 \mathrm{e}+16$ & $6.60844 \mathrm{e}+15$ \\
\hline 5 & Fe & $8.23124 \mathrm{e}+15$ & $4.14517 \mathrm{e}+15$ \\
\hline 6 & C & $2.49648 \mathrm{e}+16$ & $1.28741 \mathrm{e}+16$ \\
\hline 7 & W & $8.39496 \mathrm{e}+15$ & $4.28783 \mathrm{e}+15$ \\
\hline
\end{tabular}

Table B.2: Table of target position, target name, Raw POT and Live POT for Roadset 57.

\begin{tabular}{|c|c|c|c|}
\hline \multicolumn{4}{|c|}{ Roadset 59 } \\
\hline TargetPos & Target & Raw POT & Live POT \\
\hline 0 & All & $5.17261 \mathrm{e}+16$ & $2.14969 \mathrm{e}+16$ \\
\hline 1 & LH2 & $2.24758 \mathrm{e}+16$ & $9.42881 \mathrm{e}+15$ \\
\hline 2 & Empty & $2.26658 \mathrm{e}+15$ & $1.01688 \mathrm{e}+15$ \\
\hline 3 & LD2 & $1.11329 \mathrm{e}+16$ & $4.32787 \mathrm{e}+15$ \\
\hline 4 & None & $4.46670 \mathrm{e}+15$ & $2.00673 \mathrm{e}+15$ \\
\hline 5 & Fe & $2.31845 \mathrm{e}+15$ & $9.41273 \mathrm{e}+14$ \\
\hline 6 & C & $6.78338 \mathrm{e}+15$ & $2.83152 \mathrm{e}+15$ \\
\hline 7 & W & $2.28232 \mathrm{e}+15$ & $9.43782 \mathrm{e}+14$ \\
\hline
\end{tabular}

Table B.3: Table of target position, target name, Raw POT and Live POT for Roadset 59.

\begin{tabular}{|c|c|c|c|}
\hline \multicolumn{4}{|c|}{ Roadset 62 } \\
\hline TargetPos & Target & Raw POT & Live POT \\
\hline 0 & All & $2.94576 \mathrm{e}+17$ & $1.35076 \mathrm{e}+17$ \\
\hline 1 & LH2 & $1.25817 \mathrm{e}+17$ & $5.82119 \mathrm{e}+16$ \\
\hline 2 & Empty & $2.43685 \mathrm{e}+16$ & $1.18501 \mathrm{e}+16$ \\
\hline 3 & LD2 & $6.17881 \mathrm{e}+16$ & $2.70679 \mathrm{e}+16$ \\
\hline 4 & None & $2.56435 \mathrm{e}+16$ & $1.23643 \mathrm{e}+16$ \\
\hline 5 & Fe & $1.50687 \mathrm{e}+16$ & $6.62631 \mathrm{e}+15$ \\
\hline 6 & C & $2.67396 \mathrm{e}+16$ & $1.22319 \mathrm{e}+16$ \\
\hline 7 & W & $1.51500 \mathrm{e}+16$ & $6.72392 \mathrm{e}+15$ \\
\hline
\end{tabular}

Table B.4: Table of target position, target name, Raw POT and Live POT for Roadset 62. 


\begin{tabular}{|c|c|c|c|}
\hline \multicolumn{5}{|c|}{ Roadset 67 } \\
\hline TargetPos & Target & Raw POT & Live POT \\
\hline 0 & All & $8.12868 \mathrm{e}+17$ & $3.81305 \mathrm{e}+17$ \\
\hline 1 & LH2 & $3.42718 \mathrm{e}+17$ & $1.61288 \mathrm{e}+17$ \\
\hline 2 & Empty & $7.46929 \mathrm{e}+16$ & $3.66565 \mathrm{e}+16$ \\
\hline 3 & LD2 & $1.71274 \mathrm{e}+17$ & $7.70196 \mathrm{e}+16$ \\
\hline 4 & None & $7.45034 \mathrm{e}+16$ & $3.65750 \mathrm{e}+16$ \\
\hline 5 & Fe & $3.73450 \mathrm{e}+16$ & $1.71730 \mathrm{e}+16$ \\
\hline 6 & C & $7.46692 \mathrm{e}+16$ & $3.50933 \mathrm{e}+16$ \\
\hline 7 & W & $3.76658 \mathrm{e}+16$ & $1.75003 \mathrm{e}+16$ \\
\hline
\end{tabular}

Table B.5: Table of target position, target name, Raw POT and Live POT for Roadset 67.

\begin{tabular}{|c|c|c|c|}
\hline \multicolumn{4}{|c|}{ Roadset 70 } \\
\hline TargetPos & Target & Raw POT & Live POT \\
\hline 0 & All & $8.95383 \mathrm{e}+16$ & $4.20655 \mathrm{e}+16$ \\
\hline 1 & LH2 & $3.86889 \mathrm{e}+16$ & $1.82450 \mathrm{e}+16$ \\
\hline 2 & Empty & $7.87702 \mathrm{e}+15$ & $3.84128 \mathrm{e}+15$ \\
\hline 3 & LD2 & $1.95208 \mathrm{e}+16$ & $8.85504 \mathrm{e}+15$ \\
\hline 4 & None & $7.78976 \mathrm{e}+15$ & $3.79717 \mathrm{e}+15$ \\
\hline 5 & Fe & $3.96850 \mathrm{e}+15$ & $1.83760 \mathrm{e}+15$ \\
\hline 6 & C & $7.78891 \mathrm{e}+15$ & $3.66525 \mathrm{e}+15$ \\
\hline 7 & W & $3.90442 \mathrm{e}+15$ & $1.82418 \mathrm{e}+15$ \\
\hline
\end{tabular}

Table B.6: Table of target position, target name, Raw POT and Live POT for Roadset 70. 


\section{Appendix C}

\section{Intercepts for Nuclear dependence studies}

\section{C.1 $p_{T}$ dependence fits}

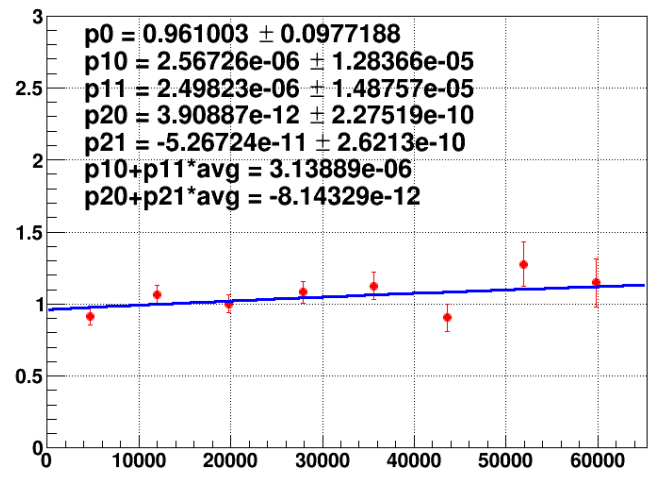

(a) $0<p_{T}<0.35$

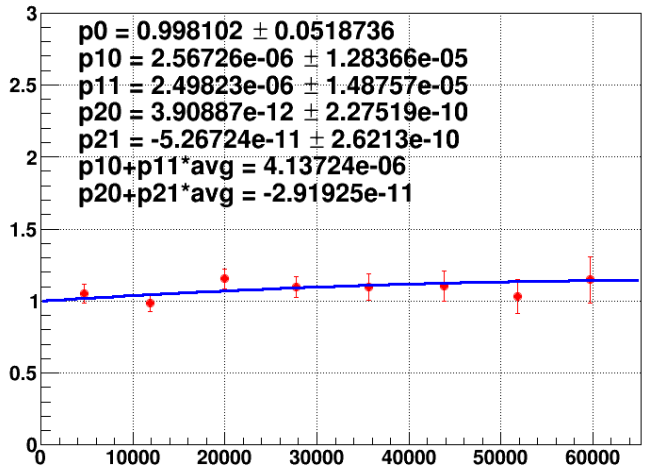

(c) $0.54<p_{T}<0.72$

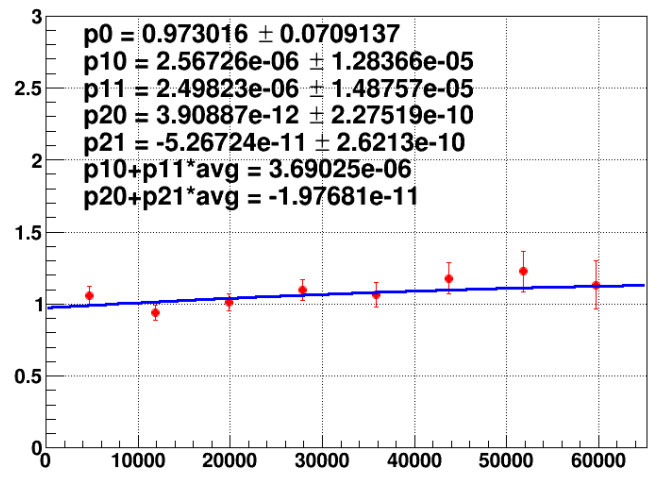

(b) $0.35<p_{T}<0.54$

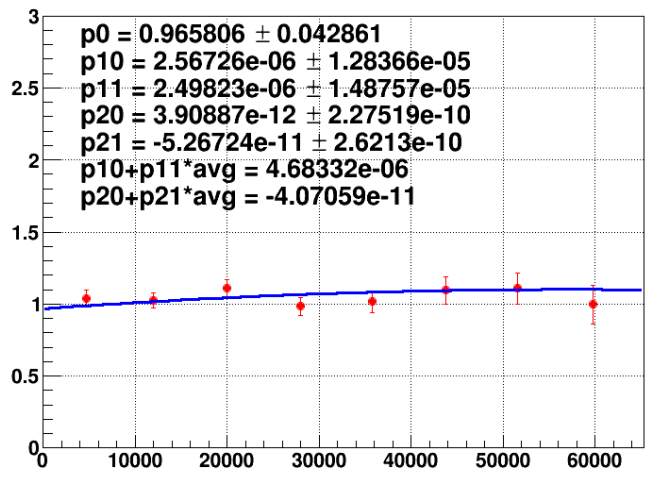

(d) $0.72<p_{T}<0.99$

Figure C.1: $R_{p A}$ for carbon for different $p_{T}$ bins. 


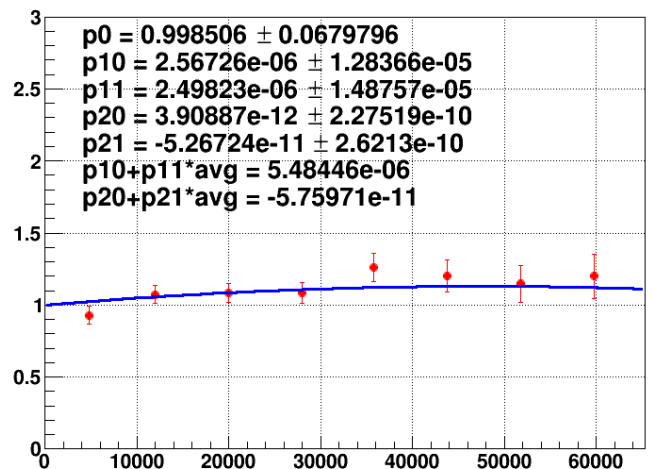

(a) $0.99<p_{T}<1.42$

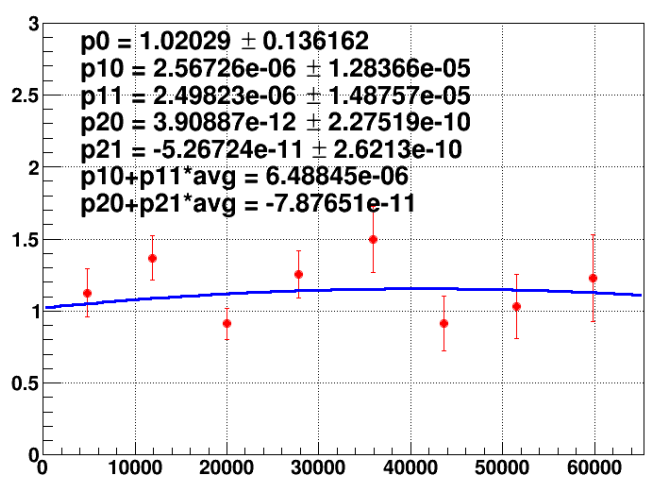

(b) $1.42<p_{T}<1.8$

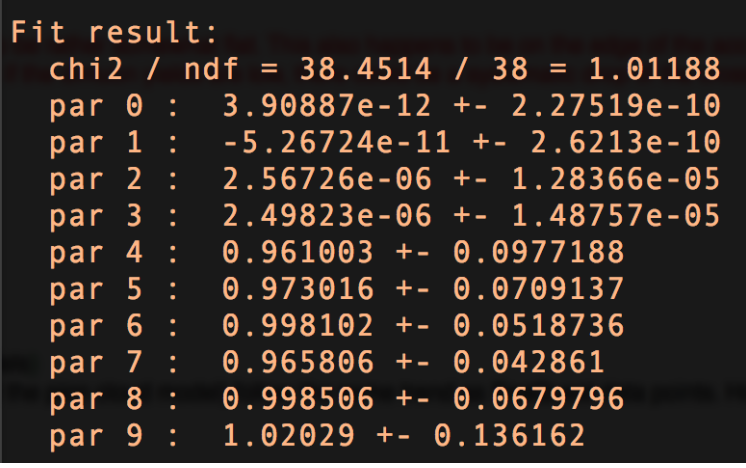

(d) Fit results

Figure C.2: $R_{p A}$ for carbon for different $p_{T}$ bins along with the fit results. 


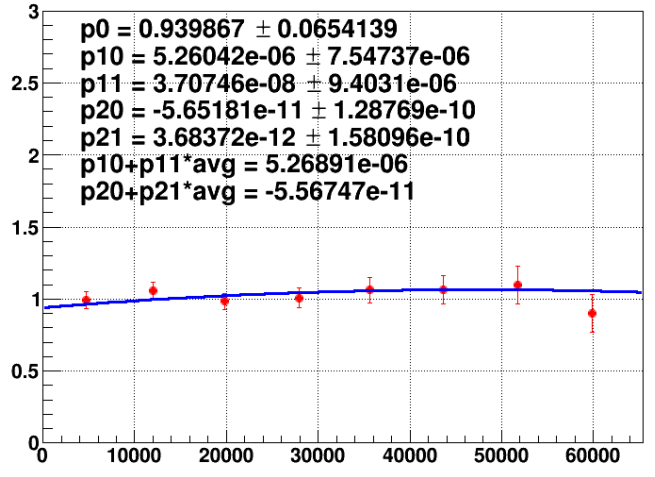

(a) $0<p_{T}<0.35$

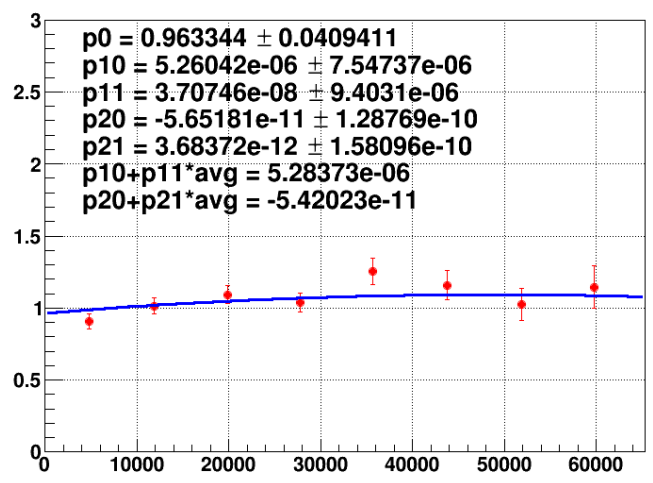

(c) $0.54<p_{T}<0.72$

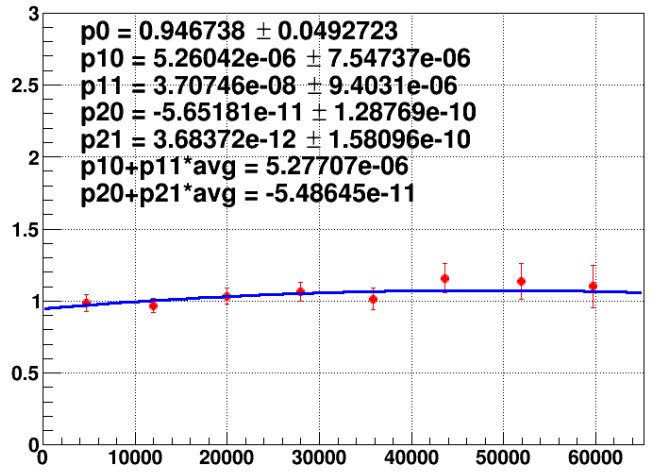

(b) $0.35<p_{T}<0.54$

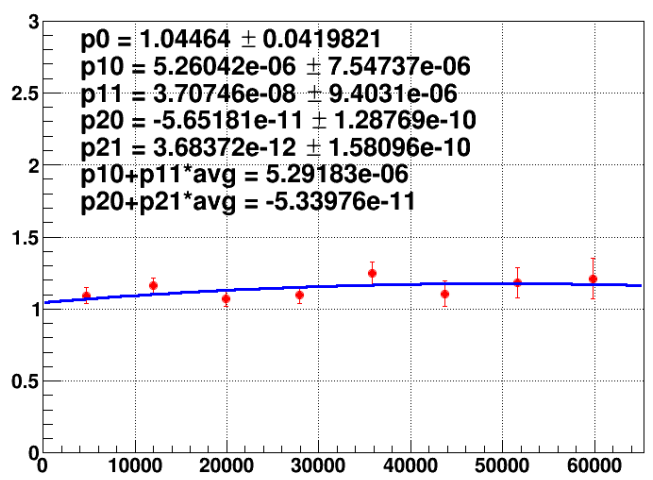

(d) $0.72<p_{T}<0.99$

Figure C.3: $R_{p A}$ for iron for different $p_{T}$ bins. 


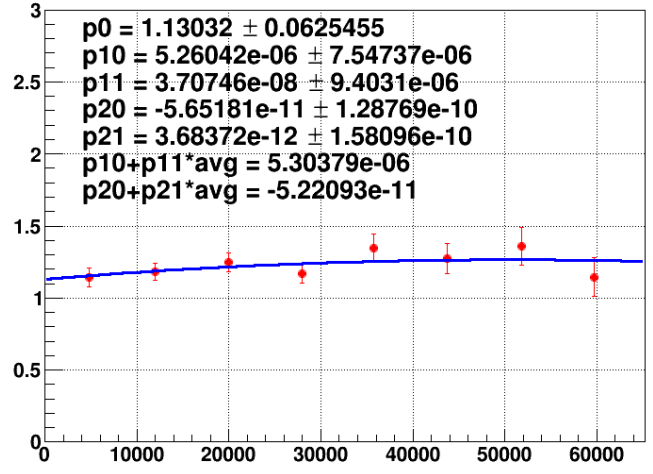

(a) $0.99<p_{T}<1.42$

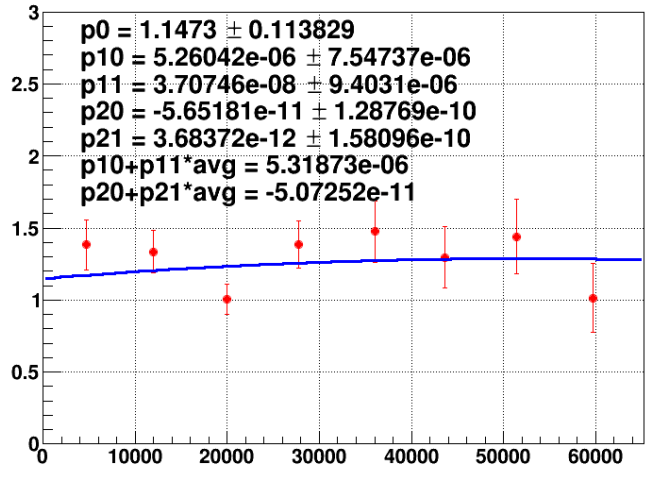

(b) $1.42<p_{T}<1.8$

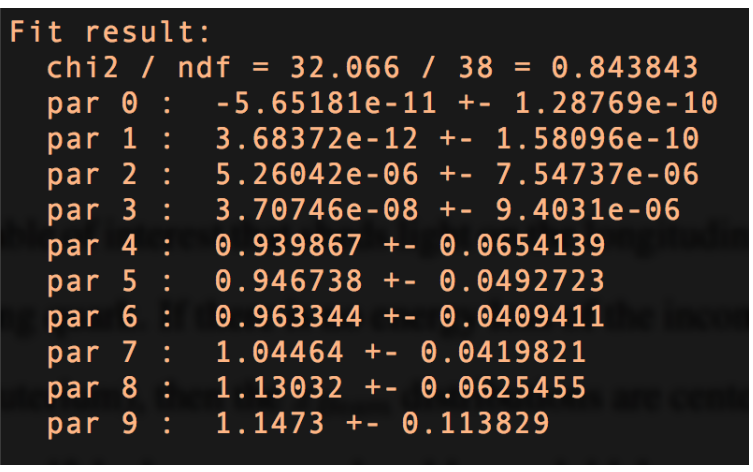

(d) Fit results

Figure C.4: $R_{p A}$ for iron for different $p_{T}$ bins along with the fit results. 


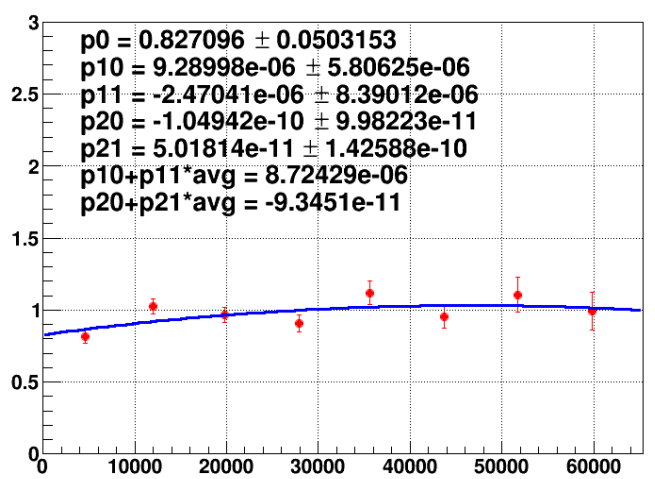

(a) $0<p_{T}<0.35$

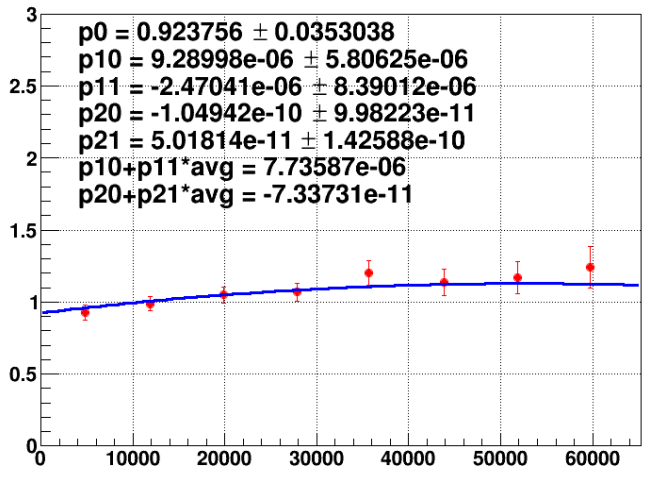

(c) $0.54<p_{T}<0.72$

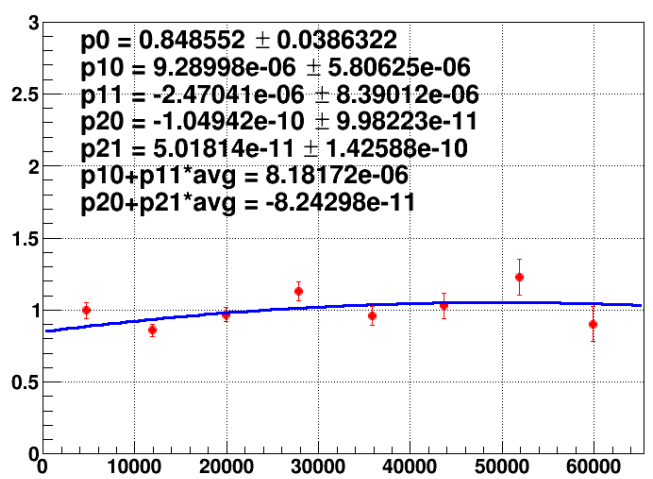

(b) $0.35<p_{T}<0.54$

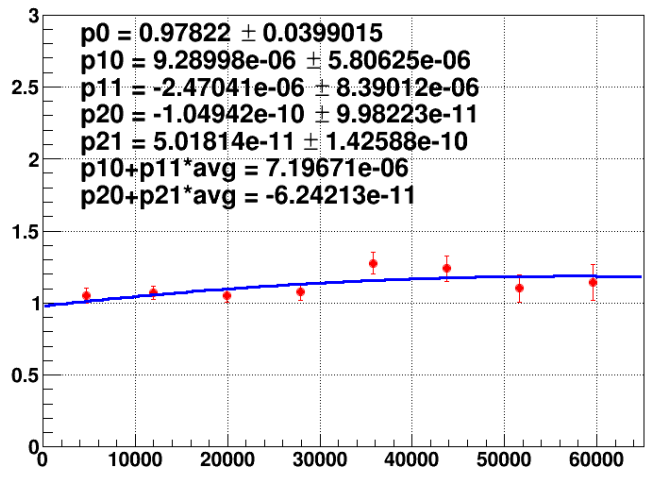

(d) $0.72<p_{T}<0.99$

Figure C.5: $R_{p A}$ for tungsten for different $p_{T}$ bins. 


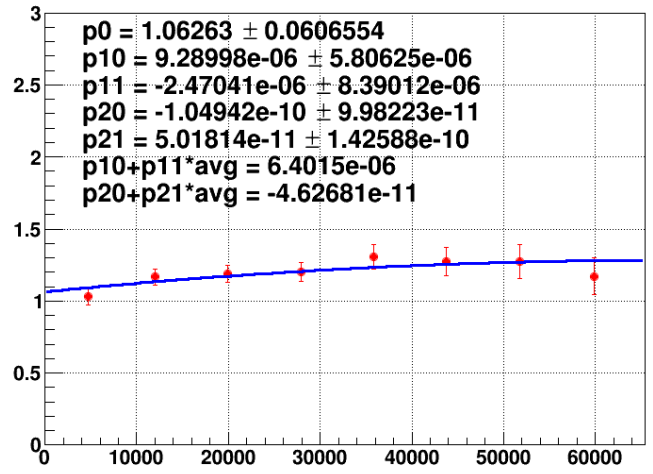

(a) $0.99<p_{T}<1.42$

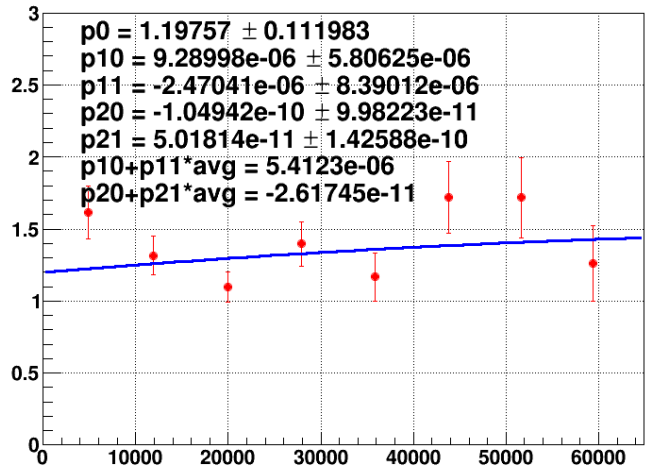

(b) $1.42<p_{T}<1.8$

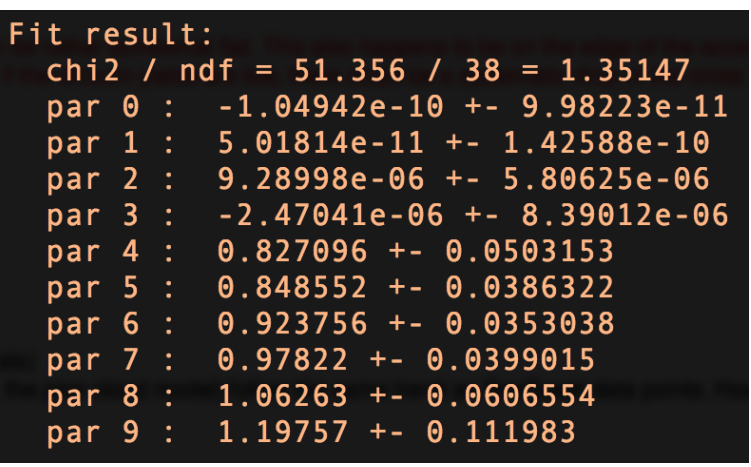

(d) Fit results

Figure C.6: $R_{p A}$ for tungsten for different $p_{T}$ bins along with the fit results. 


\section{C.2 $x_{F}$ dependence fits}

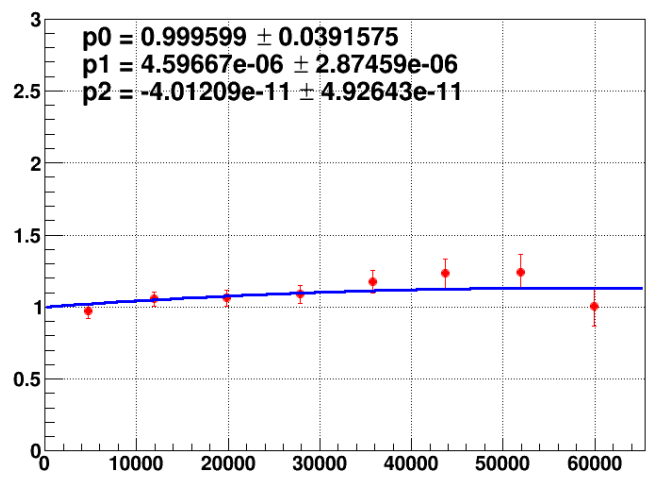

(a) $-0.1<x_{F}<0.3$

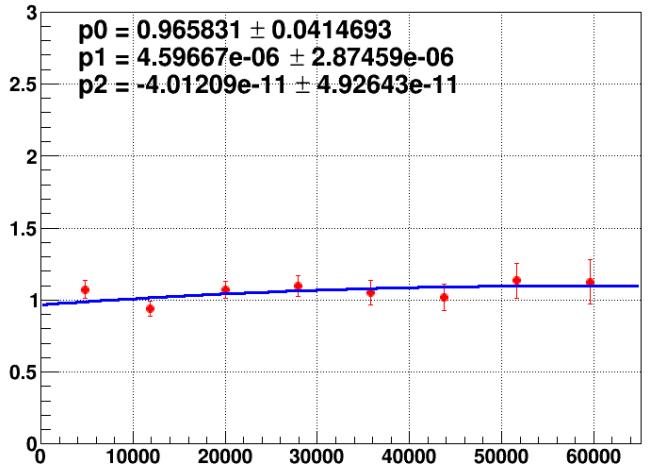

(c) $0.41<x_{F}<0.50$

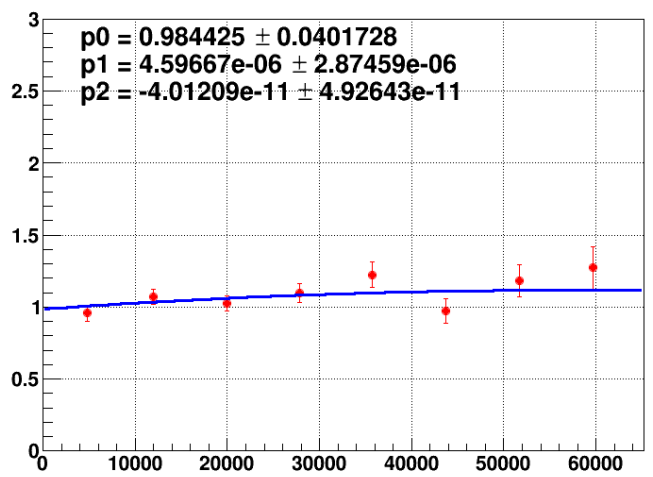

(b) $0.3<x_{F}<0.41$

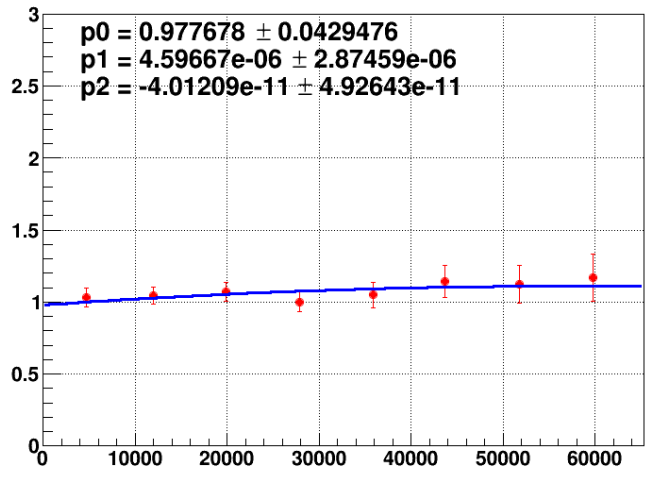

(d) $0.50<x_{F}<0.60$

Figure C.7: $R_{p A}$ for carbon for different $x_{F}$ bins. 


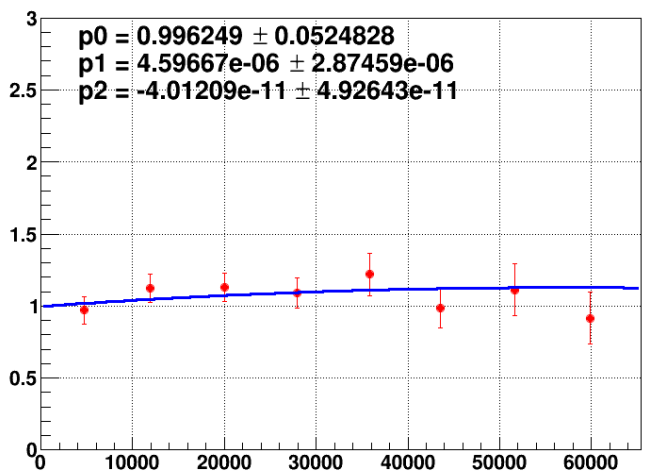

(a) $0.60<x_{F}<0.67$

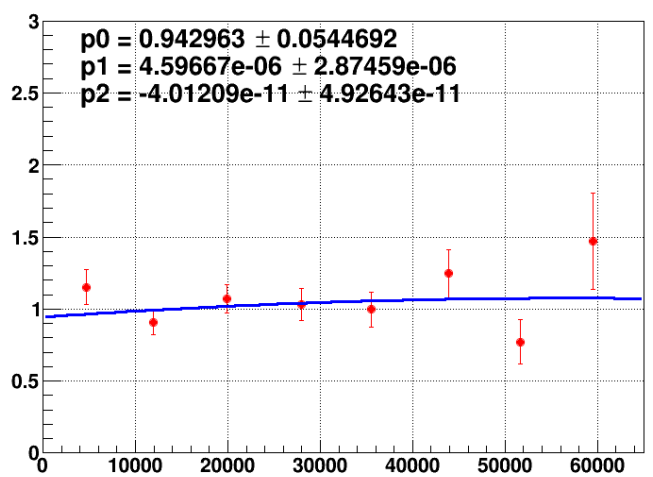

(b) $0.67<x_{F}<0.95$

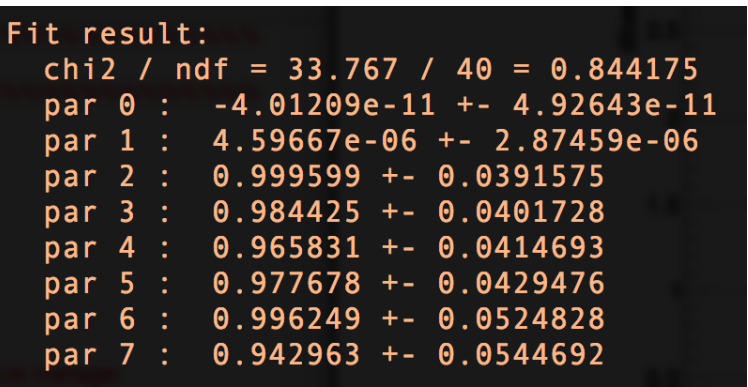

(d) Fit results

Figure C.8: $R_{p A}$ for carbon for different $x_{F}$ bins along with the fit results. 


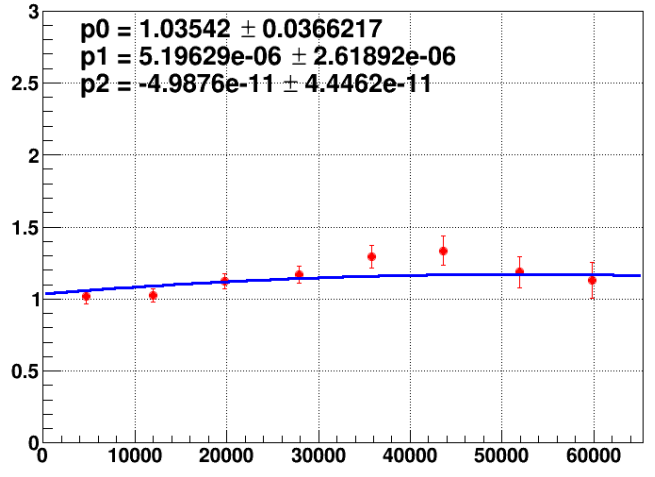

(a) $-0.1<x_{F}<0.3$

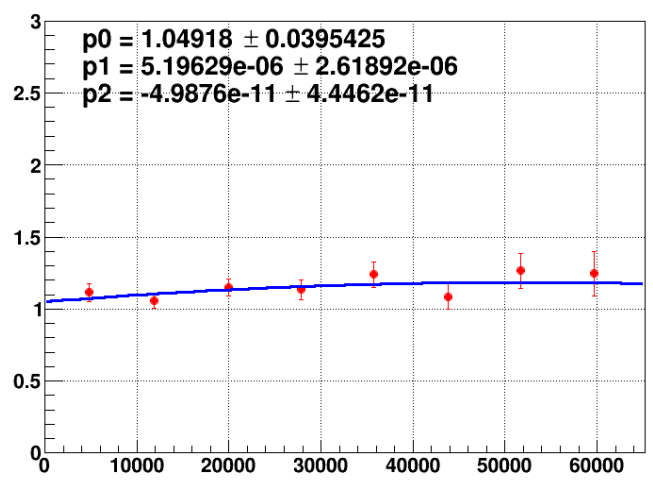

(c) $0.41<x_{F}<0.50$

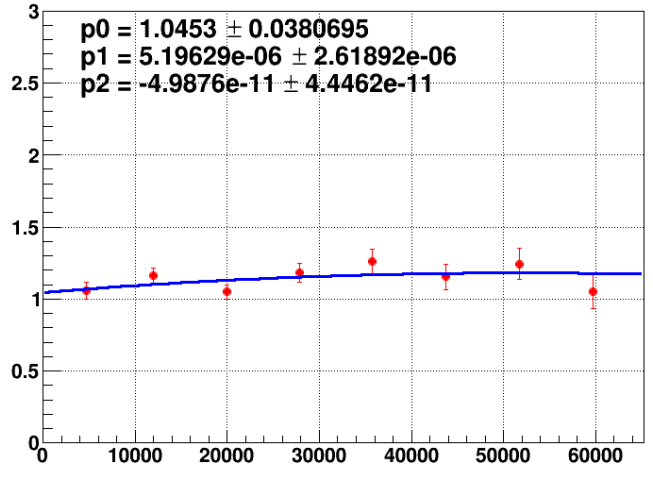

(b) $0.3<x_{F}<0.41$

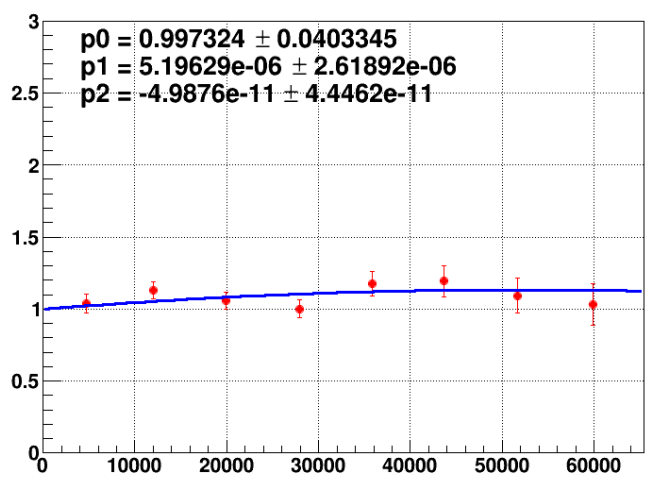

(d) $0.50<x_{F}<0.60$

Figure C.9: $R_{p A}$ for iron for different $x_{F}$ bins. 


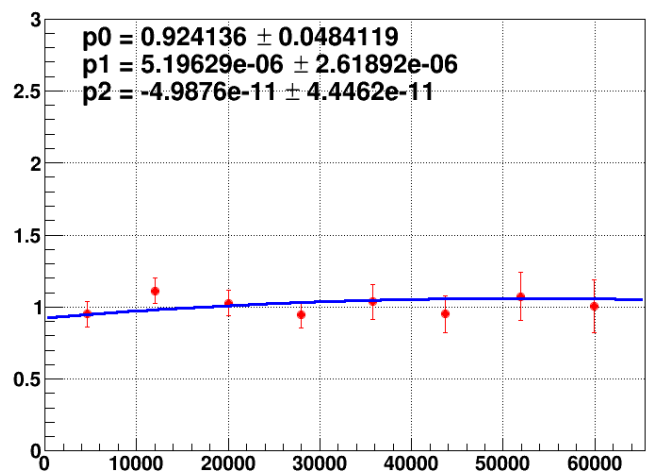

(a) $0.60<x_{F}<0.67$

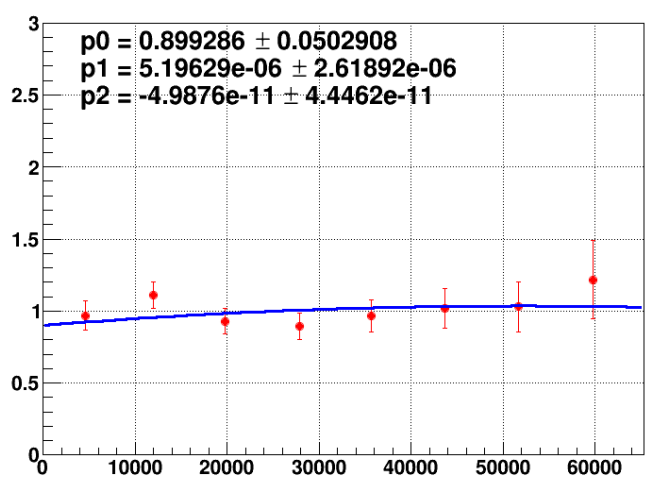

(b) $0.67<x_{F}<0.95$

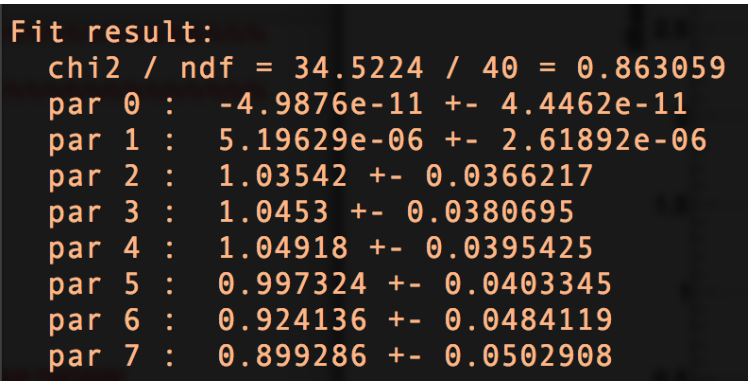

(d) Fit results

Figure C.10: $R_{p A}$ for iron for different $x_{F}$ bins along with the fit results. 


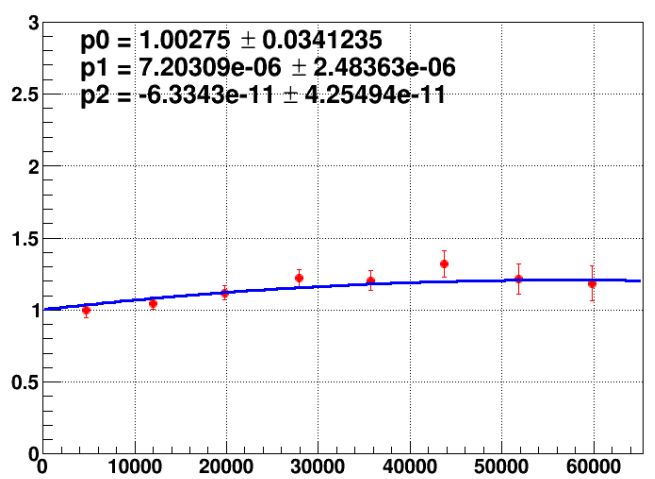

(a) $-0.1<x_{F}<0.3$

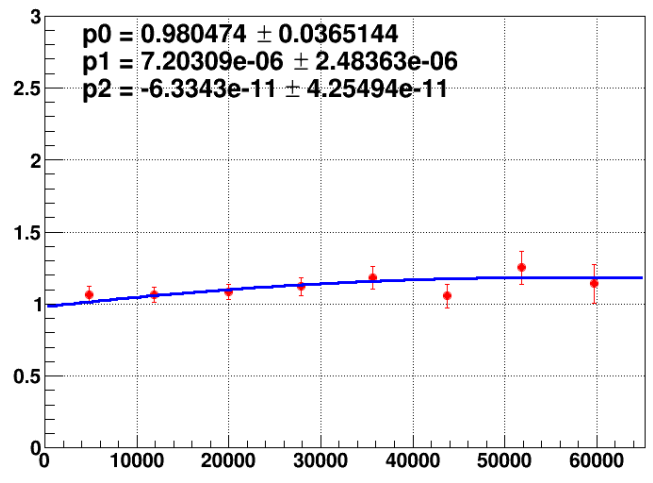

(c) $0.41<x_{F}<0.50$

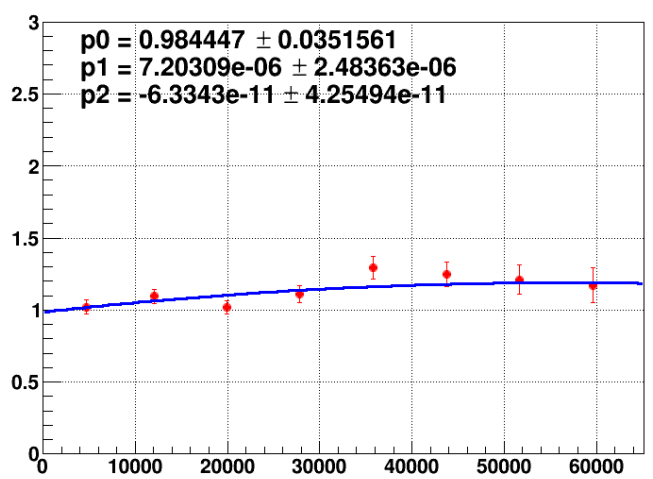

(b) $0.3<x_{F}<0.41$

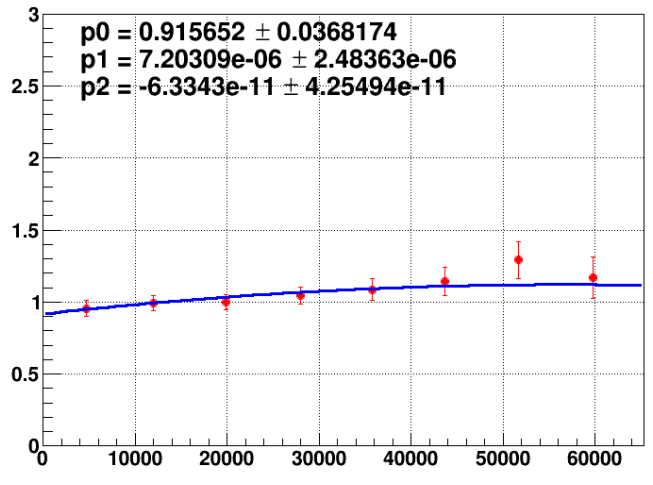

(d) $0.50<x_{F}<0.60$

Figure C.11: $R_{p A}$ for tungsten for different $x_{F}$ bins. 


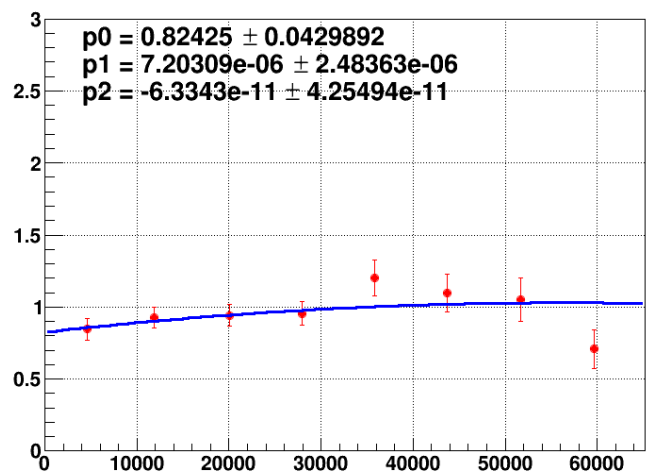

(a) $0.60<x_{F}<0.67$

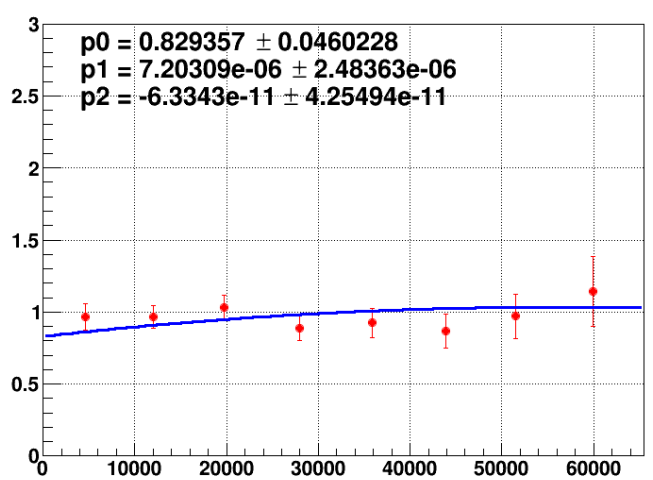

(b) $0.67<x_{F}<0.95$

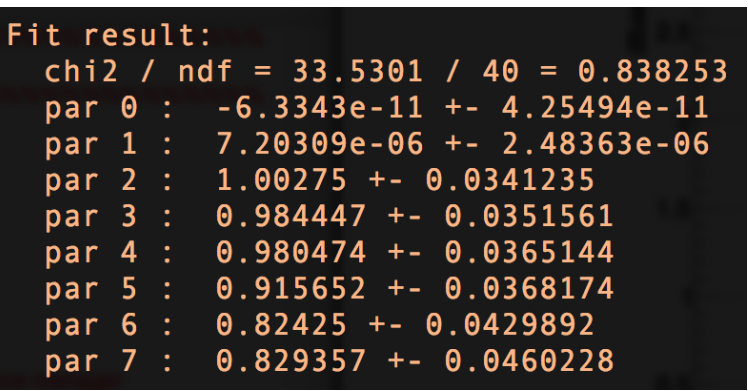

(d) Fit results

Figure C.12: $R_{p A}$ for tungsten for different $x_{F}$ bins along with the fit results. 


\section{Appendix D}

\section{Search for Dark Photons at SeaQuest}

\section{D.1 Why does the Dark Matter?}

Dark matter is a central element of cosmology and astronomy. It makes up about $27 \%$ of the energy density of the universe (as seen in D.1) and $80 \%$ of the mass of the universe, yet very little is known about it. The nature of dark matter and dark energy is a mystery and scientists all over the world are more dedicated than ever to understand them. A direct production or detection of dark matter particles in the lab will be a revolutionary discovery.

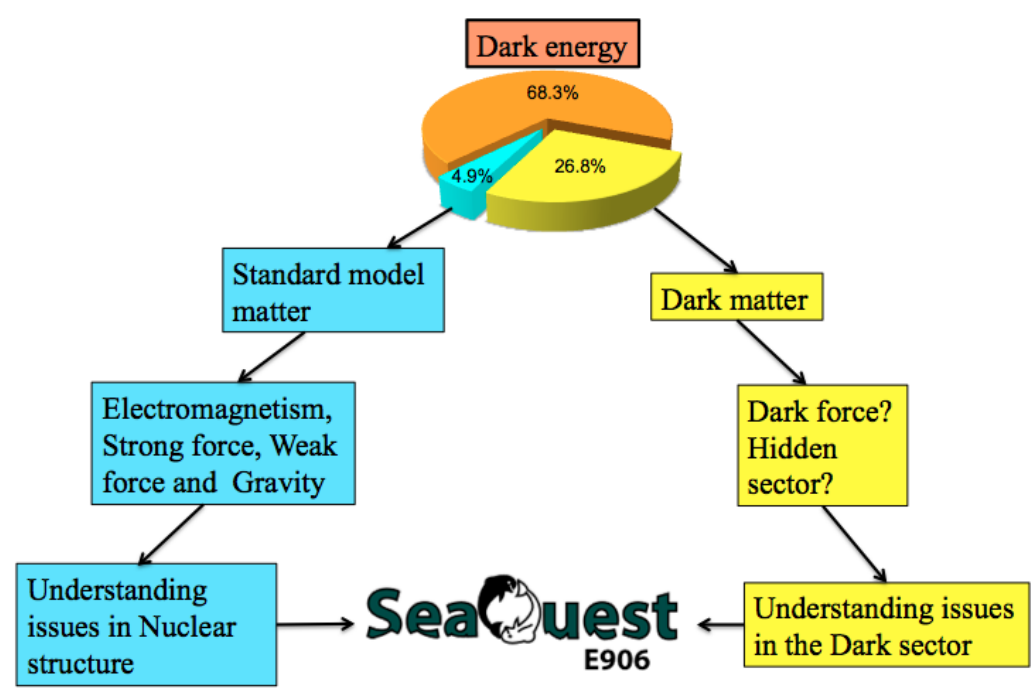

Figure D.1: Energy budget of the Universe

Probably the first mention of an unseen "darkness" existing in the universe can be traced back to as early as 1700 B.C. An english translation of the third verse of the Hymn of Creation (Nasadiya Sukta) of the Rig Veda states that [178]: 
At first there was only darkness wrapped in darkness

All this was only un-illumined water

That One which came to be, enclosed in nothing

arose at last, born of the power of heat

From the Hymn, it isn't obvious whether this cryptic "darkness" or "un-illumined cosmic water" can be identified with current day dark matter, dark energy or just an absence of radiation. Macroscopic properties (local density, temperature, physical location in space before 'creation' etc.) or microscopic particle properties (mass, spin, charge etc. that could be fundamental attributes of the Standard Model of Dark Physics) are missing a mention elsewhere. Nevertheless, it is interesting to note that such an ontologically different idea was orally transmitted from one generation of Vedic scholars to another for centuries. An excellent review on the history of dark matter can be found in [179].

It is worth mentioning that dark matter is quite different from the classical aether ${ }^{1}$. Also, black holes ${ }^{2}$ and dark energy ${ }^{3}$ are different phenomenon as compared to dark matter. The word "dark" in dark matter perhaps indicates "hidden" or "transparent" since light could pass right through it (with possible minimal interactions) [180]. It was initially coined dark as it cannot be seen directly with telescopes unlike other astronomical objects. Its indirect existence was inferred through its gravitational influence on surrounding objects.

\section{D.2 Shedding Light on Dark Matter}

\section{D.2.1 Dynamics of Galaxies in Coma Clusters}

In 1930, Fritz Zwicky carefully studied the dynamics of various galaxy clusters within the Coma cluster. He tried to estimate the mass of the Coma cluster by observing the red shifts

\footnotetext{
${ }^{1}$ Ruled out by Michelson and Morley light interferometry experiments. The unseen ethereal medium was initially proposed as a drag or accelerating force that could slow or speed up light due to its relative motion.

${ }^{2}$ Which appear "dark" as even light cannot escape its gravitational pull.

${ }^{3}$ Unseen energy accelerating the expansion of the universe.
} 
of various individual galaxies moving around on the periphery. Based on his calculations, he found that the mass of Coma cluster derived using the virial theorem ${ }^{4}$ was significantly larger than that estimated from their visible mass. He coined the term "dark matter" to explain the non luminous 'missing mass' that was essential for the galaxies to keep their trajectories. Zwicky made one of the most important observation crucial for the beginning of investigation of dark matter [181]. Yet, this remained a dormant puzzle for almost four decades.

\section{D.2.2 Rotational curves of galaxies}

In the early 1970's, Vera Rubin and collaborators carefully studied the rotational curves of various galaxies. She looked at the red shift of the stars as a function of their radii from the center of the galaxy. Her measurements extended far beyond the galaxy center, away from the visible region of the galaxy. She noted that instead of the rotational curves rapidly falling off as a function of their radii ${ }^{5}$, the rotation curves peaked at larger radii hinting the existence of unseen mass enveloping the galaxy, keeping the stars in their trajectories and preventing them from flying apart. Similar to Zwicky's observations, she also noted that 'additional gravity' is needed to explain the observed motion of stars in the galaxy [182].

\section{D.2.3 Gravitational Lensing}

It is well known from the days of Einstein that light bends in the presence of a strong gravitational field. When this happens, multiple copies (or rings and arcs) of the background galaxy emitting light are produced due to the presence of a foreground gravitational field. An example is shown in Fig. D.3 [184]. The reconstructed image depends on the geometry of the gravity lensing the light emitted from the background galaxy. The observed gravitational lensing could be used as a 'weighing scale' for the foreground galaxy. Similarly,

\footnotetext{
${ }^{4}$ The virial theorem states that the average kinetic energy of the objects is equal to - $1 / 2$ times the total gravitational potential energy for a spherically symmetric stable mass distribution.

${ }^{5} v \propto r^{-2}$ according to classical mechanics if the mass is located at the center.
} 


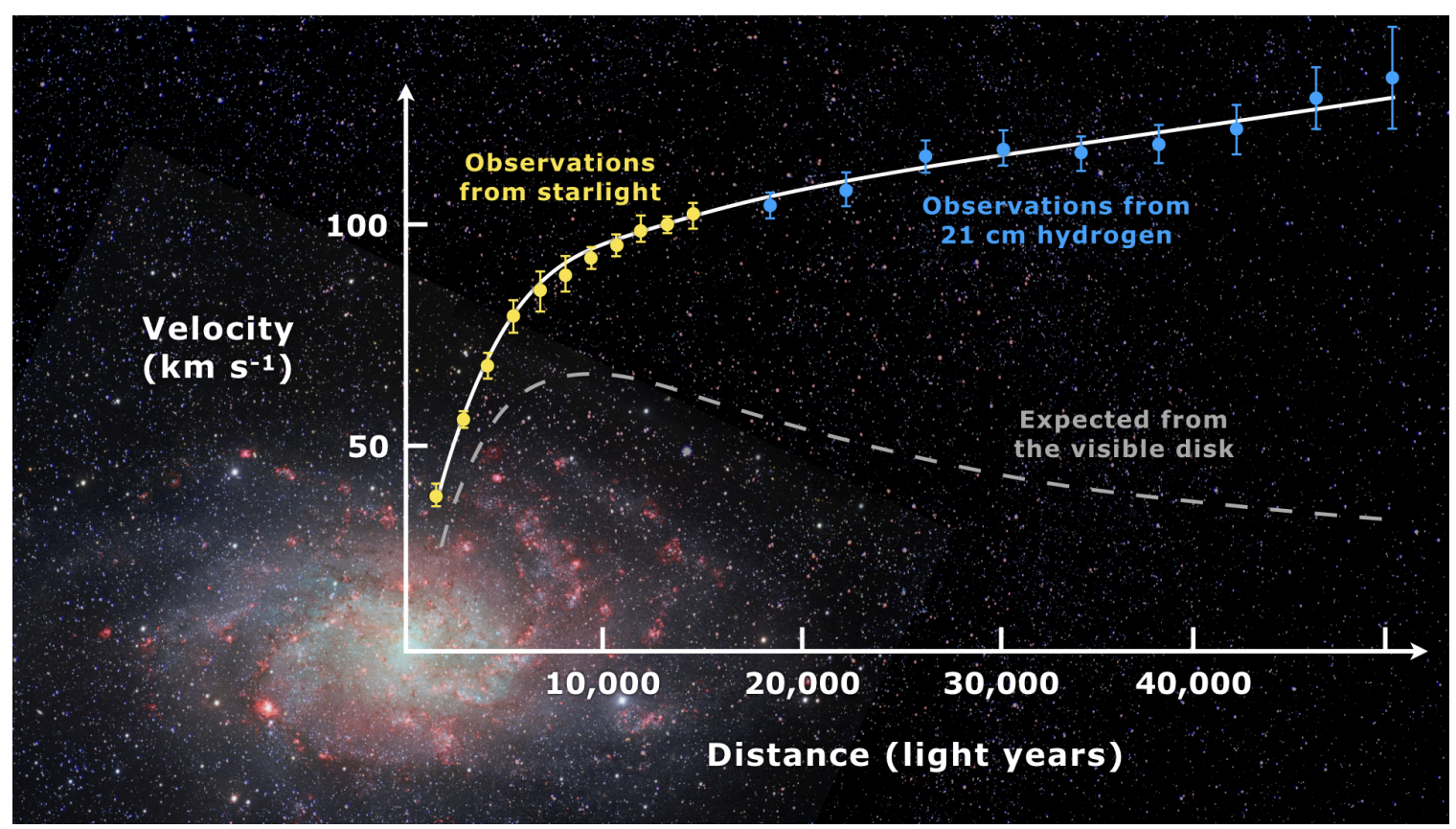

Figure D.2: Rotational curve of spiral galaxy M33 [183].

mass of the foreground galaxy can also be estimated using other independent light/mass ratio methods. A comparison of the these two methods of calculation of mass of the foreground galaxy led to the same conclusion that galaxies are more massive than expected and are surrounded by hidden matter.

\section{D.2.4 Bullet Clusters}

The bullet cluster is probably the most compelling evidence for the existence of dark matter. Even MOND theory (MOdified Newtonian Dynamics) which modifies Newtonian dynamics as a substitute for dark matter, is unable to explain the observations from the bullet cluster $[186,187]$. The bullet cluster is the aftermath of the collision of two galaxy clusters. The Chandra telescope was used to study the X-rays emitted during the collision. Red and yellow contour lines in Fig. D.4 show the X-ray maps of the collision of baryonic matter ${ }^{6}$ from the two galaxies. Green contour lines are maps from weak gravitational lensing of the X-rays. A comparison between these two maps show that mass is dominant not along the

\footnotetext{
6"Baryonic matter" in astrophysics includes electrons unlike in nuclear physics.
} 


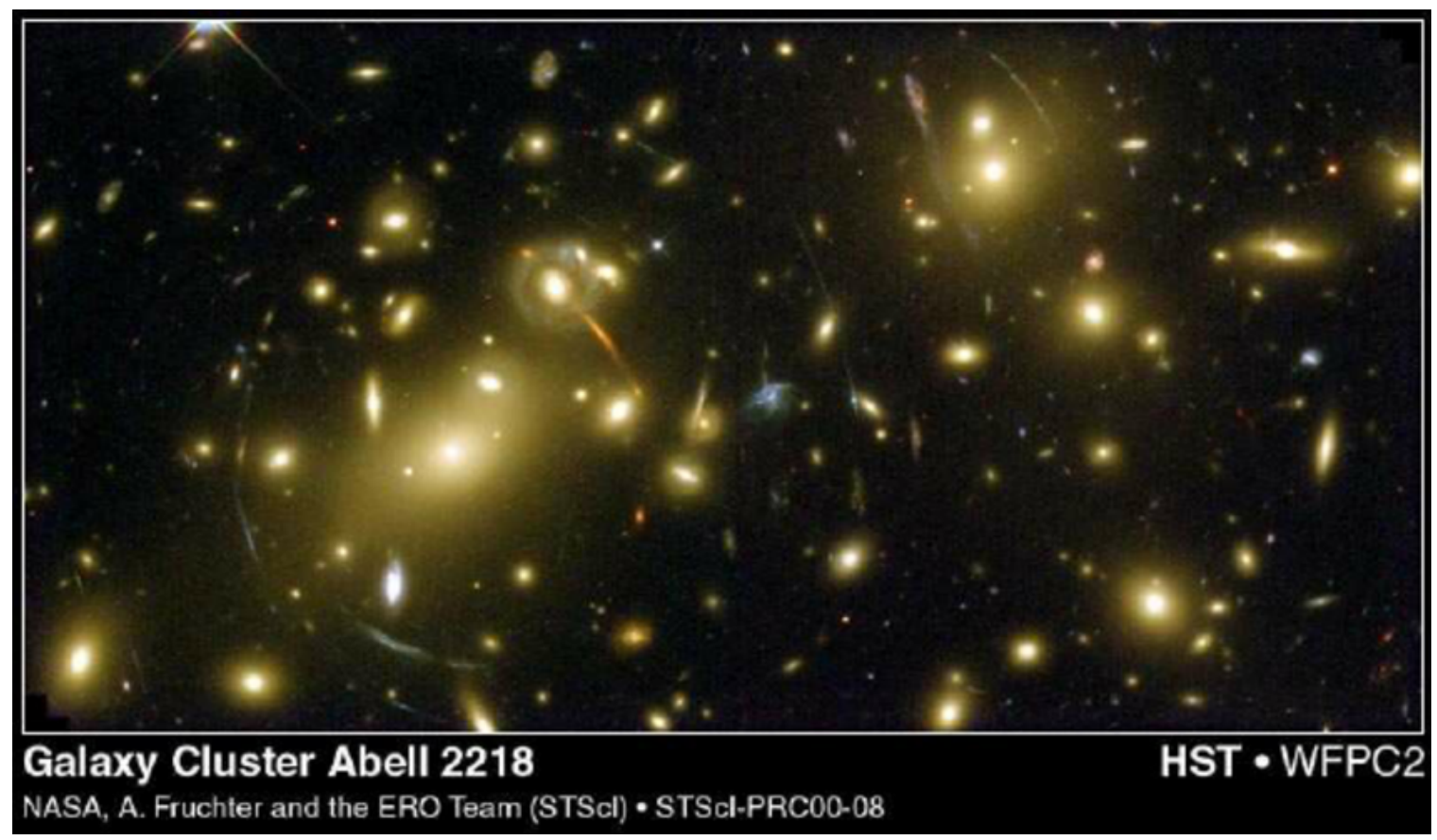

Figure D.3: Gravitational lensing of Abell 2218 Galaxy cluster. Credits: NASA, Andrew Fruchter and the ERO team [184].

distribution of the baryons but along a different kind of matter (such as dark matter) that enveloped these galaxies which moved right through each other almost without interacting [185].

\section{D.2.5 Surveys of Cosmic Microwave Background (CMB) spectrum}

During the big bang era, the universe was a hot dense 'soup' of unbound quarks, leptons and photons. High energy of these particles that constantly collided with these quarks prevented any kind of nucleosynthesis.

As the universe expanded and cooled down, the radiation was no longer energetic enough to keep these quarks and leptons from forming protons and neutrons. As the universe cooled even further, nuclei and electrons combined to form neutral atoms and eventually, the universe was left behind a background of microwave radiation at $\approx 2.75 \mathrm{~K}$. This remnant radiation is referred to as the Cosmic Microwave Background (CMB). The big bang synthesis predicted the existence of such microwave radiation and anisotropies of the 


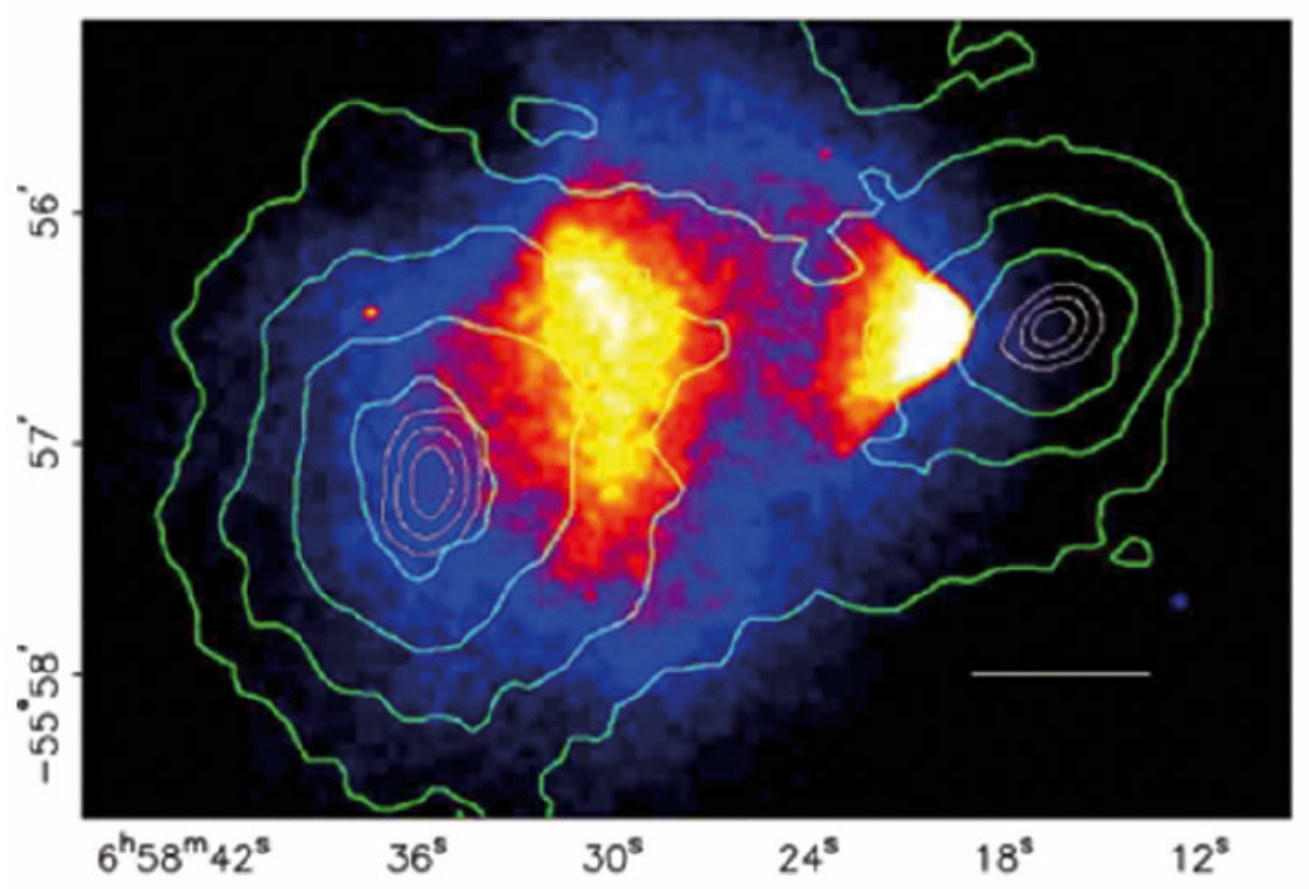

Figure D.4: Red and yellow contours show X-ray maps of the collision of baryonic matter in a bullet cluster. Green contours show the gravitational lensing of X-rays [185].

CMB have been mapped out with great precision. Fig. D.5 shows the CMB measured by the Wilkinson Microwave Anisotropy Probe (WMAP), a NASA sponsored satellite [188]. Power spectrum is plotted for this radiation and multipole moments are extracted as seen in Fig. D.6. The $\Lambda$ CDM model (solid red line) which takes into consideration dark energy and a cold dark matter component fits the data well [189] showing that dark matter was a crucial component during the formation stages of the universe.

Several other astrophysical anomalies such as the positron fraction excess in the universe [190, 191], and gamma ray excess from the galactic center [192, 193, 194, 195] (which have dark matter annihilation interpretations) suggest an exciting possibility that dark matter couples to ordinary matter more than just gravitationally. A protophobic fifthforce interpretation of the observed anomaly in ${ }^{8} \mathrm{Be}$ transition also suggests the possibility that dark matter could be studied via intensity based precision table top experiments [196]. Today, there is overwhelming evidence that dark matter not only exists, but also constitutes 


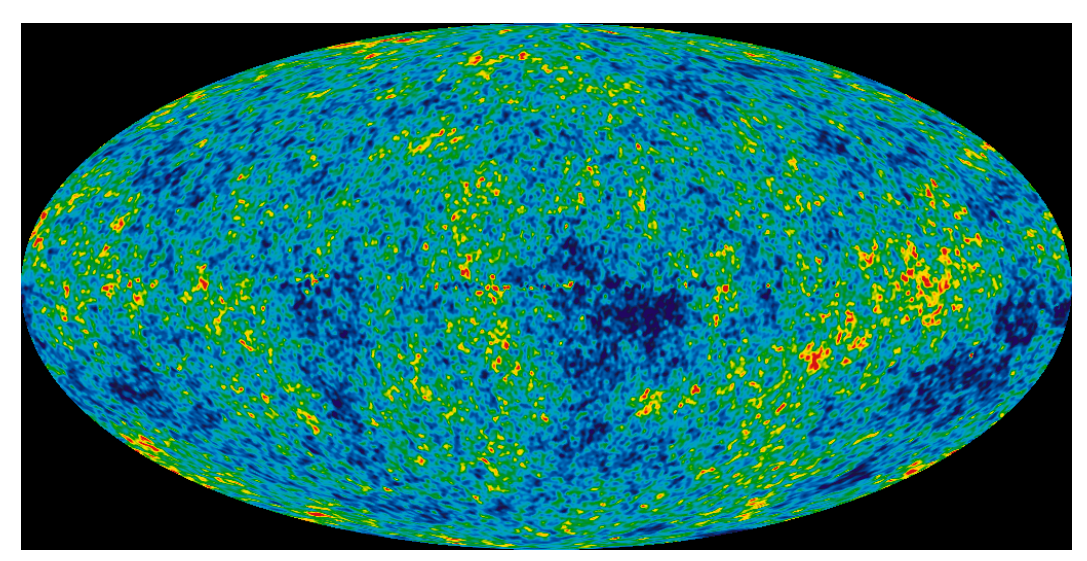

Figure D.5: The detailed, all-sky picture of the infant universe created from nine years of Wilkinson Microwave Anisotropy Probe data (WMAP). Fig. shows a temperature range of $\pm 200 \mu \mathrm{K}[188]$.

$27 \%$ of the energy density of the entire universe. The nature of dark matter remains a mystery and scientists are more determined than ever to gain a deeper understanding of dark matter.

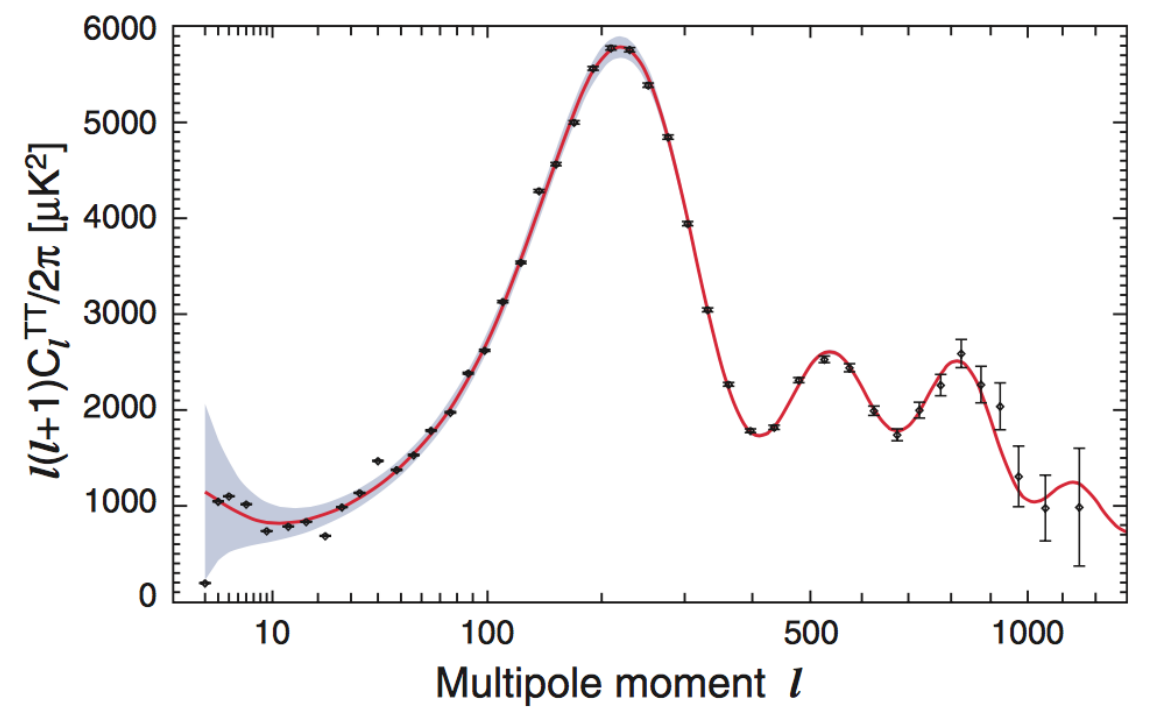

Figure D.6: Seven year temperature power spectrum from Wilkinson Microwave Anisotropy Probe [189]. 


\section{D.3 The Standard Model of Dark physics}

\section{D.3.1 What can Dark Matter be?}

Currently, the four fundamental forces that we know about do not account for the properties of dark matter. It could be that there are new dark forces that only dark matter particles feel. These particles from the dark sector might interact with regular matter very weakly. It could also be that dark matter does not interact with the regular matter except for via gravity. What we can measure possibly is the interaction between regular matter and dark matter. Various constraints have been put forward by particle physics theories on how often the dark matter particles should interact with regular matter and how massive the particles should be leaving a vast parameter space to be explored by experiments. WIMPS (Weakly Interacting Massive Particles), SIMPS (Strongly Interacting Massive Particles), neutralinos, QCD Axions, axinos, Q-balls etc. are all possible dark matter candidates. Neutrinos, due to them being electrically neutral and weakly interacting in nature, at one point were thought to be dark matter particles but were ruled out as they are too energetic ${ }^{7}$ and have too little mass to make up dark matter.

Indirect evidence for dark matter has been observed through its gravitational effects on baryonic matter in galaxies. However, the challenge undertaken by many experiments around the world is to find out if particles from the dark sector couple to those in Standard Model matter in any way. A variety of strategies have been adopted in searching for dark matter particles depending on their mass, type of coupling (scalar, pseudoscalar, vector etc.) and nature of interaction. Some collider and fixed target experiments try to make dark matter directly in the lab and detect its decay channels taking advantage of the energy and intensity frontiers. Some precision based table top experiments observe deviations from Standard Model measurements while others study the energy deposition from the scattering

\footnotetext{
${ }^{7}$ Neutrinos move close to the speed of light making it difficult for dark matter clumping essential for the formation of galaxies.
} 
of a dark matter particle with extremely sensitive detector material.

\section{D.3.2 Standard Model and Dark Sector coupling}

From a theoretical point of view, analogous to how the photons couple to the electromagnetic field (with a $U(1)$ gauge symmetry), the dark sector could also interact with ordinary matter via a similar mechanism. One could simply extend the Standard Model Lagrangian by making it $\mathrm{SU}(3)_{\text {color }} \times \mathrm{SU}(2)_{\text {weak }} \times \mathrm{U}(1)_{\gamma} \times \mathrm{U}^{\prime}(1)_{\gamma}$. Under such circumstances, a hidden gauge boson $A^{\prime}$ (or a dark photon, para photon, hidden photon etc.) would couple to ordinary matter with a small coupling constant $\varepsilon$ [197]. The Lagrangian of dark interaction is given by:

$$
\mathcal{L}_{\text {Dark }} \supset-\frac{1}{4} F_{\mu \nu}^{S M} F_{S M}^{\mu \nu}-\frac{1}{4} F_{\mu \nu}^{\text {hidden }} F_{\text {hidden }}^{\mu \nu}+\frac{1}{2} \varepsilon F_{\mu \nu}^{S M} F_{\text {hidden }}^{\mu \nu}+\frac{1}{2} m_{A^{\prime}}^{2} A_{\mu}^{\text {hidden }} A_{\text {hidden }}^{\mu}
$$

The first term in the expression is the free field Maxwell's equations in the SM sector, the second term is the analogous counterpart in the hidden sector, the third term is the coupling of the two sectors through the respective photons, the last term (usually called the kinetic mixing term) is the mass term for the dark sector. The equivalent term in the SM sector is the mass of the photon which is zero, so it doesnt appear. Fig. D.7 shows a depiction of the coupling between the Standard Model sector and the dark sector. From a theoretical

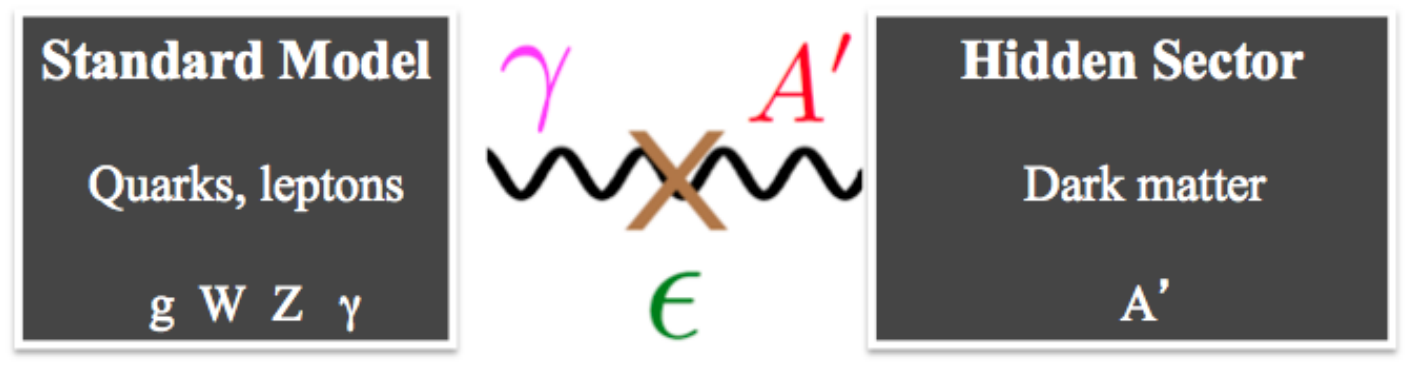

Figure D.7: Coupling between Standard Model sector and the Dark Sector

point of view, currently there could be many possibilities that make the dark sector have 
a rich underlying dynamics of interactions. From an experimental point of view, careful measurements on the decay channels of a dark photon will put constraints on the mass of the dark photon and the $\varepsilon$ variable and provide a portal into the dark sector.

\section{D.4 SeaQuest Dark Photon Search Strategy}

Since the experiment uses a dimuon trigger, three possible mechanisms could be taken advantage of in terms of searching for dark photons as shown in Fig D.9.

- Proton Bremsstrahlung: When protons traverse a nuclear medium, they decelerate due to interactions with the medium and as a consequence, emit electromagnetic radiation. This phenomenon is known as Proton Bremsstrahlung. Instead of emitting photons, the proton could emit a dark photon. This dark photon could decay into a lepton pair.

- $\eta$ decay: Many pseudoscalar mesons also decay by emitting two photons. Instead of emitting two photons, they can decay into a photon and a dark photon which can then decay into a lepton pair.

- Dark Drell-Yan process: In a Drell-Yan process, a quark from one hadron annihilates with a sea anti-quark from another hadron producing a virtual photon. Instead, they could annihilate into a dark photon which then decays into a lepton pair.

Since SeaQuest uses a dimuon trigger and the mass of the dark photon has to be $>2 m_{\mu}=$ $210 \mathrm{MeV}$, other light pseudoscalar mesons such as $\pi^{0}$ decays are not used. There are future plans of upgrading the spectrometer to include an electromagnetic calorimeter which could be used to detect $e^{+} e^{-}$pairs extending the parameter space to even lower mass regions. The dark photon thus produced from these decays could travel a distance without interacting with the FMAG (Fe Magnet) and decay into a $\mu^{+} \mu^{-}$pair in the last few interaction lengths to be opened up by the magnetic field of FMAG (shown in Fig D.8). This is a rather 


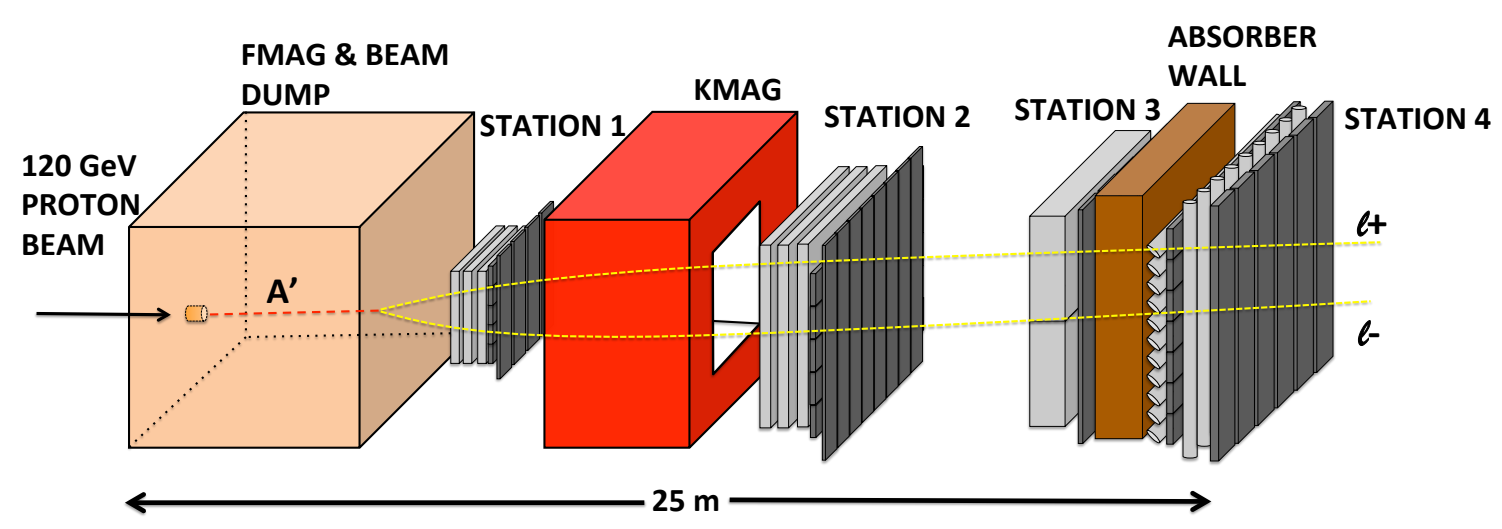

Figure D.8: Cartoon of a dark photon production-propagation-decay event

conservative approach where we have considered events where the background is known to be low, after most of the shielding. For the sake of muon pair detection, we have used part of the last meter of the Iron dump as part of the fiducial region. After detailed studies of the background, trigger optimization and track reconstruction efficiencies the fiducial region can be extended to further upstream [198].

If A decays into standard model particles, its decay length $l_{0}$ is given by [199]:

$$
l_{0} \simeq \frac{0.8 \mathrm{~cm}}{N_{\mathrm{eff}}}\left(\frac{E_{0}}{10 \mathrm{GeV}}\right)\left(\frac{10^{-4}}{\varepsilon}\right)^{2}\left(\frac{100 \mathrm{MeV}}{m_{A^{\prime}}}\right)^{2}
$$

where $N_{\text {eff }}$ is the number of available decay products, $E_{0}$ is the total energy of the incoming proton, $\varepsilon$ is the coupling constant between the dark sector and the Standard Model particles and $m_{A^{\prime}}$ is the mass of the dark photon in $\mathrm{MeV}$. 


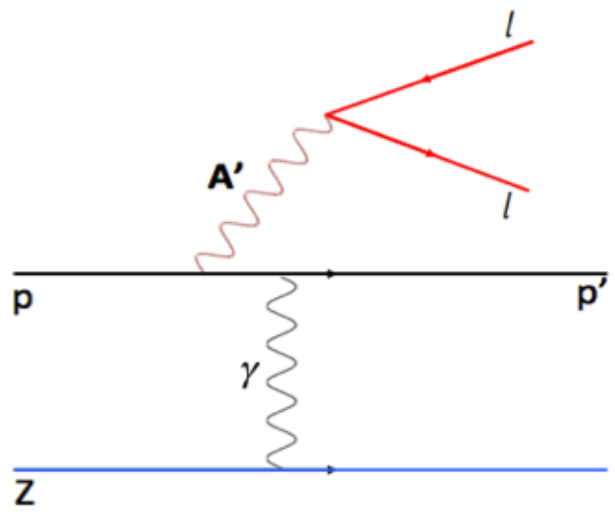

(a) Proton Bremsstrahlung

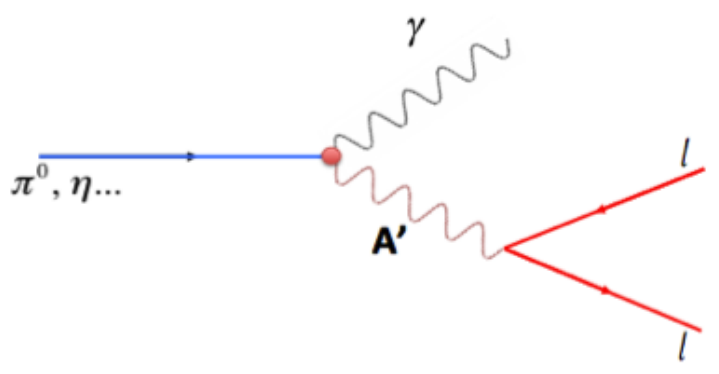

(b) $\eta$ decay

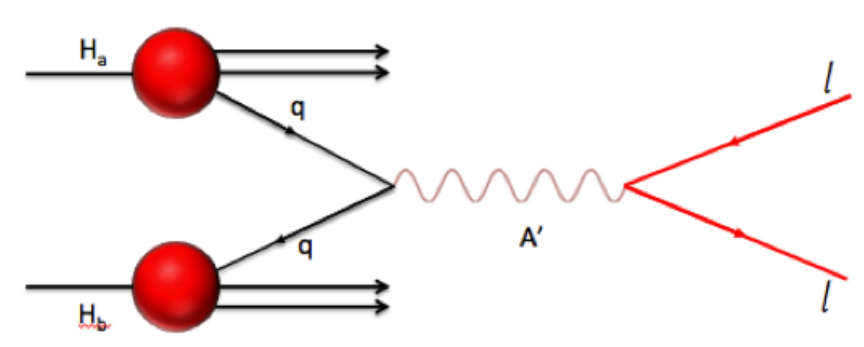

(c) Dark Drell-Yan process

Figure D.9: Dark photon production mechanisms

\section{D.5 Role of SeaQuest -III}

Understanding the structure and behavior of the constituents of matter is one of the central goals of modern physics. Many experiments all over the world continue to peel the layers of nature's rich sub-structure. SeaQuest is one such experiment that has potential for great discovery. It simultaneously addresses issues in nucleon and nuclear structure and can gain a portal into the dark sector. SeaQuest also has taken data with a trigger that has acceptance for high mass dark photon decays. A dark photon can be produced in collisions of charged particles with nuclei. These dark photons thus produced could decay into a lepton pair. Preliminary calculations show that SeaQuest covers a unique parameter space in 
coupling constant vs mass of the dark photon that is not investigated by other experiments. Simulation efforts and analysis of actual data are ongoing. No results will be presented on this topic. Only the modification of the trigger to include acceptance for dark photons is discussed.

\section{D.5.1 Trigger Modification for Dark Photon Acceptance}

As previously mentioned SeaQuest takes advantage of three processes (proton bremsstrahlung, $\eta$ decay and dark Drell-Yan process) to search for massive dark photons that could be generated in the beam dump. An $A^{\prime}$ produced by the above mentioned mechanisms could be generated after proton beam interactions with the first few interaction lengths of the magnet (one interaction length $=17 \mathrm{~cm}$ of $\mathrm{Fe}$ ). This massive dark photon could travel a certain distance up to $300-500 \mathrm{~cm}$ into the Iron beam dump and then decay into a dimuon. The difference between this and a regular Drell-Yan dimuon would be that the reconstructed events would have a displaced vertex from the generation point. Monte Carlo simulations were done with FastMC and GEANT4 - based Monte Carlo productions (SeaQuest simulation tools) assuming:

- low mass particle $\left(0.3 \mathrm{GeV}<\right.$ mass $\left._{A^{\prime}}<0.6 \mathrm{GeV}\right)$

- vertex decay position $440 \mathrm{~cm}<d z<480 \mathrm{~cm}$

$\bullet 0.05<x_{\text {beam }}<0.55$, and

- $0.002<x_{\text {target }}<0.025$.

Due to the width of the hodoscopes, the "well populated roads" did not differ by much for target materials and settings. Many dozens of new roads have to be included in order to gain 100\% acceptance. With minimal impact to the Drell-Yan program, a small list of roads was prepared and added to the main dimuon trigger roads. It turned out that the trigger roads that started out taking data already had some acceptance due to cross-over Drell-Yan 
events. These events are generated at the target or the first few interaction lengths of the beam dump and cross over in the "dark photon decay region" mimicking a displaced vertex event. The trigger event rate before and after the inclusion of these roads practically had no difference on bandwidth and DAQ deadtime.

\section{D.5.2 Finishing up the Search for Dark Photons}

This analysis requires further work on implementing the three mechanisms discussed ( $\eta$ decay, proton Bremsstrahlung and dark Drell-Yan like process) in the GEANT4 Monte Carlo used at SeaQuest. It also requires a thorough understanding of the single muon background and cross-over Drell-Yan events that are generated upstream of the target, in the target and in the region of the beam dump that could mimic dark photon like decays. The tracker software has to be modified accordingly to search for dimuon events with a displaced vertex coming from further downstream in the beam dump. Eventually all the data sets would have to be reprocessed with the upgraded tracker settings. Careful analysis cuts have to be designed to identify dark photon events coming from the region identified in the search strategy. Indeed, a discovery of dark photons would be revolutionary! 


\section{References}

[1] D. F. Geesaman and P. E. Reimer, "The sea of quarks and antiquarks in the nucleon," Rept. Prog. Phys., vol. 82, no. 4, p. $046301,2019$. arXiv: 1812.10372 [nucl-ex].

[2] H Geiger and E Marsden, "On a Diffuse Reflection of the alpha Particles," Proc. Roy. Soc., vol. 82, Dec. 2018.

[3] E. Rutherford, "The scattering of $\alpha$ and $\beta$ particles by matter and the structure of the atom," Phil. Mag. Ser.6, vol. 21, pp. 669-688, 1911.

[4] J. Chadwick, "The existence of a neutron," Proceedings of the Royal Society of London. Series A, Containing Papers of a Mathematical and Physical Character, vol. 136, no. 830, pp. 692-708, 1932.

[5] C. D. Anderson, “The Positive Electron,” Phys. Rev., vol. 43, pp. 491-494, 61933.

[6] M. Gell-Mann, "A schematic model of baryons and mesons," Physics Letters, vol. 8, no. 3, pp. $214-215,1964$.

[7] G. Zweig, "An SU(3) model for strong interaction symmetry and its breaking. version 2," in DEVELOPMENTS IN THE QUARK THEORY OF HADRONS. VOL. 1. 1964 - 1978, D. Lichtenberg and S. P. Rosen, Eds., 1964, pp. 22-101.

[8] M. Tanabashi et al., "Review of Particle Physics," Phys. Rev. D, vol. 98, p. 030 001, 32018.

[9] V. E. Barnes, and others., "Observation of a Hyperon with Strangeness Minus Three," Phys. Rev. Lett., vol. 12, pp. 204-206, 81964.

[10] J. J. Aubert et al., "Experimental Observation of a Heavy Particle J," Phys. Rev. Lett., vol. 33, pp. 1404-1406, 231974.

[11] J. E. Augustin et al., "Discovery of a Narrow Resonance in e+ e- Annihilation," Phys. Rev. Lett., vol. 33, pp. 1406-1408, 231974.

[12] M. L. Perl et al., "Evidence for anomalous lepton production in e+ e- annihilation," Physical Review Letters, vol. 35, pp. 1489-1492, Dec. 1975.

[13] Barber et al., "Discovery of Three-Jet Events and a Test of Quantum Chromodynamics at PETRA," Physical Review Letters, vol. 43, pp. 830-833, Sep. 1979. 
[14] S. W. Herb et al., "Observation of a Dimuon Resonance at $9.5 \mathrm{GeV}$ in $400-\mathrm{GeV}$ Proton-Nucleus Collisions," Physical Review Letters, vol. 39, pp. 252-255, Aug. 1977.

[15] G. Arnison et al., "Experimental observation of lepton pairs of invariant mass around $95 \mathrm{GeV} / \mathrm{c} 2$ at the CERN SPS collider," Physics Letters B, vol. 126, no. 5, pp. $398-410,1983$.

[16] M. Banner et al., "Observation of single isolated electrons of high transverse momentum in events with missing transverse energy at the CERN pp collider," Physics Letters B, vol. 122, no. 5, pp. $476-485,1983$.

[17] G. Arnison et al., "Experimental observation of isolated large transverse energy electrons with associated missing energy at $\mathrm{s}=540 \mathrm{GeV}$," Physics Letters B, vol. 122, no. 1, pp. $103-116,1983$.

[18] F. Abe et al., "Observation of Top Quark Production in p-pbar Collisions with the Collider Detector at Fermilab," Phys. Rev. Lett., vol. 74, pp. 2626-2631, 141995.

[19] S. Abachi, B. Abbott, M. Abolins, B. S. Acharya, I. Adam, D. L. Adams, M. Adams, S. Ahn, H. Aihara, J. Alitti, and et al., "Observation of the Top Quark," Physical Review Letters, vol. 74, pp. 2632-2637, Apr. 1995. eprint: hep-ex / 9503003.

[20] S. Chatrchyan, et al., "Observation of a new boson at a mass of $125 \mathrm{GeV}$ with the CMS experiment at the LHC," Physics Letters B, vol. 716, no. 1, pp. 30 -61, 2012.

[21] G. Aad et al., "Observation of a new particle in the search for the Standard Model Higgs boson with the ATLAS detector at the LHC," Physics Letters B, vol. 716, no. 1, pp. $1-29,2012$.

[22] Wikipedia, Standard Model, https://en.wikipedia.org/wiki/Standard_ Mode 1, [Online; accessed May 22, 2019].

[23] D. Griffiths, Introduction to Elementary particles, Second, Revised Edition. WileyVCH., 2008.

[24] F. Halzen and M. Alan, Quarks and Leptons. John Wiley and sons, 1984.

[25] H. Frauenfelder and E. Henley, Subatomic physics, Second. Prentice-Hall, 1991.

[26] H. Fritzsch, M. Gell-Mann, and H. Leutwyler, "Advantages of the color octet gluon picture," Physics Letters B, vol. 47, no. 4, pp. $365-368,1973$. 
[27] H. D. Politzer, "Reliable Perturbative Results for Strong Interactions?" Phys. Rev. Lett., vol. 30, pp. 1346-1349, 261973.

[28] D. J. Gross and F. Wilczek, "Ultraviolet Behavior of Non-Abelian Gauge Theories," Phys. Rev. Lett., vol. 30, pp. 1343-1346, 261973.

[29] R. Hofstadter and R. W. McAllister, "Electron Scattering from the Proton," Phys. Rev., vol. 98, pp. 217-218, 11955.

[30] M. N. Rosenbluth, "High Energy Elastic Scattering of Electrons on Protons," Phys. Rev., vol. 79, pp. 615-619, 41950.

[31] R. G. Sachs, "Magnetic and electric form factors," in High-energy physics. Proceedings, 11th International Conference, ICHEP'62, Geneva, Switzerland, Jul 411, 1962, 1962, pp. 197-199.

[32] Arnold et al., "Measurement of Elastic Electron Scattering from the Proton at High Momentum Transfer," Phys. Rev. Lett., vol. 57, pp. 174-177, 21986.

[33] R. P. Feynman, "Very High-Energy Collisions of Hadrons," Phys. Rev. Lett., vol. 23, pp. 1415-1417, 241969.

[34] J. Kuti and V. F. Weisskopf, "Inelastic Lepton-Nucleon Scattering and Lepton Pair Production in the Relativistic Quark-Parton Model," Phys. Rev. D, vol. 4, pp. 3418 3439, 111971.

[35] J. D. Bjorken, “Asymptotic Sum Rules at Infinite Momentum," Phys. Rev., vol. 179, pp. 1547-1553, 51969.

[36] C. G. Callan and D. J. Gross, "High-Energy Electroproduction and the Constitution of the Electric Current," Phys. Rev. Lett., vol. 22, pp. 156-159, 41969.

[37] J. D. Bjorken and E. A. Paschos, "Inelastic Electron-Proton and gamma - Proton Scattering and the Structure of the Nucleon," Phys. Rev., vol. 185, pp. 1975-1982, 51969.

[38] Y. L. Dokshitzer, "Calculation of the Structure Functions for Deep Inelastic Scattering and e+ e- Annihilation by Perturbation Theory in Quantum Chromodynamics.," Sov. Phys. JETP, vol. 46, pp. 641-653, 1977, [Zh. Eksp. Teor. Fiz.73,1216(1977)].

[39] V. N. Gribov and L. N. Lipatov, "Deep inelastic e p scattering in perturbation theory," Sov. J. Nucl. Phys., vol. 15, pp. 438-450, 1972, [Yad. Fiz.15,781(1972)].

[40] G. Altarelli and G. Parisi, "Asymptotic Freedom in Parton Language," Nucl. Phys., vol. B126, pp. 298-318, 1977. 
[41] J. H. Christenson, G. S. Hicks, L. M. Lederman, P. J. Limon, B. G. Pope, and E. Zavattini, "Observation of Massive Muon Pairs in Hadron Collisions," Phys. Rev. Lett., vol. 25, pp. 1523-1526, 211970.

[42] S. D. Drell and T.-M. Yan, "Massive Lepton-Pair Production in Hadron-Hadron Collisions at High Energies," Phys. Rev. Lett., vol. 25, pp. 316-320, 51970.

[43] B. Pasquini and P. Schweitzer, "Pion transverse momentum dependent parton distributions in a light-front constituent approach, and the Boer-Mulders effect in the pion-induced Drell-Yan process," Phys. Rev. D, vol. 90, p. 014 050, 12014.

[44] A. Accardi, L. T. Brady, W. Melnitchouk, J. F. Owens, and N. Sato, "Constraints on large- $x$ parton distributions from new weak boson production and deep-inelastic scattering data," Phys. Rev., vol. D93, no. 11, p. 114 017, 2016. arXiv: 1602 . 03154 [hep-ph].

[45] K. Freudenreich, "Lepton pair production in fixed target hadron hadron collisions," Int. J. Mod. Phys., vol. A5, pp. 3643-3760, 1990.

[46] G. Moreno, Brown, et al., "Dimuon production in proton-copper collisions at sqrt(s)=38.8 gev," Phys. Rev. D, vol. 43, pp. 2815-2835, 91991.

[47] McGaughey et al., "Cross sections for the production of high-mass muon pairs from $800 \mathrm{GeV}$ proton bombardment of 2H," Phys. Rev. D, vol. 50, pp. 3038-3045, 51994.

[48] P. L. McGaughey, J. M. Moss, and J. C. Peng, "High-energy hadron-induced dilepton production from nucleons and nuclei," Annual Review of Nuclear and Particle Science, vol. 49, no. 1, pp. 217-253, 1999.

[49] K. Gottfried, "Sum Rule for High-Energy Electron-Proton Scattering," Phys. Rev. Lett., vol. 18, pp. 1174-1177, 251967.

[50] Amaudruz et al., "Gottfried sum from the ratio F2n/F2p," Phys. Rev. Lett., vol. 66, pp. 2712-2715, 211991.

[51] Arneodo et al., "Reevaluation of the Gottfried sum," Phys. Rev. D, vol. 50, R1-R3, 11994.

[52] G. Preparata, P. G. Ratcliffe, and J. Soffer, "Isospin violation in quark-parton distributions," Phys. Rev. Lett., vol. 66, pp. 687-690, 61991.

[53] B.-Q. Ma, "Sea quark content of nucleons. Flavour distribution asymmetry or isospin symmetry breaking?" Physics Letters B, vol. 274, no. 1, pp. 111 -115, 1992. 
[54] S. Ellis and W. Stirling, "Constraints on isospin breaking in the light quark sea from the Drell-Yan process," Physics Letters B, vol. 256, no. 2, pp. 258 -264, 1991.

[55] A. Baldit et al., "Study of the isospin symmetry breaking in the light quark sea of the nucleon from the Drell-Yan process," Physics Letters B, vol. 332, no. 1, pp. 244 $-250,1994$.

[56] E. A. Hawker et al., "Measurement of the Light Antiquark Flavor Asymmetry in the Nucleon Sea," Phys. Rev. Lett., vol. 80, pp. 3715-3718, 171998.

[57] R. S. Towell et al., "Improved measurement of the dbar/ubar asymmetry in the nucleon sea," Phys. Rev. D, vol. 64, p. 052 002, 52001.

[58] Ackerstaff et al., "Flavor Asymmetry of the Light Quark Sea from Semi-inclusive Deep-Inelastic Scattering," Phys. Rev. Lett., vol. 81, pp. 5519-5523, 251998.

[59] R. D. Field and R. P. Feynman, "Quark elastic scattering as a source of hightransverse-momentum mesons," Phys. Rev. D, vol. 15, pp. 2590-2616, 91977.

[60] D. A. Ross and C. T. Sachrajda, "Flavor Symmetry Breaking in Anti-Quark Distributions," Nucl. Phys., vol. B149, pp. 497-516, 1979.

[61] M. Alberg and G. A. Miller, "Chiral Light Front Perturbation Theory and the Flavor Dependence of the Light-Quark Nucleon Sea," 2017. arXiv: 1712.05814 [nucl-th].

[62] W. Melnitchouk, J. Speth, and A. W. Thomas, "Dynamics of light antiquarks in the proton," Phys. Rev. D, vol. 59, p. 014 033, 11998.

[63] E. Henley and G. Miller, "Excess of d over u in the proton sea quark distribution," Physics Letters B, vol. 251, no. 3, pp. 453 -454, 1990.

[64] M. Alberg, E. M. Henley, and G. A. Miller, "Omega meson cloud and the proton's light anti-quark distributions," Physics Letters B, vol. 471, no. 4, pp. $396-399$, 2000.

[65] E. J. Eichten, I. Hinchliffe, and C. Quigg, "Flavor asymmetry in the light-quark sea of the nucleon," Phys. Rev. D, vol. 45, pp. 2269-2275, 71992.

[66] Bourrely, C., Soffer, J., and Buccella, F., "A statistical approach for polarized parton distributions," Eur. Phys. J. C, vol. 23, no. 3, pp. 487-501, 2002.

[67] H. Song, X. Zhang, and B.-Q. Ma, "Light flavor asymmetry of nucleon sea," The European Physical Journal C, vol. 71, no. 2, p. 1542, 2011. 
[68] M. Wakamatsu, "Transverse momentum distributions of quarks in the nucleon from the chiral quark soliton model," Phys. Rev. D, vol. 79, p. 094 028, 92009.

[69] A. Dorokhov and N. Kochelev, "Instanton-induced asymmetric quark configurations in the nucleon and parton sum rules," Physics Letters B, vol. 304, no. 1, pp. $167-175,1993$.

[70] P. Reimer, D. Geesaman, et al., "Drell-Yan Measurements of Nucleon and Nuclear Structure with the Fermilab Main Injector: E906," 2006.

[71] M. H. Schub, et al., "Measurement of J/ $\psi$ and $\psi^{\prime}$ production in $800 \mathrm{GeV} / \mathrm{c}$ protongold collisions," Phys. Rev. D, vol. 52, pp. 1307-1315, 31995.

[72] J. Aubert et al., "The ratio of the nucleon structure functions F2N for iron and deuterium," Physics Letters B, vol. 123, no. 3, pp. 275 -278, 1983.

[73] — - "Measurements of the nucleon structure functions F2N in deep inelastic muon scattering from deuterium and comparison with those from hydrogen and iron," Nuclear Physics B, vol. 293, pp. 740 -786, 1987.

[74] A. Bodek and J. L. Ritchie, "Further studies of Fermi-motion effects in lepton scattering from nuclear targets," Phys. Rev. D, vol. 24, pp. 1400-1402, 51981.

[75] — - "Fermi-motion effects in deep-inelastic lepton scattering from nuclear targets," Phys. Rev. D, vol. 23, pp. 1070-1091, 51981.

[76] A. Bodek et al., "Electron Scattering from Nuclear Targets and Quark Distributions in Nuclei," Phys. Rev. Lett., vol. 50, pp. 1431-1434, 191983.

[77] X. Chen, J. Ruan, R. Wang, P. Zhang, and W. Zhu, "Applications of a nonlinear evolution equation ii: the EMC effect," Int. J. Mod. Phys., vol. E23, no. 10, p. 1450058 , 2014. arXiv: 1306.1874 [hep-ph] .

[78] G. Bari et al., "A Measurement of Nuclear Effects in Deep Inelastic Muon Scattering on Deuterium, Nitrogen and Iron Targets," Phys. Lett., vol. 163B, p. 282, 1985.

[79] P. Amaudruz et al., "The ratio F2n/F2p in deep inelastic muon scattering," Nuclear Physics B, vol. 371, no. 1, pp. 3-31, 1992.

[80] M. R. Adams et al., "Saturation of shadowing at very low bjorken x," Phys. Rev. Lett., vol. 68, pp. 3266-3269, 221992.

[81] A. Bodek et al., "Comparison of the deep-inelastic Structure Functions of Deuterium and Aluminum Nuclei," Phys. Rev. Lett., vol. 51, pp. 534-537, 71983. 
[82] J. Gomez et al., "Measurement of the A dependence of deep-inelastic electron scattering," Phys. Rev. D, vol. 49, pp. 4348-4372, 91994.

[83] S. Dasu et al., "Measurement of the difference in $R=\frac{\sigma_{L}}{\sigma_{T}}$ and of $\frac{\sigma^{A}}{\sigma^{D}}$ in deepinelastic $e-D, e-F e$, and $e-A u$ scattering," Phys. Rev. Lett., vol. 60, pp. 25912594, 251988.

[84] K. Ackerstaff et al., "Nuclear effects on $\mathrm{r}=\sigma_{L} / \sigma_{T}$ in deep inelastic scattering," Phys. Lett., vol. B475, pp. 386-394, 2000, [Erratum: Phys. Lett.B567,339(2003)]. arXiv: hep-ex/9910071 [hep-ex].

[85] K. S. Egiyan et al., "Observation of nuclear scaling in the $\mathrm{A}\left(\mathrm{e}, \mathrm{e}\right.$ ') reaction at $x_{B}>$ 1," Phys. Rev. C, vol. 68, p. 014 313, 12003.

[86] K. S. Egiyan et al., "Measurement of Two- and Three-Nucleon Short-Range Correlation Probabilities in Nuclei," Phys. Rev. Lett., vol. 96, p. 082 501, 82006.

[87] J. Seely et al., "New Measurements of the European Muon Collaboration Effect in Very Light Nuclei," Phys. Rev. Lett., vol. 103, p. 202 301, 202009.

[88] N. Fomin et al., "New Measurements of High-Momentum Nucleons and ShortRange Structures in Nuclei," Phys. Rev. Lett., vol. 108, p. 092 502, 92012.

[89] H. Abramowicz et al., "Neutrino and antineutrino charged-current inclusive scattering in iron in the energy range $20 ; \mathrm{Ev} ; 300 \mathrm{GeV}$," Zeitschrift für Physik C Particles and Fields, vol. 17, no. 4, pp. 283-307, 1983.

[90] D. M. Alde et al., "Nuclear dependence of dimuon production at $800 \mathrm{GeV}$," Phys. Rev. Lett., vol. 64, pp. 2479-2482, 211990.

[91] M. A. Vasiliev et al., "Parton Energy Loss Limits and Shadowing in Drell-Yan Dimuon Production," Phys. Rev. Lett., vol. 83, pp. 2304-2307, 121999.

[92] M. Arneodo et al., "The Structure Function ratios F2(li) / F2(D) and F2(C) / F2(D) at small x," Nucl. Phys., vol. B441, pp. 12-30, 1995. arXiv: hep-ex/9504002 [hep-ex].

[93] K. Rith, "Present Status of the EMC effect," Subnucl. Ser., vol. 51, pp. 431-449, 2015. arXiv: 1402.5000 [hep-ex].

[94] S. J. Brodsky and H. J. Lu, "Shadowing and antishadowing of nuclear structure functions," Phys. Rev. Lett., vol. 64, pp. 1342-1345, 121990.

[95] D. F. Geesaman, K Saito, and A. W. Thomas, "The Nuclear EMC Effect," Annual Review of Nuclear and Particle Science, vol. 45, no. 1, pp. 337-390, 1995. 
[96] P. R. Norton, “The EMC effect,” Rept. Prog. Phys., vol. 66, pp. 1253-1297, 2003.

[97] S. Malace, D. Gaskell, D. W. Higinbotham, and I. Cloet, "The Challenge of the EMC effect: existing data and future directions," Int. J. Mod. Phys., vol. E23, no. 08, p. 1430 013, 2014. arXiv: 1405.1270 [nucl-ex].

[98] S. Dasu et al., "Measurement of kinematic and nuclear dependence of $R=\frac{\sigma_{L}}{\sigma_{T}}$ in deep inelastic electron scattering," Phys. Rev. D, vol. 49, pp. 5641-5670, 111994.

[99] M. Arneodo et al., "The A dependence of the nuclear structure function ratios," Nucl. Phys., vol. B481, pp. 3-22, 1996.

[100] H. D. Vries, C. D. Jager, and C. D. Vries, "Nuclear charge-density-distribution parameters from elastic electron scattering," Atomic Data and Nuclear Data Tables, vol. 36, no. 3, pp. $495-536,1987$.

[101] P. Amaudruz et al., "Measurements of Rd-Rp and RCa-Rc in deep inelastic muon scattering," Physics Letters B, vol. 294, no. 1, pp. 120 -126, 1992.

[102] D. W. Higinbotham, J. Gomez, and E. Piasetzky, "Nuclear scaling and the EMC Effect," 2010. arXiv: 1003.4497 [hep-ph] .

[103] O. Hen, D. W. Higinbotham, G. A. Miller, E. Piasetzky, and L. B. Weinstein, "The EMC Effect and High Momentum Nucleons in Nuclei," Int. J. Mod. Phys., vol. E22, p. 1330 017, 2013. arXiv: 1304.2813 [nucl-th].

[104] J. Arrington, A. Daniel, D. Day, N. Fomin, D. Gaskell, and P. Solvignon, “A detailed study of the nuclear dependence of the EMC effect and short-range correlations," Phys. Rev., vol. C86, p. 065 204, 2012. arXiv: 1206.6343 [nucl-ex] .

[105] O. Hen, G. A. Miller, E. Piasetzky, and L. B. Weinstein, "Nucleon-Nucleon Correlations, Short-lived Excitations, and the Quarks Within," Rev. Mod. Phys., vol. 89, no. 4, p. 045 002, 2017. arXiv: 1611.09748 [nucl-ex].

[106] O. Hen, E. Piasetzky, and L. B. Weinstein, "New data strengthen the connection between Short Range Correlations and the EMC effect," Phys. Rev., vol. C85, p. 047301,2012 arXiv: 1202.3452 [nucl-ex].

[107] L. B. Weinstein, E. Piasetzky, D. W. Higinbotham, J. Gomez, O. Hen, and R. Shneor, "Short Range Correlations and the EMC Effect," Phys. Rev. Lett., vol. 106, p. 052301,2011 . arXiv: 1009.5666 [hep-ph ] .

[108] E. Piasetzky, L. Weinstein, D. Higinbotham, J. Gomez, O. Hen, and R. Shneor, "Short range correlations and the EMC effect," Nuclear Physics A, vol. 855, no. 1, 
pp. $245-248,2011$, Proceedings of the 4th International Conference on Hard and Electromagnetic Probes of High-Energy Nuclear Collisions HP2010.

[109] J.-W. Chen, W. Detmold, J. E. Lynn, and A. Schwenk, "Short-Range Correlations and the EMC Effect in Effective Field Theory," Phys. Rev. Lett., vol. 119, p. $262502,262017$.

[110] S. A. Kulagin and R. Petti, "Global study of nuclear structure functions," Nucl. Phys., vol. A765, pp. 126-187, 2006. arXiv: hep-ph/0412425 [hep-ph].

[111] E. L. Berger, F. Coester, and R. B. Wiringa, "Pion density in nuclei and deepinelastic lepton scattering," Phys. Rev. D, vol. 29, pp. 398-411, 31984.

[112] E. L. Berger and F. Coester, "Nuclear effects in deep-inelastic lepton scattering," Phys. Rev. D, vol. 32, pp. 1071-1084, 51985.

[113] H. Jung and G. A. Miller, "Pionic contributions to deep inelastic nuclear structure functions," Phys. Rev. C, vol. 41, pp. 659-664, 21990.

[114] A. E. L. Dieperink and C. L. Korpa, "Pions in the nuclear medium and DrellYan scattering," Phys. Rev., vol. C55, pp. 2665-2674, 1997. arXiv: nucl-th / 9703025 [nucl-th].

[115] G. E. Brown, M. Buballa, Z. B. Li, and J. Wambach, "Where the nuclear pions are," Nucl. Phys., vol. A593, pp. 295-314, 1995. arXiv: nucl-th/9410049 [nucl-th].

[116] J. R. Smith and G. A. Miller, "Chiral Solitons in Nuclei: Saturation, EMC Effect, and Drell-Yan Experiments," Phys. Rev. Lett., vol. 91, p. 212 301, 212003.

[117] S. A. Kulagin and R. Petti, "Nuclear parton distributions and the Drell-Yan process," Phys. Rev., vol. C90, no. 4, p. 045 204, 2014. arXiv: 1405.2529 [ hep-ph] .

[118] Fermilab, Fermilab's Accelerator Complex, http : / www. fnal .gov/pub / science/particle-accelerators/accelerator-complex.html, [Online; accessed Feb 10, 2019].

[119] A. Watts, Slow Spill extraction - Beam to SeaQuest, DOCID - 1715, [Online; accessed Feb 12, 2019].

[120] C. Aidala et al., "The SeaQuest spectrometer at Fermilab," Nuclear Instruments and Methods in Physics Research Section A: Accelerators, Spectrometers, Detectors and Associated Equipment, vol. 930, pp. 49 -63, 2019. 
[121] M. R. Roder H.M. Childs G.E. and A. P.E., Survey of the Properties of the Hydrogen Isotopes Below Their Critical Temperatures. U.S. Department of Commerce, 1973.

[122] R. Towell, "Measurement of the antiquark flavor asymmetry in the nucleon sea," $\mathrm{PhD}$ thesis, University of Texas at Austin, 1999.

[123] D. Geesaman, Hall probe measurements of KMAG field, DOCID - 445-v1, [Online; accessed Feb 15, 2019].

[124] T. Connor, personal communication, Feb. 15, 2019.

[125] P.-J. Lin, "Measurement of Quark Energy Loss in Cold Nuclear matter," $\mathrm{PhD}$ thesis, University of Colorado, 2017.

[126] G Charpak and F Sauli, "High-Resolution Electronic Particle Detectors," Annual Review of Nuclear and Particle Science, vol. 34, no. 1, pp. 285-350, 1984. eprint: https://doi.org/10.1146/annurev.ns.34.120184.001441.

[127] F. Sauli, "Principles of Operation of multiwire Proportional and Drift Chambers," 1977.

[128] G. Charpak and F. Sauli, "Multiwire proportional chambers and drift chambers," Nuclear Instruments and Methods, vol. 162, no. 1, pp. 405 -428, 1979.

[129] W. Leo, Techniques for Nuclear and Particle Physics Experiments, Second, Revised Edition. Springer-Verlag, 1994.

[130] T. Ferbel, Experimental Techniques in High-Energy Nuclear and Particle physics, Second Edition. World Scientific, 1991.

[131] S. Wang, J. Wu, S. Yao, and W. Chang, "A Field-Programmable Gate Array (FPGA) TDC for the Fermilab SeaQuest (E906) Experiment and its Test with a Novel External Wave Union Launcher," IEEE Transactions on Nuclear Science, vol. 61, no. 6, pp. 3592-3598, 2014.

[132] S. Miyasaka, "Probing Flavor Asymmetry of Anti-quarks in the Proton by DrellYan experiment SeaQuest," PhD thesis, Tokyo Institute of Technology, 2016.

[133] S.-H. Shiu, J. Wu, R. E. McClellan, T.-H. Chang, W.-C. Chang, Y.-C. Chen, R. Gilman, K. Nakano, J.-C. Peng, and S.-Y. Wang, "FPGA-based trigger system for the Fermilab SeaQuest experimentz," Nuclear Instruments and Methods in Physics Research Section A: Accelerators, Spectrometers, Detectors and Associated Equipment, vol. 802, pp. $82-88,2015$. 
[134] W. G. J., Description and Technical Information for Version 4 Trigger Supervisor (TS) Module, https : / / coda . jlab . org/drupal / system/files / pdfs/HardwareManual/TS/TS . pdf, [Online; accessed Feb 23, 2019].

[135] E. McClellan, "Angular Distributions of High-Mass Dilepton Production in Hadron Collisions," PhD thesis, University of Illinois at Urbana Champaign, 2016.

[136] CODA documentation website. https: / / coda. jlab.org/drupal/, [Online; accessed Feb 23, 2019].

[137] B. Ramson, "Parton Dynamics inferred from High-Mass Drell-Yan Dimuons Induced by $120 \mathrm{GeV}$ p+D Interactions," PhD thesis, University of Michigan, 2018.

[138] B. Dannowitz, "Nuclear Dependence of Proton-Induced Drell-Yan Dimuon production at $120 \mathrm{GeV}$ at SeaQuest," PhD thesis, University of Illinois at Urbana Champaign, 2016.

[139] K. Nagai, Hit Cluster Removal DOCID - 1123, [Online; accessed Mar 3, 2019].

[140] K. Liu, Step-by-step walkthrough of kTracker DOCID - 1283, [Online; accessed Mar 3, 2019].

[141] K. Nagai, "Recent Measurement of Flavor Asymmetry of Anti-quarks in the Proton by Drell-Yan experiment SeaQuest at Fermilab," PhD thesis, Tokyo Institute of Technology, 2017.

[142] R. Kalman, "A New Approach to Linear Filtering and Prediction Problems," Journal of Basic Engineering, vol. 82(1), pp. 35-45, 1960.

[143] S. Gorbunov and I. Kisel, CBM-SOFT-note-2006-001, [Online; accessed Mar 6, 2019].

[144] L. Landau, "On the energy loss of fast particles by ionization," J. Phys.(USSR), vol. 8, pp. 201-205, 1944.

[145] K. Nakano, Spill Selection on Roadsets 57-70 with R006-V003 DOCID - 1307, [Online; accessed Mar 18, 2019].

[146] A. Tadepalli and C. Brown, Beam y-offset for different roadsets DOCID - 2198 -v2, [Online; accessed Mar 7, 2019].

[147] A. Tadepalli, Beam offset for roadset 78, R007DOCID - 2216 - v1, [Online; accessed Mar 7, 2019]. 
[148] A. Tadepalli et al., Comparison of Monte Carlo productions with and without $Y$ offset DOCID - 2405 -v1, [Online; accessed Mar 7, 2019].

[149] _- Practical effect of wrong KMAG position and strength DOCID - 3059 -v6, [Online; accessed Mar 7, 2019].

[150] K. Nakano, Problem with Middle 1/3 of Roadset 67 DOCID - 1584 -v1, [Online; accessed Mar 7, 2019].

[151] P. Reimer, Hydrogen and Deuterium Analysis DOCID - 1893 - v2, [Online; accessed Mar 7, 2019].

[152] K. Nakano, Definition of ktrack.chisq target DOCID - 2339 - v1, [Online; accessed Mar 7, 2019].

[153] D. Geesaman, Handling the liquid target contamination and densities DOCID 4993-v1, [Online; accessed Apr 12, 2019].

[154] Y Chen, LD2 and LH2 Vent Pressure Analysis DOCID - 1453 - v3, [Online; accessed Mar 7, 2019].

[155] K. Nagai, Ktracker-Efficiency on Extrapolation Method DOCID - 4456 - v1, [Online; accessed Apr 12, 2019].

[156] K. Nakano, Logic to Select Fitting Function for Ratio-Extrapolation Method DOCID - 5053 - v2, [Online; accessed Apr 12, 2019].

[157] _ Estimates of systematic Error of Fitting Shape in Intensity Extrapolation DOCID - 4627, [Online; accessed Apr 12, 2019].

[158] _ Numerical Simulation to Estimate Cross-Section Ratio with Roadset-70 Statistics DOCID - 5348-v1, [Online; accessed Apr 12, 2019].

[159] A. Tadepalli et al., $J / \psi$ and $\psi$ ' tail contamination into the Drell-Yan mass region DOCID - 4555-v3, [Online; accessed Apr 12, 2019].

[160] K. Nakano, Fast Current Transformer (FCT) for Measurement of Absolute Beam Intensity DOCID - 1272-v1, [Online; accessed Apr 12, 2019].

[161] H.-L. Lai, M. Guzzi, J. Huston, Z. Li, P. M. Nadolsky, J. Pumplin, and C. P. Yuan, "New parton distributions for collider physics," Phys. Rev., vol. D82, p. 074024, 2010. arXiv: 1007.2241 [hep-ph].

[162] T.-J. Hou, S. Dulat, J. Gao, M. Guzzi, J. Huston, P. Nadolsky, C. Schmidt, J. Winter, K. Xie, and C. P. Yuan, "CT14 Intrinsic Charm Parton Distribution Functions from 
CTEQ-TEA Global Analysis," JHEP, vol. 02, p. 059, 2018. arXiv: 1707.00657 [hep-ph] .

[163] L. A. Harland-Lang, A. D. Martin, P. Motylinski, and R. S. Thorne, "Parton distributions in the LHC era: MMHT 2014 PDFs," Eur. Phys. J., vol. C75, no. 5, p. 204, 2015. arXiv: 1412.3989 [hep-ph] .

[164] C. Bourrely, personal communication, May 8, 2019.

[165] M. Alberg, personal communication, May 8, 2019.

[166] W.-C. Chang and J.-C. Peng, "Flavor structure of the nucleon sea," Progress in Particle and Nuclear Physics, vol. 79, pp. 95 -135, 2014.

[167] M. B. Johnson, B. Z. Kopeliovich, M. J. Leitch, P. L. McGaughey, J. M. Moss, I. K. Potashnikova, and I. Schmidt, "Nuclear broadening of transverse momentum in Drell-Yan reactions," Phys. Rev., vol. C75, p. 035 206, 2007. arXiv: hep-ph / 0606126 [hep-ph].

[168] M. J. Leitch et al., "Nuclear dependence of $\mathrm{j} / \psi$ production by $800 \mathrm{gev} / \mathrm{c}$ protons near $x_{F}=0$," Phys. Rev. D, vol. 52, pp. 4251-4253, 71995.

[169] - "Measurement of Differences between $J / \psi$ and $\psi$ ' Suppression in $p-A$ Collisions," Phys. Rev. Lett., vol. 84, pp. 3256-3260, 152000.

[170] S. Gavin and J. Milana, "Energy loss at large $x_{f}$ in nuclear collisions," Phys. Rev. Lett., vol. 68, pp. 1834-1837, 121992.

[171] S. J. Brodsky and P. Hoyer, “A Bound on the energy loss of partons in nuclei,” Phys. Lett., vol. B298, pp. 165-170, 1993. arXiv: hep-ph/9210262 [hep-ph] .

[172] R. Baier, Y. L. Dokshitzer, A. H. Mueller, S. Peigne, and D. Schiff, "Radiative energy loss and $\mathrm{p}(\mathrm{T})$ broadening of high-energy partons in nuclei," Nucl. Phys., vol. B484, pp. 265-282, 1997. arXiv: hep-ph/9608322 [hep-ph].

[173] R. Baier, Y. L. Dokshitzer, A. H. Mueller, and D. Schiff, "Medium induced radiative energy loss: Equivalence between the BDMPS and Zakharov formalisms," Nucl. Phys., vol. B531, pp. 403-425, 1998. arXiv: hep-ph/9804212 [hep-ph] .

[174] R. B. Neufeld, I. Vitev, and B.-W. Zhang, "A possible determination of the quark radiation length in cold nuclear matter," Phys. Lett., vol. B704, pp. 590-595, 2011. arXiv: 1010.3708 [hep-ph].

[175] M. B. Johnson et al., "Energy Loss of Fast Quarks in Nuclei," Phys. Rev. Lett., vol. 86, pp. 4483-4487, 202001. 
[176] G. T. Garvey and J. C. Peng, "Partonic Energy Loss and the Drell-Yan Process," Phys. Rev. Lett., vol. 90, p. 092 302, 92003.

[177] SeaQuest New St.3- DC documentation website. https: / / sites . google. $\mathrm{com} / \mathrm{site} / \mathrm{seaquestnewdc/home,} \mathrm{[Online;} \mathrm{accessed} \mathrm{Feb} \mathrm{26,} \mathrm{2019].}$

[178] A. Basham, Creation Myths, https://www. creationmyths.org/rigveda10-129-indian-creation/rigveda-10-129-indian-creation6-basham-translation.htm, [Online; accessed Dec 26, 2018 ].

[179] G. Bertone and D. Hooper, "History of dark matter," Rev. Mod. Phys., vol. 90, p. $045002,42018$.

[180] L. Randall, Dark Matter and the Dinosaurs. HarperCollins Publishers, 2015.

[181] F. Zwicky, "On the Masses of Nebulae and of Clusters of Nebulae," APJ, vol. 86, p. 217, Oct. 1937.

[182] V. C. Rubin, N. Thonnard, and W. K. Ford Jr., "Rotational properties of 21 SC galaxies with a large range of luminosities and radii, from NGC $4605 / \mathrm{R}=4 \mathrm{kpc} /$ to UGC 2885 /R = 122 kpc/,' APJ, vol. 238, p. 471, 1980.

[183] Wikipedia, Rotational Curves of spiral galaxy M33, https : / / en.wikipedia. org/wiki/Galaxy_rotation_curve, [Online; accessed Dec 26, 2018].

[184] NASA, A. Fruchter and the ERO (STSCI), STSCI-PRC00-08, http: / / imgsrc . hubblesite.org/hvi/uploads/image_file/image_attachment/ $6060 /$ pdf .pdf, [Online; accessed Dec 26, 2018].

[185] D.-C. Dai, R. Matsuo, and G. Starkman, "Gravitational lenses in generalized Einsteinaether theory: The bullet cluster," Phys. Rev. D, vol. 78, p. 104 004, 102008.

[186] M. Milgrom, "A modification of the Newtonian dynamics as a possible alternative to the hidden mass hypothesis," Apj, vol. 270, pp. 365-370, Jul. 1983.

[187] M. Milgrom, "Solutions for the modified Newtonian dynamics field equation," Apj, vol. 302, pp. 617-625, Mar. 1986.

[188] B. Griswold., Wilkinson Microwave Anisotropy Probe. NASA, https : / / map . gsfc.nasa.gov/media/121238/index.html, [Online; accessed Dec $26,2018]$.

[189] D. Larson, J. Dunkley, G. Hinshaw, E. Komatsu, M. R. Nolta, C. L. Bennett, B. Gold, M. Halpern, R. S. Hill, N. Jarosik, A. Kogut, M. Limon, S. S. Meyer, N. Odegard, L. Page, K. M. Smith, D. N. Spergel, G. S. Tucker, J. L. Weiland, E. Wollack, 
and E. L. Wright, "Seven-year Wilkinson Microwave Anisotropy Probe (WMAP) Observations: Power Spectra and WMAP-derived Parameters," The Astrophysical Journal Supplement Series, vol. 192, no. 2, p. 16, 2011.

[190] M. Aguilar et al., "Electron and Positron Fluxes in Primary Cosmic Rays Measured with the Alpha Magnetic Spectrometer on the International Space Station," Phys. Rev. Lett., vol. 113, p. 121 102, 122014.

[191] M. S. Turner and F. Wilczek, "Positron line radiation as a signature of particle dark matter in the halo," Phys. Rev. D, vol. 42, pp. 1001-1007, 41990.

[192] B. Fields, S. Shapiro, and J. Shelton, "Galactic center gamma-ray excess from dark matter annihilation: Is there a black hole spike?" Physical Review Letters, vol. 113, no. 15, Oct. 2014.

[193] P. Agrawal, B. Batell, D. Hooper, and T. Lin, "Flavored dark matter and the Galactic Center gamma-ray excess," Phys. Rev. D, vol. 90, p. 063 512, 62014.

[194] T. Daylan, D. P. Finkbeiner, D. Hooper, T. Linden, S. K. Portillo, N. L. Rodd, and T. R. Slatyer, "The characterization of the gamma-ray signal from the central Milky Way: A case for annihilating dark matter," Physics of the Dark Universe, vol. 12, pp. $1-23,2016$.

[195] D. Hooper and T. Linden, "Origin of the gamma rays from the Galactic Center," Phys. Rev. D, vol. 84, p. 123 005, 122011.

[196] J. L. Feng, B. Fornal, I. Galon, S. Gardner, J. Smolinsky, T. M. P. Tait, and P. Tanedo, "Protophobic Fifth-Force Interpretation of the Observed Anomaly in $8 \mathrm{Be}$ Nuclear Transitions," Phys. Rev. Lett., vol. 117, p. 071 803, 72016.

[197] B. Holdom, “Two U(1)'s and eps charge shifts," Physics Letters B, vol. 166, no. 2, pp. $196-198,1986$.

[198] S. Gardner, R. J. Holt, and A. S. Tadepalli, "New Prospects in Fixed Target Searches for Dark Forces with the Seaquest Experiment at Fermilab," Phys. Rev., vol. D93, no. 11, p. 115 015, 2016. arXiv: 1509.00050 [hep-ph] .

[199] J. D. Bjorken, R. Essig, P. Schuster, and N. Toro, "New fixed-target experiments to search for dark gauge forces," Phys. Rev. D, vol. 80, p. 075 018, 72009. 